STATISTICAL SUMMARIES OF STREAMFLOW DATA IN OREGON VOLUME 1. EASTERN OREGON

By John Friday and Suzanne J. Miller

U.S. GEOLOGICAL SURVEY

OPEN-FILE REPORT $84-454$

Prepared in Cooperation with

Oregon Water Resources Department

Portland, Oregon

1984 


\author{
IJNITED STATES DEPARTMENT OF THE INTERIDR \\ WILLIAM P. CLARK, Secretary \\ GEOLOGICAL SURVEY \\ Dallas L. Peck, Director
}

Cover photo: Thielsen Creek near Diamond Lake

For additional information

write to:

District Chief

U.S. Geological Survey

847 N.E. 19th Ave., Suite 300

Portland, Oregon 97232
Copies of this report can be purchased from:

Open-File Services Section Western Distribution Branch Box 25425, Federal Center Denver, Colorado 80225

(Telephone: (303) 776-7476) 


\section{CONTENTS}

Page

Abstract-1- 1

Introduction-1. 1

Streamflow records- 2

Statistical summaries-- 2

Monthly and annual mean discharges- 2

Magnitude and probability of annual low flows-_...- 3

Magnitude and probability of annual high flows- 3

Magnitude and probability of instantaneous peak flows---.-- 3

Duration table of daily mean flows- 4

References-- 5

List of gaging stations, in downstream order-a 7

Gaging-station descriptions and statistical tables-a...... 11

Index-0.- 147

ILLUSTRATIONS

[Plate is in pocket]

Plate 1. Map showing location of streamflow gaging stations in eastern Oregon.

\section{METRIC CONVERSION TABLE}

To convert inch-pound units [in this report] to metric units, multiply by the following factors:

\begin{tabular}{|c|c|c|}
\hline Multiply inch-pound unit & By & To obtain metric unit \\
\hline acre & 0.4047 & hectometer $(\mathrm{hm})$ \\
\hline acre-foot per year (acre-ft/yr) & 0.001233 & $\begin{array}{l}\text { cubic hectometers per year } \\
\left(\mathrm{hm}^{3} / \mathrm{yr}\right)\end{array}$ \\
\hline $\begin{array}{l}\text { cubic foot per second }\left(f t^{3} / \mathrm{s}\right. \\
\text { and CFS) }\end{array}$ & 0.02832 & $\begin{array}{l}\text { cubic meter per second } \\
\left(\mathrm{m}^{3} / \mathrm{s}\right)\end{array}$ \\
\hline foot $(f t)$ & 0.3048 & meter $(m)$ \\
\hline inch per year (in/yr) & 25.40 & millimeter per year $(\mathrm{mm} / \mathrm{yr})$ \\
\hline mile $(\mathrm{mi})$ & 1.609 & kilometer $(\mathrm{km})$ \\
\hline square mile $\left(m i^{2}\right)$ & 2.590 & square kilometer $\left(\mathrm{km}^{2}\right)$ \\
\hline
\end{tabular}




\title{
STATISTICAL SUMMARIES OF STREAMFLOW DATA IN OREGON
}

\author{
VOLUME 1. EASTERN OREGON
}

By John Friday and Suzanne J. Miller

\begin{abstract}
Statistical summaries of streamflow data at 123 stream-gaging sites are presented in this report to aid in appraising the hydrology of river basins in Eastern Oregon. Records for 13 gaging stations were compiled into separate periods owing to changes in regulation during the period of data collection. The periods before and after regulation are presented for comparison.

A brief station description is given describing the physical and operational features for each gaging station. Following the station description are tables of monthly and annual flow statistics, flood frequency data, low-flow and high-flow frequency data, and flow-duration information.
\end{abstract}

\section{INTRODUCTION}

This is the first of two volumes of statistical summaries of streamflow data collected at gaging stations in Oregon. Volume 1 contains data from 123 gaging stations located in the area east of the divide of the Cascade Range. Volume 2 will cover the remainder of the State. It is anticipated these reports will be updated about every five years.

This report was prepared in cooperation with the Oregon Water Resouces Department (OWRD). The purpose is to provide water-resource managers with a knowledge of streamflow characteristics based on historical data. For the purpose of this report, gaging-station records had to satisfy one of two criteria. The data had to be hydrologically transferable (for use in estimating streamflow in ungaged basins), or it had to be a significant indicator of flow availability. For example, a gage at the outlet of a natural lake would have little hydrologic transferability (due to storage of water in the lake), but the data would be a good indication of low-flow availability as well as flood events.

Stations on canals, reservoirs, or combined-flow stations, were not used in this report. Data for combined-flow stations include diverted flow which may or may not reenter the stream downstream from the gage. A map showing the location of gaging stations used in this report is enclosed in a packet in the back of the book. 


\section{STREAMFLOW RECORDS}

Both active and discontinued gaging stations having a minimum of 10 years of daily-mean discharge values were used in this report. For active stations, the period of record extends to the end of the 1982 water year (ending on September 30). A brief station description showing the physical and operational features of the gaging station precedes the statistical summaries for each station. Except for paragraphs describing revised records and extremes for a specific year, the station descriptions are identical to the last-published version in the annual release "Water Resources Data - Oregon" (U.S. Geological Survey, 1983). A detailed explanation of the manuscript data and a definition of terms is given in that report.

Sometimes the natural flow of a stream may be altered by the construction of a dam, an irrigation diversion, or by the augmentation of flow by transbasin diversions. If this occurs during the operation of a gaging station, the statistical summaries must reflect both natural and altered states of flow, providing there are at least 10 years of data for each state. There are 13 such stations in this report.

\section{STATISTICAL SUMMARIES}

The tables of statistical data include monthly and annual mean discharges, magnitude and probability of annual low flows, magnitude and probability of annual high flows, magnitude and probability of instantaneous peak flows, and a duration table of daily mean flows. The statistics were generated by using computer programs available from the U.S. Geological Survey's "National Water Data Storage and Retrieval System" (WATSTORE).

\section{Monthly and Annual Mean Discharges}

This statistical summary is from computer program $W 4422$ by Price and Meeks (1977). The period of record shown in the heading is the first and last water year for which daily-mean discharge values are available, and does not necessarily indicate a continuous period of record (see the PERIOD OF RECORD paragraph in the station description for fragmentary records).

The standard deviation shown in the table is a measure of the variability of flows occurring during a given month during the period of record. The value represents the spread in discharges (from the mean value) that could be expected to occur two-thirds of the time based on the distribution of data during the sampling period. The coefficient of variation is the ratio of the standard deviation to the mean.

The annual mean shown in the table will sometimes vary from the average discharge shown in the manuscript because the annual mean is based only on years for which daily-mean discharge values are available, while the average discharge includes years having estimated monthly values. 


\section{Magn itude and Probability of Annual Low Flows}

This summary is from computer program A969 by Meeks (1977). The computation period is based on a climatic year which ends on March 31 thus assuring the low-flow season will be complete within a twelve-month period. The recurrence interval was generally extended to twice the period of record for stations having less than 40 years of record. If 40 or more years of record were available, the recurrence interval was extended to 100 years (a flow having a 1 percent chance of not being exceeded in any given year).

The table shows the amount of flow that probably would not be exceeded, during specified periods, for the indicated recurrence intervals. The recurrence intervals are also shown as nonexceedance probabilities which are expressed as a percent chance of the event not being exceeded in any given year. For example, the low flow during a 30-day period might not be exceeded on the average of once every 50 years, and would have a 2 percent chance of not being exceeded in any given year.

\section{Magnitude and Probability of Annual High Flows}

This summary is also from computer program A969 (Meeks, 1977). The period of record is based on water years having daily-mean discharge data. The recurrence intervals are extended in the same manner as the low-flow frequency. The table shows maximum flows that could be equalled or exceeded, during specified periods, for the indicated recurrence intervals. Occasionally, the values shown for a 1-day period will exceed the instantaneous peaks shown for the same recurrence intervals in the adjacent table. The reason for this is that different periods of record are involved. Quite often peak data are documented outside the period of systematic data collection.

\section{Magnitude and Probability of Instantaneous Peak Flow}

This summary is from computer program J407 by Kirby (1981). The period of record shows the first and last water years for which annual peak flows were documented. The table shows the magnitude of instantaneous peak flows for selected recurrence intervals as computed from a log-Pearson Type 111 probability distribution of gaging-station data. For stations having less than 25 years of record, the frequency curve was based on a generalized skew coefficient taken from the U.S. Water Resources Council Bulletin 17B (1981). For stations having more than 25 years of data, the skew coefficient was determined by weighing the generalized skew against the actual station skew as recommended by the council. The skew used for a particular station is shown at the bottom of the table.

Flood-frequency data were not determined at gaging stations immediately downstream from dams and reservoirs. At stations where low-flow statistics are divided into two periods due to the construction of a bypass structure, the flood-frequency data are computed for the entire period of record. 


\section{Duration Table of Daily Mean Flow}

This summary is from computer program A969 by Meeks (1977). The period of record indicates the first and last water year where daily-mean values of discharge were available for analysis. The table shows the amount of flow that was equalled or exceeded for the indicated percent of the time. 


\section{REFERENCES}

Kirby, William, 1981, Annual flood frequency analysis using U.S. Water Resources Council guidelines (Program J407), chapter 1, section C of WATSTORE user's guide: U.S. Geological Survey Open-File Report $76-435, v .4, \mathrm{p} . \mathrm{C}-1$ to $\mathrm{C}-57$.

Meeks, W. C., 1977, Daily values statistics (Program A969), chapter IV, section G, of WATSTORE user's guide: U.S. Geological Survey Open-File Report 75-426, v. 1, p. G-1 to G-37.

Price, W. E., Jr., and Meeks W. C., 1977, Daily values monthly and annual statistics (Program W4422), chapter IV, section F, of WATSTORE user's guide: U.S. Geological Survey Open-File Report $75-426, v .1, p . F-1$ to $F-46$.

U.S. Geological Survey, 1983, Water resources data, Oregon, water year 1982, volume 1. Eastern Oregon; Portland, Oregon, U.S. Geological Survey Water-Data Report OR-82-1, $206 \mathrm{p}$.

U.S. Water Resources Council, 1981, Guidelines for determining flood flow frequency [revised]: Hydrology Committee Bulletin 17B, 28 p. 
The Great Basin

Warner Lakes Basin

Twentymile Creek (head of Warner Lakes Basin)

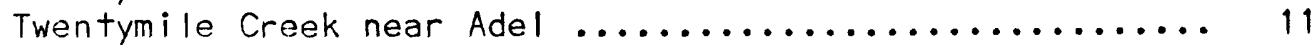

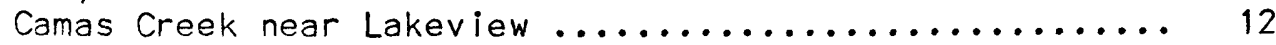

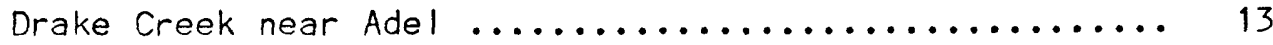

Deep Creek above Adel .......................... 14

Hart Lake

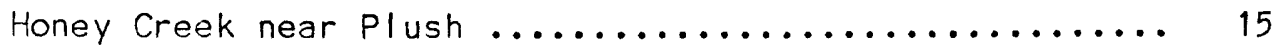

Abert Lake Basin

Chewaucan River near Paisley ............................. 16

Malheur and Harney Lakes Basin

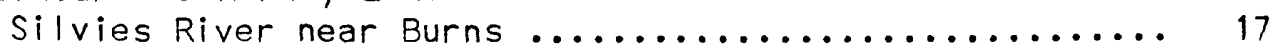

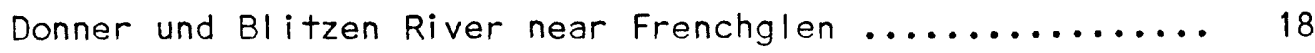

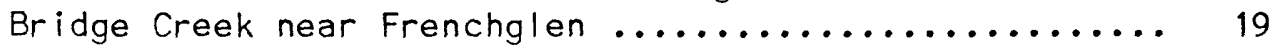

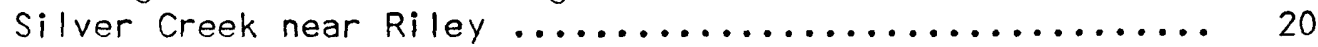

Alvord Lake Basin

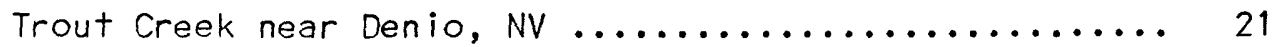

Goose Lake Basin (closed basin)

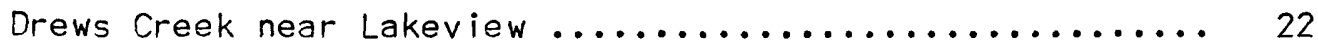

Cottonwood Creek near Lakeview .................... 23

Pacific Slope Basins in Oregon-California

Klamath River Basin

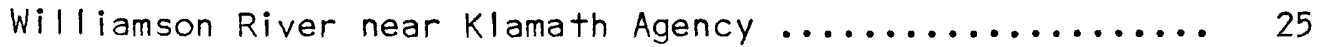

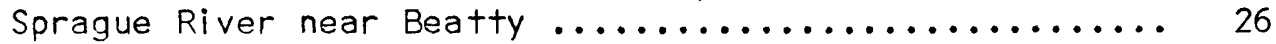

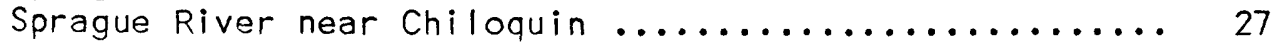

Williamson River below Sprague River, near Chiloquin ..... 28

Upper Klamath Lake (continuation of Williamson River)

Wood River:

Wood River at Fort Klamath ..................... 29

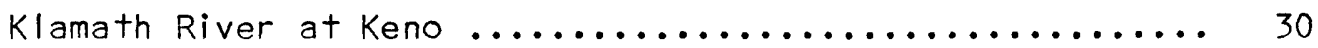

Klamath River below John C. Boyle powerplant, near Keno .. 31 Jenny Creek:

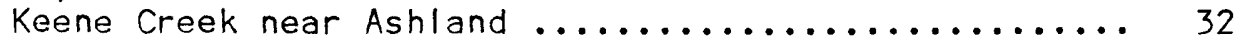

Upper Columbia River Basin

Snake River Basin

Owyhee River Basin

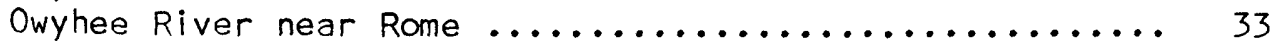

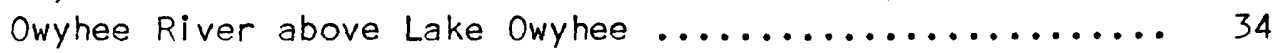

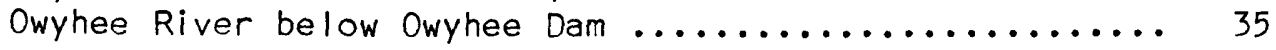

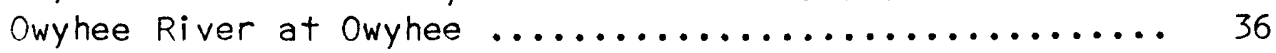

Malheur River Basin

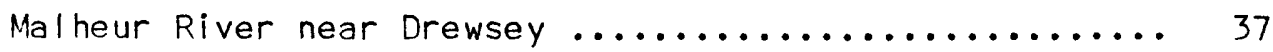

Malheur River below Warmsprings Reservoir, near

Riverside ........................... 38 
PAGE

Upper Columbia River Basin--Continued

Snake River Basin--Continued

Mal heur River Basin--Continued

North Fork Mal heur River above Beulah Reservoir,

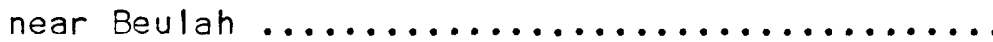

North Fork Malheur River at Beulah .............. 40

Mal heur River at Little Valley, near Hope ........... 41

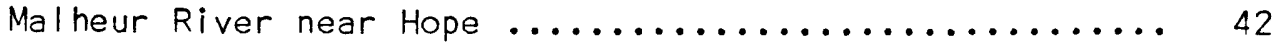

Bully Creek at Warmsprings, near Vale ............ 43

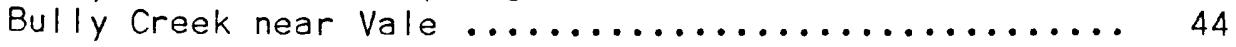

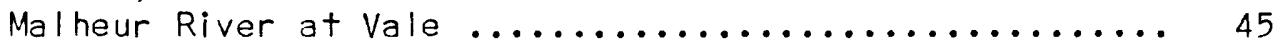

Burnt River Basin

North Fork Burnt River near Whitney .............. 46

South Fork Burnt River above Barney Creek, near Unity. 47

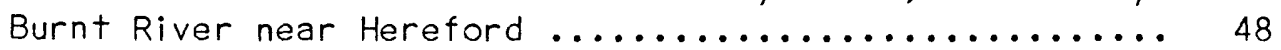

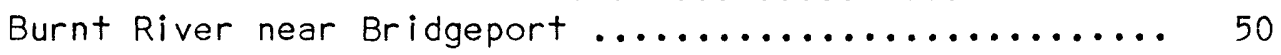

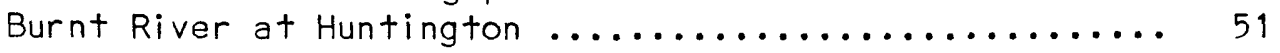

Powder River Basin

Powder River near Sumpter ................... 52

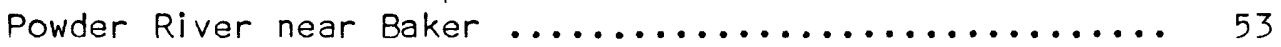

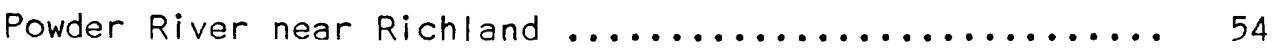

Eagle Creek above Skull Creek, near New Bridge ...... 56

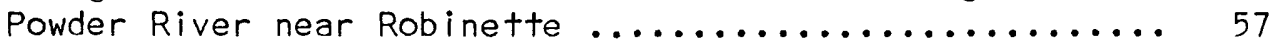

Imnaha River Basin

Imnaha River at Imnaha ....................... 58

Grande Ronde River Basin

Grande Ronde River near Hilgard ................... 59

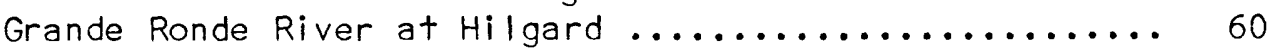

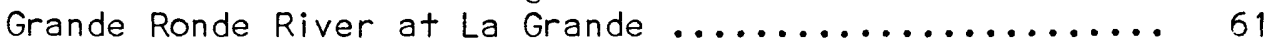

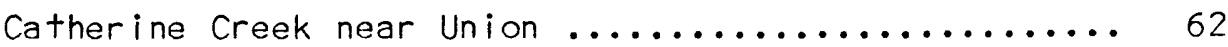

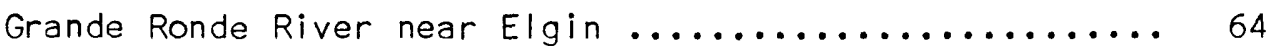

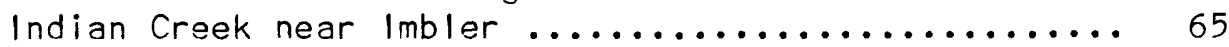

Wall lowa River

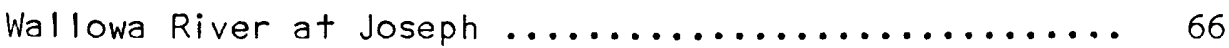

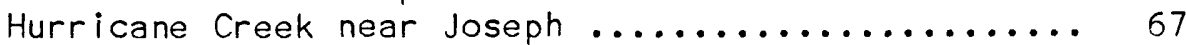

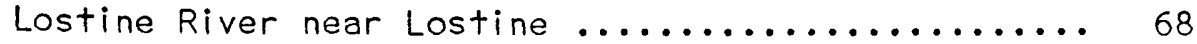

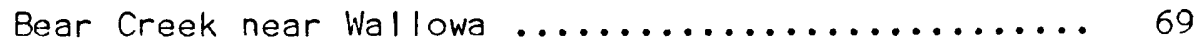

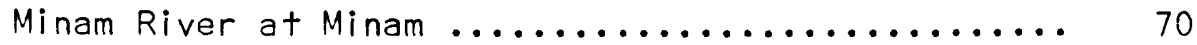

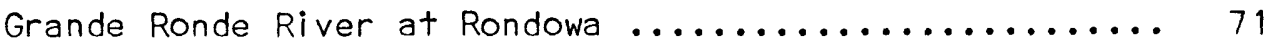

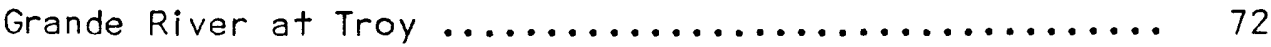


Lower Columbia River Basin

Columbia River

Walla Walla River Basin

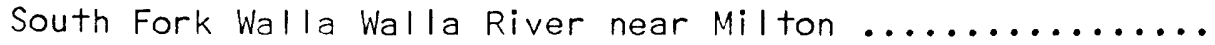

South Fork Walla Walla River below Pacific Power and Light

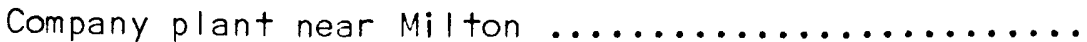

North Fork Walla Walla River near Milton-Freewater ..... 75

North Fork Walla Walla River near Milton ............ 76

Umatilla River Basin

Umatilla River above Meacham Creek, near Gibbon ........ 77

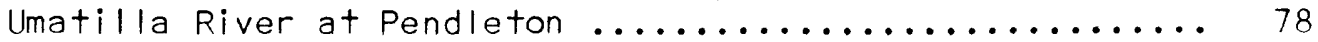

Umatilla River above Mckay Creek near Pendleton ......... 79

Mckay Creek

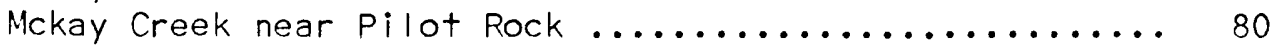

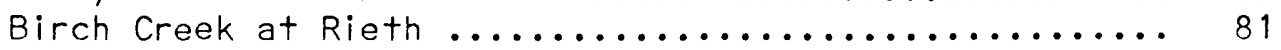

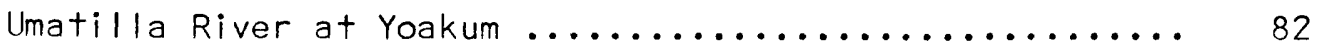

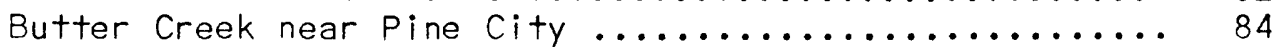

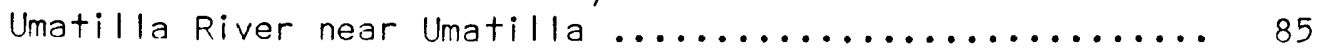

Willow Creek Basin

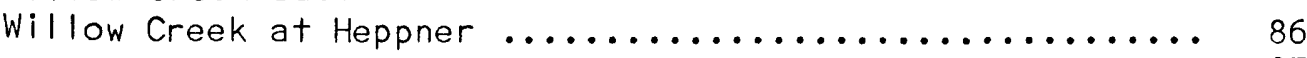

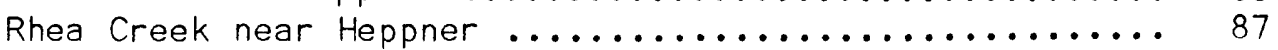

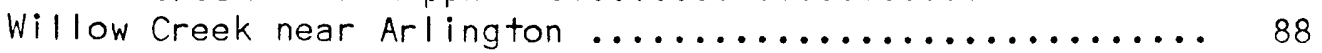

John Day River Basin

John Day River

Strawberry Creek above Slide Creek, near Prairie City .. 89

John Day River at Prairie City .................. 90

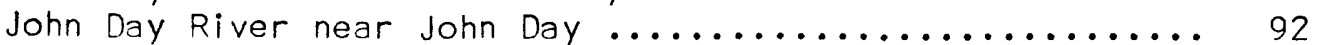

John Day River at Picture Gorge, near Dayville .......... 93

North Fork John Day River

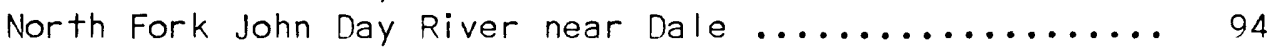

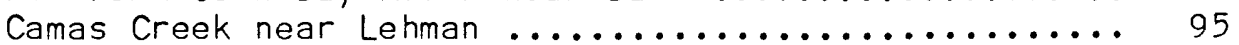

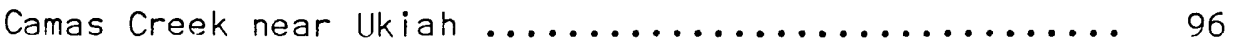

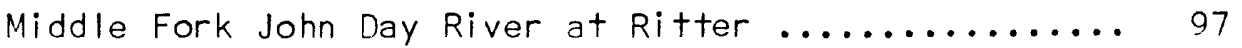

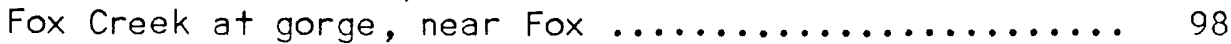

North Fork John Day River at Monument .............. 99

John Day River at Service Creek ...................... 100

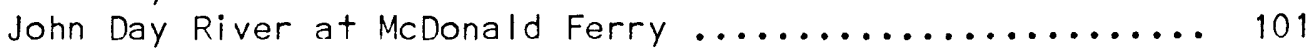

Deschutes River Basin

Deschutes River below Snow Creek, near La Pine ......... 102

Cultus River above Cultus Creek, near La Pine ......... 103

Cultus Creek above Crane Prairie Reservoir, near

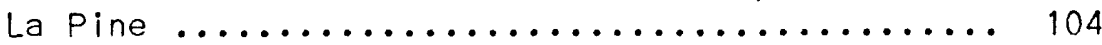

Deer Creek above Crane Prairie Reservoir, near La Pine 106

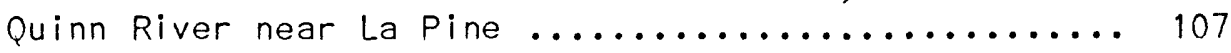

Deschutes River below Crane Prairie Reservoir, near

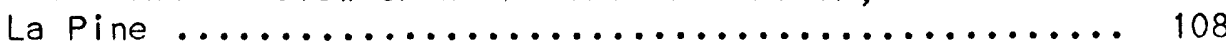

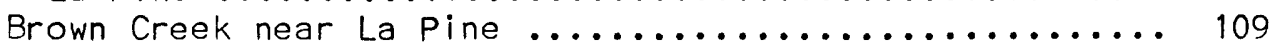

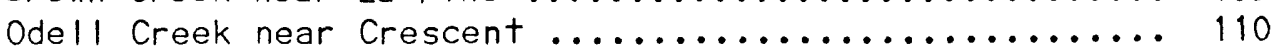

Deschutes River below Wickiup Reservoir, near La Pine .... 111 
PAGE

Lower Columbia River Basin--Continued

Deschutes River Basin--Continued

Deschutes River at Pringle Falls, near La Pine ......... 112

Fall River near La Pine ........................ 114

Little Deschutes River

Crescent Creek at Crescent Lake, near Crescent ...... 115

Big Marsh Creek at Hoey Ranch, near Crescent ....... 116

Little Deschutes River near La Pine ................ 117

Deschutes River at Benham Falls, near Bend ............. 118

Deschutes River below Lava Island, near Bend .......... 120

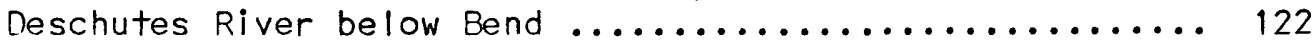

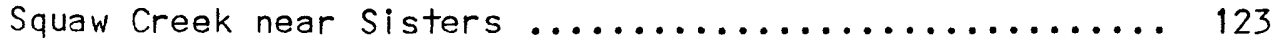

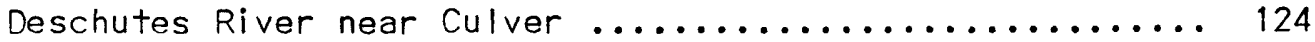

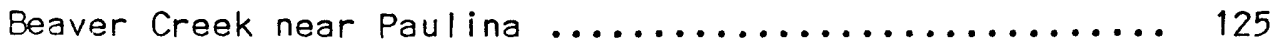

Crooked River

North Fork Crooked River above Deep Creek .......... 126

Crooked River near Post ...................... 127

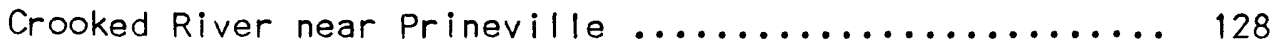

Crooked River below Opal Springs, near Culver ........ 130

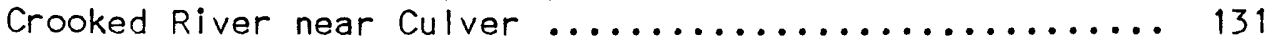

Metolius River

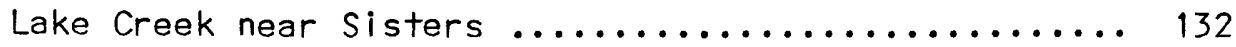

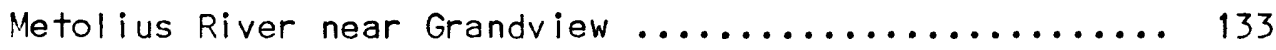

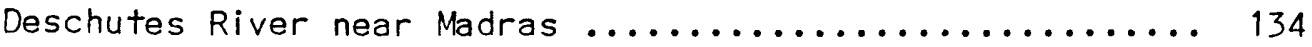

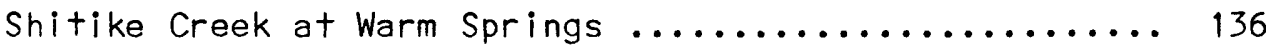

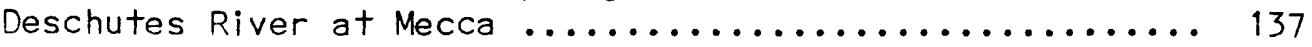

Warm Springs River near Kahneeta Hot Springs ......... 138

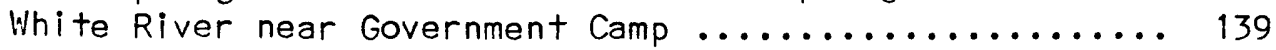

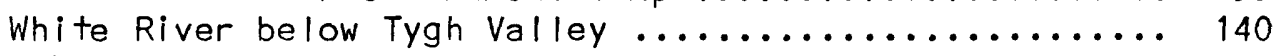

Deschutes River at Moody, near Biggs ................ 141

Mosier Creek Basin

Mosier Creek near Mosier ....................... 143

Hood River Basin

Dog River near Parkdale ....................... 144

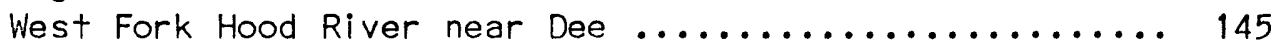

Hood River at Tucker Bridge, near Hood River .......... 146 
WARNER LAKES BASIN

10366000 TWENTYMILE CREEK NEAR ADEL, OR

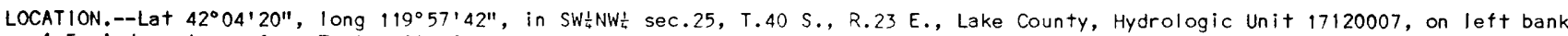
$1.5 \mathrm{mi}$ downstream from Twelvemile Creek and $8 \mathrm{mi}$ southwest of Adel.

DRAINAGE AREA.--194 $\mathrm{mi}^{2}$, including $46 \mathrm{mi}^{2}$ in Cowhead Loke ares.

PERIOD OF RECORD.-- March 1910 to July 1915, December 1917 to September 1919, and "larch 1921 to June 1922 (published as "near Warner Lake"), September 1940 to November 1944, March 1945 to september 1982.

GAGE.--Water-stage recorder and concrete control. Datum of gage is 4,560.83 ft National Geodetic Vertical Datum of 1929 . Prior to Sept. 21, 1940, nonrecording gage or water-stage recorder at sites within 1 mi downstream at various datums. Sept. 21 , 1940 , to Nov. 30, 1944, water-stage recorder at site $1.8 \mathrm{mi}$ upstream at different datums. Mar. 12, 1945, to June 28 , 1952, water-stage recorder at site $70 \mathrm{ft}$ upstream at datum $0.88 \mathrm{ft}$ higher.

REMARKS.--Some regulation by pumpage from Cowhead Lake. Diversions in Oregon for irrigation above station; considerable diversions for irrigation in Cowhead Lake area in California.

AVERAGE DISCHARGE. --47 years (water years $1911-15,1919,1941-44,1946-82), 51.9+t^{3} / \mathrm{s}, 37,600 \mathrm{acre}-t+\mathrm{yr}$.

EXTREMES FOR PERIOD OF RECORD.--Maximum discharge, 3,670 $\mathrm{ft} \mathrm{t}^{3} \mathrm{~s} \mathrm{Dec.} 23$, 1964, gage height, $16.1 \mathrm{ft}$, trom rating curve extended above $920 \mathrm{ft}^{3} / \mathrm{s}$ on basis of contracted-opening measurement of $3,260 \mathrm{ft}^{3} / \mathrm{s}$; no flow at times.

STATISTICAL SUMMARIES

MONTHLY AND ANNUAL MEAN DISCHARGES 1911-82

\begin{tabular}{|c|c|c|c|c|c|c|}
\hline MONTH & $\begin{array}{c}\text { MINIMUM } \\
\text { (CFS) }\end{array}$ & $\begin{array}{l}\text { MAXIMUM } \\
\text { (CFS) }\end{array}$ & $\begin{array}{l}\text { MEAN } \\
\text { (CFS) }\end{array}$ & $\begin{array}{l}\text { STAN- } \\
\text { DARD } \\
\text { DEVIA- } \\
\text { TION } \\
\text { (CFS) }\end{array}$ & $\begin{array}{l}\text { COEFFI- } \\
\text { CIENT OF } \\
\text { VARI- } \\
\text { ATION }\end{array}$ & $\begin{array}{c}\text { PERCENT } \\
\text { OF } \\
\text { ANNUAL } \\
\text { RUNOFF }\end{array}$ \\
\hline OCTOBER & 2.4 & $12 i$ & 6.8 & 17 & 2.42 & 1.1 \\
\hline $\begin{array}{l}\text { NOVEMBER } \\
\text { DECEMBER }\end{array}$ & $\begin{array}{l}3.8 \\
3.1\end{array}$ & $\begin{array}{r}65 \\
349\end{array}$ & 32 & $\begin{array}{l}10 \\
62\end{array}$ & $\begin{array}{l}1.14 \\
1.93\end{array}$ & $\begin{array}{l}1.5 \\
5.3\end{array}$ \\
\hline $\begin{array}{l}\text { JANUARY } \\
\text { FEBRUARY }\end{array}$ & $\begin{array}{l}2.8 \\
3.3\end{array}$ & $\begin{array}{l}330 \\
383\end{array}$ & $\begin{array}{l}52 \\
73\end{array}$ & $\begin{array}{l}82 \\
92\end{array}$ & $\begin{array}{l}1.58 \\
1.26\end{array}$ & $\begin{array}{r}8.5 \\
12.0\end{array}$ \\
\hline $\begin{array}{l}\text { MARCH } \\
\text { APRIL }\end{array}$ & $\begin{array}{l}5.9 \\
5.9\end{array}$ & $\begin{array}{l}578 \\
879\end{array}$ & $\begin{array}{l}134 \\
133\end{array}$ & $\begin{array}{l}117 \\
144\end{array}$ & $\begin{array}{r}.87 \\
1.08\end{array}$ & $\begin{array}{l}22.0 \\
21.8\end{array}$ \\
\hline $\begin{array}{l}\text { MAY } \\
\text { JUNE }\end{array}$ & $\begin{array}{l}9.4 \\
4.2\end{array}$ & $\begin{array}{l}283 \\
261\end{array}$ & $\begin{array}{l}97 \\
56\end{array}$ & $\begin{array}{l}53 \\
44\end{array}$ & $\begin{array}{l}.54 \\
.78\end{array}$ & $\begin{array}{r}15.9 \\
9.3\end{array}$ \\
\hline $\begin{array}{l}\text { JULY } \\
\text { AUGUST }\end{array}$ & $\begin{array}{l}1.5 \\
1.2\end{array}$ & $\begin{array}{l}34 \\
8.4\end{array}$ & $\begin{array}{l}9.6 \\
3.4\end{array}$ & $\begin{array}{l}7.4 \\
1.5\end{array}$ & $\begin{array}{l}.77 \\
.43\end{array}$ & $\begin{array}{l}1.6 \\
.6\end{array}$ \\
\hline SEPTEMBER & 2.1 & 6.3 & 3.5 & 1.0 & .29 & .6 \\
\hline ANNUAL & 4.2 & 125 & 52 & 30 & .58 & 100 \\
\hline
\end{tabular}

MAGNITUDE AND PROBABILITY OF INSTANTANEOUS PEAK FLOW BASED ON PERIOD OF RECORD 1911-82

DISCHARGE, IN CFS, FOR INDICATED RECURRENCE INTERVAL, IN YEARS, AND ANNUAL EXCEEDANCE PROBABILITY, IN PERCENT

\begin{tabular}{ccccccc}
1.25 & 2 & 5 & 10 & 25 & 50 & 100 \\
$80 \%$ & $50 \%$ & $20 \%$ & $10 \%$ & $4 \%$ & $2 \%$ & $1 \%$ \\
\hline 716 & 1340 & 2310 & 2970 & 3800 & 4390 & 4960
\end{tabular}

WEIGHTED SKEW $=-.508$
MAGNITUDE AND PROBABILITY OF ANNUAL LOW FLOW BASED ON PERIOD OF RECORD $1912-82$

\begin{tabular}{|c|c|c|c|c|c|c|}
\hline $\begin{array}{l}\text { PERIOD } \\
\text { (CON- }\end{array}$ & \multirow{2}{*}{\multicolumn{6}{|c|}{$\begin{array}{l}\text { DISCHARGE, IN CFS, FOR INDICATED RECURRENCE } \\
\text { INTERVAL, IN YEARS, AND ANNUAL NON- } \\
\text { EXCEEDANCE PROBABILITY, IN PERCENT }\end{array}$}} \\
\hline SECU- & & & & & & \\
\hline TIVE & 2 & 5 & 10 & 20 & 50 & 100 \\
\hline DAYSI & $50 \%$ & $20 \%$ & $10 \%$ & $5 \%$ & $2 \%$ & $1 \%$ \\
\hline 1 & 2.1 & 1.2 & .8 & . & . & \\
\hline 3 & 2.2 & 1.3 & 1.0 & .8 & . & \\
\hline 7 & 2.3 & 1.5 & 1.2 & . & . & \\
\hline 14 & 2.5 & 1.7 & 1.4 & 1. & . & \\
\hline 30 & 2.8 & 2.0 & 1.6 & 1. & 1. & \\
\hline 60 & 3.0 & 2.3 & 2.0 & 1. & 1. & \\
\hline 90 & 3.3 & 2.6 & 2.3 & 2. & 1. & \\
\hline 120 & 3.8 & 3.0 & 2.6 & 2. & 2. & \\
\hline 183 & 5.0 & 3.8 & 3.5 & 3.3 & 3.2 & \\
\hline
\end{tabular}

MAGN ITUDE AND PROBABILITY OF ANNUAL HIGH FLOW BASED ON PERIOD OF RECORD 1911-82

\begin{tabular}{ccccccc} 
PERIOD & \multicolumn{3}{c}{$\begin{array}{c}\text { DISCHARGE, IN CFS, FOR INDICATED RECURRENCE } \\
\text { INTERVAL, IN YEARS, AND ANNUAL } \\
\text { (CON- }\end{array}$} \\
$\begin{array}{c}\text { EXCEEDANCE PROBABILITY, IN PERCENT } \\
\text { SECU- }\end{array}$ TIVE & 2 & 5 & 10 & 25 & 50 & 100 \\
DAYS) & $50 \%$ & $20 \%$ & $10 \%$ & $4 \%$ & $2 \%$ & $1 \%$ \\
\hline & & & & & & \\
1 & 913 & 1650 & 2080 & 2510 & 2770 & 2980 \\
3 & 649 & 1240 & 1590 & 1970 & 2190 & 2380 \\
7 & 455 & 873 & 1130 & 1420 & 1600 & 1750 \\
15 & 318 & 594 & 762 & 943 & 1050 & 1150 \\
30 & 228 & 411 & 516 & 626 & 690 & 743 \\
60 & 165 & 281 & 343 & 404 & 438 & 465 \\
90 & 141 & 227 & 269 & 307 & 328 & 343 \\
\hline
\end{tabular}

DURATION TABLE OF DAILY MEAN FLOW FOR PERIOD OF RECORD $1911-82$

DISCHARGE, IN CFS, WHICH WAS EQUALED OR EXCEEDED FOR INDICATED PERCENT OF TIME

$\begin{array}{rllllllllllllll}5 \% & 10 \% & 15 \% & 20 \% & 25 \% & 30 \% & 40 \% & 50 \% & 60 \% & 70 \% & 75 \% & 80 \% & 85 \% & 90 \% & 95 \% \\ 205 & 125 & 89 & 67 & 48 & 33 & 15 & 7.8 & 6.0 & 4.7 & 4.3 & 3.9 & 3.4 & 3.0 & 2.4\end{array}$




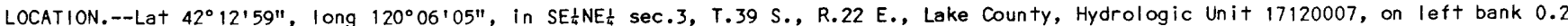
$\mathrm{mi}$ downstream from Blue Creek and $12 \mathrm{mi}$ east of Lakevlew.

DRAINAGE AREA.--63 $\mathrm{mi}^{2}$, approximately.

PERIOD OF RECORD.--September 1912 to November 1914, March to May 1915, December 1949 to September 1973.

GAGE.--Water-stage recorder. Datum of gage is 5,472.41 ft National Geodetlc Vertical Datum of 1929 (State Highway Department bench mark). Sept. 11, 1912, to May 9, 1915, water-stage recorder or nonrecording gage at site 500 ft upstream at different datum.

REMARKS.--No regulation. Diversions for irrigation above station.

AVERAGE DISCHARGE.--25 years (water years $1913-14,1951-73$ ), $46.6 \mathrm{ft} / \mathrm{s}, 33,760 \mathrm{acre}-\mathrm{ft} / \mathrm{yr}$.

EXTREMES FOR PERIOD OF RECORD.--Maximum discharge, 3,190 $\mathrm{ft}^{3} / \mathrm{s}$ Dec. 23, 1964, gage height, $7.32 \mathrm{tt}$, from rating curve extended above $410 \mathrm{ft}^{3} / \mathrm{s}$ on basis of slope-area measurement of peak flow; minlmum, $0.90 \mathrm{ft} 3 / \mathrm{s} \mathrm{Aug.} 16,1960$.

STATISTICAL SUMMARIES

MONTHLY AND ANNUAL MEAN DISCHARGES 1913-73

\begin{tabular}{|c|c|c|c|c|c|c|}
\hline MONTH & $\begin{array}{l}\text { MINIMUM } \\
\text { (CFS) }\end{array}$ & $\begin{array}{l}\text { MAXIMUM } \\
\text { (CFS) }\end{array}$ & $\begin{array}{l}\text { MEAN } \\
\text { (CFS) }\end{array}$ & $\begin{array}{l}\text { STAN- } \\
\text { DARD } \\
\text { DEVIA- } \\
\text { TION } \\
\text { (CFS) }\end{array}$ & $\begin{array}{l}\text { COEFFI- } \\
\text { CIENT OF } \\
\text { VARI- } \\
\text { ATION }\end{array}$ & $\begin{array}{c}\text { PERCENT } \\
\text { OF } \\
\text { ANNUAL } \\
\text { RUNOFF }\end{array}$ \\
\hline OCTOBER & 2.9 & 52 & 8.6 & 9.5 & 1.11 & 1.5 \\
\hline NOVEMBER & 3.3 & 42 & 14 & 8.9 & .65 & 2.4 \\
\hline DECEMBER & 2.3 & 241 & 33 & 53 & 1.62 & 5.9 \\
\hline JANUARY & 2.7 & 125 & 30 & 37 & 1.22 & 5.4 \\
\hline FEBRUARY & 6.3 & 142 & 41 & 35 & .85 & 7.3 \\
\hline MARCH & 15 & 254 & 64 & 52 & .81 & 11.4 \\
\hline APRIL & 40 & 344 & 164 & 77 & .47 & 29.3 \\
\hline MAY & 19 & 339 & 144 & 87 & .61 & 25.7 \\
\hline JUNE & 7.3 & 114 & 42 & 28 & .68 & 7.5 \\
\hline JULY & 2.0 & 23 & 10 & 5.8 & .57 & 1.8 \\
\hline AUGUST & 1.9 & 10 & 5.2 & 2.3 & .45 & .9 \\
\hline SEPTEMBER & 2.0 & 9.1 & 4.8 & 1.9 & .40 & .9 \\
\hline ANNU & 17 & 89 & 47 & 21 & .44 & 100 \\
\hline
\end{tabular}

MAGNITUDE AND PROBABILITY OF INSTANTANEOUS PEAK FLOW BASED ON PERIOD OF RECORD 1913-73

DISCHARGE, IN CFS, FOR INDICATED RECURRENCE INTERVAL, IN YEARS, AND ANNUAL EXCEEDANCE PROBABILITY, IN PERCENT

\begin{tabular}{ccccccc}
1.25 & 2 & 5 & 10 & 25 & 50 & 100 \\
$80 \%$ & $50 \%$ & $20 \%$ & $10 \%$ & $4 \%$ & $2 \%$ & $1 \%$ \\
\hline 293 & 472 & 821 & 1130 & 1630 & 2100 & - \\
\hline
\end{tabular}

WEIGHTED SKEW $=.525$
MAGNITUDE AND PROBABILITY OF ANNUAL LOW FLOW BASED ON PERIOD OF RECORD 1914-73

\begin{tabular}{|c|c|c|c|c|c|c|}
\hline \multirow{3}{*}{$\begin{array}{l}\text { PERIOD } \\
\text { (CON- } \\
\text { SECU- } \\
\text { TIVE } \\
\text { DAYS) }\end{array}$} & \multicolumn{6}{|c|}{$\begin{array}{l}\text { DISCHARGE, IN CFS, FOR IND ICATED RECURRENCE } \\
\text { INTERVAL, IN YEARS, AND ANNUAL NON- } \\
\text { EXCEEDANCE PROBABILITY, IN PERCENT }\end{array}$} \\
\hline & 2 & 5 & 10 & 20 & 50 & 100 \\
\hline & $50 \%$ & $20 \%$ & $10 \%$ & $5 \%$ & $2 \%$ & $1 \%$ \\
\hline 1 & 3.4 & 2.1 & 1.6 & 1.2 & .9 & -- \\
\hline 3 & 3.5 & 2.2 & 1.6 & 1.3 & .9 & -- \\
\hline 7 & 3.6 & 2.2 & 1.7 & 1.3 & 1.0 & -- \\
\hline 14 & 3.8 & 2.4 & 1.8 & 1.4 & 1.1 & -- \\
\hline 30 & 4.0 & 2.6 & 2.0 & 1.6 & 1.2 & -- \\
\hline 60 & 4.4 & 2.9 & 2.3 & 1.8 & 1.4 & -- \\
\hline 90 & 4.9 & 3.2 & 2.5 & 2.1 & 1.6 & -- \\
\hline 120 & 5.6 & 3.7 & 2.9 & 2.3 & 1.8 & -- \\
\hline 183 & 8.4 & 5.3 & 4.0 & 3.2 & 2.4 & -- \\
\hline
\end{tabular}

MAGNITUDE AND PROBABILITY OF ANNUAL HIGH FLOW BASED ON PERIOD OF RECORD 1913-73

\begin{tabular}{|c|c|c|c|c|c|c|}
\hline \multirow{4}{*}{$\begin{array}{l}\text { PERIOD } \\
\text { (CON- } \\
\text { SECU- } \\
\text { TIVE } \\
\text { DAYS) }\end{array}$} & \multicolumn{6}{|c|}{$\begin{array}{c}\text { DISCHARGE, IN CFS, FOR IND ICATED RECURRENCE } \\
\text { INTERVAL, IN YEARS, AND ANNUAL } \\
\text { EXCEEDANCE PROBABILITY, IN PERCENT }\end{array}$} \\
\hline & 2 & 5 & & 25 & 50 & \\
\hline & 500 & 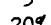 & 108 & 23 & 9 & 19 \\
\hline & & $20 \%$ & & & & 16 \\
\hline 1 & 373 & 633 & 868 & 1250 & 1610 & -- \\
\hline 3 & 336 & 541 & 706 & 951 & 1160 & -- \\
\hline 7 & 292 & 440 & 544 & 683 & 791 & -- \\
\hline 15 & 243 & 348 & 416 & 497 & 556 & -- \\
\hline 30 & 204 & 290 & 341 & 398 & 436 & -- \\
\hline 60 & 157 & 224 & 264 & 309 & 339 & -- \\
\hline 90 & 127 & 180 & 211 & 245 & 267 & -- \\
\hline
\end{tabular}

DURATION TABLE OF DAILY MEAN FLOW FOR PERIOD OF RECORD 1913-73

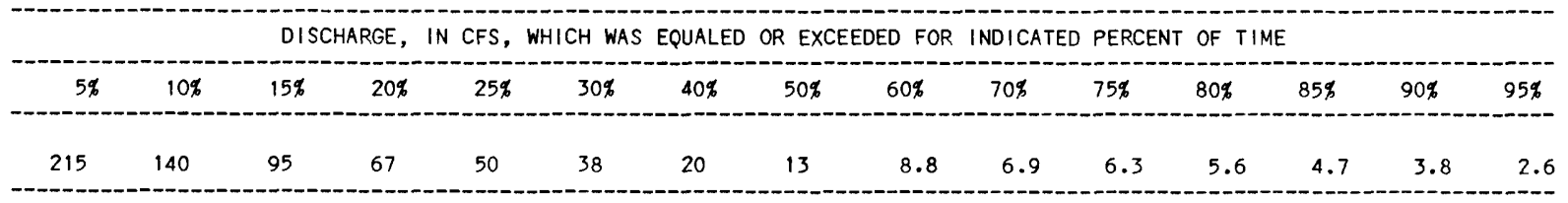


10371000 DRAKE CREEK NEAR ADEL, OR

LOCATION.--Lat 42 $12^{\prime} 00^{\prime \prime}$, long $120^{\circ} 00^{\prime} 4^{\prime \prime}$, in NE $\frac{1}{4}$ SW $\frac{1}{4}$ sec.9, T.39 S., R.23 E., Lake County, Hydrologic Unit 17120007, on right bank $800 \mathrm{ft}$ downstream from highway bridge and Parsnip Creek, $1.0 \mathrm{mi}$ upstream from mouth, and $6.5 \mathrm{mi}$ west of Adel.

DRAINAGE AREA. $--67 \mathrm{mi}^{2}$, approximately.

PERIOD OF RECORD.--March to May 1915, December 1922 to May 1923, December 1949 to December 1964 , October 1965 to September 1973.

GAGE.- Water-stage recorder. Datum of gage is 5,075.94 ft National Geodetic Vertical Datum of 1929 (State High Department benc mark). Mar 18 to May 10, 1915, and Dec. 21, 1922, to May 9, 1923, nonrecording gage at site $800 \mathrm{ft}$ upstream at different datums. Dec. 16, 1949, to June 21,1951 , at site $1,300 \mathrm{ft}$ upstream at different datum. June 22 , 1951 , to Dec. 23 , 1964 , at site $20 \mathrm{ft}$ upstream at datum $0.48 \mathrm{ft}$ higher.

REMARKS.--Some regulation by two reservoirs above station with combined capacity of 436 acre-ft. Diversions for irrigation above station.

AVERAGE DISCHARGE. - 22 years $(1951-64,1966-73), 14.9 \mathrm{ft}^{3} / \mathrm{s}, 10,800 \mathrm{acre}-\mathrm{ft} / \mathrm{yr}$.

EXTREMES FOR PERIOD OF RECORD.--Maximum discharge, $6,210 \mathrm{ft}^{3} / \mathrm{s} \mathrm{Dec} .23,1964$, gage height, $9.8 \mathrm{ft}$, result of slope-area measurement; minimum, $0.40 \mathrm{tt}^{3} / \mathrm{s}$ Jan. 11 , 1963, result of freezeup.

STATISTICAL SUMMARIES

MONTHLY AND ANNUAL MEAN DISCHARGES 1951-73

\begin{tabular}{|c|c|c|c|c|c|c|}
\hline MONTH & $\begin{array}{l}\text { MINIMIUM } \\
\text { (CFS) }\end{array}$ & $\begin{array}{l}\text { MAXIMUM } \\
\text { (CFS) }\end{array}$ & $\begin{array}{l}\text { MEAN } \\
\text { (CFS) }\end{array}$ & $\begin{array}{l}\text { STAN- } \\
\text { DARD } \\
\text { DEVIA- } \\
\text { TION } \\
\text { (CFS) }\end{array}$ & $\begin{array}{l}\text { COEFFI- } \\
\text { CIENT OF } \\
\text { VARI- } \\
\text { ATION }\end{array}$ & $\begin{array}{c}\text { PERCENT } \\
\text { OF } \\
\text { ANNUAL } \\
\text { RUNOFF }\end{array}$ \\
\hline OCTOBER & 3.1 & 12 & 6.5 & 2.1 & .32 & 3.6 \\
\hline NOVEMBER & 3.0 & 11 & 6.5 & 1.9 & .29 & 3.6 \\
\hline DECEMBER & 2.9 & 48 & 9.2 & 9.0 & .99 & 5.1 \\
\hline JANUARY & 2.4 & 88 & 17 & 21 & 1.25 & 9.3 \\
\hline FEBRUARY & 4.1 & 91 & 27 & 29 & 1.09 & 14.8 \\
\hline MARCH & 6.0 & 121 & 34 & 31 & .90 & 19.2 \\
\hline APRIL & 4.3 & 178 & 36 & 44 & 1.22 & 20.2 \\
\hline MAY & 3.5 & 48 & 15 & 11 & .74 & 8.3 \\
\hline JUNE & 3.4 & 22 & 8.7 & 4.2 & .48 & 4.8 \\
\hline JULY & 3.5 & 14 & 7.0 & 2.5 & .36 & 3.9 \\
\hline AUGUST & 3.5 & 12 & 6.5 & 2.1 & .33 & 3.6 \\
\hline SEPTEMBER & 3.6 & 12 & 6.3 & 2.1 & .33 & 3.5 \\
\hline ANNUAL & 4.7 & 32 & 15 & 7.7 & .52 & 100 \\
\hline
\end{tabular}

MAGNITUDE AND PROBABILITY OF INSTANTANEOUS PEAK FLOW BASED ON PERIOD OF RECORD 1951-73

DISCHARGE, IN CFS, FOR INDICATED RECURRENCE INTERVAL, IN YEARS, AND ANNUAL EXCEEDANCE PROBABILITY, IN PERCENT

\begin{tabular}{ccccccc}
1.25 & 2 & 5 & 10 & 25 & 50 & 100 \\
$80 \%$ & $50 \%$ & $20 \%$ & $10 \%$ & $4 \%$ & $2 \%$ & $1 \%$ \\
\hline 229 & 551 & 1380 & 2250 & 3860 & - & - \\
\hline
\end{tabular}

WEJGHTED SKEW $=.147$
MAGNITUDE AND PROBABILITY OF ANNUAL LOW FLOW BASED ON PERIOD OF RECORD 1952-73

\begin{tabular}{|c|c|c|c|c|c|c|}
\hline \multirow{2}{*}{$\begin{array}{l}\text { PERIOD } \\
\text { (CON- } \\
\text { SECU- } \\
\text { TIVE } \\
\text { DAYS) }\end{array}$} & \multicolumn{6}{|c|}{$\begin{array}{l}\text { DISCHARGE, IN CFS, FOR INDICATED RECURRENCE } \\
\text { INTERVAL, IN YEARS, AND ANNUAL NON- } \\
\text { EXCEEDANCE PROBABILITY, IN PERCENT }\end{array}$} \\
\hline & $\begin{array}{c}2 \\
50 \%\end{array}$ & $\begin{array}{c}5 \\
20 \%\end{array}$ & $\begin{array}{l}10 \\
10 \%\end{array}$ & $\begin{array}{l}20 \\
5 \%\end{array}$ & $\begin{array}{l}50 \\
2 \%\end{array}$ & $\begin{array}{r}100 \\
. \quad 1 \%\end{array}$ \\
\hline 1 & 3.8 & 2.3 & 1.7 & 1.3 & -- & -- \\
\hline 3 & 4.0 & 2.8 & 2.3 & 2.0 & -- & -- \\
\hline 7 & 4.2 & 3.0 & 2.5 & 2.1 & -- & - \\
\hline 14 & 4.5 & 3.3 & 2.8 & 2.4 & - & - \\
\hline 30 & 4.9 & 3.6 & 3.0 & 2.6 & -- & - \\
\hline 60 & 5.4 & 4.0 & 3.4 & 2.9 & -- & -- \\
\hline 90 & 5.7 & 4.2 & 3.6 & 3.1 & -- & -- \\
\hline 120 & 5.9 & 4.4 & 3.7 & 3.2 & - & -- \\
\hline 183 & 6.1 & 4.6 & 4.0 & 3.5 & -- & -- \\
\hline
\end{tabular}

MAGNITUDE AND PROBABILITY OF ANNUAL HIGH FLOW BASED ON PERIOD OF RECORD 1951-73

\begin{tabular}{|c|c|c|c|c|c|c|}
\hline $\begin{array}{l}\text { PERIOD } \\
\text { (CON- } \\
\text { SECU- }\end{array}$ & \multicolumn{6}{|c|}{$\begin{array}{c}\text { DISCHARGE, IN CFS, FOR INDICATED RECURRENCE } \\
\text { INTERVAL, IN YEARS, AND ANNUAL } \\
\text { EXCEEDANCE PROBABILITY, IN PERCENT }\end{array}$} \\
\hline TIVE & 2 & 5 & 10 & 25 & 50 & 100 \\
\hline DAYS) & $50 \%$ & $20 \%$ & $10 \%$ & $4 \%$ & $2 \%$ & $1 \%$ \\
\hline 1 & 238 & 609 & 953 & 1490 & -- & -- \\
\hline 3 & 170 & 411 & 619 & 924 & -- & -- \\
\hline 7 & 117 & 272 & 403 & 593 & - & -- \\
\hline 15 & 78 & 178 & 264 & 390 & -- & -- \\
\hline 30 & 52 & 112 & 162 & 237 & -- & - \\
\hline 60 & 36 & 70 & 96 & 132 & -- & -- \\
\hline 90 & 30 & 55 & 74 & 100 & - & - \\
\hline
\end{tabular}

DURATION TABLE OF DAILY MEAN FLOW FOR PERIOD OF RECORD 1951-73

DISCHARGE, IN CFS, WHICH WAS EQUALED OR EXCEEDED FOR INDICATED PERCENT OF TIME

\begin{tabular}{|c|c|c|c|c|c|c|c|c|c|c|c|c|c|c|}
\hline $5 \%$ & $10 \%$ & $15 \%$ & $20 \%$ & $25 \%$ & $30 \%$ & $40 \%$ & $50 \%$ & $60 \%$ & $70 \%$ & $75 \%$ & $80 \%$ & $85 \%$ & $90 \%$ & $95 \%$ \\
\hline 45 & 21 & 14 & 12 & 10 & 8.8 & 7.9 & 7.0 & 6.4 & 5.7 & 5.3 & 4.9 & 4.5 & 4.0 & 3 \\
\hline
\end{tabular}




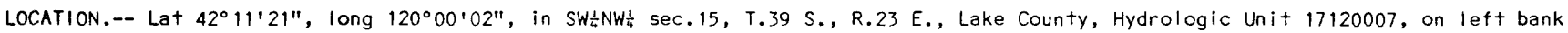
$700 \mathrm{ft}$ downstream from Drake Creek and $5 \mathrm{mi}$ west of Adel.

DRAINAGE AREA.--249 $\mathrm{mi}^{2}$.

PERIOD OF RECORD.--September 1922 to September 1923, rctober 1929 to September 1982. Monthly discharge only October 1929 to September 1932, published in WSP 1314.

GAGE.--Water-stage recorder. Datum of gage is 4,980.34 ft National Geodetic Vertical Datum of 1929 (State Highway Department bench mark). Sept. 8 to Dec. 20, 1922, nonrecording gage. Dec. 21, 1922, to Sept. 30, 1923, and Oct. 11, 1929, to Dec. 23, 1964, water-stage recorder at site $700 \mathrm{ft}$ downstream at different datums. Jan. 20 to Sept. 30, 1965, nonrecording gage at site $2,000 \mathrm{ft}$ downstream at different datum.

REMARKS.--No regulation. Diversions for irrigation above station.

AVERAGE DISCHARGE. -54 years, $130 \mathrm{ft} / \mathrm{s}, 94,180$ acre- $f+\mathrm{yr}$.

EXTREMES FOR PERIOD OF RECORD.--Maximum discharge, 9,420 $\mathrm{ft}$ /3/s Dec. 23, 1964, gage height, $10.64 \mathrm{ft}$, from $f l o o d m a r k$, from rating curve extended above $1,100 \mathrm{ft} / \mathrm{s}$ on basis of slope-area measurements at $7.3 \mathrm{ft}$ and of peak $\mathrm{flow}$; $\mathrm{minimum}$, $1.7 \mathrm{ft} / \mathrm{s} \mathrm{July} 20$, $27-29,1934$

\section{STATISTICAL SUMMARIES}

MONTHLY AND ANNUAL MEAN DISCHARGES 1923-82

\begin{tabular}{|c|c|c|c|c|c|c|}
\hline MONTH & $\begin{array}{l}\text { MINIMUM } \\
\text { (CFS) }\end{array}$ & $\begin{array}{c}\text { MAXIMUM } \\
\text { (CFS) }\end{array}$ & $\begin{array}{l}\text { MEAN } \\
\text { (CFS) }\end{array}$ & $\begin{array}{l}\text { STAN- } \\
\text { DARD } \\
\text { DEVIA- } \\
\text { TION } \\
\text { (CFS) }\end{array}$ & $\begin{array}{l}\text { COEFFI- } \\
\text { CIENT OF } \\
\text { VARI- } \\
\text { ATION }\end{array}$ & $\begin{array}{c}\text { PERCENT } \\
\text { OF } \\
\text { ANNUAL } \\
\text { RUNOFF }\end{array}$ \\
\hline OCTOBER & 5.2 & 179 & 24 & 24 & 1.00 & 1.5 \\
\hline NOVEMBER & 10 & 197 & 40 & 36 & .91 & 2.4 \\
\hline DECEMBER & 8.5 & 605 & 82 & 121 & 1.48 & 5.1 \\
\hline JANUARY & 7.0 & 451 & 86 & 109 & 1.27 & 5.3 \\
\hline FEBRUARY & 8.0 & 713 & 115 & 130 & 1.13 & 7.1 \\
\hline MARCH & 19 & 825 & 178 & 141 & .79 & 11.0 \\
\hline APRIL & 46 & 1072 & 398 & 222 & .56 & 24.7 \\
\hline MAY & 32 & 920 & 429 & 218 & .51 & 26.6 \\
\hline JUNE & 12 & 590 & 203 & 132 & .65 & 12.6 \\
\hline JULY & 2.4 & 115 & 34 & 27 & .81 & 2.1 \\
\hline AUGUST & 2.1 & 28 & 13 & 6.5 & .51 & .8 \\
\hline SEPTEMBER & 2.9 & 32 & 15 & 7.0 & .47 & .9 \\
\hline ANNUAL & 27 & 288 & 134 & 66 & .49 & 100 \\
\hline
\end{tabular}

MAGNITUDE AND PROBABILITY OF INSTANTANEOUS PEAK FLOW BASED ON PERIOD OF RECORD 1923-82

DISCHARGE, IN CFS, FOR INDICATED RECURRENCE INTERVAL, IN YEARS, AND ANNUAL EXCEEDANCE PROBABILITY, IN PERCENT

\begin{tabular}{ccccccc}
1.25 & 2 & 5 & 10 & 25 & 50 & 100 \\
$80 \%$ & $50 \%$ & $20 \%$ & $10 \%$ & $4 \%$ & $2 \%$ & $1 \%$ \\
\hline 605 & 1220 & 2480 & 3620 & 5440 & 7080 & 9000 \\
\hline
\end{tabular}

WEIGHTED SKEW $=.074$
MAGNITUDE AND PROBABILITY OF ANNUAL LOW FLOW BASED ON PERIOD OF RECORD 1934-82

\begin{tabular}{|c|c|c|c|c|c|c|}
\hline \multirow{4}{*}{$\begin{array}{l}\text { PERIOD } \\
\text { (CON- } \\
\text { SECU- } \\
\text { TIVE } \\
\text { DAYS) }\end{array}$} & \multicolumn{6}{|c|}{$\begin{array}{l}\text { DISCHARGE, IN CFS, FOR INDICATED RECURRENCE } \\
\text { INTERVAL, IN YEARS, AND ANNUAL NON- } \\
\text { EXCEEDANCE PROBABILITY, IN PERCENT }\end{array}$} \\
\hline & & & & 20 & 0 & \\
\hline & 2 & $\begin{array}{c}5 \\
209\end{array}$ & 10 & 20 & 50 & $\begin{array}{r}100 \\
1 \%\end{array}$ \\
\hline & & & & & & \\
\hline 1 & 9.0 & 5.3 & 3.9 & 3.0 & 2.2 & \\
\hline 3 & 9.2 & 5.4 & 4.0 & 3.0 & 2.2 & 1 \\
\hline 7 & 9.4 & 5.5 & 4.1 & 3.1 & 2.3 & 1 \\
\hline 14 & 9.6 & 5.7 & 4.2 & 3.3 & 2.4 & \\
\hline 30 & 10 & 6.1 & 4.6 & 3.5 & 2.6 & \\
\hline 60 & 12 & 7.0 & 5.2 & 4.1 & 3.0 & \\
\hline 90 & 13 & 8.1 & 6.2 & 4.8 & 3.6 & \\
\hline 120 & 16 & 10 & 7.7 & 6.0 & 4.2 & \\
\hline 183 & 24 & 15 & 12 & 9.3 & 7.2 & \\
\hline
\end{tabular}

MAGNITUDE AND PROBABILITY OF ANNUAL HIGH FLOW BASED ON PERIOD OF RECORD 1923-82

\begin{tabular}{|c|c|c|c|c|c|c|}
\hline \multirow{4}{*}{$\begin{array}{l}\text { PERIOD } \\
\text { (CON- } \\
\text { SECU- } \\
\text { TIVE } \\
\text { DAYS) }\end{array}$} & \multicolumn{6}{|c|}{$\begin{array}{l}\text { DISCHARGE, IN CFS, FOR INDICATED RECURRENCE } \\
\text { INTERVAL, IN YEARS, AND ANNUAL } \\
\text { EXCEEDANCE PROBABILITY, IN PERCENT }\end{array}$} \\
\hline & & & 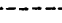 & 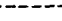 & & \\
\hline & 2 & 5 & 10 & 25 & 50 & 100 \\
\hline & $50 \%$ & $20 \%$ & $10 \%$ & $4 \%$ & $2 \%$ & $1 \%$ \\
\hline 1 & 1020 & 1960 & 2750 & 3930 & 4960 & 6110 \\
\hline 3 & 875 & 1530 & 2000 & 2650 & 3150 & 3660 \\
\hline 7 & 731 & 1170 & 1450 & 1780 & 2010 & 2230 \\
\hline 15 & 616 & 928 & 1110 & 1300 & 1420 & 1520 \\
\hline 30 & 524 & 778 & 918 & 1070 & 1160 & 1230 \\
\hline 60 & 438 & 633 & 731 & 826 & 880 & 922 \\
\hline 90 & 366 & $528^{\circ}$ & 611 & 692 & 739 & 777 \\
\hline
\end{tabular}

DURATION TABLE OF DAILY MEAN FLOW FOR PERIOD OF RECORD 1923-82

DISCHARGE, IN CFS, WHICH WAS EQUALED OR EXCEEDED FOR INDICATED PERCENT OF TIME

\begin{tabular}{|c|c|c|c|c|c|c|c|c|c|c|c|c|c|c|}
\hline $5 \%$ & $10 \%$ & $15 \%$ & $20 \%$ & $25 \%$ & $30 \%$ & $40 \%$ & $50 \%$ & $60 \%$ & $70 \%$ & $75 \%$ & $80 \%$ & $85 \%$ & $90 \%$ & $95 \%$ \\
\hline 579 & 403 & 290 & 214 & 156 & 111 & 57 & 35 & 26 & 21 & 18 & 16 & 13 & 11 & 7.0 \\
\hline
\end{tabular}


$1337259=$ HONEY RREEK NEAR PLUSH, OR

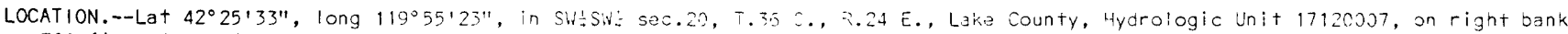
$700 \mathrm{ft}$ upstream from mouth of canyon, $1.4 \mathrm{mi}$ northwest of Plush, and $4 \mathrm{mi}$ downstream from Twelvemile Creek.

DRAINAGE AREA.--170 $\mathrm{mi}^{2}$, approximately.

PERIOD OF RECORD.--May 1909 to Septenber 1714 (prior to January 191), ajge heights only), March to "lay 1915, ilarch to September 1921, March to June 1922, May 1030 to September 1022. Jonthiv discharge only "lay 1030 to September 1949 , published in WSP 1314.

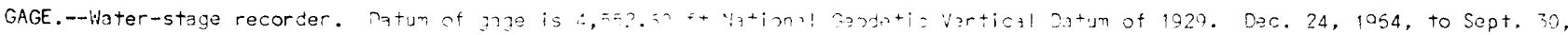
1965, nonrecording gage at site $170 \mathrm{ft}$ downstream at different datums. See isp 1927 for history of changes priar to Dec. 24 , 1964 .

REMARKS.--Slight regulation by five small reserviors, combined capacity, 870 acre-tt. Diversions for irrigation above station.

AVERAGE DISCHARGE.--56 years (water years $1911-14,1931-32$ ), $30.0 \mathrm{ft} / \mathrm{s}, 21,740 \mathrm{acre}-\mathrm{ft} / \mathrm{yr}$.

EXTREMES FOR PERIOD OF RECORD.--Maxiumum discharge, 11,000 fto/s Dac. 23, 1954, gage height, $13.4 \mathrm{f}, \mathrm{t}$ from floodmark, from rating curve extended above $250 \mathrm{ft} / \mathrm{s}$ on basis of slop?-area medsurements at gage height $10.45 \mathrm{ft}$ and of peak $\mathrm{flow}$; no flow at times.

STATISTICAL SUMNARIES

MONTHLY AND ANNUAL MEAN DISCHARGES 1750-92

\begin{tabular}{|c|c|c|c|c|c|c|}
\hline MONTH & $\begin{array}{l}\text { MINIMUA } \\
\text { (CFS) }\end{array}$ & $\begin{array}{l}\text { MAXIMUM } \\
\text { (CFS) }\end{array}$ & $\begin{array}{l}\text { MEAN } \\
\text { (CFS) }\end{array}$ & $\begin{array}{l}\text { STAN- } \\
\text { DARD } \\
\text { DEVIA- } \\
\text { TION } \\
\text { (CFS) }\end{array}$ & $\begin{array}{l}\text { COEFFI- } \\
\text { CIENT OF } \\
\text { VARI- } \\
\text { ATION }\end{array}$ & $\begin{array}{c}\text { PERCENT } \\
\text { OF } \\
\text { ANNUAL } \\
\text { RUNOFF }\end{array}$ \\
\hline OCTOBER & .1 & 39 & 3.5 & 6.2 & 1.76 & .8 \\
\hline NOVEMBER & .9 & 32 & 7.2 & 7.1 & .98 & 1.7 \\
\hline DECEMBER & 1.0 & 253 & 22 & 46 & 2.05 & 5.4 \\
\hline JANUARY & 1.1 & 107 & 22 & 29 & 1.29 & 5.4 \\
\hline FEBRUARY & 4.0 & 181 & 30 & 38 & 1.27 & 7.2 \\
\hline MARCH & 4.6 & 268 & 50 & 52 & 1.05 & 12.0 \\
\hline APRIL & 10 & 264 & 96 & 58 & .50 & 23.1 \\
\hline MAY & 11 & 318 & 122 & 68 & .56 & 29.4 \\
\hline JUNE & 3.2 & 147 & 50 & 35 & .69 & 12.1 \\
\hline JULY & .3 & 24 & 8.8 & 6.9 & .78 & 2.1 \\
\hline AUGUST & .2 & 9.5 & 1.7 & 1.9 & 1.11 & .4 \\
\hline SEPTEMBER & .2 & 7.7 & 1.3 & 1.3 & 1.25 & .3 \\
\hline NNUAL & 3.9 & 79 & 34 & 20 & .57 & 100 \\
\hline
\end{tabular}

MAGNITUDE AND PROBABILITY OF INSTANTANEOUS PEAK FLOH BASED ON PERIOD OF RECORD $1911-32$

\begin{tabular}{|c|c|c|c|c|c|c|}
\hline \multicolumn{7}{|c|}{$\begin{array}{l}\text { DISCHARGE, IN CFS, FOR INDICATEO RECURRENCE INTERVAL, IN } \\
\text { YEARS, AND ANNUAL EXCEEDANCE PROBABILITY, IN PERCENT }\end{array}$} \\
\hline $\begin{array}{l}1.25 \\
80 \%\end{array}$ & $\begin{array}{c}2 \\
50 \%\end{array}$ & $\begin{array}{c}5 \\
20^{\circ}\end{array}$ & $\begin{array}{l}10 \\
10 \%\end{array}$ & $\begin{array}{l}25 \\
4^{\prime \prime}\end{array}$ & $\begin{array}{l}50 \\
2 \pi\end{array}$ & $\begin{array}{r}100 \\
1 *\end{array}$ \\
\hline 171 & 450 & 1190 & 1990 & 3420 & 4860 & 5670 \\
\hline
\end{tabular}

WEIGHTED SKEW $=.027$
"AGNITUDE AIJ PRDBASILITY OF ANNUAL LOW FLOW BASED ON PERIOD OF RECORD 1951-82

\begin{tabular}{|c|c|c|c|c|c|c|}
\hline $\begin{array}{l}\text { PERIOD } \\
\text { (CON- }\end{array}$ & \multicolumn{6}{|c|}{$\begin{array}{l}\text { DISCHARGE, IN CFS, FOR INDICATED RECURRENCE } \\
\text { INTERVAL, IN YEARS, AND ANNUAL NON- } \\
\text { EXCEEDANCE PROBABILITY, IN PERCENT }\end{array}$} \\
\hline TIVE & 2 & 5 & 10 & 20 & 50 & 00 \\
\hline DAYS) & $50^{\circ}$ & $20^{\circ}$ & $10 \%$ & $5^{x}$ & $2^{\circ}$ & $1 \frac{3}{3}$ \\
\hline \\
\hline 1 & .3 & .2 & .1 & .1 & .1 & \\
\hline 3 & .4 & .2 & .1 & .1 & .1 & \\
\hline 7 & .4 & .2 & .1 & .1 & .1 & \\
\hline 14 & .5 & .2 & .2 & .1 & .1 & \\
\hline 30 & .6 & .3 & .2 & .1 & .1 & \\
\hline 60 & .8 & .4 & .3 & .2 & .1 & \\
\hline 90 & 1.1 & .5 & .4 & .3 & .2 & \\
\hline 120 & 1.8 & .9 & .6 & .4 & .2 & \\
\hline 183 & 3.8 & 2.1 & 1.4 & 1.1 & .7 & \\
\hline
\end{tabular}

IIAGNITUDE AND PROBABILITY OF ANNUAL HIGH FLOW BASED ON PERIOD OF RECORD 1950-82

\begin{tabular}{|c|c|c|c|c|c|c|}
\hline PERIOD & \multicolumn{6}{|c|}{$\begin{array}{c}\text { DISCHARGE, IN CFS, FOR INDICATED RECURRENCE } \\
\text { INTERVAL, IN YEARS, AND ANNUAL }\end{array}$} \\
\hline C CON- & \multicolumn{6}{|c|}{ EXCEEDANCE PROBABILITY, IN PERCENT } \\
\hline SECU- & & & - & & & \\
\hline TIVE & 2 & 5 & 10 & 25 & 50 & 100 \\
\hline DAYS) & $50 \%$ & $20^{\circ}$ & 10\% & $4:$ & $2 \%$ & $1 \%$ \\
\hline \\
\hline 1 & 327 & 728 & 1090 & 1650 & 2150 & 2710 \\
\hline 3 & 267 & 532 & 734 & 1010 & 1210 & 1420 \\
\hline 7 & 212 & 391 & 511 & 659 & 762 & 858 \\
\hline 15 & 172 & 290 & 359 & 431 & 476 & 513 \\
\hline 30 & 142 & 231 & 277 & 321 & 345 & 363 \\
\hline 60 & 113 & 182 & 218 & 254 & 274 & 291 \\
\hline 90 & 34 & 150 & 180 & 208 & 224 & 237 \\
\hline
\end{tabular}

DURATION TABLE OF DAILY MEAN FLOW FOR PERIOD OF RECORD $1911-82$

DISCHARGE, IN CFS, WHICH WAS EOUALED OR EXCEEDED FOR INDICATED PERCENT OF TIME

\begin{tabular}{|c|c|c|c|c|c|c|c|c|c|c|c|c|c|c|}
\hline $5 \%$ & $10 \%$ & $15 \%$ & $20^{\alpha}$ & $25 \%$ & $30 \%$ & $40 \%$ & $50 \%$ & $60 \%$ & $70^{\circ}$, & $75 \%$ & $80 \%$ & $85 \%$ & $90 \%$ & $95 \%$ \\
\hline 159 & 103 & 71 & 53 & 35 & 25 & 14 & 7.6 & 5.1 & 3.2 & 2.4 & 1.8 & 1.2 & .7 & .3 \\
\hline
\end{tabular}


10384000 CHEWAUCAN RIVER NEAR PAISLEY, OR

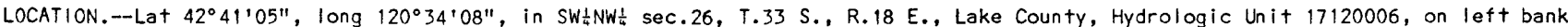
$1.2 \mathrm{mi}$ downstream from Mill Creek and $1.4 \mathrm{mi}$ southwest of Paisley.

DRAINAGE AREA. $--275 \mathrm{~m}^{2}$.

PERIOD OF RECORD.--April 1912 to September 1921 (fragmentary), May 1924 to September 1982. Published as "above Conn ditch, near Paisley" Aprii to September 1912 and May 1924 to September 1955, as "above Mill Creek, near Paisley" October 1912 to Decemb.1913; and as "at Chewaucan Land \& Cattle Co.'s gage, near Paisley" January to September 1914.

GAGE.--Water-stage recorder. Datum of gage is $4,430 \mathrm{ft}$ National Geodetic Vertical Datum of 1929 (river-profile survey). See WSP 1734 for history of changes prior to Oct. $6,1956$.

REMARKS. - No regulation. Diversions for irrigation above station.

AVERAGE DISCHARGE. -67 years, $144 \mathrm{ft}^{3} / \mathrm{s}, 104,300$ acre- $\mathrm{ft} / \mathrm{yr}$.

EXTREMES FOR PERIDD OF RECORD.--Maximum discharge, $6,490 \mathrm{ft}^{3} / \mathrm{s}$ Dec. 22,1964 , gage height, $8.35 \mathrm{ft}$, from rating curve extended above $900 \mathrm{ft}^{3} / \mathrm{s}$ on basis of slope-area measurement of peak flow; no flow for part of each day Dec. 7,1927 , Dec. 12, 1932, result of treezeup.

STATISTICAL SUMMARIES

MONTHLY AND ANNUAL MEAN DISCHARGES 1925-82

\begin{tabular}{|c|c|c|c|c|c|c|}
\hline MONTH & $\begin{array}{l}\text { MINIMUM } \\
\text { (CFS) }\end{array}$ & $\begin{array}{l}\text { MAXIMUM } \\
\text { (CFS) }\end{array}$ & $\begin{array}{l}\text { MEAN } \\
\text { (CFS) }\end{array}$ & $\begin{array}{l}\text { STAN- } \\
\text { DARD } \\
\text { DEVIA- } \\
\text { TION } \\
\text { (CFS) }\end{array}$ & $\begin{array}{l}\text { COEFFI- } \\
\text { CIENT OF } \\
\text { VARI- } \\
\text { ATION }\end{array}$ & $\begin{array}{c}\text { PERCENT } \\
\text { OF } \\
\text { ANNUAL } \\
\text { RUNOFF }\end{array}$ \\
\hline OCTOBER & 22 & 156 & 39 & 19 & .47 & 2.2 \\
\hline NOVEMBER & 21 & 205 & 54 & 34 & .62 & 3.1 \\
\hline DECEMBER & 10 & 657 & 88 & 115 & 1.30 & 5.1 \\
\hline JANUARY & 17 & 395 & 88 & 86 & .98 & 5.0 \\
\hline FEBRUARY & 23 & 566 & 112 & 98 & .87 & 6.4 \\
\hline MARCH & 42 & 659 & 160 & 103 & .64 & 9.2 \\
\hline APRIL & 54 & 828 & 338 & 189 & .56 & 19.3 \\
\hline MAY & 57 & 1211 & 494 & 283 & .57 & 28.3 \\
\hline JUNE & 25 & 672 & 252 & 173 & .69 & 14.4 \\
\hline JULY & 14 & 168 & 62 & 38 & .62 & 3.5 \\
\hline AUGUST & 9.4 & 69 & 31 & 13 & .41 & 1.8 \\
\hline SEPTEMBER & 14 & 50 & 31 & 8.5 & .28 & 1.7 \\
\hline ANNUAL & 34 & 353 & 146 & 74 & .51 & 100 \\
\hline
\end{tabular}

MAGNITUDE AND PROBABILITY OF INSTANTANEOUS PEAK FLOW BASED ON PERIOD OF RECORD $1925-82$

DISCHARGE, IN CFS, FOR INDICATED RECURRENCE INTERVAL, IN YEARS, AND ANNUAL EXCEEDANCE PROBABILITY, IN PERCENT

\begin{tabular}{|c|c|c|c|c|c|c|}
\hline $\begin{array}{l}1.25 \\
80 \%\end{array}$ & $\begin{array}{c}2 \\
50 \%\end{array}$ & $\begin{array}{c}5 \\
20 \%\end{array}$ & $\begin{array}{l}10 \\
10 \%\end{array}$ & $\begin{array}{l}25 \\
4 \%\end{array}$ & $\begin{array}{l}50 \\
2 \%\end{array}$ & $\begin{array}{r}100 \\
1 \%\end{array}$ \\
\hline 573 & 957 & 1650 & 2210 & 3060 & 3800 & 4620 \\
\hline
\end{tabular}

WEIGHTED SKEW $=.222$
MAGNITUDE AND PROBABILITY OF ANNUAL LOW FLOW BASED ON PERIOD OF RECORD $1926-82$

\begin{tabular}{ccccccc}
$\begin{array}{c}\text { PERIOD } \\
\text { (CON- }\end{array}$ & $\begin{array}{c}\text { DISCHARGE, IN CFS, FOR INDICATED RECURRENCE } \\
\text { INTERVAL, IN YEARS, AND ANNUAL NON- } \\
\text { EXCEEDANCE PROBABILITY, IN PERCENT }\end{array}$ \\
SECU- & - & -10 & 20 & 50 & 100 \\
TIVE & 2 & 5 & 10 & $5 \%$ & $2 \%$ & $1 \%$ \\
DAYS) & $50 \%$ & $20 \%$ & $10 \%$ & $5 \%$ \\
\hline 1 & 19 & 13 & 10 & 8.5 & 6.7 & 5.6 \\
3 & 21 & 14 & 11 & 9.1 & 7.1 & 6.0 \\
7 & 22 & 15 & 12 & 10 & 8.0 & 6.8 \\
14 & 23 & 16 & 13 & 11 & 8.9 & 7.6 \\
30 & 25 & 18 & 14 & 12 & 9.6 & 8.2 \\
60 & 27 & 20 & 17 & 14 & 12 & 10 \\
90 & 30 & 22 & 19 & 16 & 13 & 12 \\
120 & 33 & 25 & 21 & 18 & 15 & 13 \\
183 & 40 & 29 & 25 & 21 & 18 & 16 \\
\hline
\end{tabular}

MAGNI TUDE AND PROBABILITY OF ANNUAL HIGH FLOW BASED ON PERIOD OF RECORD $1925-82$

\begin{tabular}{ccccccc} 
DISCHARGE, IN CFS, FOR INDICATED RECURRENCE \\
INTERVAL, IN YEARS, AND ANNUAL \\
$\begin{array}{c}\text { PERIOD } \\
\text { (CON- }\end{array}$ EXCEEDANCE PROBABILITY, IN PERCENT \\
$\begin{array}{c}\text { SECU- } \\
\text { TIVE }\end{array}$ & 2 & 5 & 10 & 25 & 50 & 100 \\
DAYS) & $50 \%$ & $20 \%$ & $10 \%$ & $4 \%$ & $2 \%$ & $1 \%$ \\
\hline & & & & & & \\
1 & 788 & 1400 & 1870 & 2500 & 3000 & 3520 \\
3 & 728 & 1240 & 1580 & 2000 & 2300 & 2600 \\
7 & 663 & 1070 & 1310 & 1570 & 1740 & 1890 \\
15 & 597 & 925 & 1100 & 1290 & 1400 & 1490 \\
30 & 528 & 810 & 963 & 1120 & 1210 & 1290 \\
60 & 428 & 664 & 799 & 946 & 1040 & 1120 \\
90 & 352 & 544 & 657 & 782 & 863 & 934 \\
\hline
\end{tabular}

DURATION TABI.E OF DAILY MEAN FLOW FOR PERIOD OF RECORD 1925-82

DISCHARGE, IN CFS, WHICH WAS EQUALED OR EXCEEDED FOR INDICATED PERCENT OF TIME

\begin{tabular}{|c|c|c|c|c|c|c|c|c|c|c|c|c|c|c|}
\hline $5 \%$ & $10 \%$ & $15 \%$ & $20 \%$ & $25 \%$ & $30 \%$ & $40 \%$ & $50 \%$ & $60 \%$ & $70 \%$ & $75 \%$ & $80 \%$ & $85 \%$ & $90 \%$ & $95 \%$ \\
\hline 608 & 403 & 284 & 209 & 158 & 121 & 77 & 56 & 45 & 37 & 34 & 31 & 28 & 24 & 20 \\
\hline
\end{tabular}


10393500 SILVIES RIVER NEAR BURNS, OR

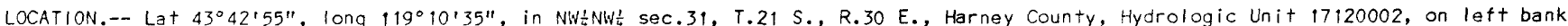
$5 \mathrm{mi}$ downstream from Emigrant Creek and $11 \mathrm{mi}$ northwest of Burns.

DRAINAGE AREA.--934 $\mathrm{mi}^{2}$.

PERIOD OF RECORD.--May 1903 to July 1906, December 1908 to December 1912, March 1913 to September 1917 (irrigation seasons only), March 1918 to October 1920, March 1921 to July 1922 (irrigation seasons only), October 1922 to September 1982.

GAGE.--Water-stage recorder. Datum of gage is 4,195 ft National Geodetic Vertical Datum of 1929 (river-profile survey). See WSP 1734 for history of changes prior to Oct. 4, 1951.

REMARKS.--No regulation. Diversions for irrigation above station during periods of high flow oniy.

AVERAGE DISCHARGE. --69 years (water years 1904-5, 1910-12, 1918-21, 1923-82), $169 \mathrm{ft} 3 / \mathrm{s}, 122,400$ acre- $f+/ \mathrm{yr}$.

EXTREMES FOR PERIOD OF RECORD.--Maximum discharge, 4,960 ft3/s Apr. 6, 1952, gage height, $15.2 \mathrm{ft}$; no flow July $19 \mathrm{fo}$ Sept. 22 , 1934.

STATISTICAL SUMMARIES

MONTHLY AND ANNUAL MEAN DISCHARGES 1904-82

\begin{tabular}{|c|c|c|c|c|c|c|}
\hline MONTH & $\begin{array}{l}\text { MINIMUM } \\
\text { (CFS) }\end{array}$ & $\begin{array}{l}\text { MAXIMUM } \\
\text { (CFS) }\end{array}$ & $\begin{array}{l}\text { MEAN } \\
\text { (CFS) }\end{array}$ & $\begin{array}{l}\text { STAN- } \\
\text { DARD } \\
\text { DEVIA- } \\
\text { TION } \\
\text { (CFS) }\end{array}$ & $\begin{array}{l}\text { COEFFI- } \\
\text { CIENT OF } \\
\text { VARI- } \\
\text { ATION }\end{array}$ & $\begin{array}{c}\text { PERCENT } \\
\text { OF } \\
\text { ANNUAL } \\
\text { RUNOFF }\end{array}$ \\
\hline OCTOBER & 3.9 & 45 & 18 & 9.1 & .50 & .9 \\
\hline NOVEMBER & 5.2 & 62 & 31 & 13 & .42 & 1.5 \\
\hline DECEMBER & 10 & 482 & 51 & 60 & 1.16 & 2.5 \\
\hline JANUARY & 10 & 715 & 81 & 107 & 1.33 & 3.9 \\
\hline FEBRUARY & 18 & 799 & 156 & 164 & 1.05 & 7.5 \\
\hline $\mathrm{MARCH}$ & 30 & 1508 & 347 & 275 & .79 & 16.7 \\
\hline APRIL & 12 & 2716 & 757 & 530 & .70 & 36.4 \\
\hline MAY & 5.6 & 1535 & 447 & $35 \%$ & .79 & 21.5 \\
\hline JUNE & 2.2 & 473 & 134 & 115 & .86 & 6.5 \\
\hline JULY & .5 & 152 & 31 & 26 & .85 & 1.5 \\
\hline AUGUST & 0.0 & 52 & 13 & 19 & .86 & .6 \\
\hline SEPTEMBER & .6 & 45 & 12 & 8.6 & .73 & .6 \\
\hline ANNUAL & 15 & 412 & 166 & 98 & .59 & 100 \\
\hline
\end{tabular}

MAGNITUDE AND PROBABILITY OF INSTANTANEOUS PEAK FLOW BASED ON PERIOD OF RECORD 1904-82

DISCHARGE, IN CFS, FOR INDICATED RECURRENCE INTERVAL, IN YEARS, AND ANNUAL EXCEEDANCE PROBABILITY, IN PERCENT

\begin{tabular}{ccccccc}
1.25 & 2 & 5 & 10 & 25 & 50 & 100 \\
$80 \%$ & $50 \%$ & $20 \%$ & $10 \%$ & $4 \%$ & $2 \%$ & $1 \%$ \\
\hline 749 & 1280 & 2130 & 2750 & 3580 & 4230 & 4900
\end{tabular}

WEIGHTED SKEW $=-.166$
MAGNITUDE AND PROBABILITY OF ANNUAL LOW FLOW BASED ON PERIOD OF RECORD 1905-82

\begin{tabular}{|c|c|c|c|c|c|c|}
\hline $\begin{array}{l}\text { PERIOD } \\
\text { (CON- } \\
\text { SECU- }\end{array}$ & \multicolumn{6}{|c|}{$\begin{array}{l}\text { DISCHARGE, IN CFS, FOR INDICATED RECURRENCE } \\
\text { INTERVAL, IN YEARS, AND ANNUAL NON- } \\
\text { EXCEEDANCE PROBABILITY, IN PERCENT }\end{array}$} \\
\hline $\begin{array}{l}\text { TIVE } \\
\text { DAYS) }\end{array}$ & $\stackrel{2}{50 \%}$ & $\begin{array}{c}5 \\
20 \%\end{array}$ & $\begin{array}{l}10 \\
10 q\end{array}$ & $\begin{array}{l}20 \\
5 \%\end{array}$ & $\begin{array}{l}50 \\
2 \%\end{array}$ & $\begin{array}{r}100 \\
1 \%\end{array}$ \\
\hline & & & & & & \\
\hline 1 & 6.1 & 2.3 & 1.2 & .7 & .3 & \\
\hline 3 & 6.4 & 2.5 & 1.3 & .7 & .4 & \\
\hline 7 & 6.7 & 2.7 & 1.5 & .9 & .4 & \\
\hline 14 & 7.1 & 3.1 & 1.9 & 1.2 & .6 & \\
\hline 30 & 7.5 & 3.7 & 2.4 & 1.7 & 1.1 & \\
\hline 60 & 8.8 & 4.6 & 3.2 & 2.3 & 1.5 & \\
\hline 90 & 12 & 5.5 & 3.3 & 2.5 & 1.6 & \\
\hline 120 & 14 & 7.0 & 4.6 & 3.0 & 1.9 & \\
\hline 183 & 21 & 12 & 8.2 & 5.9 & 3.9 & \\
\hline
\end{tabular}

MAGNITUDE AND PROBABILITY OF ANNUAL HIGH FLOW BASED ON PERIOD OF RECORD 1904-82

\begin{tabular}{|c|c|c|c|c|c|c|}
\hline $\begin{array}{l}\text { PERIOD } \\
\text { (CON- } \\
\text { SECU- }\end{array}$ & \multicolumn{6}{|c|}{$\begin{array}{l}\text { DISCHARGE, IN CFS, FOR INDICATED RECURRENCE } \\
\text { INTERVAL, IN YEARS, AND ANNUAL } \\
\text { EXCEEDANCE PROBABILITY, IN PERCENT }\end{array}$} \\
\hline TIVE & 2 & 5 & 10 & 25 & 50 & 100 \\
\hline DAYS) & $50 \%$ & $20 \%$ & $10 \%$ & $4 \%$ & $2 \%$ & $1 \%$ \\
\hline 1 & 1240 & 2050 & 2480 & 2890 & 3120 & 3300 \\
\hline 3 & 1170 & 1960 & 2390 & 2810 & 3050 & 3240 \\
\hline 7 & 1050 & 1790 & 2200 & 2600 & 2840 & 3030 \\
\hline 15 & 904 & 1560 & 1940 & 2330 & 2560 & 2750 \\
\hline 30 & 757 & 1320 & 1640 & 1980 & 2190 & 2350 \\
\hline 60 & 581 & 999 & 1240 & 1510 & 1670 & 1800 \\
\hline 90 & 465 & 793 & 987 & 1200 & 1330 & 1440 \\
\hline
\end{tabular}

DURATION TABLE OF DAILY MEAN FLOW FOR PERIOD OF RECORD $1904-82$

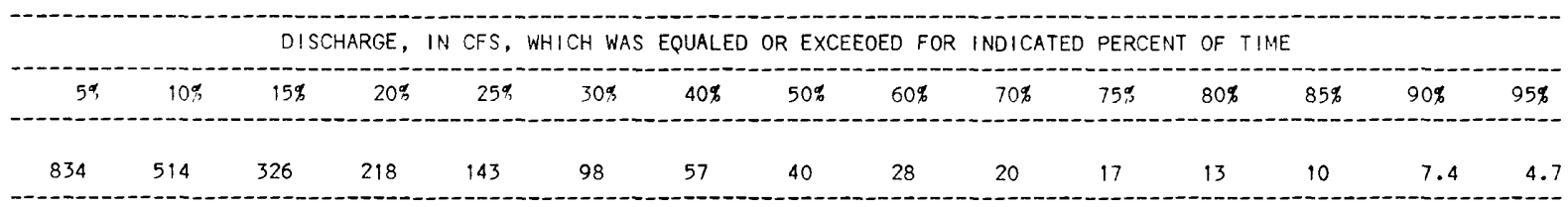




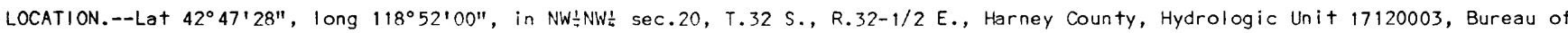
Land Management land, on left bank $1.5 \mathrm{mi}$ upstream from upper diversions for Malheur Migratory Waterfowl Refuge, $2.0 \mathrm{mi}$ downstream from Fish Creek, and $3.5 \mathrm{mi}$ southeast of Frenchglen.

DRAINAGE AREA. $--200 \mathrm{mi}^{2}$, approximately.

PERIOD OF RECORD.--March 1911 to September 1913, March 1914 to September 1916, April 1917 to September 1921 , August to November 1929, April to September 1930, December 1937 to September 1982. Monthly discharge oniy for some periods, published in wSP 1314.

Published as "near Diamond" 1911-21. Records of discharge for January 1909 to September 1910 (published in WSP 270 , 290 , and 370 , for a nonequivalent site as "near Diamond") have been found to be unreliable and should not be used.

GAGE.--Water-stage recorder and concrete control. Datum of gage is 4,254 ft National Geodetic Vertical Datum of 1929 (levels by Fish and Wildlife Service). Prior to December 1937, nonrecording gage at several sites within 2 mi downstream at different datums. Dec. 6, 1937, to Feb. 14, 1938, nonrecording gage at present site and datum.

REMARKS.--No regulation or diversion above station.

AVERAGE DISCHARGE.--52 years (water years $1912-13,1915-16,1918-21,1939-82), 123 \mathrm{ft} / \mathrm{s}, 89,110$ acre- $\mathrm{ft} / \mathrm{yr}$.

EXTREMES FOR PERIOD OF RECORD.--Maximum discharge, 4,270 $\mathrm{ft}^{3} / \mathrm{s}$ Apr. 26, 1978, gage height, $7.15 \mathrm{ft} f \mathrm{fom} \mathrm{floodmarks,} \mathrm{from} \mathrm{rating} \mathrm{curve}$ extended above $1,900 \mathrm{ft}^{3} / \mathrm{s}$ on basis of slope-area measurement of peak flow; minimum, $4.2 \mathrm{ft} / \mathrm{s}$ Dec. 9 , 1972 , result of $\mathrm{freeze} u \mathrm{p}$.

STATISTICAL SUMMARIES

MONTHLY AND ANNUAL MEAN DISCHARGES 1912-82

\begin{tabular}{|c|c|c|c|c|c|c|}
\hline MONTH & $\begin{array}{c}\text { MIN|MUM } \\
(C F S)\end{array}$ & $\begin{array}{l}\text { MAXIMUM } \\
\text { (CFS) }\end{array}$ & $\begin{array}{l}\text { MEAN } \\
\text { (CFS) }\end{array}$ & $\begin{array}{l}\text { STAN- } \\
\text { DARD } \\
\text { DEVIA- } \\
\text { TION } \\
\text { (CFS) }\end{array}$ & $\begin{array}{l}\text { COEFFI- } \\
\text { CIENT OF } \\
\text { VARI- } \\
\text { ATION }\end{array}$ & $\begin{array}{c}\text { PERCENT } \\
\text { OF } \\
\text { ANNUAL } \\
\text { RUNOFF }\end{array}$ \\
\hline OCTOBER & 25 & 64 & 43 & 9.1 & .21 & 2.8 \\
\hline NOVEMBER & 25 & 93 & 47 & 13 & .27 & 3.1 \\
\hline DECEMBER & 25 & 181 & 55 & 32 & .59 & 3.7 \\
\hline JANUARY & 25 & 239 & 65 & 44 & .67 & 4.3 \\
\hline FEBRUARY & 28 & 310 & 91 & 67 & .74 & 6.0 \\
\hline MARCH & 41 & 374 & 138 & 73 & .53 & 9.2 \\
\hline APRIL & 61 & 666 & 221 & 108 & .49 & 14.8 \\
\hline MAY & 138 & 649 & 373 & 132 & .35 & 24.9 \\
\hline JUNE & 68 & 802 & 284 & 135 & .48 & 19.0 \\
\hline JULY & 29 & 196 & 95 & 43 & .46 & 6.3 \\
\hline AUGUST & 24 & 76 & 46 & 14 & .30 & 3.1 \\
\hline SEPTEMBER & 24 & 65 & 41 & 9.9 & .24 & 2.8 \\
\hline ANNUAL & 55 & 201 & 123 & 36 & .29 & 100 \\
\hline
\end{tabular}

MAGNITUDE AND PROBABILITY OF INSTANTANEOUS PEAK FLOW BA.SED ON PERIOD OF RECORD 1912-82

DISCHARGE, IN CFS, FOR INDICATED RECURRENCE INTERVAL, IN YEARS, AND ANNUAL EXCEEDANCE PROBABILITY, IN PERCENT

\begin{tabular}{ccccccc}
1.25 & 2 & 5 & 10 & 25 & 50 & 100 \\
$80 \%$ & $50 \%$ & $20 \%$ & $10 \%$ & $4 \%$ & $2 \%$ & $1 \%$ \\
\hline 809 & 1340 & 2120 & 2630 & 3280 & 3740 & 4200 \\
\hline
\end{tabular}

WEIGHTED SKEW $=-.361$
MAGNITUDE AND PROBABILITY OF ANNUAL LOW FLOW BASED ON PERIOD OF RECORD 1913-82

\begin{tabular}{|c|c|c|c|c|c|c|}
\hline \multirow{4}{*}{$\begin{array}{l}\text { PERIOD } \\
\text { (CON- } \\
\text { SECU- } \\
\text { TIVE } \\
\text { DAYS) }\end{array}$} & \multicolumn{6}{|c|}{$\begin{array}{l}\text { DISCHARGE, IN CFS, FOR INDICATED RECURRENCE } \\
\text { INTERVAL, IN YEARS, AND ANNUAL NON- } \\
\text { EXCEEDANCE PROBABILITY, IN PERCENT }\end{array}$} \\
\hline & & & & -1 & & \\
\hline & 2 & 5 & 10 & 20 & 50 & 100 \\
\hline & & & & & & \\
\hline 1 & 23 & 17 & 15 & 13 & 11 & 10 \\
\hline 3 & 26 & 20 & 18 & 16 & 13 & 12 \\
\hline 7 & 30 & 23 & 20 & 18 & 16 & 14 \\
\hline 14 & 32 & 26 & 24 & 22 & 19 & 18 \\
\hline 30 & 35 & 29 & 26 & 24 & 22 & 20 \\
\hline 60 & 36 & 30 & 27 & 25 & 23 & 21 \\
\hline 90 & 38 & 32 & 29 & 26 & 24 & 22 \\
\hline 120 & 39 & 33 & 30 & 27 & 25 & 23 \\
\hline 183 & 43 & 36 & 32 & 30 & 27 & 26 \\
\hline
\end{tabular}

MAGNITUDE AND PROBABILITY OF ANNUAL HIGH FLOW BASED ON PERIOD OF RECORD 1912-82

\begin{tabular}{|c|c|c|c|c|c|c|}
\hline PERIOD & \multicolumn{6}{|c|}{$\begin{array}{c}\text { DISCHARGE, IN CFS, FOR INDICATED RECURRENCE } \\
\text { INTERVAL, IN YEARS, AND ANNUAL } \\
\text { EXCEEDANCE PROBABILITY, IN PERCENT }\end{array}$} \\
\hline TIVE & 2 & 5 & 10 & 25 & 50 & 100 \\
\hline DAYS) & $50 \%$ & \multicolumn{4}{|c|}{ DAYS) } & \\
\hline 1 & 831 & 1270 & 1560 & 1950 & 2240 & 2530 \\
\hline 3 & 669 & 960 & 1140 & 1340 & 1480 & 1620 \\
\hline 7 & 570 & 774 & 886 & 1010 & 1080 & 1150 \\
\hline 15 & 477 & 638 & 724 & 815 & 872 & 921 \\
\hline 30 & 410 & 550 & 627 & 711 & 765 & 812 \\
\hline 60 & 343 & 450 & 507 & 565 & 601 & 632 \\
\hline 90 & 294 & 383 & 430 & 477 & 506 & 531 \\
\hline
\end{tabular}

DURATION TABLE OF DAILY MEAN FLOW FOR PERIOD OF RECORD 1912-82

DISCHARGE, IN CFS, WHICH WAS EQUALED OR EXCEEDED FOR INDICATED PERCENT OF TIME

\begin{tabular}{|c|c|c|c|c|c|c|c|c|c|c|c|c|c|c|}
\hline $5 \%$ & $10 \%$ & $15 \%$ & $20 \%$ & $25 \%$ & $30 \%$ & $40 \%$ & $50 \%$ & $60 \%$ & $70 \%$ & $75 \%$ & $80 \%$ & $85 \%$ & $90 \%$ & $95 \%$ \\
\hline 433 & 316 & 244 & 188 & 144 & 111 & 72 & 56 & 49 & 43 & 41 & 38 & 35 & 32 & 28 \\
\hline
\end{tabular}


10397000 BRIDGE CREEK NEAR FRENCHGLEN, OR

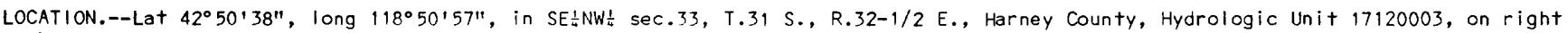
bank at mouth of canyon, $3.5 \mathrm{mi}$ nor theast of Frenchglen.

DRAINAGE AREA.- $-30 \mathrm{mi}^{2}$, approximate!y.

PERIOD OF RECORD.--March to August 1911, January 1912 to September 1916, April to June 1930 , December 1937 to September 1970. Monthly discharge only April to June 1930, publishes in WSP 1314. Published as "near Diamond" 1911-16.

GAGE.--Water-stage recorder and concrete control. Datum of gage is 4,184.93 ft National Geodetic Vertical Datum of 1929 (levels by Fish and Wildiife Service). Prior to Dec. 21, 1937, nonrecording gage at sites within 1 mi upstream at ditferent datums. Dec. 21, 1937, to May 17, 1938, nonrecording gage at site 1,000 ft downstream at difterent datum. May 18, 1938, to Aug. 22, 1939, nonrecording gage at present site and datum.

REMARKS.--No regulation or diversion above station. Low-water flow is sustained by large springs.

AVERAGE DISCHARGE.--36 years (water years $1913-16,1939-70$ ) , $13.5 \mathrm{ft} / \mathrm{s}, 9,780$ acre- $\mathrm{ft} / \mathrm{yr}$.

EXTREMES FOR PERIOD OF RECORD.--Maximum discharge, $301 \mathrm{ft} / \mathrm{s}$ May 19, 1953, gage-height, $2.73 \mathrm{ft}$, from rating curve extended above 65

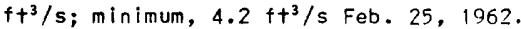

STATISTICAL SUMMARIES

MONTHLY AND ANNUAL MEAN DISCHARGES 1913-70

\begin{tabular}{|c|c|c|c|c|c|c|}
\hline MONTH & $\begin{array}{c}\text { MINIMUM } \\
\text { (CFS) }\end{array}$ & $\begin{array}{c}\text { MAXIMUM } \\
\text { (CFS) }\end{array}$ & $\begin{array}{l}\text { MEAN } \\
\text { (CFS) }\end{array}$ & $\begin{array}{l}\text { STAN- } \\
\text { DARD } \\
\text { DEVIA- } \\
\text { TION } \\
\text { (CFS) }\end{array}$ & $\begin{array}{l}\text { COEFFI- } \\
\text { CIENT OF } \\
\text { VARI- } \\
\text { ATION }\end{array}$ & $\begin{array}{c}\text { PERCENT } \\
\text { OF } \\
\text { ANNUAL } \\
\text { RUNOFF }\end{array}$ \\
\hline OCTOBER & 6.6 & 15 & 12 & 2.1 & .18 & 7.2 \\
\hline NOVEMBER & 1.2 & 15 & 11 & 2.7 & .24 & 7.0 \\
\hline DECEMBER & 6.2 & 16 & 11 & 2.4 & .21 & 7.0 \\
\hline JANUARY & 5.7 & 17 & 11 & 2.7 & .23 & 7.0 \\
\hline FEBRUARY & 5.3 & 25 & 12 & 3.7 & .31 & 7.5 \\
\hline MARCH & 7.8 & 35 & 13 & 4.8 & .36 & 8.3 \\
\hline APRIL & 8.2 & 37 & 19 & 7.2 & .38 & 11.7 \\
\hline MAY & 8.5 & 44 & 22 & 9.9 & .45 & 13.5 \\
\hline JUNE & 8.4 & 25 & 14 & 4.2 & .29 & 8.9 \\
\hline JULY & 7.9 & 16 & 12 & 1.7 & .14 & 7.3 \\
\hline AUGUST & 8.2 & 16 & 12 & 1.7 & .15 & 7.3 \\
\hline SEPTEMBER & 7.7 & 16 & 12 & 1.9 & .16 & 7.4 \\
\hline ANNUAL & 8.3 & 19 & 14 & 2.5 & .19 & 100 \\
\hline
\end{tabular}

MAGNITUDE AND PROBABILITY OF INSTANTANEOUS PEAK FLOW BASED ON PERIOD OF RECORD $1913-70$

DISCHARGE, IN CFS, FOR INDICATED RECURRENCE INTERVAL, IN YEARS, AND ANNUAL EXCEEDANCE PROBABILITY, IN PERCENT

\begin{tabular}{ccccccc}
1.25 & 2 & 5 & 10 & 25 & 50 & 100 \\
$80 \%$ & $50 \%$ & $20 \%$ & $10 \%$ & $4 \%$ & $2 \%$ & $1 \%$ \\
\hline 43 & 91 & 179 & 247 & 342 & 418 & - \\
\hline
\end{tabular}

WEIGHTED SKEW $=-.357$
MAGNITUDE AND PROBABILITY OF ANNUAL LOW FLOW BASED ON PERIOD OF RECORD 1914-70

\begin{tabular}{|c|c|c|c|c|c|c|}
\hline $\begin{array}{l}\text { PERIOD } \\
\text { (CON- } \\
\text { SECU- }\end{array}$ & \multicolumn{6}{|c|}{$\begin{array}{l}\text { DISCHARGE, IN CFS, FOR INDICATED RECURRENCE } \\
\text { INTERVAL, IN YEARS, AND ANNUAL NON- } \\
\text { EXCEEDANCE PROBABILITY, IN PERCENT }\end{array}$} \\
\hline $\begin{array}{l}\text { TIVE } \\
\text { DAYS) }\end{array}$ & $\begin{array}{c}2 \\
50 \%\end{array}$ & $\begin{array}{c}5 \\
20 \%\end{array}$ & $\begin{array}{l}10 \\
10 \%\end{array}$ & $\begin{array}{l}20 \\
5 \%\end{array}$ & $\begin{array}{l}50 \\
2 \%\end{array}$ & $\begin{array}{r}100 \\
1 \%\end{array}$ \\
\hline 1 & -- & -- & - & -- & -- & $\ldots$ \\
\hline 3 & -- & -- & -- & -- & -- & $\ldots$ \\
\hline 7 & -- & -- & -- & -- & -- & $\ldots$ \\
\hline 14 & -- & -- & -- & -- & -- & $\ldots$ \\
\hline 30 & -- & $\rightarrow$ & - & -- & -- & $\rightarrow$ \\
\hline 60 & 10 & 8.2 & 7.2 & 6.5 & 5.6 & $\cdots$ \\
\hline 90 & 10 & 8.5 & 7.5 & 6.7 & 5.8 & - \\
\hline 120 & 11 & 8.7 & 7.7 & 6.9 & 6.0 & - \\
\hline 183 & 11 & 9.3 & 8.3 & 7.5 & 6.6 & $\ldots$ \\
\hline
\end{tabular}

MAGNITUDE AND PROBABILITY OF ANNUAL HIGH FLOW BASED ON PERIOD OF RECORD $1913-70$

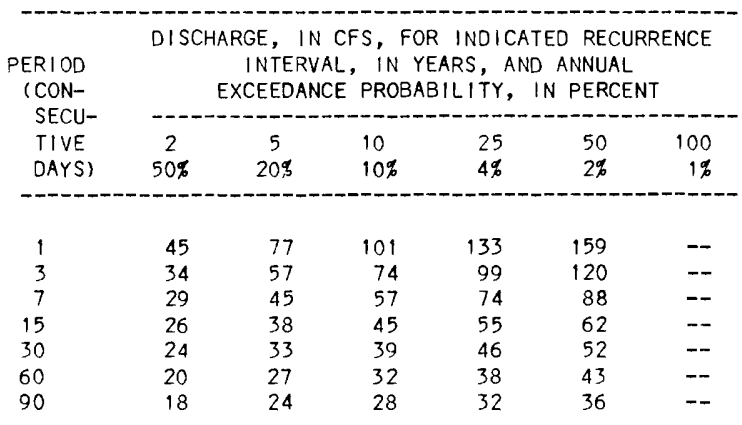

DURATION TABLE OF DAILY MEAN FLOW FOR PERIOD OF RECORD 1913-70

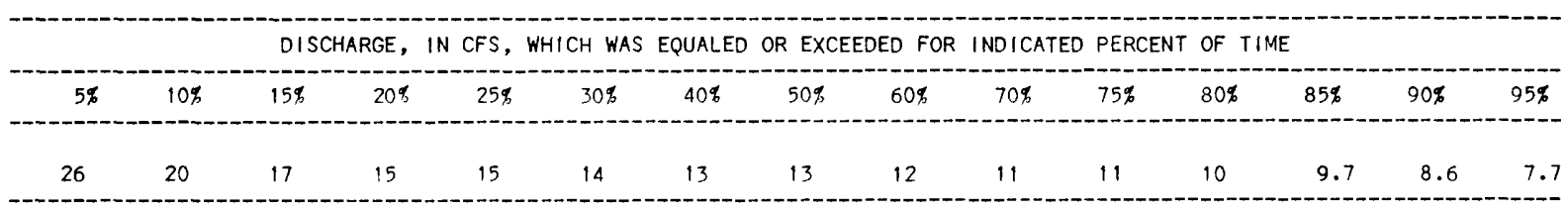




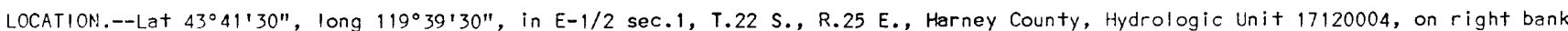

$0.4 \mathrm{mi}$ downstream from Rough Creek, $1.4 \mathrm{mi}$ upstream from Nicoll Creek, and $14 \mathrm{mi}$ northwest of Riley.

DRAINAGE AREA. $--228 \mathrm{mi}^{2}$.

PERIOD OF RECORD.--June 1951 to September 1980.

GAGE.--Water-stage recorder. Datum of gage is 4,449.70 ft National Geodetic Vertical Jatum of 1929 .

REMARKS.--No regulation. Diversions for irrigation above station.

AVERAGE DISCHARGE. - -29 years, $42.8 \mathrm{ft} / \mathrm{s}, 31,010$ acre- $\mathrm{ft} / \mathrm{yr}$.

EXTREMES FOR PERIOD OF RECORD.--Maximum discharge, 1,810 $\mathrm{ft}^{3} / \mathrm{s}$ Dec. 22 , 1964, gage height, $7.49 \mathrm{ft}$; no $\mathrm{flow}$ at $\mathrm{times}$.

STATISTICAL SUMMARIES

MONTHLY AND ANNUAL MEAN DISCHARGES 1952-80

\begin{tabular}{|c|c|c|c|c|c|c|}
\hline MONTH & $\begin{array}{l}\text { M!NIMUM } \\
\text { (CFS) }\end{array}$ & $\begin{array}{l}\text { MAXIMUM } \\
\text { (CFS) }\end{array}$ & $\begin{array}{l}\text { MEAN } \\
\text { (CFS) }\end{array}$ & $\begin{array}{c}\text { STAN- } \\
\text { DARD } \\
\text { DEVIA- } \\
T ! O N \\
\text { (CFS) }\end{array}$ & $\begin{array}{l}\text { COEFFI- } \\
\text { CIENT OF } \\
\text { VARI- } \\
\text { ATION }\end{array}$ & $\begin{array}{c}\text { PERCENT } \\
\text { OF } \\
\text { ANNUAL } \\
\text { RUNOFF }\end{array}$ \\
\hline OCTOBER & 1.9 & 7.1 & 3.4 & 1.3 & .37 & \\
\hline NOVEMBER & 2.6 & 8.8 & 4.9 & 1.5 & .30 & .9 \\
\hline DECEMBER & 1.5 & 202 & 14 & 37 & 2.60 & 2.7 \\
\hline JANUARY & 1.3 & 121 & 21 & 30 & 1.38 & 4.2 \\
\hline FEBRUARY & 1.7 & 169 & 44 & 49 & 1.11 & 8.6 \\
\hline MARCH & 5.4 & 414 & 108 & 95 & .88 & 20.9 \\
\hline APRIL & 11 & 711 & 209 & 145 & .69 & 40.5 \\
\hline MAY & 6.5 & 209 & 79 & 57 & .72 & 15.3 \\
\hline JUNE & 2.6 & 79 & 21 & 14 & .66 & 4.0 \\
\hline JULY & .4 & 15 & 6.1 & 3.5 & .57 & 1.2 \\
\hline AUGUST & .2 & 8.0 & 2.6 & 1.8 & .69 & .3 \\
\hline SEPTEMBER & .9 & 4.9 & 2.3 & 1.0 & .45 & .4 \\
\hline ANNUAL & 7.6 & 85 & 43 & 22 & .52 & 100 \\
\hline
\end{tabular}

MAGNITUDE ANO PROBABILITY OF INSTANTANEOUS PEAK FLOW BASED ON PERIOD OF RECORD 1952-80

DISCHARGE, IN CFS, FOR INDICATEO RECURRENCE INTERVAL, IN YEARS, AND ANNUAL EXCEEDANCE PROBABILITY, IN PERCENT

\begin{tabular}{ccccccc}
1.25 & 2 & 5 & 10 & 25 & 50 & 100 \\
$80 \%$ & $50 \%$ & $20 \%$ & $10 \%$ & $4 \%$ & $2 \%$ & $1 \%$ \\
315 & 590 & 1040 & 1360 & 1790 & 2110 & $\ldots$ \\
\hline WEIGHTED SKEW $=$ & -.376 &
\end{tabular}

MAGN ITUDE AND PROBABILITY OF ANNUAL LOW FLOW BASED ON PERIOD OF RECORD 1953-80

\begin{tabular}{|c|c|c|c|c|c|c|}
\hline $\begin{array}{l}\text { PERIOD } \\
\text { CCON- } \\
\text { SECU- }\end{array}$ & \multicolumn{6}{|c|}{$\begin{array}{l}\text { DISCHARGE, IN CFS, FOR INDICATED RECURRENCE } \\
\text { INTERVAL, IN YEARS, AND ANNUAL NON- } \\
\text { EXCEEDANCE PROBABILITY, IN PERCENT }\end{array}$} \\
\hline TIVE & 2 & 5 & 10 & 20 & 50 & 100 \\
\hline DAYS) & $50 \%$ & $20 \%$ & $10 \%$ & $5 \%$ & $2 \%$ & $1 \%$ \\
\hline 1 & $\sim$ & - & -- & -- & -- & -- \\
\hline 3 & - & -- & -- & -- & -- & - \\
\hline 7 & -- & -- & -- & -- & $=$ & - \\
\hline 14 & 1.6 & .5 & .2 & .1 & 0.0 & -- \\
\hline 30 & 1.7 & .7 & .3 & .2 & .1 & $-\sim$ \\
\hline 60 & 1.9 & 1.0 & .7 & .5 & .3 & - \\
\hline 90 & 2.3 & 1.4 & 1.0 & .8 & .6 & -- \\
\hline 120 & 2.7 & 1.8 & 1.4 & 1.2 & .9 & -- \\
\hline 183 & 3.8 & 2.8 & 2.3 & 1.9 & 1.6 & -- \\
\hline
\end{tabular}

MAGNITUDE AND PROBABILITY OF ANNUAL HIGH FLOW BASED ON PERIOD OF RECORD 1952-80

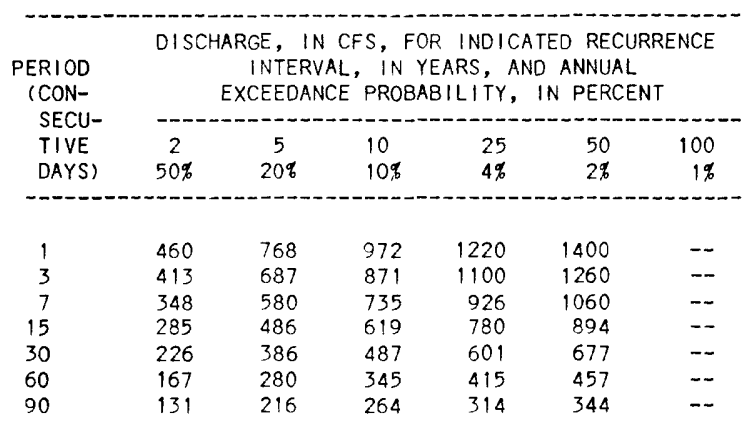

DURATION TABLE OF DAILY MEAN FLOW FOR PERIOD OF RECORD 1952-80
DISCHARGE, IN CFS, WHICH WAS EQUALED OR EXCEEDED FOR INDICATED PERCENT OF TIME

\begin{tabular}{|c|c|c|c|c|c|c|c|c|c|c|c|c|c|c|}
\hline $5 \%$ & $10 \%$ & $15 \%$ & $20 \%$ & $25 \%$ & $30 \%$ & $40 \%$ & $50 \%$ & $60 \%$ & $70 \%$ & $75 \%$ & $80 \%$ & $85 \%$ & $90 \%$ & $95 \%$ \\
\hline 233 & 128 & 77 & 47 & 30 & 21 & 11 & 6.6 & 4.8 & 3.7 & 3.3 & 2.9 & 2.4 & 1.9 & 1.3 \\
\hline
\end{tabular}


10406500 TROUT CREEK NEAR DENIO, NV

LOCATION.--Lat $42^{\circ} 09^{\prime} 20^{\prime \prime}$, long $118^{\circ} 27^{\prime} 30^{\prime \prime}$, in SW $\frac{1}{4}$ sec. 26 , T.39 S., R. 36 E., Harney County, Hydrologic Unit 17120009 , on right bank $0.4 \mathrm{mi}$ upstream from bridge at mouth of canyon, $5 \mathrm{mi}$ east of Trout Creek Panch, and $14 \mathrm{mi}$ northeast of Denio.

DRAINAGE AREA.--88 $\mathrm{mi}^{2}$, approximately.

PERIOD OF RECORD.--March 1911 to March 1912, April 1922 to November 1923, March 1925 to September 1931 (irrigation seasons only), April 1932 to September 1992. Prior to Oct. 1, 1961, published as "near Denio, Oreg."

GAGE.--Water-stage recorder. Datum of gage is 4,351.52 $\mathrm{ft}$ National Geodetic Vertical Datum of 1929. Mar. 25 , 1911, to Mar. 31 ,

1912, nonrecording gage at bridge $0.4 \mathrm{mi}$ downstream at different datum. Apr. 28, 1922, to June 14, 1932, water-stage recorder at site $10 \mathrm{ft}$ upstream at datum $0.50 \mathrm{ft}$ higher.

REMARKS.--No regulation. Diversions for irrigation above station.

AVERAGE DISCHARGE. - -51 years (water years $1923,1933-82$ ), $15.7 \mathrm{ft}^{3} / \mathrm{s}, 11,370$ acre- $\mathrm{ft} / \mathrm{yr}$.

EXTREMES FOR PERIOD OF RECORD.--Maximum discharge, $470 \mathrm{ft} / \mathrm{s}$ Aug. 1, 1933, gage height, $5.26 \mathrm{ft}$, from rating curve extended above 230

$\mathrm{ft}^{3} / \mathrm{s}$; minimum observed, $0.10 \mathrm{ft} / \mathrm{s}$ Aug. 4, 1930, Aug. 1, Sept. 12, 28, 1934. Probably no flow at times Sept. 1-19, 1931.

EXTREMES OUTSIDE PERIOD OF RECORD.--Maximum stage, $6.0 \mathrm{ft}$, caused by cloudburst, probably occurred in 1924 or 1925 .

STATISTICAL SUMMARIES

MONTHLY AND ANNUAL MEAN DISCHARGES 1933-82

\begin{tabular}{|c|c|c|c|c|c|c|}
\hline MONTH & $\begin{array}{l}\text { MIN!MUM } \\
\text { (CFS) }\end{array}$ & $\begin{array}{l}\text { MAXIMUM } \\
\text { (CFS) }\end{array}$ & $\begin{array}{l}\text { MEAN } \\
\text { (CFS) }\end{array}$ & $\begin{array}{l}\text { STAN- } \\
\text { DARD } \\
\text { DEVIA- } \\
\text { TION } \\
\text { (CFS) }\end{array}$ & $\begin{array}{l}\text { COEFFI- } \\
\text { CIENT OF } \\
\text { VARI- } \\
\text { ATION }\end{array}$ & $\begin{array}{c}\text { PERCENT } \\
\text { OF } \\
\text { ANNUAL } \\
\text { RUNOFF }\end{array}$ \\
\hline OCTOBER & 1.8 & 10 & 5.0 & 1.6 & .32 & 2.6 \\
\hline NOVEMBER & 3.0 & 9.7 & 6.2 & 1.7 & .28 & 3.3 \\
\hline DECEMBER & 2.9 & 13 & 6.4 & 2.2 & .34 & 3.4 \\
\hline JANUARY & 2.9 & 24 & 7.3 & 3.7 & .51 & 3.8 \\
\hline FEBRUARY & 3.4 & 29 & 8.8 & 5.0 & .57 & 4.6 \\
\hline MARCH & 4.5 & 69 & 14 & 11 & .76 & 7.3 \\
\hline APRIL & 7.7 & 105 & 33 & 20 & .60 & 17.6 \\
\hline MAY & 4.4 & 151 & 60 & 34 & .56 & 31.4 \\
\hline JUNE & 2.5 & 92 & 33 & 22 & .68 & 17.1 \\
\hline JULY & 1.0 & 41 & 9.7 & 6.8 & .70 & 5.1 \\
\hline AUGUST & .1 & 9.1 & 3.7 & 1.9 & .53 & 1.9 \\
\hline SEPTEMBER & $\cdot 1$ & 6.7 & 3.5 & 1.7 & .47 & 1.9 \\
\hline ANNUAL & 3.8 & 37 & 16 & 7.0 & .44 & 100 \\
\hline
\end{tabular}

MAGNITUDE AND PROBABILITY OF INSTANTANEOUS PEAK FLOW BASED ON PERIOD OF RECORD 1933-82

DISCHARGE, IN CFS, FOR INDICATED RECURRENCE INTERVAL, IN YEARS, AND ANNUAL EXCEEDANCE PROBABILITY, IN PERCENT

\begin{tabular}{ccccccc}
1.25 & 2 & 5 & 10 & 25 & 50 & 100 \\
$80 \%$ & $50 \%$ & $20 \%$ & $10 \%$ & $4 \%$ & $2 \%$ & $1 \%$ \\
\hline 66 & 115 & 195 & 257 & 343 & 413 & 488 \\
\hline
\end{tabular}

WEIGHTED SKEW $=-.147$
MAGNITUDE AND PROBABILITY OF ANNUAL LOW FLOW BASED ON PERIOD OF RECORD 1934-82

\begin{tabular}{|c|c|c|c|c|c|c|}
\hline \multirow{3}{*}{$\begin{array}{l}\text { PERIOD } \\
\text { (CON- } \\
\text { SECU- } \\
\text { TIVE } \\
\text { DAYS) }\end{array}$} & \multicolumn{6}{|c|}{$\begin{array}{l}\text { DISCHARGE, IN CFS, FOR INDICATED RECURRENCE } \\
\text { INTERVAL, IN YEARS, AND ANNUAL NON- } \\
\text { EXCEEDANCE PROBABILITY, IN PERCENT }\end{array}$} \\
\hline & & & & - & $--n$ & \\
\hline & $\begin{array}{c}2 \\
50 \%\end{array}$ & $\begin{array}{c}5 \\
20 \%\end{array}$ & $\begin{array}{l}10 \\
10 \%\end{array}$ & $\begin{array}{l}20 \\
5 \%\end{array}$ & $\begin{array}{l}50 \\
2 \%\end{array}$ & $\begin{array}{r}100 \\
1 \%\end{array}$ \\
\hline 1 & 2.1 & 1. & .6 & .4 & .2 & .1 \\
\hline 3 & 2.2 & 1. & .6 & .4 & .2 & .1 \\
\hline 7 & 2.4 & 1. & .7 & .4 & .2 & .2 \\
\hline 14 & 2.6 & 1. & .8 & .5 & .2 & .2 \\
\hline 30 & 3.0 & 1. & .9 & .6 & .3 & .2 \\
\hline 60 & 3.5 & 1. & 1.1 & .7 & .3 & .2 \\
\hline 90 & 3.9 & 2. & 1.6 & 1.2 & .7 & .5 \\
\hline 120 & 4.3 & 2. & 2.1 & 1.6 & 1.1 & .9 \\
\hline 183 & 5.0 & 3. & 2.9 & 2.4 & 1.9 & 1.6 \\
\hline
\end{tabular}

MAGNITUDE AND PROBABILITY OF ANNUAL HIGH FLOW BASED ON PERIOD OF RECORD 1933-82

\begin{tabular}{|c|c|c|c|c|c|c|}
\hline $\begin{array}{l}\text { PERIOD } \\
\text { (CON- } \\
\text { SECU- }\end{array}$ & \multicolumn{6}{|c|}{$\begin{array}{c}\text { DISCHARGE, IN CFS, FOR INDICATED RECURRENCE } \\
\text { INTERVAL, IN YEARS, AND ANNUAL } \\
\text { EXCEEDANCE PROBABILITY, IN PERCENT }\end{array}$} \\
\hline TIVE & 2 & 5 & 10 & 25 & 50 & 100 \\
\hline DAYS) & $50 \%$ & $20 \%$ & $10 \%$ & $4 \%$ & $2 \%$ & $1 \%$ \\
\hline 1 & 93 & 155 & 200 & 257 & 301 & 346 \\
\hline 3 & 90 & 147 & 183 & 226 & 255 & 282 \\
\hline 7 & 84 & 134 & 162 & 191 & 209 & 224 \\
\hline 15 & 75 & 117 & 140 & 164 & 177 & 188 \\
\hline 30 & 64 & 100 & 120 & 141 & 153 & 163 \\
\hline 60 & 50 & 78 & 94 & 111 & 121 & 130 \\
\hline 90 & 40 & 63 & 75 & 89 & 98 & 106 \\
\hline
\end{tabular}

DURATION TABLE OF DAILY MEAN FLOW FOR PERIOD OF RECORD 1933-82

\begin{tabular}{|c|c|c|c|c|c|c|c|c|c|c|c|c|c|c|}
\hline $5 \%$ & $10 \%$ & $15 \%$ & $20 \%$ & $25 \%$ & $30 \%$ & $40 \%$ & $50 \%$ & $60 \%$ & $70 \%$ & $75 \%$ & $80 \%$ & $85 \%$ & $90 \%$ & $95 \%$ \\
\hline 67 & 43 & 29 & 21 & 15 & 12 & 8.3 & 6.8 & 5.8 & 5.0 & 4.5 & 4.0 & 3.5 & 2.8 & 2.0 \\
\hline
\end{tabular}




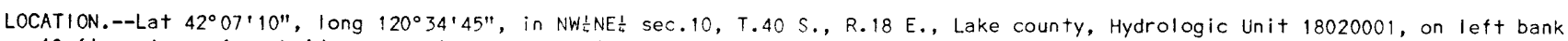
$10 \mathrm{ft}$ upstream from bridge, $2.0 \mathrm{mi}$ downstream from Willow Creek, $2.7 \mathrm{mi}$ downstream from Drews Dam, and $13 \mathrm{mi}$ southwest of Lakeview.

DRAINAGE AREA. $--212 \mathrm{mi}^{2}$.

PERIOD OF RECORD.--January 1909 to September 1930 (vearl; estinate on!y for water year 1920), March 1931 to September 1936 (irrigation seasons only), April 1937 to September 1925, iarcn 1039 to October 1941, February 1942, April 1942 to September 1952, February 1953 to September 1981. Monthly discharge only October 1921 to September 1925, published in WSP 1315-A. Published as "Drew Creek near Lakeview" October 1918 to September 1959.

GAGE.--Water-stage recorder. Datum of gage is $4,827.0 \mathrm{ft}$ National Geodetic Vertical Datum of 1929 (levels by Bureau of Reclamation). See WSP 1931 for history of changes prior to July 4, 1953.

REMARKS.--Record herein, except average discharge, not adjusted for diversion by North Drews Canal. Since 1912 , flow regulated by Drews Reservoir, capacity, 62,550 acre-ft. Diversion for irrigation above station, and since March 1914 , North Drews Canal has diverted above station for irrigation of lands west of Lakeview. Records in files of Oregon Water Resources Department.

AVERAGE DISCHARGE.--50 years (water years 1913-30, 1938, 1940-41, 1947, 1954-81), $70.6 \mathrm{ft} 3 / \mathrm{s}, 51,150 \mathrm{acre}-\mathrm{ft} / \mathrm{yr}$, including diversion by North Drews Canal.

EXTREMES FOR PERIOD OF RECORD.--Maximum discharge, about $3,000 \mathrm{ft}^{\mathrm{s}} / \mathrm{s} \mathrm{Mar}$. 1, 2, 1910, from rating curve extended above $1,200 \mathrm{ft} / \mathrm{s}$; no flow at times.

STATISTICAL SUMMARIES (RIVER DISCHARGE ONLY)

MONTHLY AND ANNUAL MEAN DISCHARGES 1913-81

\begin{tabular}{|c|c|c|c|c|c|c|}
\hline MONTH & $\begin{array}{l}\text { MINIMUM } \\
\text { (CFS) }\end{array}$ & $\begin{array}{c}\text { MAXIMUM } \\
\text { (CFS) }\end{array}$ & $\begin{array}{l}\text { MEAN } \\
\text { (CFS) }\end{array}$ & $\begin{array}{l}\text { STAN- } \\
\text { DARD } \\
\text { DEVIA- } \\
\text { TION } \\
\text { (CFS) }\end{array}$ & $\begin{array}{l}\text { COEFFI- } \\
\text { CIENT OF } \\
\text { VARI- } \\
\text { ATION }\end{array}$ & $\begin{array}{c}\text { PERCENT } \\
\text { OF } \\
\text { ANNUAL } \\
\text { RUNOFF }\end{array}$ \\
\hline OCTOBER & 0.0 & 25 & 3.2 & 4.6 & 1.46 & .5 \\
\hline NOVEMBER & 0.0 & 43 & 3.4 & 6.4 & 1.87 & .6 \\
\hline DECEMBER & 0.0 & 209 & 12 & 39 & 3.26 & 1.9 \\
\hline JANUARY & 0.0 & 361 & 20 & 59 & 2.79 & 3.2 \\
\hline FEBRUARY & .5 & 395 & 41 & 85 & 2.05 & 6.7 \\
\hline MARCH & .7 & 589 & 94 & 128 & 1.37 & 15.2 \\
\hline APRIL & .6 & 922 & 160 & 210 & 1.31 & 26.0 \\
\hline MAY & 6.9 & 531 & 114 & 117 & 1.03 & 18.5 \\
\hline JUNE & 1.8 & 201 & 56 & 33 & .60 & 9.1 \\
\hline JULY & .5 & 80 & 51 & 22 & .44 & 8.2 \\
\hline AUGUST & 0.0 & 84 & 40 & 22 & .55 & 6.6 \\
\hline SEPTEMBER & 0.0 & 63 & 21 & 15 & .72 & 3.4 \\
\hline ANNUAL & 3.9 & 172 & 52 & 42 & .82 & 100 \\
\hline
\end{tabular}

MAGNITUDE ANO PROBABILITY OF INSTANTANEOUS PEAK FLOW BASED ON PERIOD OF RECORD

\begin{tabular}{|c|c|c|c|c|c|c|}
\hline \multicolumn{7}{|c|}{$\begin{array}{l}\text { DISCHARGE, IN CFS, FOR INDICATED RECURRENCE INTERVAL, IN } \\
\text { YEARS, AND ANNUAL EXCEEDANCE PROBABILITY, IN PERCENT }\end{array}$} \\
\hline $\begin{array}{l}1.25 \\
80 \%\end{array}$ & $\begin{array}{c}2 \\
50 \%\end{array}$ & $\begin{array}{c}5 \\
20 \%\end{array}$ & $\begin{array}{l}10 \\
10 \%\end{array}$ & $\begin{array}{l}25 \\
4 \%\end{array}$ & $\begin{array}{l}50 \\
2 q\end{array}$ & $\begin{array}{r}100 \\
17\end{array}$ \\
\hline- & - & - & -- & -- & -- & -- \\
\hline
\end{tabular}

WEIGHTED SKEW $=$
MAGNITUDE AND PROBABILITY OF ANNUAL LOW FLOW BASED ON PERIOD OF RECORD 1914-81

DISCHARGE, IN CFS, FOR INDICATED RECURRENCE
PERIOD
ICON-

MAGNITUDE AND PROBABILITY OF ANNUAL HIGH FLOW BASED ON PERIOD OF RECORD 1913-81

\begin{tabular}{|c|c|c|c|c|c|c|}
\hline $\begin{array}{l}\text { PERIOD } \\
\text { (CON- }\end{array}$ & \multicolumn{6}{|c|}{$\begin{array}{l}\text { INTERVAL, IN YEARS, AND ANNUAL } \\
\text { EXCEEDANCE PROBABILITY, IN PERCENT }\end{array}$} \\
\hline SECU- & & & 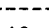 & & & \\
\hline TIVE & 2 & 5 & 10 & 25 & 50 & 100 \\
\hline DAYS) & $50 \%$ & $20 \%$ & $10 \%$ & $4 \%$ & $2 \%$ & $1 \%$ \\
\hline 1 & 255 & 636 & 993 & 1550 & 2060 & 2620 \\
\hline 3 & 250 & 616 & 951 & 1470 & 1920 & 2410 \\
\hline 7 & 240 & 582 & 885 & 1340 & 1720 & 2140 \\
\hline 15 & 215 & 509 & 767 & 1150 & 1470 & 1810 \\
\hline 30 & 176 & 404 & 602 & 898 & 1150 & 1420 \\
\hline 60 & 133 & 299 & 423 & 620 & 784 & 963 \\
\hline 90 & 111 & 231 & 329 & 469 & 582 & 702 \\
\hline
\end{tabular}

DURATION TABLE OF DAILY MEAN FLOW FOR PERIOD OF RECORD 1913-81

\begin{tabular}{|c|c|c|c|c|c|c|c|c|c|c|c|c|c|c|}
\hline $5 \%$ & $10 \%$ & $15^{\circ}$ & 209 & $25 \%$ & $30^{\circ}$, & $40^{\circ}$, & $50 \%$ & $60 \%$ & $70^{\circ}$ & $75 \%$ & 80,0 & $85 \%$ & $90 \%$ & $95 \%$ \\
\hline 266 & 106 & 78 & 67 & 56 & 45 & 25 & 12 & 5.3 & 3.3 & 2.2 & 1.6 & 1.2 & 1.0 & .6 \\
\hline
\end{tabular}


11340500 COTTONWOOD CREEK NEAR LAKEVIEW, OR

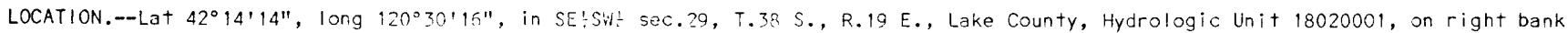
$0.5 \mathrm{mi}$ downstream from Cottonwood Dam and $9 \mathrm{mi}$ nor thwest of Lakeview.

DRAINAGE AREA. $--32.9 \mathrm{mi}^{2}$.

PERIOD OF RECORD.--November 1908 to September 1919, Nlay 1924 to November 1935, March to December 1936, April to December 1937, April 1938 to November 1942, March to November 1943, March to October 1944, February to November 1945, March 1946 to September 1981. Monthly discharge only May 1924 to september 1925 , published in WSP 1315-A.

GAGE.--Water-stage recorder. Datum of jage is $4,949.37 \mathrm{ft}$ National Ceodetic Vertical Datum of 1929 (levels by Bureau of Reclamation). Prior to June 1, 1919, and May 1, 1924, to June 3, 1932, nonrecording gage at several sites within 0.6 mi upstream at different datums. June 1 to Sept. 30, 1919, and June 4, 1932, to Sept. 14, 1961, water-stage recorder at site 0.6 mi upstream at different datums.

REMARKS.--Flow regulated since 1923 by Cottonwood Reservoir, capacity, 7,540 acre-ft. Since October 1961 , 240 acre-ft unregulated storage in Cottonwood Meadows, $9 \mathrm{mi}$ upstream. Diversions for irrigation above station. Records in files of Oregon Water Resources Department.

AVERAGE DISCHARGE.--60 years (water years 1910-19, 1925-35, 1939-42, 1947-81), $21.1 \mathrm{ft}+\mathrm{s}, 15,290 \mathrm{acre}-\mathrm{ft} / \mathrm{yr}$.

EXTREMES FOR PERIOD OF RECORD.--Maxinum discharge, between 500 and $1,000 \mathrm{ft} / \mathrm{s}$ during period Apr. 26 to May 1 , 1927 , when natural flow, estimated as $170 \mathrm{ft}^{3} / \mathrm{s}$, was augmented by water escaping from reservoir through break in outlet conduit near control gates; no flow at times.

STATISTICAL SUMHARIES (BEFORE THE CONSTRUCTION OF COTTONWOOD RESERVOIR)

MONTHLY AND ANNUAL MEAN DISCHARGES 1910-19

\begin{tabular}{|c|c|c|c|c|c|c|}
\hline MONTH & $\begin{array}{l}\text { MINIMUM } \\
\text { (CFS) }\end{array}$ & $\begin{array}{l}\text { MAXIMUM } \\
\text { (CFS) }\end{array}$ & $\begin{array}{l}\text { MEAN } \\
\text { (CFS) }\end{array}$ & $\begin{array}{l}\text { STAN- } \\
\text { DARD } \\
\text { DEV!A- } \\
\text { TION } \\
\text { (CFS) }\end{array}$ & $\begin{array}{l}\text { COEFFI- } \\
\text { CIENT OF } \\
\text { VARI- } \\
\text { ATION }\end{array}$ & $\begin{array}{c}\text { PERCENT } \\
\text { OF } \\
\text { ANNUAL } \\
\text { RUNOFF }\end{array}$ \\
\hline OCTOBER & 2.2 & 4.7 & 3.4 & .9 & .26 & 1.0 \\
\hline NOVEMBER & 5.0 & 50 & 12 & 14 & 1.12 & 3.7 \\
\hline DECEMBER & 3.7 & 34 & 11 & 9.9 & .90 & 3.3 \\
\hline JANUARY & 2.0 & 29 & 10 & 9.8 & .95 & 3.1 \\
\hline FEBRUARY & 3.4 & 41 & 14 & 13 & .93 & 4.3 \\
\hline MARCH & 8.6 & 130 & 51 & 40 & .65 & 18.7 \\
\hline APRIL & 39 & 156 & 94 & 40 & .43 & 28.6 \\
\hline MAY & 17 & 137 & 69 & 36 & .52 & 21.1 \\
\hline JUNE & 4.7 & 89 & 36 & 30 & .83 & 11.0 \\
\hline JULY & 1.7 & 18 & 9.6 & 5.9 & .61 & 2.9 \\
\hline AUGUST & 1.4 & 7.6 & 4.1 & 2.1 & .51 & 1.3 \\
\hline SEPTEMBER & 1.5 & 5.5 & 3.0 & 1.2 & .40 & .9 \\
\hline ANNUAL & 13 & 40 & 27 & 9.4 & .34 & 100 \\
\hline
\end{tabular}

MAGNITUDE AND PROBABILITY OF INSTANTANEOUS PEAK FLOW BASED ON PERIOD OF RECORD 1990-19

DISCHARGE, IN CFS, FOR INDICATED RECURRENCE INTERVAL, IN YEARS, AND ANNUAL EXCEEDANCE PROBABILITY, IN PERCENT

\begin{tabular}{ccccccc}
1.25 & 2 & 5 & 10 & 25 & 50 & 100 \\
$80 \%$ & $50 \%$ & $20 \%$ & $10 \%$ & $4 \%$ & $2 \%$ & $1 \%$ \\
\hline 185 & 239 & 304 & 343 & - & - & - \\
\hline
\end{tabular}

WEIGHTED SKEW $=-.188$
MAGNITUDE AND PROBABILITY OF ANNUAL LOW FLOW BASED ON PERIOD OF RECORD 1911-19

\begin{tabular}{|c|c|c|c|c|c|c|}
\hline $\begin{array}{r}\text { PERIOD } \\
\text { (CON- }\end{array}$ & \multicolumn{6}{|c|}{$\begin{array}{l}\text { DISCHARGE, IN CFS, FOR INDICATED RECURRENCE } \\
\text { INTERVAL, IN YEARS, AND ANNUAL NON- } \\
\text { EXCEEDANCE PROBABILITY, IN PERCENT }\end{array}$} \\
\hline SECU- & & 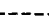 & -- & & -- & -1 \\
\hline TIVE & 2 & 5 & 10 & 20 & 50 & 100 \\
\hline DAYS) & $50 \%$ & $20 \%$ & $10 \%$ & $5 \%$ & $2 \%$ & $1 \%$ \\
\hline \multicolumn{7}{|c|}{ 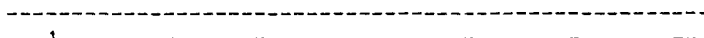 } \\
\hline i & -- & -- & -- & -- & -- & -- \\
\hline 3 & -- & -- & -- & -- & -- & -- \\
\hline 7 & -- & -- & -- & -- & $=-$ & -- \\
\hline 14 & -- & -- & -- & -- & -- & -- \\
\hline 30 & -- & -- & -- & -- & -- & -- \\
\hline 60 & -- & -- & -- & -- & -- & -- \\
\hline 90 & -- & -- & -- & -- & - & -- \\
\hline 120 & -- & -- & -- & -- & -- & -- \\
\hline 183 & -- & $=-$ & -- & $\rightarrow$ & $=-$ & -- \\
\hline
\end{tabular}

NOTE: LESS THAN 10 NON-ZERO EVENTS RECORDED.
:AAGNITUDE AND PROBABILITY OF ANNUAL HIGH FLOW BASED ON PERIOD OF RECORD 1910-19

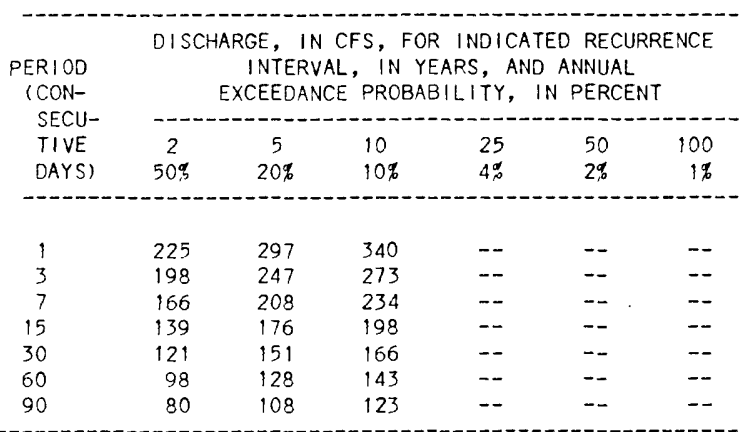

DURATION TABLE OF DAILY MEAN FLOW FOR PERIOD OF RECORD 1910-19

DISCHARGE, IN CFS, WHICH WAS EQUALED OR EXCEEDED FOR INDICATED PERCENT OF TIME

\begin{tabular}{|c|c|c|c|c|c|c|c|c|c|c|c|c|c|c|}
\hline $5 \%$ & $10 \%$ & $15 \%$ & $20 \%$ & $25 \%$ & $30 \%$ & $40 \%$ & $50 \%$ & $60 \%$ & $70 \%$ & $75 \%$ & $80 \%$ & $85 \%$ & $90 \%$ & $95 \%$ \\
\hline 122 & 90 & 65 & 42 & 31 & 20 & 12 & 7.7 & 5.7 & 4.3 & 3.8 & 3.4 & 3.1 & 2.3 & 2.0 \\
\hline
\end{tabular}


GOOSE LAKE BASIN

11340500 COTTONWOOD CREEK NEAR LAKEVIEW, OR--Continued

STATISTICAL SUMMARIES (AFTER THE COMPLETION OF COTTONWOOD RESERVOIR)

MONTHLY AND ANNUAL MEAN DISCHARGES 1925-81

\begin{tabular}{|c|c|c|c|c|c|c|}
\hline MONTH & $\begin{array}{l}\text { MINIMUM } \\
\text { (CFS) }\end{array}$ & $\begin{array}{l}\text { MAXIMUM } \\
\text { (CFS) }\end{array}$ & $\begin{array}{l}\text { MEAN } \\
\text { (CFS) }\end{array}$ & $\begin{array}{l}\text { STAN- } \\
\text { DARD } \\
\text { DEVIA- } \\
\text { TION } \\
\text { (CFS) }\end{array}$ & $\begin{array}{l}\text { COEFFI- } \\
\text { CIENT OF } \\
\text { VARI- } \\
\text { ATION }\end{array}$ & $\begin{array}{c}\text { PERCENT } \\
\text { OF } \\
\text { ANNUAL } \\
\text { RUNOFF }\end{array}$ \\
\hline OCTOBER & .3 & 16 & 2.7 & 2.9 & 1.04 & 1.1 \\
\hline NOVEMBER & .1 & 26 & 3.2 & 4.1 & 1.26 & 1.3 \\
\hline DECEMBER & .1 & 46 & 5.5 & 9.5 & 1.72 & 2.3 \\
\hline $\begin{array}{l}\text { JANUARY } \\
\text { FEBRUARY }\end{array}$ & $\begin{array}{l}0.0 \\
0.0\end{array}$ & $\begin{array}{r}62 \\
100\end{array}$ & $\begin{array}{l}5.5 \\
7.8\end{array}$ & $\begin{array}{l}12 \\
16\end{array}$ & $\begin{array}{l}2.13 \\
2.03\end{array}$ & $\begin{array}{l}2.3 \\
3.3\end{array}$ \\
\hline MARCH & 0.0 & 77 & 15 & 21 & 1.39 & 6.2 \\
\hline APRIL & .5 & 143 & 41 & 39 & .96 & 17.1 \\
\hline MAY & 1.1 & 153 & 55 & 35 & .64 & 23.0 \\
\hline JUNE & 3.0 & 100 & 44 & 21 & .47 & 18.4 \\
\hline JULY & 1.0 & 74 & 31 & 16 & .53 & 12.9 \\
\hline AUGUST & 0.0 & 61 & 20 & 17 & .85 & 8.5 \\
\hline SEPTEMBER & .1 & 39 & 8.5 & 9.9 & 1.17 & 3.5 \\
\hline ANNUAL & 2.2 & 56 & 20 & 11 & .58 & 100 \\
\hline
\end{tabular}

MAGNITUDE AND PROBABILITY OF INSTANTANEOUS PEAK FLOW BASED ON PERIOD OF RECORD

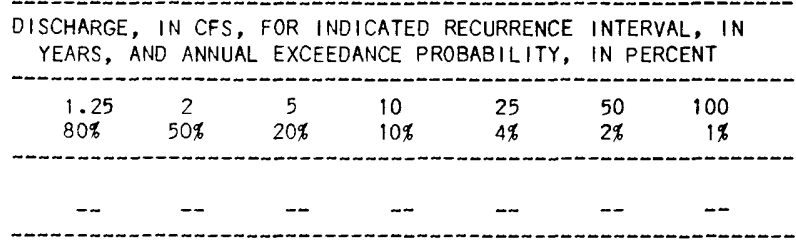

WEIGHTED SKEW $=$
MAGNITUDE AND PROBABILITY OF ANNUAL LOW FLOW BASED ON PERIOD OF RECORD 1926-81

\begin{tabular}{|c|c|c|c|c|c|c|}
\hline \multirow{4}{*}{$\begin{array}{l}\text { PERIOD } \\
\text { (CON- } \\
\text { SECU- } \\
\text { TIVE } \\
\text { DAYS) }\end{array}$} & \multicolumn{6}{|c|}{$\begin{array}{l}\text { DISCHARGE, IN CFS, FOR INDICATED RECURRENCE } \\
\text { INTERVAL, IN YEARS, AND ANNUAL NON- } \\
\text { EXCEEDANCE PROBABILITY, IN PERCENT }\end{array}$} \\
\hline & $-\infty$ & $-n$ & - & - & $\sim$ & -- \\
\hline & 2 & 5 & 10 & 20 & 50 & 100 \\
\hline & $50 \%$ & $20 \%$ & $10 \%$ & $5 \%$ & $2 \%$ & $1 \%$ \\
\hline 1 & .4 & .2 & .1 & 0.0 & 0.0 & 0.0 \\
\hline 3 & .4 & .2 & .1 & .1 & 0.0 & 0.0 \\
\hline 7 & .5 & .2 & .1 & .1 & 0.0 & 0.0 \\
\hline 14 & .5 & .2 & .1 & .1 & .1 & 0.0 \\
\hline 30 & .6 & .3 & .2 & .1 & .1 & .1 \\
\hline 60 & 1.1 & .4 & .2 & .1 & .1 & .1 \\
\hline 90 & 1.3 & .5 & .3 & .2 & .1 & .1 \\
\hline 120 & 1.6 & .7 & .4 & .2 & .1 & .1 \\
\hline 183 & 2.5 & 1.2 & .8 & .6 & .4 & .3 \\
\hline
\end{tabular}

MAGNITUDE AND PROBABILITY OF ANNUAL HIGH FLOW BASED ON PERIOD OF RECORD 1925-81

\begin{tabular}{|c|c|c|c|c|c|c|}
\hline \multirow{3}{*}{$\begin{array}{l}\text { PERIOD } \\
\text { (CON- } \\
\text { SECU- } \\
\text { TIVE } \\
\text { DAYS) }\end{array}$} & \multicolumn{6}{|c|}{$\begin{array}{c}\text { DISCHARGE, IN CFS, FOR INDICATED RECURRENCE } \\
\text { INTERVAL, IN YEARS, AND ANNUAL } \\
\text { EXCEEDANCE PROBABILITY, IN PERCENT }\end{array}$} \\
\hline & 2 & 5 & 10 & 25 & 50 & 100 \\
\hline & $50 \%$ & $20 \%$ & $10 \%$ & $4 \%$ & 28 & 18 \\
\hline 1 & 111 & 195 & 253 & 328 & 382 & 435 \\
\hline 3 & 103 & 178 & 229 & 295 & 343 & 390 \\
\hline 7 & 91 & 152 & 193 & 245 & 284 & 321 \\
\hline 15 & 78 & 124 & 155 & 193 & 220 & 246 \\
\hline 30 & 63 & 102 & 128 & 160 & 184 & 207 \\
\hline 60 & 53 & 85 & 105 & 130 & 148 & 164 \\
\hline 90 & 47 & 75 & 91 & 110 & 123 & 134 \\
\hline
\end{tabular}

DURATION TABLE OF DAILY MEAN FLOW FOR PERIOD OF RECORD 1925-81

\begin{tabular}{|c|c|c|c|c|c|c|c|c|c|c|c|c|c|c|}
\hline $5 \%$ & $10 \%$ & $15 \%$ & $20 \%$ & $25 \%$ & $30 \%$ & $40 \%$ & $50 \%$ & $60 \%$ & $70 \%$ & $75 \%$ & $80 \%$ & $85 \%$ & $90 \%$ & $95 \%$ \\
\hline 84 & 60 & 47 & 37 & 29 & 22 & 10 & 4.3 & 2.7 & 1.8 & 1.4 & 1.1 & .8 & .5 & .2 \\
\hline
\end{tabular}


11493500 WILLIAMSON RIVER NEAR KLAMATH AGENCY, OR

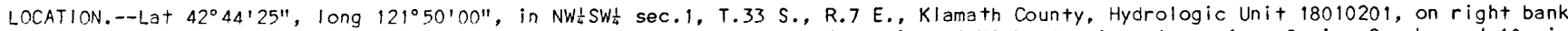
$250 \mathrm{ft}$ downstream from highway bridge, $0.6 \mathrm{mi}$ southwest of railroad station at Kirk, $10 \mathrm{mi}$ upstream from Spring Creek, and $10 \mathrm{mi}$ nor theast of Klamath Agency.

DRAINAGE AREA. $-1,290 \mathrm{mi}^{2}$, approximately.

PERIOD OF RECORO.--March 1908 to January 1909, April 1909 to June 1910, October 1954 to September 1982 . Monthly discharge only June 1910, published in WSP $1315-B$.

GAGE.--Water-stage recorder. Datum of gage is 4,483.16 ft National Geodetic Vertical Datum of 1929. Mar. 25 , 1908, to June 30, 1910, nonrecording gage or water-stage recorder at two sites about $0.5 \mathrm{mi}$ upstream at ditferent datums. Oct. 1 , 1954 , to Sept. 30. 1955, water-stage recorder at present site at datum $2.05 \mathrm{ft}$ higher.

REMARKS.--Flow affected by natural storage in Klamath Marsh. Small diversions above station for irrigation in vicinity of marsh. AVERAGE DISCHARGE. -28 years (water years $1955-82$ ), $200 \mathrm{ft} / \mathrm{s}, 144,900$ acre $-\mathrm{ft} / \mathrm{yr}$.

EXTREMES FOR PERIOD OF RECORD.--Maximum discharge observed, 1,590 ft3/s Mar. 13, 1910, gage height, $3.7 \mathrm{ft}$, site and datum then in use, from rating curve extended above $800 \mathrm{ft} / \mathrm{s}$; maximum gage height, $5.57 \mathrm{ft}$ Mar. 3, 1958; no flow at times during 1960-74, $1977-81$.

STATISTICAL SUMMARIES

MONTHLY AND ANNUAL MEAN DISCHARGES 1955-82

\begin{tabular}{|c|c|c|c|c|c|c|}
\hline MONTH & $\begin{array}{l}\text { MINIMUM } \\
\text { (CFS) }\end{array}$ & $\begin{array}{l}\text { MAXIMUM } \\
\text { (CFS) }\end{array}$ & $\begin{array}{l}\text { MEAN } \\
\text { (CFS) }\end{array}$ & $\begin{array}{l}\text { STAN- } \\
\text { DARD } \\
\text { DEVIA- } \\
\text { TION } \\
\text { (CFS) }\end{array}$ & $\begin{array}{l}\text { COEFFI- } \\
\text { CIENT OF } \\
\text { VARI- } \\
\text { ATION }\end{array}$ & $\begin{array}{c}\text { PERCENT } \\
\text { OF } \\
\text { ANNUAL } \\
\text { RUNOFF }\end{array}$ \\
\hline OCTOBER & 0.0 & 255 & 45 & 71 & 1.57 & 1.9 \\
\hline NOVEMBER & 0.0 & 391 & 127 & 114 & .90 & 5.3 \\
\hline DECEMBER & 56 & 580 & 245 & 126 & .51 & 10.2 \\
\hline JANUARY & 62 & 730 & 257 & 171 & .67 & 10.7 \\
\hline FEBRUARY & 60 & 799 & 335 & 184 & .55 & 13.9 \\
\hline MARCH & 128 & 1033 & 453 & 220 & .49 & 18.8 \\
\hline APRIL & 128 & 1080 & 470 & 230 & .49 & 19.5 \\
\hline MAY & 28 & 952 & 277 & 201 & .73 & 11.5 \\
\hline JUNE & 0.0 & 531 & 127 & 134 & 1.06 & 5.3 \\
\hline JULY & 0.0 & 332 & 46 & 81 & 1.74 & 1.9 \\
\hline AUGUST & 0.0 & 146 & 15 & 34 & 2.34 & .6 \\
\hline SEPTEMBER & 0.0 & 96 & 12 & 26 & 2.12 & .5 \\
\hline NNUAL & 62 & 468 & 200 & 99 & .50 & 100 \\
\hline
\end{tabular}

MAGNITUDE AND PROBABILITY OF INSTANTANEOUS PEAK FLOW BASED ON PERIOD OF RECORD 1955-82

DISCHARGE, IN CFS, FOR INDICATED RECURRENCE INTERVAL, IN YEARS, AND ANNUAL EXCEEDANCE PROBABILITY, IN PERCENT

\begin{tabular}{ccccccc}
1.25 & 2 & 5 & 10 & 25 & 50 & 100 \\
$80 \%$ & $50 \%$ & $20 \%$ & $10 \%$ & $4 \%$ & $2 \%$ & $1 \%$ \\
\hline 490 & 666 & 908 & 1070 & 1270 & 1420 & \\
\hline
\end{tabular}

WEIGHTED SKEW $=.017$
MAGNITUDE AND PROBABILITY OF ANNUAL LOW FLOW BASED ON PERIOD OF RECORD 1956-82

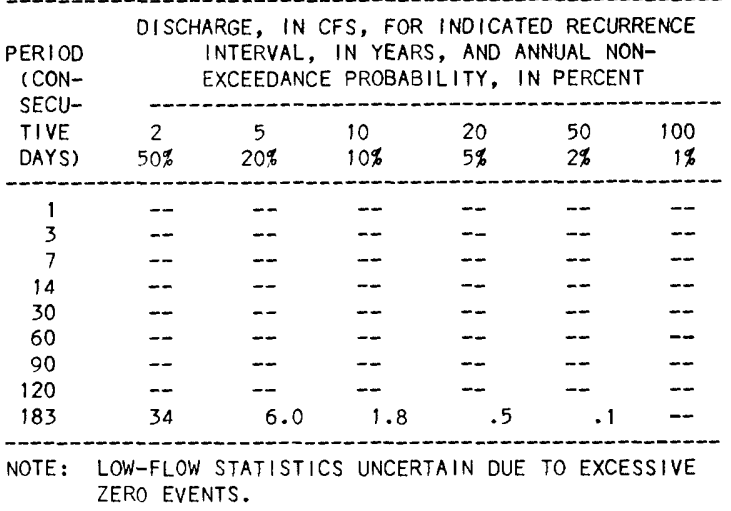

MAGNITUDE AND PROBABILITY OF ANNUAL HIGH FLOW BASED ON PERIOD OF RECORD 1955-82

\begin{tabular}{|c|c|c|c|c|c|c|}
\hline $\begin{array}{l}\text { PERIOD } \\
\text { (CON- } \\
\text { SECU- }\end{array}$ & \multicolumn{6}{|c|}{$\begin{array}{l}\text { DISCHARGE, IN CFS, FOR INDICATED RECURRENCE } \\
\text { INTERVAL, IN YEARS, AND ANNUAL } \\
\text { EXCEEDANCE PROBABILITY, IN PERCENT }\end{array}$} \\
\hline TIVE & 2 & 5 & 10 & 25 & 50 & 100 \\
\hline DAYS) & $50 \%$ & $20 \%$ & $10 \%$ & $4 \%$ & $2 \%$ & $1 \%$ \\
\hline 1 & 655 & 904 & 1050 & 1220 & 1340 & - \\
\hline 3 & 650 & 899 & 1050 & 1220 & 1330 & - \\
\hline 7 & 638 & 883 & 1030 & 1200 & 1310 & -- \\
\hline 15 & 616 & 850 & 990 & 1150 & 1260 & -- \\
\hline 30 & 571 & 783 & 910 & 1060 & 1160 & -- \\
\hline 60 & 492 & 684 & 807 & 956 & 1060 & - \\
\hline 90 & 431 & 600 & 712 & 854 & 959 & - \\
\hline
\end{tabular}

DURATION TABLE OF DAILY MEAN FLOW FOR PERIOD OF RECORD 1955-82

\begin{tabular}{|c|c|c|c|c|c|c|c|c|c|c|c|c|c|c|}
\hline & & DIS & RGE, & CFS, & $\mathrm{ICH}$ WA & QUALE & OR EXC & EO FO & NO ICA & PERC & OF & & & \\
\hline $5 \%$ & $10 \%$ & $15 \%$ & $20 \%$ & $25 \%$ & $30 \%$ & $40 \%$ & $50 \%$ & $60 \%$ & $70 \%$ & $75 \%$ & $80 \%$ & $85 \%$ & $90 \%$ & $95 \%$ \\
\hline 687 & 533 & 430 & 373 & 316 & 272 & 198 & 134 & 76 & 27 & 7.2 & .1 & 0.0 & 0.0 & 0.0 \\
\hline
\end{tabular}




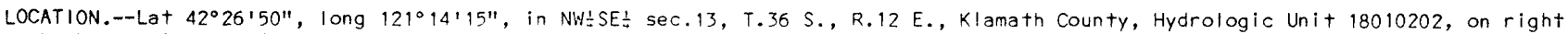
bank $1.6 \mathrm{mi}$ east of Beatty, and $4.6 \mathrm{mi}$ upstream from Sycan River.

DRAINAGE AREA. $--513 \mathrm{mi}^{2}$.

PERIOD OF RECORD.--April to September 1912 and November 1912 to September 1913 (fragmentary), October 1913 to September 1915 , February to November 1916. March 1917 to June 1918, May 1919 to October 1920, February 1921 to September 1926 (irrigation seasons only), October 1953 to September 1982. Monthly discharge only October 1913, published in WSP 1315-B. Prior to October 1917, published as "near Yainax."

GAGE.--Water-stage recorder. Datum of gage is 4,305.35 ft National Geodetic Vertical Datum of 1929. Apr. 19, 1912, to Feb. 19, 1914, nonrecording gage, Feb. 20, 1914, to Sept. 11, 1917, water-stage recorder; and Sept. 12, 1917, to Sept. 30, 1926, nonrecording gage, at site $2 \mathrm{mi}$ upstream at different datum.

REMARKS.--No regulation. Diversions for irrigation above station in the vicinity of Bly.

AVERAGE DISCHARGE.--32 years (water years 1914-15, 1920, 1954-82), $309 \mathrm{ft} / \mathrm{s}, 223,900 \mathrm{acre-ft/yr}$.

EXTREMES FOR PERIOD OF RECORD.--Maximum discharge, $6,980 \mathrm{ft}^{3} / \mathrm{s} \mathrm{Dec.} \mathrm{23,} \mathrm{1964,} \mathrm{gage} \mathrm{height,} 12.19 \mathrm{ft}$; minimum, $50 \mathrm{ft} / \mathrm{s}$ Aug. 25 , 1981 .

STATISTICAL SUMMARIES

MONTHLY AND ANNUAL MEAN DISCHARGES 1954-82

\begin{tabular}{|c|c|c|c|c|c|c|}
\hline MONTH & $\begin{array}{l}\text { MINIMUM } \\
\text { (CFS) }\end{array}$ & $\begin{array}{l}\text { MAXIMUM } \\
\text { (CFS) }\end{array}$ & $\begin{array}{l}\text { MEAN } \\
\text { (CFS) }\end{array}$ & $\begin{array}{l}\text { STAN- } \\
\text { DARD } \\
\text { DEVIA- } \\
\text { TION } \\
\text { (CFS) }\end{array}$ & $\begin{array}{l}\text { COEFFI- } \\
\text { CIENT OF } \\
\text { VARI- } \\
\text { ATION }\end{array}$ & $\begin{array}{c}\text { PERCENT } \\
\text { OF } \\
\text { ANNUAL } \\
\text { RUNOFF }\end{array}$ \\
\hline OCTOBER & 122 & 422 & 169 & 56 & .33 & 4.4 \\
\hline NOVEMBER & 140 & 410 & 197 & 64 & .32 & 5.1 \\
\hline DECEMBER & 133 & 1157 & 289 & 218 & .75 & 7.5 \\
\hline JANUARY & 128 & 898 & 307 & 218 & .71 & 8.0 \\
\hline FEBRUARY & 151 & 1102 & 366 & 225 & .61 & 9.5 \\
\hline MARCH & 147 & 1217 & 435 & 221 & .51 & 11.3 \\
\hline APRIL & 131 & 1673 & 609 & 329 & .54 & 15.9 \\
\hline MAY & 163 & 1703 & 706 & 392 & .56 & 18.4 \\
\hline JUNE & 147 & 757 & 348 & 164 & .47 & 9.1 \\
\hline JULY & 89 & 313 & 164 & 55 & .34 & 4.3 \\
\hline AUGUST & 72 & 210 & 121 & 31 & .25 & 3.2 \\
\hline SEPTEMBER & 85 & 173 & 130 & 20 & .15 & 3.4 \\
\hline ANNUAL & 139 & 643 & 320 & 124 & .39 & 100 \\
\hline
\end{tabular}

MAGNITUDE AND PROBABILITY OF INSTANTANEOUS PEAK FLOW BASED ON PERIOD OF RECORD 1914-82

\begin{tabular}{ccccccc} 
DISCHARGE, IN CFS, FOR INDICATED RECURRENCE & INTERVAL, IN \\
YEARS, AND ANNUAL EXCEEDANCE PROBABILITY, IN PERCENT \\
\hline 1.25 & 2 & 5 & 10 & 25 & 50 & 100 \\
$80 \%$ & $50 \%$ & $20 \%$ & $10 \%$ & $4 \%$ & $2 \%$ & $1 \%$ \\
\hline 680 & 1260 & 2330 & 3220 & 4550 & 5680 & -
\end{tabular}

WEIGHTED SKEW $=.010$

MAGNITUDE AND PROBABILITY OF ANNUAL LOW FLOW BASED ON PERIOD OF RECORD 1955-82

\begin{tabular}{|c|c|c|c|c|c|c|}
\hline $\begin{array}{l}\text { PERIOD } \\
\text { (CON- }\end{array}$ & \multirow{2}{*}{\multicolumn{6}{|c|}{$\begin{array}{l}\text { DISCHARGE, IN CFS, FOR INDICATED RECURRENCE } \\
\text { INTERVAL, IN YEARS, AND ANNUAL NON- } \\
\text { EXCEEDANCE PROBABILITY, IN PERCENT }\end{array}$}} \\
\hline SECU- & & & & & & \\
\hline TIVE & 2 & 5 & 10 & 20 & 50 & 100 \\
\hline DAYS) & $50 \%$ & $20 \%$ & $10 \%$ & $5 \%$ & $2 \%$ & $1 \%$ \\
\hline & & & & & & \\
\hline 1 & 97 & 78 & 69 & 62 & 54 & - \\
\hline 3 & 98 & 80 & 71 & 64 & 56 & - \\
\hline 7 & 101 & 82 & 73 & 65 & 58 & - \\
\hline 14 & 104 & 84 & 75 & 68 & 60 & $\ldots$ \\
\hline 30 & 110 & 90 & 80 & 73 & 65 & -1 \\
\hline 60 & 118 & 99 & 91 & 84 & 77 & - \\
\hline 90 & 126 & 108 & 100 & 93 & 87 & -- \\
\hline 120 & 134 & 117 & 110 & 104 & 38 & $\cdots$ \\
\hline 183 & 154 & 137 & 129 & 123 & 117 & - \\
\hline
\end{tabular}

MAGNITUDE AND PROBABILITY OF ANNUAL HIGH FLOW BASED ON PERIOD OF RECORD 1954-82

\begin{tabular}{|c|c|c|c|c|c|c|}
\hline $\begin{array}{l}\text { PERIOD } \\
\text { (CON- } \\
\text { SECU- }\end{array}$ & \multicolumn{6}{|c|}{$\begin{array}{l}\text { DISCHARGE, IN CFS, FOR INDICATED RECURRENCE } \\
\text { INTERVAL, IN YEARS, AND ANNUAL } \\
\text { EXCEEDANCE PROBABILITY, IN PERCENT }\end{array}$} \\
\hline TIVE & 2 & 5 & 10 & 25 & 50 & 100 \\
\hline DAYS) & $50 \%$ & $20 \%$ & $10 \%$ & $4 \%$ & $2 \%$ & $1 \%$ \\
\hline 1 & 1390 & 2440 & 3220 & 4290 & 5130 & -- \\
\hline 3 & 1210 & 2130 & 2840 & 3810 & 4590 & -- \\
\hline 7 & 1030 & 1760 & 2300 & 3040 & 3620 & -- \\
\hline 15 & 906 & 1450 & 1820 & 2280 & 2610 & -- \\
\hline 30 & 784 & 1220 & 1500 & 1830 & 2070 & -- \\
\hline 60 & 659 & 1010 & 1230 & 1510 & 1700 & -- \\
\hline 90 & 585 & 882 & 1070 & 1310 & 1470 & -- \\
\hline
\end{tabular}

DURATION TABLE OF DAILY MEAN FLOW FOR PERIOD OF RECORD 1954-82

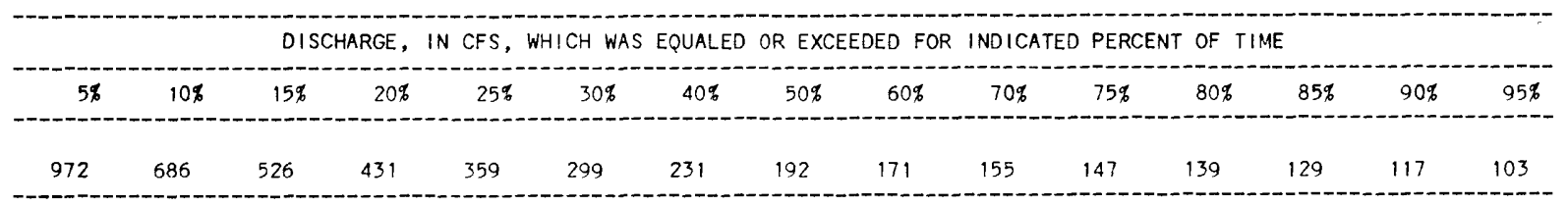


11501000 SPRAGUE RIVER NEAR CHILOQUIN, OR

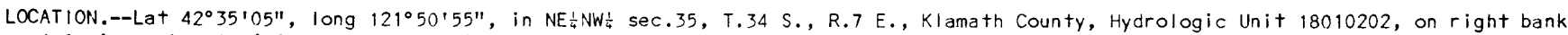

$1.0 \mathrm{mi}$ nor theast of Chiloquin, $4.6 \mathrm{mi}$ upstream from Modoc Point Canal intake, and at $\mathrm{mile} 5.4$.

DRAINAGE AREA.--1,580 $\mathrm{mi}^{2}$, approximately.

PERIOD OF RECORD.--July to October 1920, March 1921 to September 1982. Monthly discharge only July 1920 , published in WSP $1315-8$. Prior to October 1931, published as "at HcCready Fanch, near Chiloquin."

GAGE.--Water-stage recorder. Datum of gage is 4,202.43 ft National Geodetic Vertical Datum of 1929. Prior to Oct. 1, 1931, nonrecording gage at site $12 \mathrm{mi}$ upstream at different datum.

REMARKS.--Minor regulation from irrigation diversions above station.

AVERAGE DISCHARGE.--61 years (water years 1922-82), $577 \mathrm{ft}+3 / \mathrm{s}, 418,000 \mathrm{acre}-\mathrm{ft} / \mathrm{yr}$.

EXTREMES FOR PERIOD OF RECORD.--Maximum discharge, 14,900 ft³/s Dec. 26, 1964, gage height, $10.37 \mathrm{ft}$; minimum daily, $50 \mathrm{ft}+3 / \mathrm{s}$ May 26 , 1926.

STATISTICAL SUMMARIES

MONTHLY AND ANNUAL MEAN DISCHARGES 1922-82

\begin{tabular}{|c|c|c|c|c|c|c|}
\hline MONTH & $\begin{array}{l}\text { MINIMUM } \\
\text { (CFS) }\end{array}$ & $\begin{array}{l}\text { MAXIMUM } \\
\text { (CFS) }\end{array}$ & $\begin{array}{l}\text { MEAN } \\
\text { (CFS) }\end{array}$ & $\begin{array}{l}\text { STAN- } \\
\text { DARD } \\
\text { DEVIA- } \\
\text { TION } \\
\text { (CFS) }\end{array}$ & $\begin{array}{l}\text { COEFFI- } \\
\text { CIENT OF } \\
\text { VARI- } \\
\text { ATION }\end{array}$ & $\begin{array}{c}\text { PERCENT } \\
\text { OF } \\
\text { ANNUAL } \\
\text { RUNOFF }\end{array}$ \\
\hline OCTOBER & 183 & 848 & 295 & 93 & .32 & 4.3 \\
\hline $\begin{array}{l}\text { NOVEMBER } \\
\text { DECEMBER }\end{array}$ & $\begin{array}{l}219 \\
215\end{array}$ & $\begin{array}{r}789 \\
2853\end{array}$ & $\begin{array}{l}342 \\
481\end{array}$ & $\begin{array}{r}99 \\
395\end{array}$ & $\begin{array}{l}.29 \\
.82\end{array}$ & $\begin{array}{l}4.9 \\
6.9\end{array}$ \\
\hline JANUARY & 196 & 1961 & 519 & 407 & .78 & 7.5 \\
\hline FEBRUARY & 223 & 2764 & 665 & 497 & .75 & 9.6 \\
\hline MARCH & 288 & 2904 & 850 & 505 & .59 & 12.3 \\
\hline APRIL & 263 & 4250 & 1278 & 924 & .72 & 18.4 \\
\hline MAY & 184 & 3211 & 1156 & 724 & .63 & 16.7 \\
\hline JUNE & 169 & 1686 & 601 & 351 & .58 & 8.7 \\
\hline JULY & 141 & 552 & 283 & 103 & .36 & 4.1 \\
\hline AUGUST & 113 & 405 & 225 & 55 & .25 & 3.2 \\
\hline SEPTEMBER & 140 & 375 & 238 & 48 & .20 & 3.4 \\
\hline ANNUAL & 220 & 1395 & 577 & 264 & .46 & 100 \\
\hline
\end{tabular}

MAGNITUDE AND PROBABILITY OF INSTANTANEOUS PEAK FLOW BASED ON PERIOD OF RECORD 1922-82

DISCHARGE, IN CFS, FOR INDICATED RECURRENCE INTERVAL, IN YEARS, AND ANNUAL EXCEEDANCE PROBABILITY, IN PERCENT

$\begin{array}{ccccccc}1.25 & 2 & 5 & 10 & 25 & 50 & 100 \\ 80 \% & 50 \% & 20 \% & 10 \% & 4 \% & 2 \% & 1 \% \\ 1090 & 2040 & 3930 & 5590 & 8210 & 10600 & 13300\end{array}$

WEIGHTED SKEW $=.144$
MAGNITUDE AND PROBABILITY OF ANNUAL LOW FLOW BASED ON PERIOD OF RECORD 1923-82

\begin{tabular}{|c|c|c|c|c|c|c|}
\hline $\begin{array}{l}\text { PERIOD } \\
\text { (CON- }\end{array}$ & \multicolumn{6}{|c|}{$\begin{array}{l}\text { DISCHARGE, IN CFS, FOR IND ICATED RECURRENCE } \\
\text { INTERVAL, IN YEARS, AND ANNUAL NON- } \\
\text { EXCEEDANCE PROBABILITY, IN PERCENT }\end{array}$} \\
\hline SECU- & & & & & - & \\
\hline TIVE & 2 & 5 & 10 & 20 & 50 & 100 \\
\hline DAYS) & $50 \%$ & $20 \%$ & $10 \%$ & $5 \%$ & $2 \%$ & 18 \\
\hline 1 & 168 & 121 & 99 & 83 & 67 & 58 \\
\hline 3 & 172 & 131 & 114 & 101 & 88 & 81 \\
\hline 7 & 184 & 144 & 127 & 114 & 101 & 93 \\
\hline 14 & 192 & 152 & 134 & 120 & 107 & 98 \\
\hline 30 & 205 & 165 & 147 & 133 & 120 & 119 \\
\hline 60 & 217 & 177 & 160 & 146 & 133 & 124 \\
\hline 90 & 228 & 188 & 170 & 157 & 143 & 135 \\
\hline 120 & 241 & 200 & 182 & 168 & 154 & 145 \\
\hline 183 & 275 & 227 & 205 & 188 & 171 & 160 \\
\hline
\end{tabular}

MAGNITUDE AND PROBABILITY OF ANNUAL HIGH FLOW BASED ON PERIOD OF RECORD 1922-82

\begin{tabular}{|c|c|c|c|c|c|c|}
\hline \multirow{2}{*}{$\begin{array}{c}\text { PERIOD } \\
\text { (CON- } \\
\text { SECU- } \\
\text { TIVE } \\
\text { DAYS) }\end{array}$} & \multicolumn{6}{|c|}{$\begin{array}{l}\text { DISCHARGE, IN CFS, FOR INDICATED RECURRENCE } \\
\text { INTERVAL, IN YEARS, AND ANNUAL } \\
\text { EXCEEDANCE PROBABILITY, IN PERCENT }\end{array}$} \\
\hline & $\begin{array}{c}2 \\
50 \%\end{array}$ & $\begin{array}{c}5 \\
20\end{array}$ & $\begin{array}{l}10 \\
10 \%\end{array}$ & $\begin{array}{l}25 \\
4 \%\end{array}$ & $\begin{array}{l}50 \\
2 \%\end{array}$ & $\begin{array}{r}100 \\
1 \%\end{array}$ \\
\hline $\begin{array}{l}1 \\
3\end{array}$ & $\begin{array}{l}1990 \\
1930\end{array}$ & $\begin{array}{l}3850 \\
3690\end{array}$ & $\begin{array}{l}5500 \\
5220\end{array}$ & $\begin{array}{l}8110 \\
7610\end{array}$ & $\begin{array}{r}10500 \\
9730\end{array}$ & $\begin{array}{l}13200 \\
12200\end{array}$ \\
\hline 7 & 1760 & 3290 & 4590 & 6530 & 8220 & 10100 \\
\hline 15 & 1540 & 2790 & 3790 & 5240 & 6450 & 7760 \\
\hline 30 & 1330 & 2350 & 3140 & 4260 & 5170 & 6150 \\
\hline 60 & 1130 & 1940 & 2550 & 3390 & 4070 & 4790 \\
\hline 90 & 1020 & 1680 & 2170 & 2850 & 3390 & 3960 \\
\hline
\end{tabular}

DURATION TABLE OF DAILY MEAN FLOW FOR PERIOD OF RECORD 1922-82

DISCHARGE, IN CFS, WHICH WAS EQUALED OR EXCEEDED FOR INDICATED PERCENT OF TIME

\begin{tabular}{|c|c|c|c|c|c|c|c|c|c|c|c|c|c|c|}
\hline $5 \%$ & $10 \%$ & $15 \%$ & $20 \%$ & $25 \%$ & $30 \%$ & $40 \%$ & $50 \%$ & $60 \%$ & $70 \%$ & $75 \%$ & $80 \%$ & $85 \%$ & $90 \%$ & $95 \%$ \\
\hline 1780 & 1250 & 950 & 746 & 617 & 520 & 410 & 350 & 308 & 273 & 256 & 240 & 223 & 203 & 176 \\
\hline
\end{tabular}


11502500 WILLIAMSON RIVER BELOW SPRAGUE RIVER, NEAR CHILOQUIN, OR

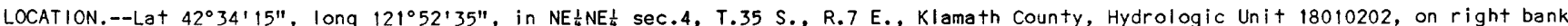
$0.2 \mathrm{mi}$ downstream from Sprague River and $0.8 \mathrm{mi}$ southwest of Chiloquin.

DRAINAGE AREA. $--3,000 \mathrm{mi}^{2}$, approximately.

PERIOD OF RECORD.-- June 1917 to September 1982.

GAGE.--Water-stage recorder. Datum of gage is $4,155.55 \mathrm{ft}$ National Geodetic Vertical Datum of 1929. Prior to Sept. 1, 1923, 3 t different datum.

REMARKS.--Some regulation by diversion dams and logpond operations of Sprague River. Diversions tor irrigation above station.

AVERAGE DISCHARGE.--65 years, $1,040 \mathrm{ft} / \mathrm{s}, 753,500$ acre- $\mathrm{ft} / \mathrm{yr}$.

EXTREMES FOR PERIOD OF RECORD.--Maximum discharge, 16,100 $\mathrm{ft}^{3} / \mathrm{s} \mathrm{Dec} .26,1964$, gage height, $10.56 \mathrm{ft}$; $\mathrm{minimum}, 320 \mathrm{ft} / \mathrm{s}$ Oct. 14 , 1920 .

STATISTICAL SUMMARIES

MONTHLY AND ANNUAL MEAN DISCHARGES 1918-82

\begin{tabular}{|c|c|c|c|c|c|c|}
\hline MONTH & $\begin{array}{l}\text { MINIMUM } \\
\text { (CFS) }\end{array}$ & $\begin{array}{l}\text { MAXIMUM } \\
\text { (CFS) }\end{array}$ & $\begin{array}{l}\text { MEAN } \\
\text { (CFS) }\end{array}$ & $\begin{array}{l}\text { STAN- } \\
\text { DARD } \\
\text { DEVIA- } \\
\text { TION } \\
\text { (CFS) }\end{array}$ & $\begin{array}{l}\text { COEFFI- } \\
\text { CIENT OF } \\
\text { VARI- } \\
\text { ATION }\end{array}$ & $\begin{array}{c}\text { PERCENT } \\
\text { OF } \\
\text { ANNUAL } \\
\text { RUNOFF }\end{array}$ \\
\hline OCTOBER & 517 & 1237 & 658 & 143 & .22 & 5.2 \\
\hline NOVEMBER & 561 & 1345 & 759 & 186 & .25 & 6.1 \\
\hline DECEMBER & 554 & 3682 & 959 & 490 & .51 & 7.6 \\
\hline JANUARY & 524 & 3075 & 993 & 539 & .54 & 7.9 \\
\hline FEBRUARY & 547 & 3846 & 1222 & 659 & .54 & 9.7 \\
\hline MARCH & 669 & 4256 & 1528 & 713 & .47 & 12.2 \\
\hline APRIL & 595 & 5488 & 1990 & 1136 & .57 & 15.9 \\
\hline MAY & 472 & 4376 & 1686 & 909 & .54 & 13.4 \\
\hline JUNE & 458 & 2658 & 1003 & 475 & .47 & 8.0 \\
\hline JULY & 399 & 1278 & 619 & 174 & .28 & 4.9 \\
\hline AUGUST & 382 & 934 & 551 & 95 & .17 & 4.4 \\
\hline SEPTEMBER & 440 & 872 & 571 & 83 & .15 & 4.6 \\
\hline ANNUAL & 548 & 2187 & 1043 & 359 & .34 & 100 \\
\hline
\end{tabular}

MAGNITUDE AND PROBABILITY OF INSTANTANEOUS PEAK FLOW BASED ON PERIOD OF RECORD 1917-82

DISCHARGE, IN CFS, FOR INDICATED RECURRENCE INTERVAL, IN YEARS, AND ANNUAL EXCEEDANCE PROBABILITY, IN PERCENT

\begin{tabular}{ccccccc}
1.25 & 2 & 5 & 10 & 25 & 50 & 100 \\
$80 \%$ & $50 \%$ & $20 \%$ & $10 \%$ & $4 \%$ & $2 \%$ & $1 \%$ \\
\hline 1800 & 2910 & 4920 & 6610 & 9180 & 11500 & 14100
\end{tabular}

MAGNITUDE AND PROBABILITY OF ANNUAL LOW FLOW BASED ON PERIOD OF RECORD 19 $19-82$

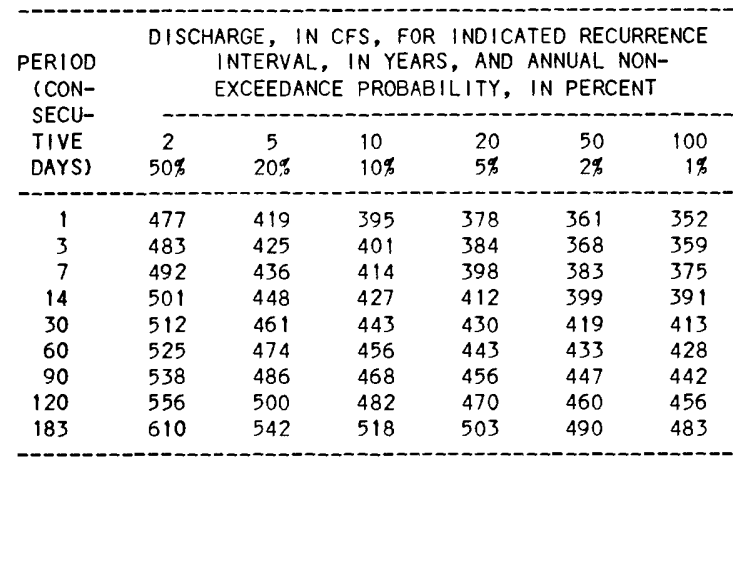

MAGNITUDE AND PROBABILITY OF ANNUAL HIGH FLOW BASED ON PERIOD OF RECORD $1918-82$

\begin{tabular}{|c|c|c|c|c|c|c|}
\hline $\begin{array}{l}\text { PERIOD } \\
\text { (CON- } \\
\text { SFCI- }\end{array}$ & \multicolumn{6}{|c|}{$\begin{array}{l}\text { DISCHARGE, IN CFS, FOR INDICATED RECURRENCE } \\
\text { INTERVAL, IN YEARS, AND ANNUAL } \\
\text { EXCEEDANCE PROBABILITY, IN PERCENT }\end{array}$} \\
\hline TIVE & 2 & 5 & 10 & 25 & 50 & 100 \\
\hline DAYS & $50 \%$ & $20 \%$ & $10 \%$ & $4 \%$ & $2 \%$ & $1 \%$ \\
\hline 1 & 2780 & 4790 & 6510 & 9160 & 11500 & 14300 \\
\hline 3 & 2690 & 4610 & 6240 & 8730 & 11000 & 13500 \\
\hline 7 & 2520 & 4230 & 5630 & 7750 & 9590 & 11700 \\
\hline 15 & 2290 & 3730 & 4850 & 6460 & 7810 & 9300 \\
\hline 30 & 2060 & 3250 & 4140 & 5390 & 6410 & 7510 \\
\hline 60 & 1820 & 2780 & 3490 & 4450 & 5230 & 6050 \\
\hline 90 & 1650 & 2460 & 3050 & 3850 & 4490 & 5160 \\
\hline
\end{tabular}

DURATION TABLE OF DAILY MEAN FLOW FOR PERIOD OF RECORD 1918-82

DISCHARGE, IN CFS, WHICH WAS EQUALED OR EXCEEDED FOR INDICATED PERCENT OF TIME

\begin{tabular}{|c|c|c|c|c|c|c|c|c|c|c|c|c|c|c|}
\hline $5 \%$ & $10 \%$ & $15 \%$ & $20 \%$ & $25 \%$ & $30 \%$ & $40 \%$ & $50 \%$ & $60 \%$ & $70 \%$ & $75 \%$ & $80 \%$ & $85 \%$ & $90 \%$ & $95 \%$ \\
\hline 2650 & 1940 & 1580 & 1340 & 1170 & 1050 & 874 & 749 & 662 & 611 & 587 & 564 & 542 & 514 & 482 \\
\hline
\end{tabular}


11504000 WOOD RIVER AT FORT KLAMATH, OR

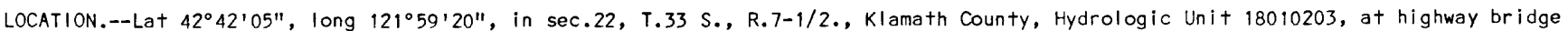
$0.2 \mathrm{mi}$ east of Fort Klamath.

DRAINAGE AREA. $--90 \mathrm{mi}^{2}$, approximately.

PERIOD OF RECORD.--April 1913 to September 1916, October 1918 to September 1919, October 1923 to September 1936.

GAGE.--Staff gage. Datum of gage is 4,166.65 ft National Geodetic Vertical Datum of 1929. Prior to Aug. 21 , 1923 , near described site at datum 0.19 ft lower. Aug. 21 to Sept. 30, 1923, $0.2 \mathrm{mi}$ downstream at different datum. Oct. 1 , 1923 to Sept. 30, 1925, at described site at datum $0.81 \mathrm{ft}$ higher.

REMARKS.--Many diversions for irrigation above station. Regulation by diversion dams.

AVERAGE DISCHARGE. -- 17 years (water years 1914-16, 1919, 1924-36), $215 \mathrm{ft}^{3} / \mathrm{s}$.

EXTREMES FOR PERIOD OF RECORD.--Maximum discharge observed, $520 \mathrm{ft} / \mathrm{s}$ Nov. 17, 1920, gage height, $2.41 \mathrm{ft}$, from rating curve extended above $350 \mathrm{ft}^{3} / \mathrm{s}$; minimum, $84 \mathrm{ft}^{3} / \mathrm{s}$ in July, August, September, 1931.

\section{STATISTICAL SUMMARIES}

MONTHLY AND ANNUAL MEAN DISCHARGES 1914-36

\begin{tabular}{|c|c|c|c|c|c|c|}
\hline MONTH & $\begin{array}{l}\text { MINIMUM } \\
\text { (CFS) }\end{array}$ & $\begin{array}{l}\text { MAXIMUM } \\
\text { (CFS) }\end{array}$ & $\begin{array}{l}\text { MEAN } \\
\text { (CFS) }\end{array}$ & $\begin{array}{l}\text { STAN- } \\
\text { DARD } \\
\text { DEVIA- } \\
\text { TION } \\
\text { (CFS) }\end{array}$ & $\begin{array}{l}\text { COEFFI- } \\
\text { CIENT OF } \\
\text { VARI- } \\
\text { ATION }\end{array}$ & $\begin{array}{c}\text { PERCENT } \\
\text { OF } \\
\text { ANNUAL } \\
\text { RUNOFF }\end{array}$ \\
\hline OCTOBER & 131 & 300 & 222 & 49 & .22 & 8.6 \\
\hline NOVEMBER & 143 & 310 & 230 & 49 & .21 & 8.9 \\
\hline DECEMBER & 143 & 308 & 226 & 49 & .22 & 8.8 \\
\hline JANUARY & 148 & 312 & 219 & 47 & .22 & 8.5 \\
\hline FEBRUARY & 145 & 323 & 225 & 47 & .21 & . 8.7 \\
\hline MARCH & 176 & 338 & 236 & 50 & .21 & 9.2 \\
\hline APRIL & 160 & 363 & 237 & 54 & .23 & 9.2 \\
\hline MAY & 112 & 340 & 211 & 57 & .27 & 8.2 \\
\hline JUNE & 101 & 345 & 208 & 63 & .30 & 8.1 \\
\hline JULY & 93 & 325 & 189 & 65 & .34 & 7.3 \\
\hline AUGUST & 87 & 293 & 176 & 59 & .34 & 6.8 \\
\hline SEPTEMBER & 110 & 282 & 199 & 55 & .27 & 7.7 \\
\hline ANNUAL & 141 & 311 & 215 & 47 & .22 & 100 \\
\hline
\end{tabular}

MAGNITUDE AND PROBABILITY OF INSTANTANEOUS PEAK FLOW BASED ON PERIOD OF RECORD 1914-36

\begin{tabular}{lcccccc} 
DISCHARGE, IN CFS, FOR INDICATED RECURRENCE & INTERVAL, IN \\
YEARS, AND ANNUAL EXCEEDANCE PROBABILITY, IN PERCENT \\
\hline 1.25 & 2 & 5 & 10 & 25 & 50 & 100 \\
$80 \%$ & $50 \%$ & $20 \%$ & $10 \%$ & $4 \%$ & $2 \%$ & $1 \%$ \\
\hline 318 & 371 & 435 & 475 & 522 & - &
\end{tabular}

$\begin{array}{llllll}318 & 371 & 435 & 475 & 522 & -\end{array}$

MAGNITUDE AND PROBABILITY OF ANNUAL LOW FLOW BASED ON PERIOD OF RECORD 1915-36

\begin{tabular}{|c|c|c|c|c|c|c|}
\hline \multirow{3}{*}{$\begin{array}{l}\text { PERIOD } \\
\text { (CON- } \\
\text { SECU- } \\
\text { TIVE } \\
\text { DAYS) }\end{array}$} & \multicolumn{6}{|c|}{$\begin{array}{l}\text { DISCHARGE, IN CFS, FOR INDICATED RECURRENCE } \\
\text { INTERVAL, IN YEARS, AND ANNUAL NON- } \\
\text { EXCEEDANCE PROBABILITY, IN PERCENT }\end{array}$} \\
\hline & 2 & 5 & 10 & 20 & 50 & 100 \\
\hline & $50 \%$ & $20 \%$ & $10 \%$ & $5 \%$ & $2 \%$ & $1 \%$ \\
\hline \multicolumn{7}{|c|}{$m-n-m-n$} \\
\hline 1 & 141 & 109 & 96 & 87 & -- & -- \\
\hline 3 & 144 & 111 & 98 & 89 & -- & -- \\
\hline 7 & 148 & 114 & 99 & 89 & -- & -- \\
\hline 14 & 151 & 116 & 102 & 92 & -- & -- \\
\hline 30 & 155 & 119 & 104 & 93 & -- & - \\
\hline 60 & 160 & 123 & 107 & 96 & - & -- \\
\hline 90 & 167 & 128 & 112 & 100 & -- & -- \\
\hline 120 & 174 & 133 & 116 & 103 & -- & -- \\
\hline 183 & 182 & 144 & 127 & 114 & -- & -- \\
\hline
\end{tabular}

MAGNITUDE AND PROBABILITY OF ANNUAL HIGH FLOW BASED ON PERIOD OF RECORD $1914-36$

\begin{tabular}{|c|c|c|c|c|c|c|}
\hline $\begin{array}{l}\text { PERIOD } \\
\text { (CON- } \\
\text { SECU- }\end{array}$ & \multicolumn{6}{|c|}{$\begin{array}{c}\text { DISCHARGE, IN CFS, FOR IND ICATED RECURRENCE } \\
\text { INTERVAL, IN YEARS, AND ANNUAL } \\
\text { EXCEEDANCE PROBABILITY, IN PERCENT }\end{array}$} \\
\hline TIVE & 2 & 5 & 10 & 25 & 50 & 100 \\
\hline DAYS) & $50 \%$ & $20^{\alpha}$ & $10 \%$ & $4 \%$ & $2 \%$ & $1 \%$ \\
\hline 1 & 351 & 407 & 435 & 464 & - & - \\
\hline 3 & 320 & 366 & 390 & 415 & -- & -- \\
\hline 7 & 296 & 342 & 367 & 396 & - & -- \\
\hline 15 & 277 & 322 & 348 & 380 & -- & - \\
\hline 30 & 260 & 305 & 332 & 366 & - & -- \\
\hline 60 & 248 & 292 & 319 & 351 & -- & -- \\
\hline 90 & 243 & 287 & 314 & 346 & - & - \\
\hline
\end{tabular}

DURATION TABLE OF DAILY MEAN FLOW FOR PERIOD OF RECORD 1914-36

OISCHARGE, IN CFS, WHICH WAS EQUALED OR EXCEEDED FOR INDICATED PERCENT OF TIME

\begin{tabular}{|c|c|c|c|c|c|c|c|c|c|c|c|c|c|c|}
\hline $5 \%$ & $10 \%$ & $15 \%$ & $20 \%$ & $25 \%$ & $30 \%$ & $40 \%$ & $50 \%$ & $60 \%$ & $70 \%$ & $75 \%$ & $80 \%$ & $85 \%$ & $90 \%$ & $95 \%$ \\
\hline 315 & 296 & 280 & 270 & 258 & 248 & 231 & 214 & 196 & 179 & 170 & 164 & 153 & 141 & 126 \\
\hline
\end{tabular}




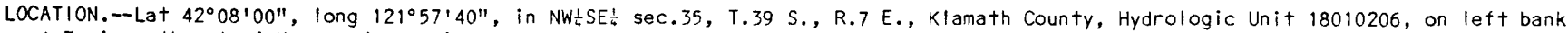
$1.7 \mathrm{mi}$ northwest of Keno and $4.5 \mathrm{mi}$ upstream from Spencer Creek.

DRAINAGE AREA.--3,920 $\mathrm{mi}^{2}$, approximately (not including Lost River or Lower Klamath Lake basins).

PERIOD OR RECORD.-- June 1904 to December 1913, October 1929 to September 1982. Monthly discharge only October to December 1929, published in WSP $1315-8$.

GAGE.- Water-stage recorder. Datum of gage is $3,961 \mathrm{ft}$ National Geodetic Vertical Datum of 1929 (from $r$ iver-profile survey). See WSP 1735 for history of changes prior to Nov. 6, 1954.

REMARKS.--Flow regulated since 1919 by Upper Klamath Lake. Diversions for irrigation above station.

AVERAGE DISCHARGE.--62 years, 1,668 $\mathrm{ft}+3 / \mathrm{s}, 1,208,000$ acre- $\mathrm{ft} / \mathrm{yr}$.

EXTREMES FOR PERIOD OF RECORD. - Maximum discharge, 10,200 $\mathrm{ft}^{3} / \mathrm{s}$ Feb. 24 , 1982, gage height, $12.74 \mathrm{ft}$, caused by regulation $\mathrm{from}$ Keno Dam $0.9 \mathrm{mi}$ upstream; minimum, $26 \mathrm{ft} 3 / \mathrm{s}$ Sept. 23, 1956; minimum daily, 60 ft $3 / \mathrm{s} \mathrm{May} 19,1934$.

EXTREMES OUTSIDE PERIOD OF RECORD.--Maximum stage, 15.3 ft, from floodmark (original datum), about May 10, 1904, discharge, 9,250 $f+{ }^{3} / \mathrm{s}$.

\section{STATISTICAL SUMMARIES}

MONTHLY AND ANNUAL MEAN DISCHARGES 1905-82

\begin{tabular}{|c|c|c|c|c|c|c|}
\hline MONTH & $\begin{array}{l}\text { MINIMUMA } \\
\text { (CFS) }\end{array}$ & $\begin{array}{l}\text { MAXIMUM } \\
\text { (CFS) }\end{array}$ & $\begin{array}{l}\text { MEAN } \\
\text { (CFS) }\end{array}$ & $\begin{array}{l}\text { STAN- } \\
\text { DARD } \\
\text { DEVIA- } \\
\text { TION } \\
\text { (CFS) }\end{array}$ & $\begin{array}{l}\text { COEFFI- } \\
\text { CIENT OF } \\
\text { VARI- } \\
\text { ATION }\end{array}$ & $\begin{array}{c}\text { PERCENT } \\
\text { OF } \\
\text { ANNUAL } \\
\text { RUNOFF }\end{array}$ \\
\hline OCTOBER & 564 & 3055 & 1447 & 472 & .33 & 7.2 \\
\hline NOVEMBER & 290 & 3976 & 1678 & 691 & .41 & 8.4 \\
\hline DECEMBER & 391 & 3859 & 1909 & 867 & .45 & 9.5 \\
\hline JANUARY & 542 & 7702 & 2045 & 1261 & .62 & 10.2 \\
\hline FEBRUARY & 324 & 7564 & 2154 & 1412 & .66 & 10.7 \\
\hline MARCH & 229 & 8197 & 2452 & 1777 & .72 & 12.2 \\
\hline APRIL & 166 & 6594 & 2287 & 1669 & .73 & 11.4 \\
\hline MAY & 109 & 5258 & 1787 & 1292 & .72 & 8.9 \\
\hline JUNE & 98 & 3713 & 1141 & 927 & .81 & 5.7 \\
\hline JULY & 114 & 2748 & 898 & 590 & .66 & 4.5 \\
\hline AUGUST & 202 & 1898 & 1006 & 424 & .42 & 5.0 \\
\hline SEPTEMBER & 334 & 2214 & 1241 & 405 & .33 & 6.2 \\
\hline ANNUAL & 546 & 3582 & 1668 & 673 & .40 & 100 \\
\hline
\end{tabular}

MAGNITUDE AND PROBABILITY OF INSTANTANEOUS PEAK FLOW BASED ON PERIOD OF RECORD 1905-82

DISCHARGE, IN CFS, FOR INDICATED RECURRENCE INTERVAL, IN YEARS, AND ANNUAL EXCEEDANCE PROBABILITY, IN PERCENT

\begin{tabular}{ccccccc}
1.25 & 2 & 5 & 10 & 25 & 50 & 100 \\
$80 \%$ & $50 \%$ & $20 \%$ & $10 \%$ & $4 \%$ & $2 \%$ & $1 \%$ \\
\hline 2810 & 4110 & 6120 & 7590 & 9610 & 11200 & 13000 \\
\hline
\end{tabular}

WEIGHTED SKEW $=.162$

MAGNITUDE AND PROBABILITY OF ANNUAL LOW FLOW BASED ON PERIOD OF RECORD 1906-82

\begin{tabular}{ccccccc} 
PERIOD & \multicolumn{1}{c}{ DISCHARGE, IN CFS, FOR INDICATED RECURRENCE } \\
(CON- & \multicolumn{5}{c}{$\begin{array}{l}\text { INTERVAL, IN YEARS, AND ANNUAL NON- } \\
\text { EXCEEDANCE PROBABILITY, IN PERCENT }\end{array}$} \\
$\begin{array}{l}\text { SECU- } \\
\text { TIVE }\end{array}$ & 2 & 5 & 10 & 20 & 50 & 100 \\
DAYS) & $50 \%$ & $20 \%$ & $10 \%$ & $5 \%$ & $2 \%$ & $1 \%$ \\
\hline 1 & 300 & 155 & 110 & 84 & 61 & 50 \\
3 & 371 & 200 & 144 & 110 & 80 & 65 \\
7 & 409 & 226 & 165 & 127 & 95 & 78 \\
14 & 443 & 248 & 182 & 140 & 105 & 86 \\
30 & 500 & 293 & 221 & 174 & 133 & 111 \\
60 & 569 & 347 & 266 & 213 & 165 & 139 \\
90 & 690 & 434 & 332 & 263 & 200 & 165 \\
120 & 811 & 521 & 399 & 313 & 233 & 189 \\
183 & 1050 & 705 & 550 & 437 & 330 & 269 \\
\hline
\end{tabular}

MAGNITUDE AND PROBABILITY OF ANNUAL HIGH FLOW BASED ON PERIOD OF RECORD 1905-82

\begin{tabular}{|c|c|c|c|c|c|c|}
\hline $\begin{array}{l}\text { PERIOD } \\
\text { (CON- } \\
\text { SECU- }\end{array}$ & \multicolumn{6}{|c|}{$\begin{array}{l}\text { DISCHARGE, IN CFS, FOR INDICATED RECURRENCE } \\
\text { INTERVAL, IN YEARS, AND ANNUAL } \\
\text { EXCEEDANCE PROBABILITY, IN PERCENT }\end{array}$} \\
\hline TIVE & 2 & 5 & 10 & 25 & 50 & 100 \\
\hline DAYS) & $50 \%$ & $20 \%$ & $10 \%$ & $4 \%$ & $2 \%$ & $1 \%$ \\
\hline 1 & 3840 & 5830 & 7310 & 9340 & 11000 & 12700 \\
\hline 3 & 3740 & 5730 & 7210 & 9260 & 10900 & 12700 \\
\hline 7 & 3590 & 5570 & 7030 & 9040 & 10700 & 12400 \\
\hline 15 & 3350 & 5210 & 6590 & 8490 & 10000 & 11600 \\
\hline 30 & 3100 & 4770 & 6000 & 7660 & 8970 & 10400 \\
\hline 60 & 2770 & 4220 & 5220 & 6520 & 7510 & 8500 \\
\hline 90 & 2530 & 3850 & 4760 & 5930 & 6830 & 7730 \\
\hline
\end{tabular}

DURATION TABLE OF DAILY MEAN FLOW FOR PERIOD OF RECORD 1905-82

DISCHARGE, IN CFS, WHICH WAS EQUALED OR EXCEEDED FOR INDICATED PERCENT OF TIME

\begin{tabular}{|c|c|c|c|c|c|c|c|c|c|c|c|c|c|c|}
\hline $5 \%$ & $10 \%$ & $15 \%$ & $20 \%$ & $25 \%$ & $30 \%$ & $40 \%$ & $50 \%$ & $60 \%$ & $70 \%$ & $75 \%$ & $80 \%$ & $85 \%$ & $90 \%$ & $95 \%$ \\
\hline 4220 & 3150 & 2710 & 2480 & 2210 & 1970 & 1610 & 1330 & 1120 & 920 & 821 & 706 & 578 & 442 & 304 \\
\hline
\end{tabular}


11510700 KLAMATH RIVER BELOW JOHN C. BOYLE POWERPLANT, NEAR KENO, OR

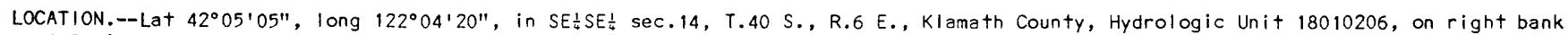

$0.7 \mathrm{mi}$ downstream from John $\mathrm{C}$. Boyle powerplant, $8 \mathrm{mi}$ downstream from Spencer Creek, and $8.5 \mathrm{mi}$ southwest of Keno.

DRAINAGE AREA.--4,080 $\mathrm{mi}^{2}$, approximately (not including Lost River or Lower Klamath Lake basins).

PERIOD OF RECORD. -- January 1959 to September 1982. Prior to Jct. 1, 1961, published as "below Big Bend powerplant."

GAGE.--Water-stage recorder. Datum of gage is $3,274.82+$ t National Geodetic Vertical Datum of 1929 (levels by Pacific Power \& Light Co.).

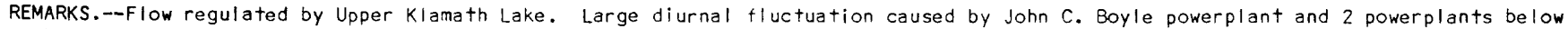
Upper Klamath Lake. Diversions for irrigation above station.

AVERAGE DISCHARGE. -23 years, $1,829+t^{3} / s, 1,325,000$ acre $-t+/ y r$.

EXTREMES FOR PERIOD OF RECORD.--Maximum discharge, 11,000 $\mathrm{ft}^{3} / \mathrm{s} \mathrm{Mar.} \mathrm{5,} \mathrm{1972,} \mathrm{gage} \mathrm{height,} 9.33 \mathrm{ft}$; $\mathrm{minimum,} 283 \mathrm{ft} / \mathrm{s}$ Feb. 17, 1968; minimum daily, $317 \mathrm{ft}^{3} / \mathrm{s}$ July $25,1968$.

STATISTICAL SUMMARIES

MONTHLY AND ANNUAL. MEAN OISCHARGES 1961-82

\begin{tabular}{|c|c|c|c|c|c|c|}
\hline MONTH & $\begin{array}{l}\text { MINIMUM } \\
\text { (CFS) }\end{array}$ & $\begin{array}{l}\text { MAXIMUM } \\
\text { (CFS) }\end{array}$ & $\begin{array}{l}\text { MEAN } \\
\text { (CFS) }\end{array}$ & $\begin{array}{l}\text { STAN- } \\
\text { DARD } \\
\text { DEVIA- } \\
\text { TION } \\
\text { (CFS) }\end{array}$ & $\begin{array}{l}\text { COEFFI- } \\
\text { CIENT OF } \\
\text { VARI- } \\
\text { ATION }\end{array}$ & $\begin{array}{c}\text { PERCENT } \\
\text { OF } \\
\text { ANNUAL } \\
\text { RUNOFF }\end{array}$ \\
\hline OCTOBER & 786 & 2568 & 1603 & 467 & .29 & 7.2 \\
\hline $\begin{array}{l}\text { NOVEMBER } \\
\text { DECEMBER }\end{array}$ & $\begin{array}{r}897 \\
1112\end{array}$ & $\begin{array}{l}3989 \\
3895\end{array}$ & $\begin{array}{l}2060 \\
2573\end{array}$ & $\begin{array}{l}772 \\
803\end{array}$ & $\begin{array}{l}.37 \\
.31\end{array}$ & $\begin{array}{r}9.3 \\
11.6\end{array}$ \\
\hline JANUARY & 1174 & 7905 & 2786 & 1454 & .52 & 12.5 \\
\hline FEBRUARY & 1091 & 7780 & 2631 & 1590 & .60 & 11.8 \\
\hline $\begin{array}{l}\text { MARCH } \\
\text { APRIL }\end{array}$ & $\begin{array}{l}634 \\
723\end{array}$ & $\begin{array}{l}8755 \\
5645\end{array}$ & $\begin{array}{l}2902 \\
2448\end{array}$ & $\begin{array}{l}1916 \\
1505\end{array}$ & $\begin{array}{l}.66 \\
.61\end{array}$ & $\begin{array}{l}13.0 \\
11.0\end{array}$ \\
\hline MAY & 591 & 3935 & 1680 & 956 & .57 & 7.6 \\
\hline JUNE & 550 & 1742 & 785 & 280 & .36 & 3.5 \\
\hline JULY & 501 & 1339 & 642 & 175 & .27 & 2.9 \\
\hline AUGUST & 590 & 1054 & 890 & 132 & .15 & 4.0 \\
\hline SEPTEMBER & 776 & 1876 & 1240 & 271 & .22 & 5.6 \\
\hline ANNUAL & 1080 & 2945 & 1850 & 531 & .29 & 100 \\
\hline
\end{tabular}

MAGNITUDE ANO PROBABILITY OF INSTANTANEOUS PEAK FLOW BASED ON PERIOD OF RECORO 1961-82

\begin{tabular}{|c|c|c|c|c|c|c|}
\hline $\begin{array}{l}\text { DISCHARGE } \\
\text { YEARS, }\end{array}$ & $\begin{array}{l}\text { IN CF } \\
D A N I\end{array}$ & $\begin{array}{l}\text { FOR } \\
\text { EXCE }\end{array}$ & $\begin{array}{l}\text { CATED } \\
\text { INCE F }\end{array}$ & $\begin{array}{l}\text { RECURR } \\
\text { OBABIL }\end{array}$ & $\begin{array}{l}\text { INT } \\
\text { IN }\end{array}$ & ENT ${ }^{11}$ \\
\hline $\begin{array}{l}1.25 \\
80 \%\end{array}$ & $\begin{array}{c}2 \\
50 \%\end{array}$ & ${ }_{20 \%}^{5}$ & $\begin{array}{l}10 \\
10 \%\end{array}$ & $\begin{array}{l}25 \\
4 \%\end{array}$ & $\begin{array}{l}50 \\
2 \%\end{array}$ & $\begin{array}{r}100 \\
1 \%\end{array}$ \\
\hline 3410 & 5130 & 7830 & 9830 & 12600 & -- & -- \\
\hline
\end{tabular}

WEIGHTED SKEW $=\quad .163$
MAGNITUDE AND PROBABILITY OF ANNUAL LOW FLOW BASED ON PERIOD OF RECORD 1962-82

\begin{tabular}{|c|c|c|c|c|c|c|}
\hline $\begin{array}{l}\text { PERIOD } \\
\text { (CON- } \\
\text { SECH- }\end{array}$ & \multicolumn{6}{|c|}{$\begin{array}{l}\text { DISCHARGE, IN CFS, FOR INDICATED RECURRENCE } \\
\text { INTERVAL, IN YEARS, AND ANNUAL NON- } \\
\text { EXCEEDANCE PROBABILITY, IN PERCENT }\end{array}$} \\
\hline TIVE & & 5 & 10 & & & \\
\hline DAYS) & $50 \%$ & $20 \%$ & $10 \%$ & $5 \%$ & $2 \%$ & $1 \%$ \\
\hline & & & & & & \\
\hline 1 & 361 & 328 & 320 & 316 & -- & -- \\
\hline 3 & 402 & 353 & 338 & 330 & -- & -- \\
\hline 7 & 477 & 428 & 413 & 404 & -- & -- \\
\hline 14 & 514 & 474 & 463 & 457 & -- & -- \\
\hline 30 & 551 & 512 & 501 & 496 & -- & -- \\
\hline 60 & 601 & 562 & 550 & 543 & -- & -- \\
\hline 90 & 704 & 633 & 601 & 577 & -- & -- \\
\hline 120 & 816 & 717 & 667 & 628 & -- & -- \\
\hline 183 & 1030 & 855 & 779 & 723 & -- & -- \\
\hline
\end{tabular}

MAGNITUDE AND PROBABILITY OF ANNUAL HIGH FLOW BASEO ON PERIOD OF RECORD 1961-82

\begin{tabular}{|c|c|c|c|c|c|c|}
\hline $\begin{array}{l}\text { PERIOO } \\
\text { (CON- } \\
\text { SECU- }\end{array}$ & \multicolumn{6}{|c|}{$\begin{array}{c}\text { DISCHARGE, IN CFS, FOR IND ICATED RECURRENCE } \\
\text { INTERVAL, IN YEARS, AND ANNUAL } \\
\text { EXCEEDANCE PROBABILITY, IN PERCENT }\end{array}$} \\
\hline TIVE & 2 & 5 & 10 & 25 & 50 & 100 \\
\hline DAYS) & $50 \%$ & $20 \%$ & $10 \%$ & $4 \%$ & $2 \%$ & $1 \%$ \\
\hline 1 & 4920 & 7470 & 9320 & 11800 & -- & -- \\
\hline 3 & 4830 & 7290 & 9070 & 11500 & -- & - \\
\hline 7 & 4640 & 6950 & 8650 & 11000 & -- & -- \\
\hline 15 & 4190 & 6300 & 7890 & 10100 & - & -- \\
\hline 30 & 3770 & 5620 & 7030 & 9030 & -- & -- \\
\hline 60 & 3290 & 4750 & 5800 & 7210 & -- & -- \\
\hline 90 & 3000 & 4280 & 5190 & 6420 & -- & -- \\
\hline
\end{tabular}

DURATION TABLE OF DAILY MEAN FLOW FOR PERIOD OF RECORD $1961-82$

DISCHARGE, IN CFS, WHICH WAS EQUALED OR EXCEEDED FOR INDICATED PERCENT OF TIME

\begin{tabular}{|c|c|c|c|c|c|c|c|c|c|c|c|c|c|c|}
\hline $5 \%$ & $10 \%$ & $15 \%$ & $20 \%$ & $25 \%$ & $30 \%$ & $40 \%$ & $50 \%$ & $60 \%$ & $70 \%$ & $75 \%$ & $80 \%$ & $85 \%$ & $90 \%$ & $95 \%$ \\
\hline 4380 & 3230 & 2910 & 2770 & 2580 & 2330 & 1710 & 1420 & 1190 & 985 & 885 & 771 & 688 & 623 & 530 \\
\hline
\end{tabular}




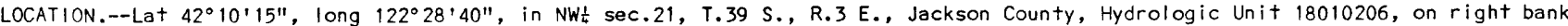
$0.3 \mathrm{mi}$ upstream from Burnt Creek, $0.6 \mathrm{mi}$ downstream from Hyatt Dam, and $12 \mathrm{mi}$ east of Ashland.

DRAINAGE AREA.- $-12.1 \mathrm{mi}^{2}$.

PERIOD OF RECORD.--April to July 1917, December 1917 to July 1920 (no low-flow records), October 1920 to June 1922 , October 1948 to September 1965. Monthly discharge only October 1948, published in WSP 1315-B. Published as "at Hyatt Prairie, near Ashland" December 1917 to July 1920, October 1920 to June 1922, October 1948 to September 1958.

GAGE.--Water-stage recorder and concrete control. Datum of gage is 4,706 ft National Geodetic Vertical Datum of 1929 (by stadia survey). Apr. 1, 1917, to June 30, 1922, at site $0.5 \mathrm{mi}$ upstream at different datum.

REMARKS.--Flow regulated since December 1922 by Hyatt Reservoir. No diversion above station. Practically entire flow diverted below station by Green Springs powerplant diversion.

AVERAGE DISCHARGE.--17 years (water years $1949-65$ ), $12.7 \mathrm{ft} / \mathrm{s}, 9,190 \mathrm{acre}-\mathrm{t} t / \mathrm{yr}$.

EXTREMES FOR PERIOD OF RECORD.--Maximum discharge, $751 \mathrm{ft}^{3} / \mathrm{s}$ May 3,1954 , gage height, $5.33 \mathrm{ft}$, caused by failure of $f$ lashboards on spillway of Hyatt Dam, from rating curve extended above $50 \mathrm{ft} / \mathrm{s}$ on basis of computation of peak $\mathrm{flow}$ over Columbus-type control; no flow at times.

\section{STATISTICAL SUMMARIES}

MONTHLY AND ANNUAL MEAN DISCHARGES 1949-65

\begin{tabular}{|c|c|c|c|c|c|c|}
\hline MONTH & $\begin{array}{l}\text { MINIMUM } \\
\text { (CFS) }\end{array}$ & $\begin{array}{l}\text { MAXIMUM } \\
\text { (CFS) }\end{array}$ & $\begin{array}{l}\text { MEAN } \\
\text { (CFS) }\end{array}$ & $\begin{array}{l}\text { STAN- } \\
\text { DARD } \\
\text { DEVIA- } \\
\text { TION } \\
\text { (CFS) }\end{array}$ & $\begin{array}{l}\text { COEFFI- } \\
\text { CIENT OF } \\
\text { VARI- } \\
\text { ATION }\end{array}$ & $\begin{array}{c}\text { PERCENT } \\
\text { OF } \\
\text { ANNUAL } \\
\text { RUNOFF }\end{array}$ \\
\hline OCTOBER & .1 & 21 & 2.3 & 5.5 & 2.36 & 1.5 \\
\hline NOVEMBER & .2 & 1.9 & .4 & .4 & .98 & .3 \\
\hline DECEMBER & .2 & 2.1 & .6 & .5 & .82 & .4 \\
\hline JANUARY & .2 & 32 & 2.4 & 7.6 & 3.18 & 1.6 \\
\hline FEBRUARY & .3 & 45 & 3.5 & 11 & 2.99 & 2.3 \\
\hline MARCH & .5 & 9.8 & 1.5 & 2.2 & 1.44 & 1.0 \\
\hline APRIL & .9 & 32 & 4.8 & 7.3 & 1.53 & 3.2 \\
\hline MAY & .6 & 38 & 13 & 12 & .91 & 8.6 \\
\hline JUNE & .4 & 55 & 24 & 17 & .71 & 15.7 \\
\hline $\begin{array}{l}\text { JULY } \\
\text { AUGUST }\end{array}$ & $\begin{array}{l}6.0 \\
8.5\end{array}$ & $\begin{array}{l}81 \\
65\end{array}$ & $\begin{array}{l}39 \\
44\end{array}$ & $\begin{array}{l}22 \\
19\end{array}$ & $\begin{array}{l}.57 \\
.44\end{array}$ & $\begin{array}{l}26.0 \\
28.8\end{array}$ \\
\hline SEPTEMBER & .5 & 37 & 16 & 11 & .70 & 10.7 \\
\hline ANNUAL & 2.8 & 17 & 13 & 3.8 & .30 & 100 \\
\hline
\end{tabular}

MAGNITUDE AND PROBABILITY OF INSTANTANEOUS PEAK FLOW BASED ON PERIOD OF RECORD

DISCHARGE, IN CFS, FOR INDICATED RECURRENCE INTERVAL, IN YEARS, AND ANNUAL EXCEEDANCE PROBABILITY, IN PERCENT

\begin{tabular}{|c|c|c|c|c|c|c|}
\hline $\begin{array}{l}1.25 \\
80 \%\end{array}$ & $\begin{array}{c}2 \\
50 \%\end{array}$ & $\begin{array}{c}5 \\
20 \%\end{array}$ & $\begin{array}{l}10 \\
10 \%\end{array}$ & $\begin{array}{l}25 \\
4_{\%}^{\alpha}\end{array}$ & $\begin{array}{l}50 \\
2 \%\end{array}$ & $\begin{array}{l}100 \\
1 \%\end{array}$ \\
\hline- & -- & - & -- & - & - & - \\
\hline
\end{tabular}

WEIGHTED SKEW =
MAGNITUDE AND PROBABILITY OF ANNUAL LOW FLOW BASED ON PERIOD OF RECORD 1950-65

\begin{tabular}{|c|c|c|c|c|c|c|}
\hline \multirow{2}{*}{$\begin{array}{l}\text { PERIOD } \\
\text { (CON- } \\
\text { SECU- } \\
\text { TIVE } \\
\text { DAYS) }\end{array}$} & \multicolumn{6}{|c|}{$\begin{array}{l}\text { DISCHARGE, IN CFS, FOR INDICATED RECURRENCE } \\
\text { INTERVAL, IN YEARS, AND ANNUAL NON- } \\
\text { EXCEEDANCE PROBABILITY, IN PERCENT }\end{array}$} \\
\hline & $\stackrel{2}{50 \%}$ & 5 & 10 & 20 & 50 & 100 \\
\hline & & & & & & \\
\hline 1 & .2 & .2 & .1 & .1 & - & - \\
\hline 3 & .2 & .2 & .1 & .1 & - & - \\
\hline 7 & .2 & .2 & .1 & .1 & - & -- \\
\hline 14 & .2 & .2 & .1 & .1 & - & - \\
\hline 30 & .2 & .2 & .2 & .2 & - & -- \\
\hline 60 & .3 & .2 & .2 & .2 & -- & -- \\
\hline 90 & .3 & .2 & .2 & .2 & - & - \\
\hline 120 & .3 & .3 & .2 & .2 & - & - \\
\hline 183 & .5 & .3 & .3 & .3 & - & - \\
\hline
\end{tabular}

MAGNITUDE AND PROBABILITY OF ANNUAL HIGH FLOW BASED ON PERIOD OF RECORD 1949-65

\begin{tabular}{|c|c|c|c|c|c|c|}
\hline $\begin{array}{l}\text { PERIOD } \\
\text { (CON- } \\
\text { SECU- }\end{array}$ & \multicolumn{6}{|c|}{$\begin{array}{c}\text { DISCHARGE, IN CFS, FOR INDICATED RECURRENCE } \\
\text { INTERVAL, IN YEARS, AND ANNUAL } \\
\text { EXCEEDANCE PROBABILITY, IN PERCENT }\end{array}$} \\
\hline TIVE & 2 & 5 & 10 & 25 & 50 & 100 \\
\hline DAYS) & $50 \%$ & $20 \%$ & $10 \%$ & $4 \%$ & $2 q$ & $1 \%$ \\
\hline 1 & 71 & 90 & 112 & 154 & -- & - \\
\hline 3 & 70 & 88 & 102 & 122 & -- & -- \\
\hline 7 & 69 & 89 & 86 & 91 & -- & - \\
\hline 15 & 65 & 79 & 84 & 87 & -- & -- \\
\hline 30 & 59 & 75 & 80 & 83 & - & -- \\
\hline 60 & 50 & 65 & 70 & 74 & -- & - \\
\hline 90 & 42 & 55 & 60 & 64 & - & -- \\
\hline
\end{tabular}

DURATION TABLE OF DAILY MEAN FLOW FOR PERIOD OF RECORD 1949-65

\begin{tabular}{|c|c|c|c|c|c|c|c|c|c|c|c|c|c|c|}
\hline $5 \%$ & $10 \%$ & $15 \%$ & $20 \%$ & $25 \%$ & $30 \%$ & $40 \%$ & $50 \%$ & $60 \%$ & $70 \%$ & $75 \%$ & $80 \%$ & $85 \%$ & $90 \%$ & $95 \%$ \\
\hline 64 & 52 & 39 & 28 & 17 & 7.8 & 1.8 & .9 & .6 & .5 & .4 & .3 & .3 & .2 & 2 \\
\hline
\end{tabular}


13181000 OWYHEE RIVER NEAR ROME, OR

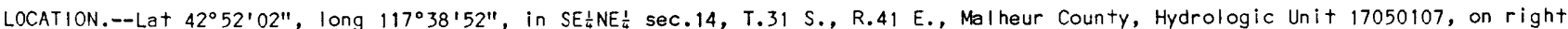
bank $0.5 \mathrm{mi}$ downstream from Jordan Creek, $2.6 \mathrm{mi}$ north of Rome, and at mile 122.4.

DRAINAGE AREA.--About $8,000 \mathrm{mi}^{2}$.

PERIOD OF RECORD.--October 1949 to September 1982.

GAGE.--Water-stage recorder. Datum of gage is 3,344.20 ft National Geodetic Vertical Datum of 1929 . Prior to Feb 10, 1960, at datum $0.24 \mathrm{ft}$ lower.

REMARKS.--Flow regulated since 1914 by Antelope Reservoir, capacity, 45,000 acre-ft (increased to 70,000 acre-ft, in 1970), and wild Horse Reservoir since 1938, capacity, 32,690 acre-ft, and numerous small reservoirs. Diversions above station for irrigation.

AVERAGE DISCHARGE. --33 years, $916 \mathrm{ft}^{3} / \mathrm{s}, 663,600$ acre- $\mathrm{t}+\mathrm{t} / \mathrm{yr}$.

EXTREMES FOR PERIOD OF RECORD.--Maximum discharge, 33,500 $\mathrm{ft}^{3} / \mathrm{s} \mathrm{Dec.} \mathrm{24,} \mathrm{1964,} \mathrm{gage} \mathrm{height,} 16.7 \mathrm{ft}$, from $\mathrm{floodmark}$; minimum, 42 $f t^{3} / \mathrm{s}$ Aug. 12, 1954, July 28, Aug. 5, 1961, July 31, 1968.

STATISTICAL SUMMARIES

MONTHLY AND ANNUAL MEAN DISCHARGES 1950-82

\begin{tabular}{|c|c|c|c|c|c|c|}
\hline MONTH & $\begin{array}{l}\text { MINIMUM } \\
\text { (CFS) }\end{array}$ & $\begin{array}{l}\text { MAXIMUM } \\
\text { (CFS) }\end{array}$ & $\begin{array}{l}\text { MEAN } \\
\text { (CFS) }\end{array}$ & $\begin{array}{l}\text { STAN- } \\
\text { DARD } \\
\text { DEVIA- } \\
\text { TION } \\
\text { (CFS) }\end{array}$ & $\begin{array}{l}\text { COEFFI- } \\
\text { CIENT OF } \\
\text { VARI- } \\
\text { ATION }\end{array}$ & $\begin{array}{c}\text { PERCENT } \\
\text { OF } \\
\text { ANNUAL } \\
\text { RUNOFF }\end{array}$ \\
\hline OCTOBER & 85 & 442 & 157 & 67 & .43 & 1.4 \\
\hline NOVEMBER & 107 & 593 & 205 & 95 & .46 & 1.9 \\
\hline DECEMBER & 104 & 2898 & 423 & 541 & 1.28 & 3.8 \\
\hline JANUARY & 114 & 4461 & 750 & 948 & 1.26 & 6.8 \\
\hline FEBRUARY & 129 & 5350 & 1206 & 1167 & .97 & $10.9^{\circ}$ \\
\hline MARCH & 233 & 9404 & 2072 & 1911 & .92 & 18.8 \\
\hline APRIL & 206 & 16960 & 2976 & 3110 & 1.05 & 26.9 \\
\hline MAY & 124 & 6736 & 1847 & 1557 & .84 & 16.7 \\
\hline JUNE & 157 & 2726 & 874 & 729 & .83 & 7.9 \\
\hline JULY & 61 & 652 & 254 & 147 & .58 & 2.3 \\
\hline AUGUST & 64 & 247 & 146 & 53 & .36 & 1.3 \\
\hline SEPTEMBER & 63 & 247 & 136 & 52 & .38 & 1.2 \\
\hline ANNUAL & 188 & 2357 & 917 & 528 & .58 & 100 \\
\hline
\end{tabular}

MAGNITUDE AND PROBABILITY OF INSTANTANEOUS PEAK FLOW BASED ON PERIOD OF RECORD

\begin{tabular}{|c|c|c|c|c|c|c|}
\hline $\begin{array}{l}\text { DISCHARGE } \\
\text { YEARS, }\end{array}$ & $\begin{array}{l}\text { IN CF } \\
D \text { ANN }\end{array}$ & $\begin{array}{l}\text { FOR } \\
\text { EXC }\end{array}$ & ATE & URR & \multicolumn{2}{|c|}{$\begin{array}{l}\text { INTERVAL, IN } \\
\text { IN PERCENT }\end{array}$} \\
\hline $\begin{array}{l}1.25 \\
80 \%\end{array}$ & $\stackrel{2}{50 \%}$ & $\begin{array}{c}5 \\
20 \%\end{array}$ & $\begin{array}{l}10 \\
10 \%\end{array}$ & $\begin{array}{l}25 \\
4 \%\end{array}$ & $\begin{array}{l}50 \\
2 \%\end{array}$ & $\begin{array}{r}100 \\
1 \%\end{array}$ \\
\hline-- & -- & -- & -- & -- & -- & -- \\
\hline
\end{tabular}

WEIGHTED SKEW = --
MAGNITUDE AND PROBABILITY OF ANNUAL LOW FLOW BASED ON PERIOD OF RECORD 1951-82

\begin{tabular}{|c|c|c|c|c|c|c|}
\hline $\begin{array}{l}\text { PERIOD } \\
\text { (CON- }\end{array}$ & \multicolumn{6}{|c|}{$\begin{array}{l}\text { DISCHARGE, IN CFS, FOR INDICATED RECURRENCE } \\
\text { INTERVAL, IN YEARS, AND ANNUAL NON- } \\
\text { EXCEEDANCE PROBABILITY, IN PERCENT }\end{array}$} \\
\hline SECU- & & $-\infty$ & -0 & -1 & & \\
\hline TIVE & 2 & 5 & 10 & 20 & 50 & 100 \\
\hline DAYS) & $50 \%$ & $20 \%$ & $10 \%$ & $5 \%$ & $2 \%$ & $1 \%$ \\
\hline 1 & 92 & 67 & 56 & 48 & 40 & -- \\
\hline 3 & 95 & 69 & 58 & 50 & 42 & -- \\
\hline 7 & 98 & 72 & 60 & 52 & 43 & -- \\
\hline 14 & 102 & 74 & 61 & 52 & 43 & -- \\
\hline 30 & 110 & 80 & 67 & 58 & 48 & -- \\
\hline 60 & 120 & 88 & 74 & 64 & 54 & -- \\
\hline 90 & 130 & 97 & 82 & 72 & 61 & -- \\
\hline 120 & 139 & 105 & 90 & 79 & 69 & - \\
\hline 183 & 166 & 123 & 106 & 95 & 84 & -- \\
\hline
\end{tabular}

MAGN ITUDE AND PROBABILITY OF ANNUAL HIGH FLOW BASED ON PERIOD OF RECORD 1950-82

\begin{tabular}{|c|c|c|c|c|c|c|}
\hline \multirow{3}{*}{$\begin{array}{l}\text { PERIOD } \\
\text { (CON- } \\
\text { SECU- } \\
\text { TIVE } \\
\text { DAYS) }\end{array}$} & \multicolumn{6}{|c|}{$\begin{array}{c}\text { DISCHARGE, IN CFS, FOR INDICATED RECURRENCE } \\
\text { INTERVAL, IN YEARS, AND ANNUAL } \\
\text { EXCEEDANCE PROBABILITY, IN PERCENT }\end{array}$} \\
\hline & 2 & 5 & 10 & 25 & 50 & 100 \\
\hline & $50 \%$ & $20 \%$ & 10, & $4 \%$ & $2 \%$ & $1 \%$ \\
\hline 1 & 9600 & 16600 & 20600 & 24500 & 26800 & -- \\
\hline 3 & 7850 & 13700 & 17200 & 21000 & 23300 & -- \\
\hline 7 & 5960 & 11000 & 14300 & 18400 & 21200 & -- \\
\hline 15 & 4460 & 8360 & 11100 & 14600 & 17100 & -- \\
\hline 30 & 3410 & 6420 & 8570 & 11300 & 13400 & -- \\
\hline 60 & 2610 & 4830 & 6350 & 8220 & 9540 & -- \\
\hline 90 & 2170 & 3970 & 5190 & 6660 & 7690 & - \\
\hline
\end{tabular}

DURATION TABLE OF DAILY MEAN FLOW FOR PERIOD OF RECORD 1950-82

\begin{tabular}{|c|c|c|c|c|c|c|c|c|c|c|c|c|c|c|}
\hline & & DIs & ARGE, & CFS, & $\mathrm{ICH}$ & EQUAL & OR EX & EED & INDIC & D PER & OF & & & \\
\hline $5 \%$ & $10 \%$ & $15 \%$ & $20 \%$ & $25 \%$ & $30 \%$ & $40 \%$ & $50 \%$ & $60 \%$ & $70 \%$ & $75 \%$ & $80 \%$ & $85 \%$ & $90 \%$ & $95 \%$ \\
\hline 3850 & 2480 & 1740 & 1180 & 797 & 576 & 332 & 236 & 194 & 166 & 152 & 138 & 124 & 107 & 87 \\
\hline
\end{tabular}




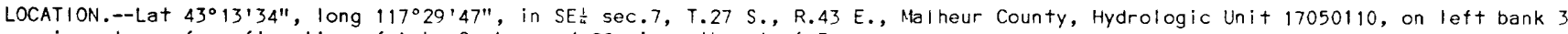

$\mathrm{mi}$ upstream from flow $\mathrm{I}$ ine of Lake Owyhee and $26 \mathrm{mi}$ nor theast of Rome.

DRAINAGE AREA. $--10,400 \mathrm{mi}^{2}$, approximately.

PERIOD OF RECORO.--April 1929 to September 1051. "lonthly jischarge only for some periods, Dublished in wSP i317.

GAGE.- Water-stage recorder. Al+itude of jage is : 6a? $\mathrm{f}+$ liational Geodetic Vertical batum of 1929 (levels by Bureau of Reclamation).

REMARKS.--Flow regulated by Antelope Reservoir, Wild Horse Reservoir, and numerous small reservoirs. Diversions above station for irrigation.

AVERAGE DISCHARGE.--22 years (water years $1930-51$ ), $851 \mathrm{ft} 3 / \mathrm{s}, 616,100 \mathrm{acre}-f+\mathrm{yr}$.

EXTREMES FOR PERIOD OF RECORR.--Maximum discharge, 16,000 $\mathrm{ft} 3 / \mathrm{s} \mathrm{Mar.} \mathrm{20,} \mathrm{1932,} \mathrm{Apr.} \mathrm{19,} \mathrm{1936;} \mathrm{maximum} \mathrm{gage} \mathrm{height,} \mathrm{12.95} \mathrm{ft}$ Mar. 20, 1932; minimum discharge, $99 \mathrm{ft} / \mathrm{s} \mathrm{Dec.} \mathrm{18,} \mathrm{1948,} \mathrm{gage} \mathrm{height,} 3.45 \mathrm{ft}$.

STATISTICAL SUMMARIES

MONTHLY AND ANNUAL MEAN DISChARGES $1931-51$

\begin{tabular}{|c|c|c|c|c|c|c|}
\hline MONTH & $\begin{array}{l}\text { MINIMUM } \\
\text { (CFS) }\end{array}$ & $\begin{array}{l}\text { MAXIMUM } \\
\text { (CFS) }\end{array}$ & $\begin{array}{l}\text { MEAN } \\
\text { (CFS) }\end{array}$ & $\begin{array}{l}\text { STAN- } \\
\text { DARD } \\
\text { DEVIA- } \\
\text { TION } \\
\text { (CFS) }\end{array}$ & $\begin{array}{l}\text { COEFFI- } \\
\text { CIENT OF } \\
\text { VARI- } \\
\text { ATION }\end{array}$ & $\begin{array}{c}\text { PERCENT } \\
\text { OF } \\
\text { ANNUAL } \\
\text { RUNOFF }\end{array}$ \\
\hline OCTOBER & 149 & 289 & 200 & 38 & .19 & 1.9 \\
\hline NOVEMBER & 159 & 450 & 252 & 73 & .29 & 2.4 \\
\hline DECEMBER & 161 & 959 & 335 & 211 & .63 & 3.2 \\
\hline JANUARY & 174 & 1885 & 389 & 375 & .96 & 3.7 \\
\hline FEBRUARY & 197 & 3554 & 889 & 889 & 1.00 & 8.4 \\
\hline MARCH & 336 & 4052 & 1841 & 1195 & .65 & 17.5 \\
\hline APRIL & 384 & 7309 & 3507 & 2050 & .58 & 33.2 \\
\hline MAY & 171 & 4000 & 1657 & 1065 & .64 & 15.7 \\
\hline JUNE & 137 & 2270 & 861 & 566 & .66 & 8.2 \\
\hline JULY & 115 & 482 & 261 & 91 & .35 & 2.5 \\
\hline AUGUST & 116 & 238 & 180 & 39 & .21 & 1.7 \\
\hline SEPTEMBER & 132 & 223 & 177 & 33 & .18 & 1.7 \\
\hline ANNUAL & 212 & 1529 & 875 & 372 & .43 & 100 \\
\hline
\end{tabular}

MAGNITUDE AND PROBABILITY OF INSTANTANEOUS PEAK FLOW BASED ON PERIOD OF RECORD 1931-51

DISCHARGE, IN CFS, FOR INDICATED RECURRENCE INTERVAL, IN YEARS, AND ANNUAL EXCEEDANCE PROBABILITY, IN PERCENT

\begin{tabular}{ccccccc}
1.25 & 2 & 5 & 10 & 25 & 50 & 100 \\
$80 \%$ & $50 \%$ & $20 \%$ & $10 \%$ & $4 \%$ & $2 \%$ & $1 \%$ \\
\hline 4310 & 7610 & 12800 & 16600 & 21400 & - & -
\end{tabular}

WEIGHTED SKEW $=-.308$
MAGNITUDE AND PROBABILITY OF ANNUAL LOW FLOW BASED ON PERIOD OF RECORD 1932-51

\begin{tabular}{|c|c|c|c|c|c|c|}
\hline $\begin{array}{l}\text { PERIOD } \\
\text { (CON- } \\
\text { SECU- }\end{array}$ & \multicolumn{6}{|c|}{$\begin{array}{l}\text { DISCHARGE, IN CFS, FOR INDICATED RECURRENCE } \\
\text { INTERVAL, IN YEARS, AND ANNUAL NON- } \\
\text { EXCEEDANCE PROBABILITY, IN PERCENT }\end{array}$} \\
\hline TIVE & 2 & 5 & 10 & 20 & 50 & 100 \\
\hline DAYS) & $50 \%$ & $20 \%$ & $10 \%$ & $5 \%$ & $2 \%$ & $1 \%$ \\
\hline 1 & 155 & 130 & 118 & 109 & -- & - \\
\hline 3 & 158 & 132 & 119 & 109 & -- & -- \\
\hline 7 & 161 & 133 & 120 & 109 & -- & -- \\
\hline 14 & 164 & 135 & 121 & 110 & -- & -- \\
\hline 30 & 168 & 138 & 124 & 112 & -- & -- \\
\hline 60 & 173 & 143 & 128 & 117 & -- & -- \\
\hline 90 & 181 & 149 & 134 & 122 & -- & -- \\
\hline 120 & 190 & 157 & 141 & 128 & -- & -- \\
\hline 183 & 214 & 174 & 155 & 142 & -- & -- \\
\hline
\end{tabular}

MAGNITUDE AND PROBABILITY OF ANNUAL HIGH FLOW BASED ON PERIOD OF RECORD 1931-51

\begin{tabular}{|c|c|c|c|c|c|c|}
\hline $\begin{array}{l}\text { PERIOD } \\
\text { (CON- }\end{array}$ & \multicolumn{6}{|c|}{$\begin{array}{l}\text { DISCHARGE, IN CFS, FOR INDICATED RECURRE } \\
\text { INTERVAL, IN YEARS, AND ANNUAL } \\
\text { EXCEEDANCE PROBABILITY, IN PERCENT }\end{array}$} \\
\hline SECU- & -0 & $\cdots$ & 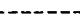 & $-\infty$ & 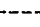 & \\
\hline TIVE & 2 & 5 & 10 & 25 & 50 & 100 \\
\hline DAYS) & $50 \%$ & $20 \%$ & $10 \%$ & $4 \%$ & $2 \%$ & 19 \\
\hline 1 & 7570 & 12100 & 14400 & 16700 & -- & - \\
\hline 3 & 6440 & 10500 & 12800 & 15100 & -- & -- \\
\hline 7 & 5330 & 9090 & 11500 & 14200 & -- & -- \\
\hline 15 & 4500 & 7760 & 9820 & 12200 & - & -- \\
\hline 30 & 3770 & 6210 & 7560 & 8950 & -- & -- \\
\hline 60 & 2970 & 4600 & 5380 & 6080 & -- & -- \\
\hline 90 & 2500 & 3780 & 4380 & 4890 & -- & -- \\
\hline
\end{tabular}

DURATION TABLE OF DAILY MEAN FLOW FOR PERIOD OF RECORD 1931-51

DISCHARGE, IN CFS, WHICH WAS EQUALED OR EXCEEDED FOR INDICATED PERCENT OF TIME

\begin{tabular}{|c|c|c|c|c|c|c|c|c|c|c|c|c|c|c|}
\hline $5 \%$ & $10 \%$ & $15 \%$ & $20 \%$ & $25 \%$ & $30 \%$ & $40 \%$ & $50 \%$ & $60 \%$ & $70 \%$ & $75 \%$ & $80 \%$ & $85 \%$ & $90 \%$ & $95 \%$ \\
\hline 3720 & 2460 & 1750 & 1230 & 797 & 541 & 335 & 264 & 230 & 206 & 196 & 185 & 172 & 160 & 138 \\
\hline
\end{tabular}


13183000 OWYHEE RIVER BELCW OWYHEE OAM, OR

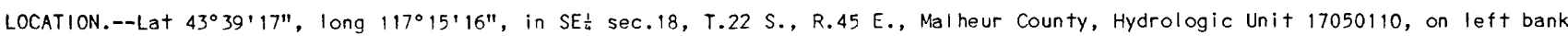
$0.8 \mathrm{mi}$ downstream trom Owyhee Dam, $20 \mathrm{mi}$ southwest of Nyssa, and at mile 27.3 .

DRAINAGE AREA. $--11,160 \mathrm{mi}^{2}$, approximately.

PERIOD OF RECORD.--February 1929 to September 1982.

GAGE.--Water-stage recorder. Datum of gage is 2,343.6) + Hational Geodetic Vertical Datum of 1929 (levels by Bureau of Reclamation).

REMARKS.--flow regulated since October 1932 by Lake Owyhee, and by many smaller reservoirs. Diversion of 429 , 100 acre-ft from Lake Owyhee during the year for irrigation of lands below station and outside the basin. Many smaller diversions above Lake Owyhe for irrigation above station.

COOPERATION.--Water-stage recorder inspected by irrigation district employees.

AVERAGE DISCHARGE. - 50 years (water years 1933-82), $356 \mathrm{ft} 3 / \mathrm{s}, 257,900$ acre- $\mathrm{ft} / \mathrm{yr}$, not adjusted for storage or diversion.

EXTREMES FOR PERIOD OF RECORD.--Maximum discharge, 22,900 $\mathrm{ft} \mathrm{t}^{3} / \mathrm{s}$ Apt. 15, 1952, gage height, $15.70 \mathrm{ft}$; no $\mathrm{flow}$ for part of Aug. 8, 9, 1932, when temporary diversion tunnel at Owyhee Dam was closed.

STATISTICAL SUMMARIES

MONTHLY AND ANNUAL MEAN DISCHARGES 1933-82

\begin{tabular}{|c|c|c|c|c|c|c|}
\hline MONTH & $\begin{array}{c}\text { MINIMUM } \\
\text { (CFS) }\end{array}$ & $\begin{array}{c}\text { MAXIMUM } \\
\text { (CFS) }\end{array}$ & $\begin{array}{l}\text { MEAN } \\
\text { (CFS) }\end{array}$ & $\begin{array}{l}\text { STAN- } \\
\text { DARD } \\
\text { DEVIA- } \\
\text { TION } \\
\text { (CFS) }\end{array}$ & $\begin{array}{l}\text { COEFFI- } \\
\text { CIENT OF } \\
\text { VARI- } \\
\text { ATION }\end{array}$ & $\begin{array}{c}\text { PERCENT } \\
\text { OF } \\
\text { ANNUAL } \\
\text { RUNOFF }\end{array}$ \\
\hline OCTOBER & 2.8 & 112 & 36 & 29 & .81 & .8 \\
\hline NOVEMBER & 1.0 & 196 & 8.6 & 27 & 3.17 & .2 \\
\hline DECEMBER & 1.7 & 51 & 5.4 & 7.0 & 1.29 & .1 \\
\hline JANUARY & 1.5 & 2751 & 112 & 458 & 4.09 & 2.6 \\
\hline FEBRUARY & 1.8 & 4468 & 287 & 893 & 3.11 & 6.7 \\
\hline MARCH & 2.0 & 7799 & 826 & 1496 & 1.81 & 19.3 \\
\hline APRIL & 28 & 12560 & 1620 & 2296 & 1.42 & 37.8 \\
\hline MAY & 40 & 4864 & 725 & 1035 & 1.43 & 16.9 \\
\hline JUNE & 46 & 1413 & 269 & 320 & 1.19 & 6.3 \\
\hline JULY & 44 & 618 & 160 & 85 & .53 & 3.7 \\
\hline AUGUST & 22 & 312 & 137 & 60 & .44 & 3.2 \\
\hline SEPTEMBER & 8.0 & 248 & 103 & 48 & .46 & 2.4 \\
\hline ANNUAL & 22 & 1610 & 356 & 370 & 1.04 & 100 \\
\hline
\end{tabular}

MAGNITUDE AND PROBABILITY OF INSTANTANEOUS PEAK FLOW BASED ON PERIOD OF RECORD

DISCHARGE, IN CFS, FOR INDICATED RECURRENCE INTERVAL, IN YEARS, AND ANNUAL EXCEEDANCE PROBABILITY, IN PERCENT

\begin{tabular}{ccccccc}
1.25 & 2 & 5 & 10 & 25 & 50 & 100 \\
$80 \%$ & $50 \%$ & $20 \%$ & $10 \%$ & $4 \%$ & $2 \%$ & $1 \%$ \\
\hline- & - & - & & & & \\
\hline & - & - & - & - & -
\end{tabular}

MAGNITUDE AND PROBABILITY OF ANNUAL LOW FLOW BASED ON PERIOD OF RECORD 1934-82

\begin{tabular}{|c|c|c|c|c|c|c|}
\hline $\begin{array}{l}\text { PERIOD } \\
\text { ICON- }\end{array}$ & \multirow{2}{*}{\multicolumn{6}{|c|}{$\begin{array}{l}\text { DISCHARGE, IN CFS, FOR INDICATED RECURRENCE } \\
\text { INTERVAL, IN YEARS, AND ANNUAL NON- } \\
\text { EXCEEDANCE PROBABILITY, IN PERCENT }\end{array}$}} \\
\hline SECU- & & & & & & \\
\hline TIVE & 2 & 5 & 10 & 20 & 50 & 100 \\
\hline DAYS) & $50 \%$ & $20 \%$ & $10 \%$ & $5 \%$ & 29 & $1 \%$ \\
\hline 1 & 3.2 & 2.1 & 1.6 & 1.4 & 1.1 & \\
\hline 3 & 3.3 & 2.1 & 1.7 & 1.4 & 1.2 & \\
\hline 7 & 3.3 & 2.1 & 1.7 & 1.4 & 1.2 & \\
\hline 14 & 3.4 & 2.2 & 1.7 & 1.4 & 1.2 & \\
\hline 30 & 3.5 & 2.2 & 1.8 & 1.5 & 1.2 & \\
\hline 60 & 3.5 & 2.4 & 2.0 & 1.7 & 1.5 & \\
\hline 90 & 3.8 & 2.5 & 2.1 & 1.8 & 1.6 & \\
\hline 120 & 4.0 & 2.6 & 2.2 & 2.0 & 1.8 & \\
\hline 183 & 13 & 5.9 & 4.0 & 2.9 & 2.0 & \\
\hline
\end{tabular}

MAGNITUDE AND PROBABILITY OF ANNUAL HIGH FLOW BASED ON FERIOD OF RECORD 1933-82

\begin{tabular}{|c|c|c|c|c|c|c|}
\hline $\begin{array}{l}\text { PER!OD } \\
\text { (CON- } \\
\text { SFCII- }\end{array}$ & \multicolumn{6}{|c|}{$\begin{array}{l}\text { DISCHARGE, IN CFS, FOR INDICATED RECURRE } \\
\text { INTERVAL, IN YEARS, AND ANNUAL } \\
\text { EXCEEDANCE PROBABILITY, IN PERCENT }\end{array}$} \\
\hline TIVE & 2 & 5 & 10 & 25 & 50 & 100 \\
\hline DAYS) & $50 \%$ & $20 \%$ & $10 \%$ & $4 \%$ & $2 \%$ & $1 \%$ \\
\hline 1 & 1290 & 6060 & 13400 & 30700 & 52200 & 83800 \\
\hline 3 & 1220 & 5670 & 12500 & 28800 & 49100 & 78900 \\
\hline 7 & 1110 & 5070 & 11100 & 25500 & 43300 & 69700 \\
\hline 15 & 979 & 4350 & 9400 & 21200 & 35800 & 57200 \\
\hline 30 & 793 & 3290 & 6890 & 15100 & 25100 & 39600 \\
\hline 60 & 617 & 2320 & 4650 & 9780 & 15800 & 24400 \\
\hline 90 & 523 & 1810 & 3480 & 6990 & 11000 & 16500 \\
\hline
\end{tabular}

DURATION TABLE OF DAILY MEAN FLOW FOR PERIOD OF RECORD 1933-82

DISCHARGE, IN CFS, WHICH WAS EQUALED OR EXCEEDED FOR INDICATED PERCENT OF TIME

\begin{tabular}{|c|c|c|c|c|c|c|c|c|c|c|c|c|c|c|}
\hline 53 & $10 \%$ & $15 \%$ & $20 \%$ & $25 \%$ & $30 \%$ & $40 \%$ & $50 \%$ & $60 \%$ & $70 \%$ & $75 \%$ & $80 \%$ & $85 \%$ & $90 \%$ & $95 \%$ \\
\hline 2090 & 428 & 220 & 194 & 168 & 148 & 104 & 57 & 8.3 & 5.2 & 4.2 & 3.6 & 3.2 & 2.8 & 2.3 \\
\hline
\end{tabular}




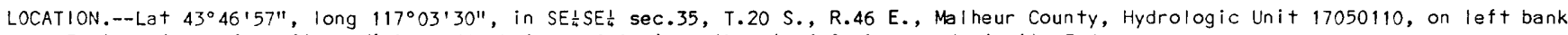
$0.3 \mathrm{mi}$ upstream from State Highway 201 bridge, $0.9 \mathrm{mi}$ southwest of Owyee, and at mile 3.1 .

DRAINAGE AREA. $--11,300 \mathrm{mi}^{2}$, approximately.

PERIOD OF RECORD.--llarch 1890 to June 1891. February to June 1892. February to July, October to December 1893 , January 1895 to May 1897. August 1903 to September 1916, May 1920 to July 1929 , July 1979 to September 1982 . Monthly discharge only for some periods publisted in WSP 1317. Published as "at Rigsby", 1890-93; "at Nyssa", 1985-96; and as "at Owyhee" in WSP 370. Records for September, October 1903, May to October 1904, Harch, April 1905, published in WSP 135 in conjunction with records for Owyhee River near Owyhee and in WSP 370, have been found in error and should not be used.

GAGE.- Water-stage recorder. Altitude of gage is 2,190 $\mathrm{ft}$, from topographic map.

REMARKS.--Flow regulated since October 1932 by Lake Owyhee, and smaller reservoirs. Diversions trom Lake Owyhee for irrigation of

lands above station and outside the basin. Many smaller diversions above Lake Owyhee for irrigation.

AVERAGE DISCHARGE.--20 years (water years $1896,1904-16,1922-27$ ), $1,048 \mathrm{ft} / \mathrm{s}, 759,300 \mathrm{acre}-\mathrm{ft} / \mathrm{yr}$.

EXTREMES FOR PERIOD OF RECORO.--Maximum discharge, 29,000 $\mathrm{ft}^{3} / \mathrm{s}$ Mar. 2, 1s: $\mathrm{C}$. gage height, $12.9 \mathrm{ft}$ site and datum then in use, from rating curve extended above $14,000 \mathrm{ft} 3 / \mathrm{s}$; no flow July 7 , 19, Aug. 14-16, 1924, July 5, 6, 1926 . Maximum discharge recorded since construction of Owyhee Dam in 1932, 7,790 ft3/s Feb. 23, 1982, gage heizht, $11.91 \mathrm{ft}$.

STATISTICAL SUMMARIES

MONTHLY AND ANNUAL MEAN DISCHARGES $1896-28$

\begin{tabular}{|c|c|c|c|c|c|c|}
\hline MONTH & $\begin{array}{l}\text { MINIMUM } \\
\text { (CFS) }\end{array}$ & $\begin{array}{l}\text { MAXIMUM } \\
\text { (CFS) }\end{array}$ & $\begin{array}{l}\text { MEAN } \\
\text { (CFS) }\end{array}$ & $\begin{array}{l}\text { STAN- } \\
\text { DARO } \\
\text { DEVIA- } \\
\text { TION } \\
\text { (CFS) }\end{array}$ & $\begin{array}{l}\text { COEFFI- } \\
\text { CIENT OF } \\
\text { VARI- } \\
\text { ATION }\end{array}$ & $\begin{array}{c}\text { PERCENT } \\
\text { OF } \\
\text { ANNUAL } \\
\text { RUNOFF }\end{array}$ \\
\hline \multicolumn{7}{|l|}{$---n$} \\
\hline OCTOBER & 32 & 162 & 86 & 42 & .49 & .7 \\
\hline NOVEMBER & 70 & 362 & 173 & 80 & .46 & 1.4 \\
\hline DECEMBER & 135 & 864 & 278 & 159 & .57 & 2.2 \\
\hline JANUARY & 141 & 4740 & 704 & 1039 & 1.48 & 5.6 \\
\hline FEBRUARY & 247 & 5559 & 1416 & 1358 & .96 & 11.4 \\
\hline MARCH & 487 & 12460 & 3053 & 2950 & .97 & 24.5 \\
\hline APRIL & 616 & 7256 & 3425 & 1942 & .57 & 27.5 \\
\hline MAY & 114 & 6075 & 2159 & 1625 & .75 & 17.3 \\
\hline JUNE & 5.6 & 2636 & 980 & 761 & .78 & 7.9 \\
\hline JULY & 3.2 & 498 & 130 & 141 & 1.08 & 1.0 \\
\hline AUGUST & 2.5 & 170 & 29 & 37 & 1.28 & .2 \\
\hline SEPTEMBER & 2.5 & 174 & 38 & 38 & .99 & .3 \\
\hline ANNUAL & 301 & 1828 & 1014 & 498 & .49 & 100 \\
\hline
\end{tabular}

MAGNITUDE AND PROBABILITY OF INSTANTANEOUS PEAK FLOW BASED ON PERIOD OF RECORO 1896-27

DISCHARGE, IN CFS, FOR INDICATEO RECURRENCE INTERVAL, IN YEARS, AND ANNUAL EXCEEOANCE PROBABILITY, IN PERCENT

\begin{tabular}{ccccccc}
1.25 & 2 & 5 & 10 & 25 & 50 & 100 \\
$80 \%$ & $50 \%$ & $20 \%$ & $10 \%$ & $4 \%$ & $2 \%$ & $1 \%$ \\
\hline 5570 & 9410 & 15800 & 20700 & 27600 & 33200 & $\ldots$
\end{tabular}

WEIGHTEO SKEW $=-.035$
MAGN ITUDE AND PROBABILITY OF ANNUAL LOW FLOW BASED ON PERIOD OF RECORD 1905-27

\begin{tabular}{|c|c|c|c|c|c|c|}
\hline $\begin{array}{l}\text { PERIOD } \\
\text { (CON- }\end{array}$ & \multicolumn{6}{|c|}{$\begin{array}{l}\text { DISCHARGE, IN CFS, FOR INDICATED RECURRENCE } \\
\text { INTERVAL, IN YEARS, AND ANNUAL NON- } \\
\text { EXCEEDANCE PROBABILITY, IN PERCENT }\end{array}$} \\
\hline SECU- & & --- & $\cdots$ & $\cdots$ & -- & 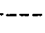 \\
\hline TIVE & 2 & 5 & 10 & 20 & 50 & 100 \\
\hline DAYS) & $50 \%$ & $20 \%$ & $10 \%$ & $5 \%$ & $2 \%$ & $1 \%$ \\
\hline 1 & 3.8 & 1.6 & 10 & 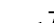 & 5 & -- \\
\hline 3 & 4.0 & 1.6 & 1.0 & .7 & .5 & - \\
\hline 7 & 5.1 & 1.8 & 1.1 & .8 & .6 & -- \\
\hline 14 & 6.0 & 2.2 & 1.2 & .8 & .6 & -- \\
\hline 30 & 7.6 & 3.8 & 2.8 & 2.2 & 1.8 & -- \\
\hline 60 & 13 & 5.9 & 4.0 & 2.8 & 2.0 & -- \\
\hline 90 & 20 & 9.4 & 6.3 & 4.6 & 3.2 & -- \\
\hline 120 & 39 & 19 & 13 & 9.1 & 5.9 & -- \\
\hline 183 & 92 & 54 & 39 & 29 & 21 & - \\
\hline
\end{tabular}

MAGNITUDE ANO PROBABILITY OF ANNUAL HIGH FLOW BASED ON PERIOD OF RECORD 1896-27

\begin{tabular}{|c|c|c|c|c|c|c|}
\hline $\begin{array}{l}\text { PERIOD } \\
\text { (CON- } \\
\text { SECU- }\end{array}$ & \multicolumn{6}{|c|}{$\begin{array}{l}\text { DISCHARGE, IN CFS, FOR IND ICATED RECURRE } \\
\text { INTERVAL, IN YEARS, AND ANNUAL } \\
\text { EXCEEDANCE PROBABILITY, IN PERCENT }\end{array}$} \\
\hline TIVE & 2 & 5 & 10 & 25 & 50 & 100 \\
\hline DAYS) & $50 \%$ & $20 \%$ & $10 \%$ & $4 \%$ & $2 \%$ & $1 \%$ \\
\hline 1 & 9030 & 14500 & 18200 & 23000 & 26600 & - \\
\hline 3 & 7700 & 12500 & 15700 & 19600 & 22400 & - \\
\hline 7 & 6570 & 10600 & 13000 & 15900 & 17800 & -- \\
\hline 15 & 5310 & 8450 & 10400 & 12600 & 14100 & -- \\
\hline 30 & 4190 & 7010 & 8870 & 11100 & 12800 & - \\
\hline 60 & 3250 & 5540 & 7050 & 8880 & 10200 & - \\
\hline 90 & 2800 & 4620 & 5810 & 7260 & 8280 & - \\
\hline
\end{tabular}

DURATION TABLE OF DAILY MEAN FLOW FOR PERIOD OF RECORD 1896-27

\begin{tabular}{|c|c|c|c|c|c|c|c|c|c|c|c|c|c|c|}
\hline 56 & $10 \%$ & $15 \%$ & $20 \%$ & $25 \%$ & $30 \%$ & $40 \%$ & $50 \%$ & $60 \%$ & $70 \%$ & $75^{\circ}$ & $80 \%$ & $85 \%$ & $90 \%$ & $95 \%$ \\
\hline 5010 & 3240 & 2110 & 1450 & 1020 & 726 & 402 & 257 & 171 & 104 & 69 & 43 & 25 & 11 & 5.6 \\
\hline
\end{tabular}


13214000 MALHEUR RIVER NEAR DREWSEY, OR

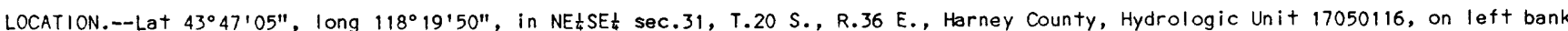
$300 \mathrm{ft}$ downstream from bridge on U.S. Highway $20,0.5 \mathrm{mi}$ downstream from Cottonwood Creek, $3.0 \mathrm{mi}$ southeast of Drewsey, and at mile 129.0 .

DRAINAGE AREA.--910 $\mathrm{mi}^{2}$, approximately.

PERIOD OF RECORD.--June 1920 to September 1921, November, December 1921, March, April 1922, April to September 1923, June 1.20 t3 September 1982. Monthly discharge only for' some periods, published in WSP 1317 . March to September 1914 at site 13 mi (21 km) upstream; records not equivalent owing to inflow from several creeks.

GAGE.--Water-stage recorder. Datum of gage is 3,479.13 ft National Geodetic Vertical Datum of 1929. Prior to Apr. 27, 1923, water-stage recorder or nonrecording gage at site $0.5 \mathrm{mi}$ downstream at different datum. Apr. 27 , 1923, to June 6 , 1939, water-stage recorder at site $7 \mathrm{mi}$ downstream at different datum.

REMARKS.--SIight regulation by small reservoirs above station. Diversions for irrigation above station.

AVERAGE DISCHARGE. -56 years (water years 1927-82), $184 \mathrm{ft} /, 133,300 \mathrm{acre}-\mathrm{ft} / \mathrm{yr}$.

EXTREMES FOR PERIOD OF RECORD.--Maximum discharge, 12,000 $\mathrm{ft}^{3} / \mathrm{s}$ Dec. 23, 1964, gage height, $13.50 \mathrm{ft}$, from rating curve extended above $4,500 \mathrm{ft} / \mathrm{s}$, on basis of contracted-opening measurement at gage height $13.20 \mathrm{ft}$; no $\mathrm{flow}$ at times.

STATISTICAL SUMMARIES

MONTHLY AND ANNUAL MEAN DISCHARGES 1927-82

\begin{tabular}{|c|c|c|c|c|c|c|}
\hline MONTH & $\begin{array}{l}\text { MINIMUM } \\
\text { (CFS) }\end{array}$ & $\begin{array}{l}\text { MAXIMUM } \\
\text { (CFS) }\end{array}$ & $\begin{array}{l}\text { MEAN } \\
\text { (CFS) }\end{array}$ & $\begin{array}{c}\text { STAN- } \\
\text { DARD } \\
\text { DEVIA- } \\
\text { TION } \\
\text { (CFS) }\end{array}$ & $\begin{array}{l}\text { COEFFI- } \\
\text { CIENT OF } \\
\text { VARI- } \\
\text { ATION }\end{array}$ & $\begin{array}{c}\text { PERCENT } \\
\text { OF } \\
\text { ANNUAL } \\
\text { RUNOFF }\end{array}$ \\
\hline OCTOBER & 5.3 & 73 & 38 & 17 & .44 & 1.7 \\
\hline $\begin{array}{l}\text { NOVEMBER } \\
\text { DECEMBER }\end{array}$ & $\begin{array}{l}5.1 \\
9.0\end{array}$ & $\begin{array}{l}178 \\
739\end{array}$ & $\begin{array}{r}66 \\
104\end{array}$ & $\begin{array}{r}26 \\
105\end{array}$ & $\begin{array}{r}.40 \\
1.01\end{array}$ & $\begin{array}{l}3.0 \\
4.7\end{array}$ \\
\hline JANUARY & 20 & 817 & 146 & 174 & 1.19 & 6.6 \\
\hline FEBRUARY & 20 & 1124 & 258 & 223 & .86 & 11.7 \\
\hline MARCH & 55 & 1314 & 419 & 273 & .65 & 19.0 \\
\hline APRIL & 44 & 2290 & 653 & 425 & .65 & 29.5 \\
\hline MAY & 18 & 1136 & 345 & 249 & .72 & 15.6 \\
\hline JUNE & 8.0 & 436 & 134 & 100 & .75 & 6.1 \\
\hline JULY & 1.9 & 158 & 26 & 27 & 1.05 & 1.2 \\
\hline AUGUST & 0.0 & 60 & 9.5 & 11 & 1.12 & .4 \\
\hline SEPTEMBER & 0.0 & 59 & 12 & 11 & .89 & .6 \\
\hline ANNUAL & 34 & 423 & 183 & 98 & .53 & 100 \\
\hline
\end{tabular}

MAGNITUDE AND PROBABILITY OF INSTANTANEOUS PEAK FLOW BASED ON PERIOD OF RECORD 1927-82

DISCHARGE, IN CFS, FOR INDICATED RECURRENCE INTERVAL, IN YEARS, AND ANNUAL EXCEEDANCE PROBABILITY, IN PERCENT

\begin{tabular}{|c|c|c|c|c|c|c|}
\hline $\begin{array}{l}1.25 \\
80 \%\end{array}$ & $\begin{array}{c}2 \\
50 \%\end{array}$ & $\begin{array}{c}5 \\
20 \%\end{array}$ & $\begin{array}{l}10 \\
10 \%\end{array}$ & $\begin{array}{l}25 \\
4 \%\end{array}$ & $\begin{array}{l}50 \\
2 \%\end{array}$ & $\begin{array}{r}100 \\
1 \%\end{array}$ \\
\hline 1020 & 1980 & 3850 & 5440 & 7890 & 10000 & 12500 \\
\hline
\end{tabular}

WEIGHTED SKEW $=.038$
MAGNITUDE AND PROBABILITY OF ANNUAL LOW FLOW BASED ON PERIOD OF RECORD 1928-82

\begin{tabular}{|c|c|c|c|c|c|c|}
\hline \multirow{4}{*}{$\begin{array}{l}\text { PERIOD } \\
\text { (CON- } \\
\text { SECU- } \\
\text { TIVE } \\
\text { DAYS) }\end{array}$} & \multicolumn{6}{|c|}{$\begin{array}{l}\text { DISCHARGE, IN CFS, FOR INDICATED RECURRENCE } \\
\text { INTERVAL, IN YEARS, AND ANNUAL NON- } \\
\text { EXCEEDANCE PROBABILITY, IN PERCENT }\end{array}$} \\
\hline & & $=-$ & - & -- & & \\
\hline & 2 & 5 & 10 & 20 & 50 & 100 \\
\hline & $50 \%$ & $20 \%$ & $10 \%$ & $5 \%$ & 28 & $1 \%$ \\
\hline 1 & -- & -- & -- & -- & -- & -- \\
\hline 3 & -- & -- & -- & - & - & -- \\
\hline 7 & -- & -- & -- & -- & -- & -- \\
\hline 14 & -- & -- & -- & -- & -. & -- \\
\hline 30 & -- & -- & -- & -- & -- & -- \\
\hline 60 & -- & -- & -- & -- & -- & -- \\
\hline 90 & 10 & 3.7 & 1.8 & 1.0 & .4 & \\
\hline 120 & 16 & 7.7 & 4.9 & 3.3 & 2.0 & \\
\hline 183 & 33 & 20 & 14 & 10 & 7.1 & \\
\hline
\end{tabular}

NOTE: LOW-FLOW STATISTICS UNCERTAIN DUE TO EXCESSIVE ZERO EVENTS.

MAGNITUDE AND PROBABILITY OF ANNUAL HIGH FLOW BASED ON PERIOD OF RECORD 1927-82

\begin{tabular}{|c|c|c|c|c|c|c|}
\hline $\begin{array}{l}\text { PERIOD } \\
\text { (CON- }\end{array}$ & \multicolumn{6}{|c|}{$\begin{array}{c}\text { DISCHARGE, IN CFS, FOR INDICATED RECURRENCE } \\
\text { INTERVAL, IN YEARS, AND ANNUAL } \\
\text { EXCEEDANCE PROBABILITY, IN PERCENT }\end{array}$} \\
\hline $\begin{array}{l}\text { TIVE } \\
\text { DAYSI) }\end{array}$ & $\begin{array}{c}2 \\
50 \%\end{array}$ & $\begin{array}{c}5 \\
20 \%\end{array}$ & $\begin{array}{l}10 \\
10 \%\end{array}$ & $\begin{array}{l}25 \\
4 \%\end{array}$ & $\begin{array}{l}50 \\
2 \%\end{array}$ & $\begin{array}{r}100 \\
1 \%\end{array}$ \\
\hline $\begin{array}{l}1 \\
3\end{array}$ & $\begin{array}{l}1530 \\
1300\end{array}$ & $\begin{array}{l}2870 \\
2380\end{array}$ & $\begin{array}{l}3830 \\
3110\end{array}$ & $\begin{array}{l}5060 \\
4020\end{array}$ & $\begin{array}{l}5970 \\
4660\end{array}$ & $\begin{array}{l}6860 \\
5280\end{array}$ \\
\hline 7 & 1070 & 1870 & 2370 & 2960 & 3350 & 3700 \\
\hline 15 & 880 & 1470 & 1820 & 2190 & 2420 & 2610 \\
\hline 30 & 707 & 1180 & 1470 & 1780 & 1990 & 2160 \\
\hline 60 & 552 & 912 & 1140 & 1390 & 1560 & 1710 \\
\hline 90 & 467 & 756 & 932 & 1130 & 1270 & 1380 \\
\hline
\end{tabular}

DURATION TABLE OF DAILY MEAN FLOW FOR PERIOD OF RECORD 1927-82

\begin{tabular}{|c|c|c|c|c|c|c|c|c|c|c|c|c|c|c|}
\hline $5 \%$ & $10 \%$ & $15 \%$ & $20 \%$ & $25 \%$ & $30 \%$ & $40 \%$ & $50 \%$ & $60 \%$ & $70 \%$ & $75 \%$ & $80 \%$ & $85 \%$ & $90 \%$ & $95 \%$ \\
\hline 789 & 508 & 358 & 259 & 189 & 142 & 90 & 67 & 50 & 32 & 24 & 17 & 11 & 6.0 & 2.7 \\
\hline
\end{tabular}




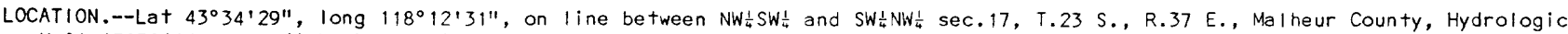
Unit 17050116, on left bank $0.9 \mathrm{mi}$ downstream from Warmsprings Dam, $3.0 \mathrm{mi}$ upstream from South Fork, $4.0 \mathrm{mi}$ northwest of Riverside, and at mile 113.0 .

DRAINAGE AREA. $--1,100 \mathrm{mi}^{2}$, approximately.

PERIOD OF RECORD.--January 1906 to March 1907 and December 1908 (gage heights on ty), January 1909 to September 1910 , December 1914 to July 1917, March 1919 to September 1982. Monthly discharge only for some periods, published in WSP 1317 . Figures of discharge for January 1906 to March 1907, published in WSP 272 and 370 , have been found to be unreliable and should not be used. Published as "Middle Fork of Malheur River at Riverside" 1906-7; as "Middle Fork of Malheur River above South Fork, at Riverside" 1909-10; as "Malheur River above South Fork, at Riverside" in WSP 370, 1906-10; and as "Ma!heur River at Warmsprings reservoir site, near Riverside" 1914-17.

GAGE.--Water-stage recorder and concrete control. Altitude of gage is 3,305 $\mathrm{ft}$, by barometer. See WSP 1317 or 1737 for history of changes prior to Sept. $29,1949$.

REMARKS.--Flow completely regulated since November 1919 by Warmsprings Reservoir. Diversions for irrigation above station.

AVERAGE DISCHARGE.--63 years (water years 1920-82), $180 \mathrm{ft}^{3} / \mathrm{s}, 130,400 \mathrm{acre}-\mathrm{ft} / \mathrm{yr}$.

EXTREMES FOR PERIOD OF RECORD.--Maximum discharge observed, $7,200 \mathrm{ft} / \mathrm{s}$ Mar. 1 , 1910, gage height, $10.7 \mathrm{ft}$, site and datum then in use, from rating curve extended above $820 \mathrm{ft}^{3} / \mathrm{s}$; no flow at times.

STATISTICAL SUMMARIES

MONTHLY AND ANNUAL MEAN DISCHARGES 1920-82

\begin{tabular}{|c|c|c|c|c|c|c|}
\hline MONTH & $\begin{array}{l}\text { MINIMUM } \\
\text { (CFS) }\end{array}$ & $\begin{array}{l}\text { MAXIMUM } \\
\text { (CFS) }\end{array}$ & $\begin{array}{l}\text { MEAN } \\
\text { (CFS) }\end{array}$ & $\begin{array}{l}\text { STAN- } \\
\text { DARD } \\
\text { DEVIA- } \\
\text { TION } \\
\text { (CFS) }\end{array}$ & $\begin{array}{l}\text { COEFFI- } \\
\text { CIENT OF } \\
\text { VARI- } \\
\text { ATION }\end{array}$ & $\begin{array}{c}\text { PERCENT } \\
\text { OF } \\
\text { ANNUAL } \\
\text { RUNOFF }\end{array}$ \\
\hline OCTOBER & 0.0 & 138 & 31 & 34 & 1.07 & 1.5 \\
\hline NOVEMBER & 0.0 & 20 & 1.0 & 2.7 & 2.72 & 0.0 \\
\hline DECEMBER & 0.0 & 8.5 & .7 & 1.2 & 1.83 & 0.0 \\
\hline JANUARY & 0.0 & 452 & 7.8 & 57 & 7.32 & .4 \\
\hline FEBRUARY & 0.0 & 749 & 24 & 107 & 4.50 & 1.1 \\
\hline MARCH & 0.0 & 741 & 42 & 138 & 3.33 & 1.9 \\
\hline APRIL & 0.0 & 1534 & 281 & 354 & 1.26 & 13.2 \\
\hline MAY & 31 & 1162 & 423 & 219 & .52 & 19.8 \\
\hline JUNE & 92 & 558 & 331 & 99 & .30 & 15.5 \\
\hline JULY & 145 & 677 & 430 & 117 & .27 & 20.2 \\
\hline AUGUST & 5.7 & 575 & 355 & 140 & .39 & 16.7 \\
\hline SEPTEMBER & .1 & 394 & 205 & 110 & .54 & 9.6 \\
\hline NNUAL & 47 & 397 & 178 & 71 & .40 & 100 \\
\hline
\end{tabular}

MAGNITUDE AND PROBABILITY OF INSTANTANEOUS PEAK FLOW BASED ON PERIOD OF RECORD

DISCHARGE, IN CFS, FOR INDICATED RECURRENCE INTERVAL, IN YEARS, AND ANNUAL EXCEEDANCE PROBABILITY, IN PERCENT

\begin{tabular}{ccccccc}
1.25 & 2 & 5 & 10 & 25 & 50 & 100 \\
$80 \%$ & $50 \%$ & $20 \%$ & $10 \%$ & $4 \%$ & $2 \%$ & $1 \%$ \\
\hline- & - & - & - & - & - & - \\
\hline
\end{tabular}

WE!GHTED SKEW = --
MAGNITUDE AND PROBABILITY OF ANNUAL LOW FLOW BASED ON PERIOD OF RECORD 1921-82

\begin{tabular}{|c|c|c|c|c|c|c|}
\hline $\begin{array}{l}\text { PERIOD } \\
\text { (CON- }\end{array}$ & \multicolumn{6}{|c|}{$\begin{array}{l}\text { DISCHARGE, IN CFS, FOR INDICATED RECURRENCE } \\
\text { INTERVAL, IN YEARS, AND ANNUAL NON- } \\
\text { EXCEEDANCE PROBABILITY, IN PERCENT }\end{array}$} \\
\hline SECU- & & & & & - & \\
\hline TIVE & 2 & 5 & 10 & 20 & 50 & 100 \\
\hline DAYS) & $50 \%$ & $20 \%$ & $10 \%$ & $5 \%$ & $2 \%$ & 19 \\
\hline 1 & -- & -- & -- & -- & -- & -- \\
\hline 3 & -- & -- & -- & -- & - & -- \\
\hline 7 & -- & -- & -- & -- & -- & -- \\
\hline 14 & -- & -- & -- & -- & -- & -- \\
\hline 30 & -- & -- & -- & -- & -- & -- \\
\hline 60 & -- & -- & -- & -- & - & -- \\
\hline 90 & -- & -- & -- & -- & -- & -- \\
\hline 120 & -- & -- & -- & -- & -- & -- \\
\hline 183 & -- & -- & -- & -- & -- & -- \\
\hline NOTE: & $\begin{array}{l}-F L O \\
0 \mathrm{EV}\end{array}$ & TIS & UNC & & & IVE \\
\hline
\end{tabular}

MAGNITUDE AND PROBABILITY OF ANNUAL HIGH FLOW BASED ON PERIOD OF RECORD 1920-82

\begin{tabular}{|c|c|c|c|c|c|c|}
\hline $\begin{array}{l}\text { PERIOD } \\
\text { (CON- } \\
\text { SFCII- }\end{array}$ & \multicolumn{6}{|c|}{$\begin{array}{c}\text { DISCHARGE, IN CFS, FOR INDICATED RECURRENCE } \\
\text { INTERVAL, IN YEARS, AND ANNUAL } \\
\text { EXCEEDANCE PROBABILITY, IN PERCENT }\end{array}$} \\
\hline TIVE & 2 & 5 & 10 & 25 & 50 & 100 \\
\hline DAYS) & $50 \%$ & $20 \stackrel{\infty}{\circ}$ & $10 \%$ & $4 \stackrel{\phi}{\phi}$ & $2 \%$ & $1 \%$ \\
\hline 1 & 677 & 1110 & 1520 & 2190 & 2840 & 3640 \\
\hline 3 & 669 & 1090 & 1480 & 2120 & 2740 & 3490 \\
\hline 7 & 648 & 1050 & 1410 & 2000 & 2570 & 3260 \\
\hline 15 & 604 & 943 & 1240 & 1720 & 2160 & 2690 \\
\hline 30 & 550 & 815 & 1030 & 1360 & 1640 & 1970 \\
\hline 60 & 491 & 672 & 793 & 945 & 1060 & 1170 \\
\hline 90 & 451 & 595 & 682 & 784 & 855 & 922 \\
\hline
\end{tabular}

DURATION TABLE OF DAILY MEAN FLOW FOR PERIOD OF RECORD 1920-82

DISCHARGE, IN CFS, WHICH WAS EOUALED OR EXCEEDED FOR INDICATED PERCENT OF TIME

\begin{tabular}{|c|c|c|c|c|c|c|c|c|c|c|c|c|c|c|}
\hline $5 \%$ & $10 \%$ & $15 \%$ & $20 \%$ & $25 \%$ & $30 \%$ & $40^{\circ}$ & $50 \%$ & $60 \%$ & $70 \%$ & $75 \%$ & $80 \%$ & $85 \%$ & $90 \%$ & $95 \%$ \\
\hline 582 & 511 & 450 & 389 & 341 & 294 & 160 & 3.3 & 1.1 & .5 & .2 & .1 & .1 & .1 & 0.0 \\
\hline
\end{tabular}


13216500 NORTH FORK MALHEUR RIVER ABOVE BEULAH RESERVOIR, NEAR BEULAH, OR

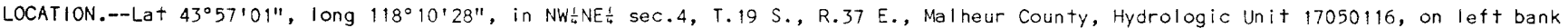
$500 \mathrm{ft}$ upstream from Beulah Reservoir, $2.5 \mathrm{mi}$ upstream from Warm Springs Creek, $3.5 \mathrm{mi}$ northwest of Beulah, and at mile 18.0 .

DRAINAGE AREA.--355 $\mathrm{mi}^{2}$.

PERIOD OF RECORD.-- January to September 1914 (published as "at Scott's Ranch, near Beulah"), June 1936 to September 1982. Published as "above Agency Valley Reservoir, near Beulah", June 1036 to September 1968.

GAGE.--Water-stage recorder. Datum of gage is 3,349.4 $\mathrm{ft}$ National Geodetic vertical Datum of 1929. Jan. 1 to Sept. 30 , 1914, nonrecording gage and June 10, 1936, to Oct. 14, 1958, water-stage recorder at site $0.5 \mathrm{mi}$ upstream at different datums. Oct. 15, 1958, to Oct. 8, 1975, water-stage recorder at present site at datum $1.6 \mathrm{ft}$ higher.

REMARKS.--No regulation. Diversions for irrigation above station.

AVERAGE DISCHARGE. - 46 years (water years 1937-82), $132 \mathrm{tt}+3 / \mathrm{s}, 95,630 \mathrm{acre}-\mathrm{ft} / \mathrm{yr}$.

EXTREMES FOR PERIOD OF RECORD.--Maximum discharge, 3,970 $\mathrm{ft} \mathrm{t}^{3} / \mathrm{s} \mathrm{Dec.} \mathrm{23,} \mathrm{1964,} \mathrm{gage} \mathrm{height,} 9.90 \mathrm{ft}$, present datum, from floodmark, from rating curve extended above $1,300 \mathrm{ft} / 3 / \mathrm{s}$, on basis of slope-area measurement of peak $\mathrm{flow}$; maximum gage height, 1 il. $0 \mathrm{ft}$, present datum, sometime during period Dec. 17-23, 1964 (icejam); minimum discharge, $8.5 \mathrm{ft} 3 / \mathrm{s}$ Dec. 13, 1967 , result of freezeup.

STATISTICAL SUMMAR!ES

MONTHLY AND ANNUAL MEAN DISCHARGES 1937-82

\begin{tabular}{|c|c|c|c|c|c|c|}
\hline MONTH & $\begin{array}{l}\text { MIN!MUM } \\
\text { (CFS) }\end{array}$ & $\begin{array}{l}\text { MAXIMUM } \\
\text { (CFS) }\end{array}$ & $\begin{array}{l}\text { MEAN } \\
\text { (CFS) }\end{array}$ & $\begin{array}{l}\text { STAN- } \\
\text { DARD } \\
\text { DEVIA- } \\
\text { TION } \\
\text { (CFS) }\end{array}$ & $\begin{array}{l}\text { COEFFI- } \\
\text { CIENT OF } \\
\text { VARI- } \\
\text { ATION }\end{array}$ & $\begin{array}{c}\text { PERCENT } \\
\text { OF } \\
\text { ANNUAL } \\
\text { RUNOFF }\end{array}$ \\
\hline OCTOBER & 32 & 66 & 51 & 7.8 & .15 & 3.2 \\
\hline NOVEMBER & 37 & 98 & 57 & 10 & .18 & 3.6 \\
\hline DECEMBER & 38 & 346 & 69 & 46 & .67 & 4.4 \\
\hline JANUARY & 32 & 280 & 78 & 56 & .71 & 4.9 \\
\hline FEBRUARY & 51 & 518 & 120 & 85 & .71 & 7.5 \\
\hline MARCH & 58 & 451 & 198 & 97 & .49 & 12.4 \\
\hline APRIL & 111 & 906 & 373 & 189 & .51 & 23.4 \\
\hline MAY & 92 & 726 & 319 & 157 & .49 & 20.1 \\
\hline JUNE & 55 & 397 & 167 & 89 & .53 & 10.5 \\
\hline JULY & 34 & 190 & 67 & 29 & .43 & 4.2 \\
\hline AUGUST & 24 & 74 & 46 & 11 & .24 & 2.9 \\
\hline SEPTEMBER & 30 & 74 & 46 & 8.3 & .18 & 2.9 \\
\hline ANNUAL & 60 & 272 & 132 & 49 & .37 & 100 \\
\hline
\end{tabular}

MAGNITUDE AND PROBABILITY OF INSTANTANEOUS PEAK FLOW BASED ON PERIOD OF RECORD 1937-82

DISCHARGE, IN CFS, FOR INDICATED RECURRENCE INTERVAL, IN YEARS, AND ANNUAL EXCEEDANCE PROBABILITY, IN PERCENT

\begin{tabular}{ccccccc}
1.25 & 2 & 5 & 10 & 25 & 50 & 100 \\
$80 \%$ & $50 \%$ & $20 \%$ & $10 \%$ & $4 \%$ & $2 \%$ & 18 \\
\hline 575 & 904 & 1440 & 1850 & 2430 & 2910 & 3420
\end{tabular}

WEIGHTED SKEW $=.110$
MAGN I TUDE AND PROBABILITY OF ANNUAL LOW FLOW BASED ON PERIOD OF RECORD 1938-82

\begin{tabular}{ccccccc} 
PERIOD & \multicolumn{5}{c}{ DISCHARGE, IN CFS, FOR INDICATED RECURRENCE } \\
(CON- & \multicolumn{5}{c}{ INTERVAL, IN YEARS, AND ANNUAL NON- } \\
SECU- & EXCEEDANCE PROBABILITY, IN PERCENT \\
TIVE & 2 & 5 & 10 & 20 & 50 & 100 \\
DAYS) & $50 \%$ & $20 \%$ & $10 \%$ & $5 \%$ & $2 \%$ & $1 \%$ \\
\hline 1 & 30 & 25 & 22 & 20 & 18 & 16 \\
3 & 32 & 26 & 23 & 21 & 18 & 17 \\
7 & 35 & 29 & 26 & 23 & 20 & 18 \\
14 & 37 & 31 & 28 & 26 & 23 & 21 \\
30 & 40 & 34 & 31 & 29 & 26 & 24 \\
60 & 44 & 37 & 34 & 31 & 28 & 26 \\
90 & 46 & 39 & 36 & 33 & 30 & 28 \\
120 & 48 & 41 & 38 & 35 & 32 & 30 \\
183 & 52 & 46 & 42 & 40 & 37 & 35
\end{tabular}

MAGNITUDE AND PROBABILITY OF ANNUAL HIGH FLOW BASED ON PERIOD OF RECORD 1937-82

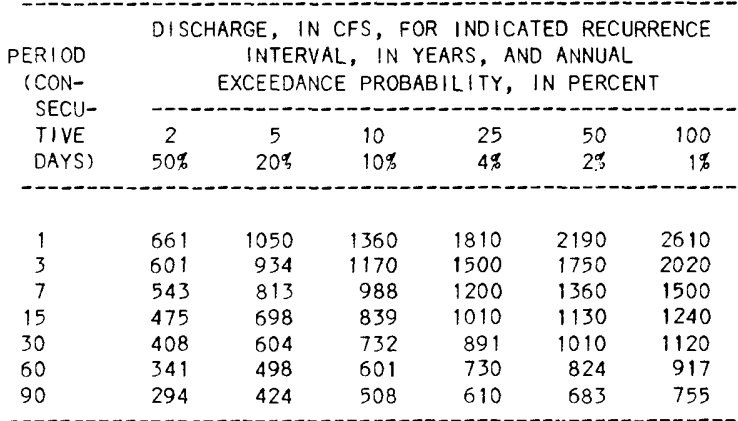

DURATION TABLE OF DAILY MEAN FLOW FOR PERIOD OF RECORD 1937-82

DISCHARGE, IN CFS, WHICH WAS EQUALED OR EXCEEDED FOR INDICATED PERCENT OF TIME

\begin{tabular}{|c|c|c|c|c|c|c|c|c|c|c|c|c|c|c|}
\hline $5 \%$ & $10 \%$ & $15 \%$ & $20 \%$ & $25 \%$ & $30 x$ & $40 \%$ & $50 \%$ & $60 \%$ & $70 \%$ & $75 \%$ & $80 \%$ & $85 \%$ & $90 \%$ & $95 \%$ \\
\hline 457 & 325 & 247 & 191 & 151 & 120 & 79 & 64 & 56 & 51 & 48 & 46 & 43 & 40 & 36 \\
\hline
\end{tabular}




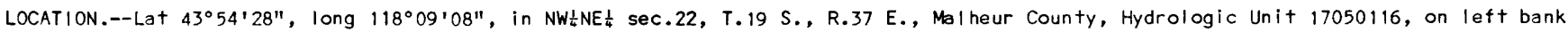
at Beulah, $0.3 \mathrm{mi}$ downstream from Agency Valley Dam, $12 \mathrm{mi}$ northwest of Juntura, and at $\mathrm{mile} 14.5$.

DRAINAGE AREA. $--440 \mathrm{mi}^{2}$, approximately.

PERIOD OF RECORD.--June 1926 to September 1982. Published as "near Beulah" June 1926 to September 1935.

GAGE.--Water-stage recorder. Datum of gage is 3,261.20 ft National Geodetic Vertical Datum of 1929. Prior to Apr. 25, 1926, water-stage recorder at site $1 \mathrm{mi}$ downstream at different datum. Apr. 25, 1936, to Sept. 30 , 1949, nonrecording gage at site 20 $\mathrm{ft}$ downstream at datum $1.0 \mathrm{ft}$ higher. Oct. 1, 1949, to June 30, 1964, at present site at datum $1.0 \mathrm{ft}$ higher.

REMARKS.--Flow regulated since 1935 by Beulah Reservoir. Dlversions for irrigation above station.

AVERAGE DISCHARGE.--47 years (water years 1936-82), $142 \mathrm{ft} / \mathrm{s}, 102,900 \mathrm{acre}-\mathrm{ft} / \mathrm{yr}$.

EXTREMES FOR PERIOD OF RECORD.--Maximum discharge, 7,000 $\mathrm{ft}^{3} / \mathrm{s}$ May 7,1942 , gage height, $9.4 \mathrm{ft}$, present datum, from floodmark, caused by failure of gates at Agency Valley Dam, from rating curve extended above $1,100 \mathrm{tt} / \mathrm{s}$ on basis of computation of peak flow over dam; no flow at times.

STATISTICAL SUMMARIES

MONTHLY AND ANNUAL MEAN DISCHARGES 1936-82

\begin{tabular}{|c|c|c|c|c|c|c|}
\hline MONTH & $\begin{array}{l}\text { MINIMUM } \\
\text { (CFS) }\end{array}$ & $\begin{array}{l}\text { MAXIMUM } \\
\text { (CFS) }\end{array}$ & $\begin{array}{l}\text { MEAN } \\
\text { (CFS) }\end{array}$ & $\begin{array}{l}\text { STAN- } \\
\text { DARO } \\
\text { DEVIA- } \\
\text { TION } \\
\text { (CFS) }\end{array}$ & $\begin{array}{l}\text { COEFF I- } \\
\text { CIENT OF } \\
\text { VARI- } \\
\text { ATION }\end{array}$ & $\begin{array}{c}\text { PERCENT } \\
\text { OF } \\
\text { ANNUAL } \\
\text { RUNOFF }\end{array}$ \\
\hline OCTOBER & .1 & 134 & 36 & 32 & .89 & 2.1 \\
\hline NOVEMBER & 0.0 & 36 & 1.3 & 5.1 & 4.05 & .1 \\
\hline DECEMBER & 0.0 & 63 & 2.3 & 9.6 & 4.27 & .1 \\
\hline JANUARY & 0.0 & 287 & 7.0 & 42 & 6.03 & .4 \\
\hline FEBRUARY & 0.0 & 478 & 25 & 98 & 3.92 & 1.5 \\
\hline MARCH & 0.0 & 518 & 55 & 113 & 2.06 & 3.2 \\
\hline APRIL & 2.3 & 856 & 289 & 230 & .80 & 17.0 \\
\hline MAY & 120 & 793 & 351 & 160 & .46 & 20.7 \\
\hline JUNE & 54 & 510 & 279 & 106 & .38 & 16.4 \\
\hline JULY & 58 & 402 & 282 & 83 & .29 & 16.6 \\
\hline AUGUST & 43 & 399 & 223 & 95 & .43 & 13.1 \\
\hline SEPTEMBER & 32 & 341 & 148 & 82 & .56 & 8.7 \\
\hline ANNUAL & 55 & 301 & 141 & 55 & .39 & 100 \\
\hline
\end{tabular}

MAGNITUDE AND PROBABILITY OF INSTANTANEOUS PEAK FLOW BASED ON PERIOD OF RECORD

DISCHARGE, IN CFS, FOR INDICATED RECURRENCE INTERVAL, IN YEARS, AND ANNUAL EXCEEDANCE PROBABILITY, IN PERCENT

\begin{tabular}{ccccccc}
1.25 & 2 & 5 & 10 & 25 & 50 & 100 \\
$80 \%$ & $50 \%$ & $20 \%$ & $10 \%$ & $4 \%$ & $2 \%$ & $1 \%$ \\
\hline- & - & - & - & - & - & - \\
\hline
\end{tabular}

WEIGHTED SKEW =
MAGNITUDE AND PROBABILITY OF ANNUAL LOW FLOW BASED ON PERIOD OF RECORD 1937-82

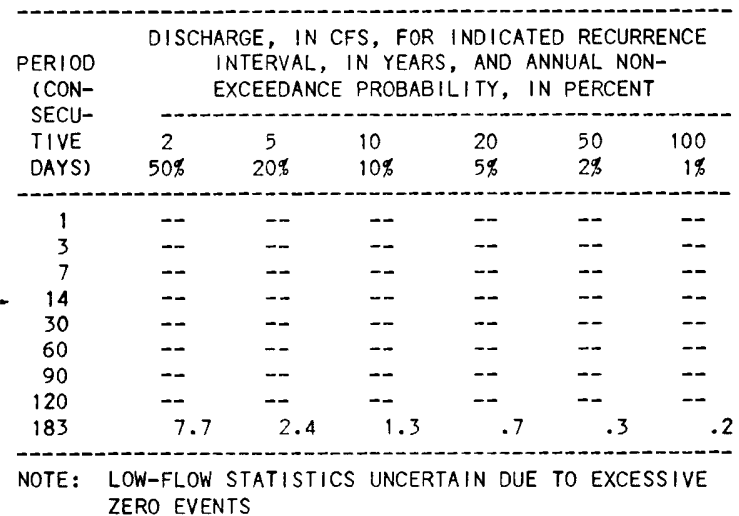

MAGNITUDE AND PROBABILITY OF ANNUAL HIGH FLOW BASED ON PERIOD OF RECORD $1936-82$

\begin{tabular}{|c|c|c|c|c|c|c|}
\hline $\begin{array}{l}\text { PERIOD } \\
\text { (CON- } \\
\text { SECU- }\end{array}$ & \multicolumn{6}{|c|}{$\begin{array}{c}\text { DISCHARGE, IN CFS, FOR INDICATED RECURRENCE } \\
\text { INTERVAL, IN YEARS, AND ANNUAL } \\
\text { EXCEEDANCE PROBABILITY, IN PERCENT }\end{array}$} \\
\hline TIVE & 2 & 5 & 10 & 25 & 50 & 100 \\
\hline DAYS) & $50 \%$ & $20 \%$ & $10 \%$ & $4:$ & $2 \%$ & $1 \%$ \\
\hline 1 & 561 & 974 & 1370 & 2060 & 2730 & 3590 \\
\hline 3 & 552 & 919 & 1240 & 1770 & 2260 & 2840 \\
\hline 7 & 522 & 811 & 1040 & 1390 & 1680 & 2010 \\
\hline 15 & 475 & 700 & 868 & 1100 & 1300 & 1500 \\
\hline 30 & 424 & 608 & 742 & 924 & 1070 & 1220 \\
\hline 60 & 368 & 510 & 607 & 736 & 834 & 936 \\
\hline 90 & 336 & 455 & 529 & 618 & 681 & 742 \\
\hline
\end{tabular}

DURATION TABLE OF DAILY MEAN FLOW FOR PERIOD OF RECORD 1936-82

DISCHARGE, IN CFS, WHICH WAS EQUALED OR EXCEEDED FOR INDICATED PERCENT OF TIME

\begin{tabular}{|c|c|c|c|c|c|c|c|c|c|c|c|c|c|c|}
\hline $5 \%$ & $10 \%$ & $15 \%$ & $20 \%$ & $25 \%$ & $30 \%$ & $40 \%$ & $50 \%$ & $60 \%$ & $70 \%$ & $75 \%$ & $80 \%$ & $85 \%$ & $90 \%$ & $95 \%$ \\
\hline 486 & 378 & 340 & 302 & 264 & 227 & 138 & 45 & 1.3 & .5 & .4 & .2 & .2 & .1 & .1 \\
\hline
\end{tabular}


13220000 MALHEUR RIVER AT LITTLE VALLEY, NEAR HOPE, OR

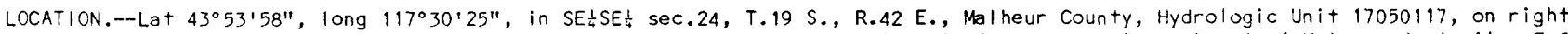
bank $500 \mathrm{ft}$ downstream from highway bridge at Little Valley, $8 \mathrm{mi}$ southwest of Hope, $14 \mathrm{mi}$ southwest of Vale, and at mile 45.6 .

DRAINAGE AREA.--3,010 $\mathrm{mi}^{2}$, approximately.

PERIOD OF RECORD.--April 1949 to September 1979.

GAGE.--Water-stage recorder. Datum of gage is $2,424.03 \mathrm{ft}$ National Geodetic Vertical Datum of 1929.

REMARKS.--Flow regulated by Warmsprings and Beulah Reservoirs. Since 1930, Vale-Oregon Canal has diverted $f$ low above station at Namorf in sec.31, T.20 S., R.41 E., for supplying Bully Creek Reservolr and for irrigation. Many small diversions for irrigation above station.

AVERAGE DISCHARGE. -30 years, $192 \mathrm{ft} / \mathrm{s}, 139,100$ acre $-\mathrm{ft} / \mathrm{yr}$.

EXTREMES FOR PERIOD OF RECORD.--Maximum discharge, 12,300 ft3/s Feb. 24, 1957, gage height, $11.5 \mathrm{ft}$, from $\mathrm{floodmark,} \mathrm{from} \mathrm{rating}$ curve extended above $5,500 \mathrm{ft}^{3} / \mathrm{s}$ on basis of slope-area measurement of peak flow; minimum, $1.6 \mathrm{ft} / \mathrm{s}$ Dec. 21 , 1977 , result of treezeup.

EXTREMES OUTSIDE PERIOD OF RECORD. --The two greatest floods occurred March 1894 and March 1910 , on basis of records for former station near Namort.

STATISTICAL SUMMARIES

MONTHLY AND ANNUAL MEAN DISCHARGES 1950-79

\begin{tabular}{|c|c|c|c|c|c|c|}
\hline MONTH & $\begin{array}{l}\text { MINIMUM } \\
\text { (CFS) }\end{array}$ & $\begin{array}{l}\text { MAXIMUM } \\
\text { (CFS) }\end{array}$ & $\begin{array}{l}\text { MEAN } \\
\text { (CFS) }\end{array}$ & $\begin{array}{l}\text { STAN- } \\
\text { DARD } \\
\text { DEVIA- } \\
\text { T/ON } \\
\text { (CFS) }\end{array}$ & $\begin{array}{l}\text { COEFFI- } \\
\text { CIENT OF } \\
\text { VARI- } \\
\text { ATION }\end{array}$ & $\begin{array}{c}\text { PERCENT } \\
\text { OF } \\
\text { ANNUAL } \\
\text { RUNOFF }\end{array}$ \\
\hline OCTOBER & 18 & 93 & 50 & 17 & .33 & 2.1 \\
\hline NOVEMBER & 14 & 72 & 42 & 13 & .31 & 1.8 \\
\hline DECEMBER & 10 & 407 & 58 & 68 & 1.17 & 2.5 \\
\hline JANUARY & 27 & 1119 & 168 & 250 & 1.49 & 7.3 \\
\hline FEBRUARY & 39 & 887 & 269 & 264 & .98 & 11.6 \\
\hline MARCH & 11 & 1053 & 268 & 274 & 1.02 & 11.6 \\
\hline APRIL & 32 & 2143 & 449 & 570 & 1.27 & 19.4 \\
\hline MAY & 108 & 1478 & 347 & 337 & .97 & 15.0 \\
\hline JUNE & 101 & 767 & 195 & 116 & .59 & 8.4 \\
\hline $\begin{array}{l}\text { JULY } \\
\text { AUGUST }\end{array}$ & $\begin{array}{r}125 \\
34\end{array}$ & $\begin{array}{l}297 \\
225\end{array}$ & $\begin{array}{l}218 \\
156\end{array}$ & $\begin{array}{l}40 \\
47\end{array}$ & $\begin{array}{l}.18 \\
.30\end{array}$ & $\begin{array}{l}9.4 \\
6.7\end{array}$ \\
\hline SEPTEMBER & 22 & 159 & 97 & 36 & .37 & 4.2 \\
\hline ANNUAL & 61 & 475 & 192 & 115 & .60 & 100 \\
\hline
\end{tabular}

MAGNITUDE AND PROBABILITY OF INSTANTANEOUS PEAK FLOW BASED ON PERIOD OF RECORD

DISCHARGE, IN CFS, FOR INDICATED RECURRENCE INTERVAL, IN YEARS, AND ANNUAL EXCEEDANCE PROBABILITY, IN PERCENT

\begin{tabular}{|c|c|c|c|c|c|c|}
\hline $\begin{array}{l}1.25 \\
80 \%\end{array}$ & $\begin{array}{c}2 \\
50 \%\end{array}$ & $\begin{array}{c}5 \\
20 \%\end{array}$ & $\begin{array}{l}10 \\
10 \%\end{array}$ & $\begin{array}{l}25 \\
4 \%\end{array}$ & $\begin{array}{l}50 \\
2 \%\end{array}$ & $\begin{array}{r}100 \\
1 \%\end{array}$ \\
\hline -- & - & - & -- & -- & -- & - \\
\hline
\end{tabular}

MAGNITUDE AND PROBABILITY OF ANNUAL LOW FLOW BASED ON PERIOD OF RECORD 1951-79

\begin{tabular}{|c|c|c|c|c|c|c|}
\hline $\begin{array}{l}\text { PERIOD } \\
\text { (CON- }\end{array}$ & DIS & $\begin{array}{l}\text { GE, } \\
\text { TERV } \\
\text { CEED }\end{array}$ & $\begin{array}{l}F S, \\
\text { IN Y } \\
\text { PRO }\end{array}$ & $\begin{array}{l}\text { NDIO } \\
\text { AND } \\
\text { ITY, }\end{array}$ & $\begin{array}{l}\text { REC } \\
\text { JAL } \\
\text { DERC }\end{array}$ & \\
\hline SECU- & & 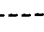 & - & $n-\infty$ & an- & -0 \\
\hline TIVE & 2 & 5 & 10 & 20 & 50 & 100 \\
\hline DAYS) & $50 \%$ & $20 \%$ & $10 \%$ & $5 \%$ & $2 \%$ & $1 \%$ \\
\hline 1 & & & & & & \\
\hline 1 & 21 & 14 & 11 & 9. & 7. & - \\
\hline 3 & 22 & 15 & 12 & 9. & 7. & - \\
\hline 7 & 23 & 16 & 12 & 10 & 7. & -- \\
\hline 14 & 27 & 18 & 14 & 11 & 8. & - \\
\hline 30 & 33 & 21 & 16 & 13 & 9. & -- \\
\hline 60 & 38 & 26 & 20 & 15 & 11 & -- \\
\hline 90 & 41 & 30 & 25 & 20 & 16 & -- \\
\hline 120 & 45 & 34 & 29 & 24 & 20 & -- \\
\hline 183 & 67 & 48 & 39 & 33 & 26 & - \\
\hline
\end{tabular}

MAGNITUDE AND PROBABILITY OF ANNUAL HIGH FLOW BASED ON PERIOD OF RECORD 1950-79

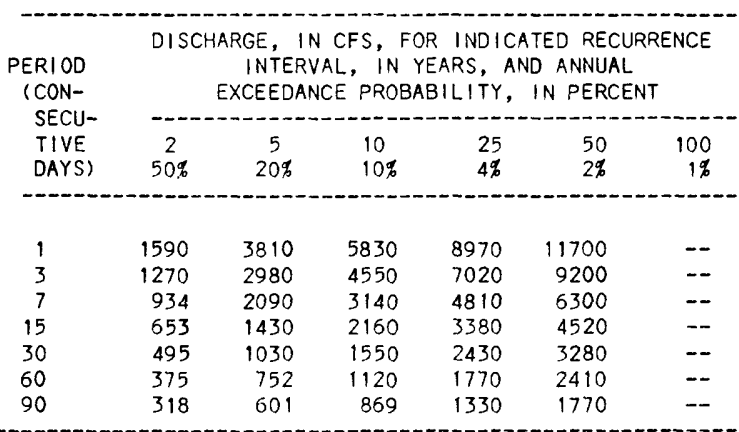

DURATION TABLE OF DAILY MEAN FLOW FOR PERIOD OF RECORD 1950-79

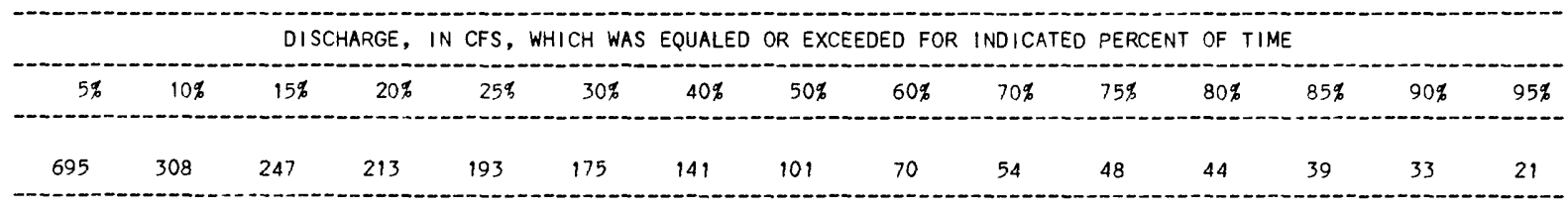




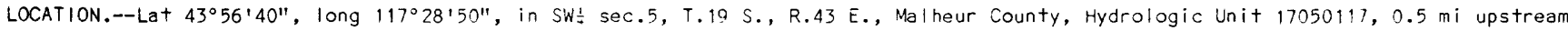

from intake of Vines Canal, $5.5 \mathrm{mi}$ west of Hope, and $12 \mathrm{mi}$ west of Vale.

DRAINAGE AREA.- $-3,030 \mathrm{mi}^{2}$, approximately.

PERIOD OF RECORD.--March 1922 to Septenber 1949.

GAGE.--Water-stage recorder. Altitude of gage is $2,270 \mathrm{ft}$ (from topographic nap).

REMARKS.--Since March 1930, Vale-Oregon Canal has divartad at Namor f for irrigation above and below station. Many small diversions for irrigation above station. Flow regulated by Warmsprings Reservoir and, since December 1935, by Agency Valley Reservoir.

AVERAGE DISCHARGE.--19 years (water years 1930-49), $187 \mathrm{ft}^{3} / \mathrm{s}$.

EXTREMES FOR PERIOD OF RECORD.--Maximum discharge, $8,100 \mathrm{ft}^{3} / \mathrm{s}$ Feb. 5, 1925, gage height, $8.1 \mathrm{ft}$, from rating curve extended above $2,800 \mathrm{ft} / \mathrm{s} ;$ minimum, $3.5 \mathrm{ft} / \mathrm{s}$ Sept. $2,1919$.

STATISTICAL SUMMARIES

MONTHLY AND ANNUAL MEAN DISCHARGES 1931-49

\begin{tabular}{|c|c|c|c|c|c|c|}
\hline MONTH & $\begin{array}{l}\text { MINIMUM } \\
\text { (CFS) }\end{array}$ & $\begin{array}{l}\text { MAXIMUM } \\
\text { (CFS) }\end{array}$ & $\begin{array}{l}\text { MEAN } \\
\text { (CFS) }\end{array}$ & $\begin{array}{l}\text { STAN- } \\
\text { DARD } \\
\text { DEVIA- } \\
\text { TION } \\
\text { (CFS) }\end{array}$ & $\begin{array}{l}\text { COEFFI- } \\
\text { CIENT OF } \\
\text { VARI- } \\
\text { ATION }\end{array}$ & $\begin{array}{c}\text { PERCENT } \\
\text { OF } \\
\text { ANNUAL } \\
\text { RUNOFF }\end{array}$ \\
\hline OCTOBER & 9.5 & 89 & 48 & 21 & .44 & 2.1 \\
\hline NOVEMBER & 12 & 95 & 53 & 20 & .38 & 2.3 \\
\hline DECEMBER & 15 & 329 & 75 & 72 & .97 & 3.3 \\
\hline JANUARY & 20 & 722 & 102 & 153 & 1.50 & 4.5 \\
\hline FEBRUARY & 30 & 768 & 236 & 205 & .87 & 10.4 \\
\hline MARCH & 23 & 1493 & 385 & 382 & .99 & 17.0 \\
\hline APRIL & 54 & 2040 & 496 & 565 & 1.14 & 22.0 \\
\hline MAY & 156 & 652 & 271 & 120 & .44 & 12.0 \\
\hline JUNE & 105 & 242 & 167 & 41 & .24 & 7.4 \\
\hline JULY & 90 & 264 & 186 & 50 & .27 & 8.2 \\
\hline AUGUST & 18 & 196 & 139 & 46 & .33 & 6.2 \\
\hline SEPTEMBER & 10 & 158 & 102 & 47 & .46 & 4.5 \\
\hline ANNUAL & 72 & 520 & 188 & 114 & .61 & 100 \\
\hline
\end{tabular}

MAGNITUDE AND PROBABILITY OF INSTANTANEOUS PEAK FLOW BASED ON PERIOD OF RECORD

DISCHARGE, IN CFS, FOR INDICATED RECURRENCE INTERVAL, IN YEARS, AND ANNUAL EXCEEDANCE PROBABILITY, IN PERCENT

\begin{tabular}{ccccccc}
1.25 & 2 & 5 & 10 & 25 & 50 & 100 \\
$80 \%$ & $50 \%$ & $20 \%$ & $10 \%$ & $4 \%$ & $2 \%$ & 19 \\
\hline & & & & & & \\
\hline & - & - & - & - & - & -
\end{tabular}

WEIGHTED SKEW =
MAGNITUDE ANO PROBABILITY OF ANNUAL LOW FLOW BASED ON PERIOD OF RECORD 1932-49

\begin{tabular}{|c|c|c|c|c|c|c|}
\hline $\begin{array}{l}\text { PERIOD } \\
\text { (CON- } \\
\text { SECU- }\end{array}$ & \multicolumn{6}{|c|}{$\begin{array}{l}\text { DISCHARGE, IN CFS, FOR INDICATED RECURRENCE } \\
\text { INTERVAL, IN YEARS, AND ANNUAL NON- } \\
\text { EXCEEDANCE PROBABILITY, IN PERCENT }\end{array}$} \\
\hline $\begin{array}{l}\text { TIVE } \\
\text { DAYS) }\end{array}$ & $\begin{array}{c}2 \\
50 \%\end{array}$ & $\begin{array}{c}5 \\
20 \%\end{array}$ & $\begin{array}{l}10 \\
10 \%\end{array}$ & $\begin{array}{l}20 \\
5 \%\end{array}$ & $\begin{array}{l}50 \\
2 \%\end{array}$ & $\begin{array}{r}100 \\
1 \%\end{array}$ \\
\hline 1 & 22 & 14 & 19 & 87 & & -- \\
\hline 3 & 24 & 15 & 11 & 8.7 & -- & -- \\
\hline 7 & 25 & 15 & 11 & 8.8 & -- & -- \\
\hline 14 & 28 & 17 & 12 & 9.2 & -- & -- \\
\hline 30 & 33 & 19 & 13 & 10.0 & -- & -- \\
\hline 60 & 42 & 25 & 18 & 13 & -- & -- \\
\hline 90 & 45 & 28 & 20 & 15 & -- & - \\
\hline 120 & 52 & 32 & 24 & 18 & -- & - \\
\hline 183 & 69 & 45 & 35 & 28 & $\cdots$ & - \\
\hline
\end{tabular}

MAGNITUDE AND PROBABILITY OF ANNUAL HIGH FLOH BASED ON PERIOD OF RECORD 1931-49

\begin{tabular}{|c|c|c|c|c|c|c|}
\hline $\begin{array}{l}\text { PERIOD } \\
\text { (CON- } \\
\text { SFCU- }\end{array}$ & DIS & $\begin{array}{l}\text { RGE, } \\
\text { INTE } \\
\text { XCEED }\end{array}$ & $\begin{array}{l}\mathrm{CFS} \text {, } \\
\mathrm{L}, \text { IN }\end{array}$ & $\begin{array}{l}\text { INDIC } \\
\text { RS, } \\
\text { LITY }\end{array}$ & $\begin{array}{l}\text { NNU } \\
\text { PER } \\
\end{array}$ & \\
\hline SECU- & 2 & 5 & 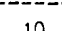 & 25 & 50 & 100 \\
\hline TIVE & ${ }^{2}$ & ? & 10 & 23 & 20 & 100 \\
\hline נדורנה & & & & & & \\
\hline 1 & 1370 & 2910 & 4150 & 5910 & -- & $=$ \\
\hline 3 & 1030 & 2050 & 2860 & 4020 & - & - \\
\hline 7 & 746 & 1440 & 2030 & 2940 & - & - \\
\hline 15 & 576 & 1150 & 1710 & 2660 & -- & - \\
\hline 30 & 443 & 897 & 1360 & 2190 & -- & - \\
\hline 60 & 359 & 709 & 1060 & 1680 & -- & - \\
\hline 90 & 317 & 593 & 863 & 1330 & -- & -- \\
\hline
\end{tabular}

DURATION TABLE OF DAILY MEAN FLOW FOR PERIOD OF RECORD 1931-49

DISCHARGE, IN CFS, WHICH WAS EQUALED OR EXCEEDED FOR INDICATED PERCENT OF TIME

\begin{tabular}{|c|c|c|c|c|c|c|c|c|c|c|c|c|c|c|}
\hline $5 \%$ & $10 \%$ & $15 \%$ & $20 \%$ & $25 \%$ & $30 \%$ & $40 \%$ & $50 \%$ & $60 \%$ & $70 \%$ & $75 \%$ & $80 \%$ & $85 \%$ & $90 \%$ & $95 \%$ \\
\hline 617 & 337 & 265 & 226 & 201 & 178 & 141 & 104 & 77 & 61 & 54 & 48 & 40 & 33 & 21 \\
\hline
\end{tabular}


13226500 BULLY CREEK AT WARMSPRINGS, NEAR VALE, OR

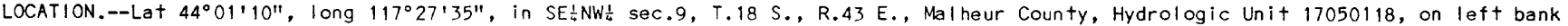
$400 \mathrm{ft}$ downstream from Cottonwood Creek, $4.7 \mathrm{mi}$ upstream from Bully Creek Dam, $11.4 \mathrm{mi}$ northwest of Vale, and at mile 17.2 .

DRAINAGE AREA.--539 $\mathrm{mi}^{2}$.

PERIOD OF RECORD.--September 1903 to February 1924, February 1905 to Yarch 1907, February 1910 , January 1911 to May 1917 , March 1922 to June 1923, October 1963 to September 1982. Monthly discharge only for some periods, published in WSP 1317 . Published as "near Vale" 1903, 1907, and as "above Vale" 1904-6, 1910.

GAGE.--Water-stage recorder. Datum of gage is 2,527.21 ft National Geodetic Vertical Datum of 1929 (Bureau of Reclamation bench mark). Prior to July 1, 1923, nonrecording gages within $0.5 \mathrm{mi}$ downstream at different datums.

REMARKS.--No regulation. Many diversions for irrigation above station.

AVERAGE DISCHARGE.--25 years (water years $1906,1912-16,1964-82$ ), $46.0 \mathrm{ft} / \mathrm{s}, 33,330 \mathrm{acre}-\mathrm{ft} / \mathrm{yr}$.

EXTREMES FOR PERIOD OF RECORD.--1laximum discharge, 12,800 $\mathrm{ft}^{3} / \mathrm{s}$ Dec. 22,1964 , gage height, $8.68 \mathrm{ft}$, from rating curve extended above $200 \mathrm{ft}^{3} / \mathrm{s}$ on basis of slope-area measurement of peak flow; no flow at times.

STATISTICAL SUMMARIES

MONTHLY AND ANNUAL MEAN DISCHARGES 1964-82

\begin{tabular}{|c|c|c|c|c|c|c|}
\hline MONTH & $\begin{array}{l}\text { MINIMUM } \\
\text { (CFS) }\end{array}$ & $\begin{array}{l}\text { MAXIMUM } \\
\text { (CFS) }\end{array}$ & $\begin{array}{l}\text { MEAN } \\
\text { (CFS) }\end{array}$ & $\begin{array}{l}\text { STAN- } \\
\text { DARD } \\
\text { DEVIA- } \\
\text { TION } \\
\text { (CFS) }\end{array}$ & $\begin{array}{l}\text { COEFF! - } \\
\text { CIENT OF } \\
\text { VARI- } \\
\text { ATION }\end{array}$ & $\begin{array}{c}\text { PERCENT } \\
\text { OF } \\
\text { ANNUAL } \\
\text { RUNOFF }\end{array}$ \\
\hline OCTOBER & .6 & 11 & 4.1 & 2.3 & .55 & .8 \\
\hline NOVEMBER & 2.9 & 12 & 6.7 & 2.4 & .36 & 1.2 \\
\hline DECEMBER & 3.6 & 331 & 35 & 77 & 2.16 & 6.5 \\
\hline JANUARY & 6.6 & 330 & 87 & 106 & 1.22 & 16.0 \\
\hline FEBRUARY & 7.7 & 708 & 135 & 154 & 1.21 & 24.8 \\
\hline MARCH & .8 & 340 & 126 & 98 & .78 & 23.2 \\
\hline APRIL & .5 & 367 & 97 & 110 & 1.14 & 17.8 \\
\hline MAY & .9 & 105 & 26 & 32 & 1.23 & 4.8 \\
\hline JUNE & 1.9 & 70 & 14 & 16 & 1.16 & 2.6 \\
\hline JULY & .3 & 51 & 7.9 & 14 & 1.77 & 1.4 \\
\hline AUGUST & .3 & 6.7 & 2.4 & 1.9 & .79 & .4 \\
\hline SEPTEMBER & .1 & 17 & 3.0 & 3.6 & 1.19 & .6 \\
\hline ANNUAL & 3.4 & 138 & 45 & 34 & .75 & 100 \\
\hline
\end{tabular}

MAGNITUDE AND PROBABILITY OF INSTANTANEOUS PEAK FLOW BASED ON PERIOD OF RECORD 1954-82

DISCHARGE, IN CFS, FOR INDICATEO RECURRENCE INTERVAL, IN YEARS, AND ANNUAL EXCEEDANCE PROBABILITY, IN PERCENT

\begin{tabular}{ccccccc}
1.25 & 2 & 5 & 10 & 25 & 50 & 100 \\
$80 \%$ & $50 \%$ & $20 \%$ & $10 \%$ & $4 \%$ & $2 \%$ & $1 \%$ \\
\hline 922 & 2270 & 5310 & 8110 & 12500 & $\ldots$ & -
\end{tabular}

WEIGHTED SKEW $=-.161$
MAGNITUDE AND PROBABILITY OF ANNUAL LOW FLOW BASED ON PERIOD OF RECORD 1965-82

\begin{tabular}{|c|c|c|c|c|c|c|}
\hline $\begin{array}{l}\text { PERIOD } \\
\text { (CON- }\end{array}$ & \multicolumn{6}{|c|}{$\begin{array}{l}\text { DISCHARGE, IN CFS, FOR INDICATED RECURRENCE } \\
\text { INTERVAL, IN YEARS, AND ANNUAL NON- } \\
\text { EXCEEDANCE PROBABILITY, IN PERCENT }\end{array}$} \\
\hline SECU- & 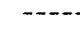 & --- & & & & \\
\hline TIVE & 2 & 5 & 10 & 20 & 50 & 100 \\
\hline DAYS) & $50 \%$ & $20 \%$ & $10 \%$ & $5 \%$ & $2{ }_{\mu}^{\sigma}$ & $1 \%$ \\
\hline 1 & .5 & .2 & .2 & .1 & -- & -- \\
\hline 3 & .5 & .3 & .2 & .2 & -- & -- \\
\hline 7 & .6 & .3 & .2 & .2 & -- & -- \\
\hline 14 & .7 & .3 & .2 & .2 & -- & -- \\
\hline 30 & 1.0 & .4 & .3 & .2 & -- & -- \\
\hline 60 & 1.5 & .7 & .5 & .3 & -- & -- \\
\hline 90 & 1.9 & 1.0 & .6 & .4 & -- & -- \\
\hline 120 & 2.6 & 1.4 & .9 & .6 & -- & -- \\
\hline 183 & 4.0 & 2.3 & 1.7 & 1.2 & -- & -- \\
\hline
\end{tabular}

MAGNITUDE AND PROBABILITY OF ANNUAL HIGH FLOW BASED ON PERIOD OF RECORD 1964-82

\begin{tabular}{|c|c|c|c|c|c|c|}
\hline $\begin{array}{l}\text { PERIOD } \\
\text { (CON- } \\
\text { SECU- }\end{array}$ & \multicolumn{6}{|c|}{$\begin{array}{c}\text { DISCHARGE, IN CFS, FOR INDICATED RECURRENCE } \\
\text { INTERVAL, IN YEARS, AND ANNUAL } \\
\text { EXCEEDANCE PROBAB!LITY, IN PERCENT }\end{array}$} \\
\hline TIVE & 2 & 5 & 10 & 25 & 50 & 100 \\
\hline DAYS) & $50 \%$ & $20 \%$ & $10_{\rho}^{\alpha}$ & $4 \%$ & 2,6 & $1 \%$ \\
\hline 1 & 1080 & 2230 & 2960 & 3750 & - & -- \\
\hline 3 & 762 & 1520 & 1950 & 2370 & -- & - \\
\hline 7 & 540 & 1060 & 1320 & 1550 & -- & -- \\
\hline 15 & 350 & 669 & 827 & 964 & -- & -- \\
\hline 30 & 223 & 427 & 540 & 647 & -- & -- \\
\hline 60 & 154 & 300 & 387 & 476 & -- & -- \\
\hline 90 & 124 & 244 & 319 & 399 & -- & -- \\
\hline
\end{tabular}

DURATION TABLE OF DAILY MEAN FLOW FOR PERIOD OF RECORD 1964-82

\begin{tabular}{|c|c|c|c|c|c|c|c|c|c|c|c|c|c|c|}
\hline $5 \%$ & $10 \%$ & $15 \%$ & $20 \%$ & $25 \%$ & $30 \%$ & $40 \%$ & $50 \%$ & $60 \%$ & $70 \%$ & $75 \%$ & $80 \%$ & $85 \%$ & $90 \%$ & $95 \%$ \\
\hline 204 & 107 & 64 & 42 & 26 & 18 & 11 & 7.3 & 5.1 & 3.6 & 2.9 & 2.3 & 1.7 & 1.1 & .6 \\
\hline
\end{tabular}




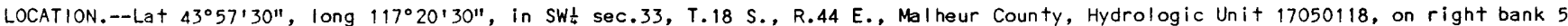
$\mathrm{mi}$ southwest of Vale and $7 \mathrm{mi}$ upstream from mouth.

DRAINAGE AREA. $--570 m \mathrm{i}^{2}$, approximately.

PERIOD OF RECORD.--May 1933 to September 1934, November 1934, March 1935, March, April 1936, June 1937 to September 1962. Monthly discharge only for some periods, published in WSP 1317.

GAGE.-- Hater-stage recorder and concrete control. Altitude of gage is 2,313 ft (by levels to reference point furnished by Union Pacitic Railroad). Prior to Mar. 15, 1937, water-stage recorder or staft gage at site $2 \mathrm{mi}$ upstream at different datum. Mar. 15, 1937, to Jan. 1, 1940, water-stage recorder at present site at datum 0.38 ft higher.

REMARKS.--Occasional fluctuations caused by releases from Vale-Oregon Canal which diverts water from Malheur River for irrigation of lands west of Vale; considerable return flow at times enters Bully Creek above station. Diversions for irrigation of about 7,000 acres above station.

AVERAGE DISCHARGE.--26 years (water years $1934,1938-62$ ) , 40.4 $\mathrm{ft}^{3} / \mathrm{s}, 29,250 \mathrm{acre}-\mathrm{ft} / \mathrm{yr}$.

EXTREMES FOR PERIOD OF RECORD.--Maximum discharge, $8,980 \mathrm{ft}^{3} / \mathrm{s}$ Feb. 24 , 1957, gage height, $10.5 \mathrm{ft}$, from $\mathrm{floodmarks,} \mathrm{from} \mathrm{rating}$ curve extended above $2,000 \mathrm{ft}^{3} / \mathrm{s}$ on basis of slope-area measurement of peak flow; no flow at times.

STATISTICAL SUMMARIES

MONTHIY AND ANNUAL MEAN DISCHARGES 1934-62

\begin{tabular}{|c|c|c|c|c|c|c|}
\hline MONTH & $\begin{array}{c}M I N I M U M \\
\text { (CFS) }\end{array}$ & $\begin{array}{l}\text { MAXIMUM } \\
\text { (CFS) }\end{array}$ & $\begin{array}{l}\text { MEAN } \\
\text { (CFS) }\end{array}$ & $\begin{array}{l}\text { STAN- } \\
\text { DARD } \\
\text { DEVIA- } \\
\text { TION } \\
\text { (CFS) }\end{array}$ & $\begin{array}{l}\text { COEFFI- } \\
\text { CIENT OF } \\
\text { VARI- } \\
\text { ATION }\end{array}$ & $\begin{array}{c}\text { PERCENT } \\
\text { OF } \\
\text { ANNUAL } \\
\text { RUNOFF }\end{array}$ \\
\hline OCTOBER & 1.7 & 54 & 14 & 10 & .73 & 2.9 \\
\hline NOVEMBER & 2.0 & 46 & 12 & 8.9 & .75 & 2.4 \\
\hline DECEMBER & 2.2 & 55 & 17 & 15 & .88 & 3.4 \\
\hline JANUARY & 2.4 & 166 & 27 & 41 & 1.51 & 5.6 \\
\hline FEBRUARY & 1.8 & 374 & 95 & 105 & 1.10 & 19.5 \\
\hline MARCH & 1.0 & 519 & 142 & 134 & .94 & 29.0 \\
\hline APRIL & 1.1 & 426 & 86 & 104 & 1.21 & 17.5 \\
\hline MAY & 1.9 & 74 & 23 & 18 & .79 & 4.7 \\
\hline JUNE & 3.8 & 207 & 29 & 39 & 1.35 & 5.9 \\
\hline JULY & 1.0 & 30 & 15 & 7.1 & .47 & 3.1 \\
\hline AUGUST & 1.0 & 38 & 15 & 8.0 & .55 & 3.0 \\
\hline SEPTEMBER & 1.0 & 32 & 15 & 7.9 & .52 & 3.1 \\
\hline ANNUAL & 2.3 & 101 & 40 & 29 & .72 & 100 \\
\hline
\end{tabular}

MAGNITUDE AND PROBABILITY OF INSTANTANEOUS PEAK FLOW BASED ON PERIOD OF RECORD 1934-62

DISCHARGE, IN CFS, FOR INDICATED RECURRENCE INTERVAL, IN YEARS, AND ANNUAL EXCEEDANCE PROBABILITY, IN PERCENT

\begin{tabular}{ccccccc}
1.25 & 2 & 5 & 10 & 25 & 50 & 100 \\
$80 \%$ & $50 \%$ & $20 \%$ & $10 \%$ & $4 \%$ & $2 \%$ & $1 \%$ \\
\hline 279 & 843 & 2340 & 3850 & 6400 & 8760 & - \\
\hline
\end{tabular}

WEIGHTED SKEW $=-.290$
MAGNITUDE AND PROBABILITY OF ANNUAL LOW FLOW BASED ON PERIOD OF RECORD 1939-62

\begin{tabular}{|c|c|c|c|c|c|c|}
\hline $\begin{array}{l}\text { PER IOD } \\
\text { (CON- }\end{array}$ & \multicolumn{6}{|c|}{$\begin{array}{l}\text { DISCHARGE, IN CFS, FOR INDICATED RECURRENCE } \\
\text { INTERVAL, IN YEARS, AND ANNUAL NON- } \\
\text { EXCEEDANCE PROBABILITY, IN PERCENT }\end{array}$} \\
\hline SECU- & & - & & $=$ & & 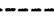 \\
\hline $\begin{array}{l}\text { TIVE } \\
\text { DAYS) }\end{array}$ & $\begin{array}{c}2 \\
50 \%\end{array}$ & $\begin{array}{c}5 \\
20 \%\end{array}$ & $\begin{array}{l}10 \\
10 \%\end{array}$ & $\begin{array}{l}20 \\
5 \%\end{array}$ & $\begin{array}{l}50 \\
2 \%\end{array}$ & $\begin{array}{r}100 \\
1 \%\end{array}$ \\
\hline 1 & 4.7 & 2. & 1. & 1. & 1.1 & -- \\
\hline 3 & 5.3 & 2. & 2. & 1.5 & 1.1 & -- \\
\hline 7 & 6.1 & 3. & 2. & 1.7 & 1.2 & -- \\
\hline 14 & 6.7 & 3. & 2.6 & 1.8 & 1.2 & -- \\
\hline 30 & 7.8 & 4. & 2. & 2. & 1.3 & -- \\
\hline 60 & 8.8 & 4. & 3. & 2. & 1.4 & -- \\
\hline 90 & 9.5 & 5. & 3. & 2. & 1.6 & -- \\
\hline 120 & 10 & 5. & 4. & 3. & 1.9 & -- \\
\hline 183 & 12 & 7. & 5. & 3.6 & 2.4 & -- \\
\hline
\end{tabular}

MAGNITUDE AND PROBABILITY OF ANNUAL HIGH FLOW BASED ON PERIOD OF RECORD 1934-62

\begin{tabular}{|c|c|c|c|c|c|c|}
\hline $\begin{array}{l}\text { PERIOD } \\
\text { (CON- }\end{array}$ & \multicolumn{6}{|c|}{$\begin{array}{c}\text { DISCHARGE, IN CFS, FOR INDICATED RECURRENCE } \\
\text { INTERVAL, IN YEARS, AND ANNUAL } \\
\text { EXCEEDANCE PROBABILITY, IN PERCENT }\end{array}$} \\
\hline SECU- & & & 10 & 25 & 50 & 100 \\
\hline $\begin{array}{l}\text { TIVE } \\
\text { DAYS) }\end{array}$ & $\begin{array}{c}2 \\
50 \%\end{array}$ & $\begin{array}{c}5 \\
20 \%\end{array}$ & $\begin{array}{l}10 \% \\
10 \%\end{array}$ & $\begin{array}{l}25 \\
4 \%\end{array}$ & $2 \%$ & $1 \%$ \\
\hline 1 & 447 & 1160 & 1760 & 2600 & 3240 & -- \\
\hline 3 & 327 & 897 & 1410 & 2170 & 2800 & - \\
\hline 7 & 237 & 649 & 1020 & 1560 & 2000 & -- \\
\hline 15 & 186 & 496 & 766 & 1150 & 1460 & -- \\
\hline 30 & 136 & 353 & 543 & 817 & 1040 & -- \\
\hline 60 & 97 & 250 & 384 & 576 & 730 & - \\
\hline 90 & 75 & 187 & 282 & 417 & 524 & -- \\
\hline
\end{tabular}

DURATION TABLE OF DAILY MEAN FLOW FOR PERIOD OF RECORD 1934-62

\begin{tabular}{|c|c|c|c|c|c|c|c|c|c|c|c|c|c|c|}
\hline $5 \%$ & $10 \%$ & $15 \%$ & $20 \%$ & $25 \%$ & $30 \%$ & $40 \%$ & $50 \%$ & $60 \%$ & $70 \%$ & $75 \%$ & $80 \%$ & $85 \%$ & $90 \%$ & $95 \%$ \\
\hline 185 & 82 & 48 & 31 & 25 & 21 & 17 & 15 & 12 & 9.7 & 8.3 & 7.1 & 5.5 & 3.6 & 2.2 \\
\hline
\end{tabular}


13228000 MALHEUR RIVER AT VALE, OR

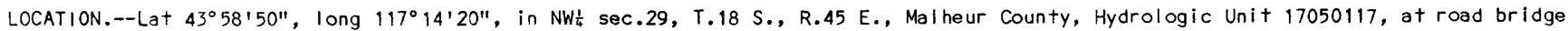
at vale and $0.2 \mathrm{mi}$ downstream from Buliy Creek.

DRAINAGE AREA. $--3,880 \mathrm{mi}^{2}$, approximately.

PERIOD OF RECORD.--April 1890 to September 1891, January 1895 to September 1896, June 1903 to September 1906 , June 1908 to September 1914.

GAGE.--Staff gage. Altitude of gage is 2,230 ft (from topographic map). Prior to Mar. 20, 1919, statf or chain gages at different datums.

REMARKS.--Many diversions above station for irrigation above and below station. Flow slightly regulated since 1915 by Vale-Oregon Irrigation Co. dam on Bully Creek.

AVERAGE DISCHARGE.--11 years (water years 1896, 1904-1906, 1909-1914), $541 \mathrm{ft}^{3} / \mathrm{s}$.

EXTREMES FOR PERIDD OF RECORD.--Maximum discharge, 22,800 $\mathrm{ft}^{3} / \mathrm{s}$ Mar. 2, 1910, gage height, $19.5 \mathrm{ft}$, datum then in use, from floodmark, from rating curve extended above 3,600 $\mathrm{ft}^{3} / \mathrm{s}$; minimum, $4 \mathrm{ft}^{3} / \mathrm{s}$ July 19-21, 1895, Aug. 23 , 1906.

STATISTICAL SUMMARIES

MONTHLY AND ANNUAL MEAN DISCHARGES $1891-1914$

\begin{tabular}{|c|c|c|c|c|c|c|}
\hline MONTH & $\begin{array}{l}\text { MINIMUM } \\
\text { (CFS) }\end{array}$ & $\begin{array}{l}\text { MAXIMUM } \\
\text { (CFS) }\end{array}$ & $\begin{array}{l}\text { MEAN } \\
\text { (CFS) }\end{array}$ & $\begin{array}{l}\text { STAN- } \\
\text { DARD } \\
\text { DEVIA- } \\
\text { TION } \\
\text { (CFS) }\end{array}$ & $\begin{array}{l}\text { COEFFI- } \\
\text { CIENT OF } \\
\text { VARI- } \\
\text { ATION }\end{array}$ & $\begin{array}{c}\text { PERCENT } \\
\text { OF } \\
\text { ANNUAL } \\
\text { RUNOFF }\end{array}$ \\
\hline OCTOBER & 41 & 144 & 90 & 36 & .40 & 1.5 \\
\hline NOVEMBER & 89 & 192 & 145 & 38 & .26 & 2.4 \\
\hline DECEMBER & 72 & 242 & 139 & 47 & .34 & 2.3 \\
\hline JANUARY & 87 & 1775 & 384 & 440 & 1.15 & 6.3 \\
\hline FEBRUARY & 264 & 2245 & 785 & 679 & .86 & 12.9 \\
\hline MARCH & 397 & 3461 & 1423 & 978 & .69 & 23.4 \\
\hline APRIL & 511 & 5698 & 1700 & 1655 & .97 & 28.0 \\
\hline MAY & 211 & 2319 & 790 & 694 & .88 & 13.0 \\
\hline JUNE & 22 & 1161 & 373 & 304 & .82 & 6.1 \\
\hline JULY & 13 & 517 & 121 & 140 & 1.16 & 2.0 \\
\hline AUGUST & 8.5 & 516 & 69 & 142 & 2.05 & 1.1 \\
\hline SEPTEMBER & 19 & 133 & 54 & 34 & .64 & .9 \\
\hline NNUAL & 185 & 1232 & 516 & 288 & .56 & 100 \\
\hline
\end{tabular}

MAGNITUDE AND PROBABILITY OF INSTANTANEOUS PEAK FLOW BASED ON PERIOD OF RECORD 1891-14

DISCHARGE, IN CFS, FOR INDICATED RECURRENCE INTERVAL, IN YEARS, AND ANNUAL EXCEEDANCE PROBABILITY, IN PERCENT

\begin{tabular}{ccccccc}
1.25 & 2 & 5 & 10 & 25 & 50 & 100 \\
$80 \%$ & $50 \%$ & $20 \%$ & $10 \%$ & $4 \%$ & $2 \%$ & $1 \%$ \\
\hline 3880 & 7500 & 14300 & 19800 & 28100 & -1 & -
\end{tabular}

WEIGHTED SKEW $=-.087$
BASED ON PERIOD OF RECORD 1896-1914

\begin{tabular}{|c|c|c|c|c|c|c|}
\hline \multirow{4}{*}{$\begin{array}{l}\text { PERIOD } \\
\text { (CON- } \\
\text { SECU- } \\
\text { TIVE } \\
\text { DAYS) }\end{array}$} & \multicolumn{6}{|c|}{$\begin{array}{l}\text { DISCHARGE, IN CFS, FOR INDICATED RECURRENCE } \\
\text { INTERVAL, IN YEARS, AND ANNUAL NON- } \\
\text { EXCEEDANCE PROBABILITY, IN PERCENT }\end{array}$} \\
\hline & & $-\infty$ & $-\infty$ & & & $-\cdots$ \\
\hline & 2 & 5 & 10 & 20 & 50 & 100 \\
\hline & $50 \%$ & $20 \%$ & $10 \%$ & $5 \%$ & $2 \%$ & $1 \%$ \\
\hline 1 & -- & -- & - & -- & -- & -- \\
\hline 3 & - & -- & -- & -- & -- & -- \\
\hline 7 & -- & -- & -- & -- & - & -- \\
\hline 14 & -- & -- & -- & -- & -- & -- \\
\hline 30 & - & -- & -- & $\rightarrow$ & - & -- \\
\hline 60 & - & - & -- & -- & -- & - \\
\hline 90 & -- & -- & - & -- & -- & -- \\
\hline 120 & -- & -- & -- & -- & -- & - \\
\hline 183 & -- & -- & -- & -- & -- & -- \\
\hline NOTE: & $S T H$ & $10 \mathrm{NO}$ & ERO & RE & ED. & \\
\hline
\end{tabular}

MAGNITUDE AND PROBABILITY OF ANNUAL HIGH FLOW BASED ON PERIOD OF RECORD 1891-14

\begin{tabular}{|c|c|c|c|c|c|c|}
\hline \multirow{2}{*}{$\begin{array}{l}\text { PERIOD } \\
\text { (CON- } \\
\text { SECU- } \\
\text { TIVE } \\
\text { DAYS) }\end{array}$} & \multicolumn{6}{|c|}{$\begin{array}{l}\text { DISCHARGE, IN CFS, FOR INDICATED RECURRENCE } \\
\text { INTERVAL, IN YEARS, AND ANNUAL } \\
\text { EXCEEDANCE PROBABILITY, IN PERCENT }\end{array}$} \\
\hline & $\begin{array}{c}2 \\
50 \%\end{array}$ & $\begin{array}{c}5 \\
20 \%\end{array}$ & $\begin{array}{l}10 \\
10^{q}\end{array}$ & $\begin{array}{l}25 \\
4 \%\end{array}$ & $\begin{array}{l}50 \\
2 \not p\end{array}$ & $\begin{array}{r}100 \\
1 \%\end{array}$ \\
\hline 1 & 4420 & 7950 & 11200 & 16500 & -- & -- \\
\hline 3 & 3420 & 6040 & 8430 & 12400 & -- & - \\
\hline 7 & 2760 & 5050 & 7050 & 10200 & -- & -- \\
\hline 15 & 2370 & 4300 & 5980 & 8630 & - & -- \\
\hline 30 & 1880 & 3350 & 4690 & 6890 & -- & -- \\
\hline 60 & 1460 & 2490 & 3440 & 5010 & -- & -- \\
\hline 90 & 1210 & 2070 & 2860 & 4170 & -- & -- \\
\hline
\end{tabular}

DURATION TABLE OF DAILY MEAN FLOW FOR PERIOD OF RECORD 1891-14

\begin{tabular}{|c|c|c|c|c|c|c|c|c|c|c|c|c|c|c|}
\hline $5 \%$ & $10 \%$ & $15 \%$ & $20 \%$ & $25 \%$ & $30 \%$ & $40 \%$ & $50 \%$ & $60 \%$ & $70 \%$ & $75 \%$ & $80 \%$ & $85 \%$ & $90 \%$ & $95 \%$ \\
\hline 2160 & 1300 & 933 & 662 & 483 & 365 & 235 & 164 & 127 & 98 & 81 & 62 & 43 & 30 & 19 \\
\hline
\end{tabular}




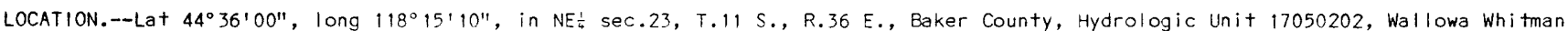
National Forest, on right bank $950 \mathrm{ft}$ upstream from Petticoat Creek, $1.0 \mathrm{mi}$ downstream from U.S. Bureau of Reclamation damsite,

$4.5 \mathrm{mi}$ southeast of Whitney, and $11.5 \mathrm{mi}$ nor thwest of Unity.

DRAINAGE AREA. $--110 \mathrm{mi}^{2}$, approximately.

PERIOD OF RECORD.--June 1964 to June 1978, January 1079 to June 1980, January to June only, each year.

GAGE. - Water-stage recorder. Altitude of gage is $4,0,0 \mathrm{ft}$, from topographic map.

REMARKS.- Some regulations from irrigation and mining operations upstream. A transmountain diversion from headwaters of Middle Fork John Day River delivers as much as $12 \mathrm{ft}^{3} / \mathrm{s}$ to North Fork Burnt River above station.

AVERAGE DISCHARGE.--13 years (water years 1965-77), $49.8 \mathrm{ft}^{3} / \mathrm{s}, 36,080 \mathrm{acre}-\mathrm{ft} / \mathrm{yr}$.

EXTREMES FOR PERIOD OF RECORD.--Maximum discharge, 1,190 $\mathrm{ft}^{3} / \mathrm{s} \mathrm{Apr.} 6$, 1971, gage height, $4.31 \mathrm{ft}$; maximum gage height, 4.95 $\mathrm{ft}$ Jan. 29, 1965 (ice jam); minimum discharge, $0.14 \mathrm{ft}^{3} / \mathrm{s}$ Aug. 15, 1977, but may have been less when stage fell below inlets July 19 to Aug. 17, 1966

STATISTICAL SUMMARIES

MONTHLY AND ANNUAL MEAN DISCHARGES 1965-77

\begin{tabular}{|c|c|c|c|c|c|c|}
\hline MONTH & $\begin{array}{l}\text { MINIMUUM } \\
\text { (CFS) }\end{array}$ & $\begin{array}{l}\text { MAXIMUM } \\
\text { (CFS) }\end{array}$ & $\begin{array}{l}\text { MEAN } \\
\text { (CFS) }\end{array}$ & $\begin{array}{l}\text { STAN- } \\
\text { DARD } \\
\text { DEVIA- } \\
\text { TION } \\
\text { (CFS) }\end{array}$ & $\begin{array}{l}\text { COEFFI- } \\
\text { CIENT OF } \\
\text { VARI- } \\
\text { ATION }\end{array}$ & $\begin{array}{c}\text { PERCENT } \\
\text { OF } \\
\text { ANNUAL } \\
\text { RUNOFF }\end{array}$ \\
\hline OCTOBER & 1.1 & 5.2 & 2.9 & 1.1 & .38 & .5 \\
\hline NOVEMBER & 3.0 & 26 & 7.4 & 6.6 & .88 & 1.2 \\
\hline DECEMBER & 3.6 & 51 & $12^{4}$ & 14 & 1.11 & 2.0 \\
\hline JANUARY & 3.9 & 95 & 27 & 32 & 1.19 & 4.5 \\
\hline FEBRUARY & 5.3 & 93 & 36 & 34 & .97 & 5.9 \\
\hline MARCH & 8.9 & 322 & 39 & 86 & .87 & 16.5 \\
\hline APRIL & 22 & 414 & 215 & 133 & .62 & 35.9 \\
\hline MAY & 9.7 & 370 & 153 & 105 & .69 & 25.5 \\
\hline JUNE & 9.2 & 69 & 34 & 19 & .55 & 5.7 \\
\hline JULY & 1.0 & 17 & 8.4 & 5.5 & .66 & 1.4 \\
\hline AUGUST & .3 & 8.5 & 2.7 & 2.7 & 1.00 & .5 \\
\hline SEPTEMBER & .4 & 4.8 & 1.6 & 1.3 & .77 & .3 \\
\hline ANNUAL & 6.2 & 99 & 50 & 27 & .55 & 100 \\
\hline
\end{tabular}

MAGNITUDE AND PROBABILITY OF INSTANTANEOUS PEAK FLOW BASED ON PERIOD OF RECORD 1965-77

DISCHARGE, IN CFS, FOR INDICATED RECURRENCE INTERVAL, IN YEARS, AND ANNUAL EXCEEDANCE PROBABIL!TY, IN PERCENT

\begin{tabular}{ccccccc}
1.25 & 2 & 5 & 10 & 25 & 50 & 100 \\
$80 \%$ & $50 \%$ & $20 \%$ & $10 \%$ & $4 \%$ & $2 \%$ & $1 \%$ \\
\hline 409 & 661 & 1010 & 1230 & 1490 & - & \\
\hline WEIGHTED SKEW $=$ & -.468 & &
\end{tabular}

MAGNITUDE AND PROBABILITY OF ANNUAL LOW FLOW BASED ON PERIOD OF RECORD 1966-77

\begin{tabular}{|c|c|c|c|c|c|c|}
\hline $\begin{array}{l}\text { PERIOD } \\
\text { (CON- }\end{array}$ & \multicolumn{6}{|c|}{$\begin{array}{l}\text { DISCHARGE, IN CFS, FOR INDICATED RECURRENCE } \\
\text { INTERVAL, IN YEARS, AND ANNUAL NON- } \\
\text { EXCEEDANCE PROBABILITY, IN PERCENT }\end{array}$} \\
\hline SECU- & \multicolumn{6}{|c|}{ - } \\
\hline TIVE & 2 & 5 & 10 & 20 & 50 & 100 \\
\hline DAYS) & $50 \%$ & $20^{d}$ & $10 \%$ & $5 \%$ & $2 \%$ & $1 \%$ \\
\hline 1 & .6 & .3 & .2 & .2 & -- & - \\
\hline 3 & .6 & .3 & .3 & .2 & -- & -- \\
\hline 7 & .7 & .4 & .3 & .2 & -- & -- \\
\hline 14 & .8 & .4 & .3 & .2 & -- & - \\
\hline 30 & 1.0 & .5 & .4 & .3 & - & - \\
\hline 60 & 1.3 & .7 & .5 & .4 & -- & -- \\
\hline 90 & 1.9 & 1.0 & .7 & .5 & -- & - \\
\hline 120 & 2.5 & 1.6 & 1.2 & .9 & -- & -- \\
\hline 183 & 4.5 & 3.2 & 2.7 & 2.2 & -- & -- \\
\hline
\end{tabular}

MAGNITUDE AND PROBABIL! TY OF ANNUAL HIGH FLOW BASED ON PERIOD OF RECORD 1965-77

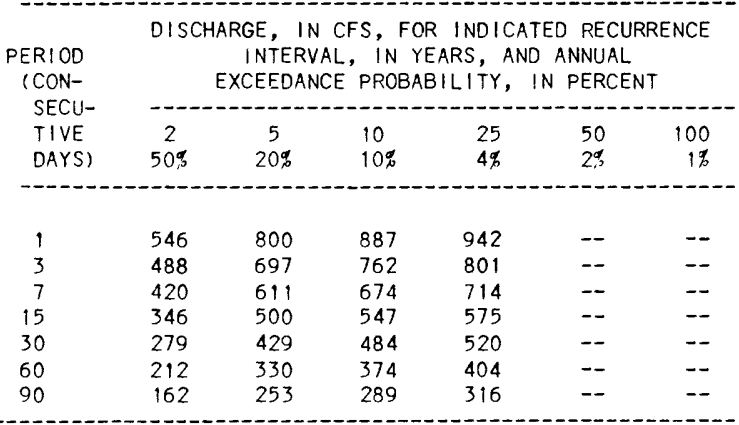

DURATION TABLE OF DAILY MEAN FLOW FOR PERIOD OF RECORD 1965-77

DISCHARGE, IN CFS, WHICH WAS EQUALED OR EXCEEDED FOR INDICATED PERCENT OF TIME

\begin{tabular}{|c|c|c|c|c|c|c|c|c|c|c|c|c|c|c|}
\hline $5 \%$ & $10 \%$ & $15 \%$ & $20 \%$ & $25 \%$ & $30 \%$ & $40_{\infty}^{\alpha}$ & $50 \%$ & $60 \%$ & $70 \%$ & $75 \%$ & $80 \%$ & $85 \%$ & $90 \%$ & $95 \%$ \\
\hline 276 & 158 & 91 & 60 & 41 & 28 & 15 & 9.0 & 6.1 & 4.0 & 3.3 & 2.5 & 1.7 & 1.3 & 7 \\
\hline
\end{tabular}


$132708^{\circ} \%$ SOUTH FORK BURNT RIVER ABOVE BARNEY CREEK, NEAR UNITY, JR

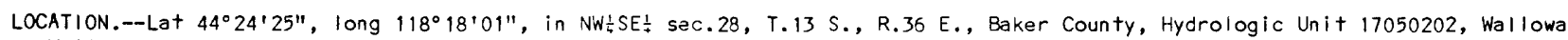
Whitman National Forest, on right bank $84 \mathrm{ft}$ upstream from Barney Creek and 6 mi southwest of Unity.

DRAINAGE AREA.--38.5 $\mathrm{mi}^{2}$.

PERIOD OF RECORD.--March 1963 to September 1981.

GAGE.--Water-stage recorder. Datum of gage is 4,341.75 t+ National Geodetic Vertical Datum of 1929 (Ievels by Bureau of

Reclamation). Prior to July 18, 1963, nonrecording gage at site $220 \mathrm{ft}$ upstream at datum $5.47 \mathrm{ft}$ higher, Juiy 18 , 1963, to July

18,1979 , at site $215 \mathrm{ft}$ upstream at datum $5.31 \mathrm{ft}$ higher.

REMARKS.-No regulation or diversion above station.

AVERAGE DISCHARGE.--18 years, $27.2 \mathrm{ft}+3 / \mathrm{s}, 19,710$ acre- $\mathrm{ft} / \mathrm{yr}$.

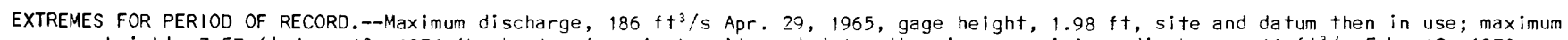
gage height, $3.57 \mathrm{ft}$ Jan. 10, 1974 (backwater from ice), site and datum then in use; minimum discharge, $11 \mathrm{ft} 3 / \mathrm{s}$ Feb. 12, 1978, but may have been less during period of no gage-height record Nov. 20 to Dec. $5,1977$.

STATISTICAL SUMHARIES

MONTHLY AND ANNUAL MEAN DISCHARGES 1964-81

\begin{tabular}{|c|c|c|c|c|c|c|}
\hline MONTH & $\begin{array}{l}\text { MINIMUM } \\
\text { (CFS) }\end{array}$ & $\begin{array}{l}\text { MAXIMUM } \\
\text { (CFS) }\end{array}$ & $\begin{array}{l}\text { MEAN } \\
\text { (CFS) }\end{array}$ & $\begin{array}{l}\text { STAN- } \\
\text { DARD } \\
\text { DEVIA- } \\
\text { TION } \\
\text { (CFS) }\end{array}$ & $\begin{array}{l}\text { COEFFI- } \\
\text { CIENT OF } \\
\text { VARI- } \\
\text { ATION }\end{array}$ & $\begin{array}{c}\text { PERCENT } \\
\text { OF } \\
\text { ANNUAL } \\
\text { RUNOFF }\end{array}$ \\
\hline OCTOBER & 19 & 25 & 22 & 2.2 & .10 & 6.7 \\
\hline NOVEMBER & 18 & 27 & 22 & 2.3 & .11 & 6.6 \\
\hline DECEMBER & 18 & 28 & 21 & 2.8 & .13 & 6.5 \\
\hline JANUARY & 16 & 29 & 21 & 2.8 & .13 & 6.4 \\
\hline FEBRUARY & 16 & 31 & 21 & 3.0 & .14 & 6.5 \\
\hline MARCH & 20 & 39 & 24 & 4.5 & .18 & 7.4 \\
\hline APRIL & 23 & 86 & 36 & 15 & .42 & 11.1 \\
\hline MAY & 23 & 84 & 52 & 19 & .35 & 16.0 \\
\hline JUNE & 21 & 68 & 37 & 13 & .36 & 11.3 \\
\hline JULY & 19 & 32 & 25 & 3.8 & .15 & 7.7 \\
\hline AUGUST & 19 & 30 & 23 & 3.0 & .13 & 7.0 \\
\hline SEPTEMBER & 18 & 27 & 22 & 2.5 & .11 & 6.7 \\
\hline ANNUAL & 21 & 39 & 27 & 4.7 & .17 & 100 \\
\hline
\end{tabular}

MAGNITUDE AND PROBABILITY OF INSTANTANEOUS PEAK FLOW BASED ON PERIOD OF RECORD 1964-81

DISCHARGE, IN CFS, FOR INDICATED RECURRENCE INTERVAL, IN YEARS, AND ANNUAL EXCEEDANCE PROBABILITY, IN PERCENT

\begin{tabular}{ccccccc}
1.25 & 2 & 5 & 10 & 25 & 50 & 100 \\
$80 \%$ & $50 \%$ & $20 \%$ & $10 \%$ & $4 \%$ & $2 \%$ & $1 \%$ \\
\hline 49 & 74 & 110 & 135 & 165 & - & - \\
\hline
\end{tabular}

WEIGHTED SKEW $=-.146$
MAGNITUDE AND PROBABILITY OF ANNUAL LOW FLOW BASED ON PERIOD OF RECORD 1965-81

\begin{tabular}{|c|c|c|c|c|c|c|}
\hline $\begin{array}{r}\text { PERIOD } \\
\text { ICON- } \\
\text { SECU- }\end{array}$ & \multicolumn{6}{|c|}{$\begin{array}{l}\text { OISCHARGE, IN CFS, FOR INDICATED RECURRENCE } \\
\text { INTERVAL, IN YEARS, AND ANNUAL NON- } \\
\text { EXCEEDANCE PROBABILITY, IN PERCENT }\end{array}$} \\
\hline $\begin{array}{l}\text { TIVE } \\
\text { DAYS) }\end{array}$ & $\begin{array}{c}2 \\
50 \%\end{array}$ & $\begin{array}{c}5 \\
20 \%\end{array}$ & $\begin{array}{l}10 \\
10 \%\end{array}$ & $\begin{array}{l}20 \\
5 \%\end{array}$ & $\begin{array}{l}50 \\
2 \%\end{array}$ & $\begin{array}{r}100 \\
1 \%\end{array}$ \\
\hline 1 & 16 & 14 & 13 & 12 & -- & -- \\
\hline 3 & 17 & 15 & 14 & 13 & -- & -- \\
\hline 7 & 18 & 16 & 14 & 13 & -- & -- \\
\hline 14 & 19 & 17 & 16 & 15 & -- & -- \\
\hline 30 & 19 & 17 & 17 & 16 & -- & -- \\
\hline 60 & 20 & 18 & 18 & 17 & -- & - \\
\hline 90 & 20 & 19 & 18 & 18 & -- & -- \\
\hline 120 & 21 & 19 & 18 & 18 & -- & -- \\
\hline 183 & 21 & 20 & 19 & 18 & -- & -- \\
\hline
\end{tabular}

MAGNITUDE AND PROBABILITY OF ANNUAL HIGH FLOW BASED ON PERIOD OF RECORD 1964-81

\begin{tabular}{|c|c|c|c|c|c|c|}
\hline $\begin{array}{l}\text { PERIOD } \\
\text { (CON- }\end{array}$ & \multicolumn{6}{|c|}{$\begin{array}{l}\text { DISCHARGE, IN CFS, FOR IND ICATED RECURRENCE } \\
\text { INTERVAL, IN YEARS, AND ANNUAL } \\
\text { EXCEEDANCE PROBABILITY, IN PERCENT }\end{array}$} \\
\hline SECU- & & & P & 2 & $\overrightarrow{0}$ & \\
\hline TIVE & 2 & 5 & 10 & 25 & 50 & 100 \\
\hline DAYS) & $50 \%$ & $20 \%$ & $10 \%$ & $4 \%$ & $2 \%$ & 18 \\
\hline 1 & 71 & 106 & 129 & 158 & -- & -- \\
\hline 3 & 68 & 102 & 125 & 153 & $\rightarrow$ & -- \\
\hline 7 & 65 & 96 & 116 & 140 & -- & -- \\
\hline 15 & 60 & 87 & 104 & 127 & - & -- \\
\hline 30 & 53 & 75 & 89 & 106 & -- & -- \\
\hline 60 & 46 & 62 & 73 & 86 & - & -- \\
\hline 90 & 41 & 53 & 62 & 72 & -- & -- \\
\hline
\end{tabular}

DURATION TABLE OF DAILY MEAN FLOW FOR PERIOD OF RECORD 1964-81

DISCHARGE, IN CFS, WHICH WAS EQUALED OR EXCEEDED FOR INDICATED PERCENT OF TIME

\begin{tabular}{|c|c|c|c|c|c|c|c|c|c|c|c|c|c|c|}
\hline $5 \%$ & $10 \%$ & $15 \%$ & $20 \%$ & $25 \%$ & $30 \%$ & $40 \%$ & $50 \%$ & $60 \%$ & $70 \%$ & $75 \%$ & $80 \%$ & $85 \%$ & $90 \%$ & $95 \%$ \\
\hline 55 & 42 & 34 & 30 & 27 & 26 & 25 & 23 & 22 & 21 & 21 & 20 & 20 & 19 & 18 \\
\hline
\end{tabular}




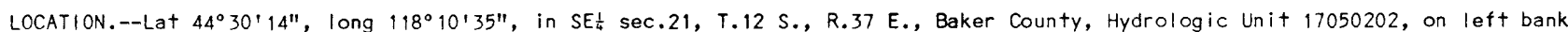
$800 \mathrm{ft}$ downstream from Unity Dam, $0.4 \mathrm{mi}$ upstream from Van Cleve ditch, $7 \mathrm{mi}$ west of Hereford, and at mile 63.5 .

DRAINAGE AREA.--309 $\mathrm{mi}^{2}$.

PERIOD OF RECORD.--March to September 1915, April to September 1916, October 1928 to September 1982 . Monthly discharge only for some periods, published in WSP 1317.

GAGE.--Water-stage recorder. Datum of gage is 3,758.19 ft National Geodetic Vertical Datum of 1929. Oct. 1, 1943, to Oct. 31, 1966, water-stage recorder at site $450 \mathrm{ft}$ downstream at datum $1.44 \mathrm{ft}$ lower. See WSP 1317 or 1737 for history of changes prior to oct. 1, 1943.

REMARKS.--Flow regulated since 1938 by Unity Reservoir. Diversions for irrigation above station.

AVERAGE DISCHARGE. -54 years (water years $1929-82$ ), $84.6 \mathrm{ft}^{3} / \mathrm{s}, 61,290$ acre- $\mathrm{ft} / \mathrm{yr}$.

EXTREMES FOR PERIOD OF RECORD.--Maximum discharge, 2,220 $\mathrm{ft}^{3} / \mathrm{s} \mathrm{Apr}$. 17, 1943, gage height, $5.91 \mathrm{ft}$, site and datum then in use, from rating curve extended above $1,300 \mathrm{ft} / \mathrm{s}$; maximum gage height, $9.07 \mathrm{ft}$ Apr. 8 , 197 ; no flow at times; minimum discharge before construction of Unity Dam, $1.6 \mathrm{ft}^{3} / \mathrm{s}$ Aug. 31, 1935.

STATISTICAL SUMMARIES (BEFORE THE CONSTRUCTION OF UNITY RESERVOIR)

MONTHLY AND ANNUAL MEAN DISCHARGES 1929-38

\begin{tabular}{|c|c|c|c|c|c|c|}
\hline MONTH & $\begin{array}{l}\text { MINIMUM } \\
\text { (CFS) }\end{array}$ & $\begin{array}{l}\text { MAXIMUM } \\
\text { (CFS) }\end{array}$ & $\begin{array}{l}\text { MEAN } \\
\text { (CFS) }\end{array}$ & $\begin{array}{l}\text { STAN- } \\
\text { DARD } \\
\text { DEVIA- } \\
\text { TION } \\
\text { (CFS) }\end{array}$ & $\begin{array}{l}\text { COEFFI- } \\
\text { CIENT OF } \\
\text { VARI- } \\
\text { ATION }\end{array}$ & $\begin{array}{c}\text { PERCENT } \\
\text { OF } \\
\text { ANNUAL } \\
\text { RUNOFF }\end{array}$ \\
\hline OCTOBER & 6.5 & 19 & 12 & 3.9 & .33 & 1.7 \\
\hline NOVEMBER & 5.0 & 34 & 21 & 8.7 & .42 & 2.0 \\
\hline DECEMBER & 15 & 73 & 32 & 18 & .56 & 4.5 \\
\hline JANUARY & 14 & 58 & 29 & 13 & .44 & 4.1 \\
\hline FEBRUARY & 24 & 62 & 39 & 14 & .36 & 5.4 \\
\hline MARCH & 55 & 169 & 99 & 40 & .41 & 13.8 \\
\hline APRIL & 41 & 567 & 288 & 180 & .63 & 40.1 \\
\hline MAY & 19 & 316 & 119 & 95 & .80 & 16.6 \\
\hline JUNE & 16 & 70 & 32 & 16 & .50 & 4.4 \\
\hline JULY & 8.1 & 47 & 18 & 11 & .65 & 2.4 \\
\hline AUGUST & 6.1 & 67 & 16 & 18 & 1.09 & 2.3 \\
\hline SEPTEMBER & 6.1 & 43 & 13 & 11 & .84 & 1.8 \\
\hline ANNUAL & 31 & 94 & 60 & 22 & .37 & 100 \\
\hline
\end{tabular}

MAGNITUDE AND PROBABILITY OF INSTANTANEOUS PEAK FLOW BASED ON PERIOD OF RECORD 1929-38

DISCHARGE, IN CFS, FOR INDICATED RECURRENCE INTERVAL, IN YEARS, AND ANNUAL EXCEEDANCE PROBABILITY, IN PERCENT

\begin{tabular}{ccccccc}
1.25 & 2 & 5 & 10 & 25 & 50 & 100 \\
$80 \%$ & $50 \%$ & $20 \%$ & $10 \%$ & $4 \%$ & $2 \%$ & 19 \\
\hline 441 & 651 & 967 & 1190 & & & \\
\hline
\end{tabular}

WEIGHTED SKEW $=.061$

MAGNITUDE AND PROBABILITY OF ANNUAL LOW FLOW BASED ON PERIOD OF RECORD 1930-38

\begin{tabular}{|c|c|c|c|c|c|c|}
\hline $\begin{array}{l}\text { PERIOD } \\
\text { (CON- }\end{array}$ & \multicolumn{6}{|c|}{$\begin{array}{l}\text { DISCHARGE, IN CFS, FOR INDICATED RECURRENCE } \\
\text { INTERVAL, IN YEARS, AND ANNUAL NON- } \\
\text { EXCEEDANCE PROBABILITY, IN PERCENT }\end{array}$} \\
\hline SECU- & -- & $\cdots$ & $--\infty$ & --- & --1 & \\
\hline $\begin{array}{l}\text { TIVE } \\
\text { DAYS }\end{array}$ & $\begin{array}{c}2 \\
50 \%\end{array}$ & $\begin{array}{c}5 \\
20 \%\end{array}$ & $\begin{array}{l}10 \\
10 \%\end{array}$ & $\begin{array}{l}20 \\
5 \%\end{array}$ & $\begin{array}{l}50 \\
29\end{array}$ & $\begin{array}{r}100 \\
1 \%\end{array}$ \\
\hline & & & & & & \\
\hline 1 & -- & -- & -- & -- & -- & -- \\
\hline 3 & -- & -- & -- & -- & -- & -- \\
\hline 7 & -- & - & - & -- & -- & -- \\
\hline 14 & - & -- & -- & -- & -- & -- \\
\hline 30 & -- & - & -- & -- & -- & - \\
\hline 60 & -- & -- & -- & -- & -- & - \\
\hline 90 & -- & -- & -- & -- & - & -- \\
\hline 120 & -- & -- & -- & -- & -- & -- \\
\hline 183 & -- & -- & -- & -- & -- & -- \\
\hline OTE : & ILYS & & DF & AVA & & \\
\hline
\end{tabular}

MAGNITUDE AND PROBABILITY OF ANNUAL HIGH FLOW BASED ON PERIOD OF RECORD 1929-38

\begin{tabular}{|c|c|c|c|c|c|c|}
\hline $\begin{array}{l}\text { PERIOD } \\
\text { (CON- } \\
\text { SFCU- }\end{array}$ & \multicolumn{6}{|c|}{$\begin{array}{c}\text { DISCHARGE, IN CFS, FOR INDICATED RECURRENCE } \\
\text { INTERVAL, IN YEARS, AND ANNUAL } \\
\text { EXCEEDANCE PROBABILITY, IN PERCENT }\end{array}$} \\
\hline $\begin{array}{l}\text { TIVE } \\
\text { DAYS) }\end{array}$ & $\begin{array}{c}2 \\
50 \%\end{array}$ & $\begin{array}{c}5 \\
20 \%\end{array}$ & $\begin{array}{l}10 \\
10 \%\end{array}$ & $\begin{array}{l}25 \\
4 \%\end{array}$ & $\begin{array}{l}50 \\
2 \%\end{array}$ & $\begin{array}{r}100 \\
1 \%\end{array}$ \\
\hline 1 & 586 & 882 & 1030 & -- & -- & - \\
\hline 3 & 498 & 794 & 964 & -- & -- & -- \\
\hline 7 & 428 & 706 & 884 & -- & -- & - \\
\hline 15 & 364 & 586 & 724 & -- & -- & -- \\
\hline 30 & 293 & 464 & 569 & -- & -- & -- \\
\hline 60 & 213 & 330 & 406 & -- & -- & -- \\
\hline 90 & 161 & 241 & 295 & -- & -- & -- \\
\hline
\end{tabular}

DURATION TABLE OF DAILY MEAN FLOW FOR PERIOD OF RECORD $1929-38$

DISCHARGE, IN CFS, WHICH WAS EQUALED OR EXCEEDED FOR INDICATED PERCENT OF TIME

\begin{tabular}{|c|c|c|c|c|c|c|c|c|c|c|c|c|c|c|}
\hline $5 \%$ & $10 \%$ & $15 \%$ & $20 \%$ & $25 \%$ & $30 \%$ & $40 \%$ & $50 \%$ & $60 \%$ & $70 \%$ & $75 \%$ & $80 \%$ & $85 \%$ & $90 \%$ & $95 \%$ \\
\hline 281 & 141 & 89 & 67 & 51 & 42 & 32 & 24 & 19 & 15 & 13 & 11 & 10 & 8.3 & 5.7 \\
\hline
\end{tabular}


13273000 BURNT RIVER NEAR HEREFORD, OR--Continued

STATISTICAL SUMMARIES (AFTER THE COMPLETION OF UNITY RESERVOIR)

MONTHLY AND ANNUAL MEAN DISCHARGES 1939-82

\begin{tabular}{|c|c|c|c|c|c|c|}
\hline MONTH & $\begin{array}{l}\text { MINIMUM } \\
\text { (CFS) }\end{array}$ & $\begin{array}{l}\text { MAXIMUM } \\
\text { (CFS) }\end{array}$ & $\begin{array}{l}\text { MEAN } \\
\text { (CFS) }\end{array}$ & $\begin{array}{l}\text { STAN- } \\
\text { DARD } \\
\text { DEVIA- } \\
\text { TION } \\
\text { (CFS) }\end{array}$ & $\begin{array}{l}\text { COEFFI- } \\
\text { CIENT OF } \\
\text { VARI- } \\
\text { ATION }\end{array}$ & $\begin{array}{c}\text { PERCENT } \\
\text { OF } \\
\text { ANNUAL } \\
\text { RUNOFF }\end{array}$ \\
\hline OCTOBER & .2 & 93 & 27 & 24 & .88 & 2.5 \\
\hline NOVEMBER & .5 & 63 & 15 & 16 & 1.09 & 1.4 \\
\hline DECEMBER & .7 & 66 & 18 & 18 & 1.01 & 1.6 \\
\hline JANUARY & 1.7 & 87 & 23 & 23 & 1.02 & 2.1 \\
\hline FEBRUARY & 0.0 & 287 & 39 & 51 & 1.28 & 3.6 \\
\hline MARCH & 1.4 & 379 & 94 & 94 & 1.00 & 8.6 \\
\hline APRIL & .2 & 618 & 257 & 173 & .67 & 23.7 \\
\hline MAY & 87 & 484 & 200 & 94 & .47 & 18.4 \\
\hline JUNE & 42 & 326 & 125 & 46 & .37 & 11.5 \\
\hline JULY & 60 & 134 & 102 & 19 & .19 & 9.4 \\
\hline AUGUST & 47 & 148 & 107 & 20 & .19 & 9.9 \\
\hline SEPTEMBER & .2 & 128 & 78 & 29 & .38 & 7.2 \\
\hline ANNUAL & 32 & 166 & 91 & 36 & .39 & 100 \\
\hline
\end{tabular}

MAGNITUDE ANO PROBABILITY OF INSTANTANEOUS PEAK FLOW BASED ON PERIOD OF RECORD

\begin{tabular}{|c|c|c|c|c|c|c|}
\hline \multicolumn{7}{|c|}{$\begin{array}{l}\text { DISCHARGE, IN CFS, FOR INDICATED RECURRENCE INTERVAL, IN } \\
\text { YEARS, AND ANNUAL EXCEEDANCE PROBABILITY, IN PERCENT }\end{array}$} \\
\hline $\begin{array}{l}1.25 \\
80 \%\end{array}$ & $\begin{array}{c}2 \\
50 \%\end{array}$ & $\begin{array}{c}5 \\
20 \%\end{array}$ & $\begin{array}{l}10 \\
10 \%\end{array}$ & $\begin{array}{l}25 \\
4 \%\end{array}$ & $\begin{array}{l}50 \\
2 \%\end{array}$ & $\begin{array}{r}100 \\
1 \%\end{array}$ \\
\hline-- & -- & -- & -- & -- & -. & -- \\
\hline
\end{tabular}

WEIGHTED SKEW =
MAGNITUDE AND PROBABILITY OF ANNUAL LOW FLOW BASED ON PERIOD OF RECORD 1940-82

\begin{tabular}{|c|c|c|c|c|c|c|}
\hline $\begin{array}{l}\text { PERIOD } \\
\text { (CON- }\end{array}$ & \multicolumn{6}{|c|}{$\begin{array}{l}\text { DISCHARGE, IN CFS, FOR INDICATED RECURRENCE } \\
\text { INTERVAL, IN YEARS, AND ANNUAL NON- } \\
\text { EXCEEDANCE PROBABIL!TY, IN PERCENT }\end{array}$} \\
\hline SECU- & & & & & & 100 \\
\hline DAYS) & $\begin{array}{c}2 \\
50 \%\end{array}$ & ${ }_{20 \%}^{5}$ & $10 \%$ & $5 \%$ & $2 \%$ & $1 \%$ \\
\hline & & & & & & \\
\hline 1 & 1.4 & .3 & .1 & .1 & 0.0 & 0.0 \\
\hline 3 & 1.4 & .4 & .2 & .1 & 0.0 & 0.0 \\
\hline 7 & 1.8 & .4 & .2 & .1 & 0.0 & 0.0 \\
\hline 14 & 2.1 & .6 & .3 & .2 & .1 & .1 \\
\hline 30 & 3.3 & .8 & .4 & .2 & .1 & .1 \\
\hline 60 & 4.6 & 1.5 & .8 & .5 & .3 & .2 \\
\hline 90 & 6.9 & 2.3 & 1.3 & .7 & .4 & .3 \\
\hline 120 & 10 & 3.8 & 2.2 & 1.3 & .7 & .5 \\
\hline 183 & 24 & 11 & 7.2 & 4.7 & 2.8 & 1.9 \\
\hline
\end{tabular}

MAGNITUDE AND PROBABILITY OF ANNUAL HIGH FLOW BASED ON PERIOD OF RECORD 1940-82

\begin{tabular}{|c|c|c|c|c|c|c|}
\hline $\begin{array}{l}\text { PERIOD } \\
\text { (CON- } \\
\text { SFCI- }\end{array}$ & \multicolumn{6}{|c|}{$\begin{array}{l}\text { DISCHARGE, IN CFS, FOR INDICATED RECURRENCE } \\
\text { INTERVAL, IN YEARS, AND ANNUAL } \\
\text { EXCEEDANCE PROBABILITY, IN PERCENT }\end{array}$} \\
\hline TIVE & 2 & 5 & 10 & 25 & 50 & 100 \\
\hline DAYS) & $50 \%$ & $20 \%$ & $10 \%$ & $4 \%$ & $2 \%$ & $1 \%$ \\
\hline 1 & 444 & 729 & 932 & 1200 & 1400 & 1600 \\
\hline 3 & 431 & 706 & 901 & 1160 & 1350 & 1540 \\
\hline 7 & 400 & 648 & 821 & 1040 & 1210 & 1380 \\
\hline 15 & 344 & 549 & 696 & 889 & 1040 & 1190 \\
\hline 30 & 285 & 446 & 560 & 712 & 829 & 949 \\
\hline 60 & 227 & 341 & 419 & 518 & 592 & 666 \\
\hline 90 & 194 & 283 & 343 & 419 & 476 & 534 \\
\hline
\end{tabular}

DURATION TABLE OF DAILY MEAN FLOW FOR PERIOD OF RECORD 1940-82

DISCHARGE, IN CFS, WHICH WAS EQUALED OR EXCEEDED FOR INDICATED PERCENT OF TIME

\begin{tabular}{|c|c|c|c|c|c|c|c|c|c|c|c|c|c|c|}
\hline $5 q$ & $10 \%$ & $15 \%$ & $20 \%$ & $25^{\circ}$ & $30 \%$ & $40 \%$ & $50 \%$ & $60 \%$ & $70 \%$ & $75 \%$ & $80^{\circ}$, & $35 \%$ & $90 \%$ & $95 \%$ \\
\hline 298 & 193 & 149 & 138 & 126 & 115 & 93 & 70 & 42 & 22 & 12 & 7.9 & 5.0 & 2.9 & 1.4 \\
\hline
\end{tabular}




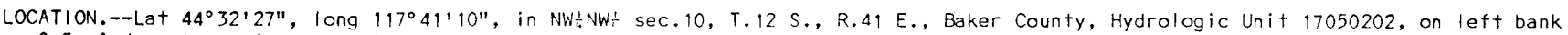

$0.5 \mathrm{mi}$ downstream from Dark Canyon, $4.6 \mathrm{mi}$ upstream from Deer Creek, $5.0 \mathrm{mi}$ northeast of Bridgeport, and at mile 37.1 .

DRAINAGE AREA. $--650 \mathrm{mi}^{2}$, aporoximately.

PERIOD OF RECORD.--October 1955 to Septembor 198 .

GAGE.--Water-stage recorder. Datum of gage is? $22^{2} .23++$ iational Geodetic vertical Datum of 1929 (levels by Bureau of Reclamation).

REMARKS. -Flow regulated since 1938 by Unity Reservoir. llany diversions for irrigation above station.

AVERAGE DISCHARGE. -24 years, $105 \mathrm{ft} / \mathrm{s}, 76,070$ acre- $+\mathrm{t} / \mathrm{yr}$.

EXTREMES FOR PERIOD OF RECORD.--Maximum discharge, 1,600 $\mathrm{ft}^{3} / \mathrm{s} \mathrm{Apr} .9,1071$, gage height, $6.40 \mathrm{ft}$; minimum, $5.2 \mathrm{ft} / \mathrm{s}$ Dec. 5 , 1972.

STATISTICAL SUPMARIES

MONTHLY AND ANNUAL MEAN MISCHARGES 1957-80

\begin{tabular}{|c|c|c|c|c|c|c|}
\hline MONTH & $\begin{array}{l}\text { MINIMUM } \\
\text { (CFS) }\end{array}$ & $\begin{array}{l}\text { MAXIMUM } \\
\text { (CFS) }\end{array}$ & $\begin{array}{l}\text { MEAN } \\
\text { (CFS) }\end{array}$ & $\begin{array}{l}\text { STAN- } \\
\text { DARD } \\
\text { DEVIA- } \\
\text { TION } \\
\text { (CFS) }\end{array}$ & $\begin{array}{l}\text { COEFFI- } \\
\text { CIENT OF } \\
\text { VARI- } \\
\text { ATION }\end{array}$ & $\begin{array}{c}\text { PERCENT } \\
\text { OF } \\
\text { ANNUAL } \\
\text { RUNOFF }\end{array}$ \\
\hline OCTOBER & 11 & 77 & 45 & 16 & .36 & 3.5 \\
\hline NOVEMBER & 11 & 63 & 28 & 10 & .37 & 2.3 \\
\hline DECEMBER & 15 & 65 & 31 & 13 & .43 & 2.5 \\
\hline JANUARY & 14 & 146 & 44 & 34 & .78 & 3.5 \\
\hline FEBRUARY & 17 & 354 & 83 & 76 & .92 & 6.6 \\
\hline MARCH & 17 & 490 & 134 & 114 & .85 & 10.7 \\
\hline APRIL & 21 & 797 & 311 & 223 & .72 & 24.8 \\
\hline MAY & 38 & 678 & 237 & 168 & .71 & 19.9 \\
\hline JUNE & 27 & 200 & 117 & 52 & .44 & 9.3 \\
\hline JULY & 29 & 124 & 81 & 26 & .32 & 6.5 \\
\hline AUGUST & 26 & 107 & 75 & 22 & .29 & 6.0 \\
\hline SEPTEMBER & 12 & 108 & 71 & 25 & .37 & 5.5 \\
\hline ANNUAL & 26 & 209 & 105 & 53 & .50 & 100 \\
\hline
\end{tabular}

MAGNITUDE AND PROBABILITY OF INSTANTANEOUS PEAK FLOW BASED ON PERIOD OF RECORD

\begin{tabular}{|c|c|c|c|c|c|c|}
\hline \multicolumn{7}{|c|}{$\begin{array}{l}\text { DISCHARGE, IN CFS, FOR INDICATED RECURRENCE INTERVAL, IN } \\
\text { YEARS, AND ANNUAL EXCEEDANCE PROBABILITY, IN PERCENT }\end{array}$} \\
\hline $\begin{array}{l}1.25 \\
80 \%\end{array}$ & $\begin{array}{c}2 \\
50 \%\end{array}$ & $\begin{array}{c}5 \\
20 \%\end{array}$ & $\begin{array}{l}10 \\
10^{\circ}\end{array}$ & $\begin{array}{l}25 \\
4 \%\end{array}$ & $\begin{array}{l}50 \\
24\end{array}$ & $\begin{array}{r}100 \\
19\end{array}$ \\
\hline- & -- & -- & -- & $\ldots$ & - & -- \\
\hline
\end{tabular}

WEIGHTED SKEW =
: $A$ AGNITUDE AND PROBABILITY OF ANNUAL LOW FLOW BASED ON PERIOD OF RECORD 1958-80

\begin{tabular}{|c|c|c|c|c|c|c|}
\hline $\begin{array}{l}\text { PERIOD } \\
\text { (CON- }\end{array}$ & \multirow{2}{*}{\multicolumn{6}{|c|}{$\begin{array}{l}\text { DISCHARGE, IN CFS, FOR INDICATED RECURRENCE } \\
\text { INTERVAL, IN YEARS, AND ANNUAL NON- } \\
\text { EXCEEDANCE PROBABILITY, IN PERCENT }\end{array}$}} \\
\hline SECU- & & -1 & $-\cdots$ & & & \\
\hline TIVE & 2 & 5 & 10 & 20 & 50 & 100 \\
\hline DAYS) & $50^{\alpha}$. & $20^{\circ}$ & $10^{\circ}$ & $5 \%$ & $2 \%$ & 10 \\
\hline 1 & 15 & 10 & 8.3 & 5.9 & - - & -- \\
\hline 3 & 16 & 11 & 9.0 & 7.6 & -- & -- \\
\hline 7 & 17 & 12 & 9.9 & 8.3 & -- & -- \\
\hline 14 & 18 & 14 & 11 & 10 & - & -- \\
\hline 30 & 21 & 16 & 14 & 12 & -- & -- \\
\hline 60 & 24 & 18 & 15 & 13 & -- & -- \\
\hline 90 & 26 & 19 & 16 & 14 & - & -- \\
\hline 120 & 29 & 22 & 18 & 16 & -- & -- \\
\hline 183 & 40 & 30 & 25 & 21 & -- & -- \\
\hline
\end{tabular}

MAGNITUDE AND PROBABILITY OF ANNUAL HIGH FLOW BASED ON PER 100 OF RECORD 1957-80

\begin{tabular}{|c|c|c|c|c|c|c|}
\hline $\begin{array}{l}\text { PERIOD } \\
\text { (CON- }\end{array}$ & \multicolumn{6}{|c|}{$\begin{array}{c}\text { DISCHARGE, IN CFS, FOR INDICATED RECURRENCE } \\
\text { INTERVAL, IN YEARS, AND ANNUAL } \\
\text { EXCEEDANCE PROBABILITY, IN PERCENT }\end{array}$} \\
\hline SECU- & & 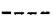 & . & . & -- & - \\
\hline TIVE & 2 & 5 & 10 & 25 & 50 & 100 \\
\hline DAYS) & $50^{\circ}$ & $20^{\circ}$ & $10 \%$ & $4^{\alpha}$ & $2^{\infty}$ & $1 \%$ \\
\hline 1 & 533 & 988 & 1310 & 1720 & -- & -- \\
\hline 3 & 501 & 928 & 1230 & 1510 & -- & -- \\
\hline 7 & 448 & 826 & 1100 & 1440 & -- & -- \\
\hline 15 & 387 & 703 & 929 & 1220 & -- & - \\
\hline 30 & 324 & 590 & 781 & 1030 & -- & -- \\
\hline 50 & 250 & 446 & 584 & 758 & $\cdots$ & - \\
\hline 90 & 208 & 365 & 472 & 605 & -- & -- \\
\hline
\end{tabular}

DURATION TAELE OF DAILY MEAN FLOW FOR PERIOD OF RECORD 1957-80

DISCHARGE, IN CFS, WHICH WAS EOUALEO OR EXCEEDED FOR INDICATED PERCENT OF TIME

\begin{tabular}{|c|c|c|c|c|c|c|c|c|c|c|c|c|c|c|}
\hline $5 \%$ & 109 & $15^{\circ}$, & $20^{\circ}$ & $25^{\alpha}$, & $30 \%$ & $40^{\prime}$ 。 & $50 \%$ & $60 \%$ & $70 \%$ & $75 \%$ & $80 \%$ & $85 \%$ & $90 \%$ & $95 \%$ \\
\hline 363 & 225 & 167 & 131 & 108 & 95 & 78 & 63 & 48 & 37 & 32 & 28 & 25 & 22 & 18 \\
\hline
\end{tabular}


1:275nก0 3UR:UT RIVER AT HUNTINGTON, OR

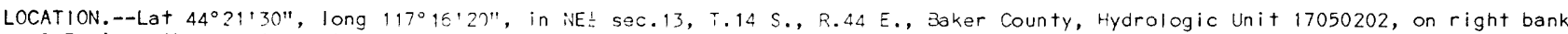
$0.5 \mathrm{mi}$ northwest of Huntington and at mile 2.0 .

DRAINAGE AREA. $--1,093 \mathrm{mi}^{2}$.

PERIOD OF RECORD.--September 1928 to Sentember 1032 , Crtober 1956 to September 1959 , June 1962 to September 1990.

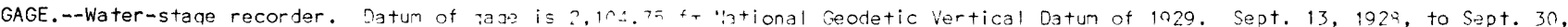
1932, nonrecording gage at site $200 \mathrm{ft}$ upstream at different datum. Oct. 1, 1956, to Sept. 30, 1959, water-stage recorder and Oct. 1, 1959, to Aug. 20, 1962, crest-stage gage.

REMARKS. --Flow regulated since 1938 by Unity Reservoir. Diversions for irrigation above station.

AVERAGE DISCHARGE. -25 years, $132 \mathrm{ft}^{3} / \mathrm{s}, 95,630$ acre $-\mathrm{ft} / \mathrm{yr}$.

EXTREMES FOR PERIOD OF RECORD.--llaximum discharge, $2,220 \mathrm{ft} / \mathrm{s}$ Dec. 22,1964 , gage height, $5.94 \mathrm{ft} ;$ maximum gage height, $6.80 \mathrm{ft}$ Feb. 3, 1963 (ice jam); no flow at times.

STATISTICAL SUMMARIES

MONTHLY AND ANNUAL MEAN DISCHARGES 1929-80

\begin{tabular}{|c|c|c|c|c|c|c|}
\hline MONTH & $\begin{array}{l}\text { MINIMUM } \\
\text { (CFS) }\end{array}$ & $\begin{array}{l}\text { MAXIMUM } \\
\text { (CFS) }\end{array}$ & $\begin{array}{l}\text { MEAN! } \\
\text { (CFS) }\end{array}$ & $\begin{array}{l}\text { STAN- } \\
\text { DARD } \\
\text { DEVIA- } \\
\text { TION } \\
\text { (CFS) }\end{array}$ & $\begin{array}{l}\text { COEFFI- } \\
\text { CIENT OF } \\
\text { VARI- } \\
\text { ATION }\end{array}$ & $\begin{array}{c}\text { PERCENT } \\
\text { OF } \\
\text { ANNUAL } \\
\text { RUNOFF }\end{array}$ \\
\hline OCTOBER & 0.0 & 92 & 55 & 27 & .50 & 3.4 \\
\hline NOVEMBER & 2.9 & 96 & 52. & 20 & .39 & 3.3 \\
\hline DECEMBER & 19 & 112 & 60 & 22 & .37 & 3.7 \\
\hline JANUARY & 20 & 262 & 83 & 56 & .68 & 5.2 \\
\hline FEBRUARY & 33 & 447 & 145 & 105 & .72 & 9.1 \\
\hline MARCH & 35 & 688 & 224 & 149 & .67 & 14.1 \\
\hline APRIL & 21 & 1069 & 398 & 298 & .75 & 25.0 \\
\hline MAY & 8.0 & 820 & 270 & 221 & .82 & 17.0 \\
\hline JUNE & 6.1 & 271 & 121 & 77 & .54 & 7.6 \\
\hline JULY & 1.0 & 133 & 65 & 41 & .63 & 4.1 \\
\hline AUGUST & 0.0 & 106 & 57 & 34 & .59 & 3.6 \\
\hline SEPTEMBER & 0.0 & 122 & 63 & 40 & .63 & 4.0 \\
\hline ANNUAL & 35 & 280 & 132 & 73 & .55 & 100 \\
\hline
\end{tabular}

MAGNITUDE AND PROBABILITY OF INSTANTANEOUS PEAK FLOH BASED ON PERIOD OF RECORD 1929-80

DISCHARGE, IN CFS, FOR INDICATED RECURRENCE INTERVAL, IN YEARS, AND ANNUAL EXCEEDANCE PROBABILITY, IN PERCENT

\begin{tabular}{|c|c|c|c|c|c|c|}
\hline $\begin{array}{l}1.25 \\
80 \%\end{array}$ & $\begin{array}{c}2 \\
50^{\circ},\end{array}$ & $\begin{array}{c}5 \\
20^{\circ}\end{array}$ & $\begin{array}{l}10 \\
10^{\alpha}\end{array}$ & $\begin{array}{l}25 \\
49\end{array}$ & $\begin{array}{l}50 \\
2^{\circ},\end{array}$ & $\begin{array}{r}100 \\
1 \%\end{array}$ \\
\hline- & - & -- & -. & - & -- & -- \\
\hline
\end{tabular}

MAGNITUDE AND PROBABILITY OF ANNUAL LOW FLOW BASED ON PERIOD OF RECORD $1930-80$

\begin{tabular}{|c|c|c|c|c|c|c|}
\hline $\begin{array}{l}\text { PERIOD } \\
\text { (CON- }\end{array}$ & \multirow{2}{*}{\multicolumn{6}{|c|}{$\begin{array}{l}\text { DISCHARGE, IN CFS, FOR INDICATED RECURRENCE } \\
\text { INTERVAL, IN YEARS, AND ANNUAL NON- } \\
\text { EXCEEDANCE PROBABILITY, IN PERCENT }\end{array}$}} \\
\hline SECU- & & & & & & \\
\hline TIVE & 2 & 5 & 10 & 20 & 50 & 100 \\
\hline DAYS) & $50^{x}$ & $20^{\pi}$ & $10 \%$ & $5 \%$ & 2, & $1 \%$ \\
\hline 1 & 27 & 9.4 & 4.0 & 1.7 & .5 & - \\
\hline 3 & 29 & 10 & 4.4 & 1.8 & .5 & -- \\
\hline 7 & 33 & 12 & 5.4 & 2.3 & .7 & - \\
\hline 14 & 37 & 15 & 7.0 & 3. & 1.0 & -- \\
\hline 30 & 44 & 19 & 8.8 & $4 . C$ & 1.3 & -- \\
\hline 60 & 49 & 23 & 12 & 6.4 & 2.6 & -- \\
\hline 90 & -- & -- & -- & -- & -- & -- \\
\hline 120 & -- & -- & - & -- & -- & -- \\
\hline 183 & -- & -- & -- & -- & -- & -- \\
\hline
\end{tabular}

NOTE: LONG-DURATION STATISTICS UNCERTAIN DUE TO EXCESS IVE SKEW.

MAGNITUDE AND PROBABILITY OF ANNUAL HIGH FLOW BASED ON PERIOD OF RECORD 1929-80

\begin{tabular}{|c|c|c|c|c|c|c|}
\hline $\begin{array}{l}\text { PER!OD } \\
\text { (CON- } \\
\text { SECU- }\end{array}$ & \multicolumn{6}{|c|}{$\begin{array}{c}\text { DISCHARGE, IN CFS, FOR INDICATED RECURRENCE } \\
\text { INTERVAL, IN YEARS, AND ANNUAL } \\
\text { EXCEEDANCE PROBABILITY, IN PERCENT }\end{array}$} \\
\hline TIVE & 2 & 5 & 10 & 25 & 50 & 100 \\
\hline DAYS) & $50^{\circ}$ & $20 \%$ & $10 \%$ & $4 \%$ & $2 \%$ & $1 \%$ \\
\hline 1 & 731 & 1310 & 1720 & 2230 & 2610 & -- \\
\hline 3 & 671 & 1190 & 1550 & 2010 & 2340 & -- \\
\hline 7 & 573 & 1010 & 1330 & 1750 & 2050 & -- \\
\hline 15 & 471 & 847 & 1130 & 1510 & 1800 & -- \\
\hline 30 & 394 & 723 & 974 & 1320 & 1600 & -- \\
\hline 60 & 308 & 559 & 752 & 1020 & 1240 & -- \\
\hline 90 & 259 & 466 & 624 & 844 & 1020 & -- \\
\hline
\end{tabular}

DURATION TABLE OF DAILY MEAN FLOW FOR PERIOD OF RECORD 1929-80

DISCHARGE, IN CFS, WHICH WAS EOUALED OR EXCEEDED FOR INDICATED PERCENT OF TIME

\begin{tabular}{|c|c|c|c|c|c|c|c|c|c|c|c|c|c|c|}
\hline $5 \%$ & $10 \%$ & $15 \%$ & $20 \%$ & $25^{\circ}$ & $30 \%$ & $40 \%$ & $50 \%$ & 60,5 & $70 \%$ & $75 \%$ & $80 \%$ & $85 \%$ & $90 \%$ & $95 \%$ \\
\hline 492 & 311 & 230 & 172 & 133 & 112 & 88 & 74 & 61 & 51 & 46 & 40 & 31 & 19 & 5 \\
\hline
\end{tabular}




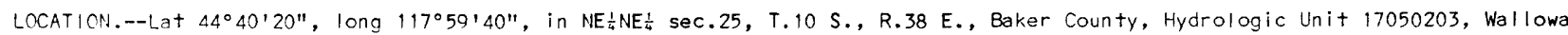
Whitman National Forest, on left bank 1,200 ft downstream from Mason Dam, $1.4 \mathrm{mi}$ upstream from California Gulch, $11.4 \mathrm{mi}$ southeast of Sumpter, and at mile 123.2.

SRAINAGE AREA.--168 $\mathrm{mi}^{2}$, approximately. Prior to Oct. 1, 1970, $170 \mathrm{mi}^{2}$ at cableway, $0.5 \mathrm{mi}$ downstream.

PER1OD OF RECORD.--April 1965 to September 1982.

GAGE.--Water-stage recorder. Datum of gage is 3,898.47 ft National Geodetic Vertical Datum of 1929 (Bureau of Reclamation bench mark). Prior to July 29 , 1965, nonrecording gage at datum $1.03 \mathrm{ft}$ higher.

REMARKS.--Flow completely regulated since Oct. 31, 1967, by Phillips Lake, active capacity, 90,540 acre-ft. Many small diversions for irrigation above station.

AVERAGE DISCHARGE.-- 17 years, $107 \mathrm{ft3} / \mathrm{s}, 77,520$ acre-ft/yr, not adjusted for storage in Phil! ips Lake.

EXTREMES FOR PERIOD OF RECORD. -Maximum discharge observed, $971 \mathrm{ft} 3 / \mathrm{s} \mathrm{Apr.} \mathrm{30,} \mathrm{1965,} \mathrm{gage} \mathrm{height,} 4.43 \mathrm{ft}$; no $\mathrm{flow} \mathrm{Nov.} 12$, 1967.

EXTREMES OUTSIDE PERIOD OF RECORD.--Maximum discharge, 1,600 $\mathrm{ft}+3 / \mathrm{s}$, approximately, Mar. 20, 1910, based on comparison with records for station downstream, near Baker.

\section{STATISTICAL SUMMARIES}

MONTHLY AND ANNUAL MEAN DISCHARGES 1968-82

\begin{tabular}{|c|c|c|c|c|c|c|}
\hline MONTH & $\begin{array}{l}\text { MINIMUM } \\
\text { (CFS) }\end{array}$ & $\begin{array}{l}\text { MAXIMUM } \\
\text { (CFS) }\end{array}$ & $\begin{array}{l}\text { MEAN } \\
\text { (CFS) }\end{array}$ & $\begin{array}{l}\text { STAN- } \\
\text { DARD } \\
\text { DEVIA- } \\
\text { TION } \\
\text { (CFS) }\end{array}$ & $\begin{array}{l}\text { COEFFI- } \\
\text { CIENT OF } \\
\text { VARI- } \\
\text { ATION }\end{array}$ & $\begin{array}{c}\text { PERCENT } \\
\text { OF } \\
\text { ANNUAL } \\
\text { RUNOFF }\end{array}$ \\
\hline DCTOBER & 2.6 & 20 & 11 & 5.3 & .46 & .8 \\
\hline NOVEMBER & .5 & 15 & 7.6 & 3.8 & .50 & .6 \\
\hline DECEMBER & .5 & 14 & 7.0 & 3.6 & .52 & .5 \\
\hline JANUARY & .4 & 13 & 6.6 & 3.9 & .59 & .5 \\
\hline FEBRUARY & .8 & 53 & 13 & 14 & 1.06 & 1.0 \\
\hline MARCH & 1.1 & 317 & 51 & 89 & 1.73 & 3.9 \\
\hline APRIL & 11 & 311 & 110 & 97 & .88 & 8.3 \\
\hline MAY & 157 & 519 & 326 & 111 & .34 & 24.5 \\
\hline JUNE & 148 & 529 & 299 & 127 & .42 & 22.5 \\
\hline JULY & 86 & 273 & 197 & 48 & .24 & 14.8 \\
\hline AUGUST & 56 & 301 & 209 & 72 & .34 & 15.7 \\
\hline SEPTEMBER & 22 & 171 & 94 & 39 & .41 & 7.0 \\
\hline ANNUAL & 60 & 177 & 112 & 36 & .33 & 100 \\
\hline
\end{tabular}

MAGN ITUDE AND PROBABILITY OF INSTANTANEOUS PEAK FLOW BASED ON PERIOD OF RECDRD

\begin{tabular}{lcccccc} 
DISCHARGE, IN CFS, FOR INDICATED RECURRENCE INTERVAL, IN \\
YEARS, AND ANNUAL EXCEEDANCE PROBAB ILITY, IN PERCENT \\
\hline 1.25 & 2 & 5 & 10 & 25 & 50 & 100 \\
$80 \%$ & $50 \%$ & $20 \%$ & $10 \%$ & $4 \%$ & $2 \%$ & $1 \%$ \\
\hline & & & & & & \\
\hline
\end{tabular}

MAGNITUDE AND PROBABILITY OF ANNUAL LOW FLOW BASED ON PERIOD OF RECORD 1969-82

\begin{tabular}{|c|c|c|c|c|c|c|}
\hline \multirow{3}{*}{$\begin{array}{l}\text { PERIOD } \\
\text { (CON- } \\
\text { SECU- } \\
\text { TIVE } \\
\text { DAYS) }\end{array}$} & \multicolumn{6}{|c|}{$\begin{array}{l}\text { DISCHARGE, IN CFS, FOR IND ICATED RECURRENCE } \\
\text { INTERVAL, IN YEARS, AND ANNUAL NON- } \\
\text { EXCEEDANCE PROBABILITY, IN PERCENT }\end{array}$} \\
\hline & 2 & 5 & 10 & 20 & 50 & 100 \\
\hline & $50 \%$ & $20 \%$ & $10 \%$ & $5 \%$ & $2 \%$ & $1 \%$ \\
\hline 1 & 3.2 & 1.1 & .5 & .2 & -- & -- \\
\hline 3 & 4.9 & 9.4 & .7 & .3 & - & -- \\
\hline 7 & 4.2 & 1.5 & .7 & .3 & -- & - \\
\hline 14 & 5.0 & 2.0 & 1.0 & .5 & -- & $m$ \\
\hline 30 & 5.4 & 2.6 & 1.5 & .9 & -- & -- \\
\hline 60 & 5.8 & 3.4 & 2.4 & 1.8 & -- & - \\
\hline 90 & 6.5 & 4.0 & 3.0 & 2.3 & - & - \\
\hline 120 & 7.1 & 4.6 & 3.5 & 2.7 & -- & -- \\
\hline 183 & 11 & 6.2 & 4.4 & 3.3 & -- & -- \\
\hline
\end{tabular}

MAGNITUDE AND PROBABILITY OF ANNUAL HIGH FLOW BASED ON PERIOD OF RECORD 1968-82

\begin{tabular}{|c|c|c|c|c|c|c|}
\hline $\begin{array}{l}\text { PERIOD } \\
\text { (CON- }\end{array}$ & \multicolumn{6}{|c|}{$\begin{array}{c}\text { DISCHARGE, IN CFS, FOR INDICATED RECURRENCE } \\
\text { INTERVAL, IN YEARS, AND ANNUAL } \\
\text { EXCEEDANCE PROBABILITY, IN PERCENT }\end{array}$} \\
\hline SECU- & & & & & & $\cdots$ \\
\hline TIVE & 2 & 5 & 10 & 25 & 50 & 100 \\
\hline DAYS) & $50 \%$ & $20 \%$ & $10 \%$ & $4 \%$ & $2 \%$ & $1 \%$ \\
\hline 1 & 461 & 538 & 575 & 612 & ــ & -- \\
\hline 3 & 447 & 526 & 565 & 604 & - & - \\
\hline 7 & 428 & 511 & 555 & 602 & - & -- \\
\hline 15 & 395 & 486 & 539 & 601 & -- & - \\
\hline 30 & 352 & 447 & 508 & 582 & -- & -- \\
\hline 60 & 301 & 404 & 477 & 575 & - & - \\
\hline 90 & 273 & 358 & 415 & 488 & -- & -- \\
\hline
\end{tabular}

DURATION TABLE OF DAILY MEAN FLOW FOR PERIOD OF RECORD 1968-82
DISCHARGE, IN CFS, WHICH WAS EQUALED OR EXCEEDED FOR INDICATED PERCENT OF TIME

\begin{tabular}{|c|c|c|c|c|c|c|c|c|c|c|c|c|c|c|}
\hline $5 \%$ & $10 \%$ & $15^{\alpha}$, & $20 \%$ & $25 \%$ & $30 \%$ & $40 \%$ & $50 \%$ & $60 \%$ & $70 \%$ & $75 \%$ & $80 \%$ & $85 \%$ & $90 \%$ & $95 \%$ \\
\hline 428 & 323 & 270 & 234 & 202 & 168 & 97 & 17 & 11 & 9.0 & 7.9 & 6.8 & 5.6 & 3.9 & 2.0 \\
\hline
\end{tabular}


13275500 POWDER RIVER NEAR BAKER, OR

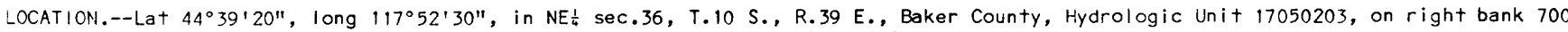
$\mathrm{ft}$ downstream from Stices Gulch, $8.5 \mathrm{mi}$ south of Baker, and at mile 116.2 .

DRAINAGE AREA. $--219 \mathrm{mi}^{2}$.

PERIOD OF RECORD.--December 1903 to August 1914, July 1926 to September 1968. Monthly discharge only for some periods, published in WSP 1317. Published as "near Baker City" December 1903 to December 1905, and as "at Salisbury" January 1906 to August 1914 , October 1928 to September 1951.

GAGE.--Water-stage recorder and concrete-filled bag control. Datum of gage is 3,632.31 ft National Geodetic Vertical Datum of 1929. Dec. 20, 1903, to Feb. 29, 1912, staft gage at site $400 \mathrm{ft}$ upstream at different datum. Mar. 1, 1912, to Aug. 1, 1914, and June 16, 1926, to Oct. 15, 1933, staff gage at site $0.4 \mathrm{mi}$ downstream at different datum. Oct. 16, 1933, to Sept. 3, 1965, graphic water-stage recorder at present site and datum.

REMARKS.--Flow regulated since Oct. 31, 1967, by Phillips Lake (active capacity, 90,540 acre-ft). Many small diversions for irrigation above station. At times Auburn ditch diverts water into basin above station.

AVERAGE DISCHARGE.--51 years (water years 1905-13, 1927-68), $110 \mathrm{ft} 3 / \mathrm{s}, 79,640 \mathrm{acre}-\mathrm{ft} / \mathrm{yr}$.

EXTREMES FOR PERIOD OF RECORD.--Maximum discharge, 1,820 $\mathrm{ft}^{3} / \mathrm{s}$ Mar. 20, 1910, gage height, $7.05 \mathrm{ft}$, site and datum then in use; no flow Aug. 31, 1909, Sept. 7, 1931.

STATISTICAL SUMMARIES

MONTHLY AND ANNUAL MEAN DISCHARGES 1905-67

\begin{tabular}{|c|c|c|c|c|c|c|}
\hline MONTH & $\begin{array}{l}\text { MINIMUM } \\
\text { (CFS) }\end{array}$ & $\begin{array}{l}\text { MAXIMUM } \\
\text { (CFS) }\end{array}$ & $\begin{array}{l}\text { MEAN } \\
\text { (CFS) }\end{array}$ & $\begin{array}{l}\text { STAN- } \\
\text { DARD } \\
\text { DEVIA- } \\
\text { TION } \\
\text { (CFS) }\end{array}$ & $\begin{array}{l}\text { COEFFI- } \\
\text { CIENT OF } \\
\text { VARI- } \\
\text { ATION }\end{array}$ & $\begin{array}{c}\text { PERCENT } \\
\text { OF } \\
\text { ANNUAL } \\
\text { RUNOFF }\end{array}$ \\
\hline OCTOBER & 1.9 & 40 & 15 & 9.2 & .60 & 1.2 \\
\hline NOVEMBER & 5.6 & 111 & 27 & 17 & .65 & 2.0 \\
\hline DECEMBER & 10 & 114 & 36 & 25 & .69 & 2.7 \\
\hline JANUARY & 8.5 & 161 & 38 & 26 & .68 & 2.8 \\
\hline FEBRUARY & 12 & 237 & 60 & 45 & .75 & 4.5 \\
\hline MARCH & 28 & 671 & 153 & 101 & .66 & 11.5 \\
\hline APRIL & 88 & 658 & 344 & 162 & .47 & 25.9 \\
\hline MAY & 52 & 833 & 356 & 148 & .42 & 26.8 \\
\hline JUNE & 22 & 574 & 231 & 126 & .55 & 17.4 \\
\hline JULY & 3.9 & 157 & 46 & 31 & .67 & 3.5 \\
\hline AUGUST & .5 & 49 & 13 & 9.8 & .75 & 1.0 \\
\hline SEPTEMBER & .3 & 41 & 8.5 & 7.1 & .83 & .6 \\
\hline ANNUAL & 40 & 192 & 110 & 40 & .36 & 100 \\
\hline
\end{tabular}

MAGNITUDE AND PROBABILITY OF INSTANTANEOUS PEAK FLOW BASED ON PERIOD OF RECORD $1905-67$

DISCHARGE, IN CFS, FOR INDICATED RECURRENCE INTERVAL, IN YEARS, AND ANNUAL EXCEEDANCE PROBABILITY, IN PERCENT

\begin{tabular}{ccccccc}
1.25 & 2 & 5 & 10 & 25 & 50 & 100 \\
$80 \%$ & $50 \%$ & $20 \%$ & $10 \%$ & $4 \%$ & $2 \%$ & $1 \%$ \\
\hline 479 & 725 & 1050 & 1250 & 1480 & 1640 & 1800 \\
\hline
\end{tabular}

MAGNITUDE AND PROBABILITY OF ANNUAL LOW FLOW BASED ON PERIOD OF RECORD 1906-67

\begin{tabular}{|c|c|c|c|c|c|c|}
\hline $\begin{array}{l}\text { PERIOD } \\
\text { (CON- } \\
\text { SECU- }\end{array}$ & \multicolumn{6}{|c|}{$\begin{array}{l}\text { DISCHARGE, IN CFS, FOR INDICATED RECURRENCE } \\
\text { INTERVAL, IN YEARS, AND ANNUAL NON- } \\
\text { EXCEEDANCE PROBABILITY, IN PERCENT }\end{array}$} \\
\hline $\begin{array}{l}\text { TIVE } \\
\text { DAYS) }\end{array}$ & $\stackrel{2}{2}$ & $\begin{array}{c}5 \\
20 \%\end{array}$ & $\begin{array}{l}10 \\
10 \%\end{array}$ & $\begin{array}{l}20 \\
5 q\end{array}$ & $\begin{array}{l}50 \\
2 \%\end{array}$ & $\begin{array}{r}100 \\
1 \%\end{array}$ \\
\hline 1 & 4.2 & 1.0 & .5 & .2 & . & .1 \\
\hline 3 & 4.5 & 1.3 & .6 & .3 & . & .1 \\
\hline 7 & 5.0 & 1.6 & .7 & .3 & . & .1 \\
\hline 14 & 5.6 & 1.9 & .9 & .4 &. & .1 \\
\hline 30 & 6.2 & 2.3 & 1.2 & .6 & . & .1 \\
\hline 60 & 8.1 & 3.6 & 2.1 & 1.2 &. & .4 \\
\hline 90 & 10 & 5.1 & 3.2 & 2.1 & 1. & .8 \\
\hline 120 & 13 & 7.2 & 5.0 & 3.5 & 2. & 1.7 \\
\hline 183 & 19 & 12 & 9.2 & 7.2 & 5. & 4.3 \\
\hline
\end{tabular}

MAGNITUDE AND PROBABILITY OF ANNUAL HIGH FLOW BASED ON PERIOD OF RECORD 1905-67

\begin{tabular}{|c|c|c|c|c|c|c|}
\hline $\begin{array}{l}\text { PERIOD } \\
\text { (CON- } \\
\text { SECU- }\end{array}$ & \multicolumn{6}{|c|}{$\begin{array}{c}\text { DISCHARGE, IN CFS, FOR IND ICATED RECURRENCE } \\
\text { INTERVAL, IN YEARS, AND ANNUAL } \\
\text { EXCEEDANCE PROBABILITY, IN PERCENT }\end{array}$} \\
\hline TIVE & 2 & 5 & 10 & 25 & 50 & 100 \\
\hline DAYS) & $50 \%$ & $20 \%$ & $10 \%$ & $4 \%$ & $2 \%$ & $1 \%$ \\
\hline 1 & 675 & 952 & 1110 & 1290 & 1410 & 1510 \\
\hline 3 & 641 & 893 & 1030 & 1180 & 1270 & 1350 \\
\hline 7 & 589 & 810 & 925 & 1040 & 1110 & 1170 \\
\hline 15 & 525 & 717 & 813 & 908 & 964 & 1010 \\
\hline 30 & 447 & 609 & 694 & 782 & 836 & 881 \\
\hline 60 & 373 & 512 & 591 & 677 & 732 & 782 \\
\hline 90 & 320 & 438 & 504 & 575 & 622 & 662 \\
\hline
\end{tabular}

DURATION TABLE OF DAILY MEAN FLOW FOR PERIOD DF RECORD 1905-67

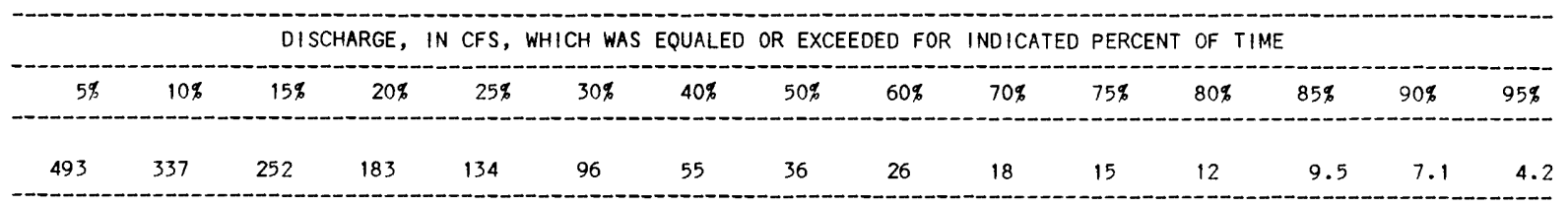


13286700 POWDER RIVER NEAR RICHLAND, OR

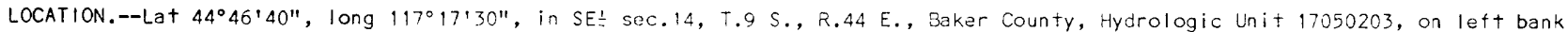

$0.4 \mathrm{mi}$ upstream from Upper Timber Canyon, $5.0 \mathrm{mi}$ west of Richland, and at mile 20.3 .

DRAINAGE AREA.--1,310 $\mathrm{mi}^{2}$, approximately.

PERIOD OF RECORD.--October 1957 to September 1982.

GAGE.--Water-stage recorder. Datum of gage is 2,277.42 ft National Geodetic Vertical Datum of 1929.

REMARKS.--Regulation by several reservoirs, the largest being Phillips Lake since Oct. 31 , 1967, active capacity, 90,540 acre-ft,

Thief Valley Reservoir, capacity, 17,400 acre-ft, and since April 1975, Wolf Creek Reservoir, capacity, 10,400 acre-ft.

Diversions for irrigation above and below station.

AVERAGE DISCHARGE.--25 years, $255 \mathrm{ft} / \mathrm{s}, 134,700$ acre- $\mathrm{ft} / \mathrm{yr}$.

EXTREMES FOR PERIOD OF RECORD.--Maximum discharge, 4,090 $\mathrm{ft} \hat{\mathrm{s}} / \mathrm{s}$ Feb. 21,1992 , gage height, $7.50 \mathrm{ft}$, from $\mathrm{floodmark;}$ maximum gage height, $9.29 \mathrm{ft}$ Jan. 15, 1974 (ice jam); minimum discharge, 0.30 ft3/s Aug. 11, $12,1966$.

STATISTICAL SUMPAARIES (BEFORE THE CONSTRUCTION OF PHILLIPS LAKE)

MONTHLY AND ANNUAL MEAN DISCHARGES 1959-67

\begin{tabular}{|c|c|c|c|c|c|c|}
\hline MONTH & $\begin{array}{l}\text { MINIMUM } \\
\text { (CFS) }\end{array}$ & $\begin{array}{c}\text { MAXIMUM } \\
\text { (CFS) }\end{array}$ & $\begin{array}{l}\text { MEAN } \\
\text { (CFS) }\end{array}$ & $\begin{array}{l}\text { STAN- } \\
\text { DARD } \\
\text { DEVIA- } \\
\text { TION } \\
\text { (CFS) }\end{array}$ & $\begin{array}{l}\text { COEFFI- } \\
\text { CIENT OF } \\
\text { VARI- } \\
\text { ATION }\end{array}$ & $\begin{array}{c}\text { PERCENT } \\
\text { OF } \\
\text { ANNUAL } \\
\text { RUNOFF }\end{array}$ \\
\hline & & & & & & - \\
\hline OCTOBER & 12 & 76 & 42 & 20 & .46 & 1.6 \\
\hline NOVEMBER & 38 & 72 & 56 & 10 & .18 & 2.1 \\
\hline DECEMBER & 33 & 198 & 104 & 54 & .52 & 3.8 \\
\hline JANUARY & 28 & 557 & 173 & 165 & .95 & 6.3 \\
\hline FEBRUARY & 87 & 1081 & 370 & 319 & .86 & 13.6 \\
\hline MARCH & 177 & 909 & 405 & 229 & .57 & 14.9 \\
\hline APRIL & 174 & 1092 & 591 & 347 & .59 & 21.7 \\
\hline MAY & 40 & 1533 & 425 & 452 & 1.06 & 15.6 \\
\hline JUNE & 52 & 917 & 401 & 328 & .82 & 14.7 \\
\hline JULY & 33 & 128 & 56 & 32 & .49 & 2.4 \\
\hline AUGUST & 7.7 & 85 & 43 & 20 & .40 & 1.8 \\
\hline SEPTEMBER & 10 & 83 & 44 & 25 & .55 & 1.6 \\
\hline ANNUAL & 116 & 455 & 225 & 125 & .56 & 100 \\
\hline
\end{tabular}

MAGNITUDE AND PROBABILITY OF INSTANTANEOUS PEAK FLOW BASED ON PERIOD OF RECORD 1958-67

DISCHARGE, IN CFS, FOR INDICATED RECURRENCE INTERVAL, IN YEARS, AND ANNUAL EXCEEDANCE PROBABILITY, IN PERCENT

\begin{tabular}{ccccccc}
1.25 & 2 & 5 & 10 & 25 & 50 & 100 \\
$80 \%$ & $50 \%$ & $20 \%$ & $10 \%$ & $4 \%$ & $2 \%$ & $1 \%$ \\
\hline 803 & 1290 & 2080 & 2680 & - & - & - \\
\hline
\end{tabular}

WEIGHTED SKEW $=.046$
MAGNITUDE AND PROBABILITY OF ANNUAL LOW FLOW BASED ON PERIOD OF RECORD 1959-67

\begin{tabular}{|c|c|c|c|c|c|c|}
\hline $\begin{array}{l}\text { PERIOD } \\
\text { (CON- }\end{array}$ & \multicolumn{6}{|c|}{$\begin{array}{l}\text { DISCHARGE, IN CFS, FOR INDICATED RECURRENCE } \\
\text { INTERVAL, IN YEARS, AND ANNUAL NON- } \\
\text { EXCEEDANCE PROBABILITY, IN PERCENT }\end{array}$} \\
\hline SECU- & & -- & $\ldots$ & -- & $\ldots$ & \\
\hline TIVE & 2 & 5 & 10 & 20 & 50 & 100 \\
\hline DAYS) & $50 \%$ & $20^{d}$ & $10 \frac{9}{5}$ & $5 \not$ & $2^{q}$ & $1 \%$ \\
\hline 1 & 22 & 8.4 & 3.7 & 1.6 & -- & -- \\
\hline 3 & 23 & 11 & 6.2 & 3.5 & -- & -- \\
\hline 7 & 25 & 13 & 7.7 & 4.7 & -- & -- \\
\hline 14 & 28 & 14 & 8.6 & 5.3 & -- & -- \\
\hline 30 & 31 & 17 & 11 & 7.6 & - & - \\
\hline 60 & 37 & 21 & 14 & 10.0 & - & -- \\
\hline 90 & 43 & 25 & 17 & 12 & - & -- \\
\hline 120 & 45 & 28 & 20 & 15 & $-\infty$ & - \\
\hline 183 & 53 & 36 & 29 & 24 & -- & -- \\
\hline
\end{tabular}

MAGNITUDE AND PROBABILITY OF ANNUAL HIGH FLOW BASED ON PERIOD OF RECORD $1958-67$

\begin{tabular}{|c|c|c|c|c|c|c|}
\hline $\begin{array}{c}\text { PERIOD } \\
\text { CCON- } \\
\text { SFCH- }\end{array}$ & \multicolumn{6}{|c|}{$\begin{array}{l}\text { DISCHARGE, IN CFS, FOR INDICATED RECURRENCE } \\
\text { INTERVAL, IN YEARS, AND ANNUAL } \\
\text { EXCEEDANCE PROBABILITY, IN PERCENT }\end{array}$} \\
\hline $\begin{array}{l}\text { TIVE } \\
\text { DAYS) }\end{array}$ & $\begin{array}{c}2 \\
50 \%\end{array}$ & $\begin{array}{c}5 \\
20 \%\end{array}$ & $\begin{array}{l}10 \\
10 \%\end{array}$ & $\begin{array}{l}25 \\
4 \%\end{array}$ & $\begin{array}{l}50 \\
2 \%\end{array}$ & $\begin{array}{r}100 \\
1 \%\end{array}$ \\
\hline 1 & 1120 & 1840 & 2480 & -- & -- & - \\
\hline 3 & 1070 & 1770 & 2380 & -- & -- & -- \\
\hline 7 & 1000 & 1620 & 2140 & - & -- & 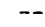 \\
\hline 15 & 894 & 1400 & 1800 & -- & - & -1 \\
\hline 30 & 736 & 1140 & 1460 & -- & -- & -- \\
\hline 60 & 541 & 836 & 1090 & -- & -- & -- \\
\hline 90 & 467 & 738 & 971 & -- & -- & - \\
\hline
\end{tabular}

DURATION TABLE OF DAILY MEAN FLOW FOR PERIOD OF RECORD 1958-6?

DISCHARGE, IN CFS, WHICH WAS EQUALED OR EXCEEDED FOR INDICATED PERCENT OF TIME

\begin{tabular}{|c|c|c|c|c|c|c|c|c|c|c|c|c|c|c|}
\hline 5a, & $10 \%$ & $15^{\circ}$ & $20 \%$ & $25^{\circ}$ & $30 \%$ & $40^{\infty}$ & $50^{\alpha}$ & $60 \%$ & $70^{\circ}$ & $75^{\circ}$ & $80^{\circ}$ & $85 \%$ & $90 \%$ & $95 \%$ \\
\hline 863 & 618 & 435 & 341 & 271 & 200 & 124 & 86 & 65 & 53 & 48 & 42 & 37 & 31 & 23 \\
\hline
\end{tabular}


POWDER RIVER BASIN

13286700 POWDER RIVER NEAR RICHLAND, OR--Continued

STATISTICAL SUMMARIES (AFTER THE COMPLETION OF PHILLIPS LAKE)

MONTHLY AND ANNUAL MEAN DISCHARGES 1968-82

MAGNITUDE AND PROBABILITY OF ANNUAL LOW FLOW BASED ON PERIOD OF RECORD 1969-82

\begin{tabular}{|c|c|c|c|c|c|c|}
\hline MONTH & $\begin{array}{l}\text { MIN IMUM } \\
\text { (CFS) }\end{array}$ & $\begin{array}{c}\text { MAXIMUM } \\
\text { (CFS) }\end{array}$ & $\begin{array}{l}\text { MEAN } \\
\text { (CFS) }\end{array}$ & $\begin{array}{l}\text { STAII- } \\
\text { DARD } \\
\text { DEVIA- } \\
\text { TION } \\
\text { (CFS) }\end{array}$ & $\begin{array}{l}\text { COEFF:- } \\
\text { CIENT OF } \\
\text { VARI- } \\
\text { ATION }\end{array}$ & $\begin{array}{c}\text { PERCENT } \\
\text { OF } \\
\text { ANNUAL } \\
\text { RUNOFF }\end{array}$ \\
\hline OCTOBER & 17 & 103 & 57 & 25 & .43 & 1.7 \\
\hline NOVEMBER & 20 & 91 & 55 & 21 & .38 & 1.7 \\
\hline DECEMBER & 34 & 237 & 111 & 72 & .65 & 3.4 \\
\hline JANUARY & 42 & 750 & 267 & 205 & .77 & 8.1 \\
\hline FEBRUARY & 86 & 1034 & 375 & 223 & .59 & 11.4 \\
\hline MARCH & 132 & 1277 & 531 & 316 & .60 & 16.1 \\
\hline APRIL & 38 & 981 & 556 & 333 & .60 & 16.8 \\
\hline MAY & 23 & 1095 & 527 & 336 & .64 & 16.0 \\
\hline JUNE & 24 & 1141 & 539 & 398 & .74 & 16.3 \\
\hline JULY & 9.2 & 798 & 167 & 201 & 1.20 & 5.1 \\
\hline AUGUST & 14 & 118 & 50 & 24 & .48 & 1.5 \\
\hline SEPTEMBER & 16 & 106 & 66 & 26 & .39 & 2.0 \\
\hline ANNUAL & 58 & 520 & 274 & 142 & .52 & 100 \\
\hline
\end{tabular}

MAGNITUDE AND PROBABILITY OF INSTANTANEOUS PEAK FLOW BASED ON PERIOD OF RECORD

DISCHARGE, IN CFS, FOR INDICATED RECURRENCE INTERVAL, IN YEARS, AND ANNUAL EXCEEDANCE PROBABILITY, IN PERCENT

\begin{tabular}{|c|c|c|c|c|c|c|}
\hline $\begin{array}{l}1.25 \\
80 \%\end{array}$ & $\begin{array}{c}2 \\
50 \%\end{array}$ & $\begin{array}{c}5 \\
20 \%\end{array}$ & $\begin{array}{l}10 \\
10 \%\end{array}$ & $\begin{array}{l}25 \\
4 \%\end{array}$ & $\begin{array}{l}50 \\
2 \%\end{array}$ & $\begin{array}{r}100 \\
1 \%\end{array}$ \\
\hline-- & -- & - & -- & -- & -- & -- \\
\hline
\end{tabular}

\begin{tabular}{|c|c|c|c|c|c|c|}
\hline \multirow{4}{*}{$\begin{array}{l}\text { PERIOD } \\
\text { (CON- } \\
\text { SECU- } \\
\text { TIVE } \\
\text { OAYS) }\end{array}$} & \multirow{2}{*}{\multicolumn{6}{|c|}{$\begin{array}{l}\text { DISCHARGE, IN CFS, FOR INDICATED RECURRENCE } \\
\text { INTERVAL, IN YEARS, AND ANNUAL IION- } \\
\text { EXCEEDANCE PROBABILITY, IN PERCENT }\end{array}$}} \\
\hline & & & & & & \\
\hline & 2 & 5 & 10 & 20 & 50 & 100 \\
\hline & $50 \%$ & $20 \%$ & $10 \%$ & 5,0 & 20 & $1 \%$ \\
\hline \multirow{2}{*}{\multicolumn{7}{|c|}{ 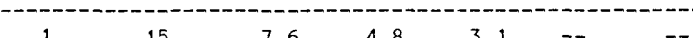 }} \\
\hline & 15 & 7. & 4.8 & 3.1 & - & -- \\
\hline 3 & 19 & 9. & 5.6 & 3.5 & -- & -- \\
\hline 7 & 27 & 13 & 8.1 & 4.9 & -- & -- \\
\hline 14 & 32 & 17 & 11 & 6.8 & -- & - \\
\hline 30 & 36 & 21 & 15 & 11 & - & -- \\
\hline 60 & 45 & 28 & 21 & 16 & -- & -- \\
\hline 90 & 51 & 32 & 24 & 17 & -- & - \\
\hline 120 & 53 & 35 & 26 & 19 & -- & -- \\
\hline 183 & 63 & 39 & 29 & 22 & - & -- \\
\hline
\end{tabular}

MAGNITUDE AND PROBABILITY OF ANNUAL HIGH FLOW BASED ON PERIOD OF RECORD $1968-82$

\begin{tabular}{|c|c|c|c|c|c|c|}
\hline $\begin{array}{l}\text { PERIOD } \\
\text { (CON- } \\
\text { SECU- }\end{array}$ & \multicolumn{6}{|c|}{$\begin{array}{c}\text { DISCHARGE, IN CFS, FOR INDICATED RECURRENCE } \\
\text { INTERVAL, IN YEARS, AND ANNUAL } \\
\text { EXCEEDANCE PROBABILITY, IN PERCENT }\end{array}$} \\
\hline TIVE & 2 & 5 & 10 & 25 & 50 & 100 \\
\hline DAYS) & $50 \%$ & $20 \%$ & $10 \%$ & $4 \%$ & $2 \%$ & $1 \%$ \\
\hline 1 & 1390 & 2190 & 2580 & 2930 & - & - \\
\hline 3 & 1270 & 2030 & 2420 & 2780 & - & -- \\
\hline 7 & 1100 & 1740 & 2050 & 2340 & -- & -- \\
\hline 15 & 912 & 1400 & 1640 & 1860 & - & -- \\
\hline 30 & 783 & 1200 & 1410 & 1600 & - & -- \\
\hline 60 & 660 & 1020 & 1210 & 1380 & - & -- \\
\hline 90 & 591 & 934 & 1110 & 1280 & - & -- \\
\hline
\end{tabular}

DURATION TABLE OF DAILY MEAN FLOW FOR PERIOD OF RECORD 1968-82

\begin{tabular}{|c|c|c|c|c|c|c|c|c|c|c|c|c|c|c|}
\hline & & 015 & ARGE, & CFS, & $\mathrm{CH}$ W. & EQUAL & OR EXC & ED F & NDIC & PER & OF & & & \\
\hline $5 \%$ & $10 \%$ & $15^{\circ}$ & $20 \%$ & $25^{\circ}$ & $30 \%$ & 408 & $50 \%$ & $60 \%$ & $70 \%$ & $75 \%$ & $80 \%$ & $85 \%$ & $90 \%$ & $95 \%$ \\
\hline 1010 & 796 & 623 & 489 & 380 & 301 & 185 & 107 & 70 & 55 & 50 & 45 & 39 & 30 & 20 \\
\hline
\end{tabular}


13288200 EAGLE CREEK ABOVE SKULL CREEK, NEAR NEW BRIDGE, OR

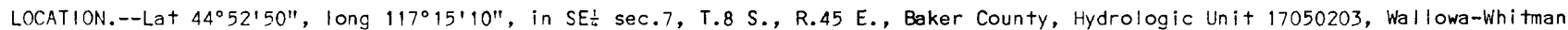
National Forest, on left bank $0.5 \mathrm{mi}$ upstream from Skuli Creek, $6.5 \mathrm{mi}$ northwest of New Bridge, and at mile 10.5 .

DRAINAGE AREA.-- $156 \mathrm{mi}^{2}$.

PERIOD OF RECORD.--October 1957 to September 1982.

GAGE.--Water-stage recorder. Altitude of gage is 2,800 ft, from topographic map.

REMARKS.--No regulation. Some diversions above station for irrigation and one small interbasin diversion for irrigation supply. All diversions are small compared to flow at station during irrigation season.

AVERAGE DISCHARGE. -25 years, $322 \mathrm{ft} / \mathrm{s}, 233,300$ acre- $\mathrm{ft} / \mathrm{yr}$.

EXTREMES FOR PERIOD OF RECORD.--Maximum discharge, $5,310 \mathrm{ft}^{3} / \mathrm{s}$ July 12,1975 , gage height, $5.06 \mathrm{ft}$, from rating curve extended above $2,500 \mathrm{ft}^{3} / \mathrm{s}$ on basis of slope-area measurement of peak flow; maximum gage height, $6.88 \mathrm{ft} \mathrm{Jan}$. 25 , 1962 (ice jam); minimum daily discharge, $30 \mathrm{ft}^{3} / \mathrm{s}$ Nov. $28,1976$.

STATISTICAL SUMMARIES

MONTHLY AND ANNUAL MEAN DISCHARGES 1958-82

\begin{tabular}{|c|c|c|c|c|c|c|}
\hline MONTH & $\begin{array}{l}\text { MINIMUM } \\
\text { (CFS) }\end{array}$ & $\begin{array}{l}\text { MAXIMUM } \\
\text { (CFS) }\end{array}$ & $\begin{array}{l}\text { MEAN } \\
\text { (CFS) }\end{array}$ & $\begin{array}{l}\text { STAN- } \\
\text { DARD } \\
\text { DEVIA- } \\
\text { TION } \\
\text { (CFS) }\end{array}$ & $\begin{array}{l}\text { COEFFI- } \\
\text { CIENT OF } \\
\text { VARI- } \\
\text { ATION }\end{array}$ & $\begin{array}{c}\text { PERCENT } \\
\text { OF } \\
\text { ANNUAL } \\
\text { RUNOFF }\end{array}$ \\
\hline OCTOBER & 69 & 323 & 113 & 53 & .47 & 2.9 \\
\hline NOVEMBER & 78 & 264 & 128 & 45 & .35 & 3.3 \\
\hline DECEMBER & 72 & 211 & 120 & 38 & .32 & 3.1 \\
\hline JANUARY & 59 & 191 & 114 & 31 & .27 & 3.0 \\
\hline FEBRUARY & 72 & 230 & 128 & 42 & .32 & 3.3 \\
\hline MARCH & 65 & 306 & 170 & 60 & .35 & 4.4 \\
\hline APRIL & 191 & 649 & 392 & 138 & .35 & 10.2 \\
\hline IIAY & 252 & 1747 & 941 & 276 & .29 & 24.4 \\
\hline JUNE & 276 & 2134 & 1069 & 389 & .36 & 27.7 \\
\hline JULY & 84 & 1011 & 426 & 231 & .54 & 11.0 \\
\hline AUGUST & 63 & 252 & 148 & 42 & .28 & 3.8 \\
\hline SEPTEMBER & 66 & 172 & 109 & 26 & .24 & 2.8 \\
\hline ANNUAL & 118 & 519 & 322 & 79 & .25 & 100 \\
\hline
\end{tabular}

MAGNITUDE AND PROBABILITY OF INSTANTANEOUS PEAK FLOW BASED ON PERIOD OF RECORD 1958-82

DISCHARGE, IN CFS, FOR INDICATED RECURRENCE INTERVAL, IN YEARS, AND ANNUAL EXCEEDANCE PROBABILITY, IN PERCENT

\begin{tabular}{ccccccc}
1.25 & 2 & 5 & 10 & 25 & 50 & 100 \\
$80 \%$ & $50 \%$ & $20 \%$ & $10 \%$ & $4 \%$ & $2 \%$ & $1 \%$ \\
\hline 1570 & 2040 & 2730 & 3220 & 3870 & 4380 & -
\end{tabular}

WEIGHTED SKEW $=.376$
MAGNITUDE AND PROBABILITY OF ANNUAL LOW FLOW BASED ON PERIOD OF RECORD 1959-82

\begin{tabular}{|c|c|c|c|c|c|c|}
\hline $\begin{array}{l}\text { PERIOD } \\
\text { (CON- }\end{array}$ & \multicolumn{6}{|c|}{$\begin{array}{l}\text { DISCHARGE, IN CFS, FOR INDICATED RECURRENCE } \\
\text { INTERVAL, IN YEARS, AND ANNUAL NON- } \\
\text { EXCEEDANCE PROBABILITY, IN PERCENT }\end{array}$} \\
\hline SECU- & & & 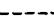 & $--n$ & $\cdots$ & \\
\hline TIVE & 2 & 5 & 10 & 20 & 50 & 100 \\
\hline DAYS) & $50 \%$ & $20 \%$ & $10 \%$ & $5 \%$ & $2 \%$ & $1 \%$ \\
\hline 1 & 63 & 50 & 44 & 39 & 33 & - \\
\hline 3 & 65 & 56 & 52 & 49 & 46 & -- \\
\hline 7 & 70 & 59 & 55 & 52 & 48 & -- \\
\hline 14 & 74 & 64 & 59 & 55 & 51 & -- \\
\hline 30 & 81 & 70 & 65 & 61 & 57 & - \\
\hline 60 & 88 & 76 & 70 & 65 & 60 & -- \\
\hline 90 & 93 & 79 & 73 & 68 & 64 & -- \\
\hline 120 & 98 & 83 & 78 & 73 & 69 & -- \\
\hline 183 & 107 & 91 & 84 & 79 & 75 & -- \\
\hline
\end{tabular}

MAGN I TUDE AND PROBABILITY OF ANNUAL HIGH FLOW BASED ON PERIOD OF RECORD 1958-82

\begin{tabular}{|c|c|c|c|c|c|c|}
\hline $\begin{array}{l}\text { PERIOD } \\
\text { (CON- } \\
\text { SECU- }\end{array}$ & & $\begin{array}{l}\text { RGE, } \\
\text { INTE } \\
\text { XCEED }\end{array}$ & CFS, IN & $\begin{array}{l}\text { INDIC } \\
\text { RSS, } \\
\text { LITY }\end{array}$ & $\begin{array}{l}\text { ED RE } \\
\text { ANNU } \\
\text { P PER }\end{array}$ & ENCE \\
\hline TIVE & 2 & 5 & 10 & 25 & 50 & 100 \\
\hline DAYS) & $50 \%$ & 20,5 & $10 \%$ & $4 \%$ & $2 \%$ & $1 \%$ \\
\hline & & & & & & \\
\hline 1 & 1830 & 2270 & 2470 & 2670 & 2790 & -- \\
\hline 3 & 1720 & 2160 & 2370 & 2590 & 2710 & -- \\
\hline 7 & 1590 & 2000 & 2180 & 2360 & 2450 & - \\
\hline 15 & 1450 & 1800 & 1950 & 2070 & 2130 & -- \\
\hline 30 & 1300 & 1570 & 1660 & 1720 & 1750 & -- \\
\hline 60 & 1080 & 1310 & 1390 & 1450 & 1470 & -- \\
\hline 90 & 901 & 1080 & 1140 & 1190 & 1200 & - \\
\hline
\end{tabular}

DURATION TABLE OF DAILY MEAN FLOW FOR PERIOD OF RECORD 1958-82

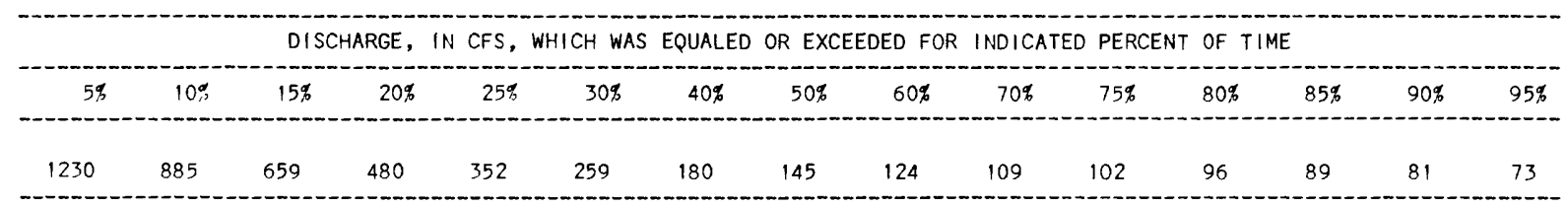


13289500 POWDER RIVER NEAR ROBINETTE, OR

LOCATION.--Lat $44^{\circ} 46^{\prime} 10^{\prime \prime}$, long $117^{\circ} 04^{\prime} 10^{\prime \prime}$, in E-1/2 sec.22, T.9 S., R. 46 E., Baker County, Hydrologic Unit 17050203 , on left bank 2.2 $\mathrm{mi}$ northwest of Robinette and $2.5 \mathrm{mi}$ upstream from mouth.

DRAINAGE AREA. $--1,660 \mathrm{mi}^{2}$, approximately.

PERIOD OF RECORD.--September 1928 to September 1957.

GAGE.--Water-stage recorder. Datum of gage is 1,937.10 $\mathrm{ft}$ National Geodetic Vertical Datum of 1929. Prior to Aug. 24, 1936, staft gage at site $0.5 \mathrm{mi}$ upstream at different datum. Aug. 24, 1936, to Oct. 31, 1948, staff gage at site $50 \mathrm{ft}$ upstream at present datum.

REMARKS.--FIOw partly regulated by several reservoirs, the largest being Thief Valley Reservoir (capacity, 17,000 acre-ft). Many diversions for irrigation above station. One canal with capacity of about $5 \mathrm{ft} / \mathrm{s}$ diverts around station on left bank.

AVERAGE DISCHARGE.--29 years (water years $1929-57$ ), $534 \mathrm{ft} / \mathrm{s}, 386,600 \mathrm{acre}-\mathrm{ft} / \mathrm{yr}$.

EXTREMES FOR PERIOD OF RECORD.--Maximum discharge, $5,500 \mathrm{ft}^{3} / \mathrm{s} \mathrm{May} 27,1956$, gage height, $6.38 \mathrm{ft} ; \mathrm{minimum} \mathrm{observed,} 18 \mathrm{ft} 3 / \mathrm{s}$ Sept. 2-10, 1931.

STATISTICAL SUMMARIES

MONTHLY AND ANNUAL MEAN DISCHARGES 1929-57

\begin{tabular}{|c|c|c|c|c|c|c|}
\hline MONTH & $\begin{array}{l}M|N| M U M \\
(C F S)\end{array}$ & $\begin{array}{l}\text { MAXIMUM } \\
\text { (CFS) }\end{array}$ & $\begin{array}{l}\text { MEAN } \\
\text { (CFS) }\end{array}$ & $\begin{array}{l}\text { STAN- } \\
\text { DARD } \\
\text { DEVIA- } \\
\text { T/ON } \\
\text { (CFS) }\end{array}$ & $\begin{array}{l}\text { COEFF I- } \\
\text { CIENT OF } \\
\text { VARI- } \\
\text { ATION }\end{array}$ & $\begin{array}{c}\text { PERCENT } \\
\text { OF } \\
\text { ANNUAL } \\
\text { RUNOFF }\end{array}$ \\
\hline OCTOBER & 35 & 253 & 139 & 62 & .45 & 2.2 \\
\hline NOVEMBER & 70 & 416 & 184 & 75 & .41 & 2.9 \\
\hline DECEMBER & 80 & 755 & 229 & 140 & .61 & 3.6 \\
\hline JANUARY & 58 & 711 & 240 & 142 & .59 & 3.7 \\
\hline FEBRUARY & 86 & 769 & 377 & 213 & .56 & 5.9 \\
\hline MARCH & 174 & 1302 & 719 & 352 & .49 & 11.2 \\
\hline APRIL & 379 & 2978 & 1254 & 656 & .52 & 19.6 \\
\hline MAY & 490 & 2824 & 1452 & 656 & .45 & 22.6 \\
\hline JUNE & 235 & 2971 & 1245 & 719 & .58 & 19.4 \\
\hline JULY & 48 & 1106 & 358 & 260 & .73 & 5.6 \\
\hline AUGUST & 26 & 253 & 109 & 53 & .49 & 1.7 \\
\hline SEPTEMBER & 22 & 215 & 107 & 52 & .48 & 1.7 \\
\hline NNUAL & 216 & 916 & 534 & 212 & .40 & 100 \\
\hline
\end{tabular}

MAGNITUDE AND PROBABILITY OF INSTANTANEOUS PEAK FLOW BASED ON PERIOD OF RECORD 1929-57

DISCHARGE, IN CFS, FOR INDICATED RECURRENCE INTERVAL, IN YEARS, AND ANNUAL EXCEEDANCE PROBABILITY, IN PERCENT

\begin{tabular}{ccccccc}
1.25 & 2 & 5 & 10 & 25 & 50 & 100 \\
$80 \%$ & $50 \%$ & $20 \%$ & $10 \%$ & $4 \%$ & $2 \%$ & $1 \%$ \\
\hline 1660 & 2630 & 3960 & 4800 & 5810 & 6520 & - \\
\hline WEIGHTED SKEW $=$ & -.439 & & &
\end{tabular}

MAGNITUDE AND PROBABILITY OF ANNUAL LOW FLOW BASED ON PERIOD OF RECORD 1930-57

\begin{tabular}{|c|c|c|c|c|c|c|}
\hline $\begin{array}{l}\text { PERIOD } \\
\text { (CON- } \\
\text { SECU- }\end{array}$ & \multicolumn{6}{|c|}{$\begin{array}{l}\text { DISCHARGE, IN CFS, FOR INDICATED RECURRENCE } \\
\text { INTERVAL, IN YEARS, AND ANNUAL NON- } \\
\text { EXCEEDANCE PROBABILITY, IN PERCENT }\end{array}$} \\
\hline TIVE & 2 & 5 & 10 & 20 & 50 & 100 \\
\hline DAYS) & $50 \%$ & $20 \%$ & $10 \%$ & $5 \%$ & $2 \%$ & $1 \%$ \\
\hline 1 & 66 & 39 & 29 & 22 & 16 & -- \\
\hline 3 & 69 & 42 & 31 & 23 & 17 & - \\
\hline 7 & 73 & 44 & 33 & 25 & 18 & -- \\
\hline 14 & 78 & 47 & 34 & 26 & 19 & -- \\
\hline 30 & 84 & 50 & 37 & 28 & 20 & -- \\
\hline 60 & 95 & 57 & 42 & 32 & 23 & - \\
\hline 90 & 107 & 65 & 48 & 36 & 26 & - \\
\hline 120 & 122 & 77 & 58 & 44 & 32 & - \\
\hline 183 & 150 & 101 & 81 & 66 & 52 & - \\
\hline
\end{tabular}

MAGNITUDE AND PROBABILITY OF ANNUAL HIGH FLOW BASED ON PERIOD OF RECORD 1929-57

\begin{tabular}{|c|c|c|c|c|c|c|}
\hline $\begin{array}{l}\text { PERI OD } \\
\text { (CON- } \\
\text { SECU- }\end{array}$ & \multicolumn{6}{|c|}{$\begin{array}{l}\text { DISCHARGE, IN CFS, FOR INDICATEO RECURRENCE } \\
\text { INTERVAL, IN YEARS, AND ANNUAL } \\
\text { EXCEEDANCE PROBABILITY, IN PERCENT }\end{array}$} \\
\hline TIVE & 2 & 5 & 10 & 25 & 50 & 100 \\
\hline DAYS) & $50 \%$ & $20 \%$ & $10 \%$ & $4 \%$ & $2 \%$ & $1 \%$ \\
\hline 1 & 2510 & 3680 & 4360 & 5130 & 5630 & -- \\
\hline 3 & 2370 & 3480 & 4130 & 4860 & 5340 & -- \\
\hline 7 & 2190 & 3280 & 3930 & 4670 & 5170 & -- \\
\hline 15 & 1980 & 2980 & 3590 & 4280 & 4750 & -- \\
\hline 30 & 1700 & 2540 & 3070 & 3680 & 4100 & -- \\
\hline 60 & 1480 & 2150 & 2560 & 3020 & 3330 & -- \\
\hline 90 & 1290 & 1870 & 2230 & 2640 & 2930 & -- \\
\hline
\end{tabular}

DURATION TABLE OF DAILY MEAN FLOW FOR PERIOD OF RECORO 1929-57

\begin{tabular}{|c|c|c|c|c|c|c|c|c|c|c|c|c|c|c|}
\hline $5 \%$ & $10 \%$ & $15 \%$ & $20 \%$ & $25 \%$ & $30 \%$ & $40 \%$ & $50 \%$ & $60 \%$ & $70 \%$ & $75 \%$ & $80 \%$ & $85 \%$ & $90 \%$ & $95 \%$ \\
\hline 1990 & 1470 & 1120 & 867 & 683 & 548 & 344 & 232 & 180 & 148 & 133 & 117 & 101 & 81 & 54 \\
\hline
\end{tabular}




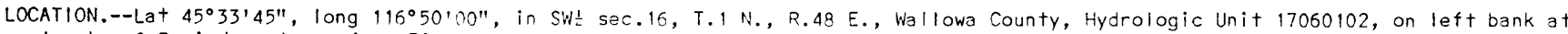
Imnaha, $0.3 \mathrm{mi}$ downstream from Big Sheep Creek, and at mile 19.3 .

DRAINAGE AREA. $--622 \mathrm{mi}^{2}$.

PERIOD OF RECORD.--June 1928 to September 1982.

GAGE.--Water-stage recorder. Datum of gage is 1,941.14 4t National Geodetic Vertical Datum of 1929. Prior to Aug. 6, 1934, nonrecording gage at site $0.25 \mathrm{mi}$ upstream at different datum. Aug. 6-31, 1934, nonrecording gage at present site and datum.

REMARKS.--No regulation. Diversions for irrigation above station. Water is diverted from Big Sheep Creek and tributaries above station for irrigation in Wallowa River basin.

AVERAGE DISCHARGE. --54 years, $517 \mathrm{ft}^{3} / \mathrm{s}, 374,600$ acre- $\mathrm{ft} / \mathrm{yr}$.

EXTREMES FOR PERIOD OF RECORD.--Maximum discharge, 10,100 $\mathrm{ft}+3 / \mathrm{s} \mathrm{Jan.} \mathrm{17,} \mathrm{1974,} \mathrm{gage} \mathrm{height,} 7.86 \mathrm{ft}$, from rating curve extended above $3,500 \mathrm{ft} / \mathrm{s}$; minimum observed, $16 \mathrm{ft} / \mathrm{s}$ Nov. 22, 1931, result of freezeup; minimum daily, $25 \mathrm{ft} / \mathrm{s}$ Nov. 22,23 , 1931.

STATISTICAL SUMMARIES

MONTHLY AND ANNUAL MEAN DISCHARGES 1929-82

\begin{tabular}{|c|c|c|c|c|c|c|}
\hline MONTH & $\begin{array}{l}M|N| A \mid U M \\
\text { (CFS) }\end{array}$ & $\begin{array}{l}\text { MAXIMUM } \\
\text { (CFS) }\end{array}$ & $\begin{array}{l}\text { MEAN } \\
\text { (CFS) }\end{array}$ & $\begin{array}{l}\text { STAN- } \\
\text { DARD } \\
\text { DEVIA- } \\
\text { TION } \\
\text { (CFS) }\end{array}$ & $\begin{array}{l}\text { COEFFI- } \\
\text { CHENT OF } \\
\text { VARI- } \\
\text { ATION }\end{array}$ & $\begin{array}{c}\text { PERCEN } \\
\text { OF } \\
\text { ANNUAL } \\
\text { RUNOFF }\end{array}$ \\
\hline OCTOBER & 82 & 501 & 159 & 63 & .40 & 2.6 \\
\hline NOVEMBER & 80 & 625 & 187 & 91 & .49 & 3.0 \\
\hline DECEMBER & 89 & 806 & 219 & 136 & .62 & 3.5 \\
\hline JANUARY & 69 & 393 & 193 & 34 & .43 & 3.1 \\
\hline FEBRUARY & 82 & 569 & 236 & 112 & .47 & 3.8 \\
\hline MARCH & 114 & 869 & 377 & 162 & .43 & 6.1 \\
\hline APRIL & 345 & 1760 & 942 & 345 & .37 & 15.2 \\
\hline MAY & 445 & 2804 & 1603 & 535 & .33 & 25.9 \\
\hline JUNE & 423 & 2612 & 1365 & 529 & .39 & 22.1 \\
\hline JULY & 123 & 1348 & 567 & 308 & .54 & 9.2 \\
\hline AUGUST & 79 & 380 & 193 & 70 & .36 & 3.1 \\
\hline SEPTEMBER & 83 & 256 & 143 & 39 & .27 & 2.3 \\
\hline ANNUAL & 184 & 829 & 516 & 150 & .29 & 100 \\
\hline
\end{tabular}

MAGNITUDE AND PROBABILITY OF INSTANTANEOUS PEAK FLOW BASED ON PERIOD OF RECORD 1929-82

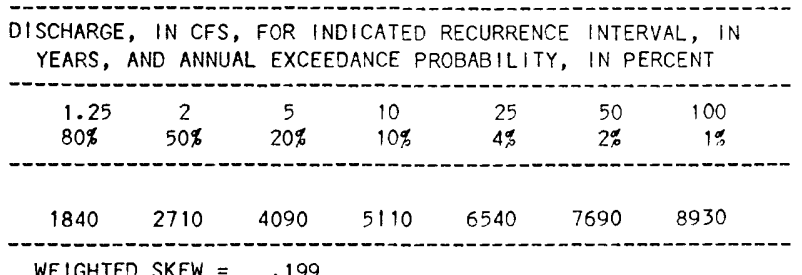

MAGNITUDE AND PROBABILITY OF ANNUAL LOW FLOW BASED ON PERIOD OF RECORD 1930-82

\begin{tabular}{|c|c|c|c|c|c|c|}
\hline $\begin{array}{l}\text { PERIOD } \\
\text { (CON- }\end{array}$ & \multicolumn{6}{|c|}{$\begin{array}{l}\text { DISCHARGE, IN CFS, FOR INDICATED RECURRENCE } \\
\text { INTERVAL, IN YEARS, AND ANNUAL NON- } \\
\text { EXCEEDANCE PROBABILITY, IN PERCENT }\end{array}$} \\
\hline SECU- & & . & 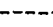 & $-\cdots$ & & \\
\hline TIVE & 2 & 5 & 10 & 20 & 50 & 100 \\
\hline DAYS) & $50 \%$ & $20 \%$ & $10 \%$ & $5 \frac{d}{8}$ & $2, q$ & $1 \%$ \\
\hline & & & & 23 & 7 & 7 \\
\hline 1 & 70 & 48 & 39 & 35 & 21 & 25 \\
\hline 3 & 84 & 61 & 51 & 44 & 36 & 32 \\
\hline 7 & 98 & 76 & 66 & 59 & 51 & 46 \\
\hline 14 & 108 & 88 & 79 & 71 & 64 & 59 \\
\hline 30 & 119 & 98 & 88 & 80 & 71 & 66 \\
\hline 60 & 128 & 105 & 94 & 85 & 76 & 71 \\
\hline 90 & 136 & 111 & 99 & 91 & 81 & 75 \\
\hline 120 & 144 & 116 & 104 & 95 & 85 & 80 \\
\hline 183 & 165 & 128 & 111 & 100 & 88 & 81 \\
\hline
\end{tabular}

MAGNITUDE AND PROBABILITY OF ANNUAL HIGH FLOW BASED ON PERIOD OF RECORD 1929-82

\begin{tabular}{|c|c|c|c|c|c|c|}
\hline $\begin{array}{l}\text { PERIOD } \\
\text { (CON- }\end{array}$ & \multicolumn{6}{|c|}{$\begin{array}{c}\text { DISCHARGE, IN CFS, FOR INDICATED RECURRENCE } \\
\text { INTERVAL, IN YEARS, AND ANNUAL } \\
\text { EXCEEDANCE PROBABILITY, IN PERCENT }\end{array}$} \\
\hline SECU- & \multicolumn{6}{|c|}{ 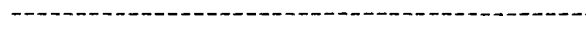 } \\
\hline TIVE & 2 & 5 & 10 & 25 & 50 & 100 \\
\hline DAYS) & $50 \%$ & $20 \%$ & $10 \%$ & $4 \%$ & $2 \%$ & $1 \%$ \\
\hline \\
\hline 1 & 2470 & 3460 & 4090 & 4870 & 5430 & 5970 \\
\hline 3 & 2300 & 3170 & 3710 & 4350 & 4810 & 5240 \\
\hline 7 & 2130 & 2880 & 3330 & 3840 & 4190 & 4520 \\
\hline 15 & 1930 & 2570 & 2950 & 3370 & 3660 & 3920 \\
\hline 30 & 1740 & 2290 & 2580 & 2910 & 3110 & 3290 \\
\hline 60 & 1550 & 2000 & 2230 & 2460 & 2610 & 2730 \\
\hline 90 & 1350 & 1720 & 1910 & 2100 & 2210 & 2300 \\
\hline
\end{tabular}

DURATION TABLE OF DAILY MEAN FLOW FOR PERIOD OF RECORD 1929-82

DISCHARGE, IN CFS, WHICH WAS EQUALED OR EXCEEDED FOR INDICATED PERCENT OF TIME

\begin{tabular}{|c|c|c|c|c|c|c|c|c|c|c|c|c|c|c|}
\hline $5 \not q$ & $10 \%$ & $15 \%$ & $20 \%$ & $25^{\circ}$ & $30 \%$ & $40 \%$ & $50^{\alpha}$ & $60 \%$ & $70 \%$ & $75 \%$ & $80 \%$ & $85 \%$ & $90 \%$ & $95 \%$ \\
\hline 1860 & 1420 & 1120 & 861 & 653 & 496 & 310 & 230 & 188 & 158 & 147 & 137 & 126 & 113 & 94 \\
\hline
\end{tabular}


13318500 GRANDE RONDE RIVER NEAR HILGARD, CR

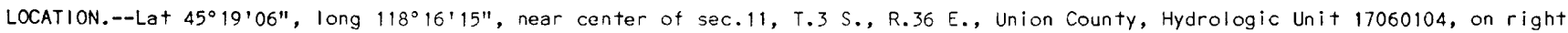
bank $0.5 \mathrm{mi}$ upstream from lower reservoir site of Bureau of Reclamation, $0.8 \mathrm{mi}$ upstream from Spring Creek, and $3 \mathrm{mi}$ southwest of Hi lgard.

DRAINAGE AREA. $--505 \mathrm{mi}^{2}$.

PERIOD OF RECORD.--January 1938 to September 1956. Monthly discharge only prior to October 1945 , published in WSP 1317.

GAGE.--Water-stage recorder. Datum of gage is 3,059.05 $\mathrm{ft}$ National Geodetic Vertical Datum of 1929. Prior to Sept. 16, 1946, water-stage recorder at site $800 \mathrm{ft}$ upstream at different datum.

REMARKS.--Several small diversions for irrigation above station. Since 1909, city of La Grande has diverted about 3 ft ${ }^{3 / s}$ for municipal use at Beaver Creek Reservoir (capacity, about 900 acre- $f t$ ).

AVERAGE DISCHARGE.--18 years (water years 1939-56), $274 \mathrm{ft}^{3} / \mathrm{s}, 198,400$ acre- $\mathrm{ft} / \mathrm{yr}$.

EXTREMES FOR PERIOD OF RECORD.--Maximum discharge, 5,060 $\mathrm{ft}+3 / \mathrm{s}$ May 3 , 1956, gage height, $6.48 \mathrm{tt}$, from rating curve extended above 3,100 $\mathrm{ft}^{3} / \mathrm{s}$; minimum, $6 \mathrm{ft} / \mathrm{s}$ Aug. 10, 12-29, Sept. 1-4, 1940.

STATISTICAL SUMMARIES

MONTHLY AND ANNUAL MEAN DISCHARGES 1938-56

\begin{tabular}{|c|c|c|c|c|c|c|}
\hline MONTH & $\begin{array}{l}\text { MINIMUM } \\
\text { (CFS) }\end{array}$ & $\begin{array}{l}\text { MAXIMUM } \\
\text { (CFS) }\end{array}$ & $\begin{array}{l}\text { MEAN } \\
\text { (CFS) }\end{array}$ & $\begin{array}{l}\text { STAN- } \\
\text { DARD } \\
\text { DEVIA- } \\
\text { TION } \\
\text { (CFS) }\end{array}$ & $\begin{array}{l}\text { COEFFI- } \\
\text { CIENT OF } \\
\text { VARI- } \\
\text { ATION }\end{array}$ & $\begin{array}{c}\text { PERCENT } \\
\text { OF } \\
\text { ANNUAL } \\
\text { RUNOFF }\end{array}$ \\
\hline OCTOBER & 18 & 128 & 35 & 24 & .68 & 1.1 \\
\hline NOVEMBER & 20 & 323 & 73 & 71 & .98 & 2.2 \\
\hline DECEMBER & 17 & 467 & 145 & 139 & .96 & 4.4 \\
\hline JANUARY & 25 & 301 & 118 & 89 & .76 & 3.6 \\
\hline FEBRUARY & 29 & 493 & 200 & 134 & .67 & 6.1 \\
\hline MARCH & 72 & 841 & 455 & 200 & .44 & 13.9 \\
\hline APRIL & 387 & 1590 & 878 & 322 & .37 & 26.9 \\
\hline MAY & 230 & 1922 & 788 & 423 & .54 & 24.1 \\
\hline JUNE & 48 & 1096 & 420 & 282 & .67 & 12.9 \\
\hline JULY & 13 & 246 & 98 & 65 & .66 & 3.0 \\
\hline AUGUST & 7.0 & 55 & 28 & 14 & .50 & .9 \\
\hline SEP TEMBER & 14 & 71 & 25 & 12 & .49 & .8 \\
\hline ANNUAL & 129 & 478 & 272 & 100 & .37 & 100 \\
\hline
\end{tabular}

MAGN ITUDE AND PROBABILITY OF INSTANTANEOUS PEAK FLOW BASED ON PERIOD OF RECORD 1938-56

DISCHARGE, IN CFS, FOR INDICATED RECURRENCE INTERVAL, IN YEARS, AND ANNUAL EXCEEDANCE PROBABILITY, IN PERCENT

\begin{tabular}{ccccccc}
1.25 & 2 & 5 & 10 & 25 & 50 & 100 \\
$80 \%$ & $50 \%$ & $20 \%$ & $10 \%$ & $4 \%$ & $2 \%$ & $1 \%$ \\
\hline 1620 & 2190 & 3010 & 3590 & 4340 & - & - \\
\hline
\end{tabular}

WEIGHTED SKEW $=.187$
MAGNITUDE AND PROBABILITY OF ANNUAL LOW FLOW BASED ON PERIOD OF RECORD $1939-56$

\begin{tabular}{|c|c|c|c|c|c|c|}
\hline $\begin{array}{l}\text { PERIOD } \\
\text { (CON- }\end{array}$ & \multicolumn{6}{|c|}{$\begin{array}{l}\text { DISCHARGE, IN CFS, FOR INDICATED RECURRENCE } \\
\text { INTERVAL, IN YEARS, AND ANNUAL NON- } \\
\text { EXCEEDANCE PROBABILITY, IN PERCENT }\end{array}$} \\
\hline SECU- & & - & -...- & $\cdots$ & -- & -- \\
\hline TIVE & 2 & 5 & 10 & 20 & 50 & 100 \\
\hline DAYS) & $50 \%$ & $20 \%$ & $10 \%$ & $5 \%$ & $2 \%$ & $1 \%$ \\
\hline 1 & 14 & 9.7 & 8. & 6.9 & - & -- \\
\hline 3 & 14 & 9.9 & 8. & 7.0 & -- & -- \\
\hline 7 & 15 & 11 & 8. & 7.1 & -- & $-\rightarrow$ \\
\hline 14 & 17 & 11 & 9. & 7.5 & -- & -- \\
\hline 30 & 18 & 12 & 9. & 8.1 & -- & -- \\
\hline 60 & 21 & 15 & 12 & 11 & - & -- \\
\hline 90 & 23 & 17 & 15 & 14 & -- & -- \\
\hline 120 & 26 & 21 & 19 & 18 & -- & -- \\
\hline 183 & 42 & 27 & 23 & 20 & -- & -- \\
\hline
\end{tabular}

MAGNITUDE AND PROBABILITY OF ANNUAL HIGH FLOW BASED ON PERIOD OF RECORD 1938-56

\begin{tabular}{|c|c|c|c|c|c|c|}
\hline $\begin{array}{c}\text { PERIOD } \\
\text { (CON- } \\
\text { SECU- }\end{array}$ & \multicolumn{6}{|c|}{$\begin{array}{c}\text { DISCHARGE, IN CFS, FOR INDICATED RECURRENCE } \\
\text { INTERVAL, IN YEARS, AND ANNUAL } \\
\text { EXCEEDANCE PROBABILITY, IN PERCENT }\end{array}$} \\
\hline TIVE & 2 & 5 & 10 & 25 & 50 & 100 \\
\hline DAYS) & $50 \%$ & $20 \%$ & 103 & 4.0 & $2 \%$ & $1 \%$ \\
\hline 1 & 1700 & 2310 & 2720 & 3250 & -- & -- \\
\hline 3 & 1500 & 2060 & 2470 & 3020 & -- & -- \\
\hline 7 & 1320 & 1820 & 2190 & 2690 & -- & - \\
\hline 15 & 1150 & 1580 & 1870 & 2230 & -- & -- \\
\hline 30 & 981 & 1350 & 1610 & 1960 & -- & -- \\
\hline 60 & 860 & 1170 & 1390 & 1670 & -- & -- \\
\hline 90 & 731 & 991 & 1170 & 1390 & -- & -- \\
\hline
\end{tabular}

DURATION TABLE OF DAILY MEAN FLOW FOR PERIOD OF RECORD 1938-56

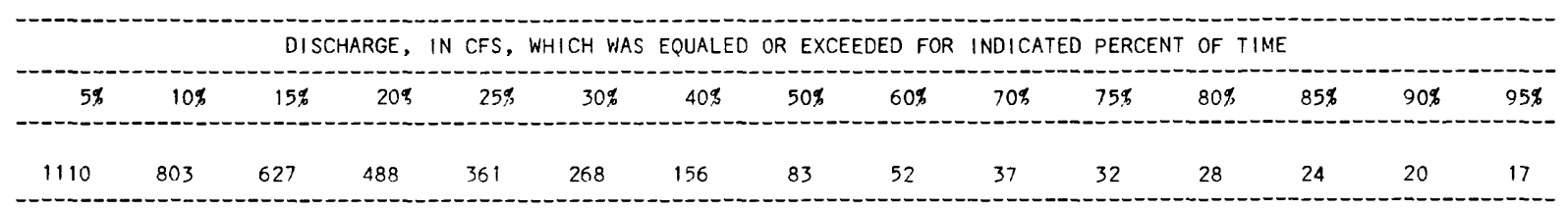




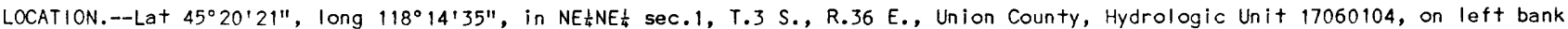

$8.8 \mathrm{mi}$ northwest of La Grande, $1.6 \mathrm{mi}$ upstream from Fivepoint Creek, and at $\mathrm{mile} 171.3$.

DRAINAGE AREA. $--555 \mathrm{mi}^{2}$.

PERIOD OF RECORD.--October 1966 to September 1982.

GAGE.--Water-stage recorder. Datum of gage is 2,993.62 ft National Geodetic Vertical Datum of 1929.

REMARKS.--SIight regulation by city of La Grande reservoir on Beaver Creek, capacity, about 900 acre-ft. Diversions for irrigation above station. Since 1909, city of La Grande has diverted about $3 \mathrm{ft} / \mathrm{s}$ from Beaver Creek above station for domestic water

supply.

AVERAGE DISCHARGE. --16 years, $299 \mathrm{ft}^{3} / \mathrm{s}, 216,600$ acre- $\mathrm{ft} / \mathrm{yr}$.

EXTREMES FOR PERIOD OF RECORD.--Maximum discharge, $4,740 \mathrm{ft}+3 / \mathrm{s} \mathrm{Mar} .13,1972$, gage height, $7.18 \mathrm{ft}$; maximum gage height, $12.25 \mathrm{ft}$ Jan. 15, 1974 (ice jam); minimum discharge, $9.6 \mathrm{ft}^{3} / \mathrm{s}$ Aug. 17, 18, 23, 1973

EXTREMES DUTSIDE PERIOD OF RECORD.--Flood of Jan. 30, 1965, reached a stage of about $9+\mathrm{ft}$, from $f$ loodmark, discharge not determined.

STATISTICAL SUMMARIES

MONTHLY AND ANNUAL MEAN DISCHARGES 1967-82

\begin{tabular}{|c|c|c|c|c|c|c|}
\hline MONTH & $\begin{array}{l}\text { MINIMUM } \\
\text { (CFS) }\end{array}$ & $\begin{array}{l}\text { MAXIMUM } \\
\text { (CFS) }\end{array}$ & $\begin{array}{l}\text { MEAN } \\
\text { (CFS) }\end{array}$ & $\begin{array}{l}\text { STAN- } \\
\text { DARD } \\
\text { DEVIA- } \\
\text { TION } \\
\text { (CFS) }\end{array}$ & $\begin{array}{l}\text { COEFFI- } \\
\text { CIENT OF } \\
\text { VARI- } \\
\text { ATION }\end{array}$ & $\begin{array}{c}\text { PERCENT } \\
\text { OF } \\
\text { ANNUAL } \\
\text { RUNOFF }\end{array}$ \\
\hline OCTOBER & 25 & 56 & 36 & 8.6 & .24 & 1.0 \\
\hline NOVEMBER & 31 & 236 & 71 & 56 & .79 & 2.0 \\
\hline DECEMBER & 33 & 481 & 159 & 143 & .90 & 4.4 \\
\hline JAMUARY & 27 & 681 & 272 & 197 & .72 & 7.5 \\
\hline FEBRUARY & 49 & 521 & 288 & 133 & .46 & 7.9 \\
\hline MARCH & 75 & 1668 & 544 & 374 & .69 & 15.0 \\
\hline APRIL & 181 & 1481 & 787 & 389 & .49 & 21.7 \\
\hline MAY & 256 & 1340 & 882 & 333 & .38 & 24.3 \\
\hline JUNE & 85 & 825 & 421 & 229 & .54 & 11.6 \\
\hline JULY & 22 & 253 & 101 & 59 & .58 & 2.8 \\
\hline AUGUST & 12 & 81 & 36 & 17 & .47 & 1.0 \\
\hline SEPTEMBER & 17 & 51 & 31 & 10.0 & .32 & .9 \\
\hline ANNUAL & 122 & 454 & 302 & 99 & .33 & 100 \\
\hline
\end{tabular}

MAGNITUDE AND PROBABILITY OF INSTANTANEOUS PEAK FLOW BASED ON PERIOD OF RECORD 1967-81

DISCHARGE, IN CFS, FOR INDICATED RECURRENCE INTERVAL, IN YEARS, AND ANNUAL EXCEEDANCE PROBABILITY, IN PERCENT

\begin{tabular}{ccccccc}
1.25 & 2 & 5 & 10 & 25 & 50 & 100 \\
$80 \%$ & $50 \%$ & $20 \%$ & $10 \%$ & $4 \%$ & $2 \%$ & $1 \%$ \\
1730 & 2360 & 3220 & 3790 & 4520 & - & - \\
\hline WEIGHTED SKEW & & 040 &
\end{tabular}

MAGNITUDE AND PROBABILITY OF ANNUAL LOW FLOW BASED ON PERIOD OF RECORO 1968-81

\begin{tabular}{|c|c|c|c|c|c|c|}
\hline $\begin{array}{l}\text { PERIOD } \\
\text { (CON- } \\
\text { SECU- }\end{array}$ & \multicolumn{6}{|c|}{$\begin{array}{l}\text { DISCHARGE, IN CFS, FOR INDICATED RECURRENCE } \\
\text { INTERVAL, IN YEARS, AND ANNUAL NON- } \\
\text { EXCEEDANCE PROBABILITY, IN PERCENT }\end{array}$} \\
\hline $\begin{array}{l}\text { TIVE } \\
\text { DAYS) }\end{array}$ & $\begin{array}{c}2 \\
50 \%\end{array}$ & $\begin{array}{c}5 \\
20 \%\end{array}$ & $\begin{array}{l}10 \\
10 \%\end{array}$ & $\begin{array}{l}20 \\
5 \%\end{array}$ & $\begin{array}{l}50 \\
2 \%\end{array}$ & $\begin{array}{r}100 \\
1 \%\end{array}$ \\
\hline 1 & 18 & 14 & 12 & 11 & -- & -- \\
\hline 3 & 19 & 15 & 13 & 11 & - & -- \\
\hline 7 & 21 & 16 & 13 & 11 & - & -- \\
\hline 14 & 22 & 17 & 14 & 12 & -- & - \\
\hline 30 & 24 & 19 & 16 & 14 & - & - \\
\hline 60 & 29 & 22 & 19 & 16 & - & -- \\
\hline 90 & 31 & 24 & 21 & 18 & - & - \\
\hline 120 & 33 & 26 & 23 & 21 & -- & -- \\
\hline 183 & 52 & 40 & 35 & 32 & - & -- \\
\hline
\end{tabular}

MAGNITUDE AND PROBABILITY OF ANNUAL HIGH FLOW BASED ON PERIOD OF RECORD 1967-81

\begin{tabular}{|c|c|c|c|c|c|c|}
\hline $\begin{array}{l}\text { PERIOD } \\
\text { (CON- } \\
\text { SECU- }\end{array}$ & \multicolumn{6}{|c|}{$\begin{array}{c}\text { DISCHARGE, IN CFS, FOR ; NDICATED RECURRENCE } \\
\text { INTERVAL, IN YEARS, AND ANNUAL } \\
\text { EXCEEDANCE PROBABILITY, IN PERCENT }\end{array}$} \\
\hline TIVE & 2 & 5 & 10 & 25 & 50 & 100 \\
\hline DAYS) & $50 \%$ & $20 \%$ & $10 \%$ & $4 \%$ & $2 \%$ & $1 \%$ \\
\hline 1 & 2000 & 2650 & 3110 & 3720 & -- & -- \\
\hline 3 & 1790 & 2330 & 2690 & 3130 & -- & - \\
\hline 7 & 1500 & 1970 & 2290 & 2700 & -- & -- \\
\hline 15 & 1260 & 1690 & 1960 & 2290 & - & -- \\
\hline 30 & 1080 & 1450 & 1650 & 1870 & - & -- \\
\hline 60 & 914 & 1230 & 1380 & 1540 & -- & -- \\
\hline 90 & 801 & 1080 & 1210 & 1340 & -- & -- \\
\hline
\end{tabular}

DURATION TABLE OF DAILY MEAN FLOW FOR PERIOD OF RECORD 1967-81

\begin{tabular}{|c|c|c|c|c|c|c|c|c|c|c|c|c|c|c|}
\hline $5 \%$ & $10 \%$ & $15 \%$ & $20 \%$ & $25 \%$ & $30 \%$ & $40 \%$ & $50 \%$ & $60 \%$ & $70 \%$ & $75 \%$ & $80 \%$ & $85 \%$ & $90 \%$ & $95 \%$ \\
\hline 1200 & 903 & 683 & 524 & 394 & 317 & 202 & 114 & 65 & 45 & 39 & 34 & 30 & 26 & 21 \\
\hline
\end{tabular}


13319000 GRANDE RONDE RIVER AT LA GRANDE, OR

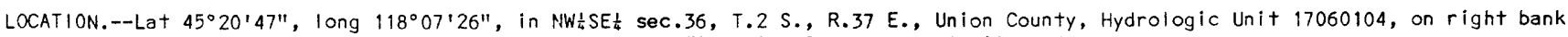
$1.8 \mathrm{mi}$ northwest of La Grande, $5.7 \mathrm{mi}$ downstream from Fivepoint Creek, and at mile 164.0 .

DRAINAGE AREA. $--678 \mathrm{mi}^{2}$.

PERIOD OF RECORD. - October 1903 to September 1915. February 1918 to September 1923, October 1925 to September 1982. Monthly discharge only for some periods, published in WSP 1317. Published as "at Hilgard" $1903-15$.

GAGE.--llater-stage recorder. Datum of gage is 2,826.25 $\mathrm{ft}$ National Geodetic Vertical Datum of 1929. Nov. 6, 1903, to Sept. 30, 1915, nonrecording gage at site $5.5 \mathrm{mi}$ upstream at various datums. Feb. 16, 1918, to June 28 , 1923, and Oct. 1, 1925, to Nov. 23, 1931, nonrecording gage at site $0.7 \mathrm{mi}$ downstream at various datums. Nov. 24, 1931, to Oct. 8, 1965, water-stage recorder at site $0.3 \mathrm{mi}$ upstream at datum $4.61 \mathrm{ft}$ higher.

REMARKS.--Since 1915, slight regulation by city of La Grande reservoir on Beaver Creek, capacity, about 900 acre-ft. Diversions for

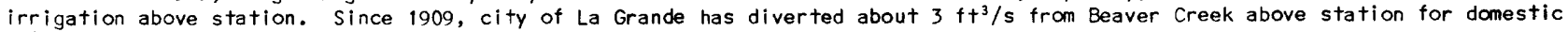
water supply.

AVERAGE DISCHARGE. -74 years, $384 \mathrm{ft} / \mathrm{s}, 278,200$ acre- $f+\mathrm{yr}$.

EXTREMES FOR PERIOD OF RECORD.--Maximum discharge, 14,100 $\mathrm{ft}^{3} / \mathrm{s}$ Jan. 30,1965 , gage height, $11.44 \mathrm{ft}$, site and datum then in use, from rating curve extended above $7,200 \mathrm{ft}^{3} / \mathrm{s}$; minimum, $3.9 \mathrm{ft}^{3} / \mathrm{s}$ Aug. $26,1940$.

\section{STATISTICAL SUMMARIES}

MONTHLY AND ANNUAL MEAN DISCHARGES 1904-82

\begin{tabular}{|c|c|c|c|c|c|c|}
\hline MONTH & $\begin{array}{l}\text { MINIMUM } \\
\text { (CFS) }\end{array}$ & $\begin{array}{c}\text { MAXIMUM } \\
\text { (CFS) }\end{array}$ & $\begin{array}{l}\text { MEAN } \\
\text { (CFS) }\end{array}$ & $\begin{array}{l}\text { STAN- } \\
\text { DARD } \\
\text { DEVIA- } \\
\text { TION } \\
\text { (CFS) }\end{array}$ & $\begin{array}{l}\text { COEFFI- } \\
\text { CIENT OF } \\
\text { VARI- } \\
\text { ATION }\end{array}$ & $\begin{array}{c}\text { PERCENT } \\
\text { OF } \\
\text { ANNUAL } \\
\text { RUNOFF }\end{array}$ \\
\hline OCTOBER & 16 & 145 & 42 & 24 & .57 & .9 \\
\hline NOVEMBER & 22 & 700 & 93 & 105 & 1.13 & 2.0 \\
\hline DECEMBER & 20 & 669 & 178 & 178 & 1.00 & 3.9 \\
\hline JANUARY & 12 & 1295 & 218 & 237 & 1.09 & 4.7 \\
\hline FEBRUARY & 25 & 1379 & 334 & 302 & .90 & 7.3 \\
\hline MARCH & 83 & 3700 & 757 & 521 & .69 & 16.4 \\
\hline APRIL & 237 & 3372 & 1291 & 589 & .46 & 28.0 \\
\hline MAY & 118 & 2675 & 1068 & 551 & .52 & 23.2 \\
\hline JUNE & 58 & 1328 & 459 & 285 & .62 & 10.0 \\
\hline JULY & 18 & 600 & 104 & 90 & .86 & 2.3 \\
\hline AUGUST & 7.0 & 88 & 31 & 17 & .54 & .7 \\
\hline SEPTEMBER & 8.8 & 82 & 30 & 14 & .47 & .6 \\
\hline ANNUAL & 141 & 706 & 381 & 145 & .38 & 100 \\
\hline
\end{tabular}

MAGNITUDE AND PROBABILITY OF INSTANTANEOUS PEAK FLOW BASED ON PERIOD OF RECORD 1904-82

DISCHARGE, IN CFS, FOR INDICATED RECURRENCE INTERVAL, IN YEARS, AND ANNUAL EXCEEDANCE PROBABILITY, IN PERCENT

\begin{tabular}{ccccccc}
1.25 & 2 & 5 & 10 & 25 & 50 & 100 \\
$80 \%$ & $50 \%$ & $20 \%$ & $10 \%$ & $4 \%$ & $2 \%$ & $1 \%$ \\
\hline 2150 & 3180 & 4800 & 6000 & 7660 & 8990 & 10400 \\
\hline
\end{tabular}

WEIGHTED SKEW $=.174$
MAGNITUDE AND PROBABILITY OF ANNUAL LOW FLOW BASED ON PERIOD OF RECORD 1905-82

\begin{tabular}{|c|c|c|c|c|c|c|}
\hline \multirow{2}{*}{$\begin{array}{l}\text { PERIOD } \\
\text { (CON- } \\
\text { SECU- } \\
\text { TIVE } \\
\text { DAYS) }\end{array}$} & \multicolumn{6}{|c|}{$\begin{array}{l}\text { DISCHARGE, IN CFS, FOR INDICATED RECURRENCE } \\
\text { INTERVAL, IN YEARS, AND ANNUAL NON- } \\
\text { EXCEEDANCE PROBABILITY, IN PERCENT }\end{array}$} \\
\hline & $\begin{array}{c}2 \\
50 \%\end{array}$ & $\begin{array}{c}5 \\
20 \%\end{array}$ & $\begin{array}{l}10 \\
10 \%\end{array}$ & $\begin{array}{l}20 \\
5 \%\end{array}$ & $\begin{array}{l}50 \\
2 \%\end{array}$ & $\begin{array}{r}100 \\
1 \%\end{array}$ \\
\hline 1 & 15 & 9.8 & 7.3 & 5.7 & 4.1 & 3.2 \\
\hline 3 & 16 & 10 & 7.7 & 5.9 & 4.3 & 3.4 \\
\hline 7 & 17 & 11 & 8.7 & 6.8 & 5.1 & 4.1 \\
\hline 14 & 19 & 12 & 9.7 & 7.8 & 5.9 & 4.9 \\
\hline 30 & 21 & 14 & 11 & 9.2 & 7.2 & 6.0 \\
\hline 60 & 25 & 18 & 15 & 12 & 9.9 & 8.6 \\
\hline 90 & 28 & 21 & 17 & 15 & 13 & 11 \\
\hline 120 & 32 & 24 & 21 & 19 & 17 & 16 \\
\hline 183 & 52 & 34 & 28 & 24 & 20 & 18 \\
\hline
\end{tabular}

MAGNITUDE AND PROBABILITY OF ANNUAL HIGH FLOW BASED ON PERIOD OF RECORD 1904-82

\begin{tabular}{|c|c|c|c|c|c|c|}
\hline \multirow{4}{*}{$\begin{array}{l}\text { PERIOD } \\
\text { (CON- } \\
\text { SECU- } \\
\text { TIVE } \\
\text { DAYS) }\end{array}$} & \multicolumn{6}{|c|}{$\begin{array}{c}\text { DISCHARGE, IN CFS, FOR INDICATED RECURRENCE } \\
\text { INTERVAL, IN YEARS, AND ANNUAL } \\
\text { EXCEEDANCE PROBABILITY, IN PERCENT }\end{array}$} \\
\hline & & 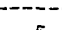 & 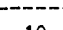 & - & - & \\
\hline & 2 & 5 & 10 & 25 & 50 & 100 \\
\hline & $50 \%$ & $20 \%$ & $10 \%$ & $4 \%$ & $2 \%$ & $1 \%$ \\
\hline 1 & 2720 & 4120 & 5110 & 6440 & 7480 & 8560 \\
\hline 3 & 2380 & 3520 & 4320 & 5370 & 6180 & 7010 \\
\hline 7 & 2040 & 2920 & 3490 & 4200 & 4720 & 5240 \\
\hline 15 & 1710 & 2420 & 2870 & 3430 & 3830 & 4220 \\
\hline 30 & 1440 & 2050 & 2450 & 2950 & 3310 & 3670 \\
\hline 60 & 1220 & 1720 & 2040 & 2420 & 2690 & 2960 \\
\hline 90 & 1050 & 1460 & 1720 & 2020 & 2240 & 2440 \\
\hline
\end{tabular}

DURATION TABLE OF DAILY MEAN FLOW FOR PERIOD OF RECORD 1904-82

DISCHARGE, IN CFS, WHICH WAS EQUALED OR EXCEEDED FOR INDICATED PERCENT OF TIME

\begin{tabular}{|c|c|c|c|c|c|c|c|c|c|c|c|c|c|c|}
\hline $5 \%$ & $10 \%$ & $15 \%$ & $20 \%$ & $25 \%$ & $30 \%$ & $40 \%$ & $50 \%$ & $60 \%$ & $70 \%$ & $75 \%$ & $80 \%$ & $85 \%$ & $90 \%$ & $95 \%$ \\
\hline 1650 & 1150 & 852 & 650 & 495 & 365 & 193 & 103 & 62 & 43 & 38 & 33 & 28 & 23 & 18 \\
\hline
\end{tabular}




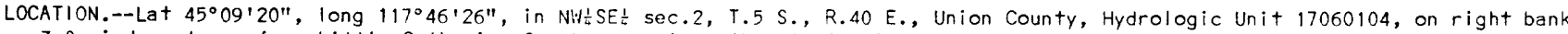
$3.0 \mathrm{mi}$ downstream from Little Catherine Creek, $5.5 \mathrm{mi}$ southeast of Union, and at mile 25.4 .

DRAINAGE AREA. $--105 \mathrm{mi}^{2}$.

PERIOD OF RECORD.--May 1906 to May 1007 (gage heights on!y). August 1911 to December 1912 , March to September 1915 , February 1918 to September 1919, October 1925 to September 1092. Monthly discharge only for some periods, published in ivSP 1317 .

GAGE.--Water-stage recorder. Datum of gage is 3,081.75 ft National Geodetic Vertical Datum of 1929 (Oregon State Highway Department bench mark). Prior to Nov. 28, 1939, nonrecording gage at several sites within $1.8 \mathrm{mi}$ of present site at various datums. Nov. 28,1938 , to May 16, 1939, water-stage recorder at site $400 \mathrm{ft}$ downstream at datum $4.29 \mathrm{ft}$ lower.

REMARKS.--No regulation. Several small diversions for irrigation above station. Since 1937, diversion to Big Creek in Powder River basin provides a small part of the water used for irrigation in that basin.

AVERAGE DISCHARGE.--59 years (water years $1912,1919,1926-82$ ) , $118 \mathrm{ft} 3 / \mathrm{s}, 85,490 \mathrm{acre}-\mathrm{ft} / \mathrm{yr}$.

EXTREMES FOR PERIOD OF RECORD.--Maximum discharge, 1,740 ft3/s May 27,1948 , gage height, $4.57 \mathrm{ft}$; $\mathrm{minimum,} 6.5 \mathrm{ft}{ }^{3} / \mathrm{s}$ Feb. 4 , 1955 , result of treezeup; minimum daily, $8 \mathrm{ft}^{3} / \mathrm{s}$ Nov. $7,1925$.

STATISTICAL SUMMARIES (BEFORE CONSTRUCTION OF BIG CREEK DIVERSION)

MONTHLY AND ANNUAL MEAN DISCHARGES 1912-36

\begin{tabular}{|c|c|c|c|c|c|c|}
\hline MONTH & $\begin{array}{l}\text { MINIMUM } \\
\text { (CFS) }\end{array}$ & $\begin{array}{l}\text { MAXIMUM } \\
\text { (CFS) }\end{array}$ & $\begin{array}{l}\text { MEAN } \\
\text { (CFS) }\end{array}$ & $\begin{array}{l}\text { STAN- } \\
\text { DARD } \\
\text { DEVIA- } \\
\text { TION } \\
\text { (CFS) }\end{array}$ & $\begin{array}{l}\text { COEFFI- } \\
\text { CIENT OF } \\
\text { VARI- } \\
\text { ATION }\end{array}$ & $\begin{array}{c}\text { PERCENT } \\
\text { OF } \\
\text { ANNUAL } \\
\text { RUNOFF }\end{array}$ \\
\hline OCTOBER & 21 & 103 & 34 & 21 & .60 & 2.4 \\
\hline NOVEMBER & 14 & 157 & 41 & 35 & .86 & 2.9 \\
\hline DECEMBER & 13 & 105 & 37 & 23 & .62 & 2.6 \\
\hline JANUARY & 12 & 95 & 38 & 22 & .59 & 2.7 \\
\hline FEBRUARY & 22 & 74 & 42 & 18 & .42 & 3.0 \\
\hline MARCH & 51 & 203 & 95 & 48 & .50 & 6.7 \\
\hline APRIL & 158 & 375 & 268 & 60 & .23 & 19.0 \\
\hline MAY & 139 & 776 & 440 & 194 & .44 & 31.1 \\
\hline JUNE & 66 & 632 & 289 & 194 & .57 & 20.4 \\
\hline JULY & 26 & 145 & 76 & 44 & .58 & 5.4 \\
\hline AUGUST & 11 & 63 & 29 & 13 & .44 & 2.1 \\
\hline SEPTEMBER & 16 & 50 & 26 & 9.4 & .37 & 1.8 \\
\hline ANNUAL & 67 & 185 & 116 & 39 & .34 & 100 \\
\hline
\end{tabular}

MAGN I TUDE AND PROBABILITY OF INSTANTANEOUS PEAK FLOW BASED ON PERIOD OF RECORD 1912-82

DISCHARGE, IN CFS, FOR INDICATED RECURRENCE INTERVAL, IN YEARS, AND ANNUAL EXCEEDANCE PROBABILITY, IN PERCENT

\begin{tabular}{ccccccc}
1.25 & 2 & 5 & 10 & 25 & 50 & 100 \\
$80 \%$ & $50 \%$ & $20 \%$ & $10 \%$ & $4 \%$ & $2 \%$ & $1 \%$ \\
\hline 563 & 757 & 1000 & 1160 & 1340 & 1470 & 1590 \\
\hline
\end{tabular}

WEIGHTED SKEW $=-.191$
MAGNITUDE AND PROBABILITY OF ANNUAL LOW FLOW BASED ON PERIOD OF RECORD 1919-36

\begin{tabular}{|c|c|c|c|c|c|c|}
\hline $\begin{array}{l}\text { PERIOD } \\
\text { ICON- } \\
\text { SECU- }\end{array}$ & \multicolumn{6}{|c|}{$\begin{array}{l}\text { DISCHARGE, IN CFS, FOR INDICATED RECURRENCE } \\
\text { INTERVAL, IN YEARS, AND ANNUAL NON- } \\
\text { EXCEEDANCE PROBABILITY, IN PERCENT }\end{array}$} \\
\hline TIVE & 2 & 5 & 10 & 20 & 50 & 100 \\
\hline DAYS) & $50 \%$ & $20 \%$ & $10 \%$ & $5 \%$ & $2 q$ & $1 \%$ \\
\hline 1 & 17 & 13 & 11 & 10 & -- & -- \\
\hline 3 & 17 & 13 & 11 & 10 & - & - \\
\hline 7 & 18 & 13 & 12 & 10 & -- & -- \\
\hline 14 & 18 & 14 & 12 & 10 & -- & - \\
\hline 30 & 20 & 15 & 13 & 11 & -- & -- \\
\hline 60 & 22 & 17 & 15 & 14 & -- & -- \\
\hline 90 & 22 & 18 & 16 & 15 & -- & - \\
\hline 120 & 24 & 20 & 18 & 18 & -- & -- \\
\hline 183 & 27 & 21 & 19 & 18 & -- & -- \\
\hline
\end{tabular}

MAGNITUDE AND PROBABILITY OF ANNUAL HIGH FLOW BASED ON PERIOD OF RECORD 1912-36

\begin{tabular}{|c|c|c|c|c|c|c|}
\hline $\begin{array}{l}\text { PERIOD } \\
\text { (CON- } \\
\text { SFCI- }\end{array}$ & \multicolumn{6}{|c|}{$\begin{array}{c}\text { DISCHARGE, IN CFS, FOR INDICATED RECURRENCE } \\
\text { INTERVAL, IN YEARS, AND ANNUAL } \\
\text { EXCEEDANCE PROBABILITY, IN PERCENT }\end{array}$} \\
\hline SECU- & & & & & -1 & \\
\hline TIVE & 2 & 5 & 10 & 25 & 50 & 100 \\
\hline DAYS) & $50 \%$ & 209 & $10 \%$ & $4 q$ & 28 & $1 \%$ \\
\hline 1 & 799 & 1090 & 1230 & 1380 & -- & -- \\
\hline 3 & 755 & 1050 & 1190 & 1340 & -- & -- \\
\hline 7 & 683 & 954 & 1100 & 1240 & -- & -- \\
\hline 15 & 582 & 809 & 938 & 1080 & -- & -- \\
\hline 30 & 500 & 692 & 808 & 943 & -- & -- \\
\hline 60 & 405 & 550 & 635 & 732 & -- & -- \\
\hline 90 & 326 & 441 & 511 & 596 & -- & -- \\
\hline
\end{tabular}

DURATION TABLE OF DAILY MEAN FLOW FOR PERIOD OF RECORD 1912-36

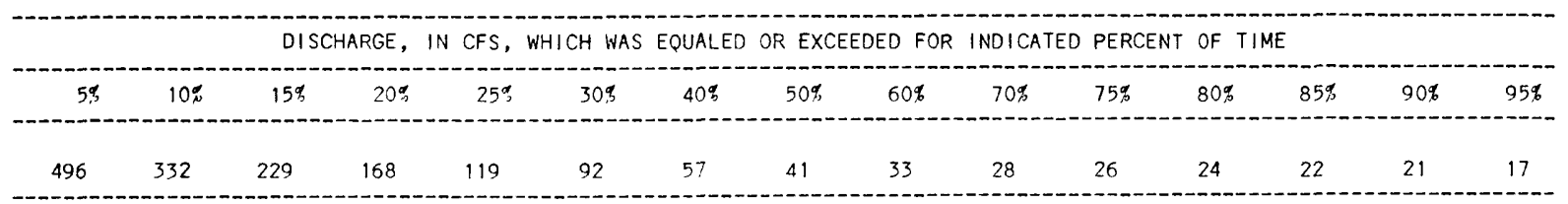


13320000 CATHERINE CREEK NEAR UNION, OR--Continued

STATISTICAL SUMMARIES (AFTER COMPLETION OF BIG CREEK DIVERSION)

MONTHLY AND ANNUAL MEAN DISCHARGES 1939-82

\begin{tabular}{|c|c|c|c|c|c|c|}
\hline MONTH & $\begin{array}{l}\text { MINIMUN } \\
\text { (CFS) }\end{array}$ & $\begin{array}{l}\text { MAXIMUM } \\
\text { (CFS) }\end{array}$ & $\begin{array}{l}\text { MEAN } \\
\text { (CFS) }\end{array}$ & $\begin{array}{l}\text { STAN- } \\
\text { DARD } \\
\text { DEVIA- } \\
\text { TION } \\
\text { (CFS) }\end{array}$ & $\begin{array}{l}\text { COEFFI- } \\
\text { CIENT OF } \\
\text { VARI- } \\
\text { ATION }\end{array}$ & $\begin{array}{c}\text { PERCENT } \\
\text { OF } \\
\text { ANNUAL } \\
\text { RUNOFF }\end{array}$ \\
\hline OCTOBER & 20 & 138 & 33 & 17 & .52 & 2.3 \\
\hline NOVEMBER & 22 & 100 & 39 & 15 & .39 & 2.7 \\
\hline DECEMBER & 20 & 125 & 48 & 24 & .51 & 3.3 \\
\hline JANUARY & 23 & 101 & 48 & 20 & .42 & 3.4 \\
\hline FEBRUARY & 23 & 135 & 60 & 27 & .45 & 4.1 \\
\hline MARCH & 28 & 213 & 90 & 40 & .44 & 6.3 \\
\hline APRIL & 83 & 445 & 230 & 93 & .40 & 16.0 \\
\hline MAY & 121 & 742 & 412 & 126 & .31 & 28.7 \\
\hline JUNE & 86 & 686 & 313 & 138 & .44 & 21.8 \\
\hline JULY & 34 & 226 & 96 & 44 & .45 & 6.7 \\
\hline AUGUST & 19 & 62 & 38 & 9.6 & .25 & 2.7 \\
\hline SEPTEMBER & 18 & 58 & 31 & 7.5 & .24 & 2.1 \\
\hline ANNUAL & 49 & 180 & 120 & 29 & .24 & 100 \\
\hline
\end{tabular}

MAGNITUDE AND PROBABILITY OF INSTANTANEOUS PEAK FLOW BASED ON PERIOD OF RECORD

\begin{tabular}{|c|c|c|c|c|c|c|}
\hline \multicolumn{7}{|c|}{$\begin{array}{l}\text { DISCHARGE, IN CFS, FOR INDICATED RECURPENCE INTERVAL, IN } \\
\text { YEARS, AND ANNUAL EXCEEDANCE PROBABILITY, IN PERCENT }\end{array}$} \\
\hline $\begin{array}{l}1.25 \\
808\end{array}$ & $\begin{array}{c}2 \\
50 \%\end{array}$ & $\begin{array}{c}5 \\
208\end{array}$ & $\begin{array}{l}10 \\
10 \%\end{array}$ & $\begin{array}{l}25 \\
4 \%\end{array}$ & $\begin{array}{l}50 \\
2 \%\end{array}$ & $\begin{array}{r}100 \\
19\end{array}$ \\
\hline -- & -- & - & - & - & - & -- \\
\hline
\end{tabular}

WEIGHTED SKEW =
MAGNITUDE AND PROBABILITY OF ANNUAL LOW FLOW BASED ON PERIOD OF RECORD 1939-82

\begin{tabular}{|c|c|c|c|c|c|c|}
\hline $\begin{array}{l}\text { PERIOD } \\
\text { ICON- } \\
\text { SECU- }\end{array}$ & \multicolumn{6}{|c|}{$\begin{array}{l}\text { DISCHARGE, IN CFS, FOR, INDICATED RECURRENCE } \\
\text { INTERVAL, IN YEARS, AND ANNUAL NON- } \\
\text { EXCEEDANCE PROBABILITY, IN PERCENT }\end{array}$} \\
\hline TIVE & 2 & 5 & 10 & 20 & 50 & 100 \\
\hline DAYS) & 50,3 & $20 \%$ & $10 \%$ & $5 \%$ & 2, & $1 \%$ \\
\hline 1 & 19 & 15 & 14 & 12 & 11 & 11 \\
\hline 3 & 20 & 17 & 16 & 15 & 14 & 13 \\
\hline 7 & 22 & 18 & 17 & 16 & 15 & 14 \\
\hline 14 & 23 & 20 & 18 & 17 & 16 & 16 \\
\hline 30 & 25 & 21 & 20 & 19 & 18 & 18 \\
\hline 60 & 27 & 23 & 22 & 21 & 20 & 20 \\
\hline 90 & 28 & 24 & 23 & 22 & 21 & 21 \\
\hline 120 & 30 & 26 & 24 & 23 & 22 & 22 \\
\hline 183 & 35 & 29 & 27 & 26 & 25 & 24 \\
\hline
\end{tabular}

MAGNITUDE AND PROBABILITY OF ANNUAL HIGH FLOW BASED ON PERIOD OF RECORD 1938-82

\begin{tabular}{|c|c|c|c|c|c|c|}
\hline $\begin{array}{l}\text { PERIOD } \\
\text { (CON- }\end{array}$ & \multicolumn{6}{|c|}{$\begin{array}{l}\text { DISCHARGE, IN CFS, FOR INDICATED RECURRE } \\
\text { INTERVAL, IN YEARS, AND ANNUAL } \\
\text { EXCEEDANCE PROBABILITY, IN PERCENT }\end{array}$} \\
\hline SECU- & & & & - & $\cdots$ & \\
\hline TIVE & 2 & 5 & 10 & 25 & 50 & 100 \\
\hline DAYS) & $50 \%$ & $20 \%$ & $10 \%$ & $4 \stackrel{q}{\prime}$ & $2 q$ & $1 \%$ \\
\hline 1 & 676 & 888 & 1020 & 1170 & 1270 & 1370 \\
\hline 3 & 647 & 849 & 965 & 1100 & 1190 & 1270 \\
\hline 7 & 598 & 787 & 894 & 1010 & 1090 & 1160 \\
\hline 15 & 532 & 695 & 784 & 880 & 941 & 996 \\
\hline 30 & 466 & 604 & 677 & 752 & 798 & 838 \\
\hline 60 & 397 & 498 & 546 & 591 & 616 & 636 \\
\hline 90 & 334 & 410 & 443 & 473 & 489 & 501 \\
\hline
\end{tabular}

DURATION TABLE OF DAILY MEAN FLOW FOR PERIOD OF RECORD 1938-82

DISCHARGE, IN CFS, WHICH WAS EQUALED OR EXCEEDED FOR INDICATED PERCENT OF TIME

\begin{tabular}{|c|c|c|c|c|c|c|c|c|c|c|c|c|c|c|}
\hline $5 \%$ & $10 \%$ & $15 \%$ & $20 \%$ & $25^{\circ}$ & $30 \%$ & $40 \%$ & $50 \%$ & $60 \%$ & $70 \%$ & $75 \%$ & $80 \%$ & $85 \%$ & $90 \%$ & $95 \$$ \\
\hline 455 & 334 & 258 & 191 & 136 & 105 & 68 & 51 & 42 & 35 & 32 & 30 & 28 & 26 & 23 \\
\hline
\end{tabular}




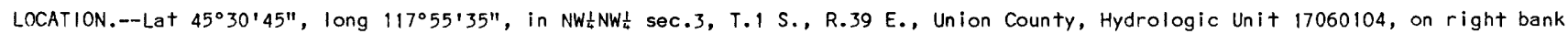
$700 \mathrm{ft}$ upstream from abandoned highway bridge, $1.5 \mathrm{mi}$ downstream from Willow Creek, $3.6 \mathrm{mi}$ south of Elgin, and at mile 104.2 .

DRA INAGE AREA.-- 1,250 $\mathrm{mi}^{2}$, approximately.

PERIOD OF RECORD.--August 1955 to September 1981.

GAGE.--Water-stage recorder. Datum of gage is 2,660.31 ft National Geodetic Vertical Datum of 1929 .

REMARKS.--No regulation. Many diversions for irrigation above station.

AVERAGE DISCHARGE.--26 years, $668 \mathrm{ft}^{3} / \mathrm{s}, 484,000$ acre $-\mathrm{ft} / \mathrm{yr}$.

EXTREMES FOR PERIOD OF RECORD.--Maximum discharge, 6,480 $\mathrm{ft}^{3} / \mathrm{s}$ Feb. 2, 1965, gage height, $13.79 \mathrm{ft}$; no $\mathrm{flow}$ Aug. $20-24$, 1973 .

EXTREMES OUTSIDE PERIOD OF RECORD.--Outstanding floods occurred in 1894 and 1917 , based on Corps of Engineers flood profiles. Flood

in May 1948 reached an elevation of $2,672.9 \mathrm{ft}$ on Corps of Engineers gage at bridge $700 \mathrm{ft}$ downstream, discharge, $5,690 \mathrm{ft} / \mathrm{s}$, result of discharge measurement.

STATISTICAL SUMMARIES

MONTHLY AND ANNUAL MEAN DISCHARGES

1956-81

\begin{tabular}{|c|c|c|c|c|c|c|}
\hline MONTH & $\begin{array}{l}\text { MINIMUM } \\
\text { (CFS) }\end{array}$ & $\begin{array}{l}\text { MAXIMUM } \\
\text { (CFS) }\end{array}$ & $\begin{array}{l}\text { MEAN } \\
\text { (CFS) }\end{array}$ & $\begin{array}{l}\text { STAN- } \\
\text { DARD } \\
\text { DEVIA- } \\
\text { TION } \\
\text { (CFS) }\end{array}$ & $\begin{array}{l}\text { COEFFI- } \\
\text { CIENT OF } \\
\text { VARI- } \\
\text { ATION }\end{array}$ & $\begin{array}{c}\text { PERCENT } \\
\text { OF } \\
\text { ANNUAL } \\
\text { RUNOFF }\end{array}$ \\
\hline OCTOBER & 31 & 325 & 87 & 55 & .63 & 1.1 \\
\hline NOVEMBER & 65 & 469 & 164 & 97 & .59 & 2.0 \\
\hline DECEMBER & 86 & 1176 & 398 & 333 & .84 & 5.0 \\
\hline J ANUARY & 90 & 1438 & 559 & 408 & .73 & 7.0 \\
\hline FEBRUARY & 106 & 2760 & 822 & 584 & .71 & 10.2 \\
\hline MARCH & 171 & 3605 & 1108 & 687 & .62 & 13.8 \\
\hline APRIL & 487 & 3395 & 1764 & 782 & .44 & 22.0 \\
\hline MAY & 439 & 3393 & 1879 & 792 & .42 & 23.4 \\
\hline JUNE & 144 & 1927 & 997 & 475 & .48 & 12.4 \\
\hline JULY & 11 & 629 & 175 & 136 & .78 & 2.2 \\
\hline AUGUST & 1.7 & 140 & 40 & 32 & .80 & .5 \\
\hline SEPTEMBER & 6.9 & 117 & 43 & 30 & .69 & .5 \\
\hline ANNUAL & 211 & 1100 & 668 & 254 & .38 & 100 \\
\hline
\end{tabular}

MAGNITUDE AND PROBABILITY OF INSTANTANEOUS PEAK FLOW BASED ON PERIOD OF RECORD 1956-81

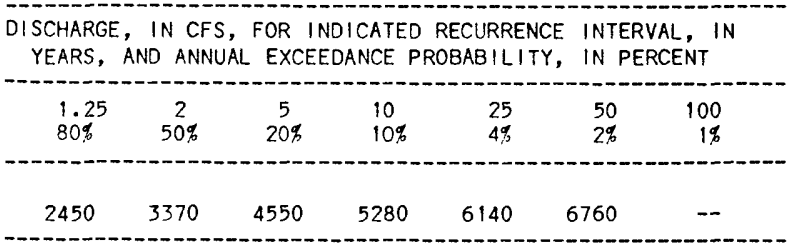

WEIGHTED SKEW $=-.234$
MAGNITUDE AND PROBABILITY OF ANNUAL LOW FLOW BASED ON PERIOD OF RECORD 1957-81

\begin{tabular}{|c|c|c|c|c|c|c|}
\hline \multirow{4}{*}{$\begin{array}{l}\text { PERIOD } \\
\text { (CON- } \\
\text { SECU- } \\
\text { TIVE } \\
\text { DAYS) }\end{array}$} & \multicolumn{6}{|c|}{$\begin{array}{l}\text { DISCHARGE, IN CFS, FOR INDICATED RECURRENCE } \\
\text { INTERVAL, IN YEARS, AND ANNUAL NON- } \\
\text { EXCEEDANCE PROBABILITY, IN PERCENT }\end{array}$} \\
\hline & \multicolumn{6}{|c|}{ 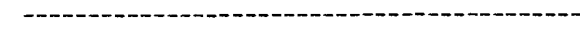 } \\
\hline & 2 & 5 & 10 & 20 & 50 & 100 \\
\hline & $50 \%$ & $20 \%$ & $10 \%$ & $5 \%$ & $2 \%$ & $1 \%$ \\
\hline & & & & & & \\
\hline 1 & -- & -- & -- & -- & -- & -- \\
\hline 3 & -- & -- & -- & - & -- & -- \\
\hline 7 & -- & -- & -- & -- & -- & -- \\
\hline 14 & 21 & 6.0 & 2.1 & .7 & .2 & -- \\
\hline 30 & 26 & 9.5 & 4.6 & 2.2 & .8 & -- \\
\hline 60 & 33 & 15 & 8.9 & 5.5 & 2.9 & -- \\
\hline 90 & 43 & 22 & 15 & 10 & 6.5 & -- \\
\hline 120 & 57 & 35 & 26 & 20 & 15 & -- \\
\hline 183 & 109 & 75 & 62 & 53 & 44 & -- \\
\hline
\end{tabular}

MAGNITUDE AND PROBABILITY OF ANNUAL HIGH FLOW BASED ON PERIOD OF RECORD 1956-81

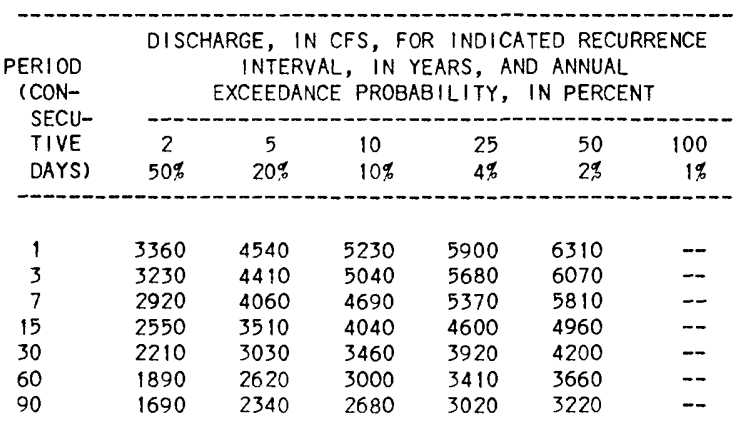

DURATION TABLE OF DAILY MEAN FLOW FOR PERIOD OF RECORD 1956-81

\begin{tabular}{|c|c|c|c|c|c|c|c|c|c|c|c|c|c|c|}
\hline $5 \%$ & $10 \%$ & $15 \%$ & $20 \%$ & $25 \%$ & $30 \%$ & $40 \%$ & $50 \%$ & $60 \%$ & $70 \%$ & $75 \%$ & $80 \%$ & $85 \%$ & $90 \%$ & $95 \%$ \\
\hline 2610 & 1950 & 1580 & 1250 & 963 & 790 & 480 & 255 & 144 & 98 & 82 & 64 & 45 & 30 & 15 \\
\hline
\end{tabular}


13323600 INDIAN CREEK NEAR IMBLER, OR

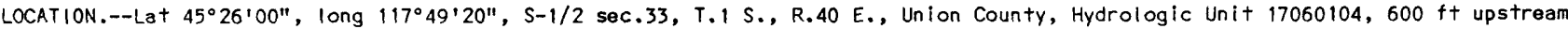
from North Fork and 7 mi southeast of imbler.

DRAINAGE AREA.--22 $\mathrm{mi}^{2}$, approximately.

PERIOD OF RECORD.--March 1938 to September 1950.

GAGE.--Water-stage recorder. Altitude of gage is $3,800 \mathrm{ft}$, from topographic map.

REMARKS.--No diversion or regulation above station.

COOPERATION.--Records for 1938-45 furnished by Oregon Water Resources Department.

AVERAGE DISCHARGE.--12 years (water years $1939-50$ ), $41.5 \mathrm{ft}^{3} / \mathrm{s}$.

EXTREMES FOR PERIOD OF RECORD.--Maximum discharge, $818 \mathrm{ft}^{3} / \mathrm{s}$ May 27,1948 , from rating curve extended above $400 \mathrm{ft} 3 / \mathrm{s}$; maximum gage height, $4.09 \mathrm{ft}$ sometime during period Jan. 4-7, 1947 (ice jam); minimum observed, $0.1 \mathrm{ft}{ }^{3} / \mathrm{s}$ Nov. 15 , 1939.

STATISTICAL SUMMARIES

MONTHIY AND ANNUAL MEAN DISCHARGES 1939-50

\begin{tabular}{|c|c|c|c|c|c|c|}
\hline MONTH & $\begin{array}{l}\text { MINIMUM } \\
\text { (CFS) }\end{array}$ & $\begin{array}{l}\text { MAXIMUM } \\
\text { (CFS) }\end{array}$ & $\begin{array}{l}\text { MEAN } \\
\text { (CFS) }\end{array}$ & $\begin{array}{l}\text { STAN- } \\
\text { DARD } \\
\text { DEVIA- } \\
\text { TION } \\
\text { (CFS) }\end{array}$ & $\begin{array}{l}\text { COEFFI- } \\
\text { CIENT OF } \\
\text { VARI- } \\
\text { ATION }\end{array}$ & $\begin{array}{c}\text { PERCENT } \\
\text { OF } \\
\text { ANNUAL } \\
\text { RUNOFF }\end{array}$ \\
\hline OCTOBER & 3.1 & 14 & 5.6 & 3.0 & .54 & 1.1 \\
\hline $\begin{array}{l}\text { NOVEMBER } \\
\text { OECEMBER }\end{array}$ & $\begin{array}{l}1.7 \\
2.1\end{array}$ & $\begin{array}{l}20 \\
39\end{array}$ & $11^{7.7}$ & $10^{4.9}$ & $\begin{array}{l}.63 \\
.93\end{array}$ & $\begin{array}{l}1.6 \\
2.2\end{array}$ \\
\hline $\begin{array}{l}\text { JANUARY } \\
\text { FEBRUARY }\end{array}$ & $\begin{array}{l}3.5 \\
4.0\end{array}$ & $\begin{array}{l}30 \\
35\end{array}$ & 8.5 & $\begin{array}{l}7.4 \\
8.4\end{array}$ & $\begin{array}{l}.86 \\
.84\end{array}$ & 1.7 \\
\hline MARCH & 6.0 & 29 & 15 & 7.5 & .49 & 3.1 \\
\hline APRIL & 19 & 140 & 66 & 35 & .53 & 13.2 \\
\hline $\begin{array}{l}\text { MAY } \\
\text { JUNE }\end{array}$ & $\begin{array}{r}107 \\
27\end{array}$ & $\begin{array}{l}316 \\
269\end{array}$ & $\begin{array}{l}189 \\
140\end{array}$ & $\begin{array}{l}60 \\
78\end{array}$ & $\begin{array}{l}.32 \\
.56\end{array}$ & $\begin{array}{l}38.1 \\
28.2\end{array}$ \\
\hline $\begin{array}{l}\text { JULY } \\
\text { AUGUST }\end{array}$ & $\begin{array}{l}6.3 \\
3.3\end{array}$ & $\begin{array}{l}95 \\
10\end{array}$ & $\begin{array}{l}32 \\
6.9\end{array}$ & $\begin{array}{r}24 \\
2.2\end{array}$ & $\begin{array}{l}.73 \\
.32\end{array}$ & $\begin{array}{l}6.5 \\
1.4\end{array}$ \\
\hline SEPTEMBER & 2.8 & 11 & 4.8 & 2.1 & .43 & 1.0 \\
\hline NNUAL & 23 & 56 & 42 & 11 & .27 & 100 \\
\hline
\end{tabular}

MAGNITUDE AND PROBABILITY OF INSTANTANEOUS PEAK FLOW BASED ON PERIOD OF RECORD 1939-50

DISCHARGE, IN CFS, FOR INDICATED RECURRENCE INTERVAL, IN YEARS, AND ANNUAL EXCEEDANCE PROBABILITY, IN PERCENT

\begin{tabular}{ccccccc}
1.25 & 2 & 5 & 10 & 25 & 50 & 100 \\
$80 \%$ & $50 \%$ & $20 \%$ & $10 \%$ & $4 \%$ & $2 \%$ & $1 \%$ \\
\hline 304 & 424 & 589 & 698 & - & - & - \\
\hline WEIGHTED SKEW $=$ & -.042 & &
\end{tabular}

MAGNITUDE AND PROBABILITY OF ANNUAL LOW FLOW BASED ON PERIOD OF RECORD 1940-50

\begin{tabular}{|c|c|c|c|c|c|c|}
\hline \multirow{3}{*}{$\begin{array}{l}\text { PERIOD } \\
\text { (CON- } \\
\text { SECU- } \\
\text { TIVE } \\
\text { DAYS) }\end{array}$} & \multicolumn{6}{|c|}{$\begin{array}{l}\text { DISCHARGE, IN CFS, FOR INDICATED RECURRENCE } \\
\text { INTERVAL, IN YEARS, AND ANNUAL NON- } \\
\text { EXCEEDANCE PROBABILITY, IN PERCENT }\end{array}$} \\
\hline & & 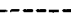 & - & - & -1 & \\
\hline & $\stackrel{2}{20 \%}$ & $\begin{array}{c}5 \\
20 \%\end{array}$ & $\begin{array}{l}10 \\
10 \%\end{array}$ & $\begin{array}{l}20 \\
5 \%\end{array}$ & $\begin{array}{l}50 \\
2 \%\end{array}$ & $\begin{array}{r}100 \\
1 \%\end{array}$ \\
\hline 1 & 28 & 18 & 94 & 12 & -- & -- \\
\hline 3 & $\begin{array}{l}2.0 \\
2.9\end{array}$ & 1.9 & 1.5 & 1.2 & - & - \\
\hline 7 & 3.1 & 2.0 & 1.5 & 1.2 & -- & -- \\
\hline 14 & 3.4 & 2.2 & 1.7 & 1.3 & - & -- \\
\hline 30 & 3.6 & 2.3 & 1.8 & 1.4 & -- & -- \\
\hline 60 & 3.9 & 2.8 & 2.4 & 2.1 & -- & -- \\
\hline 90 & 4.2 & 3.2 & 2.9 & 2.6 & -- & -- \\
\hline 120 & 4.7 & 3.5 & 3.1 & 2.8 & -- & - \\
\hline 183 & 6.1 & 4.2 & 3.6 & 3.2 & - & -- \\
\hline
\end{tabular}

MAGNITUDE AND PROBABILITY OF ANNUAL HIGH FLOW BASED ON PERIOD OF RECORD $1939-50$

\begin{tabular}{|c|c|c|c|c|c|c|}
\hline \multirow{3}{*}{$\begin{array}{l}\text { PERIOO } \\
\text { (CON- } \\
\text { SECU- } \\
\text { TIVE } \\
\text { DAYS) }\end{array}$} & \multicolumn{6}{|c|}{$\begin{array}{c}\text { DISCHARGE, IN CFS, FOR INDICATED RECURRENCE } \\
\text { INTERVAL, IN YEARS, AND ANNUAL } \\
\text { EXCEEDANCE PROBABILITY, IN PERCENT }\end{array}$} \\
\hline & 2 & 5 & 10 & 25 & 50 & 100 \\
\hline & $50 \%$ & $20 \%$ & $10 \%$ & $4 \%$ & $2 \%$ & $1 \%$ \\
\hline 1 & 369 & 501 & 591 & -- & -- & - \\
\hline 3 & 350 & 465 & 538 & -- & -- & -- \\
\hline 7 & 314 & 406 & 457 & -- & -- & -- \\
\hline 15 & 266 & 347 & 393 & - & - & - \\
\hline 30 & 227 & 298 & 339 & - & -- & - \\
\hline 60 & 178 & 222 & 243 & -- & -- & - \\
\hline 90 & 138 & 170 & 186 & -- & -- & - \\
\hline
\end{tabular}

DURATION TABLE OF DAILY MEAN FLOW FOR PERIOD OF RECORD 1939-50

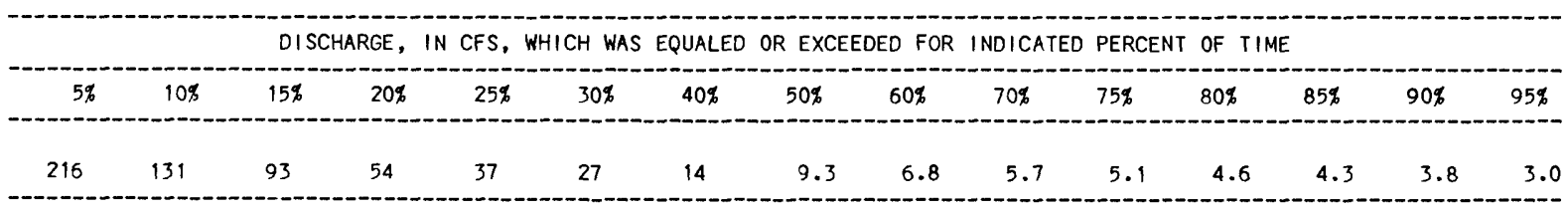




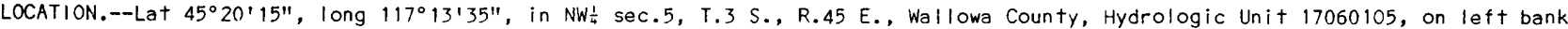
$0.2 \mathrm{mi}$ downstream from Wallowa lake dam, $1.1 \mathrm{mi}$ south of Joseph, and at mile 50.0 .

DRAINAGE AREA. $--50.9 \mathrm{mi}^{2}$.

PERIOD OF RECORD.--November 1903 to August 1907, June 1908 to March 1914, May to September 1915 , December 1926 to September 1982. Monthly discharge only for some periods, published in WSP 1317 . Published as "near Joseph" 1911.

GAGE.--Water-stage recorder. Datum of gage is 4,326.86 $\mathrm{ft}$ National Geodetic vertical Datum of 1929. Nov. 12, 1903, to Sept. 25 , 1915, nonrecording gage at several sites at lake outlet or near present site at different datums.

REMARKS.--Monthly discharge adjusted for storage in Wallowa Lake and diversion from Wallowa Lake by Silver Lake ditch. Silver Lake ditch diverts at Wallowa Lake dam for irrigation northeast of Joseph. City of Joseph diverts less than 1.0 ft ${ }^{3} / \mathrm{s} \mathrm{from}$ Wallowa Lake for municipal use.

AVERAGE DISCHARGE.--55 years (water years 1928-82), $134 \mathrm{ft} \mathrm{t}^{3} / \mathrm{s}, 35.75 \mathrm{in} / \mathrm{yr}, 97,080$ acre- $\mathrm{ft} / \mathrm{yr}$, adjusted for storage and diversion. EXTREMES FOR PERIOD OF RECORD.--Maximum discharge, 1,550 $\mathrm{ft}+3 / \mathrm{s}$ June 10, 1969, gage height, $5.15 \mathrm{ft}$; no $\mathrm{flow}$ at $+\mathrm{imes}$ in some years.

STATISTICAL SUMMARIES

MONTHLY AND ANNUAL MEAN DISCHARGES 1905-82

\begin{tabular}{|c|c|c|c|c|c|c|}
\hline MONTH & $\begin{array}{l}\text { MINIMUM } \\
\text { (CFS) }\end{array}$ & $\begin{array}{l}\text { MAXIMUMM } \\
\text { (CFS) }\end{array}$ & $\begin{array}{l}\text { MEAN } \\
\text { (CFS) }\end{array}$ & $\begin{array}{l}\text { STAN- } \\
\text { DARD } \\
\text { DEVIA- } \\
\text { TION } \\
\text { (CFS) }\end{array}$ & $\begin{array}{l}\text { COEFFI- } \\
\text { CIENT OF } \\
\text { VARI- } \\
\text { ATION }\end{array}$ & $\begin{array}{c}\text { PERCENT } \\
\text { OF } \\
\text { ANNUAL } \\
\text { RUNOFF }\end{array}$ \\
\hline OCTOBER & 5.2 & 89 & 36 & 21 & .59 & 2.6 \\
\hline NOVEMBER & 6.7 & 159 & 33 & 27 & .83 & 2.4 \\
\hline DECEMBER & 5.4 & 135 & 31 & 26 & .83 & 2.3 \\
\hline JANUARY & 6.1 & 99 & 28 & 19 & .68 & 2.0 \\
\hline FEBRUARY & 5.0 & 61 & 25 & 16 & .63 & 1.9 \\
\hline MARCH & 2.8 & 109 & 27 & 21 & .80 & 2.0 \\
\hline APRIL & 3.1 & 173 & 34 & 34 & 1.01 & 2.5 \\
\hline MAY & 16 & 354 & 168 & 79 & .47 & 12.3 \\
\hline JUNE & 159 & 567 & 376 & 111 & .30 & 27.6 \\
\hline JULY & 91 & 614 & 343 & 100 & .29 & 25.2 \\
\hline AUGUST & 22 & 397 & 202 & 86 & .43 & 14.8 \\
\hline SEPTEMBER & 9.0 & 151 & 59 & 34 & .58 & 4.3 \\
\hline ANNUAL & 51 & 172 & 112 & 31 & .28 & 100 \\
\hline
\end{tabular}

MAGNITUDE AND PROBABILITY OF INSTANTANEOUS PEAK FLOW BASED ON PERIOD OF RECORD

DISCHARGE, IN CFS, FOR INDICATED RECURRENCE INTERVAL, IN YEARS, AND ANNUAL EXCEEDANCE PROBABILITY, IN PERCENT

\begin{tabular}{ccccccc}
1.25 & 2 & 5 & 10 & 25 & 50 & 100 \\
$80 \%$ & $50 \%$ & $20 \%$ & $10 \%$ & $4 \%$ & $2 \%$ & $1 \%$ \\
\hline- & & & & & & \\
\hline & & - & - & - & - & - \\
\hline WEIGHTED SKEW & & &
\end{tabular}

MAGNITUDE AND PROBABILITY OF ANNUAL LOW FLOW BASED ON PERIOD OF RECORD 1906-82

\begin{tabular}{|c|c|c|c|c|c|c|}
\hline $\begin{array}{l}\text { PERIOD } \\
\text { (CON- } \\
\text { SFCI- }\end{array}$ & \multicolumn{6}{|c|}{$\begin{array}{l}\text { DISCHARGE, IN CFS, FOR INDICATED RECURRENCE } \\
\text { INTERVAL, IN YEARS, AND ANNUAL NON- } \\
\text { EXCEEDANCE PROBABILITY, IN PERCENT }\end{array}$} \\
\hline TIVE & 2 & 5 & 10 & 20 & 50 & 100 \\
\hline DAYS) & $50 \%$ & $20 \%$ & $10 \%$ & $5 \%$ & $2 \%$ & $1 \%$ \\
\hline 1 & 11 & 57 & 35 & 25 & 17 & \\
\hline $\begin{array}{l}1 \\
3\end{array}$ & 12 & 5.6 & $\begin{array}{l}3.5 \\
3.7\end{array}$ & 2.9 & 8 & \\
\hline 7 & 12 & 6.1 & 4.2 & 3.0 & 2.1 & 1.6 \\
\hline 14 & 13 & 6.9 & 4.8 & 3.6 & 2.5 & 2 \\
\hline 30 & 15 & 7.8 & 5.6 & 4.2 & 3.0 & 2.4 \\
\hline 60 & 19 & 10 & 7.6 & 5.8 & 4.2 & 3 \\
\hline 90 & 20 & 11 & 8.4 & 6.5 & 4.9 & 4. \\
\hline 120 & 21 & 12 & 9.4 & 7.6 & 6.0 & 5. \\
\hline 183 & 24 & 15 & 11 & 8.8 & 6.8 & 5.7 \\
\hline
\end{tabular}

MAGNITUDE AND PROBABILITY OF ANNUAL HIGH FLOW BASED ON PERIOD OF RECORD 1905-82

\begin{tabular}{|c|c|c|c|c|c|c|}
\hline \multirow{4}{*}{$\begin{array}{l}\text { PERIOD } \\
\text { (CON- } \\
\text { SECU- } \\
\text { TIVE } \\
\text { DAYS) }\end{array}$} & \multicolumn{6}{|c|}{$\begin{array}{c}\text { DISCHARGE, IN CFS, FOR INDICATED RECURRENCE } \\
\text { INTERVAL, IN YEARS, AND ANNUAL } \\
\text { EXCEEDANCE PROBABILITY, IN PERCENT }\end{array}$} \\
\hline & & & 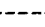 & & $-1-1$ & \\
\hline & 2 & 5 & 10 & 25 & 50 & 100 \\
\hline & $50 \%$ & $20 \%$ & $10 \%$ & $4 \%$ & $2 \%$ & $1 \%$ \\
\hline 1 & 559 & 728 & 855 & 1030 & 1180 & 1340 \\
\hline 3 & 552 & 708 & 821 & 975 & 1100 & 1230 \\
\hline 7 & 527 & 661 & 755 & 878 & 973 & 1070 \\
\hline 15 & 484 & 590 & 657 & 739 & 799 & 858 \\
\hline 30 & 421 & 513 & 567 & 628 & 669 & 709 \\
\hline 60 & 365 & 439 & 480 & 525 & 554 & 580 \\
\hline 90 & 330 & 394 & 429 & 465 & 488 & 508 \\
\hline
\end{tabular}

DURATION TABLE OF DAILY MEAN FLOW FOR PERIOD OF RECORD 1905-82

\begin{tabular}{|c|c|c|c|c|c|c|c|c|c|c|c|c|c|c|}
\hline & & DIS & ARGE, & CFS, & $\mathrm{ICH} \mathrm{W}$ & $\begin{array}{l}\text { EUA } \\
\end{array}$ & $R$ EX & ED & NDIC & $\begin{array}{l}\text { PER } \\
-\end{array}$ & OF & & & \\
\hline $5 \%$ & $10 \%$ & $15 \%$ & $20 \%$ & $25 \%$ & $30 \%$ & $40 \%$ & $50 \%$ & $60 \%$ & $70 \%$ & $75 \%$ & $80 \%$ & $85 \%$ & $90 \%$ & $95 \%$ \\
\hline 446 & 365 & 296 & 214 & 140 & 100 & 56 & 35 & 28 & 23 & 21 & 17 & 13 & 11 & 7.4 \\
\hline
\end{tabular}


13329500 HURRICANE CREEK NEAR JOSEPH, OR

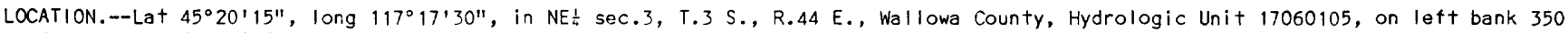
$\mathrm{ft}$ upstream from intake of Moonshine ditch, $3.5 \mathrm{mi}$ southwest of Joseph, and at mile 7.5 .

DRAINAGE AREA. $--29.6 \mathrm{mi}^{2}$.

PERIOD OF RECORD.--April to September 1915, April 1924 to September 1978. Monthly discharge on!y for some periods, published in wSP 1317 .

GAGE.--Water-stage recorder. Altitude of gage is 4,500 ft, by barometer. Apr. 27 to Sept. 3 , 1915, nonrecording gage at site $250 \mathrm{ft}$ downstream at different datum. Apr. 23, 1924, to June 13, 1933, water-stage recorder at site $150 \mathrm{tt}$ downstream at different datum.

REMARKS.--No regulation or diversion above station.

AVERAGE DISCHARGE. --54 years, $74.3 \mathrm{ft} / \mathrm{s}, 34.09 \mathrm{in} / \mathrm{yr}, 53,830 \mathrm{acre}-\mathrm{ft} / \mathrm{yr}$.

EXTREMES FOR PERIOD OF RECORD.--Maximum discharge, $1,110 \mathrm{ft}+3 / \mathrm{s}$ June 9 , 1948, gage height, $3.55 \mathrm{ft}$; maximum gage height, $6.94 \mathrm{ft}$ June 4, 1977, backwater from debris; minimum discharge, $2.8 \mathrm{ft}^{3} / \mathrm{s}$ Mar. 2, 1955, result of ice jam upstream; minimum daily, 6.0 $\mathrm{ft}+\mathrm{s}$ Jan. 6, Apr. 13, 1945 .

STATISTICAL SUMMARIES

MONTHLY AND ANNUAL MEAN DISCHARGES 1925-78

\begin{tabular}{|c|c|c|c|c|c|c|}
\hline MONTH & $\begin{array}{l}\text { MINIMUM } \\
\text { (CFS) }\end{array}$ & $\begin{array}{l}\text { MAXIMUUM } \\
\text { (CFS) }\end{array}$ & $\begin{array}{l}\text { MEAN } \\
\text { (CFS) }\end{array}$ & $\begin{array}{l}\text { STAN- } \\
\text { DARD } \\
\text { DEVIA- } \\
\text { TION } \\
\text { (CFS) }\end{array}$ & $\begin{array}{l}\text { COEFFI- } \\
\text { CIENT OF } \\
\text { VARI- } \\
\text { ATION }\end{array}$ & $\begin{array}{c}\text { PERCENT } \\
\text { OF } \\
\text { ANNUAL } \\
\text { RUNOFF }\end{array}$ \\
\hline OCTOBER & 16 & 96 & 33 & 14 & .44 & 3.7 \\
\hline NOVEMBER & 13 & 69 & 31 & 13 & .41 & 3.5 \\
\hline DECEMBER & 11 & 67 & 27 & 11 & .42 & 3.0 \\
\hline JANUARY & 10 & 44 & 22 & 7.7 & .35 & 2.5 \\
\hline FEBRUARY & 10 & 37 & 20 & 6.9 & .34 & 2.3 \\
\hline MARCH & 9.0 & 42 & 20 & 6.4 & .33 & 2.2 \\
\hline APRIL & 14 & 111 & 46 & 23 & .49 & 5.2 \\
\hline MAY & 64 & 292 & 165 & 57 & .34 & 18.6 \\
\hline JUNE & 116 & 439 & 266 & 79 & .30 & 29.9 \\
\hline JULY & 41 & 355 & 164 & 72 & .44 & 18.4 \\
\hline AUGUST & 25 & 113 & 60 & 22 & .36 & 6.7 \\
\hline SEPTEMBER & 20 & 62 & 36 & 9.5 & .27 & 4.0 \\
\hline ANNUAL & 37 & 103 & 74 & 16 & .21 & 100 \\
\hline
\end{tabular}

MAGNITUDE AND PROBABILITY OF INSTANTANEOUS PEAK FLOW BASED ON PERIOD OF RECORD 1925-78

DISCHARGE, IN CFS, FOR INDICATED RECURRENCE INTERVAL, IN YEARS, AND ANNUAL EXCEEDANCE PROBABILITY, IN PERCENT

\begin{tabular}{ccccccc}
1.25 & 2 & 5 & 10 & 25 & 50 & 100 \\
$80 \%$ & $50 \%$ & $20 \%$ & $10 \%$ & $4 \%$ & $2 \%$ & $1 \%$ \\
\hline 402 & 548 & 738 & 858 & 1010 & 1110 & 1220 \\
\hline
\end{tabular}

WE IGHTED SKEW $=-.129$
MAGNITUDE AND PROBABILITY OF ANNUAL LOW FLOW BASED ON PERIOD OF RECORD 1926-78

\begin{tabular}{|c|c|c|c|c|c|c|}
\hline $\begin{array}{l}\text { PER!OD } \\
\text { (CON- }\end{array}$ & \multicolumn{6}{|c|}{$\begin{array}{l}\text { DISCHARGE, IN CFS, FOR INDICATED RECURRENCE } \\
\text { INTERVAL, IN YEARS, AND ANNUAL NON- } \\
\text { EXCEEDANCE PROBABILITY, IN PERCENT }\end{array}$} \\
\hline SECU- & & & $n-\cdots$ & 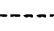 & -- & \\
\hline TIVE & 2 & 5 & 10 & 20 & 50 & 100 \\
\hline DAYS) & $50 \%$ & $20 \%$ & $10 \%$ & $5 \%$ & 2,6 & $1 \%$ \\
\hline 1 & 13 & 9.9 & 8.4 & 7.2 & 6. & \\
\hline 3 & 14 & 10 & 8.9 & 7. & 6. & \\
\hline 7 & 14 & 11 & 9.3 & 8.1 & 6. & \\
\hline 14 & 15 & 11 & 9.8 & 8.5 & 7. & \\
\hline 30 & 16 & 13 & 11 & 9.7 & 8. & \\
\hline 60 & 18 & 14 & 12 & 11 & 9. & \\
\hline 90 & 19 & 15 & 13 & 11 & 9. & \\
\hline 120 & 21 & 16 & 14 & 12 & 11 & \\
\hline 183 & 24 & 19 & 16 & 15 & 13 & 12 \\
\hline
\end{tabular}

MAGNITUDE AND PROBABILITY OF ANNUAL HIGH FLOW BASED ON PERIOD OF RECORD 1925-78

DISCHARGE, IN CFS, FOR INDICATED RECURRENCE

$\begin{array}{ll}\text { PERIOD } & \text { INTERVAL, IN YEARS, AND ANNUAL } \\ \text { (CON- } & \text { EXCEEDANCE PROBABILITY, IN PERCENT }\end{array}$

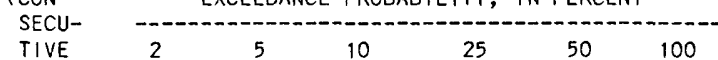

DAYS) $50 \% \quad 20 \% \quad 10 \% \quad 4 \% \quad 2 \% \quad 1 \%$

$436 \quad 561 \quad 635 \quad 722 \quad 782$

$\begin{array}{rllllll}1 & 436 & 561 & 635 & 722 & 782 & 838 \\ 3 & 407 & 522 & 590 & 669 & 724 & 775 \\ 7 & 369 & 474 & 535 & 606 & 655 & 701 \\ 15 & 327 & 418 & 471 & 530 & 571 & 608 \\ 30 & 293 & 365 & 403 & 443 & 469 & 492 \\ 60 & 247 & 301 & 328 & 355 & 372 & 386 \\ 90 & 204 & 246 & 267 & 287 & 298 & 308\end{array}$

DURATION TABLE OF DAILY MEAN FLOW FOR PERIOD OF RECORD 1925-78

\begin{tabular}{|c|c|c|c|c|c|c|c|c|c|c|c|c|c|c|}
\hline $5 \%$ & $10 \%$ & $15 \%$ & $20 \%$ & $25 \%$ & $30 \%$ & $40 \%$ & $50 \%$ & $60 \%$ & $70 \%$ & $75 \%$ & $80 \%$ & $85 \%$ & $90 \%$ & $95 \%$ \\
\hline 285 & 210 & 157 & 113 & 81 & 61 & 42 & 33 & 28 & 24 & 23 & 21 & 19 & 16 & 14 \\
\hline
\end{tabular}


LOCATION.--Lat $45^{\circ} 26^{\prime} 20^{\prime \prime}$, long $117^{\circ} 25^{\prime} 35^{\prime \prime}$, in NW/ sec.34, T.1 S., R.43 E., Wallowa County, Hydrologic Unit 17060105 , on left bank 3.5 $\mathrm{mi}$ south of Lostine and at $\mathrm{mile} 10.0$.

DRAINAGE AREA. $--70.9 \mathrm{mi}^{2}$.

PERIOD OF RECORD.--August 1912 to March 1914, April to September 1915, July 1925 to September 1982. Monthly discharge only for some periods, published in WSP 1317.

GAGE.--Water-stage recorder. Altitude of gage is 3,650 ft, by barometer. See WSP 1317 or 1737 for history of changes prior to Dec. 16, 1953. Dec. 16, 1953, to Aug. 23, 1977, at datum $1.04 \mathrm{ft}$ higher.

REMARKS.--Minam Lake, capacity 440 acre-ft, has stored and diverted flow from Minam River since 1917 for irrigation in Lostine River basin. Diversions for irrigation above station.

AVERAGE DISCHARGE. - -58 years (water years 1913, 1926-82), $196 \mathrm{ft}^{3} / \mathrm{s}, 142,000$ acre- $\mathrm{ft} / \mathrm{yr}$.

EXTREMES FOR PERIOD OF RECORD.--Maximum discharge, $2,550 \mathrm{ft}^{3} / \mathrm{s}$ June 16,1974 , gage height, $8.59 \mathrm{ft}$, present datum; minimum, $7.5 \mathrm{ft} 3 / \mathrm{s}$ Mar. 2, 1966, result of freezeup; minimum daily, $10 \mathrm{ft}^{3} / \mathrm{s}$ Nov. 28-30, 1936.

\section{STATISTICAL SUMMARIES}

MONTHLY AND ANNUAL MEAN DISCHARGES $1913-82$

\begin{tabular}{|c|c|c|c|c|c|c|}
\hline MONTH & $\begin{array}{l}\text { MINIMUM } \\
\text { (CFS) }\end{array}$ & $\begin{array}{l}\text { MAXIMUMA } \\
\text { (CFS) }\end{array}$ & $\begin{array}{l}\text { MEAN } \\
\text { (CFS) }\end{array}$ & $\begin{array}{l}\text { STAN- } \\
\text { DARD } \\
\text { DEVIA- } \\
\text { TION } \\
\text { (CFS) }\end{array}$ & $\begin{array}{l}\text { COEFFI- } \\
\text { CIENT OF } \\
\text { VARI- } \\
\text { ATION }\end{array}$ & $\begin{array}{c}\text { PERCENT } \\
\text { OF } \\
\text { ANNUAL } \\
\text { RUNOFF }\end{array}$ \\
\hline OCTOBER & 18 & 291 & 58 & 45 & .77 & 2.5 \\
\hline NOVEMBER & 15 & 226 & 64 & 45 & .70 & 2.7 \\
\hline DECEMBER & 15 & 212 & 60 & 39 & .65 & 2.6 \\
\hline JANUARY & 15 & 158 & 49 & 27 & .54 & 2.1 \\
\hline FEBRUARY & 15 & 117 & 46 & 22 & .47 & 2.0 \\
\hline MARCH & 16 & 155 & 53 & 23 & .44 & 2.2 \\
\hline APRIL & 36 & 393 & 158 & 79 & .50 & 6.8 \\
\hline MAY & 203 & 909 & 525 & 162 & .31 & 22.4 \\
\hline JUNE & 332 & 1427 & 805 & 258 & .32 & 34.4 \\
\hline JULY & 60 & 913 & 385 & 207 & .54 & 16.5 \\
\hline AUGUST & 31 & 180 & 85 & 39 & .45 & 3.7 \\
\hline SEPTEMBER & 23 & 104 & 50 & 19 & .38 & 2.1 \\
\hline ANNUAL & 91 & 288 & 196 & 43 & .22 & 100 \\
\hline
\end{tabular}

MAGNITUDE AND PROBABILITY OF INSTANTANEOUS PEAK FLOW BASED ON PERIOD OF RECORD 1913-82

\begin{tabular}{|c|c|c|c|c|c|c|}
\hline $\begin{array}{l}1.25 \\
80 \%\end{array}$ & $\begin{array}{c}2 \\
50 \%\end{array}$ & $\begin{array}{c}5 \\
20 \%\end{array}$ & $\begin{array}{l}10 \\
10 \%\end{array}$ & $\begin{array}{l}25 \\
4 \%\end{array}$ & $\begin{array}{l}50 \\
2 \%\end{array}$ & $\begin{array}{r}100 \\
1 \%\end{array}$ \\
\hline 1280 & 1570 & 1930 & 2150 & 2400 & 2580 & 2750 \\
\hline
\end{tabular}

WEIGHTED SKEW $=-.055$
MAGNITUDE AND PROBABILITY OF ANNUAL LOW FLOW BASED ON PERIOD OF RECORD 1914-82

\begin{tabular}{|c|c|c|c|c|c|c|}
\hline $\begin{array}{l}\text { PERIOD } \\
\text { (CON- } \\
\text { SECU- }\end{array}$ & \multicolumn{6}{|c|}{$\begin{array}{l}\text { DISCHARGE, IN CFS, FOR INDICATED RECURRENCE } \\
\text { INTERVAL, IN YEARS, AND ANNUAL NON- } \\
\text { EXCEEDANCE PROBABILITY, IN PERCENT }\end{array}$} \\
\hline TIVE & 2 & 5 & 10 & 20 & 50 & 100 \\
\hline DAYS) & $50 \%$ & $20 \%$ & $10 \%$ & $5 \%$ & $2 \%$ & $1 \%$ \\
\hline 1 & 23 & 18 & 15 & 14 & 12 & 11 \\
\hline 3 & 24 & 19 & 16 & 14 & 12 & 11 \\
\hline 7 & 26 & 20 & 17 & 15 & 13 & 12 \\
\hline 14 & 27 & 21 & 18 & 16 & 14 & 13 \\
\hline 30 & 30 & 23 & 20 & 17 & 15 & 14 \\
\hline 60 & 34 & 25 & 22 & 19 & 17 & 15 \\
\hline 90 & 37 & 27 & 23 & 20 & 17 & 15 \\
\hline 120 & 41 & 29 & 24 & 20 & 17 & 15 \\
\hline 183 & 47 & 32 & 27 & 23 & 20 & 18 \\
\hline
\end{tabular}

MAGNITUDE AND PROBABILITY OF ANNUAL HIGH FLOW BASED ON PERIOD OF RECORD 1913-82

\begin{tabular}{|c|c|c|c|c|c|c|}
\hline \multirow{3}{*}{$\begin{array}{l}\text { PERIOD } \\
\text { (CON- } \\
\text { SECU- } \\
\text { TIVE } \\
\text { DAYS) }\end{array}$} & \multicolumn{6}{|c|}{$\begin{array}{c}\text { DISCHARGE, IN CFS, FOR INDICATED RECURRENCE } \\
\text { INTERVAL, IN YEARS, AND ANNUAL } \\
\text { EXCEEDANCE PROBABILITY, IN PERCENT }\end{array}$} \\
\hline & 2 & 5 & 10 & 25 & 50 & 100 \\
\hline & $50 \%$ & $20 \%$ & $10 \%$ & $4 \%$ & $2 \%$ & $1 \%$ \\
\hline 1 & 1360 & 1700 & 1890 & 2120 & 2280 & 2430 \\
\hline 3 & 1280 & 1600 & 1780 & 1990 & 2130 & 2260 \\
\hline 7 & 1150 & 1460 & 1640 & 1860 & 2010 & 2150 \\
\hline 15 & 1010 & 1290 & 1460 & 1660 & 1810 & 1950 \\
\hline 30 & 903 & 1130 & 1250 & 1400 & 1490 & 1580 \\
\hline 60 & 738 & 893 & 975 & 1060 & 1120 & 1160 \\
\hline 90 & 596 & 715 & 774 & 836 & 871 & 902 \\
\hline
\end{tabular}

DURATION TABLE OF DAILY MEAN FLOW FOR PERIOD OF RECORD 1913-82

\begin{tabular}{|c|c|c|c|c|c|c|c|c|c|c|c|c|c|c|}
\hline $5 \%$ & $10 \%$ & $15 \%$ & $20 \%$ & $25 \%$ & $30 \%$ & $40 \%$ & $50 \%$ & $60 \%$ & $70 \%$ & $75 \%$ & $80 \%$ & $85 \%$ & $90 \%$ & $95 \%$ \\
\hline 866 & 614 & 439 & 295 & 196 & 138 & 85 & 64 & 52 & 43 & 40 & 36 & 32 & 28 & 24 \\
\hline
\end{tabular}


13330500 BEAR CREEK NEAR WALLOWA, OR

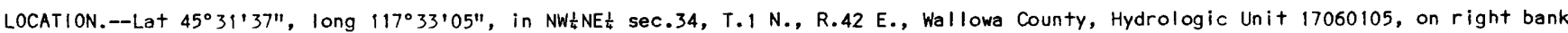
$30 \mathrm{ft}$ downstream from road bridge, $3.0 \mathrm{mi}$ southwest of Wallowa, and at mile 4.4.

DRAINAGE AREA. $--68 \mathrm{mi}^{2}$, approximately.

PERIOD OF RECORD.--April to September 1915, April 1924 to September 1982. Monthly discharge only for some periods, published in WSP 1317.

GAGE.--Water-stage recorder. Altitude of gage is 3,250 ft, by barometer. Apr. 13 to Sept. 16 , 1915, nonrecording gage at site 1.0 mi upstream at different datum. Apr. 22, 1924, to Nov. 2, 1931, water-stage recorder at site $1.5 \mathrm{mi}$ upstream at different datum.

REMARKS.--No regulation. Diversions for irrigation above statlon. Water for irrigation in Lostine River basin diverted from Little Bear Creek, a tributary above station, in sec.32, T.1 S., R. 43 E.

AVERAGE DISCHARGE.--58 years (water years 1925-82), $114 \mathrm{ft}^{3} / \mathrm{s}, 82,590 \mathrm{acre}-\mathrm{ft} / \mathrm{yr}$.

EXTREMES FOR PERIOD OF RECORD.--Maximum discharge, 1,730 ft3/s June 15, 1974; maximum gage height, $3.82 \mathrm{ft}$ Apr. 22 , 1936 (from $f$ loodmark); minimum daily discharge, $3 \mathrm{ft} / \mathrm{s}$ Jan. 20, Feb. 1, 1937.

STATISTICAL SUMMARIES

MONTHLY AND ANNUAL MEAN DISCHARGES 1925-82

\begin{tabular}{|c|c|c|c|c|c|c|}
\hline MONTH & $\begin{array}{l}\text { MINIMUM } \\
\text { (CFS) }\end{array}$ & $\begin{array}{l}\text { MAXIMUM } \\
\text { (CFS) }\end{array}$ & $\begin{array}{l}\text { MEAN } \\
\text { (CFS) }\end{array}$ & $\begin{array}{l}\text { STAN- } \\
\text { DARO } \\
\text { DEVIA- } \\
\text { TION } \\
\text { (CFS) }\end{array}$ & $\begin{array}{l}\text { COEFFI- } \\
\text { CIENT OF } \\
\text { VARI- } \\
\text { ATION }\end{array}$ & $\begin{array}{c}\text { PERCENT } \\
\text { OF } \\
\text { ANNUAL } \\
\text { RUNOFF }\end{array}$ \\
\hline OCTOBER & 7.6 & 160 & 25 & 29 & 1.13 & 1.9 \\
\hline NOVEMBER & 8.2 & 220 & 40 & 37 & .92 & 2.9 \\
\hline DECEMBER & 7.3 & 194 & 50 & 41 & .82 & 3.7 \\
\hline JANUARY & 5.2 & 136 & 43 & 30 & .70 & 3.1 \\
\hline FEBRUARY & 4.5 & 147 & 47 & 31 & .66 & 3.4 \\
\hline $\begin{array}{l}\text { MARCH } \\
\text { APRIL }\end{array}$ & $\begin{array}{l}11 \\
50\end{array}$ & $\begin{array}{l}186 \\
422\end{array}$ & $\begin{array}{r}65 \\
171\end{array}$ & $\begin{array}{l}33 \\
74\end{array}$ & $\begin{array}{l}.51 \\
.43\end{array}$ & $\begin{array}{r}4.7 \\
12.5\end{array}$ \\
\hline MAY & 138 & 682 & 374 & 102 & .27 & 27.3 \\
\hline JUNE & 112 & 869 & 402 & 160 & .40 & 29.4 \\
\hline JULY & 19 & 388 & 116 & 80 & .69 & 8.5 \\
\hline AUGUST & 8.1 & 38 & 19 & 8.0 & .42 & 1.4 \\
\hline SEPTEMBER & 6.3 & 44 & 16 & 8.5 & .55 & 1.1 \\
\hline ANNUAL & 46 & 178 & 114 & 27 & .24 & 100 \\
\hline
\end{tabular}

MAGNITUDE AND PROBABILITY OF INSTANTANEOUS PEAK FLOW BASED ON PERIOD OF RECORD 1925-82

DISCHARGE, IN CFS, FOR INDICATED RECURRENCE INTERVAL, IN YEARS, AND ANNUAL EXCEEDANCE PROBABILITY, IN PERCENT

\begin{tabular}{ccccccc}
1.25 & 2 & 5 & 10 & 25 & 50 & 100 \\
$80 \%$ & $50 \%$ & $20 \%$ & $10 \%$ & $4 \%$ & $2 \%$ & $1 \%$ \\
\hline 685 & 907 & 1200 & 1380 & 1610 & 1780 & 1950 \\
\hline
\end{tabular}

WEIGHTED SKEW $=-.022$
MAGN ITUDE AND PROBABILITY OF ANNUAL LOW FLOW BASED ON PERIOD OF RECORD 1926-82

\begin{tabular}{|c|c|c|c|c|c|c|}
\hline $\begin{array}{l}\text { PERIOD } \\
\text { ICON- } \\
\text { SECU- }\end{array}$ & \multicolumn{6}{|c|}{$\begin{array}{l}\text { DISCHARGE, IN CFS, FOR INDICATED RECURRENCE } \\
\text { INTERVAL, IN YEARS, AND ANNUAL NON- } \\
\text { EXCEEDANCE PROBABILITY, IN PERCENT }\end{array}$} \\
\hline $\begin{array}{l}\text { TIVE } \\
\text { DAYS) }\end{array}$ & $\begin{array}{c}2 \\
50 \%\end{array}$ & $\begin{array}{c}5 \\
20 \%\end{array}$ & $\begin{array}{l}10 \\
10 \%\end{array}$ & $\begin{array}{l}20 \\
5 \$\end{array}$ & $\begin{array}{l}50 \\
2 q\end{array}$ & $\begin{array}{r}100 \\
1 \%\end{array}$ \\
\hline 1 & 9.3 & 7. & 6. & 5.1 & 4.3 & \\
\hline 7 & 9.6 & 7. & 6. & 5. & 4.9 & 4. \\
\hline 14 & 10 & 7. & 6. & 5. & 5.1 & \\
\hline 30 & 11 & 8. & 7. & 6.5 & 5.6 & 30 \\
\hline 60 & 12 & 9. & 8. & 7. & 6.2 & 3 \\
\hline 90 & 14 & 10 & 9.0 & 8.1 & 7.2 & 0. \\
\hline 120 & 17 & 12 & 10 & 8.8 & 7.6 & 7. \\
\hline 183 & 25 & 16 & 13 & 10 & 8.3 & \\
\hline
\end{tabular}

MAGNITUDE AND PROBABILITY OF ANNUAL HIGH FLOW BASED ON PERIOD OF RECORD 1925-82

\begin{tabular}{|c|c|c|c|c|c|c|}
\hline $\begin{array}{l}\text { PERIOD } \\
\text { (CON- }\end{array}$ & \multicolumn{6}{|c|}{$\begin{array}{c}\text { DISCHARGE, IN CFS, FOR INDICATED RECURRENCE } \\
\text { INTERVAL, IN YEARS, AND ANNUAL } \\
\text { EXCEEDANCE PROBABILITY, IN PERCENT }\end{array}$} \\
\hline SECU- & 2 & 5 & 10 & 25 & 50 & 100 \\
\hline $\begin{array}{l}\text { TIVE } \\
\text { DAYS) }\end{array}$ & $50 \%$ & $20 \%$ & $10 \%$ & $4 \%$ & $2 \%$ & $1 \%$ \\
\hline \\
\hline 1 & 750 & 982 & 1130 & 1330 & 1470 & 1610 \\
\hline 3 & 693 & 898 & 1030 & 1210 & 1340 & 1470 \\
\hline 7 & 621 & 805 & 929 & 1090 & 1210 & 1330 \\
\hline 15 & 540 & 696 & 799 & 929 & 1030 & 1130 \\
\hline 30 & 484 & 610 & 686 & 776 & 839 & 899 \\
\hline 60 & 407 & 495 & 542 & 592 & 625 & 653 \\
\hline 90 & 336 & 403 & 435 & 467 & 486 & 503 \\
\hline
\end{tabular}

DURATION TABLE OF DAILY MEAN FLOW FOR PERIOD OF RECORD 1925-82

DISCHARGE, IN CFS, WHICH WAS EQUALED OR EXCEEDED FOR INDICATED PERCENT OF TIME

\begin{tabular}{|c|c|c|c|c|c|c|c|c|c|c|c|c|c|c|}
\hline $5 \%$ & $10 \%$ & $15 \%$ & $20 \%$ & $25 \%$ & $30 \%$ & $40 \%$ & $50 \%$ & $60 \%$ & $70 \%$ & $75 \%$ & $80 \%$ & $85 \%$ & $90 \%$ & $95 \%$ \\
\hline 477 & 352 & 259 & 187 & 133 & 96 & 60 & 42 & 29 & 21 & 18 & 16 & 13 & 11 & 9.0 \\
\hline
\end{tabular}




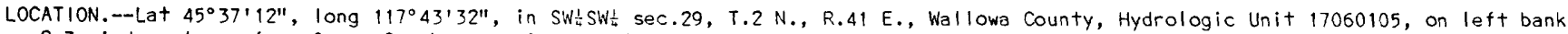
$2.3 \mathrm{mi}$ downstream from Squaw Creek, $0.3 \mathrm{mi}$ west of iMinam, and at mile 0.3 .

DRAINAGE AREA.--240 $\mathrm{mi}^{2}$, approximately.

PERIOD OF RECORD.--June 1912 to larch 1914, September 1955 to September 1982. Monthiy discharge only for some periods, published in WSP 1317.

GAGE.-Water-stage recorder. Datum of gage is 2,540.48 ft National Geodetic Vertical Datum of 1929 . June 1912 to March 1914 , nonrecording gage at approximately same site at different datum.

REMARKS.- No regulation or diversion above station.

AVERAGE DISCHARGE. -18 years, $476 \mathrm{ft} / \mathrm{s}, 26.93 \mathrm{in} / \mathrm{yr}, 344,900 \mathrm{acre}-\mathrm{ft} / \mathrm{yr}$.

EXTREMES FOR PERIOD OF RECORD.--Maximum discharge, $5,250 \mathrm{ft}^{3} / \mathrm{s}$ June 16, 1974, gage height, $5.89 \mathrm{ft}$; maximum gage height, $7.3 \mathrm{ft}$ May 28, 1913, datum then in use; minimum discharge, $10 \mathrm{ft}+3 / \mathrm{s} \mathrm{Dec.} \mathrm{6,} \mathrm{1972,} \mathrm{Jan.} \mathrm{10,} \mathrm{1973,} \mathrm{result} \mathrm{of} \mathrm{freezeup.}$

\section{STATISTICAL SUMMARIES}

MONTHLY AND ANNUAL MEAN DISCHARGES $1966-82$

\begin{tabular}{|c|c|c|c|c|c|c|}
\hline MONTH & $\begin{array}{l}\text { MINIMUM } \\
\text { (CFS) }\end{array}$ & $\begin{array}{l}\text { MAXIMUM } \\
\text { (CFS) }\end{array}$ & $\begin{array}{l}\text { MEAN } \\
\text { (CFS) }\end{array}$ & $\begin{array}{l}\text { STAN- } \\
\text { OARD } \\
\text { OEVIA- } \\
\text { TION } \\
\text { (CFS) }\end{array}$ & $\begin{array}{l}\text { COEFFI- } \\
\text { CIENT OF } \\
\text { VARI- } \\
\text { ATION }\end{array}$ & $\begin{array}{c}\text { PERCENT } \\
\text { OF } \\
\text { ANNUAL } \\
\text { RUNOFF }\end{array}$ \\
\hline OCTOBER & 65 & 173 & 96 & 28 & .29 & 1.7 \\
\hline NOVEMBER & 62 & 493 & 155 & 115 & .74 & 2.8 \\
\hline DECEMBER & 62 & 604 & 215 & 155 & .72 & 3.8 \\
\hline JANUARY & 60 & 412 & 228 & 116 & .51 & 4.1 \\
\hline FEBRUARY & 57 & 504 & 246 & 131 & .53 & 4.4 \\
\hline MARCH & 67 & 672 & 237 & 132 & .46 & 5.1 \\
\hline APRIL & 235 & 862 & 464 & 182 & .39 & 8.3 \\
\hline MAY & 484 & 2016 & 1289 & 342 & .27 & 23.0 \\
\hline JUNE & 668 & 3125 & 1702 & 666 & .39 & 30.3 \\
\hline JULY & 125 & 1392 & 680 & 371 & .55 & 12.1 \\
\hline AUGUST & 73 & 257 & 154 & 61 & .40 & 2.7 \\
\hline SEPTEMBER & 53 & 180 & 99 & 31 & .31 & 1.8 \\
\hline ANNUAL & 189 & 713 & 468 & 134 & .29 & 100 \\
\hline
\end{tabular}

MAGNITUDE AND PROBABILITY OF INSTANTANEOUS PEAK FLOW BASED ON PERIOD OF RECORD $1966-82$

\begin{tabular}{|c|c|c|c|c|c|c|}
\hline \multicolumn{7}{|c|}{$\begin{array}{l}\text { DISCHARGE, IN CFS, FOR INDICATED RECURRENCE INTERVAL, IN } \\
\text { YEARS, AND ANIIUAL EXCEEDANCE PROBABILITY, IN PERCENT }\end{array}$} \\
\hline $\begin{array}{l}1.25 \\
80 \%\end{array}$ & $\begin{array}{c}2 \\
50 \%\end{array}$ & $\begin{array}{c}5 \\
20 \%\end{array}$ & $\begin{array}{l}10 \\
10 \%\end{array}$ & $\begin{array}{l}25 \\
4 \%\end{array}$ & $\begin{array}{l}50 \\
2 \%\end{array}$ & $\begin{array}{r}100 \\
18\end{array}$ \\
\hline 2400 & 3190 & 4260 & 4970 & 5860 & -- & -- \\
\hline
\end{tabular}

MAGN ITUDE AND PROBABILITY OF ANNUAL LOW FLOW BASED ON PERIOD OF RECORD 1967-82

\begin{tabular}{|c|c|c|c|c|c|c|}
\hline $\begin{array}{r}\text { PERIOD } \\
\text { (CON- }\end{array}$ & \multicolumn{6}{|c|}{$\begin{array}{l}\text { DISCHARGE, IN CFS, FOR INDICATED RECURRENCE } \\
\text { INTERVAL, IN YEARS, AND ANNUAL NON- } \\
\text { EXCEEDANCE PROBABILITY, IN PERCENT }\end{array}$} \\
\hline SECU- & & & 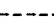 & 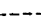 & -- & \\
\hline TIVE & 2 & 5 & 10 & 20 & 50 & 100 \\
\hline DAYS) & $50 \%$ & $20 \%$ & $10 \%$ & $5 \%$ & $2 \%$ & $1 \%$ \\
\hline 1 & 57 & 32 & 23 & 16 & -- & -- \\
\hline 3 & 57 & 37 & 27 & 20 & -- & -- \\
\hline 7 & 62 & 42 & 32 & 24 & -- & -- \\
\hline 14 & 63 & 48 & 41 & 36 & -- & -- \\
\hline 30 & 69 & 57 & 53 & 50 & -- & -- \\
\hline 60 & 77 & 63 & 58 & 55 & -- & -- \\
\hline 90 & 84 & 68 & 62 & 58 & -- & -- \\
\hline 120 & 94 & 74 & 67 & 61 & $\cdots$ & -- \\
\hline 183 & 135 & 100 & 86 & 75 & -- & -- \\
\hline
\end{tabular}

MAGNITUDE AND PROBABILITY OF ANNUAL HIGH FLOW BASED ON PERIOD OF RECORD 1966-82

\begin{tabular}{|c|c|c|c|c|c|c|}
\hline $\begin{array}{l}\text { PER! OD } \\
\text { (CON- }\end{array}$ & \multicolumn{6}{|c|}{$\begin{array}{c}\text { DISCHARGE, IN CFS, FOR INDICATED RECURRENCE } \\
\text { INTERVAL, IN YEARS, AND ANNUAL } \\
\text { EXCEEDANCE PROBABILITY, IN PERCENT }\end{array}$} \\
\hline SECU- & & . & 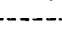 & ---- & $-\ldots$ & $\ldots$ \\
\hline TIVE & 2 & 5 & 10 & 25 & 50 & 100 \\
\hline DAYS) & $50 \%$ & $20 \%$ & $10 \%$ & $4 \%$ & 2,5 & 19 \\
\hline 1 & 2750 & 3650 & 4260 & 5040 & -- & -- \\
\hline 3 & 2570 & 3420 & 4020 & 4820 & -- & -- \\
\hline 7 & 2350 & 3140 & 3680 & 4400 & -- & -- \\
\hline 15 & 2060 & 2760 & 3210 & 3760 & -- & -- \\
\hline 30 & 1890 & 2420 & 2700 & 2990 & -- & -- \\
\hline 60 & 1600 & 2000 & 2190 & 2360 & -- & -- \\
\hline 90 & 1300 & 1620 & 1750 & 1870 & -- & -- \\
\hline
\end{tabular}

DURATION TABLE OF DAILY MEAN FLOW FOR PERIOD OF RECORD 1966-82

\begin{tabular}{|c|c|c|c|c|c|c|c|c|c|c|c|c|c|c|}
\hline $5 \%$ & $10 \%$ & $15 \%$ & $20 \%$ & $25 \%$ & $30 \%$ & $40 \%$ & $50 \%$ & $60 \%$ & $70 \%$ & $75 \%$ & $80 \%$ & $85 \%$ & $90 \%$ & $95 \%$ \\
\hline 1860 & 1350 & 991 & 713 & 529 & 417 & 285 & 210 & 156 & 117 & 103 & 91 & 81 & 72 & 61 \\
\hline
\end{tabular}


13332500 GRANDE RONDE RIVER AT RONDOWA, OR

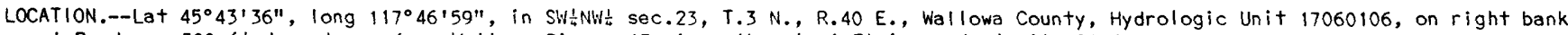
at Rondowa, $500 \mathrm{ft}$ downstream from Wallowa River, $13 \mathrm{mi}$ northeast of Elgin, and at mile 81.4 .

DRAINAGE AREA.--2,555 $\mathrm{mi}^{2}$.

PERIOD OF RECORD.--October 1926 to September 1982.

GAGE.--Water-stage recorder. Datum of gage is 2,281.87 $\mathrm{ft}$ National Geodetic Vertical Datum of 1929 .

REMARKS.--Flow slightly regulated by Wallowa Lake and small reservoirs. Diversions for irrigation above station, chiefly in vicinity of La Grande, Enterprise, and Wallowa; one transbasin diversion from Sheep Creek in Imnaha River basin for irrigation in Wallowa Valley.

AVERAGE DISCHARGE. --56 years, $2,158 \mathrm{ft}+3 / \mathrm{s}, 1,563,000$ acre $-\mathrm{ft} / \mathrm{yr}$.

EXTREMES FOR PERIOD OF RECORD.--Maximum discharge, $24,700 \mathrm{ft}^{3} / \mathrm{s} \mathrm{Jan.} \mathrm{30,} \mathrm{1965,} \mathrm{gage} \mathrm{height,} 10.93 \mathrm{ft}$; $\mathrm{minimum,} 179 \mathrm{ft} / \mathrm{s} \mathrm{Aug.} 24$, 1977.

STATISTICAL SUMMARIES

MONTHLY AND ANNUAL MEAN DISCHARGES 1927-82

\begin{tabular}{|c|c|c|c|c|c|c|}
\hline MONTH & $\begin{array}{l}\text { MINIMUM } \\
\text { (CFS) }\end{array}$ & $\begin{array}{l}\text { MAXIMUM } \\
\text { (CFS) }\end{array}$ & $\begin{array}{l}\text { MEAN } \\
\text { (CFS) }\end{array}$ & $\begin{array}{l}\text { STAN- } \\
\text { DARD } \\
\text { DEVIA- } \\
\text { TION } \\
\text { (CFS) }\end{array}$ & $\begin{array}{l}\text { COEFFI- } \\
\text { CIENT OF } \\
\text { VARI- } \\
\text { ATION }\end{array}$ & $\begin{array}{c}\text { PERCENT } \\
\text { OF } \\
\text { ANNUAL } \\
\text { RUNOFF }\end{array}$ \\
\hline OCTOBER & 343 & 1978 & 641 & 266 & .41 & 2.5 \\
\hline $\begin{array}{l}\text { NOVEMBER } \\
\text { DECEMBER }\end{array}$ & $\begin{array}{l}342 \\
358\end{array}$ & $\begin{array}{l}3346 \\
3942\end{array}$ & $\begin{array}{r}858 \\
1256\end{array}$ & $\begin{array}{l}491 \\
858\end{array}$ & $\begin{array}{l}.57 \\
.68\end{array}$ & $\begin{array}{l}3.3 \\
4.8\end{array}$ \\
\hline $\begin{array}{l}\text { JANUARY } \\
\text { FEBRUARY }\end{array}$ & $\begin{array}{l}298 \\
395\end{array}$ & $\begin{array}{l}3554 \\
5029\end{array}$ & $\begin{array}{l}1326 \\
1791\end{array}$ & $\begin{array}{r}845 \\
1055\end{array}$ & $\begin{array}{l}.64 \\
.59\end{array}$ & $\begin{array}{l}5.1 \\
6.9\end{array}$ \\
\hline $\begin{array}{l}\text { MARCH } \\
\text { APRIL }\end{array}$ & $\begin{array}{r}611 \\
1498\end{array}$ & $\begin{array}{l}7600 \\
8089\end{array}$ & $\begin{array}{l}2674 \\
4274\end{array}$ & $\begin{array}{l}1215 \\
1540\end{array}$ & $\begin{array}{l}.45 \\
.36\end{array}$ & $\begin{array}{l}10.3 \\
16.5\end{array}$ \\
\hline $\begin{array}{l}\text { MAY } \\
\text { JUNE }\end{array}$ & $\begin{array}{l}1965 \\
1561\end{array}$ & $\begin{array}{r}10010 \\
9662\end{array}$ & $\begin{array}{l}5576 \\
4709\end{array}$ & $\begin{array}{l}1795 \\
1914\end{array}$ & $\begin{array}{l}.32 \\
.41\end{array}$ & $\begin{array}{l}21.5 \\
18.1\end{array}$ \\
\hline $\begin{array}{l}\text { JULY } \\
\text { AUGUST }\end{array}$ & $\begin{array}{l}345 \\
269\end{array}$ & $\begin{array}{l}4692 \\
1098\end{array}$ & $\begin{array}{r}1712 \\
589\end{array}$ & $\begin{array}{l}933 \\
210\end{array}$ & $\begin{array}{l}.54 \\
.36\end{array}$ & $\begin{array}{l}6.6 \\
2.3\end{array}$ \\
\hline SEPTEMBER & 318 & 933 & 543 & 151 & .28 & 2.1 \\
\hline NNUAL & 855 & 3416 & 2160 & 608 & .28 & 100 \\
\hline
\end{tabular}

MAGNITUDE AND PROBABILITY OF INSTANTANEOUS PEAK FLOW BASED ON PERIOD OF RECORD $1927-82$

\begin{tabular}{|c|c|c|c|c|c|c|}
\hline $\begin{array}{l}1.25 \\
80 \%\end{array}$ & $\begin{array}{c}2 \\
50 \%\end{array}$ & $\begin{array}{c}5 \\
20 \%\end{array}$ & $\begin{array}{l}10 \\
10 \%\end{array}$ & $\begin{array}{l}25 \\
4 \%\end{array}$ & $\begin{array}{l}50 \\
2 \%\end{array}$ & $\begin{array}{r}100 \\
18\end{array}$ \\
\hline 7080 & 9650 & 13000 & 15100 & 17700 & 19500 & 21400 \\
\hline
\end{tabular}

WEIGHTED SKEW $=-.138$

MAGNITUDE AND PROBABILITY OF ANNUAL LOW FLOW BASED ON PERIOD OF RECORD $1928-82$

\begin{tabular}{|c|c|c|c|c|c|c|}
\hline $\begin{array}{l}\text { PERIOD } \\
\text { (CON- }\end{array}$ & \multicolumn{6}{|c|}{$\begin{array}{l}\text { DISCHARGE, IN CFS, FOR INDICATED RECURRENCE } \\
\text { INTERVAL, IN YEARS, AND ANNUAL NON- } \\
\text { EXCEEDANCE PROBAB ILITY, IN PERCENT }\end{array}$} \\
\hline SECU- & & & & -1 & $\cdots$ & \\
\hline TIVE & 2 & 5 & 10 & 20 & 50 & 100 \\
\hline DAYS) & $50 \%$ & 20,5 & 10,5 & $5 \%$ & 2,8 & $1 \%$ \\
\hline 1 & 378 & 306 & 269 & 240 & 209 & 190 \\
\hline 3 & 391 & 318 & 280 & 250 & 217 & 197 \\
\hline 7 & 408 & 331 & 291 & 258 & 223 & 201 \\
\hline 14 & 428 & 348 & 306 & 271 & 234 & 210 \\
\hline 30 & 455 & 368 & 323 & 286 & 248 & 223 \\
\hline 60 & 490 & 398 & 353 & 318 & 281 & 257 \\
\hline 90 & 524 & 426 & 382 & 348 & 314 & 293 \\
\hline 120 & 561 & 455 & 410 & 378 & 346 & 327 \\
\hline 183 & 720 & 544 & 472 & 421 & 371 & 341 \\
\hline
\end{tabular}

MAGNITUDE AND PROBABILITY OF ANNUAL HIGH FLOW BASED ON PERIOD OF RECORD 1927-82

\begin{tabular}{ccccccc} 
PERIOD & \multicolumn{2}{c}{ DISCHARGE, IN CFS, FOR INDICATED RECURRENCE } \\
INTERVAL, IN YEARS, AND ANNUAL \\
(CON-
\end{tabular}

DURATION TABLE OF DAILY MEAN FLOW FOR PERIOD OF RECORD 1927-82

DISCHARGE, IN CFS, WHICH WAS EQUALED OR EXCEEDED FOR INDICATED PERCENT OF TIME

\begin{tabular}{|c|c|c|c|c|c|c|c|c|c|c|c|c|c|c|}
\hline $5 \%$ & $10 \%$ & $15 \%$ & $20 \%$ & $25 \%$ & $30 \%$ & $40 \%$ & $50 \%$ & $60 \%$ & $70 \%$ & $75 \%$ & $80 \%$ & $85 \%$ & $90 \%$ & $95 \%$ \\
\hline 7000 & 5490 & 4430 & 3710 & 3110 & 2540 & 1700 & 1140 & 811 & 651 & 597 & 552 & 508 & 456 & 388 \\
\hline
\end{tabular}




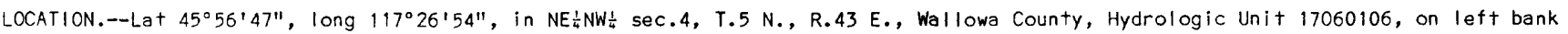
$500 \mathrm{ft}$ downstream from bridge at Troy, $600 \mathrm{ft}$ downstream trom Wenaha River, and at mile 45.2 .

DRAINAGE AREA. $--3,275 \mathrm{mi}^{2}$.

PERIOD OF RECORD.--August 1944 to September 1982. Monthly discharge only August 1944, published in WSP 1317.

GAGE.--Water-stage recorder. Datum of gage is $1,585.98 \mathrm{ft}$ National Geodetic Vertical Datum of 1929. Aug. 17, 1944, to Sept. 30 , 1949, nonrecording gage at site $500 \mathrm{ft}$ upstream at datum $10.85 \mathrm{ft}$ lower. Oct. 1, 1949, to Sept. 5, 1963, water-stage recorder at site $500 \mathrm{ft}$ upstream at datum $1.15 \mathrm{ft}$ higher.

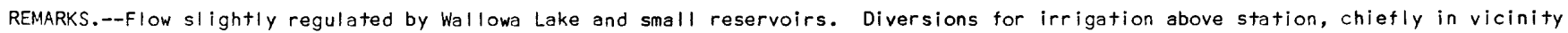
of La Grande. Enterprise, and Wallowa; one transbasin diversion from Big Sheep Creek and tributaries in Imnaha River basin for irrigation in Wallowa Valley.

AVERAGE DISCHARGE. --38 years, $3,125 \mathrm{ft}+3 / \mathrm{s}, 2,264,000$ acre- $\mathrm{ft} / \mathrm{yr}$.

EXTREMES FOR PERIOD OF RECORD.--Maximum discharge, 42,200 ft3/s Dec. 23, 1964, gage height, $11.25 \mathrm{ft}$; minimum, 344 $\mathrm{ft} / \mathrm{s} \mathrm{Aug}$. 19-21, $23,1977$.

STATISTICAL SUMMARIES

MONTHLY AND ANNUAL MEAN DISCHARGES 1945-82

\begin{tabular}{|c|c|c|c|c|c|c|}
\hline MONTH & $\begin{array}{l}\text { MINIMUM } \\
\text { (CFS) }\end{array}$ & $\begin{array}{l}\text { MAXIMUM } \\
\text { (CFS) }\end{array}$ & $\begin{array}{l}\text { MEAN } \\
\text { (CFS) }\end{array}$ & $\begin{array}{l}\text { STAN- } \\
\text { DARD } \\
\text { DEVIA- } \\
\text { TION } \\
\text { (CFS) }\end{array}$ & $\begin{array}{l}\text { COEFFI- } \\
\text { CIENT OF } \\
\text { VARI- } \\
\text { ATION }\end{array}$ & $\begin{array}{c}\text { PERCENT } \\
\text { OF } \\
\text { ANNUAL } \\
\text { RUNOFF }\end{array}$ \\
\hline OCTOBER & 603 & 2559 & 915 & 320 & .35 & 2.4 \\
\hline $\begin{array}{l}\text { NOVEMBER } \\
\text { DECEMBER }\end{array}$ & $\begin{array}{l}688 \\
685\end{array}$ & $\begin{array}{l}3023 \\
6295\end{array}$ & $\begin{array}{l}1239 \\
2158\end{array}$ & $\begin{array}{r}519 \\
1544\end{array}$ & $\begin{array}{l}.42 \\
.72\end{array}$ & $\begin{array}{l}3.3 \\
5.7\end{array}$ \\
\hline $\begin{array}{l}\text { JANUARY } \\
\text { FEBRUARY }\end{array}$ & $\begin{array}{l}702 \\
769\end{array}$ & $\begin{array}{l}6280 \\
7386\end{array}$ & $\begin{array}{l}2273 \\
3095\end{array}$ & $\begin{array}{l}1363 \\
1612\end{array}$ & $\begin{array}{l}.60 \\
.52\end{array}$ & $\begin{array}{l}6.1 \\
8.2\end{array}$ \\
\hline $\begin{array}{l}\text { MARCH } \\
\text { APRIL }\end{array}$ & $\begin{array}{r}888 \\
2257\end{array}$ & $\begin{array}{l}11520 \\
10780\end{array}$ & $\begin{array}{l}3893 \\
6335\end{array}$ & $\begin{array}{l}1855 \\
2251\end{array}$ & $\begin{array}{l}.48 \\
.36\end{array}$ & $\begin{array}{l}10.4 \\
16.9\end{array}$ \\
\hline MAY & 2368 & 13820 & 7656 & 2496 & .33 & 20.4 \\
\hline JUNE & 2159 & 11610 & 6035 & 2112 & .35 & 16.1 \\
\hline JULY & 520 & 4951 & 2291 & 990 & .43 & 6.1 \\
\hline AUGUST & 448 & 1375 & 873 & 219 & .25 & 2.3 \\
\hline SEP TEMBER & 574 & 1990 & 798 & 140 & .18 & 2.1 \\
\hline ANNUAL & 1136 & 4912 & 3125 & 816 & .26 & 100 \\
\hline
\end{tabular}

MAGNI TUDE AND PROBABILITY OF INSTANTANEOUS PEAK FLOW BASED ON PERIOD OF RECORD 1945-82

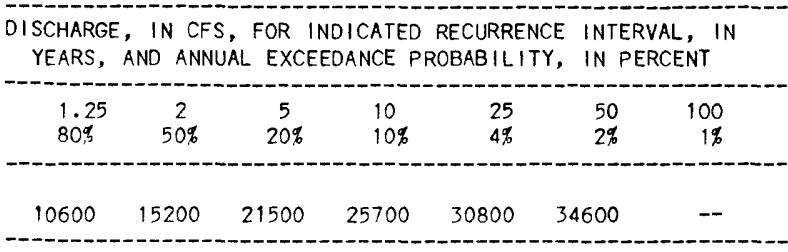

WEIGHTED SKEW $=-.141$

MAGNITUDE AND PROBABILITY OF ANNUAL LOW FLOW BASED ON PERIOD OF RECORD $1946-82$

\begin{tabular}{ccccccc} 
PERIOD & \multicolumn{1}{c}{$\begin{array}{c}\text { DISCHARGE, IN CFS, FOR INOICATED RECURRENCE } \\
\text { (CON- }\end{array}$} & $\begin{array}{c}\text { INTERVAL, IN YEARS, AND ANNUAL NON- } \\
\text { EXCEEDANCE PROBABIL ITY, IN PERCENT }\end{array}$ \\
$\begin{array}{c}\text { SECU- } \\
\text { TIVE }\end{array}$ & 2 & 5 & 10 & 20 & 50 & 100 \\
DAYS) & $50 \%$ & $20 \%$ & $10 \%$ & $5 \%$ & $2 \%$ & $1 \%$ \\
\hline 1 & 617 & 527 & 475 & 431 & 382 & -- \\
3 & 634 & 542 & 488 & 443 & 391 & - \\
7 & 654 & 562 & 506 & 458 & 403 & - \\
14 & 677 & 582 & 526 & 478 & 423 & - \\
30 & 707 & 607 & 548 & 499 & 443 & - \\
60 & 756 & 656 & 601 & 556 & 504 & - \\
90 & 789 & 691 & 644 & 608 & 569 & - \\
120 & 833 & 734 & 695 & 670 & 646 & - \\
183 & 1110 & 911 & 827 & 767 & 706 & - \\
-
\end{tabular}

MAGNITUDE AND PROBABILITY OF ANNUAL HIGH FLOW BASED ON PERIOD OF RECORD 1945-82

\begin{tabular}{|c|c|c|c|c|c|c|}
\hline $\begin{array}{l}\text { PERIOD } \\
\text { (CON- } \\
\text { SECU- }\end{array}$ & \multicolumn{6}{|c|}{$\begin{array}{l}\text { DISCHARGE, IN CFS, FOR IND ICATED RECURRENCE } \\
\text { INTERVAL, IN YEARS, AND ANNUAL } \\
\text { EXCEEDANCE PROBABILITY, IN PERCENT }\end{array}$} \\
\hline TIVE & 2 & 5 & 10 & 25 & 50 & 100 \\
\hline DAYS) & $50 \%$ & $20 \%$ & $10 \%$ & $4 \%$ & $2 \%$ & $1 \%$ \\
\hline 1 & 13900 & 19500 & 23100 & 27400 & 30600 & -- \\
\hline 3 & 12700 & 17100 & 19800 & 22900 & 25100 & - \\
\hline 7 & 11400 & 14800 & 16700 & 18600 & 19800 & -- \\
\hline 15 & 9940 & 12500 & 13700 & 14900 & 15700 & -- \\
\hline 30 & 8650 & 10900 & 12000 & 13100 & 13700 & $\rightarrow$ \\
\hline 60 & 7720 & 9740 & 10700 & 11600 & 12100 & -- \\
\hline 90 & 7020 & 8800 & 9600 & 10300 & 10700 & - \\
\hline
\end{tabular}

DURATION TABLE OF DAILY MEAN FLOW FOR PERIOD OF RECORD 1945-82

DISCHARGE, IN CFS, WHICH WAS EQUALED OR EXCEEDED FOR INDICATED PERCENT OF TIME

\begin{tabular}{|c|c|c|c|c|c|c|c|c|c|c|c|c|c|c|}
\hline $5 \%$ & $10 \%$ & $15 \%$ & $20 \%$ & $25 \%$ & $30 \%$ & $40 \%$ & $50 \%$ & $60 \%$ & $70 \%$ & $75 \%$ & $80 \%$ & $85 \%$ & $90 \%$ & $95 \%$ \\
\hline 9480 & 7650 & 6380 & 5410 & 4570 & 3750 & 2560 & 1740 & 1220 & 971 & 892 & 834 & 777 & 720 & 652 \\
\hline
\end{tabular}


14010000 SOUTH FORK WALLA WALLA RIVER NEAR MILTON, OR

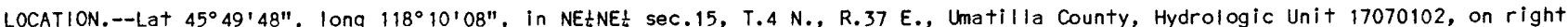
bank $1.0 \mathrm{mi}$ downstream from Elbow Creek, $13 \mathrm{mi}$ southeast of Milton, and at mile 59.1 .

DRAINAGE AREA. $--63 \mathrm{mi}^{2}$, approximately.

PERIOD OF RECORD.--February to October 1903. August 1906 to November 1917, May 1931 to September 1982. Monthly discharge only for some periods, published in WSP 1318. Published as "12 mi above Milton" 1903 and as "above Pacitic Power \& Light Co.'s intake near Milton" 1907-10.

GAGE.--Water-stage recorder. Altitude of gage is 2,050 ft, from river-profile map. Prior to Mar. 23, 1934, water-stage recorder or nonrecording gage at several sites within $1.5 \mathrm{mi}$ of present site at various datums.

REMARKS.--No regulation or diversion above station.

AVERAGE DISCHARGE.--61 years (water years 1908-17, 1932-82), $178 \mathrm{ft} / \mathrm{s}, 38.37 \mathrm{in} / \mathrm{yr}, 129,000 \mathrm{acre}-\mathrm{ft} / \mathrm{yr}$.

EXTREMES FOR PERIOD OF RECORD.--Maximum discharge, 2,530 ft3/s Jan. 29, 1965, gage height, $5.60 \mathrm{ft}$; minimum, $72 \mathrm{ft} / \mathrm{s}$ Feb 14,1932 . EXTREMES OUTSIDE PERIOD OF RECORD.--Maximum stage about $6 \mathrm{ft}$ Mar. 31, 1931, present site and datum.

STATISTICAL SUMMARIES

MONTHLY AND ANNUAL MEAN DISCHARGES $1907-82$

\begin{tabular}{|c|c|c|c|c|c|c|}
\hline MONTH & $\begin{array}{l}\text { MINIMUM } \\
\text { (CFS) }\end{array}$ & $\begin{array}{l}\text { MAXIMUM } \\
\text { (CFS) }\end{array}$ & $\begin{array}{l}\text { MEAN } \\
\text { (CFS) }\end{array}$ & $\begin{array}{l}\text { STAN- } \\
\text { DARD } \\
\text { DEVIA- } \\
\text { TION } \\
\text { (CFS) }\end{array}$ & $\begin{array}{l}\text { COEFFI- } \\
\text { CIENT OF } \\
\text { VARI- } \\
\text { ATION }\end{array}$ & $\begin{array}{c}\text { PERCENT } \\
\text { OF } \\
\text { ANNUAL } \\
\text { RUNOFF }\end{array}$ \\
\hline OCTOBER & 84 & 180 & 111 & 19 & .17 & 5.2 \\
\hline NOVEMBER & 89 & 245 & 135 & 34 & .25 & 6.3 \\
\hline DECEMBER & 93 & 376 & 171 & 66 & .38 & 8.0 \\
\hline JANUARY & 92 & 378 & 175 & 60 & .34 & 8.2 \\
\hline FEBRUARY & 102 & 326 & 188 & 54 & .29 & 8.8 \\
\hline MARCH & 111 & 399 & 213 & 52 & .24 & 10.0 \\
\hline APRIL & 147 & 458 & 282 & 60 & .21 & 13.3 \\
\hline MAY & 123 & 569 & 306 & 86 & .28 & 14.4 \\
\hline JUNE & 110 & 484 & 206 & 77 & .37 & 9.7 \\
\hline JULY & 85 & 193 & 124 & 22 & .17 & 5.8 \\
\hline AUGUST & 81 & 143 & 108 & 14 & .13 & 5.1 \\
\hline SEPTEMBER & 82 & 151 & 107 & 14 & .13 & 5.0 \\
\hline ANNUAL & 123 & 255 & 177 & 28 & .16 & 100 \\
\hline
\end{tabular}

MAGNITUDE AND PROBABILITY OF INSTANTANEOUS PEAK FLOW BASED ON PERIOD OF RECORD 1908-82

DISCHARGE, IN CFS, FOR INDICATED RECURRENCE INTERVAL, IN YEARS, AND ANNUAL EXCEEDANCE PROBABILITY, IN PERCENT

\begin{tabular}{|c|c|c|c|c|c|c|}
\hline $\begin{array}{l}1.25 \\
80 \%\end{array}$ & $\begin{array}{c}2 \\
50 \%\end{array}$ & $\begin{array}{c}5 \\
20 \%\end{array}$ & $\begin{array}{l}10 \\
10 \%\end{array}$ & $\begin{array}{l}25 \\
4 \%\end{array}$ & $\begin{array}{l}50 \\
2 \%\end{array}$ & $\begin{array}{r}100 \\
1 \%\end{array}$ \\
\hline 550 & 802 & 1220 & 1540 & 2000 & 2380 & 2800 \\
\hline
\end{tabular}

WEIGHTED SKEW $=.357$
MAGNI TUDE AND PROBABILITY OF ANNUAL LOW FLOW BASED ON PERIOD OF RECORD $1908-82$

\begin{tabular}{|c|c|c|c|c|c|c|}
\hline \multirow{3}{*}{$\begin{array}{l}\text { PERIOD } \\
\text { (CON- } \\
\text { SECU- } \\
\text { TIVE } \\
\text { DAYS) }\end{array}$} & \multicolumn{6}{|c|}{$\begin{array}{l}\text { DISCHARGE, IN CFS, FOR INDICATED RECURRENCE } \\
\text { INTERVAL, IN YEARS, AND ANNUAL NON- } \\
\text { EXCEEDANCE PROBABILIITY, IN PERCENT }\end{array}$} \\
\hline & 2 & 5 & 10 & 20 & 50 & 100 \\
\hline & $50 \%$ & $20 \%$ & $10 \%$ & $5 \%$ & $2 \%$ & $1 \%$ \\
\hline 1 & 98 & 89 & 84 & 81 & 78 & 76 \\
\hline 3 & 99 & 89 & 85 & 82 & 78 & 76 \\
\hline 7 & 99 & 90 & 86 & 83 & 79 & 77 \\
\hline 14 & 100 & 91 & 86 & 83 & 80 & 78 \\
\hline 30 & 102 & 92 & 88 & 84 & 81 & 79 \\
\hline 60 & 103 & 94 & 89 & 86 & 82 & 80 \\
\hline 90 & 105 & 95 & 90 & 87 & 83 & 81 \\
\hline 120 & 107 & 97 & 92 & 89 & 86 & 83 \\
\hline 183 & 117 & 105 & 100 & 96 & 93 & 90 \\
\hline
\end{tabular}

MAGNITUDE AND PROBABILITY OF ANNUAL HIGH FLOW BASED ON PERIOD OF RECORD 1908-82

\begin{tabular}{|c|c|c|c|c|c|c|}
\hline \multirow{4}{*}{$\begin{array}{l}\text { PERIOD } \\
\text { (CON- } \\
\text { SECU- } \\
\text { TIVE } \\
\text { DAYS) }\end{array}$} & \multicolumn{6}{|c|}{$\begin{array}{c}\text { DISCHARGE, IN CFS, FOR IND ICATED RECURRENCE } \\
\text { INTERVAL, IN YEARS, AND ANNUAL } \\
\text { EXCEEDANCE PROBABILITY, IN PERCENT }\end{array}$} \\
\hline & & $-\ldots$ & 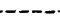 & - & & 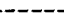 \\
\hline & 2 & 5 & 10 & 25 & 50 & 100 \\
\hline & $50 \%$ & $20 \%$ & $10 \%$ & $4 \%$ & $2 \%$ & $1 \%$ \\
\hline 1 & 627 & 886 & 1090 & 1380 & 1620 & 1890 \\
\hline 3 & 539 & 715 & 841 & 1010 & 1140 & 1280 \\
\hline 7 & 455 & 580 & 661 & 764 & 840 & 916 \\
\hline 15 & 392 & 481 & 533 & 594 & 636 & 676 \\
\hline 30 & 347 & 421 & 465 & 516 & 551 & 584 \\
\hline 60 & 311 & 373 & 408 & 448 & 476 & 501 \\
\hline 90 & 284 & 336 & 365 & 398 & 421 & 441 \\
\hline
\end{tabular}

DURATION TABLE OF DAILY MEAN FLOW FOR PERIOD OF RECORD 1908-82

$\begin{array}{lllllllllllllll}5 \% & 10 \% & 15 \% & 20 \% & 25 \% & 30 \% & 40 \% & 50 \% & 60 \% & 70 \% & 75 \% & 80 \% & 85 \% & 90 \% & 95 \% \\ 385 & 310 & 269 & 237 & 213 & 193 & 161 & 141 & 126 & 116 & 111 & 107 & 102 & 98 & 92\end{array}$


14010500 SOUTH FORK WALLA WALLA RIVER BELOW PACIFIC POWER \& LIGHT CO.'S PLANT, NEAR MILTON, OR

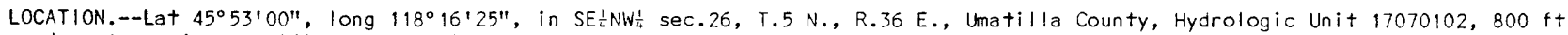
downstream from Pacific Power \& Light Co. powerplant, $1.2 \mathrm{mi}$ upstream from intake of Milton city powerplant, 2 mi upstream from confluence with North Fork, and $5.8 \mathrm{mi}$ southeast of Mi!ton.

ORAINAGE AREA. $--80 \mathrm{mi}^{2}$, approximately. At site 1903-6, 1929-38, $81 \mathrm{mi}^{2}$, approximately.

PERIOD OF RECORD.--October 1904 to M3y 1906, January 1930 to September 1945.

GAGE.--Water-stage recorder. Datum of gage is 1,49n. 3C ft National Geodetic Vertical Datun of 1929 (Pacific Power \& Light Co. benchmark). Nov. 1, 1903, to May 29, 1906, staff gage and Dec. 18, 1929, to May 26, 1938, water-stage recorder at several sites within $1.2 \mathrm{mi}$ of described site at various datums.

REMARKS.--Small diversions for irrigation of about 300 acres above station. Since 1905, some diurnal fluctuation caused by powerplant above station.

COOPERATION.--Records for 1929-40, furnished by the Oregon Water Resources Department.

AVERAGE DISCHARGE. -16 years (water years 1905, 1931-45), $169 \mathrm{ft}^{3} / \mathrm{s}$.

EXTREMES FOR PERIOD OF RECORD. - Maximum discharge not determined, probably occurred during $f(00 d s$ of ilay 30 , 31 , 1906 , or Mar. 31, 1931; maximum daily discharge, 3,000 $\mathrm{ft}^{3} / \mathrm{s}$ (estimated) May 30, 1906, and Mar. 31, 1931; minimum, , ft3/s (regulated) June 23, 1940; minimum daily, $57 \mathrm{ft} / \mathrm{s}$ July $22,1930$.

STATISTICAL SUMMARIES

MONTHLY AND ANNUAL MEAN DISCHARGES 1904-45

\begin{tabular}{|c|c|c|c|c|c|c|}
\hline MONTH & $\begin{array}{l}\text { MINIMUM } \\
\text { (CFS) }\end{array}$ & $\begin{array}{l}\text { MAXIMUM } \\
\text { (CFS) }\end{array}$ & $\begin{array}{l}\text { MEAN } \\
\text { (CFS) }\end{array}$ & $\begin{array}{l}\text { STAN- } \\
\text { DARD } \\
\text { DEVIA- } \\
\text { TION } \\
\text { (CFS) }\end{array}$ & $\begin{array}{l}\text { COEFFI- } \\
\text { CIENT OF } \\
\text { VARI- } \\
\text { ATION }\end{array}$ & $\begin{array}{c}\text { PERCENT } \\
\text { OF } \\
\text { ANNUAL } \\
\text { RUNOFF }\end{array}$ \\
\hline OCTOBER & 81 & 180 & 101 & 23 & .23 & 5.0 \\
\hline NOVEMBER & 85 & 239 & 126 & 39 & .31 & 6.2 \\
\hline DECEMBER & 100 & 335 & 160 & 63 & .39 & 7.9 \\
\hline JANUARY & 92 & 283 & 158 & 45 & .29 & 7.8 \\
\hline FEBRUARY & 105 & 290 & 169 & 45 & .27 & 8.4 \\
\hline $\begin{array}{l}\text { MARCH } \\
\text { APRIL }\end{array}$ & $\begin{array}{l}149 \\
152\end{array}$ & $\begin{array}{l}360 \\
547\end{array}$ & $\begin{array}{l}237 \\
316\end{array}$ & $\begin{array}{l}56 \\
96\end{array}$ & $\begin{array}{l}.24 \\
.30\end{array}$ & $\begin{array}{l}11.7 \\
15.6\end{array}$ \\
\hline MAY & 123 & 447 & 289 & 90 & .31 & 14.3 \\
\hline JUNE & 97 & 349 & 180 & 64 & .36 & 8.9 \\
\hline JULY & 78 & 143 & 104 & 19 & .18 & 5.2 \\
\hline AUGUST & 74 & 119 & 89 & 11 & .13 & 4.4 \\
\hline SEPTEMBER & 75 & 114 & 90 & 11 & .12 & 4.5 \\
\hline ANNUAL & 129 & 230 & 169 & 26 & .16 & 100 \\
\hline
\end{tabular}

MAGNITUDE AND PROBABILITY OF INSTANTANEOUS PEAK FLOW BASED ON PERIOD OF RECORD 1904-45

DISCHARGE, IN CFS, FOR INDICATED RECURRENCE INTERVAL, IN YEARS, AND ANNUAL EXCEEDANCE PROBABILITY, IN PERCENT

\begin{tabular}{ccccccc}
1.25 & 2 & 5 & 10 & 25 & 50 & 100 \\
$80 \%$ & $50 \%$ & $20 \%$ & $10 \%$ & $4 \%$ & $2 \%$ & $1 \%$ \\
\hline 535 & 759 & 1090 & 1330 & 1640 & - & - \\
\hline
\end{tabular}

WEIGHTED SKEW $=.124$
MAGNITUDE AND PROBABILITY OF ANNUAL LOW FLOW BASED ON PERIOD OF RECORD 1905-45

\begin{tabular}{|c|c|c|c|c|c|c|}
\hline $\begin{array}{l}\text { PERIOD } \\
\text { (CON- }\end{array}$ & \multicolumn{6}{|c|}{$\begin{array}{l}\text { DISCHARGE, IN CFS, FOR INDICATED RECURRENCE } \\
\text { INTERVAL, IN YEARS, AND ANNUAL NON- } \\
\text { EXCEEDANCE PROBABILITY, IN PERCENT }\end{array}$} \\
\hline SECU- & & - & - & --- & - & \\
\hline TIVE & 2 & 5 & 10 & 20 & 50 & 100 \\
\hline DAYS) & $50 \%$ & $20 \%$ & $10 \%$ & $5 \%$ & $2 \%$ & $1 \%$ \\
\hline 1 & 82 & 72 & 67 & 63 & -- & - \\
\hline 3 & 82 & 73 & 69 & 66 & -- & -- \\
\hline 7 & 83 & 75 & 71 & 69 & -- & - \\
\hline 14 & 84 & 77 & 74 & 71 & -- & -- \\
\hline 30 & 86 & 78 & 75 & 72 & -- & -- \\
\hline 60 & 87 & 80 & 76 & 74 & -- & -- \\
\hline 90 & 89 & 81 & 78 & 75 & -- & -- \\
\hline 120 & 91 & 83 & 80 & 77 & -- & -- \\
\hline 183 & 103 & 92 & 87 & 83 & -- & -- \\
\hline
\end{tabular}

MAGNITUDE AND PROBABILITY OF ANNUAL HIGH FLOW BASED ON PERIOD OF RECORD 1904-45

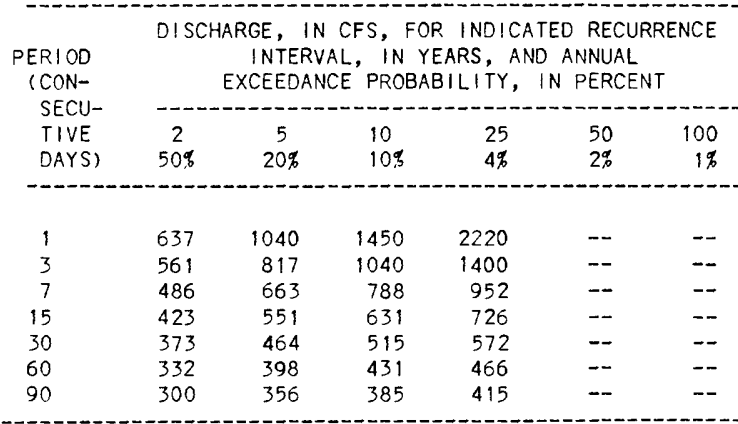

DURATION TABLE OF DAILY MEAN FLOW FOR PERIOD OF RECORD 1904-45

\begin{tabular}{|c|c|c|c|c|c|c|c|c|c|c|c|c|c|c|}
\hline $5 \%$ & $10 \%$ & $15 \%$ & $20 \%$ & $25 \%$ & $30 \%$ & 40.5 & $50 \%$ & $60 \%$ & $70 \%$ & $75 \%$ & $80 \%$ & $85 \%$ & $90 \%$ & $95 \%$ \\
\hline 386 & 314 & 270 & 235 & 206 & 183 & 148 & 128 & 113 & 101 & 97 & 93 & 90 & 86 & 80 \\
\hline
\end{tabular}


14010800 NORTH FORK WALLA WALLA RIVER NEAR MILTON-FREEWATER, OR

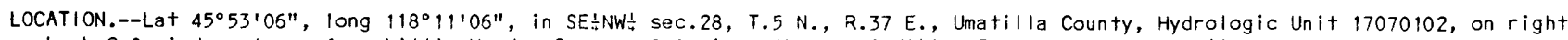
bank $2.8 \mathrm{mi}$ downstream from Little Meadow Canyon, $8.9 \mathrm{mi}$ southeast of Milton-Freewater, and at mile 5.6 .

DRAINAGE AREA.--34.4 $\mathrm{mi}^{2}$.

PERIOD OF RECORD.--October 1969 to Septenber 1932.

GAGE.--Water-stage recorder. Altitude of gage is $1,942 \mathrm{ft}$, from topographic map.

REMARKS.--No regulation or diversion above station.

AVERAGE DISCHARGE. --13 years, $54.3 \mathrm{ft}^{3} / \mathrm{s}, 21.44 \mathrm{in} / \mathrm{yr}, 39,340 \mathrm{acre}-\mathrm{f} t / \mathrm{yr}$.

EXTREMES FOR PERIOD OF RECORD.--Maximum discharge, 1,040 $\mathrm{ft} \mathrm{t}^{3} / \mathrm{s}$ Jan. 25, 1975, gage height, $6.58 \mathrm{ft}$, from rating curve extended above $400 \mathrm{ft}^{3} / \mathrm{s}$ on basis of discharge measurement at gage height $5.67 \mathrm{ft}$ and slope-area measurement at gage height $5.30 \mathrm{ft}$; minimum, 3.9 $\mathrm{ft}^{3} / \mathrm{s}$ July 19-21, 1979.

STATISTICAL SUMMARIES

MONTHLY AND ANNUAL MEAN DISCHARGES 1970-92

\begin{tabular}{|c|c|c|c|c|c|c|}
\hline MONTH & $\begin{array}{c}\text { MINIMUM } \\
\text { (CFS) }\end{array}$ & $\begin{array}{l}\text { MAXIMUM } \\
\text { (CFS) }\end{array}$ & $\begin{array}{l}\text { MEAN } \\
\text { (CFS) }\end{array}$ & $\begin{array}{l}\text { STAN- } \\
\text { DARD } \\
\text { DEVIA- } \\
\text { TION } \\
\text { (CFS) }\end{array}$ & $\begin{array}{l}\text { COEFFI- } \\
\text { CIENT OF } \\
\text { VARI- } \\
\text { ATION }\end{array}$ & $\begin{array}{c}\text { PERCENT } \\
\text { OF } \\
\text { ANNUAL } \\
\text { RUNOFF }\end{array}$ \\
\hline OCTOBER & 6.3 & 16 & 9.4 & 2.5 & .26 & 1.4 \\
\hline NOVEMBER & 8.3 & 85 & 26 & 24 & .92 & 4.0 \\
\hline DECEMBER & 11 & 170 & 62 & 47 & .75 & 9.5 \\
\hline JANUARY & 12 & 175 & 82 & 51 & .62 & 12.5 \\
\hline FEBRUARY & 15 & 181 & 85 & 41 & .49 & 12.9 \\
\hline MARCH & 43 & 236 & 96 & 52 & .54 & 14.6 \\
\hline APRIL & 55 & 222 & 119 & 42 & .35 & 18.2 \\
\hline MAY & 26 & 198 & 102 & 56 & .55 & 15.6 \\
\hline JUNE & 11 & 136 & 45 & 41 & .91 & 6.8 \\
\hline JULY & 6.7 & 21 & 13 & 4.7 & .36 & 2.0 \\
\hline AUGUST & 5.8 & 11 & 3.3 & 1.8 & .21 & 1.3 \\
\hline SEPTEMBER & 4.7 & 10 & 7.7 & 1.7 & .22 & 1.2 \\
\hline ANNUAL & 22 & 95 & 54 & 19 & .35 & 100 \\
\hline
\end{tabular}

MAGNITUDE AND PROBABILITY OF INSTANTANEOUS PEAK FLO'N BASED ON PERIOD OF RECORD 1970-82

DISCHARGE, IN CFS, FOR INDICATED RECURRENCE INTERVAL, IN YEARS, AND ANNUAL EXCEEOANCE PROBABILITY, IN PERCENT

\begin{tabular}{ccccccc}
1.25 & 2 & 5 & 10 & 25 & 50 & 100 \\
$80 \%$ & $50 \%$ & $20 \%$ & $10 \%$ & $4 \%$ & $2 \%$ & $1 \%$ \\
\hline 349 & 536 & 795 & 966 & 1180 & - & - \\
\hline
\end{tabular}

WEIGHTED SKEW $=-.285$
MAGNITUDE AND PROBABILITY OF ANNUAL LOW FLOW BASED ON PERIOD OF RECORD 1971-82

\begin{tabular}{|c|c|c|c|c|c|c|}
\hline $\begin{array}{l}\text { PERIOD } \\
\text { (CON- } \\
\text { SECU- }\end{array}$ & \multicolumn{6}{|c|}{$\begin{array}{l}\text { DISCHARGE, IN CFS, FOR INDICATED RECURRENCE } \\
\text { INTERVAL, IN YEARS, AND ANNUAL NON- } \\
\text { EXCEEDANCE PROBABILITY, IN PERCENT }\end{array}$} \\
\hline TIVE & 2 & 5 & 10 & 20 & 50 & 100 \\
\hline DAYS) & $50^{\circ}$ & $20 \%$ & $10 \%$ & $5 \%$ & $2^{a}$ & 10 \\
\hline 1 & 5.4 & 5.2 & 4.6 & 4.2 & -- & - \\
\hline 3 & 6.5 & 5.2 & 4.6 & 4.2 & -- & -- \\
\hline 7 & 6.5 & 5.3 & 4.8 & 4.3 & -- & -- \\
\hline 14 & 6.6 & 5.5 & 4.9 & 4.5 & -- & -- \\
\hline 30 & 6.9 & 5.7 & 5.1 & 4.7 & -- & -- \\
\hline 60 & 7.4 & 6.1 & 5.5 & 5.0 & -- & $\sim$ \\
\hline 90 & 7.9 & 6.6 & 5.9 & 5.4 & -- & - \\
\hline 120 & 8.7 & 7.3 & 6.6 & 6.1 & -- & -- \\
\hline 183 & 13 & 9.9 & 8.8 & 8.1 & -- & - \\
\hline
\end{tabular}

MAGNITUDE AND PROBABILITY OF ANNUAL HIGH FLOW BASED ON PERIOD OF RECORD 1970- 32

\begin{tabular}{|c|c|c|c|c|c|c|}
\hline $\begin{array}{l}\text { PERIOD } \\
\text { (CON- } \\
\text { SECU- }\end{array}$ & \multicolumn{6}{|c|}{$\begin{array}{c}\text { DISCHARGE, IN CFS, FOR IND ICATED RECURRENCE } \\
\text { INTERVAL, IN YEARS, AND ANNUAL } \\
\text { EXCEEDANCE PROBABILITY, IN PERCENT }\end{array}$} \\
\hline TIVE & 2 & 5 & 10 & 25 & 50 & 100 \\
\hline DAYS) & $50 \%$ & $20 \%$ & $10 \%$ & $4 \%$ & $2 q$ & $1 \%$ \\
\hline 1 & 393 & 549 & 645 & 761 & -- & -- \\
\hline 3 & 338 & 441 & 496 & 555 & -- & - \\
\hline 7 & 278 & 360 & 402 & 445 & -- & - \\
\hline 15 & 223 & 288 & 318 & 344 & -- & -- \\
\hline 30 & 161 & 214 & 244 & 275 & -- & -- \\
\hline 60 & 128 & 171 & 195 & 219 & -- & - \\
\hline 90 & 116 & 158 & 181 & 207 & -- & - \\
\hline
\end{tabular}

DURATION TABLE OF DAILY MEAN FLOW FOR PERIOD OF RECORD $1970-82$

DISCHARGE, IN CFS, WHICH WAS EQUALED OR EXCEEDED FOR INDICATED PERCENT OF TIME

\begin{tabular}{|c|c|c|c|c|c|c|c|c|c|c|c|c|c|c|}
\hline $5 \%$ & $10 \%$ & $15 \%$ & $20 \%$ & $25 \%$ & $30 s$ & $40 \%$ & $50 \%$ & $60 \%$ & $70 \%$ & $75^{\circ}$ & $80 \%$ & $85 \%$ & $90 \%$ & $95 \%$ \\
\hline 202 & 147 & 113 & 92 & 74 & 59 & 39 & 26 & 16 & 11 & 9.6 & 8.6 & 7.9 & 7.2 & 6.4 \\
\hline
\end{tabular}




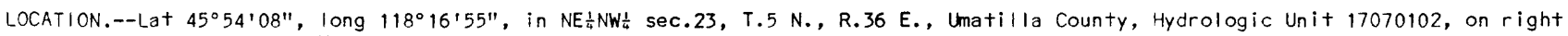
bank $5 \mathrm{mi}$ southeast of Milton, and at mile 1.2 .

DRAINAGE AREA. $--43.8 \mathrm{mi}^{2}$.

PERIOD OF RECORD.--January 1930 to October 1969. Monthly discharge only for some periods, published in WSP 1318.

GAGE. - Water-stage recorder. Datum of gage is 1,467 $\mathrm{t}+\mathrm{National}$ Geodetic Vertical Datum of 1929 (levels by U.S.G.S. National Mapping Division). Prior to Oct. 23, 1948, at several sites $0.7 \mathrm{mi}$ downstream at various datums. Oct. 23 , 1948 to Mar. 4 , 1968, at site $200 \mathrm{ft}$ downstream at same datum.

REMARKS.--No regulation. Diversions above station for irrigation.

AVERAGE DISCHARGE. --39 years, $47.3 \mathrm{ft} / \mathrm{s}, 34,270$ acre- $\mathrm{ft} / \mathrm{yr}$.

EXTREMES FOR PERIOD OF RECORD.--Maximum discharge, 2,050 $\mathrm{ft}^{3} / \mathrm{s} \mathrm{Jan.} \mathrm{30,} \mathrm{1965,} \mathrm{gage} \mathrm{height,} 8.05 \mathrm{ft}$; minimum, $0.90 \mathrm{ft} / \mathrm{s} \mathrm{Aug.} \mathrm{17,}$ 1955, Aug. 28, 29, 1961.

STATISTICAL SUMMARIES

MONTHLY AND ANNUAL MEAN DISCHARGES 1931-69

\begin{tabular}{|c|c|c|c|c|c|c|}
\hline MONTH & $\begin{array}{c}\text { MINIMUM } \\
\text { (CFS) }\end{array}$ & $\begin{array}{l}\text { MAXIMUM } \\
\text { (CFS) }\end{array}$ & $\begin{array}{l}\text { MEAN } \\
\text { (CFS) }\end{array}$ & $\begin{array}{l}\text { STAN- } \\
\text { DARD } \\
\text { DEVIA- } \\
\text { TION } \\
\text { (CFS) }\end{array}$ & $\begin{array}{l}\text { COEFFI- } \\
\text { CIENT OF } \\
\text { VARI- } \\
\text { ATION }\end{array}$ & $\begin{array}{c}\text { PERCENT } \\
\text { OF } \\
\text { ANNUAL } \\
\text { RUNOFF }\end{array}$ \\
\hline OCTOBER & 5.2 & 38 & 11 & 7.6 & .70 & 1.9 \\
\hline NOVEMBER & 7.2 & 107 & 27 & 20 & .76 & 4.7 \\
\hline DECEMBER & 9.0 & 195 & 52 & 42 & .82 & 9.1 \\
\hline JANUARY & 6.9 & 171 & 57 & 38 & .66 & 10.0 \\
\hline FEBRUARY & 13 & 143 & 66 & 34 & .52 & 11.6 \\
\hline MARCH & 25 & 230 & 82 & 38 & .47 & 14.4 \\
\hline APRIL & 24 & 204 & 120 & 39 & .32 & 21.1 \\
\hline MAY & 9.6 & 249 & 97 & 49 & .51 & 17.1 \\
\hline JUNE & 4.4 & 170 & 41 & 35 & .85 & 7.2 \\
\hline JULY & 2.3 & 28 & 7.9 & 6.0 & .77 & 1.4 \\
\hline AUGUST & 1.9 & 7.8 & 3.5 & 1.4 & .40 & .6 \\
\hline SEPTEMBER & 2.1 & 26 & 5.3 & 3.8 & .71 & .9 \\
\hline ANNUAL & 25 & 87 & 47 & 14 & .29 & 100 \\
\hline
\end{tabular}

MAGNITUDE AND PROBABILITY OF INSTANTANEOUS PEAK FLOW BASED ON PERIOD OF RECORD 1931-69

DISCHARGE, IN CFS, FOR INDICATEO RECURRENCE INTERVAL, IN YEARS, AND ANNUAL EXCEEDANCE PROBABILITY, IN PERCENT

\begin{tabular}{ccccccc}
1.25 & 2 & 5 & 10 & 25 & 50 & 100 \\
$80 \%$ & $50 \%$ & $20 \%$ & $10 \%$ & $4 \%$ & $2 \%$ & $1 \%$ \\
\hline 309 & 492 & 814 & 1080 & 1470 & 1800 & - \\
\hline
\end{tabular}

WEIGHTED SKEW $=.282$
MAGNITUDE AND PROBABILITY OF ANNUAL LOW FLOW BASED ON PERIOD OF RECORD 1932-69

\begin{tabular}{|c|c|c|c|c|c|c|}
\hline \multirow{3}{*}{$\begin{array}{l}\text { PERIOD } \\
\text { (CON- } \\
\text { SECU- } \\
\text { TIVE } \\
\text { DAYS) }\end{array}$} & \multicolumn{6}{|c|}{$\begin{array}{l}\text { DISCHARGE, IN CFS, FOR INDICATED RECURRENCE } \\
\text { INTERVAL, IN YEARS, AND ANNUAL NON- } \\
\text { EXCEEDANCE PROBABILITY, IN PERCENT }\end{array}$} \\
\hline & 2 & 5 & 10 & 20 & 50 & 100 \\
\hline & $50 \%$ & $20 \%$ & $10 \%$ & $5 \%$ & $2 \%$ & $1 \%$ \\
\hline 1 & 2.1 & 1.5 & 1. & 1.0 & .8 & -- \\
\hline 3 & 2.2 & 1.6 & 1. & 1. & 1.0 & -- \\
\hline 7 & 2.5 & 1.8 & 1. & 1. & 1.1 & -- \\
\hline 14 & 2.7 & 2.0 & 1. & 1.5 & 1.4 & - \\
\hline 30 & 2.9 & 2.2 & 1. & 1.8 & 1.6 & -- \\
\hline 60 & 3.4 & 2.6 & 2. & 2.0 & 1.7 & -- \\
\hline 90 & 4.2 & 3.1 & 2. & 2.3 & 1.9 & -- \\
\hline 120 & 5.4 & 4.0 & 3. & 3.1 & 2.7 & -- \\
\hline 183 & 11 & 7.2 & 5.8 & 4.9 & 4.1 & -- \\
\hline
\end{tabular}

MAGNITUDE AND PROBABILITY OF ANNUAL HIGH FLOW BASED ON PERIOD OF RECORD 1931-69

\begin{tabular}{|c|c|c|c|c|c|c|}
\hline \multirow{4}{*}{$\begin{array}{l}\text { PERIOD } \\
\text { (CON- } \\
\text { SECU- } \\
\text { TIVE } \\
\text { DAYS) }\end{array}$} & \multicolumn{6}{|c|}{$\begin{array}{c}\text { DISCHARGE, IN CFS, FOR INDICATED RECURRENCE } \\
\text { INTERVAL, IN YEARS, AND ANNUAL } \\
\text { EXCEEDANCE PROBABILITY, IN PERCENT }\end{array}$} \\
\hline & & & 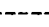 & - &.- & \\
\hline & 2 & 5 & 10 & 25 & 50 & 100 \\
\hline & $50 \%$ & $20 \%$ & $10 \%$ & $4 \%$ & $2 \%$ & $1 \%$ \\
\hline 1 & 368 & 596 & 811 & 1180 & 1530 & -- \\
\hline 3 & 292 & 448 & 600 & 867 & 1140 & -- \\
\hline 7 & 223 & 318 & 406 & 554 & 695 & -- \\
\hline 15 & 180 & 243 & 290 & 356 & 410 & - \\
\hline 30 & 150 & 194 & 222 & 258 & 284 & -- \\
\hline 60 & 127 & 160 & 180 & 202 & 216 & -- \\
\hline 90 & 110 & 139 & 155 & 173 & 185 & - \\
\hline
\end{tabular}

DURATION TABLE OF DAILY MEAN FLOW FOR PERIOD OF RECORD 1931-69

\begin{tabular}{|c|c|c|c|c|c|c|c|c|c|c|c|c|c|c|}
\hline & & & RGE, & CFS, & $\mathrm{CH}$ W & QUALE & OR EX & ED F & INDICA & PERCE & OF T & & & \\
\hline $5 \%$ & $10 \%$ & $15 \%$ & $20 \%$ & $25 \%$ & $30 \%$ & $40 \%$ & $50 \%$ & $60 \%$ & $70 \%$ & $75_{\%}^{\circ}$ & $80 \%$ & $85 \%$ & $90 \%$ & $95 \%$ \\
\hline 169 & 124 & 99 & 81 & 67 & 54 & 35 & 23 & 14 & 8.4 & 6.8 & 5.8 & 4.6 & 3.5 & 2.6 \\
\hline
\end{tabular}


14020000 UMATILLA RIVER ABOVE MEACHAM CREEK, NEAR GIBBON, OR

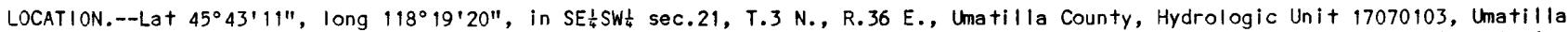
Indian Reservation, on right bank $0.8 \mathrm{mi}$ downstream from Ryan Creek, $2.2 \mathrm{mi}$ upstream from Meacham Creek, $2.5 \mathrm{mi}$ northeast of Gibbon, and at mile 83.1 .

DRAINAGE AREA. $--131 \mathrm{mi}^{2}$.

PERIOD OF RECORD.--April 1933 to September 1982.

GAGE.- Water-stage recorder. Datum of gage is 1,854.81 t+ National Geodetic Vertical Datum of 1929. Prior to June 27, 1939, at site $1 \mathrm{mi}$ downstream at datum $43.94 \mathrm{ft}$ lower.

REMARKS. - No regulation or diversion above station.

AVERAGE DISCHARGE. -49 years, $227 \mathrm{ft} / \mathrm{s}, 23.53 \mathrm{in} / \mathrm{yr}, 164,500 \mathrm{acre}-\mathrm{ft} / \mathrm{yr}$.

EXTREMES FOR PERIOD OF RECORD.--Maximum discharge, $5,930 \mathrm{ft}+\mathrm{s}$ Jan. 25,1975 , gage height, $9.18 \mathrm{t} t$, from rating curve extended above $3,500 \mathrm{ft}^{3} / \mathrm{s}$; maxinum gage height, $9.50 \mathrm{ft}$ Jan. 29, 1965; minimum discharge, $16 \mathrm{ft} / \mathrm{s}$ Nov. 9 , 1965, momentary regulation trom unknown source.

STATISTICAL SUMMARIES

MONTHLY AND ANNUAL MEAN DISCHARGES 1934-82

\begin{tabular}{|c|c|c|c|c|c|c|}
\hline MONTH & $\begin{array}{l}\text { MINIMUM } \\
\text { (CFS) }\end{array}$ & $\begin{array}{l}\text { MAXIMUHA } \\
\text { (CFS) }\end{array}$ & $\begin{array}{l}\text { MEAN } \\
\text { (CFS) }\end{array}$ & $\begin{array}{l}\text { STAN- } \\
\text { DARD } \\
\text { DEV } \mid A- \\
\text { TION } \\
\text { (CFS) }\end{array}$ & $\begin{array}{l}\text { COEFFI- } \\
\text { CIENT OF } \\
\text { VARI- } \\
\text { ATION }\end{array}$ & $\begin{array}{c}\text { PERCENT } \\
\text { OF } \\
\text { ANNUAL } \\
\text { RUNOFF }\end{array}$ \\
\hline OCTOBER & 39 & 169 & 59 & 25 & .42 & 2.2 \\
\hline NOVEMBER & 40 & 405 & 125 & 81 & .64 & 4.6 \\
\hline DECEMBER & 44 & 716 & 250 & 175 & .70 & 9.1 \\
\hline JANUARY & 46 & 656 & 267 & 163 & .61 & 9.8 \\
\hline FEBRUARY & 72 & 910 & 316 & 170 & .54 & 11.5 \\
\hline MARCH & 189 & 989 & 370 & 149 & .40 & 13.5 \\
\hline APRIL & 162 & 885 & 540 & 177 & .33 & 19.7 \\
\hline MAY & 67 & 1135 & 450 & 205 & .46 & 16.4 \\
\hline JUNE & 54 & 591 & 197 & 114 & .58 & 7.2 \\
\hline JULY & 40 & 110 & 66 & 19 & .28 & 2.4 \\
\hline AUGUST & 37 & 63 & 48 & 6.1 & .13 & 1.8 \\
\hline SEP TEMBER & 35 & 82 & 48 & 8.3 & .17 & 1.7 \\
\hline ANNUAL & 114 & 415 & 227 & 59 & .26 & 100 \\
\hline
\end{tabular}

MAGN I TUDE AND PROBAB ILITY OF INSTANTANEOUS PEAK FLOW BASED ON PERIOD OF RECORD 1934-82

DISCHARGE, IN CFS, FOR INDICATED RECURRENCE INTERVAL, IN YEARS, AND ANNUAL EXCEEDANCE PROBABILITY, IN PERCENT

\begin{tabular}{ccccccc}
1.25 & 2 & 5 & 10 & 25 & 50 & 100 \\
$80 \%$ & $50 \%$ & $20 \%$ & $10 \%$ & $4 \%$ & $2 \%$ & $1 \%$ \\
\hline 1400 & 2000 & 2910 & 3570 & 4480 & 5210 & 5970
\end{tabular}

WEIGHTED SKEW $=.221$
MAGNITUDE AND PROBABILITY OF ANNUAL LOW FLOW BASED ON PERIOD OF RECORD 1935-82

\begin{tabular}{ccccccc} 
PERIOD & \multicolumn{1}{c}{$\begin{array}{c}\text { DISCHARGE, IN CFS, FOR INDICATED RECURRENCE } \\
\text { (CON- }\end{array}$} \\
INTERVAL, IN YEARS, AND ANNUAL NON- \\
SECU- & \multicolumn{3}{c}{ EXCEEDANCE PROBABILITY, IN PERCENT } \\
TIVE & 2 & 5 & 10 & 20 & 50 & 100 \\
DAYS) & $50 \%$ & $20 \%$ & $10 \%$ & $5 \%$ & $2 \%$ & $1 \%$ \\
\hline 1 & 42 & 37 & 34 & 32 & 29 & 27 \\
3 & 42 & 37 & 35 & 32 & 30 & 28 \\
7 & 42 & 38 & 35 & 33 & 31 & 29 \\
14 & 43 & 39 & 37 & 35 & 32 & 31 \\
30 & 44 & 40 & 38 & 36 & 34 & 32 \\
60 & 46 & 42 & 40 & 38 & 35 & 34 \\
90 & 48 & 43 & 41 & 39 & 37 & 36 \\
120 & 51 & 45 & 43 & 41 & 39 & 37 \\
183 & 71 & 56 & 50 & 45 & 41 & 38
\end{tabular}

MAGNITUDE AND PROBABILITY OF ANNUAL HIGH FLOW BASED ON PERIOD OF RECORD 1934-82

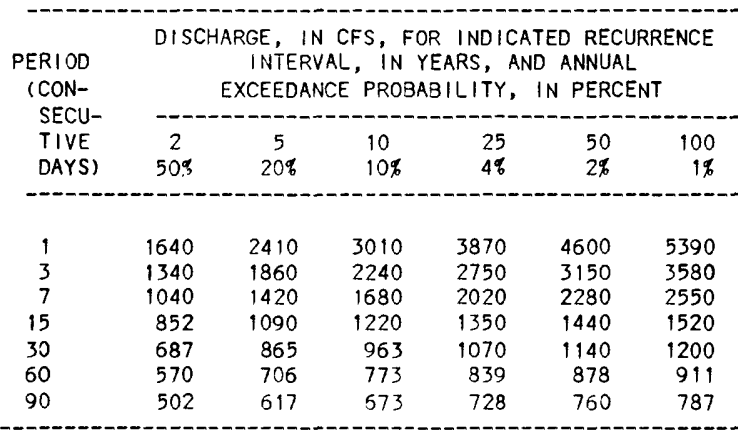

DURATION TABLE OF DAILY MEAN FLOW FOR PERIOD OF RECORD 1934-82

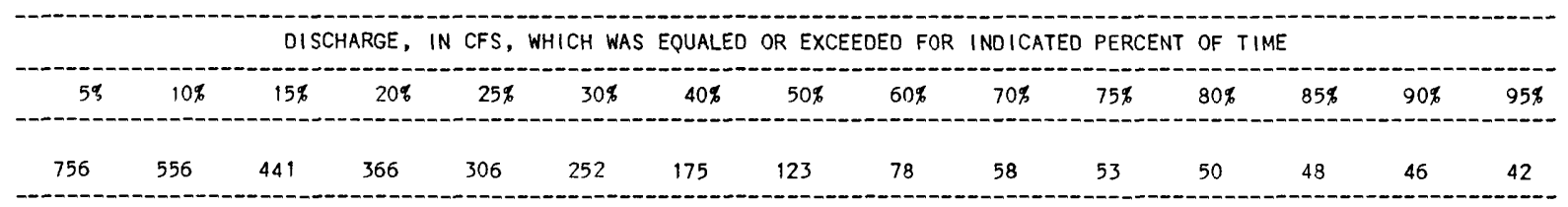




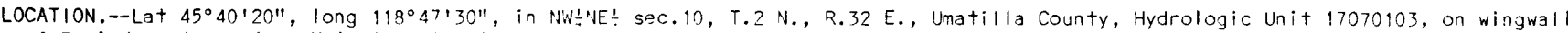
$0.3 \mathrm{mi}$ downstream from Main Street bridge at Pendleton, $1.5 \mathrm{ni}$ downstream from Wildhorse Creek, $2.8 \mathrm{mi}$ upstream from Mckay Creek, and at mile 55.2.

DRAINAGE AREA. $\rightarrow 637 \mathrm{mi}^{2}$.

PERIOD OF RECORD.--February 1891 to July 1892, May 1903 to June 1905 (gage heights and discharge measurements only June to December 1904), October 1934 to September 1982. Yonthly discharge only February 1991 to July 1892, published in WSP 1318.

GAGE.- Water-stage recorder. Datun of gage is $1,054.3 \mathrm{ft}$ National Geodetic Vertical Datun of 1929 (levels by Oregon Department of Transportation). Apr. 24 to Aug. 25, 1959, nonrecarding gage and Aug. 27, 1959, to Feb. 4, 1965, watar-stage recorder 3t 8th Street Bridge $0.7 \mathrm{mi}$ upstream at 1,067.01 $\mathrm{ft}$ Nationa! jaodetic Vertical Datum of 1929. Feb. 5 to Nov. 18, 1965, nonrecording gage at Main Street Bridge 1,600 ft upstream at different Jatum. Nov. 19, 1965, to Sept. 30, 1969, water-stage recorder at 3th Street Bridge $0.7 \mathrm{mi}$ upstream at 1,067.50 ft National Geodetic Vertical Datum of 1929. Nov. 19, 1965, to Mar. 28 , 1967, and Mar. 29, 1967, to Sept. 30, 1969 at 1,054.02 ft National Geodetic Vertical Datum of 1929. See wSP 1738 for history of changes prior to Apr. 24, 1959.

REMARKS.--No regulation. Many diversions for irrigation above station.

AVERAGE DISCHARGE. - 48 years (water years 1935-82), $503 \mathrm{ft}+3 / \mathrm{s}, 354,400$ acre $-\mathrm{ft} / \mathrm{yr}$.

EXTREMES FOR PERIOD OF RECORD.--Maximum discharge, 15,500 $\mathrm{ft}{ }^{3} / \mathrm{s} \mathrm{Jan.} \mathrm{30,} \mathrm{1965,} \mathrm{gage} \mathrm{height,} 9.40 \mathrm{ft}$, datun then in use; minimum, 10 $t t^{3} / \mathrm{s}$ July $13-16,1940$.

EXTREMES OUTSIDE PERIOD OF RECORD.--Maximum discharge, 17,000 $\mathrm{ft}^{3} / \mathrm{s}$ Dec. 14, 1882 (date and discharge from data furnished by Corps of Engineers). Flood of May 30, 31, 1906, reached a stage of $11.0 \mathrm{ft}, 1934-58$ site and datum, but before channel was improved, discharge, $15,500 \mathrm{ft}^{3} / \mathrm{s}$, estimated by Corps of Engineers.

STATISTICAL SUMMARIES

MONTHLY AND ANNUAL MEAN DISCHARGES 1935-82

\begin{tabular}{|c|c|c|c|c|c|c|}
\hline MONTH & $\begin{array}{l}\text { MINIMUM } \\
\text { (CFS) }\end{array}$ & $\begin{array}{l}\text { MAXIMUM } \\
\text { (CFS) }\end{array}$ & $\begin{array}{l}\text { MEAN } \\
\text { (CFS) }\end{array}$ & $\begin{array}{l}\text { STAN- } \\
\text { DARD } \\
\text { DEVIA- } \\
\text { TION } \\
\text { (CFS) }\end{array}$ & $\begin{array}{l}\text { COEFFI- } \\
\text { CIENT OF } \\
\text { VARI- } \\
\text { ATION }\end{array}$ & $\begin{array}{c}\text { PERCENT } \\
\text { OF } \\
\text { ANNUAL } \\
\text { RUNOFF }\end{array}$ \\
\hline OCTOBER & 37 & 246 & 74 & 42 & .58 & 1.2 \\
\hline NOVEMBER & 52 & 845 & 227 & 182 & .80 & 3.7 \\
\hline DECEMBER & 69 & 1786 & 581 & 456 & .78 & 9.6 \\
\hline JANUARY & 70 & 2088 & 658 & 477 & .72 & 10.9 \\
\hline FEBRUARY & 107 & 2375 & 943 & 455 & .54 & 13.9 \\
\hline MARCH & 410 & 2672 & 1003 & 465 & .46 & 16.6 \\
\hline APRIL & 299 & 2347 & 1321 & 469 & .36 & 21.8 \\
\hline MAY & 198 & 2519 & 876 & 458 & .52 & 14.5 \\
\hline JUNE & 64 & 892 & 319 & 199 & .62 & 5.3 \\
\hline JULY & 19 & 189 & 76 & 36 & .48 & 1.2 \\
\hline AUGUST & 17 & 76 & 38 & 12 & .31 & .5 \\
\hline SEPTEMBER & 23 & 85 & 45 & 12 & .28 & .7 \\
\hline ANNUAL & 190 & 934 & 503 & 157 & .31 & 100 \\
\hline
\end{tabular}

MAGNITUDE AND PROBABILITY OF INSTANTANEOUS PEAK FLOW BASED ON PERIOD OF RECORD 1935-82

\begin{tabular}{|c|c|c|c|c|c|c|}
\hline $\begin{array}{c}\text { DISCHARGE } \\
\text { YEARS, }\end{array}$ & $\begin{array}{l}\text { IN CF } \\
\text { ND ANI }\end{array}$ & $\begin{array}{l}\text { FOR } \\
\text { EXC }\end{array}$ & $\begin{array}{l}\text { ICATEL } \\
\text { ANCE }\end{array}$ & $\begin{array}{l}\text { ECURF } \\
\text { BABIL }\end{array}$ & $\begin{array}{ll}E & \text { INT } \\
Y, & \text { IN }\end{array}$ & $\begin{array}{l}\text { VAL, I } \\
\text { RCENT }\end{array}$ \\
\hline $\begin{array}{l}1.25 \\
80 \%\end{array}$ & $\stackrel{2}{50 s}$ & $\begin{array}{c}5 \\
20 \%\end{array}$ & $\begin{array}{l}10 \\
10 \%\end{array}$ & $\begin{array}{l}25 \\
4 \%\end{array}$ & $\begin{array}{l}50 \\
2 \%\end{array}$ & $\begin{array}{r}100 \\
16\end{array}$ \\
\hline 3430 & 5350 & 8510 & 10900 & 14400 & 17300 & 20400 \\
\hline
\end{tabular}

WEIGHTED SKEW $=.167$
MAGN ITUDE AND PROBABILITY OF ANNUAL LOW FLOW BASED ON PERIOD OF RECORD $1936-82$

\begin{tabular}{|c|c|c|c|c|c|c|}
\hline $\begin{array}{l}\text { PERIOD } \\
\text { (CON- }\end{array}$ & \multirow{2}{*}{\multicolumn{6}{|c|}{$\begin{array}{l}\text { DISCHARGE, IN CFS, FOR INDICATED RECURRENCE } \\
\text { INTERVAL, IN YEARS, AND ANNUAL NON- } \\
\text { EXCEEDANCE PROBABILLITY, IN PERCENT }\end{array}$}} \\
\hline SECU- & & & & & & \\
\hline TIVE & 2 & 5 & 10 & 20 & 50 & 100 \\
\hline DAYS) & $50 \%$ & $20 \%$ & $10 \%$ & $5 \%$ & $2 \%$ & 19 \\
\hline 1 & רט & & 7 & 18 & 17 & \\
\hline 1 & 21 & 21 & 17 & 15 & 13 & 11 \\
\hline 3 & 29 & 22 & 18 & 16 & 13 & 11 \\
\hline 7 & 30 & 24 & 20 & 18 & 15 & 13 \\
\hline 14 & 32 & 25 & 22 & 19 & 16 & 15 \\
\hline 30 & 33 & 27 & 23 & 21 & 19 & 17 \\
\hline 60 & 38 & 30 & 26 & 23 & 20 & 18 \\
\hline 90 & 43 & 34 & 30 & 27 & 24 & 22 \\
\hline 120 & 50 & 40 & 36 & 33 & 29 & 27 \\
\hline 183 & 95 & 66 & 54 & 46 & 39 & 35 \\
\hline
\end{tabular}

MAGNITUDE AND PROBABILITY OF ANNUAL HIGH FLOW BASED ON PERIOD OF RECORD 1935-82

\begin{tabular}{|c|c|c|c|c|c|c|}
\hline $\begin{array}{l}\text { PERIOD } \\
\text { (CON- } \\
\text { SECU- }\end{array}$ & \multicolumn{6}{|c|}{$\begin{array}{l}\text { DISCHARGE, IN CFS, FOR INDICATED RECURRENCE } \\
\text { INTERVAL, IN YEARS, AND ANNUAL } \\
\text { EXCEEDANCE PROBABILITY, IN PERCENT }\end{array}$} \\
\hline $\begin{array}{l}T \text { !VE } \\
\text { DAYS) }\end{array}$ & $\begin{array}{c}2 \\
50 \%\end{array}$ & $\begin{array}{c}5 \\
20 \%\end{array}$ & $\begin{array}{l}10 \\
10 \%\end{array}$ & $\begin{array}{l}25 \\
4 \%\end{array}$ & $\begin{array}{l}50 \\
2 \%\end{array}$ & $\begin{array}{r}100 \\
1 \%\end{array}$ \\
\hline $\begin{array}{l}1 \\
3 \\
7\end{array}$ & $\begin{array}{l}4240 \\
3420 \\
2610\end{array}$ & $\begin{array}{l}6480 \\
4940 \\
3670\end{array}$ & $\begin{array}{l}8200 \\
6040 \\
4420\end{array}$ & $\begin{array}{r}10600 \\
7550 \\
5430\end{array}$ & $\begin{array}{r}12700 \\
8750 \\
6210\end{array}$ & $\begin{array}{r}14900 \\
10000 \\
7030\end{array}$ \\
\hline $\begin{array}{l}15 \\
30 \\
60 \\
90\end{array}$ & $\begin{array}{l}2070 \\
1680 \\
1380 \\
1200\end{array}$ & $\begin{array}{l}2770 \\
2190 \\
1780 \\
1540\end{array}$ & $\begin{array}{l}3200 \\
2470 \\
1980 \\
1710\end{array}$ & $\begin{array}{l}3710 \\
2770 \\
2180 \\
1880\end{array}$ & $\begin{array}{l}4060 \\
2960 \\
2310 \\
1990\end{array}$ & $\begin{array}{l}4390 \\
3130 \\
2410 \\
2070\end{array}$ \\
\hline
\end{tabular}

DURATION TABLE OF DAILY MEAN FLOW FOR PERIOD OF RECORD 1935-82

\begin{tabular}{|c|c|c|c|c|c|c|c|c|c|c|c|c|c|c|}
\hline $5 \%$ & $10 \%$ & $15 \%$ & $20 \%$ & $25 \%$ & $30 \%$ & $40 \%$ & $50 \%$ & $60 \%$ & 70, & $75 \%$ & $80 \%$ & $85 \%$ & $90 \%$ & $95 \%$ \\
\hline 1840 & 1330 & 1050 & 861 & 709 & 577 & 370 & 224 & 116 & 70 & 61 & 53 & 45 & 39 & 31 \\
\hline
\end{tabular}


14022000 UMATILLA RIVER ABOVE MCKAY CREEK, NEAR PENDLETON, OR

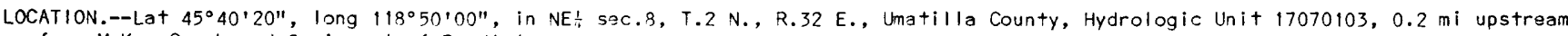
from Mckay Craek and $2 \mathrm{mi}$ west of Pendieton.

DRAINAGE AREA.--700 $\mathrm{mi}^{2}$, approximately.

PERIOD OF RECORD.--January 1923 to September 1934.

GAGE.--Water-stage recorder. Datum of gage is $997.68 \mathrm{ft}$ National Geodetic Vertical Datum of 1929. May 8 to Oct. 12 , 1921 , staff gage, and Oct. 13, 1921, to Mar. 3, 1923, water-stage racorder, $200 \mathrm{ft}$ downstream at different datum. Mar. 6, 1923, to Sept. 30, 1930, at about described datum, and Oct. 1, 1930, to Mar. 31, 1931, at datum about $2.0 \mathrm{ft}$ higher.

REMARKS.--Many diversions for irrigation above station. Slight regulation caused by mills upstream.

AVERAGE DISCHARGE.--11 years (water years 1924-34), $451 \mathrm{ft}^{3} / \mathrm{s}$.

EXTREMES FOR PERIOD OF RECORD.--Maximum discharge, 13,500 $\mathrm{ft}^{3} / \mathrm{s}$ (estimated), Apr. 1, 1931; minimum, $7 \mathrm{ft}{ }^{3} / \mathrm{s}$ Aug. 14, 1924, gage height, $1.87 \mathrm{ft}$.

STATISTICAL SUMMARIES

MONTHLY AND ANNUAL MEAN DISCHARGES 1924-34

\begin{tabular}{|c|c|c|c|c|c|c|}
\hline MONTH & $\begin{array}{l}\text { MINIMUM } \\
\text { (CFS) }\end{array}$ & $\begin{array}{c}\text { MAXIMUM } \\
\text { (CFS) }\end{array}$ & $\begin{array}{l}\text { MEAN } \\
\text { (CFS) }\end{array}$ & $\begin{array}{l}\text { STAN- } \\
\text { DARD } \\
\text { DEVIA- } \\
\text { TION } \\
\text { (CFS) }\end{array}$ & $\begin{array}{l}\text { COEFFI- } \\
\text { CIENT OF } \\
\text { VARI- } \\
\text { ATION }\end{array}$ & $\begin{array}{c}\text { PERCENT } \\
\text { OF } \\
\text { ANNUAL } \\
\text { RUNOFF }\end{array}$ \\
\hline \multicolumn{7}{|c|}{ 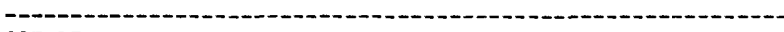 } \\
\hline OCTOBER & 47 & 451 & 100 & 119 & 1.19 & 1.8 \\
\hline NOVEMBER & 57 & 1466 & 293 & 406 & 1.39 & 5.4 \\
\hline DECEMBER & 79 & 886 & 413 & 280 & .68 & 7.6 \\
\hline JANUARY & 85 & 1532 & 564 & 433 & .77 & 10.3 \\
\hline FEBRUARY & 164 & 1494 & 767 & 416 & .54 & 14.1 \\
\hline MARCH & 510 & 2470 & 1125 & 497 & .44 & 20.6 \\
\hline APRIL & 452 & 2022 & 1199 & 516 & .43 & 22.0 \\
\hline MAY & 104 & 1680 & 660 & 494 & .75 & 12.1 \\
\hline JUNE & 50 & 631 & 223 & 179 & .80 & 4.1 \\
\hline JULY & 23 & 77 & 44 & 19 & .43 & .8 \\
\hline AUGUST & 14 & 32 & 24 & 5.5 & .23 & .4 \\
\hline SEPTEMBER & 27 & 129 & 46 & 29 & .63 & .8 \\
\hline ANNUAL & 293 & 746 & 453 & 154 & .34 & 100 \\
\hline
\end{tabular}

MAGNITUDE AND PROBABILITY OF INSTANTANEOUS PEAK FLOW BASED ON PERIOD OF RECORD 1924-34

DISCHARGE, IN CFS, FOR INDICATED RECURRENCE INTERVAL, IN YEARS, AND ANNUAL EXCEEDANCE PROBABILITY, IN PERCENT

\begin{tabular}{ccccccc}
1.25 & 2 & 5 & 10 & 25 & 50 & 100 \\
$80 \%$ & $50 \%$ & $20 \%$ & $10 \%$ & $4 \%$ & $2 \%$ & 13 \\
\hline 3470 & 5190 & 3050 & 10300 & - & - & - \\
\hline
\end{tabular}

WEIGHTED SKEW $=.310$

MAGNITUDE AND PROBABILITY OF ANNUAL LOW FLOW BASED ON PERIOD OF RECORD 1925-34

\begin{tabular}{|c|c|c|c|c|c|c|}
\hline $\begin{array}{l}\text { PERIOD } \\
\text { (CON- }\end{array}$ & \multicolumn{6}{|c|}{$\begin{array}{l}\text { DISCHARGE, IN CFS, FOR INDICATED RECURRENCE } \\
\text { INTERVAL, IN YEARS, AND ANNUAL NON- } \\
\text { EXCEEDANCE PROBABILITY, IN PERCENT }\end{array}$} \\
\hline SECU- & & & -1 & - & 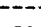 & \\
\hline $\begin{array}{l}\text { TIVE } \\
\text { DAYS) }\end{array}$ & $\begin{array}{c}2 \\
50 \%\end{array}$ & $\begin{array}{c}5 \\
20 \%\end{array}$ & $\begin{array}{l}10 \\
10 \%\end{array}$ & $\begin{array}{l}20 \\
5 q\end{array}$ & $\begin{array}{l}50 \\
29\end{array}$ & $\begin{array}{r}100 \\
1 \%\end{array}$ \\
\hline 1 & 18 & 15 & 13 & 11 & -- & -- \\
\hline 3 & 19 & 16 & 14 & 13 & -- & -- \\
\hline 7 & 20 & 17 & 15 & 14 & -. & -- \\
\hline 14 & 22 & 18 & 16 & 14 & -- & -- \\
\hline 30 & 24 & 20 & 17 & 15 & -- & -- \\
\hline 50 & 28 & 23 & 20 & 18 & -- & -- \\
\hline 90 & 33 & 27 & 25 & 23 & -- & -- \\
\hline 120 & 38 & 33 & 32 & 32 & -- & - \\
\hline 183 & 66 & 50 & 46 & 44 & -- & -- \\
\hline
\end{tabular}

MAGNITUDE AND PROBABILITY OF ANNUAL HIGH FLOW BASED ON PERIOD OF RECORD 1924-34

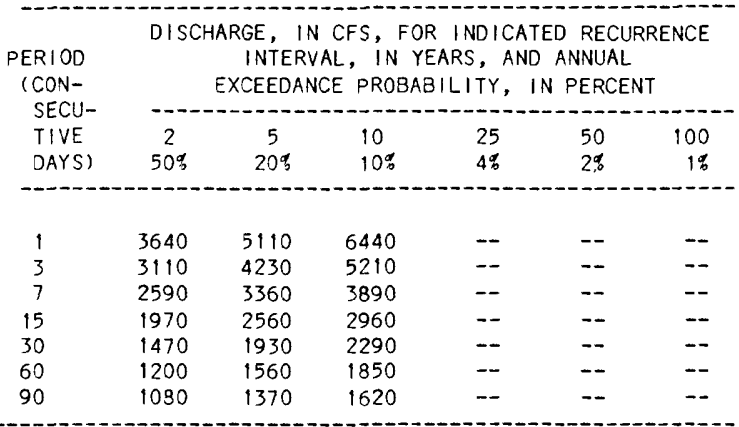

DURATION TABLE OF DAILY MEAN FLOW FOR PERIOD OF RECORD 1924-34

DISCHARGE, IN CFS, WHICH WAS EQUALED OR EXCEEDED FOR INDICATED PERCENT OF TIME

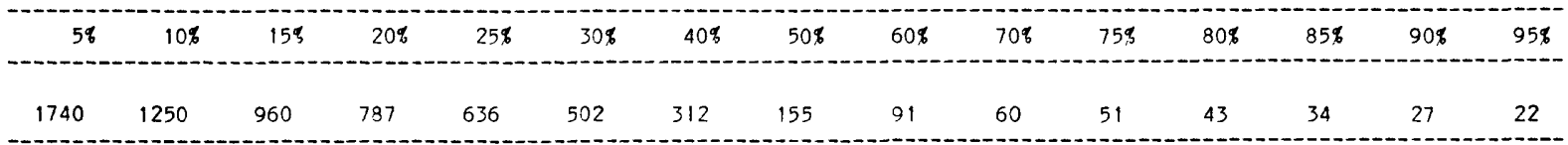




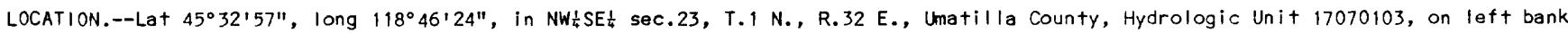
$500 \mathrm{ft}$ upstream from county road bridge, $5.5 \mathrm{mi}$ northeast of Pilot Rock, and at mile 8.2 .

DRAINAGE AREA. $--180 \mathrm{mi}^{2}$.

PERIOD OF RECORD.--May to August 1921, October 1926 to June 1928, December 1928 to July 1929 , October 1929 to September 1982.

Monthly discharge only for some periods, published in WSP 1318.

GAGE.--Water-stage recorder. Datum of gage is 1,343.60 ft National Geodetic Vertical Datum of 1929 . See WSP 1318 or 1738 for

history of changes prior to Apr. 9, 1941. Apr. 9, 1941, to July 24, 1963, at site 1,000 ft downstream at datum 7.92 ft lower.

REMARKS.--No regulation. Many small diversions for irrigation above station.

AVERAGE DISCHARGE. -54 years (water years $1927,1930-82$ ), $101 \mathrm{ft}^{3} / \mathrm{s}, 73,170 \mathrm{acre}-\mathrm{ft} / \mathrm{yr}$.

EXTREMES FOR PERIOD DF RECORD.--Maximum discharge, 7,400 $\mathrm{ft} / \mathrm{s}$ Jan. 30, 1965, gage height, $8.40 \mathrm{ft}$; no $f$ low at $+\mathrm{times}$.

STATISTICAL SUMMARIES

MONTHLY AND ANNUAL MEAN DISCHARGES 1927-82

\begin{tabular}{|c|c|c|c|c|c|c|}
\hline MONTH & $\begin{array}{l}\text { MINIMUM } \\
\text { (CFS) }\end{array}$ & $\begin{array}{l}\text { MAXIMUM } \\
\text { (CFS) }\end{array}$ & $\begin{array}{l}\text { MEAN } \\
\text { (CFS) }\end{array}$ & $\begin{array}{l}\text { STAN- } \\
\text { DARD } \\
\text { DEVIA- } \\
\text { TION } \\
\text { (CFS) }\end{array}$ & $\begin{array}{l}\text { COEFFI- } \\
\text { CIENT OF } \\
\text { VARI- } \\
\text { ATION }\end{array}$ & $\begin{array}{c}\text { PERCENT } \\
\text { OF } \\
\text { ANNUAL } \\
\text { RUNOFF }\end{array}$ \\
\hline OCTOBER & 0.0 & 97 & 7.2 & 17 & 2.32 & .6 \\
\hline NOVEMBER & .5 & 257 & 40 & 53 & 1.33 & 3.3 \\
\hline DECEMBER & 3.8 & 460 & 119 & 109 & .92 & 9.7 \\
\hline JANUARY & 5.0 & 716 & 161 & 140 & .87 & 13.2 \\
\hline FEBRUARY & 11 & 502 & 192 & 113 & .59 & 15.7 \\
\hline MARCH & 67 & 757 & 261 & 130 & .50 & 21.3 \\
\hline APRIL & 42 & 782 & 278 & 135 & .49 & 22.7 \\
\hline MAY & 8.4 & 500 & 121 & 97 & .80 & 9.9 \\
\hline JUNE & 1.0 & 173 & 37 & 46 & 1.26 & 3.0 \\
\hline JULY & 0.0 & 45 & 4.6 & 7.1 & 1.55 & .4 \\
\hline AUGUST & 0.0 & 3.7 & .7 & .9 & 1.27 & .1 \\
\hline SEPTEMBER & 0.0 & 28 & 1.8 & 4.1 & 2.25 & .1 \\
\hline ANNUAL & 27 & 188 & 101 & 40 & .39 & 100 \\
\hline
\end{tabular}

MAGNITUDE AND PROBABILITY OF INSTANTANEOUS PEAK FLOW BASED ON PERIOD OF RECORD 1927-82

DISCHARGE, IN CFS, FOR INDICATED RECURRENCE INTERVAL, IN YEARS, AND ANNUAL EXCEEDANCE PROBABILITY, IN PERCENT

\begin{tabular}{|c|c|c|c|c|c|c|}
\hline $\begin{array}{l}1.25 \\
80 \%\end{array}$ & $\begin{array}{c}2 \\
50 \%\end{array}$ & $\begin{array}{c}5 \\
20 \%\end{array}$ & $\begin{array}{l}10 \\
10 \%\end{array}$ & $\begin{array}{l}25 \\
48\end{array}$ & $\begin{array}{l}50 \\
2 \%\end{array}$ & $\begin{array}{r}100 \\
1 \%\end{array}$ \\
\hline 775 & 1350 & 2380 & 3210 & 4440 & 5480 & 6630 \\
\hline
\end{tabular}

WEIGHTED SKEW $=.071$
MAGNITUDE AND PROBABILITY OF ANNUAL LOW FLOW BASED ON PERIOD OF RECORD 1928-82

\begin{tabular}{|c|c|c|c|c|c|c|}
\hline $\begin{array}{l}\text { PERIOD } \\
\text { (CON- }\end{array}$ & \multicolumn{6}{|c|}{$\begin{array}{l}\text { DISCHARGE, IN CFS, FOR INDICATED RECURRENCE } \\
\text { INTERVAL, IN YEARS, AND ANNUAL NON- } \\
\text { EXCEEDANCE PROBABILITY, IN PERCENT }\end{array}$} \\
\hline SECU- & & -- & $-\cdots$ & -- & - & \\
\hline TIVE & 2 & 5 & 10 & 20 & 50 & 100 \\
\hline DAYS) & $50 \%$ & $20 \%$ & $10 \%$ & $5 \%$ & $2 \%$ & $1 \%$ \\
\hline 1 & 3 & 1 & 1 & 0.0 & 0.0 & 0.0 \\
\hline 3 & .3 & .1 & .1 & .1 & 0.0 & 0. \\
\hline 7 & .3 & .1 & .1 & .1 & .1 & 0.0 \\
\hline 14 & .4 & .2 & .1 & .1 & .1 & .1 \\
\hline 30 & .4 & .2 & .1 & .1 & .1 & • \\
\hline 60 & .6 & .3 & .2 & .1 & .1 & $\bullet$ \\
\hline 90 & 1.0 & .4 & .3 & .2 & .1 & .1 \\
\hline 120 & 1.5 & .5 & .4 & .3 & .1 & .1 \\
\hline 183 & 7.0 & 2.5 & 1.4 & .8 & .4 & . \\
\hline
\end{tabular}

MAGNITUDE AND PROBABILITY OF ANNUAL HIGH FLOW BASED ON PERIOD OF RECORD 1927-82

\begin{tabular}{|c|c|c|c|c|c|c|}
\hline $\begin{array}{l}\text { PERIOD } \\
\text { (CON- } \\
\text { SECU- }\end{array}$ & \multicolumn{6}{|c|}{$\begin{array}{l}\text { DISCHARGE, IN CFS, FOR INDICATED RECURRENCE } \\
\text { INTERVAL, IN YEARS, AND ANNUAL } \\
\text { EXCEEDANCE PROBAB ILITY, IN PERCENT }\end{array}$} \\
\hline TIVE & 2 & 5 & 10 & 25 & 50 & 100 \\
\hline DAYS) & $50 \%$ & $20 \%$ & $10 \%$ & $4 \%$ & $2 \%$ & 18 \\
\hline 1 & 1040 & 1700 & 2190 & 2880 & 3440 & 4030 \\
\hline 3 & 829 & 1330 & 1720 & 2300 & 2790 & 3330 \\
\hline 7 & 630 & 958 & 1200 & 1530 & 1800 & 2080 \\
\hline 15 & 477 & 597 & 846 & 1040 & 1180 & 1320 \\
\hline 30 & 382 & 535 & 627 & 731 & 802 & 868 \\
\hline 60 & 313 & 430 & 494 & 559 & 600 & 635 \\
\hline 90 & 268 & 364 & 415 & 467 & 498 & 525 \\
\hline
\end{tabular}

DURATION TABLE OF DAILY MEAN FLOW FOR PERIOD OF RECORD $1927-82$

DISCHARGE, IN CFS, WHICH WAS EQUALED OR EXCEEDED FOR INDICATED PERCENT OF TIME

\begin{tabular}{|c|c|c|c|c|c|c|c|c|c|c|c|c|c|c|}
\hline $5 \%$ & $10 \%$ & $15 \%$ & $20 \%$ & $25 \%$ & $30 \%$ & $40 \%$ & $50 \%$ & $60 \%$ & $70 \%$ & $75 \%$ & $80 \%$ & $85 \%$ & $90 \%$ & $95 \%$ \\
\hline 434 & 302 & 225 & 172 & 134 & 103 & 58 & 25 & 9.2 & 3.6 & 2.2 & 1.2 & .6 & .2 & 0.0 \\
\hline
\end{tabular}


14025000 BIRCH CREEK AT RIETH, OR

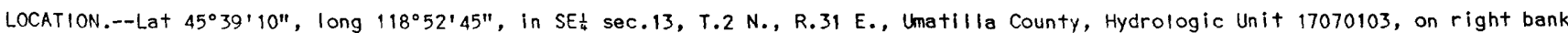
$600 \mathrm{ft}$ downstream from road bridge, $0.3 \mathrm{mi}$ southwest of Rieth, and at mile 0.2 .

DRAINAGE AREA. $--291 \mathrm{mi}^{2}$.

PERIOD OF RECORD. --May to August 1921. March to July 1922, April to September 1923, April to September 1927, January to June 1928, November 1928 to August 1929, October 1929 to September 1976. Monthly discharge only for some periods, published in WSP 1318 .

GAGE.--Water-stage recorder. Datum of gage is $951.04 \mathrm{ft}$ National Geodetic vertical Datum of 1929 . See WSP 1738 for history of changes prior to July $24,1957$.

REMARKS.--No regulation. Many diversions for irrigation above station.

AVERAGE DISCHARGE.--47 years (water years $1930-76$ ), $48.9 \mathrm{ft} / \mathrm{s}, 35,430$ acre- $\mathrm{ft} / \mathrm{yr}$.

EXTREMES FOR PERIOD OF RECORD.--Maximum discharge, 2,200 $\mathrm{ft}^{3} / \mathrm{s} \mathrm{Jan.} \mathrm{30,} \mathrm{1965,} \mathrm{gage} \mathrm{height,} 6.40 \mathrm{ft}$; no $\mathrm{flow}$ at $+\mathrm{imes}$

STATISTICAL SUMMARIES

MONTHLY AND ANNUAL MEAN DISCHARGES 1930-76

\begin{tabular}{|c|c|c|c|c|c|c|}
\hline MONTH & $\begin{array}{l}\text { MINIMUM } \\
\text { (CFS) }\end{array}$ & $\begin{array}{l}\text { MAXIMUM } \\
\text { (CFS) }\end{array}$ & $\begin{array}{l}\text { MEAN } \\
\text { (CFS) }\end{array}$ & $\begin{array}{l}\text { STAN- } \\
\text { DARD } \\
\text { DEVIA- } \\
\text { TION } \\
\text { (CFS) }\end{array}$ & $\begin{array}{l}\text { COEFFI- } \\
\text { CIENT OF } \\
\text { VARI- } \\
\text { ATION }\end{array}$ & $\begin{array}{c}\text { PERCENT } \\
\text { OF } \\
\text { ANNUAL } \\
\text { RUNOFF }\end{array}$ \\
\hline OCTOBER & 0.0 & 48 & 3.6 & 7.6 & 2.14 & .6 \\
\hline NOVEMBER & 0.0 & 96 & 15 & 19 & 1.28 & 2.5 \\
\hline DECEMBER & 0.0 & 172 & 44 & 48 & 1.08 & 7.5 \\
\hline FEBRUARY & 12 & 268 & 79 & 58 & .74 & 13.3 \\
\hline MARCH & 18 & 355 & 106 & 61 & .57 & 18.0 \\
\hline $\begin{array}{l}\text { APRIL } \\
\text { MAY }\end{array}$ & $\begin{array}{r}3.3 \\
.1\end{array}$ & $\begin{array}{l}315 \\
434\end{array}$ & $\begin{array}{r}155 \\
97\end{array}$ & $\begin{array}{l}76 \\
88\end{array}$ & $\begin{array}{l}.49 \\
.91\end{array}$ & $\begin{array}{l}26.3 \\
16.4\end{array}$ \\
\hline JUNE & 0.0 & 161 & 25 & 33 & 1.31 & 4.3 \\
\hline JULY & 0.0 & 27 & 1.6 & 4.2 & 2.60 & .3 \\
\hline AUGUST & 0.0 & 5.4 & .3 & .8 & 2.67 & .1 \\
\hline SEPTEMBER & 0.0 & 9.5 & .7 & 2.0 & 2.93 & .1 \\
\hline ANNUAL & 11 & 109 & 49 & 26 & .54 & 100 \\
\hline
\end{tabular}

MAGN ITUDE AND PROBABILITY OF INSTANTANEOUS PEAK FLOW BASED ON PERIOD OF RECORD 1930-76

\begin{tabular}{|c|c|c|c|c|c|c|}
\hline \multicolumn{7}{|c|}{$\begin{array}{l}\text { DISCHARGE, IN CFS, FOR INDICATED RECURRENCE INTERVAL, IN } \\
\text { YEARS, AND ANNUAL EXCEEDANCE PROBABILITY, IN PERCENT }\end{array}$} \\
\hline$\frac{1.25}{80 \%}$ & $\stackrel{2}{50 \%}$ & $\stackrel{5}{20 \%}$ & $\begin{array}{l}10 \\
10 \%\end{array}$ & 25 & $\begin{array}{l}50 \\
2 \%\end{array}$ & $\begin{array}{r}100 \\
1 \%\end{array}$ \\
\hline 293 & 536 & 976 & 1330 & 1850 & 2290 & 2780 \\
\hline
\end{tabular}

WEIGHTED SKEW $=-.029$

MAGNITUDE AND PROBABILITY OF ANNUAL LOW FLOW BASED ON PERIOD OF RECORD 1931-76

\begin{tabular}{|c|c|c|c|c|c|c|}
\hline \multirow{3}{*}{$\begin{array}{l}\text { PERIOD } \\
\text { (CON- } \\
\text { SECU- } \\
\text { TIVE } \\
\text { DAYS) }\end{array}$} & \multirow{2}{*}{\multicolumn{6}{|c|}{$\begin{array}{l}\text { DISCHARGE, IN CFS, FOR INDICATED RECURRENCE } \\
\text { INTERVAL, IN YEARS, AND ANNUAL NON- } \\
\text { EXCEEDANCE PROBABILITY, IN PERCENT }\end{array}$}} \\
\hline & & & & & & \\
\hline & $\begin{array}{c}2 \\
50 \%\end{array}$ & $\begin{array}{c}5 \\
20 \%\end{array}$ & $\begin{array}{l}10 \\
10 \%\end{array}$ & $\begin{array}{l}20 \\
5 \%\end{array}$ & $\begin{array}{l}50 \\
2 \%\end{array}$ & $\begin{array}{r}100 \\
1 \%\end{array}$ \\
\hline & & & & & & \\
\hline 1 & 0.0 & 0.0 & 0.0 & 0.0 & 0.0 & \\
\hline 3 & .1 & .1 & 0.0 & 0.0 & 0.0 & \\
\hline 7 & .1 & .1 & .1 & 0.0 & 0.0 & \\
\hline 30 & .1 & .1 & .1 & .1 & 0.0 & \\
\hline 60 & .1 & .1 & .1 & .1 & .1 & 0. \\
\hline 90 & .2 & .1 & .1 & .1 & .1 & \\
\hline 120 & .5 & .2 & .1 & .1 & .1 & \\
\hline 183 & 3.3 & .8 & .3 & .1 & .1 & \\
\hline
\end{tabular}

MAGNITUDE AND PROBABILITY OF ANNUAL HIGH FLOW BASED ON PERIOD OF RECORD $1930-76$

\begin{tabular}{|c|c|c|c|c|c|c|}
\hline \multirow{2}{*}{$\begin{array}{l}\text { PERIOD } \\
\text { (CON- } \\
\text { SECU- } \\
\text { TIVE } \\
\text { DAYS) }\end{array}$} & \multicolumn{6}{|c|}{$\begin{array}{l}\text { DISCHARGE, IN CFS, FOR INDICATED RECURRENCE } \\
\text { INTERVAL, IN YEARS, AND ANNUAL } \\
\text { EXCEEDANCE PROBABILITY, IN PERCENT }\end{array}$} \\
\hline & $\begin{array}{c}2 \\
50 \%\end{array}$ & $\begin{array}{c}5 \\
20 \%\end{array}$ & $\begin{array}{l}10 \\
10 \%\end{array}$ & $\begin{array}{l}25 \\
4 \%\end{array}$ & $\begin{array}{l}50 \\
2 \%\end{array}$ & $\begin{array}{r}100 \\
1 \%\end{array}$ \\
\hline \multicolumn{7}{|c|}{ (-20-1 } \\
\hline 1 & 412 & 692 & 906 & 1210 & 1450 & 1710 \\
\hline 3 & 351 & 559 & 710 & 914 & 1070 & 1240 \\
\hline 7 & 294 & 457 & 570 & 719 & 832 & 948 \\
\hline 15 & 238 & 357 & 436 & 535 & 608 & 680 \\
\hline 30 & 192 & 281 & 334 & 397 & 439 & 478 \\
\hline 60 & 152 & 222 & 262 & 307 & 337 & 364 \\
\hline 90 & 128 & 188 & 223 & 261 & 287 & 309 \\
\hline
\end{tabular}

DURATION TABLE OF DAILY MEAN FLOW FOR PERIOD OF RECORD 1930-76

DISCHARGE, IN CFS, WHICH WAS EQUALED OR EXCEEDED FOR INDICATED PERCENT OF TIME

\begin{tabular}{|c|c|c|c|c|c|c|c|c|c|c|c|c|c|c|}
\hline $5 \%$ & $10 \%$ & $15 \%$ & $20 \%$ & $25 \%$ & $30 \%$ & $40 \%$ & $50 \%$ & $60 \%$ & $70 \%$ & $75 \%$ & $80 \%$ & $85 \%$ & $90 \%$ & $95 \%$ \\
\hline 216 & 152 & 113 & 87 & 66 & 48 & 23 & 13 & 3.1 & .3 & .2 & .1 & .1 & 0.0 & 0 \\
\hline
\end{tabular}




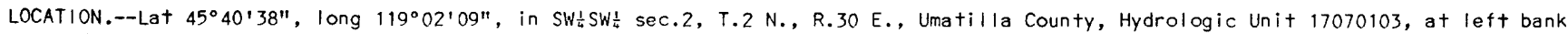
on downstream side of highway bridge, $0.5 \mathrm{mi}$ nor theast of Yoakum, $2.5 \mathrm{mi}$ downstream from abandoned Furnish Reservoir, $12.0 \mathrm{mi}$ downstream from Birch Creek, and at mile 37.7 .

DRAINAGE AREA.--1,280 $\mathrm{mi}^{2}$, approximately.

PERIOD OF RECORD.--May 1903 to September 1982. Records published as "above Furnish Reservoir, near Yoakum" October 1916 to September 1934 are equivalent.

GAGE.--Water-stage recorder. Datum of gage is $768.21 \mathrm{tt}$ National Geodetic Vertical Datum of 1929 . See WSP 1318 or 1738 for history of changes prior to Oct. $21,1948$.

REMARKS.--Slight regulation by Furnish Reservoir 1910-34, capacity, 3,900 acre-ft prior to filling with silt. Flow regulated to some extent since 1927 by Mckay Reservoir. Many diversions for irrigation above station.

AVERAGE DISCHARGE.- 79 years, $675 \mathrm{ft}^{3} / \mathrm{s}, 489,000$ acre- $\mathrm{ft} / \mathrm{yr}$.

EXTREMES FOR PERIOD OF RECORD.--Maximum discharge, 20,000 $\mathrm{ft}+\mathrm{s}$ May 30, 1906, gage height, about $15.0 \mathrm{ft}$, site and datum then in use, from floodmarks, from rating curve extended above $6,600 \mathrm{ft} / \mathrm{s} ; \mathrm{minimum}, 12 \mathrm{ft} 3 / \mathrm{s} \mathrm{Aug.} \mathrm{10-12,} \mathrm{1908,} \mathrm{Aug.} 4$, 1910.

STATISTICAL SUMMARIES (BEFORE THE CONSTRUCTION OF MCKAY RESERVOIR)

MONTHLY AND ANNUAL MEAN DISCHARGES 1904-26

\begin{tabular}{|c|c|c|c|c|c|c|}
\hline MONTH & $\begin{array}{l}\text { MINIMUM } \\
\text { (CFS) }\end{array}$ & $\begin{array}{l}\text { MAXIMUM } \\
\text { (CFS) }\end{array}$ & $\begin{array}{l}\text { MEAN } \\
\text { (CFS) }\end{array}$ & $\begin{array}{l}\text { STAN- } \\
\text { DARD } \\
\text { DEV!A- } \\
\text { TION } \\
\text { (CFS) }\end{array}$ & $\begin{array}{l}\text { COEFFI- } \\
\text { CIENT OF } \\
\text { VARI- } \\
\text { ATION }\end{array}$ & $\begin{array}{c}\text { PERCENT } \\
\text { OF } \\
\text { ANNUAL } \\
\text { RUNOFF }\end{array}$ \\
\hline OCTOBER & 40 & 224 & 91 & 50 & .55 & 1.1 \\
\hline NOVEMBER & 58 & 827 & 263 & 210 & .80 & 3.1 \\
\hline DECEMBER & 107 & 1362 & 580 & 425 & .73 & 6.8 \\
\hline JANUARY & 123 & 2761 & 772 & 621 & .80 & 9.1 \\
\hline FEBRUARY & 273 & 2878 & 1154 & 785 & .68 & 13.5 \\
\hline MARCH & 450 & 3581 & 1640 & 828 & .50 & 19.2 \\
\hline APRIL & 843 & 4636 & 2177 & 1074 & .49 & 25.5 \\
\hline MAY & 230 & 4786 & 1266 & 1027 & .81 & 14.8 \\
\hline JUNE & 48 & 1622 & 411 & 413 & 1.00 & 4.8 \\
\hline $\begin{array}{l}\text { JULY } \\
\text { AUGUST }\end{array}$ & $\begin{array}{l}20 \\
19\end{array}$ & $\begin{array}{l}255 \\
109\end{array}$ & $\begin{array}{l}85 \\
40\end{array}$ & $\begin{array}{l}60 \\
22\end{array}$ & $\begin{array}{l}.70 \\
.54\end{array}$ & $\begin{array}{r}1.0 \\
.5\end{array}$ \\
\hline SEPTEMBER & 30 & 92 & 49 & 16 & .31 & .6 \\
\hline ANNUAL & 340 & 1140 & 707 & 242 & .34 & 100 \\
\hline
\end{tabular}

MAGNITUDE AND PROBABILITY OF INSTANTANEOUS PEAK FLOW BASED ON PERIOD OF RECORD 1904-26

DISCHARGE, IN CFS, FOR INDICATED RECURRENCE INTERVAL, IN YEARS, AND ANNUAL EXCEEDANCE PROBABILITY, IN PERCENT

\begin{tabular}{ccccccc}
1.25 & 2 & 5 & 10 & 25 & 50 & 100 \\
$80 \%$ & $50 \%$ & $20 \%$ & $10 \%$ & $4 \%$ & $2 \%$ & $1 \%$ \\
\hline 4150 & 6470 & 10100 & 12800 & 16500 & - & - \\
\hline WEIGHTED SKEW $=$ & .038 & &
\end{tabular}

MAGNITUDE AND PROBABILITY OF ANNUAL LOW FLOW BASED ON PERIOD OF RECORD 1905-26

\begin{tabular}{|c|c|c|c|c|c|c|}
\hline $\begin{array}{l}\text { PERIOD } \\
\text { (CON- }\end{array}$ & \multicolumn{6}{|c|}{$\begin{array}{l}\text { DISCHARGE, IN CFS, FOR INDICATED RECURRENCE } \\
\text { INTERVAL, IN YEARS, AND ANNUAL NON- } \\
\text { EXCEEDANCE PROBABILITY, IN PERCENT }\end{array}$} \\
\hline SECU- & & $-\infty$ & - & - & -- & \\
\hline $\begin{array}{l}\text { TIVE } \\
\text { DAYS) }\end{array}$ & $\begin{array}{c}2 \\
50 \%\end{array}$ & $\begin{array}{c}5 \\
20 \%\end{array}$ & $\begin{array}{l}10 \\
10 \%\end{array}$ & $\begin{array}{l}20 \\
5 \%\end{array}$ & $\begin{array}{l}50 \\
2 \%\end{array}$ & $\begin{array}{r}100 \\
1 \%\end{array}$ \\
\hline & & & & & & \\
\hline 1 & 25 & 18 & 15 & 13 & -- & -- \\
\hline 3 & 26 & 19 & 16 & 14 & -- & -- \\
\hline 7 & 27 & 20 & 17 & 14 & - & - \\
\hline 14 & 28 & 21 & 18 & 16 & -- & -- \\
\hline 30 & 31 & 23 & 20 & 18 & -- & -- \\
\hline 60 & 37 & 29 & 26 & 23 & -- & -- \\
\hline 90 & 44 & 35 & 31 & 29 & -- & -- \\
\hline 120 & 54 & 42 & 37 & 34 & - & - \\
\hline 183 & 102 & 72 & 61 & 53 & -- & $=$ \\
\hline
\end{tabular}

MAGNITUDE AND PROBABILITY OF ANNUAL HIGH FLOW BASED ON PERIOD OF RECORD 1904-26

\begin{tabular}{|c|c|c|c|c|c|c|}
\hline $\begin{array}{l}\text { PERIOD } \\
\text { (CON- }\end{array}$ & \multicolumn{6}{|c|}{$\begin{array}{c}\text { DISCHARGE, IN CFS, FOR INDICATED RECURRENCE } \\
\text { INTERVAL, IN YEARS, AND ANNUAL } \\
\text { EXCEEDANCE PROBABILITY, IN PERCENT }\end{array}$} \\
\hline SECU- & & & & & 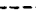 & \\
\hline TIVE & 2 & 5 & 10 & 25 & 50 & 100 \\
\hline DAYS) & $50 \%$ & $20 \%$ & $10 \%$ & $4 \%$ & 29 & $1 \%$ \\
\hline 1 & 5790 & 8990 & 11400 & 14700 & -- & -- \\
\hline 3 & 4950 & 7260 & 8830 & 10900 & -- & -- \\
\hline 7 & 4160 & 5670 & 6570 & 7600 & -- & -- \\
\hline 15 & 3340 & 4480 & 5100 & 5770 & - & - \\
\hline 30 & 2690 & 3710 & 4350 & 5110 & - & -- \\
\hline 60 & 2120 & 2970 & 3530 & 4230 & - & -- \\
\hline 90 & 1840 & 2500 & 2920 & 3440 & -- & -- \\
\hline
\end{tabular}

DURATION TABLE OF DAILY MEAN FLOW FOR PERIOD OF RECORD 1904-26

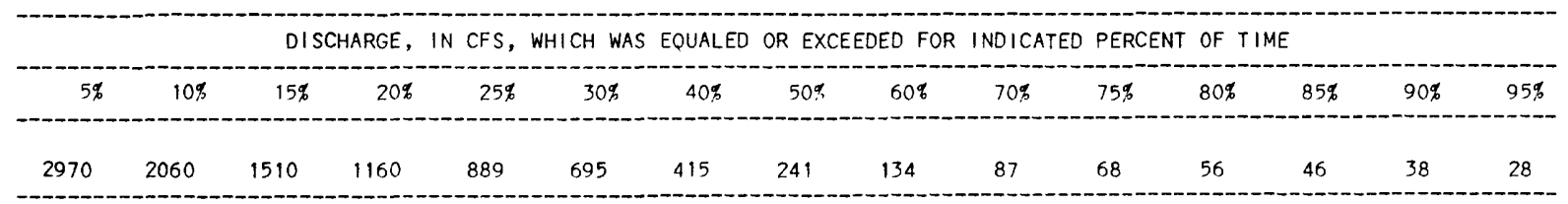


14026000 UMATILLA RIVER AT YOAKUM, OR--Continued

STATISTICAL SUMMARIES (AFTER THE COMPLETION OF MCKAY RESERVOIR)

MONTHLY AND ANNUAL MEAN DISCHARGES 1928-82

\begin{tabular}{|c|c|c|c|c|c|c|}
\hline MONTH & $\begin{array}{l}\text { MINIMUM } \\
\text { (CFS) }\end{array}$ & $\begin{array}{c}\text { MAXIMUM } \\
\text { (CFS) }\end{array}$ & $\begin{array}{l}\text { MEAN } \\
\text { (CFS) }\end{array}$ & $\begin{array}{l}\text { STAN- } \\
\text { DARO } \\
\text { DEVIA- } \\
\text { TION } \\
\text { (CFS) }\end{array}$ & $\begin{array}{l}\text { COEFFI- } \\
\text { CIENT OF } \\
\text { VARI- } \\
\text { ATION }\end{array}$ & $\begin{array}{c}\text { PERCENT } \\
\text { OF } \\
\text { ANNUAL } \\
\text { RUNOFF }\end{array}$ \\
\hline \multicolumn{7}{|c|}{ 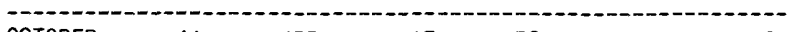 } \\
\hline OCTOBER & 44 & 455 & 97 & 72 & .74 & 1.2 \\
\hline NOVEMBER & 59 & 1539 & 265 & 256 & .97 & 3.3 \\
\hline DECEMBER & 76 & 2054 & 628 & 512 & .82 & 7.9 \\
\hline JANUARY & 82 & 2284 & 755 & 559 & .74 & 9.5 \\
\hline FEBRUARY & 115 & 3057 & 956 & 573 & .60 & 12.0 \\
\hline MARCH & 413 & 3654 & 1249 & 627 & .50 & 15.6 \\
\hline APRIL & 358 & 3334 & 1661 & 678 & .41 & 20.8 \\
\hline MAY & 256 & 3445 & 1072 & 600 & .56 & 13.4 \\
\hline JUNE & 244 & 1220 & 485 & 195 & .40 & 6.1 \\
\hline JULY & 211 & 456 & 352 & 64 & .18 & 4.4 \\
\hline AUGUST & 123 & 416 & 304 & 74 & .24 & 3.8 \\
\hline SEPTEMBER & 22 & 305 & 163 & 75 & .46 & 2.0 \\
\hline ANNUAL & 248 & 1237 & 664 & 218 & .33 & 100 \\
\hline
\end{tabular}

MAGNITUDE AND PROBABILITY OF INSTANTANEOUS PEAK FLOW BASED ON PERIOD OF RECORD

DISCHARGE, IN CFS, FOR INDICATED RECURRENCE INTERVAL, IN YEARS, AND ANNUAL EXCEEDANCE PROBABILITY, IN PERCENT

\begin{tabular}{|c|c|c|c|c|c|c|}
\hline $\begin{array}{l}1.25 \\
80 \%\end{array}$ & $\begin{array}{c}2 \\
50 \%\end{array}$ & $\begin{array}{c}5 \\
20 \%\end{array}$ & $\begin{array}{l}10 \\
10 \%\end{array}$ & $\begin{array}{l}25 \\
4 \%\end{array}$ & $\begin{array}{l}50 \\
2 \%\end{array}$ & $\begin{array}{r}100 \\
1 \%\end{array}$ \\
\hline-- & -- & - & -- & -- & -- & -- \\
\hline
\end{tabular}

MAGNITUDE AND PROBABILITY OF ANNUAL LOW FLOW BASED ON PERIOD OF RECORD 1929-82

\begin{tabular}{|c|c|c|c|c|c|c|}
\hline \multirow{2}{*}{$\begin{array}{l}\text { PERIOD } \\
\text { (CON- } \\
\text { SECU- } \\
\text { TIVE } \\
\text { DAYS) }\end{array}$} & \multicolumn{6}{|c|}{$\begin{array}{l}\text { DISCHARGE, IN CFS, FOR INDICATED RECURRENCE } \\
\text { INTERVAL, IN YEARS, AND ANNUAL NON- } \\
\text { EXCEEDANCE PROBABILITY, IN PERCENT }\end{array}$} \\
\hline & $\begin{array}{c}2 \\
50 \%\end{array}$ & ${ }^{5}{ }^{5} \%$ & $\begin{array}{l}10 \\
10 \%\end{array}$ & $\begin{array}{l}20 \\
5 \%\end{array}$ & $\begin{array}{l}50 \\
2 \%\end{array}$ & $\begin{array}{r}100 \\
1 \%\end{array}$ \\
\hline & & & & & & \\
\hline 1 & 47 & 33 & 27 & 23 & 18 & 16 \\
\hline 3 & 48 & 35 & 28 & 23 & 19 & 16 \\
\hline 7 & 51 & 36 & 30 & 25 & 20 & 17 \\
\hline 14 & 54 & 38 & 31 & 26 & 21 & 18 \\
\hline 30 & 62 & 44 & 37 & 31 & 25 & 22 \\
\hline 60 & 83 & 58 & 48 & 41 & 34 & 30 \\
\hline 90 & 117 & 81 & 66 & 56 & 45 & 39 \\
\hline 120 & 156 & 109 & 88 & 73 & 58 & 50 \\
\hline 183 & 223 & 168 & 143 & 125 & 106 & 95 \\
\hline
\end{tabular}

MAGNITUDE AND PROBABILITY OF ANNUAL HIGH FLOW BASED ON PERIOD OF RECORD 1928-82

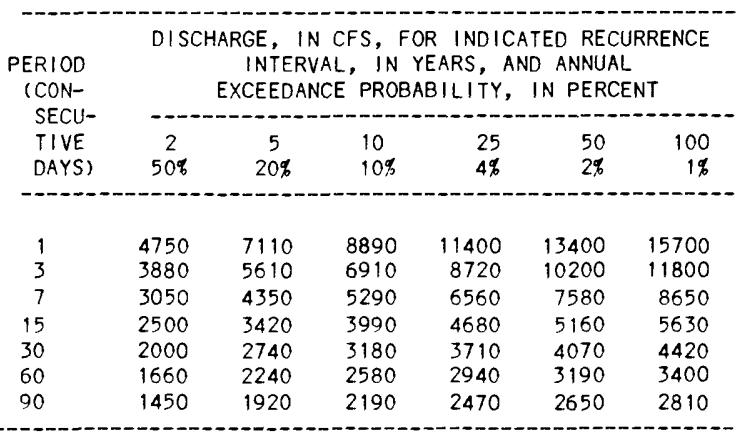

DURATION TABLE OF DAILY MEAN FLOW FOR PERIOD OF RECORO 1928-82

DISCHARGE, IN CFS, WHICH WAS EQUALED OR EXCEEDED FOR INDICATED PERCENT OF TIME

\begin{tabular}{|c|c|c|c|c|c|c|c|c|c|c|c|c|c|c|}
\hline $5 \%$ & $10 \%$ & $15 \%$ & $20 \%$ & $25 \%$ & $30 \%$ & $40 \%$ & $50 \%$ & $60 \%$ & $70 \%$ & $75 \%$ & $80 \%$ & $85 \%$ & $90 \%$ & $95 \%$ \\
\hline 2300 & 1640 & 1280 & 1020 & 819 & 651 & 454 & 376 & 316 & 248 & 202 & 146 & 103 & 79 & 58 \\
\hline
\end{tabular}




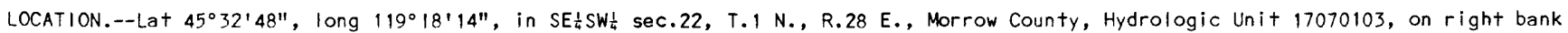

$0.3 \mathrm{mi}$ downstream from Mattlock Canyon, $6.0 \mathrm{mi}$ southeast of Pine City, $15 \mathrm{mi}$ southwest of Echo, and at mile 28.4 .

DRAINAGE AREA. $--291 \mathrm{mi}^{2}$.

PERIOD OF RECORD.--April to June 1928, November 1928 to June 1929 , October 1929 to September 1930 , January 1931 to September 1932 February to June 1933, October 1933 to September 1941, January to June 1942, October 1942 to September 1982 . Prior to October 1945 , monthly discharge only, published in WSP 1318.

GAGE.- Water-stage recorder. Altitude of gage is 1,400 ft, by barometer. Prior to Oct. 1 , 1944 , at datum $i .1 \mathrm{ft}$ higher and Oct. 1 , 1944, to Sept. 6, 1949, at datum $1.0 \mathrm{ft}$ higher.

REMARKS.--No regulation. Several small diversions for irrigation above station. Water is diverted into headwaters of Butter Creek from Fivemile Creek, a tributary of Camas Creek in John Day River basin, for irrigation below station.

AVERAGE DISCHARGE.--50 years (water years 1930, 1932, 1934-41, 1943-82), 26.4 $\mathrm{ft}+3 / \mathrm{s}, 19,130 \mathrm{acre}-\mathrm{ft} / \mathrm{yr}$.

EXTREMES FOR PERIOD OF RECORD. --Maximum discharge, 3,800 ft3/s Feb. 21, 1949, gage height, $12.4 \mathrm{ft}$, present datum, from floodmark, from rating curve extended above $440 \mathrm{ft} / 3 / \mathrm{s}$ on basis of computation of peak flow over dam; no $f$ low at times.

\section{STATISTICAL SUMMARIES}

MONTHLY AND ANNUAL MEAN DISCHARGES 1930-82

\begin{tabular}{|c|c|c|c|c|c|c|}
\hline MONTH & $\begin{array}{l}\text { MINIMUM } \\
\text { (CFS) }\end{array}$ & $\begin{array}{l}\text { MAXIMUM } \\
\text { (CFS) }\end{array}$ & $\begin{array}{l}\text { MEAN } \\
\text { (CFS) }\end{array}$ & $\begin{array}{l}\text { STAN- } \\
\text { DARD } \\
\text { DEVIA- } \\
\text { TION } \\
\text { (CFS) }\end{array}$ & $\begin{array}{l}\text { COEFFI- } \\
\text { CIENT OF } \\
\text { VARI- } \\
\text { ATION }\end{array}$ & $\begin{array}{c}\text { PERCENT } \\
\text { OF } \\
\text { ANNUAL } \\
\text { RUNOFF }\end{array}$ \\
\hline OCTOBER & 0.0 & 6.8 & 2.1 & 1.9 & .91 & .7 \\
\hline NOVEMBER & 0.0 & 48 & 6.9 & 8.6 & 1.24 & 2.2 \\
\hline DECEMBER & 0.0 & 142 & 22 & $3 i$ & 1.38 & 7.0 \\
\hline JANUARY & 0.0 & 259 & 36 & 46 & 1.28 & 11.3 \\
\hline FEBRUARY & 3.0 & 148 & 50 & 41 & .81 & 15.8 \\
\hline MARCH & 7.8 & 151 & 65 & 36 & .54 & 20.5 \\
\hline APRIL & 4.5 & 148 & 73 & 34 & .47 & 22.9 \\
\hline MAY & .9 & 128 & 45 & 30 & .66 & 14.0 \\
\hline JUNE & 0.0 & 54 & 14 & 11 & .83 & 4.3 \\
\hline JULY & 0.0 & 13 & 2.7 & 3.1 & 1.15 & .9 \\
\hline AUGUST & 0.0 & 5.1 & .7 & 1.1 & 1.55 & .2 \\
\hline SEPTEMBER & 0.0 & 6.7 & .9 & 1.4 & 1.50 & .3 \\
\hline ANNUAL & 5.1 & 64 & 26 & 15 & .56 & 100 \\
\hline
\end{tabular}

MAGN ITUDE AND PROBABILITY OF INSTANTANEOUS PEAK FLOW BASED ON PERIOD OF RECORD 1930-82

DISCHARGE, IN CFS, FOR INDICATED RECURRENCE INTERVAL, IN YEARS, AND ANNUAL EXCEEDANCE PROBABILITY, IN PERCENT

\begin{tabular}{ccccccc}
1.25 & 2 & 5 & 10 & 25 & 50 & 100 \\
$80 \%$ & $50 \%$ & $20 \%$ & $10 \%$ & $4 \%$ & $2 \%$ & $1 \%$ \\
\hline 182 & 310 & 578 & 830 & 1260 & 1670 & 2190 \\
\hline
\end{tabular}

WEIGHTED SKEW $=\quad .544$
MAGNITUDE AND PROBABILITY OF ANNUAL LOW FLOW BASED ON PERIOD OF RECORD 1932-82

\begin{tabular}{|c|c|c|c|c|c|c|}
\hline $\begin{array}{l}\text { PERIOD } \\
\text { (CON- } \\
\text { SECU- }\end{array}$ & \multicolumn{6}{|c|}{$\begin{array}{l}\text { DISCHARGE, IN CFS, FOR INDICATED RECURRENCE } \\
\text { INTERVAL, IN YEARS, AND ANNUAL NON- } \\
\text { EXCEEDANCE PROBABILITY, IN PERCENT }\end{array}$} \\
\hline $\begin{array}{l}\text { TIVE } \\
\text { DAYS) }\end{array}$ & $\stackrel{2}{50 \%}$ & $\begin{array}{c}5 \\
20 \%\end{array}$ & $\begin{array}{l}10 \\
10 \%\end{array}$ & $\begin{array}{l}20 \\
5 \%\end{array}$ & $\begin{array}{l}50 \\
2 \%\end{array}$ & $\begin{array}{r}100 \\
1 \%\end{array}$ \\
\hline & & & & & & \\
\hline $\begin{array}{l}1 \\
3\end{array}$ & .4 & - 1 & 0.0 & 0.0 & 0.0 & \\
\hline 7 & $\cdot 4$ & $\because$ & $\because$ & 0.0 & 0. & 0.0 \\
\hline 14 & .4 & 1 & .1 &. & .1 & 0. \\
\hline 30 & .5 & .2 & .1 & .1 & 1 & 0. \\
\hline 60 & .6 & .2 & .1 & .1 & .1 & \\
\hline 90 & .8 & .3 & .1 & .1 & .1 & \\
\hline 120 & 1.4 & .5 & .2 & .1 & .1 & \\
\hline 183 & 3.8 & 1.4 & .6 & .3 & .1 & \\
\hline
\end{tabular}

NOTE: LOW-FLOW STATISTICS UNCERTAIN DUE TO EXCESSIVE ZERO EVENTS.

MAGNITUDE AND PROBABILITY OF ANNUAL HIGH FLOW BASED ON PERIOD OF RECORD 1930-82

\begin{tabular}{|c|c|c|c|c|c|c|}
\hline $\begin{array}{l}\text { PERIOD } \\
\text { (CON- } \\
\text { SECU- }\end{array}$ & \multicolumn{6}{|c|}{$\begin{array}{l}\text { DISCHARGE, IN CFS, FOR IND ICATED RECURRENCE } \\
\text { INTERVAL, IN YEARS, AND ANNUAL } \\
\text { EXCEEDANCE PROBABILITY, IN PERCENT }\end{array}$} \\
\hline TIVE & 2 & 5 & 10 & 25 & 50 & 100 \\
\hline DAYS) & $50 \%$ & $20 \%$ & $10 \%$ & $4 \%$ & $2 \%$ & $1 \%$ \\
\hline 1 & 207 & 376 & 541 & 833 & 1130 & 1500 \\
\hline 3 & 169 & 302 & 426 & 632 & 830 & 1070 \\
\hline 7 & 138 & 237 & 317 & 437 & 540 & 654 \\
\hline 15 & 114 & 184 & 233 & 297 & 346 & 395 \\
\hline 30 & 94 & 145 & 178 & 217 & 244 & 269 \\
\hline 60 & 78 & 120 & 146 & 175 & 194 & 212 \\
\hline 90 & 66 & 103 & 126 & 152 & 170 & 186 \\
\hline
\end{tabular}

DURATION TABLE OF DAILY MEAN FLOW FOR PERIOD OF RECORD 1930-82

\begin{tabular}{|c|c|c|c|c|c|c|c|c|c|c|c|c|c|c|}
\hline $5 \%$ & $10 \%$ & $15 \%$ & $20 \%$ & $25 \%$ & $30 \%$ & $40 \%$ & $50 \%$ & $60 \%$ & $70 \%$ & $75 \%$ & $80 \%$ & $85 \%$ & $90 \%$ & $95 \%$ \\
\hline 112 & 81 & 61 & 47 & 35 & 25 & 13 & 7.3 & 4.5 & 2.0 & 1.0 & .5 & .1 & 0.0 & \\
\hline
\end{tabular}




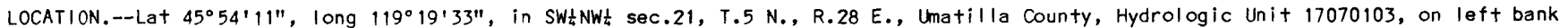

$1.6 \mathrm{mi}$ downstream from West Division main canal of Umatilla project, $1.2 \mathrm{mi}$ southeast of Umatilla, and at mile 2.1 .

DRAINAGE AREA. $--2,290 \mathrm{mi}^{2}$, approximately.

PERIOD OF RECORD.--November 1903 to September 1982.

GAGE.--Water-stage recorder. Datum of gage is $330.47 \mathrm{ft}$ National Geodetic Vertical Datum of 1929. Oct. 21 , 1903 to Jan. 25 , 1931, nonrecording gage.

REMARKS.--Some regulation since 1927 by McKay Reservoir. Many diversions above station for irrigation of lands above and below station; Brownell Canal diverts below station. Diversions since 1908 to Cold Springs Reservoir, an off-channel reservoir, capacity, 52,380 acre-ft.

AVERAGE DISCHARGE.--55 years (water years 1928-82), $451 \mathrm{ft}+3 / \mathrm{s}, 326,700$ acre-ft/yr Water years $p r i o r$ to 1928 not included in computation of average discharge owing to increased regulation and diversion since 1927.

EXTREMES FOR PERIOD OF RECORD.--Maximum discharge, 19,800 $\mathrm{ft}^{3} / \mathrm{s}$ Jan. 30, 1965, gage height, $10.75 \mathrm{ft}$; no $\mathrm{flow}$ at times.

STATISTICAL SUMMARIES

MONTHLY AND ANNUAL MEAN DISCHARGES 1929-82

\begin{tabular}{|c|c|c|c|c|c|c|}
\hline MONTH & $\begin{array}{l}\text { MINIMUM } \\
\text { (CFS) }\end{array}$ & $\begin{array}{l}\text { MAXIMUM } \\
\text { (CFS) }\end{array}$ & $\begin{array}{l}\text { MEAN } \\
\text { (CFS) }\end{array}$ & $\begin{array}{l}\text { STAN- } \\
\text { DARD } \\
\text { DEVIA- } \\
\text { TION } \\
\text { (CFS) }\end{array}$ & $\begin{array}{l}\text { COEFFI- } \\
\text { CIENT OF } \\
\text { VARI- } \\
\text { ATION }\end{array}$ & $\begin{array}{c}\text { PERCENT } \\
\text { OF } \\
\text { ANNUAL } \\
\text { RUNOFF }\end{array}$ \\
\hline OCTOBER & 4.2 & 331 & 77 & 65 & .85 & 1.4 \\
\hline $\begin{array}{l}\text { NOVEMBER } \\
\text { DECEMBER }\end{array}$ & $\begin{array}{l}40 \\
79\end{array}$ & $\begin{array}{r}878 \\
1948\end{array}$ & $\begin{array}{l}226 \\
551\end{array}$ & $\begin{array}{l}157 \\
472\end{array}$ & $\begin{array}{l}.69 \\
.86\end{array}$ & $\begin{array}{r}4.2 \\
10.3\end{array}$ \\
\hline $\begin{array}{l}\text { JANUARY } \\
\text { FEBRUARY }\end{array}$ & $\begin{array}{r}89 \\
87\end{array}$ & $\begin{array}{l}2366 \\
2994\end{array}$ & $\begin{array}{r}689 \\
898\end{array}$ & $\begin{array}{l}562 \\
612 \\
645\end{array}$ & $\begin{array}{r}.82 \\
.68 \\
.63\end{array}$ & $\begin{array}{l}12.9 \\
16.8\end{array}$ \\
\hline $\begin{array}{l}\text { MARCH } \\
\text { APRIL }\end{array}$ & $\begin{array}{r}154 \\
4.6\end{array}$ & $\begin{array}{l}3678 \\
3056\end{array}$ & $\begin{array}{l}1028 \\
1134\end{array}$ & $\begin{array}{l}645 \\
698\end{array}$ & $\begin{array}{l}.63 \\
.62\end{array}$ & $\begin{array}{l}19.2 \\
21.2\end{array}$ \\
\hline $\begin{array}{l}\text { MAY } \\
\text { JUNE }\end{array}$ & $\begin{array}{l}1.6 \\
1.3\end{array}$ & $\begin{array}{r}3362 \\
805\end{array}$ & $\begin{array}{l}560 \\
111\end{array}$ & $\begin{array}{l}581 \\
167\end{array}$ & $\begin{array}{l}1.04 \\
1.50\end{array}$ & $\begin{array}{r}10.5 \\
2.1\end{array}$ \\
\hline $\begin{array}{l}\text { JULY } \\
\text { AUGUST } \\
\text { SEPTEMBER }\end{array}$ & $\begin{array}{l}1.3 \\
1.3 \\
1.3\end{array}$ & $\begin{array}{r}149 \\
68 \\
124\end{array}$ & $\begin{array}{l}21 \\
23 \\
35\end{array}$ & $\begin{array}{l}21 \\
17 \\
29\end{array}$ & $\begin{array}{l}.99 \\
.75 \\
.85\end{array}$ & $\begin{array}{l}.4 \\
.4 \\
.6\end{array}$ \\
\hline ANNUAL & 78 & 981 & 443 & 210 & .47 & 100 \\
\hline
\end{tabular}

MAGNITUDE AND PROBABILITY OF INSTANTANEOUS PEAK FLOW BASED ON PERIOD OF RECORD 1929-82

DISCHARGE, IN CFS, FOR INDICATED RECURRENCE INTERVAL, IN YEARS, AND ANNUAL EXCEEDANCE PROBABILITY, IN PERCENT

\begin{tabular}{ccccccc}
1.25 & 2 & 5 & 10 & 25 & 50 & 100 \\
$80 \%$ & $50 \%$ & $20 \%$ & $10 \%$ & $4 \%$ & $2 \%$ & $1 \%$ \\
\hline 2930 & 4460 & 7200 & 9500 & 13000 & 16100 & 19700
\end{tabular}

WEIGHTED SKEW $=.488$
MAGNITUDE AND PROBABILITY OF ANNUAL LOW FLOW BASED ON PERIOD OF RECORD 1930-82

\begin{tabular}{|c|c|c|c|c|c|c|}
\hline \multirow{3}{*}{$\begin{array}{l}\text { PERIOD } \\
\text { (CON- } \\
\text { SECU- } \\
\text { TIVE } \\
\text { DAYS) }\end{array}$} & \multicolumn{6}{|c|}{$\begin{array}{l}\text { DISCHARGE, IN CFS, FOR INDICATED RECURRENCE } \\
\text { INTERVAL, IN YEARS, AND ANNUAL NON- } \\
\text { EXCEEDANCE PROBABILITY, IN PERCENT }\end{array}$} \\
\hline & 2 & 5 & 10 & 20 & 50 & 100 \\
\hline & $50 \%$ & $20 \%$ & $10 \%$ & $5 \%$ & $2 \%$ & $1 \%$ \\
\hline & & & & & & \\
\hline 1 & 3.5 & 1. & .8 & .5 & .3 & .2 \\
\hline 3 & 3.8 & 1. &. & .6 & .3 & .2 \\
\hline 7 & 5.2 & 2. & 1. & .8 & .5 & .3 \\
\hline 14 & 6.8 & 2. & 1.7 & 1.0 & .5 & .3 \\
\hline 30 & 9.5 & 4. & 2. & 1.7 & 1.0 & .6 \\
\hline 50 & 14 & 6. & 3. & 2.5 & 1.5 & 1.0 \\
\hline 90 & 19 & 9. & 5.6 & 3.7 & 2.1 & 1.5 \\
\hline 120 & 23 & 11 & 6.6 & 4.2 & 2.4 & 1.6 \\
\hline 183 & 52 & 21 & 12 & 7.1 & 3.7 & 2.3 \\
\hline
\end{tabular}

MAGNITUDE AND PROBABILITY OF ANNUAL HIGH FLOW BASED ON PERIOD OF RECORD 1929-82

\begin{tabular}{|c|c|c|c|c|c|c|}
\hline \multirow{2}{*}{$\begin{array}{l}\text { PERIOD } \\
\text { (CON- } \\
\text { SECU- } \\
\text { TIVE } \\
\text { DAYS) }\end{array}$} & \multicolumn{6}{|c|}{$\begin{array}{c}\text { OISCHARGE, IN CFS, FOR INDICATED RECURRENCE } \\
\text { INTERVAL, IN YEARS, AND ANNUAL } \\
\text { EXCEEDANCE PROBABILITY, IN PERCENT }\end{array}$} \\
\hline & $\begin{array}{c}2 \\
50 \%\end{array}$ & $\begin{array}{c}5 \\
20 \%\end{array}$ & $\begin{array}{l}10 \\
10 \%\end{array}$ & $\begin{array}{l}25 \\
4 \%\end{array}$ & $\begin{array}{l}50 \\
2 \%\end{array}$ & $\begin{array}{r}100 \\
1 \%\end{array}$ \\
\hline 1 & 4190 & 6620 & 8550 & 11400 & 13700 & 16400 \\
\hline 3 & 3510 & 5390 & 6830 & 8880 & 10600 & 12400 \\
\hline 7 & 2760 & 4160 & 5180 & 6540 & 7610 & 8730 \\
\hline 15 & 2140 & 3150 & 3810 & 4600 & 5170 & 5720 \\
\hline 30 & 1690 & 2440 & 2860 & 3310 & 3600 & 3860 \\
\hline 60 & 1320 & 1910 & 2240 & 2600 & 2830 & 3030 \\
\hline 90 & 1130 & 1630 & 1900 & 2200 & 2380 & 2540 \\
\hline
\end{tabular}

DURATION TABLE OF DAILY MEAN FLOW FOR PERIOD OF RECORD 1929-82

DISCHARGE, IN CFS, WHICH WAS EQUALED OR EXCEEDED FOR INDICATEO PERCENT OF TIME

\begin{tabular}{|c|c|c|c|c|c|c|c|c|c|c|c|c|c|c|}
\hline $5 \%$ & $10 \%$ & $15 \%$ & $20 \%$ & $25 \%$ & $30 \%$ & $40 \%$ & $50 \%$ & $60 \%$ & $70 \%$ & $75 \%$ & $80 \%$ & $85 \%$ & $90 \%$ & $95 \%$ \\
\hline 1960 & 1280 & 947 & 729 & 552 & 409 & 218 & 133 & 69 & 25 & 17 & 13 & 9.6 & 7.1 & 4.1 \\
\hline
\end{tabular}




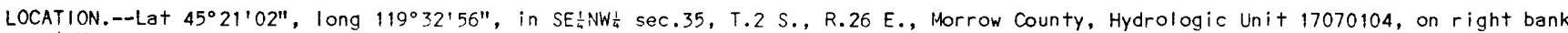
at Heppner, $100 \mathrm{ft}$ upstream from Court Street bridge, $800 \mathrm{ft}$ southeast of Morrow County courthouse, $0.3 \mathrm{mi}$ downstream from Balm Fork and at mile 52.2.

DRAINAGE AREA. $--96.8 \mathrm{mi}^{2}$, revised.

PERIOD OF RECORD.--May 1951 to September 1982.

GAGE.--Water-stage recorder. Datum of gage is $1,952.73 \mathrm{ft}$ National Geodetic Vertical Datum of 1929.

REMARKS.--No regulation. Many diversions for irrigation above station. Part of flow of Ditch Creek (John Day River basin) is diverted to Willow Creek above station.

AVERAGE DISCHARGE. --3 ! years, $19.1 \mathrm{ft} / \mathrm{s}, 13,840$ acre $-\mathrm{t} t / \mathrm{yr}$.

EXTREMES FOR PERIOD OF RECORD.--Maximum discharge, $812+t^{3} / \mathrm{s}$ May 10, 1957, gage height, $6.15 \mathrm{ft}$, trom rating curve extended above 230 $\mathrm{ft}^{3} / \mathrm{s}$; maximum gage height, $6.46 \mathrm{ft}$ May 25, 1971, backwater from Shobe Canyon; no flow at times.

EXTREMES OUTSIDE PERIOD OF RECORD.--Maximum discharge, about $36,000 \mathrm{ft}^{3} / \mathrm{s}$ June 14 , 1903, result of slope-area measurement (see WSP 96). Discharge for flood of Feb. 22, 1949, was $1,700 \mathrm{ft} / \mathrm{s}$, result of slope-area measurement.

STATISTICAL SUMMARIES

MONTHLY AND ANNUAL MEAN DISCHARGES 1952-82

\begin{tabular}{|c|c|c|c|c|c|c|}
\hline MONTH & $\begin{array}{l}\text { MINIMUM } \\
\text { (CFS) }\end{array}$ & $\begin{array}{c}\text { MAXIMUM } \\
(\text { CFS })\end{array}$ & $\begin{array}{l}\text { MEAN } \\
\text { (CFS) }\end{array}$ & $\begin{array}{l}\text { STAN- } \\
\text { DARD } \\
\text { DEVIA- } \\
\text { TION } \\
\text { (CFS) }\end{array}$ & $\begin{array}{l}\text { COEFFI- } \\
\text { CIENT OF } \\
\text { VARI- } \\
\text { ATION }\end{array}$ & $\begin{array}{c}\text { PERCENT } \\
\text { OF } \\
\text { ANNUAL } \\
\text { RUNOFF }\end{array}$ \\
\hline OCTOBER & 0.0 & 8.3 & 1.6 & 1.6 & 1.04 & .7 \\
\hline NOVEMBER & 1.4 & 27 & 5.8 & 4.5 & .78 & 2.5 \\
\hline DECEMBER & 2.0 & 82 & 18 & 20 & 1.11 & 8.0 \\
\hline JANUARY & 1.7 & 102 & 20 & 27 & .96 & 12.4 \\
\hline FEBRUARY & 1.2 & 89 & 32 & 23 & .72 & 13.9 \\
\hline MARCH & 6.6 & 83 & 38 & 23 & .60 & 16.7 \\
\hline APRIL & 3.6 & 127 & 49 & 28 & .56 & 21.3 \\
\hline MAY & 1.0 & 120 & 40 & 26 & .66 & 17.5 \\
\hline JUNE & .9 & 41 & 13 & 9.6 & .73 & 5.7 \\
\hline JULY & .1 & 7.0 & 2.3 & 1.8 & .80 & 1.0 \\
\hline AUGUST & 0.0 & 1.2 & .3 & .2 & .86 & .1 \\
\hline SEPTEMBER & 0.0 & 3.6 & .4 & .7 & 1.66 & .2 \\
\hline ANNUAL & 2.6 & 37 & 19 & 9.8 & .51 & 100 \\
\hline
\end{tabular}

MAGNITUDE AND PROBABILITY OF INSTANTANEOUS PEAK FLOW BASED ON PERIOD OF RECORD 1952-82

\begin{tabular}{|c|c|c|c|c|c|c|}
\hline \multicolumn{7}{|c|}{$\begin{array}{l}\text { DISCHARGE, IN CFS, FOR INDICATED RECURRENCE INTERVAL, IN } \\
\text { YEARS, AND ANNUAL EXCEEDANCE PROBABILITY, IN PERCENT }\end{array}$} \\
\hline $\begin{array}{l}1.25 \\
80 \%\end{array}$ & $\begin{array}{c}2 \\
50 \%\end{array}$ & $\begin{array}{c}5 \\
20 \%\end{array}$ & $\begin{array}{l}10 \\
10 \%\end{array}$ & $\begin{array}{l}25 \\
4 \%\end{array}$ & $\begin{array}{l}50 \\
2 \%\end{array}$ & $\begin{array}{r}100 \\
1 \%\end{array}$ \\
\hline 85 & 185 & 385 & 556 & 810 & 1030 & -- \\
\hline
\end{tabular}

WEIGHTED SKEW $=-.216$
MAGNITUDE AND PROBABILITY OF ANNUAL LOW FLOW BASED ON PERIOD OF RECORD $1953-82$

\begin{tabular}{|c|c|c|c|c|c|c|}
\hline $\begin{array}{l}\text { PERIOD } \\
\text { (CON- }\end{array}$ & \multicolumn{6}{|c|}{$\begin{array}{l}\text { DISCHARGE, IN CFS, FOR IND ICATED RECURRENCE } \\
\text { INTERVAL, IN YEARS, AND ANNUAL NON- } \\
\text { EXCEEDANCE PROBABILITY, IN PERCENT }\end{array}$} \\
\hline SECU- & & -1 & -- & - & -0 & \\
\hline TIVE & 2 & 5 & 10 & 20 & 50 & 100 \\
\hline DAYS) & $50 \%$ & $20 \%$ & $10 \%$ & $5 \%$ & $2 \%$ & $1 \%$ \\
\hline \\
\hline 3 & 1 & 0.0 & 0.0 & 0.0 & 0.0 & -- \\
\hline 7 & .1 & 0.0 & 0.0 & 0.0 & 0.0 & -- \\
\hline 14 & .1 & 0.0 & 0.0 & 0.0 & 0.0 & -- \\
\hline 30 & .1 & 0.0 & 0.0 & 0.0 & 0.0 & -- \\
\hline 60 & .2 & .1 & 0.0 & 0.0 & 0.0 & -- \\
\hline 90 & .3 & .1 & .1 & 0.0 & 0.0 & - \\
\hline 120 & .7 & .3 & .2 & .1 & 0.0 & -- \\
\hline 183 & 2.3 & 1.2 & .8 & .6 & .4 & -- \\
\hline
\end{tabular}

MAGNITUDE AND PROBABILITY OF ANNUAL HIGH FLOW BASED ON PERIOD OF RECORD 1952-82

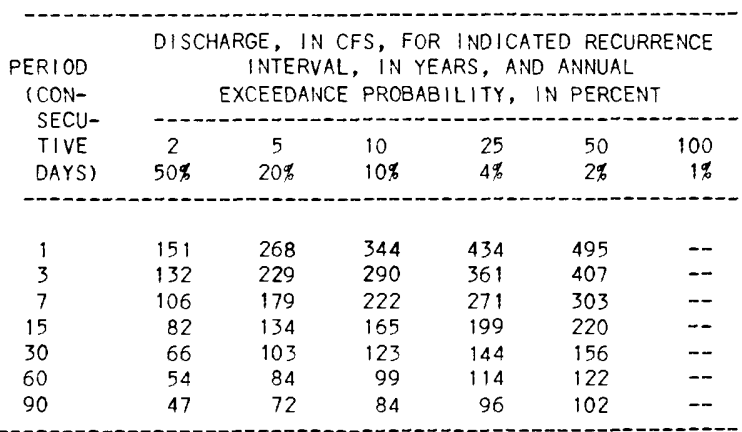

DURATION TABLE OF DAILY MEAN FLOW FOR PERIOD OF RECORD 1952-82

DISCHARGE, IN CFS, WHICH WAS EQUALED OR EXCEEDED FOR INDICATED PERCENT OF TIME

\begin{tabular}{|c|c|c|c|c|c|c|c|c|c|c|c|c|c|c|}
\hline $5 \%$ & $10 \%$ & $15 \%$ & $20 \%$ & $25 \%$ & $30 \%$ & $40 \%$ & $50 \%$ & $60 \%$ & $70 \%$ & $75 \%$ & $80 \%$ & $85 \%$ & $90 \%$ & $95 \%$ \\
\hline 77 & 55 & 42 & 34 & 27 & 20 & 11 & 6.6 & 4.1 & 1.8 & .9 & .5 & .3 & .1 & 1 \\
\hline
\end{tabular}


14034800 RHEA CREEK NEAR HEPPNER, OR

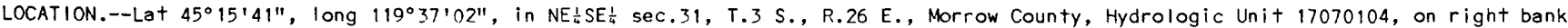

$1.0 \mathrm{mi}$ downstream from Sanford Canyon, $8 \mathrm{mi}$ southwest of Heppner, and at mile 25.4 .

DRAINAGE AREA.-- $120 \mathrm{mi}^{2}$, approximately.

PERIOD OF RECORD. - August 1960 to September 1082.

GAGE.--Water-stage recorder. Altitude of gage is $2,320 \mathrm{ft}$, from topographic map. Prior to May 28 , 1976 , at site $0.4 \mathrm{mi}$ downstream at different datum.

REMARKS.--No regulation. Many diversions for irrigation above station.

AVERAGE DISCHARGE. -22 years, $19.8 \mathrm{ft} / \mathrm{s}, 14,350$ acre- $\mathrm{ft} / \mathrm{yr}$.

EXTREMES FOR PERIOD OF RECORD.--Maximum discharge, 1,280 $\mathrm{ft}^{3} / \mathrm{s}$ june 10,1969 , gage height, $7.05 \mathrm{ft}$, site and datum then in use, from rating curve extended above $130 \mathrm{ft}^{3} / \mathrm{s}$ on basis of slope-area measurement at gage height $6.72 \mathrm{ft}$; maximum gage height, $7.41 \mathrm{ft}$ Dec. 22, 1964, site and datum then in use; no flow at times.

STATISTICAL SUMMARIES

MONTHLY AND ANNUAL MEAN DISCHARGES 1961-82

\begin{tabular}{|c|c|c|c|c|c|c|}
\hline MONTH & $\begin{array}{l}\text { MINIMUM } \\
\text { (CFS) }\end{array}$ & $\begin{array}{l}\text { MAXIMUM } \\
\text { (CFS) }\end{array}$ & $\begin{array}{l}\text { MEAN } \\
\text { (CFS) }\end{array}$ & $\begin{array}{l}\text { STAN- } \\
\text { DARD } \\
\text { DEVIA- } \\
\text { TION } \\
\text { (CFS) }\end{array}$ & $\begin{array}{l}\text { COEFFI- } \\
\text { CIENT OF } \\
\text { VARI- } \\
\text { ATION }\end{array}$ & $\begin{array}{c}\text { PERCENT } \\
\text { OF } \\
\text { ANNUAL } \\
\text { RUNOFF }\end{array}$ \\
\hline OCTOBER & .8 & 5.0 & 2.5 & 1.1 & .43 & 1.0 \\
\hline NOVEMBER & 2.5 & 49 & 7.5 & 9.6 & 1.28 & 3.1 \\
\hline DECEMBER & 2.9 & 87 & 22 & 24 & 1.10 & 9.3 \\
\hline JANUARY & 3.2 & 124 & 39 & 35 & .92 & 16.1 \\
\hline FEBRUARY & 3.2 & 116 & 42 & 32 & .76 & 17.4 \\
\hline MARCH & 7.2 & 104 & 43 & 28 & .64 & 18.1 \\
\hline APRIL & 4.7 & 138 & 43 & 32 & .76 & 17.9 \\
\hline MAY & 2.4 & 77 & 27 & 22 & .81 & 11.4 \\
\hline JUNE & .7 & 37 & 9.1 & 8.2 & .90 & 3.8 \\
\hline JULY & .1 & 6.9 & 2.3 & 2.0 & .89 & .9 \\
\hline AUGUST & 0.0 & 2.9 & .9 & .9 & .96 & .4 \\
\hline SEPTEMBER & .2 & 3.7 & 1.3 & 1.0 & .73 & .5 \\
\hline ANNUAL & 3.6 & 41 & 20 & 11 & .56 & 100 \\
\hline
\end{tabular}

MAGNITUDE AND PROBABILITY OF INSTANTANEOUS PEAK FLOW BASED ON PERIOD OF RECORD 1961-82

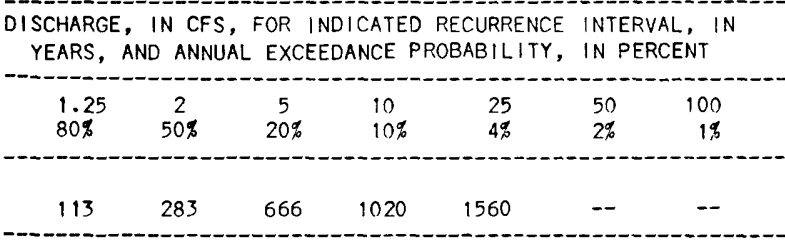

WEIGHTED SKEW $=-.266$
MAGNITUDE AND PROBABILITY OF ANNUAL LOW FLOW BASED ON PERIOD OF RECORD 1962-82

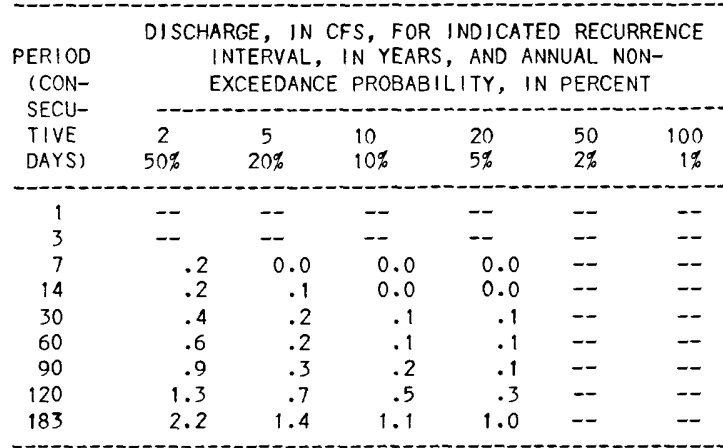

MAGNITUDE AND PROBABILITY OF ANNUAL HIGH FLOW BASED ON PERIOD OF RECORD 1961-82

\begin{tabular}{|c|c|c|c|c|c|c|}
\hline $\begin{array}{l}\text { PERIOD } \\
\text { (CON- } \\
\text { SECU- }\end{array}$ & \multicolumn{6}{|c|}{$\begin{array}{l}\text { DISCHARGE, IN CFS, FOR INDICATED RECURRENCE } \\
\text { INTERVAL, IN YEARS, AND ANNUAL } \\
\text { EXCEEDANCE PROBABILITY, IN PERCENT }\end{array}$} \\
\hline TIVE & 2 & 5 & 10 & 25 & 50 & 100 \\
\hline DAYS) & $50 \%$ & $20 \%$ & $10 \%$ & $4 \%$ & $2 \%$ & $1 \%$ \\
\hline 1 & 208 & 415 & 567 & 762 & -- & - \\
\hline 3 & 170 & 327 & 439 & 581 & - & -- \\
\hline 7 & 127 & 242 & 326 & 436 & -- & - \\
\hline 15 & 93 & 168 & 219 & 281 & -- & -- \\
\hline 30 & 69 & 121 & 154 & 192 & -- & - \\
\hline 60 & 53 & 92 & 117 & 147 & -- & -- \\
\hline 90 & 46 & 80 & 101 & 125 & $=-$ & -- \\
\hline
\end{tabular}

DURATION TABLE OF DAILY MEAN FLOW FOR PERIOD OF RECORD 1961-82

\begin{tabular}{|c|c|c|c|c|c|c|c|c|c|c|c|c|c|c|}
\hline $5 \%$ & $10 \%$ & $15 \%$ & $20 \%$ & $25 \%$ & $30 \%$ & $40 \%$ & $50 \%$ & $60 \%$ & $70 \%$ & $75 \%$ & $80 \%$ & $85 \%$ & $90 \%$ & $95 \%$ \\
\hline 82 & 55 & 40 & 30 & 23 & 18 & 10 & 6.1 & 3.9 & 2.6 & 2.0 & 1.5 & 1.0 & .5 & .1 \\
\hline
\end{tabular}




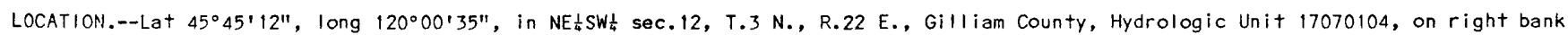
at bridge on abandoned highway, $3.8 \mathrm{mi}$ downstream from Eightmile Canyon, $10 \mathrm{mi}$ east of Arlington, and at mile 3.7 .

DRAINAGE AREA.--850 $\mathrm{mi}^{2}$, approximately.

PERIOD OF RECORD.--March to July 1906, August 1960 to September 1979. Records for March to August 1905 at site just upstream from Eightmile Canyon not equivalent owing to diversions and inflow.

GAGE.-Water-stage recorder and concrete control. Datum of gage is 291.26 ft National Geodetic Vertical Datum of 1929 . Mar. 1 to July 21, 1906, nonrecording gage at site $2.6 \mathrm{mi}$ upstream at different datum. Aug. 24 , 1960 to July 1 , 1964 , water-stage recorder at site $430 \mathrm{ft}$ downstream at same datum.

REMARKS.--No regulation. Many diversions for irrigation above station.

AVERAGE DISCHARGE.--19 years, $31.3 \mathrm{ft} 3 / \mathrm{s}, 22,680$ acre $-\mathrm{ft} / \mathrm{yr}$.

EXTREMES FOR PERIOD OF RECORD.--Maximum discharge, 16,900 $\mathrm{ft}^{3} / \mathrm{s}$ probably occurred Jan. 14, 1974, gage height, $11.18 \mathrm{ft}$, from floodmark, from rating curve extended above $1,900 \mathrm{ft}^{3} / \mathrm{s}$ on bas is of slope-area measurement at gage height $11.05 \mathrm{ft}$; maximum gage height, $13.50 \mathrm{ft}$ Feb. 7, 1979, from floodmarks; no flow at times.

\section{STATISTICAL SUMMARIES}

MONTHLY AND ANNUAL MEAN DISCHARGES 1961-79

\begin{tabular}{|c|c|c|c|c|c|c|}
\hline MONTH & $\begin{array}{l}\text { M! NIMUM } \\
\text { (CFS) }\end{array}$ & $\begin{array}{l}\text { MAXIMUM } \\
\text { (CFS) }\end{array}$ & $\begin{array}{l}\text { MEAN } \\
\text { (CFS) }\end{array}$ & $\begin{array}{l}\text { STAN- } \\
\text { DARD } \\
\text { DEVIA- } \\
\text { TION } \\
\text { (CFS) }\end{array}$ & $\begin{array}{l}\text { COEFFI- } \\
\text { CIENT OF } \\
\text { VARI- } \\
\text { ATION }\end{array}$ & $\begin{array}{c}\text { PERCENT } \\
\text { OF } \\
\text { ANNUAL } \\
\text { RUNOFF }\end{array}$ \\
\hline OCTOBER & 0.0 & 2.8 & .7 & .7 & .99 & .2 \\
\hline NOVEMBER & .1 & 86 & 6.6 & 19 & 2.94 & 1.7 \\
\hline DECEMBER & .6 & 311 & 41 & 82 & 1.99 & 10.9 \\
\hline JANUARY & .7 & 478 & 84 & 124 & 1.47 & 22.3 \\
\hline FEBRUARY & .5 & 430 & 73 & 103 & 1.41 & 19.3 \\
\hline MARCH & .1 & 130 & 50 & 50 & 1.02 & 13.1 \\
\hline APRIL & 0.0 & 211 & 63 & 65 & 1.04 & 16.5 \\
\hline MAY & 0.0 & 194 & 47 & 57 & 1.22 & 12.3 \\
\hline JUNE & 0.0 & 76 & 10 & 18 & 1.75 & 2.7 \\
\hline JULY & 0.0 & 10 & 1.3 & 2.4 & 1.84 & .3 \\
\hline AUGUST & 0.0 & 12 & 1.7 & 3.5 & 2.01 & .5 \\
\hline SEPTEMBER & 0.0 & 3.3 & .7 & .9 & 1.33 & .2 \\
\hline ANNUAL & .4 & 93 & 31 & 29 & .92 & 100 \\
\hline
\end{tabular}

MAGNITUDE AND PROBABILITY OF INSTANTANEOUS PEAK FLOW BASED ON PERIOD OF RECORD 1961-79

DISCHARGE, IN CFS, FOR INDICATED RECURRENCE INTERVAL, IN YEARS, AND ANNUAL EXCEEDANCE PROBABILITY, IN PERCENT

$\begin{array}{ccccccc}1.25 & 2 & 5 & 10 & 25 & 50 & 100 \\ 80 \% & 50 \% & 20 \% & 10 \% & 4 \% & 2 \% & 1 \% \\ 204 & 986 & 4510 & 9760 & 21900 & - & \end{array}$

WEIGHTED SKEW $=-.130$
MAGNITUDE AND PROBABILITY OF ANNUAL LOW FLOW BASED ON PERIOD OF RECORD 1962-79

\begin{tabular}{|c|c|c|c|c|c|c|}
\hline \multirow{3}{*}{$\begin{array}{l}\text { PERIOD } \\
\text { (CON- } \\
\text { SECU- } \\
\text { TIVE } \\
\text { DAYS) }\end{array}$} & \multicolumn{6}{|c|}{$\begin{array}{l}\text { DISCHARGE, IN CFS, FOR IND ICATED RECURRENCE } \\
\text { INTERVAL, IN YEARS, AND ANNUAL NON- } \\
\text { EXCEEDANCE PROBABILITY, IN PERCENT }\end{array}$} \\
\hline & & & $-\cdots$ & - & $-\infty$ & -- \\
\hline & $\frac{2}{50 \%}$ & $\begin{array}{c}5 \\
203\end{array}$ & $\begin{array}{l}10 \\
10 \%\end{array}$ & $\begin{array}{l}20 \\
5 \%\end{array}$ & $\begin{array}{l}50 \\
2 \%\end{array}$ & $\begin{array}{r}100 \\
1 \%\end{array}$ \\
\hline & & -1 & - & -1 & & - \\
\hline 1 & - & -- & - & -- & -- & -- \\
\hline 3 & - & - & - & -- & -- & -- \\
\hline 7 & -- & -- & -- & -- & -- & -- \\
\hline 14 & 0.0 & 0.0 & 0.0 & 0.0 & -- & -- \\
\hline 30 & .1 & .1 & 0.0 & 0.0 & -- & -- \\
\hline 60 & .2 & .1 & 0.0 & 0.0 & -- & - \\
\hline 90 & .3 & .1 & .1 & 0.0 & -- & - \\
\hline 120 & .4 & .2 & .1 & .1 & - & - \\
\hline 183 & .9 & .4 & .2 & .2 & -- & -- \\
\hline
\end{tabular}

MAGNITUDE AND PROBABILITY OF ANNUAL HIGH FLOW BASED ON PERIOD OF RECORD 1961-79

\begin{tabular}{|c|c|c|c|c|c|c|}
\hline $\begin{array}{c}\text { PERIOD } \\
\text { (CON- } \\
\text { SFCI- }\end{array}$ & \multicolumn{6}{|c|}{$\begin{array}{l}\text { DISCHARGE, IN CFS, FOR INDICATED RECURRENCE } \\
\text { INTERVAL, IN YEARS, AND ANNUAL } \\
\text { EXCEEDANCE PROBABILITY, IN PERCENT }\end{array}$} \\
\hline TIVE & 2 & 5 & 10 & 25 & 50 & 100 \\
\hline DAYS) & $50 \%$ & $20 \%$ & $10 \%$ & $4 \%$ & $2 \%$ & $1 \%$ \\
\hline \multicolumn{7}{|c|}{ 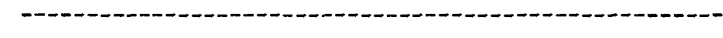 } \\
\hline 1 & 357 & 1610 & 3160 & 5970 & -- & - \\
\hline 3 & 262 & 995 & 1750 & 2910 & -- & -- \\
\hline 7 & 194 & 663 & 1090 & 1680 & -- & - \\
\hline 15 & 148 & 460 & 702 & 990 & -- & -- \\
\hline 30 & 107 & 303 & 444 & 600 & -- & - \\
\hline 60 & 78 & 218 & 319 & 432 & -- & -- \\
\hline 90 & 61 & 172 & 256 & 353 & -- & -- \\
\hline
\end{tabular}

DURATION TABLE OF DAILY MEAN FLOW FOR PERIOD OF RECORD $1961-79$

\begin{tabular}{|c|c|c|c|c|c|c|c|c|c|c|c|c|c|c|}
\hline $5 \%$ & $10 \%$ & $15 \%$ & $20 \%$ & $25 \%$ & $30 \%$ & $40 \%$ & $50 \%$ & $60 \%$ & $70 \%$ & $75 \%$ & $80 \%$ & $85 \%$ & $90 \%$ & $95 \%$ \\
\hline 147 & 82 & 55 & 37 & 24 & 13 & 3.9 & 1.6 & .7 & .3 & .2 & .1 & 0.0 & 0.0 & 0 \\
\hline
\end{tabular}


14037500 STRAWBERRY CREEK ABOVE SLIDE CREEK, NEAR PRAIRIE CITY, OR

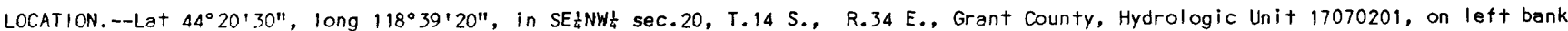
$100 \mathrm{ft}$ upstream from Slide Creek, $8.5 \mathrm{mi}$ south of Prairie City, and at mile 9.0.

DRAINAGE AREA. $-7.00 \mathrm{mi}^{2}$.

PERIOD OF RECORD.--October 1930 to September 1982. Prior to October 1944, published as "above South Fork, near Prairie City." GAGE.--Water-stage recorder and log control. Datum of gage is 4,909.57 ft National Geodetic Vertical Datum of 1929.

REMARKS.--FIOW affected by natural storage in Strawberry Lake. No diversion above station.

AVERAGE DISCHARGE. -52 years, $12.8 \mathrm{ft}^{3} / \mathrm{s}, 24.83 \mathrm{in} / \mathrm{yr}, 9,270 \mathrm{acre}-\mathrm{ft} / \mathrm{yr}$.

EXTREMES FOR PERIOD OF RECORD.--Maximum discharge, $274 \mathrm{ft} / \mathrm{s}$ June 14, 1974, gage height, $2.20 \mathrm{ft}$, from rating curve extended above $190 \mathrm{ft} / \mathrm{s}$; maximum gage height, $3.23 \mathrm{ft}$ May 24, 1956 (backwater from logs); minimum discharge, $1.0 \mathrm{ft} 3 / \mathrm{s}$ Mar. $20,1955$.

\section{STATISTICAL SUMMARIES}

MONTHLY AND ANNUAL MEAN DISCHARGES 1931-82

\begin{tabular}{|c|c|c|c|c|c|c|}
\hline MONTH & $\begin{array}{l}\text { MINIMUM } \\
\text { (CFS) }\end{array}$ & $\begin{array}{c}\text { MAXIMUM } \\
\text { (CFS) }\end{array}$ & $\begin{array}{l}\text { MEAN } \\
\text { (CFS) }\end{array}$ & $\begin{array}{c}\text { STAN- } \\
\text { DARD } \\
\text { DEVIA- } \\
\text { TION } \\
\text { (CFS) }\end{array}$ & $\begin{array}{l}\text { COEFFI- } \\
\text { CIENT OF } \\
\text { VARI- } \\
\text { ATION }\end{array}$ & $\begin{array}{c}\text { PERCENT } \\
\text { OF } \\
\text { ANNUAL } \\
\text { RUNOFF }\end{array}$ \\
\hline OCTOBER & 1.4 & 7.0 & 3.2 & 1.0 & .32 & 2.1 \\
\hline NOVEMBER & 1.7 & 11 & 3.6 & 1.7 & .47 & 2.3 \\
\hline DECEMBER & 1.6 & 14 & 4.2 & 2.6 & .62 & 2.7 \\
\hline JANUARY & 1.7 & 8.4 & 3.7 & 1.7 & .47 & 2.4 \\
\hline FEBRUARY & 1.5 & 13 & 3.5 & 2.1 & .59 & 2.3 \\
\hline MARCH & 1.3 & 7.9 & 3.5 & 1.5 & .43 & 2.3 \\
\hline APRIL & 1.9 & 23 & 9.1 & 4.7 & .52 & 5.9 \\
\hline MAY & 9.6 & 71 & 37 & 14 & .38 & 24.0 \\
\hline JUNE & 16 & 130 & 54 & 21 & .40 & 34.9 \\
\hline JULY & 6.7 & 55 & 21 & 11 & .54 & 13.7 \\
\hline AUGUST & 2.8 & 15 & 7.3 & 2.4 & .32 & 4.8 \\
\hline SEPTEMBER & 1.6 & 7.0 & 4.0 & 1.1 & .27 & 2.6 \\
\hline ANNUAL & 4.8 & 22 & 13 & 3.4 & .27 & 100 \\
\hline
\end{tabular}

MAGN ITUDE AND PROBABILITY OF INSTANTANEOUS PEAK FLOW BASED ON PERIOD OF RECORD 1931-82

DISCHARGE, IN CFS, FOR INDICATED RECURRENCE INTERVAL, IN YEARS, AND ANNUAL EXCEEDANCE PROBABILITY, IN PERCENT

\begin{tabular}{ccccccc}
1.25 & 2 & 5 & 10 & 25 & 50 & 100 \\
$80 \%$ & $50 \%$ & $20 \%$ & $10 \%$ & $4 \%$ & $2 \%$ & $1 \%$ \\
\hline 61 & 87 & 125 & 151 & 184 & 209 & 235 \\
\hline & $8 \%$ & & &
\end{tabular}

WEIGHTED SKEW $=-.017$
MAGNITUDE AND PROBABILITY OF ANNUAL LOW FLOW BASED ON PERIOD OF RECORD 1932-82

\begin{tabular}{|c|c|c|c|c|c|c|}
\hline \multirow{2}{*}{$\begin{array}{l}\text { PERIOD } \\
\text { (CON- } \\
\text { SECU- } \\
\text { TIVE } \\
\text { DAYS) }\end{array}$} & \multicolumn{6}{|c|}{$\begin{array}{l}\text { DISCHARGE, IN CFS, FOR INDICATED RECURRENCE } \\
\text { INTERVAL, IN YEARS, AND ANNUAL NON- } \\
\text { EXCEEDANCE PROBABILITY, IN PERCENT }\end{array}$} \\
\hline & $\begin{array}{c}2 \\
50 \%\end{array}$ & $\begin{array}{c}5 \\
200\end{array}$ & 10 & 20 & $\begin{array}{l}50 \\
2 \%\end{array}$ & $\begin{array}{r}100 \\
1 \%\end{array}$ \\
\hline & & & & & & \\
\hline 1 & 2.0 & 1.6 & 1.4 & 1.3 & 1.2 & 1.1 \\
\hline 3 & 2.0 & 1.6 & 1.5 & 1.3 & 1.2 & 1.1 \\
\hline 7 & 2.1 & 1.6 & 1.5 & 1.3 & 1.2 & 1.1 \\
\hline 14 & 2.1 & 1.7 & 1.5 & 1.4 & 1.3 & 1.2 \\
\hline 30 & 2.3 & 1.8 & 1.6 & 1.5 & 1.3 & 1.3 \\
\hline 60 & 2.5 & 2.0 & 1.8 & 1.6 & 1.5 & 1.4 \\
\hline 90 & 2.7 & 2.1 & 1.9 & 1.7 & 1.6 & 1.5 \\
\hline 120 & 2.9 & 2.3 & 2.0 & 1.8 & 1.7 & 1.6 \\
\hline 183 & 3.2 & 2.5 & 2.2 & 2.0 & 1.8 & 1.6 \\
\hline
\end{tabular}

MAGNITUDE AND PROBABILITY OF ANNUAL HIGH FLOW BASED ON PERIOD OF RECORD 1931-82

\begin{tabular}{|c|c|c|c|c|c|c|}
\hline \multirow{2}{*}{$\begin{array}{c}\text { PERIOD } \\
\text { (CON- } \\
\text { SECU- } \\
\text { TIVE } \\
\text { OAYS) }\end{array}$} & \multicolumn{6}{|c|}{$\begin{array}{c}\text { DISCHARGE, IN CFS, FOR INDICATED RECURRENCE } \\
\text { INTERVAL, IN YEARS, AND ANNUAL } \\
\text { EXCEEDANCE PROBABILITY, IN PERCENT }\end{array}$} \\
\hline & $\stackrel{2}{20 \%}$ & $\begin{array}{c}5 \\
20 \%\end{array}$ & $\begin{array}{l}10 \\
10 \%\end{array}$ & $\begin{array}{l}25 \\
4 \%\end{array}$ & $\begin{array}{l}50 \\
2 \%\end{array}$ & $\begin{array}{r}100 \\
1 \%\end{array}$ \\
\hline 1 & 83 & 114 & 135 & 160 & 178 & 195 \\
\hline 3 & 80 & 109 & 127 & 150 & 166 & 181 \\
\hline 7 & 74 & 101 & 117 & 138 & 152 & 166 \\
\hline 15 & 67 & 90 & 104 & 121 & 133 & 144 \\
\hline 30 & 59 & 78 & 89 & 101 & 109 & 117 \\
\hline 60 & 48 & 61 & 68 & 74 & 79 & 82 \\
\hline 90 & 38 & 48 & 53 & 58 & 61 & 64 \\
\hline
\end{tabular}

DURATION TABLE OF DAILY MEAN FLOW FOR PERIOD OF RECORD 1931-82

\begin{tabular}{|c|c|c|c|c|c|c|c|c|c|c|c|c|c|c|}
\hline & & DIS & GE, & FS, & H WAS & UALED & $R$ EXCE & ED FOR & NDICAT & PERCE & OF $T$ & & & \\
\hline $5 \%$ & $10 \%$ & $15 \%$ & $20 \%$ & $25 \%$ & $30 \%$ & $40 \%$ & $50 \%$ & $60 \%$ & $70 \%$ & $75 \%$ & $80 \%$ & $85 \%$ & $90 \%$ & $95 \%$ \\
\hline 57 & 39 & 27 & 18 & 13 & 9.0 & 6.1 & 4.5 & 3.8 & 3.3 & 3.0 & 2.8 & 2.5 & 2.3 & 1.9 \\
\hline
\end{tabular}


14038500 JOHN DAY RIVER AT PRAIRIE CITY, OR

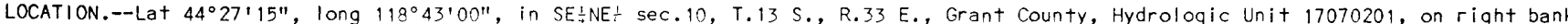
$600 \mathrm{ft}$ upstream from outlet of Prairie power canal, $0.3 \mathrm{mi}$ downstream from Dixie Creek, $0.8 \mathrm{mi}$ southwest of Prairie City, and at mile 262.0 .

DRAINAGE AREA. $--231 \mathrm{mi}^{2}$

PERIOD OF RECORD.--October 1916 to September 1917 (gage heights only), March 1925 to September 1968 . Monthiy discharge only March 1925 published in WSP 1318.

GAGE.--Water-stage recorder. Datun of gage is 3,494.5? ft National Geodetic Vertical Datum of 1929. Prior to Mar. 30 , 1926, staff gage at site $600 \mathrm{ft}$ downstream, just below outlet of Prairie power canal, at different datum. Mar. 30, 1926, to Aug. 23, 1943, staff gage at various sites and datums just above outlet of Prairie power canal. Aug. 24, 1943, to Sept. 22 , 1965, water-stage recorder at datum $2.07 \mathrm{ft}$ higher. Sept. 23, 1965, to July 27, 1966, water-stage recorder at present site and datum.

REMARKS.--No regulation. Several diversions upstream including prairie power canal (not used for power since February 1952) which diverts above station in SE $\frac{1}{4} \sec .7, T .13 \mathrm{~S} .$, R. 34 E., for irrigation above and below station.

AVERAGE DISCHARGE. - - 27 years (water years 1926-52), $113 \mathrm{ft} 3 / \mathrm{s}$, including $\mathrm{flow}$ of prairie power canal, 81,810 acre- $\mathrm{ft} / \mathrm{yr}$; $15 \mathrm{fears}$ (water years 1953-68), $114 \mathrm{ft} 3 / \mathrm{s}$, river only, 82,530 acre-ft/yr.

EXTREMES FOR PERIOD OF RECORD.--Maximum discharge, 2,400 $\mathrm{ft}^{3} / \mathrm{s}$ Dec. 22, 1964, gage height, $8.07 \mathrm{ft}$, present datum, from rating curve extended above $1,400 \mathrm{ft}^{3} / \mathrm{s}$; minimum, $2 \mathrm{ft} 3 / \mathrm{s}$ Dec. 8, 21, 22, 1932, Aug. 10, 1934.

STATISTICAL SUMMARIES (RIVER DISCHARGE ONLY DURING POWER CANAL DIVERSION)

MONTHLY AND ANNUAL MEAN DISCHARGES 1926-52

\begin{tabular}{|c|c|c|c|c|c|c|}
\hline MONTH & $\begin{array}{l}\text { MINIMUM } \\
\text { (CFS) }\end{array}$ & $\begin{array}{c}\text { MAXIMUM } \\
\text { (CFS) }\end{array}$ & $\begin{array}{l}\text { MEAN } \\
\text { (CFS) }\end{array}$ & $\begin{array}{c}\text { STAN- } \\
\text { DARD } \\
\text { DEV|A- } \\
\text { T!ON } \\
\text { (CFS) }\end{array}$ & $\begin{array}{l}\text { COEFFI- } \\
\text { CIENT OF } \\
\text { VARI- } \\
\text { ATION }\end{array}$ & $\begin{array}{c}\text { PERCENT } \\
\text { OF } \\
\text { ANNUAL } \\
\text { RUNOFF }\end{array}$ \\
\hline OCTOBER & 8.7 & 108 & 43 & 27 & .62 & 4.0 \\
\hline NOVEMBER & 6.5 & 124 & 51 & 29 & .57 & 4.8 \\
\hline DECEMBER & 8.4 & 163 & 65 & 38 & .58 & 6.2 \\
\hline JANUARY & 13 & 220 & 65 & 45 & .70 & 6.2 \\
\hline FEBRUARY & 12 & 174 & 85 & 43 & .51 & 8.0 \\
\hline MARCH & 27 & 310 & 139 & 74 & .53 & 13.1 \\
\hline APRIL & 45 & 377 & 188 & 82 & .44 & 17.8 \\
\hline MAY & 39 & 531 & 203 & 115 & .57 & 19.2 \\
\hline JUNE & 22 & 392 & 136 & 98 & .72 & 12.9 \\
\hline JULY & 9.5 & 87 & 34 & 19 & .57 & 3.2 \\
\hline AUGUST & 4.0 & 48 & 20 & 12 & .59 & 1.9 \\
\hline SEPTEMBER & 11 & 77 & 29 & 18 & .63 & 2.7 \\
\hline ANNUAL & 42 & 162 & 90 & 34 & .37 & 100 \\
\hline
\end{tabular}

MAGNITUDE AND PROBABILITY OF INSTANTANEOUS PEAK FLOW BASED ON PERIOD OF RECORD $1926-52$

DISCHARGE, IN CFS, FOR INDICATED RECURRENCE INTERVAL, IN YEARS, AND ANNUAL EXCEEDANCE PROBABILITY, IN PERCENT

$\begin{array}{ccccccc}1.25 & 2 & 5 & 10 & 25 & 50 & 100 \\ 80 \% & 50 \% & 20 \% & 10 \% & 4 \% & 2 \% & 1 \% \\ 329 & 540 & 921 & 1240 & 1720 & 2140 & -\end{array}$

WEIGHTED SKEW $=.276$
MAGNITUDE AND PROBABILITY OF ANNUAL LOW FLOW BASED ON PERIOD OF RECORD 1927-52

\begin{tabular}{|c|c|c|c|c|c|c|}
\hline $\begin{array}{r}\text { PERIOD } \\
\text { ICON- } \\
\text { SECU- }\end{array}$ & \multicolumn{6}{|c|}{$\begin{array}{l}\text { DISCHARGE, IN CFS, FOR INDICATED RECURRENCE } \\
\text { INTERVAL, IN YEARS, AND ANNUAL NON- } \\
\text { EXCEEDANCE PROBABILITY, IN PERCENT }\end{array}$} \\
\hline TIVE & 2 & 5 & 10 & 20 & 50 & 100 \\
\hline DAYS) & $50 \%$ & $20 \%$ & $10 \%$ & $5 \%$ & $2 \%$ & $1 \%$ \\
\hline 1 & 85 & 5 & 4.1 & 35 & 20 & - \\
\hline 3 & 9.3 & 5.6 & 4. & 3. & 3.1 & - \\
\hline 7 & 9.8 & 5.9 & 4.7 & 3.9 & 3.3 & - \\
\hline 14 & 11 & 6.6 & 5.2 & 4.3 & 3.5 & - \\
\hline 30 & 13 & 7.9 & 6.2 & 5.2 & 4.3 & -- \\
\hline 60 & 17 & 11 & 8.4 & 7.0 & 5.7 & -- \\
\hline 90 & 20 & 12 & 9.8 & 8.1 & 6.7 & -- \\
\hline 120 & 23 & 14 & 11 & 8.7 & 6.9 & -- \\
\hline 183 & 33 & 20 & 15 & 12 & 9.1 & -- \\
\hline
\end{tabular}

MAGNITUDE AND PROBABILITY OF ANNUAL HIGH FLOW BASED ON PERIOD OF RECORD $1926-52$

\begin{tabular}{|c|c|c|c|c|c|c|}
\hline $\begin{array}{l}\text { PERIOD } \\
\text { (CON- } \\
\text { SECU- }\end{array}$ & \multicolumn{6}{|c|}{$\begin{array}{c}\text { DISCHARGE, IN CFS, FOR INDICATED RECURRENCE } \\
\text { INTERVAL, IN YEARS, AND ANNUAL. } \\
\text { EXCEEDANCE PROBABILITY, IN PERCENT }\end{array}$} \\
\hline TIVE & 2 & 5 & 10 & 25 & 50 & 100 \\
\hline DAYS) & $50 \%$ & $20 \%$ & $10 \%$ & $4 \%$ & $2 \not t$ & $1 \%$ \\
\hline 1 & 461 & 744 & 975 & 1320 & 1620 & - \\
\hline 3 & 375 & 606 & 796 & 1080 & 1330 & - \\
\hline 7 & 327 & 512 & 659 & 871 & 1050 & -- \\
\hline 15 & 287 & 431 & 535 & 676 & 787 & $\rightarrow$ \\
\hline 30 & 250 & 364 & 439 & 533 & 601 & -- \\
\hline 50 & 217 & 310 & 370 & 443 & 496 & - \\
\hline 90 & 189 & 269 & 320 & 383 & 428 & -- \\
\hline
\end{tabular}

DURATION TABLE OF DAILY MEAN FLOW FOR PERIOD OF RECORD 1926-52

\begin{tabular}{|c|c|c|c|c|c|c|c|c|c|c|c|c|c|c|}
\hline $5 \%$ & $10 \%$ & $15 \%$ & $20 \%$ & $25 \%$ & $30 \%$ & $40 \%$ & $50^{d}$ & $60^{\alpha}$ & $70 \%$ & $75 \%$ & $80 \%$ & $85 \%$ & $90 \%$ & $95 \%$ \\
\hline 287 & 219 & 174 & 139 & 115 & 96 & 74 & 60 & 46 & 32 & 25 & 20 & 16 & 13 & 5 \\
\hline
\end{tabular}


14038500 JOHN DAY RIVER AT PRAIRIE CITY, OR--Continued

STATISTICAL SUMMARIES (POWER CANAL ABANDONED)

MONTHLY AND ANNUAL MEAN DISCHARGES 1953-58

\begin{tabular}{|c|c|c|c|c|c|c|}
\hline MONTH & $\begin{array}{l}\text { MINIMUM } \\
\text { (CFS) }\end{array}$ & $\begin{array}{l}\text { MAXIMUM } \\
\text { (CFS) }\end{array}$ & $\begin{array}{l}\text { MEAN } \\
\text { (CFS) }\end{array}$ & $\begin{array}{l}\text { STAN- } \\
\text { DARD } \\
\text { DEVIA- } \\
\text { TION } \\
\text { (CFS) }\end{array}$ & $\begin{array}{l}\text { COEFFI- } \\
\text { CIENT OF } \\
\text { VARI- } \\
\text { ATION }\end{array}$ & $\begin{array}{c}\text { PERCENT } \\
\text { OF } \\
\text { ANNUAL } \\
\text { RUNOFF }\end{array}$ \\
\hline OCTOBER & 45 & 102 & 68 & 14 & .20 & 5.0 \\
\hline NOVEMBER & 62 & 90 & 75 & 8.0 & .11 & 5.4 \\
\hline DECEMBER & 62 & 274 & 103 & 55 & .53 & 7.5 \\
\hline JANUARY & 64 & 316 & 109 & 64 & .58 & 7.9 \\
\hline FEBRUARY & 65 & 264 & $\$ 37$ & 64 & .47 & 10.0 \\
\hline MARCH & 92 & 293 & 144 & 57 & .39 & 10.5 \\
\hline APRIL & 79 & 359 & 199 & 80 & .40 & 14.5 \\
\hline MAY & 65 & 438 & 223 & 112 & .50 & 16.2 \\
\hline JUNE & 72 & 315 & 185 & 67 & .36 & 13.5 \\
\hline JULY & 24 & 105 & 59 & 24 & .41 & 4.3 \\
\hline AUGUST & 19 & 81 & 34 & 15 & .43 & 2.5 \\
\hline SEPTEMBER & 27 & 67 & 39 & 10 & .26 & 2.9 \\
\hline ANNUAL & 73 & 192 & 114 & 35 & .31 & 100 \\
\hline
\end{tabular}

MAGNITUDE AND PROBABILITY OF INSTANTANEOUS PEAK FLOW BASED ON PERIOD OF RECORD

DISCHARGE, IN CFS, FOR INDICATED RECURRENCE INTERVAL, IN YEARS, AND ANNUAL EXCEEOANCE PROBABILITY, IN PERCENT

\begin{tabular}{ccccccc}
1.25 & 2 & 5 & 10 & 25 & 50 & 100 \\
$80 \%$ & $50 \%$ & $20 \%$ & $10 \%$ & $4 \%$ & $2 \%$ & $1 \%$ \\
\hline & & & & & & \\
\hline & - & - & $\ldots$ & $\ldots$ & - & -
\end{tabular}

WEIGHTED SKEW =
MAGNITUDE AND PROBABILITY OF ANNUAL LOW FLOW BASED ON PERIOD OF RECORO 1954-68

\begin{tabular}{|c|c|c|c|c|c|c|}
\hline $\begin{array}{l}\text { PERIOD } \\
\text { ICON- } \\
\text { CFCH- }\end{array}$ & \multirow{2}{*}{\multicolumn{6}{|c|}{$\begin{array}{l}\text { DISCHARGE, IN CFS, FOR INDICATED RECURRENCE } \\
\text { INTERVAL, IN YEARS, AND ANNUAL NON- } \\
\text { EXCEEDANCE PROBABILITY, IN PERCENT }\end{array}$}} \\
\hline SECU- & & & & & & \\
\hline $\begin{array}{l}\text { TIVE } \\
\text { DAYS) }\end{array}$ & $\begin{array}{c}2 \\
50 \%\end{array}$ & ${ }^{5}$ & $\begin{array}{l}10 \\
10 \%\end{array}$ & $\begin{array}{l}20 \\
5 \%\end{array}$ & $\begin{array}{l}50 \\
2 \%\end{array}$ & $\begin{array}{r}100 \\
1 \%\end{array}$ \\
\hline 1 & 15 & 10 & 8. & 7.4 & - & - \\
\hline 3 & 16 & 11 & 9. & 8.1 & -- & - \\
\hline 7 & 17 & 13 & 11 & 10 & -- & - \\
\hline 14 & 20 & 15 & 13 & 12 & $-\infty$ & $\cdots$ \\
\hline 30 & 26 & 20 & 17 & 16 & -- & $\cdots$ \\
\hline 60 & 32 & 27 & 25 & 23 & -- & - \\
\hline 90 & 39 & 32 & 29 & 27 & -- & - \\
\hline 120 & 48 & 40 & 36 & 33 & - & $\cdots$ \\
\hline 183 & 59 & 51 & 47 & 44 & -- & - \\
\hline
\end{tabular}

MAGNITUDE AND PROBABILITY OF ANNUAL HIGH FLOW BASED ON PERIOD OF RECORD 1953-68

\begin{tabular}{|c|c|c|c|c|c|c|}
\hline \multirow{4}{*}{$\begin{array}{l}\text { PERIOD } \\
\text { (CON- } \\
\text { SECU- } \\
\text { TIVE } \\
\text { OAYS) }\end{array}$} & \multicolumn{6}{|c|}{$\begin{array}{l}\text { INTERVAL, IN YEARS, AND ANNUAL } \\
\text { EXCEEDANCE PROBABILITY, IN PERCENT }\end{array}$} \\
\hline & \multicolumn{6}{|c|}{ (1) } \\
\hline & 2 & 5 & 10 & 25 & 50 & 100 \\
\hline & $50 \%$ & $20 \%$ & $10 \%$ & $4 \%$ & & $1 \%$ \\
\hline & & & & & & \\
\hline 1 & 434 & 683 & 908 & 1280 & -- & - \\
\hline 3 & 393 & 596 & 774 & 1060 & -- & -- \\
\hline 7 & 366 & 520 & 634 & 794 & $\sim$ & - \\
\hline 15 & 310 & 425 & 506 & 613 & - & - \\
\hline 30 & 264 & 361 & 424 & 503 & -- & -- \\
\hline 60 & 226 & 310 & 364 & 432 & -- & - \\
\hline 90 & 205 & 277 & 324 & 381 & - & - \\
\hline
\end{tabular}

DURATION TABLE OF DAILY MEAN FLOW FOR PERIOD OF RECORO 1953-68

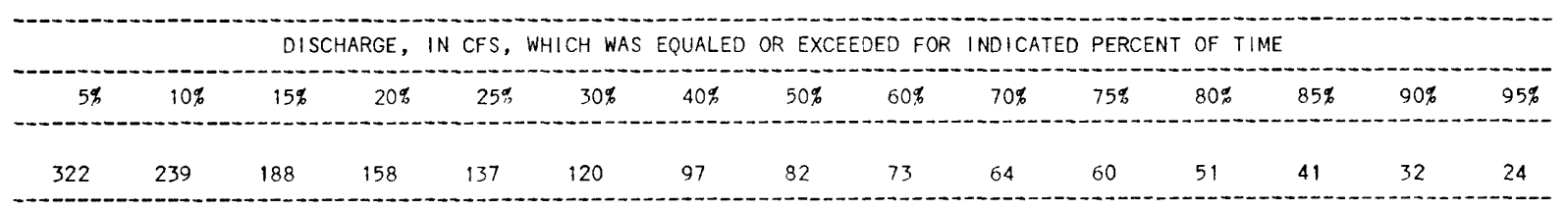




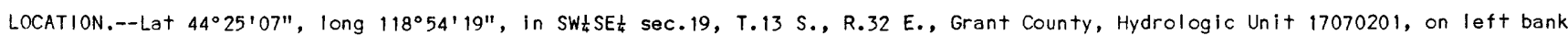

$1,200 \mathrm{ft}$ downstream from Dog Creek, $2.5 \mathrm{mi}$ east of John Day, and at mile 250.8 .

DRAINAGE AREA. $-386 \mathrm{mi}^{2}$.

PERIOD OF RECORD.--October 1968 to September 1982.

GAGE.-Water-stage recorder. Datum of gage is 3,130.56 ft National Geodetic Vertical Datum of 1929 .

REMARKS.--Some regulation from irrigation ditches upstream. Many diversions above station for irrigation.

AVERAGE DISCHARGE. -14 years, $207 \mathrm{ft}^{3} / \mathrm{s}, 150,000$ acre- $\mathrm{ft} / \mathrm{yr}$.

EXTREMES FOR PERIOD OF RECORO.--Maximum discharge, 5,830 $\mathrm{ft}^{3} / \mathrm{s}$ June 9, 1969, gage height, $10.80 \mathrm{ft}$, from $\mathrm{floodmark}$; minimum, 3.5

$f t^{3} / \mathrm{s}$ Aug. $26-28,1969$.

STATISTICAL SUMMARIES

MONTHLY AND ANNUAL MEAN DISCHARGES 1969-82

\begin{tabular}{|c|c|c|c|c|c|c|}
\hline MONTH & $\begin{array}{l}\text { MINIPAUM } \\
\text { (CFS) }\end{array}$ & $\begin{array}{l}\text { MAXIMUM } \\
\text { (CFS) }\end{array}$ & $\begin{array}{l}\text { MEAN } \\
\text { (CFS) }\end{array}$ & $\begin{array}{l}\text { STAN- } \\
\text { DARD } \\
\text { DEVIA- } \\
\text { TION } \\
\text { (CFS) }\end{array}$ & $\begin{array}{l}\text { COEFFI- } \\
\text { CIENT OF } \\
\text { VARI- } \\
\text { ATION }\end{array}$ & $\begin{array}{c}\text { PERCENT } \\
\text { OF } \\
\text { ANNUAL } \\
\text { RUNOFF }\end{array}$ \\
\hline OCTOBER & 73 & 112 & 88 & 12 & .13 & 3.5 \\
\hline NOVEMBER & 88 & 244 & 123 & 42 & .34 & 4.9 \\
\hline DECEMBER & 92 & 385 & 167 & 81 & .48 & 6.7 \\
\hline $\begin{array}{l}\text { JANUARY } \\
\text { FEBRUARY }\end{array}$ & $\begin{array}{l}88 \\
89\end{array}$ & $\begin{array}{l}514 \\
689\end{array}$ & $\begin{array}{l}235 \\
245\end{array}$ & $\begin{array}{l}117 \\
142\end{array}$ & $\begin{array}{l}.50 \\
.58\end{array}$ & $\begin{array}{l}9.5 \\
9.9\end{array}$ \\
\hline $\mathrm{MARCH}$ & 89 & 531 & 279 & 121 & .43 & 11.2 \\
\hline APRIL & 88 & 516 & 317 & 113 & .36 & 12.8 \\
\hline MAY & 108 & 741 & 443 & 161 & .36 & 17.8 \\
\hline JUNE & 74 & 810 & 381 & 209 & .55 & 15.3 \\
\hline $\begin{array}{l}\text { JULY } \\
\text { AUGUST }\end{array}$ & $\begin{array}{l}26 \\
10\end{array}$ & $\begin{array}{l}314 \\
116\end{array}$ & $\begin{array}{r}115 \\
41\end{array}$ & $\begin{array}{l}75 \\
30\end{array}$ & $\begin{array}{l}.65 \\
.71\end{array}$ & $\begin{array}{l}4.6 \\
1.7\end{array}$ \\
\hline SEPTEMBER & 26 & 101 & 51 & 20 & .39 & 2.1 \\
\hline ANNUAL & 74 & 370 & 207 & 71 & .34 & 100 \\
\hline
\end{tabular}

MAGNITUDE AND PROBABILITY OF INSTANTANEOUS PEAK FLOW BASED ON PERIOD OF RECORD 1969-82

\begin{tabular}{|c|c|c|c|c|c|c|}
\hline \multicolumn{7}{|c|}{$\begin{array}{l}\text { DISCHARGE, IN CFS, FOR INDICATED RECURRENCE INTERVAL, IN } \\
\text { YEARS, AND ANNUAL EXCEEDANCE PROBABILITY, IN PERCENT }\end{array}$} \\
\hline $\begin{array}{l}1.25 \\
80 \%\end{array}$ & $\stackrel{2}{2}$ & $\begin{array}{c}5 \\
20 \%\end{array}$ & $\begin{array}{l}10 \\
10 \%\end{array}$ & $\begin{array}{l}25 \\
4 \%\end{array}$ & $\begin{array}{l}50 \\
2 \%\end{array}$ & $\begin{array}{r}100 \\
1 \%\end{array}$ \\
\hline 1010 & 1670 & 2860 & 3840 & 5320 & -- & -- \\
\hline
\end{tabular}

WEIGHTED SKEW $=.231$

MAGNITUDE AND PROBABILITY OF ANNUAL LOW FLOW BASED ON PERIOD OF RECORD 1970-82

\begin{tabular}{|c|c|c|c|c|c|c|}
\hline \multirow{4}{*}{$\begin{array}{l}\text { PERIOD } \\
\text { (CON- } \\
\text { SECU- } \\
\text { TIVE } \\
\text { DAYS) }\end{array}$} & \multicolumn{6}{|c|}{$\begin{array}{l}\text { DISCHARGE, IN CFS, FOR INDICATED RECURRENCE } \\
\text { INTERVAL, IN YEARS, AND ANNUAL NON- } \\
\text { EXCEEDANCE PROBABILITY, IN PERCENT }\end{array}$} \\
\hline & & & & & & \\
\hline & 2 & 5 & 10 & 20 & 50 & 100 \\
\hline & $50 \%$ & $20 \%$ & $10 \%$ & $5 \%$ & $2 \%$ & $1 \%$ \\
\hline 1 & 11 & 6. & 4. & 3.7 & -- & -- \\
\hline 3 & 12 & 7. & 5. & 4.2 & -- & -- \\
\hline 7 & 13 & 8. & 6. & 4.7 & -- & - \\
\hline 14 & 15 & 9. & 6.8 & 5.4 & -- & -- \\
\hline 30 & 22 & 14 & 10 & 7.9 & -- & - \\
\hline 60 & 37 & 26 & 21 & 18 & -- & -- \\
\hline 90 & 51 & 37 & 29 & 24 & -- & -- \\
\hline 120 & 62 & 49 & 42 & 36 & -- & -- \\
\hline 183 & 83 & 70 & 64 & 59 & -- & -- \\
\hline
\end{tabular}

MAGNITUDE AND PROBABILITY OF ANNUAL HIGH FLOW BASED ON PERIOD OF RECORD 1969-82

\begin{tabular}{|c|c|c|c|c|c|c|}
\hline $\begin{array}{l}\text { PERIOD } \\
\text { (CON- } \\
\text { SECU- }\end{array}$ & \multicolumn{6}{|c|}{$\begin{array}{c}\text { DISCHARGE, IN CFS, FOR INDICATED RECURRENCE } \\
\text { INTERVAL, IN YEARS, AND ANNUAL } \\
\text { EXCEEDANCE PROBABILITY, IN PERCENT }\end{array}$} \\
\hline TIVE & 2 & 5 & 10 & 25 & 50 & 100 \\
\hline DAYS) & $50 \%$ & $20 \%$ & $10 \%$ & $4 \%$ & $2 \%$ & $1 \%$ \\
\hline 1 & 1230 & 1800 & 2030 & 2210 & -- & - \\
\hline 3 & 998 & 1450 & 1640 & 1790 & -- & -- \\
\hline 7 & 790 & 1130 & 1270 & 1380 & -- & -- \\
\hline 15 & 649 & 860 & 932 & 979 & - & -- \\
\hline 30 & 561 & 721 & 768 & 796 & -- & -- \\
\hline 60 & 468 & 608 & 653 & 682 & -- & - \\
\hline 90 & 419 & 550 & 596 & 628 & - & - \\
\hline
\end{tabular}

DURATION TABLE OF DAILY MEAN FLOW FOR PERIOD OF RECORD 1969-82

DISCHARGE, IN CFS, WHICH WAS EQUALED OR EXCEEDED FOR INDICATED PERCENT OF TIME

\begin{tabular}{|c|c|c|c|c|c|c|c|c|c|c|c|c|c|c|}
\hline $5 \%$ & $10 \%$ & $15 \%$ & $20 \%$ & $25 \%$ & $30 \%$ & $40 \%$ & $50 \%$ & $60 \%$ & $70 \%$ & $75 \%$ & $80 \%$ & $85 \%$ & $90 \%$ & $95 \%$ \\
\hline 626 & 469 & 387 & 325 & 276 & 234 & 174 & 132 & 108 & 90 & 82 & 73 & 60 & 39 & 23 \\
\hline
\end{tabular}


14040500 JOHN DAY RIVER AT PICTURE GORGE, NEAR DAYVILLE, OR

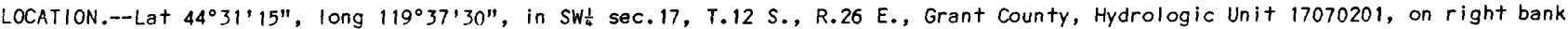
$0.7 \mathrm{mi}$ upstream from Rock Creek, $5.5 \mathrm{mi}$ northwest of Dayville, and at mile 205.1.

DRAINAGE AREA.--1,680 $\mathrm{mi}^{2}$, approximately.

PERIOD OF RECORD.--April 1926 to September 1982. Monthly discharge only April 1926, published in WSP 1318.

GAGE.--Water-stage recorder. Datum of gage is 2,229.84 ft National Geodetic Vertical Datum of 1929. Prior to Oct. 11, 1926, nonrecording gage and Oct. 11, 1926, to Sept. 30, 1930, water-stage recorder at same site at datum 2.50 ft higher. Oct. 1, 1930, to Aug. 28 , 1970, at datum $2.00 \mathrm{ft}$ higher.

REMARKS.--No regulation. Many diversions for irrigation above station.

AVERAGE DISCHARGE. -56 years, $478 \mathrm{ft} / \mathrm{s}, 346,300$ acre- $\mathrm{ft} / \mathrm{yr}$.

EXTREMES FOR PERIOD OF RECORO.--Maximum discharge, 8,170 $\mathrm{ft}^{3} / \mathrm{s} \mathrm{Dec.} \mathrm{22,} \mathrm{1964,} \mathrm{gage} \mathrm{height,} 14.97 \mathrm{ft}$; minimum, $1.0 \mathrm{ft} / \mathrm{s}$ for several days in August and September 1930, Aug. 8, 9, 1936, Sept. 9, 1966.

\section{STATISTICAL SUMMARIES}

MONTHLY AND ANNUAL MEAN DISCHARGES 1927-82

\begin{tabular}{|c|c|c|c|c|c|c|}
\hline MONTH & $\begin{array}{c}\text { MINIMUM } \\
\text { (CFS) }\end{array}$ & $\begin{array}{c}\text { MAXIMUM } \\
\text { (CFS) }\end{array}$ & $\begin{array}{l}\text { MEAN } \\
\text { (CFS) }\end{array}$ & $\begin{array}{c}\text { STAN- } \\
\text { DARD } \\
\text { DEVIA- } \\
\text { TION } \\
\text { (CFS) }\end{array}$ & $\begin{array}{l}\text { COEFFI- } \\
\text { CIENT OF } \\
\text { VARI- } \\
\text { ATION }\end{array}$ & $\begin{array}{c}\text { PERCENT } \\
\text { OF } \\
\text { ANNUAL } \\
\text { RUNOFF }\end{array}$ \\
\hline OCTOBER & 15 & 282 & 135 & 58 & .43 & 2.3 \\
\hline $\begin{array}{l}\text { NOVEMBER } \\
\text { DECEMBER } \\
\text { LNWARY }\end{array}$ & $\begin{array}{r}75 \\
105 \\
97\end{array}$ & $\begin{array}{r}561 \\
1377 \\
1706\end{array}$ & $\begin{array}{l}220 \\
366 \\
467\end{array}$ & $\begin{array}{r}83 \\
258 \\
368\end{array}$ & $\begin{array}{l}.38 \\
.70 \\
.79\end{array}$ & $\begin{array}{l}3.8 \\
6.4 \\
8.1\end{array}$ \\
\hline $\begin{array}{l}\text { JANUARY } \\
\text { FEBRUARY }\end{array}$ & $\begin{array}{r}97 \\
136\end{array}$ & $\begin{array}{l}1706 \\
2493\end{array}$ & $\begin{array}{l}467 \\
638\end{array}$ & $\begin{array}{l}368 \\
450\end{array}$ & $\begin{array}{l}.79 \\
.71\end{array}$ & $\begin{array}{r}8.1 \\
11.1\end{array}$ \\
\hline $\begin{array}{l}\text { MARCH } \\
\text { APRIL }\end{array}$ & $\begin{array}{l}196 \\
146\end{array}$ & $\begin{array}{l}2380 \\
3310\end{array}$ & $\begin{array}{r}882 \\
1204\end{array}$ & $\begin{array}{l}450 \\
596\end{array}$ & $\begin{array}{l}.51 \\
.50\end{array}$ & $\begin{array}{l}15.3 \\
20.9\end{array}$ \\
\hline $\begin{array}{l}\text { MAY } \\
\text { JUNE }\end{array}$ & $\begin{array}{l}79 \\
78\end{array}$ & $\begin{array}{l}2955 \\
1899\end{array}$ & $\begin{array}{r}1034 \\
591\end{array}$ & $\begin{array}{l}570 \\
371\end{array}$ & $\begin{array}{l}.55 \\
.63\end{array}$ & $\begin{array}{l}18.0 \\
10.3\end{array}$ \\
\hline JULY & 7.2 & 577 & 125 & 113 & .90 & 2.2 \\
\hline AUGUST & 1.6 & 213 & 43 & 45 & 1.06 & .7 \\
\hline SEPTEMBER & 2.6 & 210 & 56 & 46 & .84 & 1.0 \\
\hline ANNUAL & 124 & 1043 & 478 & 210 & .44 & 100 \\
\hline
\end{tabular}

MAGNITUDE AND PROBABILITY OF INSTANTANEOUS PEAK FLOW BASED ON PERIOD OF RECORD 1927-82

\begin{tabular}{|c|c|c|c|c|c|c|}
\hline \multicolumn{7}{|c|}{$\begin{array}{l}\text { DISCHARGE, IN CFS, FOR INDICATED RECURRENCE INTERVAL, IN } \\
\text { YEARS, AND ANNUAL EXCEEDANCE PROBABILITY, IN PERCENT }\end{array}$} \\
\hline $\begin{array}{l}1.25 \\
80 \%\end{array}$ & $\begin{array}{c}2 \\
50 \%\end{array}$ & $\begin{array}{c}5 \\
20 \%\end{array}$ & $\begin{array}{l}10 \\
10 \%\end{array}$ & $\begin{array}{l}25 \\
4 \%\end{array}$ & $\begin{array}{l}50 \\
2 \%\end{array}$ & $\begin{array}{r}100 \\
1 \%\end{array}$ \\
\hline 1650 & 2680 & 4360 & 5610 & 7340 & 8720 & 10200 \\
\hline
\end{tabular}

WEIGHTED SKEW $=-.018$
MAGNITUDE AND PROBABILITY OF ANNUAL LOW FLOW BASED ON PERIOD OF RECORD 1928-82

\begin{tabular}{|c|c|c|c|c|c|c|}
\hline $\begin{array}{l}\text { PERIOD } \\
\text { ICON- } \\
\text { SECII }\end{array}$ & \multicolumn{6}{|c|}{$\begin{array}{l}\text { DISCHARGE, IN CFS, FOR INDICATED RECURRENCE } \\
\text { INTERVAL, IN YEARS, AND ANNUAL NON- } \\
\text { EXCEEDANCE PROBABILITY, IN PERCENT }\end{array}$} \\
\hline SELU- & 2 & 5 & 10 & 20 & & \\
\hline TIVE & 2 & 5 & 10 & 20 & 50 & 100 \\
\hline DAYS) & $50 \%$ & $20 \%$ & $10 \%$ & $5 \%$ & $2 \%$ & $1 \%$ \\
\hline \multicolumn{7}{|c|}{7} \\
\hline 1 & 13 & 4.5 & 2.5 & 1.5 & .8 & .5 \\
\hline 3 & 14 & 4.9 & 2.7 & 1.6 & .9 & .6 \\
\hline 7 & 15 & 5.4 & 3.1 & 1.8 & 1.0 & .7 \\
\hline 14 & 16 & 6.0 & 3.4 & 2.0 & 1.1 & .7 \\
\hline 30 & 20 & 7.3 & 4.1 & 2.4 & 1.3 & .9 \\
\hline 60 & 28 & 10 & 5.5 & 3.2 & 1.7 & 1.1 \\
\hline 90 & 42 & 16 & 9.0 & 5.3 & 2.8 & 1.7 \\
\hline 120 & 71 & 33 & 20 & 13 & 7.0 & 4.6 \\
\hline 183 & 130 & 82 & 61 & 46 & 33 & 26 \\
\hline
\end{tabular}

MAGN ITUDE AND PROBABILITY OF ANNUAL HIGH FLOW BASED ON PERIOD OF RECORD 1927-82

\begin{tabular}{|c|c|c|c|c|c|c|}
\hline PERI OD & \multicolumn{6}{|c|}{$\begin{array}{l}\text { DISCHARGE, IN CFS, FOR INDICATED RECURRENCE } \\
\text { INTERVAL, IN YEARS, AND ANNUAL }\end{array}$} \\
\hline 'CON- & \multicolumn{6}{|c|}{ EXCEEDANCE PROBABILITY, IN PERCENT } \\
\hline TIVE & 2 & 5 & 10 & 25 & 50 & 100 \\
\hline DAYS) & $50 \%$ & $20 \%$ & $10 \%$ & $4 \%$ & $2 \%$ & $1 \%$ \\
\hline 1 & 2390 & 3860 & 4880 & 6200 & 7200 & 8200 \\
\hline 3 & 2210 & 3500 & 4330 & 5320 & 6010 & 6660 \\
\hline 7 & 1970 & 3070 & 3740 & 4500 & 5010 & 5460 \\
\hline 15 & 1720 & 2590 & 3060 & 3560 & 3870 & 4120 \\
\hline 30 & 1460 & 2160 & 2540 & 2940 & 3180 & 3380 \\
\hline 60 & 1220 & 1800 & 2130 & 2470 & 2680 & 2860 \\
\hline 90 & 1080 & 1580 & 1860 & 2150 & 2330 & 2480 \\
\hline
\end{tabular}

DURATION TABLE OF DAILY MEAN FLOW FOR PERIOD OF RECORD 1927-82

$\begin{array}{ccccccccccccccc}5 \% & 10 \% & 15 \% & 20 \% & 25 \% & 30 \% & 40 \% & 50 \% & 60 \% & 70 \% & 75 \% & 80 \% & 85 \% & 90 \% & 95 \%\end{array}$




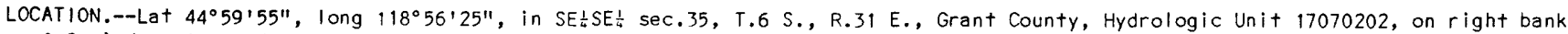

$0.2 \mathrm{mi}$ downstream from Desolation Creek and $0.8 \mathrm{mi}$ northeast of Dale.

DRAINAGE AREA.---525 $\mathrm{mi}^{2}$.

PERIOD OF RECORD.---October 1929 to September 1958.

GAGE.--Water-stage recorder. Datum of gage is 2,775.63 $\mathrm{ft}$ National Geodetic Vertical Datum of 1929.

REMARKS.--Flow regulated by 0 live Lake (capacity, about 5,500 acre- $f+$ ) and Upper Reservoir on Lake Creek (capacity, about 700 acre- $f t$ ). Some diurnal fluctuation at low flow caused by logging operations above station. Several small diversions for irrigation and mining above station. Since 1865 water has been diverted above station at times to North Fork Burnt River.

AVERAGE DISCHARGE.--29 years (water years 1929-58), $404 \mathrm{ft}+3 / \mathrm{s}, 292,500$ acre- $f+/ \mathrm{yr}$.

EXTREMES FOR PERIOD OF RECORD.--Maximum discharge, $8,170 \mathrm{ft}^{3} / \mathrm{s} \mathrm{May} 26,1948$, gage height, $10.48 \mathrm{ft} ; \mathrm{minimum}$, $6 \mathrm{ft}+\mathrm{s}^{\mathrm{s}} \mathrm{s}$ Nov. 3 , 1936, gage height, $1.40 \mathrm{ft}$, result of freezeup; minimum daily, $10 \mathrm{ft} / \mathrm{s}$ Nov. 11, Nov. 29 to Dec. $1,1936, \mathrm{Jan}$. 7 , 8 , 1937.

STATISTICAL SUMMARIES

MONTHLY AND ANNUAL MEAN DISCHARGES 1930-58

\begin{tabular}{|c|c|c|c|c|c|c|}
\hline MONTH & $\begin{array}{l}\text { MINIMUM } \\
\text { (CFS) }\end{array}$ & $\begin{array}{l}\text { MAXIMUM } \\
\text { (CFS) }\end{array}$ & $\begin{array}{l}\text { MEAN } \\
\text { (CFS) }\end{array}$ & $\begin{array}{l}\text { STAN- } \\
\text { DARD } \\
\text { DEVIA- } \\
\text { TION } \\
\text { (CFS) }\end{array}$ & $\begin{array}{l}\text { COEFFI- } \\
\text { CIENT OF } \\
\text { VARI- } \\
\text { ATION }\end{array}$ & $\begin{array}{c}\text { PERCENT } \\
\text { OF } \\
\text { ANNUAL } \\
\text { RUNOFF }\end{array}$ \\
\hline OCTOBER & 39 & 147 & 71 & 28 & .39 & 1.5 \\
\hline $\begin{array}{l}\text { NOVEMBER } \\
\text { DECEMBER }\end{array}$ & $\begin{array}{l}31 \\
37\end{array}$ & $\begin{array}{l}274 \\
463\end{array}$ & $\begin{array}{r}96 \\
145\end{array}$ & $\begin{array}{r}48 \\
111\end{array}$ & $\begin{array}{l}.50 \\
.77\end{array}$ & $\begin{array}{l}2.0 \\
3.0\end{array}$ \\
\hline JANUARY & 29 & 343 & 130 & 65 & .50 & 2.7 \\
\hline $\begin{array}{l}\text { FEBRUARY } \\
\text { MARCH }\end{array}$ & $\begin{array}{l}35 \\
83\end{array}$ & $\begin{array}{l}496 \\
593\end{array}$ & $\begin{array}{l}178 \\
276\end{array}$ & $\begin{array}{r}97 \\
128\end{array}$ & $\begin{array}{l}.54 \\
.46\end{array}$ & $\begin{array}{l}3.7 \\
5.7\end{array}$ \\
\hline APRIL & 198 & 2052 & 1009 & 457 & .45 & 20.8 \\
\hline $\begin{array}{l}\text { MAY } \\
\text { JUNE }\end{array}$ & $\begin{array}{l}253 \\
212\end{array}$ & $\begin{array}{l}3296 \\
2589\end{array}$ & $\begin{array}{r}1708 \\
906\end{array}$ & $\begin{array}{l}766 \\
557\end{array}$ & $\begin{array}{l}.45 \\
.61\end{array}$ & $\begin{array}{l}35.2 \\
18.7\end{array}$ \\
\hline JULY & 60 & 544 & 206 & 120 & .58 & 4.2 \\
\hline AUG & 28 & 13 & 69 & 28 & .41 & 1.4 \\
\hline SEPTEMBER & 31 & 117 & 56 & 19 & .34 & 1.1 \\
\hline INNUAL & 191 & 682 & 405 & 139 & .34 & 100 \\
\hline
\end{tabular}

MAGNITUDE AND PROBABILITY OF INSTANTANEOUS PEAK FLOW BASED ON PERIOD OF RECORD 1930-58

DISCHARGE, IN CFS, FOR INDICATED RECURRENCE INTERVAL, IN YEARS, AND ANNUAL EXCEEDANCE PROBABILITY, IN PERCENT

\begin{tabular}{ccccccc}
1.25 & 2 & 5 & 10 & 25 & 50 & 100 \\
$80 \%$ & $50 \%$ & $20 \%$ & $10 \%$ & $4 \%$ & $2 \%$ & $1 \%$ \\
\hline 2030 & 3080 & 4500 & 5410 & 6530 & 7330 & - \\
\hline WEIGHTED SKEW & $=$ & -.309 & &
\end{tabular}

MAGN! TUDE AND PROBABILITY OF ANNUAL LOW FLOW BASED ON PERIOD OF RECORD 1931-58

\begin{tabular}{|c|c|c|c|c|c|c|}
\hline $\begin{array}{r}\text { PERIOD } \\
\text { ICON- } \\
\text { SECU- }\end{array}$ & \multicolumn{6}{|c|}{$\begin{array}{l}\text { DISCHARGE, IN CFS, FOR INDICATED RECURRENCE } \\
\text { INTERVAL, IN YEARS, AND ANNUAL NON- } \\
\text { EXCEEDANCE PROBABILITY, IN PERCENT }\end{array}$} \\
\hline TIVE & 2 & 5 & 10 & 20 & 50 & 100 \\
\hline DAYS) & $50 \%$ & $20 \%$ & $10 \%$ & $5 \%$ & $2 \%$ & $1 \%$ \\
\hline 1 & 33 & 21 & 16 & 13 & 9.6 & -- \\
\hline 3 & 37 & 24 & 18 & 14 & 11 & -- \\
\hline 7 & 40 & 27 & 22 & 18 & 15 & - \\
\hline 14 & 42 & 32 & 27 & 24 & 20 & -- \\
\hline 30 & 47 & 35 & 31 & 27 & 24 & -- \\
\hline 60 & 52 & 39 & 34 & 31 & 27 & - \\
\hline 90 & 58 & 44 & 37 & 33 & 29 & -- \\
\hline 120 & 64 & 49 & 42 & 37 & 32 & -- \\
\hline 183 & 83 & 59 & 50 & 43 & 37 & -- \\
\hline
\end{tabular}

MAGNITUDE AND PROBABILITY OF ANNUAL HIGH FLOW BASED ON PERIOD OF RECORD 1930-58

\begin{tabular}{|c|c|c|c|c|c|c|}
\hline PERIOD & \multicolumn{6}{|c|}{$\begin{array}{c}\text { DISCHARGE, IN CFS, FOR INDICATED RECURRENCE } \\
\text { INTERVAL, IN YEARS, AND ANNUAL }\end{array}$} \\
\hline (CON- & \multicolumn{6}{|c|}{ EXCEEDANCE PROBABILITY, IN PERCENT } \\
\hline SECU- & \multicolumn{6}{|c|}{ ( } \\
\hline TIVE & 2 & 5 & 10 & 25 & 50 & 100 \\
\hline DAYS) & $50 \%$ & $20 \%$ & $10 \%$ & $4 \%$ & $2 \%$ & $1 \%$ \\
\hline \multicolumn{7}{|c|}{ 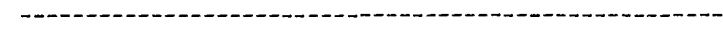 } \\
\hline 1 & 2850 & 4090 & 4840 & 5720 & 6330 & - \\
\hline 3 & 2690 & 3830 & 4510 & 5280 & 5800 & - \\
\hline 7 & 2480 & 3520 & 4130 & 4800 & 5240 & -- \\
\hline 15 & 2190 & 3110 & 3640 & 4230 & 4620 & -- \\
\hline 30 & 1900 & 2670 & 3110 & 3600 & 3930 & -- \\
\hline 60 & 1520 & 2080 & 2400 & 2740 & 2970 & -- \\
\hline 90 & 1200 & 1630 & 1880 & 2160 & 2340 & - \\
\hline
\end{tabular}

DURATION TABLE OF DAILY MEAN FLOW FOR PERIOD OF RECORD 1930-58

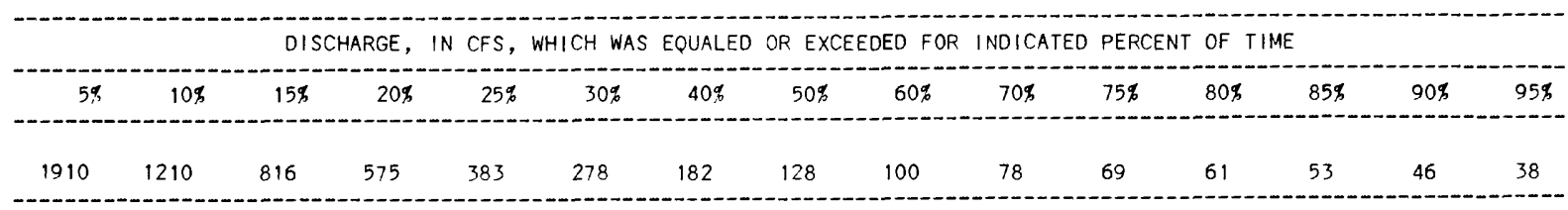


14042000 CAMAS CREEK NEAR LEHH:AN, OR

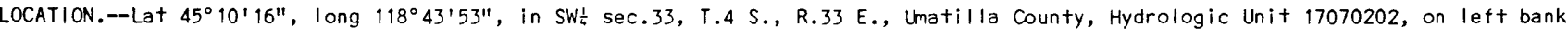

$2.1 \mathrm{mi}$ downstream from Bowman Creek, $3.5 \mathrm{mi}$ northwest of Lehman, and at mile 25.5 .

DRA INAGE AREA.--60.7 $\mathrm{mi}^{2}$.

PERIOD OF RECORD.--October 1950 to September 1970.

GAGE.--Water-stage recorder. Datum of gage is $3,969.53 \mathrm{ft}$ National Geodetic Vertical Datum of 1929 (levels by State Highway

Department).

REMARKS.--No regulation. A tew small diversions for irrigation above station.

AVERAGE DISCHARGE. --20 years, $43.3 \mathrm{ft}^{3} / \mathrm{s}, 31,370$ acre- $\mathrm{ft} / \mathrm{yr}$.

EXTREMES FOR PERIOD OF RECORD.--Maximum discharge, 1,880 $\mathrm{t}^{3} / \mathrm{s}$ Dec. 21 , 1955, gage height, $4.56 \mathrm{ft}$, from rating curve extended above $900 \mathrm{ft}^{3} / \mathrm{s}$; minimum, $0.23 \mathrm{ft}^{3} / \mathrm{s}$ Aug. 24, 1966 .

STATISTICAL SUMMARIES

MONTHLY AND ANNUAL. MEAN DISCHARGES 1951-70

\begin{tabular}{|c|c|c|c|c|c|c|}
\hline MONTH & $\begin{array}{l}\text { MINIMUM } \\
\text { (CFS) }\end{array}$ & $\begin{array}{l}\text { MAXIMUM } \\
\text { (CFS) }\end{array}$ & $\begin{array}{l}\text { MEAN } \\
\text { (CFS) }\end{array}$ & $\begin{array}{l}\text { STAN- } \\
\text { DARD } \\
\text { DEVIA- } \\
\text { TION } \\
\text { (CFS) }\end{array}$ & $\begin{array}{l}\text { COEFFI- } \\
\text { CIENT OF } \\
\text { VARI- } \\
\text { ATION }\end{array}$ & $\begin{array}{c}\text { PERCEN } \\
\text { OF } \\
\text { ANNUAL } \\
\text { RUNOF }\end{array}$ \\
\hline OCTOBER & 1.2 & 11 & 3.2 & 2.5 & .78 & .6 \\
\hline NOVEMBER & 1.8 & 36 & 11 & 11 & (99. & 2.0 \\
\hline DECEMBER & 1.9 & 155 & 34 & 39 & 1.13 & 6.5 \\
\hline JANUARY & 2.7 & 156 & 42 & 44 & 1.05 & 8. \\
\hline FEBRUARY & 2.5 & 207 & 62 & 54 & .86 & 11.9 \\
\hline MARCH & 5.9 & 196 & 81 & 47 & .58 & 15. \\
\hline APRIL & 27 & 269 & 167 & 72 & .43 & 32.0 \\
\hline $\begin{array}{l}\text { MAY } \\
\text { JUNE }\end{array}$ & $\begin{array}{l}13 \\
6.4\end{array}$ & $\begin{array}{r}203 \\
76\end{array}$ & $\begin{array}{l}91 \\
23\end{array}$ & $\begin{array}{l}51 \\
19\end{array}$ & $\begin{array}{l}.56 \\
.81\end{array}$ & $\begin{array}{r}17.5 \\
4.5\end{array}$ \\
\hline $\begin{array}{l}\text { JULY } \\
\text { AUGUST }\end{array}$ & $\begin{array}{r}1.4 \\
.5\end{array}$ & $\begin{array}{r}11 \\
2.2\end{array}$ & $\begin{array}{l}4.3 \\
1.4\end{array}$ & $\begin{array}{r}2.7 \\
.5\end{array}$ & $\begin{array}{l}.62 \\
.32\end{array}$ & .8 \\
\hline SEPTEMBER & .8 & 2.7 & 1.5 & .5 & .32 & \\
\hline ANNUAL & 18 & 81 & 43 & 16 & .36 & 100 \\
\hline
\end{tabular}

MAGNITUDE AND PROBABILITY OF INSTANTANEOUS PEAK FLOW BASED ON PERIOD OF RECORD 1951-70

DISCHARGE, IN CFS, FOR INDICATED RECURRENCE INTERVAL, IN YEARS, AND ANNUAL EXCEEDANCE PROBABILITY, IN PERCENT

\begin{tabular}{ccccccc}
1.25 & 2 & 5 & 10 & 25 & 50 & 100 \\
$80 \%$ & $50 \%$ & $20 \%$ & $10 \%$ & $4 \%$ & $2 \%$ & $1 \%$ \\
\hline 418 & 634 & 994 & 1280 & 1680 & - & - \\
\hline
\end{tabular}

WEIGHTED SKEW $=.279$
MAGN I TUDE AND PROBABILIITY OF ANNUAL LOW FLOW BASED ON PERIOD OF RECORD 1952-70

\begin{tabular}{|c|c|c|c|c|c|c|}
\hline $\begin{array}{l}\text { PERIOD } \\
\text { (CON- } \\
\text { SECU- }\end{array}$ & \multicolumn{6}{|c|}{$\begin{array}{l}\text { DISCHARGE, IN CFS, FOR INDICATED RECURRENCE } \\
\text { INTERVAL, IN YEARS, AND ANNUAL NON- } \\
\text { EXCEEDANCE PROBABILIITY, IN PERCENT }\end{array}$} \\
\hline $\begin{array}{l}\text { TIVE } \\
\text { DAYS }\end{array}$ & $\begin{array}{c}2 \\
50 \%\end{array}$ & $\begin{array}{c}5 \\
20 \%\end{array}$ & $\begin{array}{l}10 \\
10 \%\end{array}$ & $\begin{array}{l}20 \\
5 \%\end{array}$ & $\begin{array}{l}50 \\
2 \%\end{array}$ & $\begin{array}{r}100 \\
1 \%\end{array}$ \\
\hline 1 & .8 & .6 & .5 & .4 & -- & - \\
\hline 3 & 9.9 & .6 & .5 & .4 & -- & -- \\
\hline 7 & .9 & .6 & .5 & .4 & -- & -- \\
\hline 14 & 1.0 & .7 & .6 & .5 & -- & -- \\
\hline 30 & 1.1 & .8 & .7 & .6 & -- & - \\
\hline 60 & 1.3 & 1.0 & .9 & .8 & -- & -- \\
\hline 90 & 1.5 & 1.2 & 1.1 & 1.0 & -- & -- \\
\hline 120 & 1.9 & 1.5 & 1.4 & 1.3 & $\cdots$ & -- \\
\hline 183 & 4.1 & 2.7 & 2.1 & 1.7 & $\rightarrow$ & - \\
\hline
\end{tabular}

MAGNITUDE AND PROBABILITY OF ANNUAL HIGH FLON BASED ON PERIOD OF RECORD 1951-70

\begin{tabular}{|c|c|c|c|c|c|c|}
\hline $\begin{array}{l}\text { PERIOD } \\
\text { (CON- }\end{array}$ & \multicolumn{6}{|c|}{$\begin{array}{c}\text { DISCHARGE, IN CFS, FOR INDICATED RECURRENCE } \\
\text { INTERVAL, IN YEARS, AND ANNUAL } \\
\text { EXCEEDANCE PROBABILITY, IN PERCENT }\end{array}$} \\
\hline $\begin{array}{l}\text { TIVE } \\
\text { DAYS }\end{array}$ & $\begin{array}{c}2 \\
50 \%\end{array}$ & $\begin{array}{c}5 \\
20 \%\end{array}$ & $\begin{array}{l}10 \\
10 \%\end{array}$ & $\begin{array}{l}25 \\
4 \%\end{array}$ & $\begin{array}{l}50 \\
2 \%\end{array}$ & $\begin{array}{r}100 \\
1 \%\end{array}$ \\
\hline 1 & 449 & 690 & 891 & 1200 & - & $-\infty$ \\
\hline 3 & 381 & 549 & 680 & 869 & -- & $\rightarrow$ \\
\hline 7 & 312 & 419 & 488 & 574 & $\cdots$ & $\cdots$ \\
\hline 15 & 252 & 329 & 372 & 421 & -- & -- \\
\hline 30 & 192 & 254 & 290 & 330 & - & - \\
\hline 60 & 145 & 195 & 224 & 259 & -- & - \\
\hline 90 & 122 & 164 & 190 & 220 & -- & $\cdots$ \\
\hline
\end{tabular}

DURATION TABLE OF DAILY MEAN FLOW FOR PERIOD OF RECORD 1951-70
OISCHARGE, IN CFS, WHICH WAS EQUALED OR EXCEEDED FOR INDICATED PERCENT OF TIME

\begin{tabular}{|c|c|c|c|c|c|c|c|c|c|c|c|c|c|c|}
\hline $5 \%$ & $10 \%$ & $15 \%$ & $20 \%$ & $25 \%$ & $30 \%$ & $40 \%$ & $50 \%$ & $60 \%$ & $70 \%$ & $75 \%$ & $80 \%$ & $85 \%$ & $90 \%$ & $95 \%$ \\
\hline 201 & 129 & 92 & 67 & 49 & 36 & 19 & 9.5 & 4.5 & 2.8 & 2.3 & 1.9 & 1.6 & 1.3 & 1.1 \\
\hline
\end{tabular}




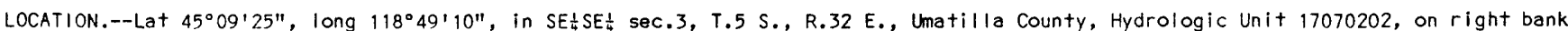

$1.2 \mathrm{mi}$ upstream from Cable Creek, $5.8 \mathrm{mi}$ east of Uklah, and at mile 18.7 .

DRAINAGE AREA.--121 $\mathrm{mi}^{2}$.

PERIOD OF RECORD. - May 1914 to September 1917, November 1919 to July 1920, November 1920 to June 1924 , March 1932 to June 1940 (fragmentary), November 1940 to September 1982. Monthly discharge only for some periods, published in WSP 1318. Published as

"above Cable Creek, near Ukiah" 1914-17, 1919-24.

GAGE.--Water-stage recorder. Datum of gage is $3.588 .61 \mathrm{ft}$ National Geodetic Vertical Datum of 1929 (levels by State Highway Department). May 1, 1914, to June 30, 1924, nonrecording gage and Mar. 1, 1932, to July 2, 1940, water-stage recorder at site 1.2 mi downstream at different datum.

REMARKS.--No regulation. Diversions for irrigation above station.

AVERAGE DISCHARGE.--46 years (water years 1915-17, 1922-23, 1942-82), $95.9 \mathrm{ft} 3 / \mathrm{s}, 69,480 \mathrm{acre}-\mathrm{ft} / \mathrm{yr}$.

EXTREMES FOR PERIOD OF RECORD.--Maximum discharge, 3,840 $\mathrm{ft}^{3} / \mathrm{s}$ Jan. 30,1965, gage height, $5.21 \mathrm{ft}$; maximum gage height, $5.92 \mathrm{ft}$ Jan. 24, 1982 (ice jam); minimum discharge recorded, $1.0 \mathrm{ft}^{3} / \mathrm{s}$ Aug. 9, 1932, June 24 to July 2 , 1940.

\section{STATISTICAL SUMMARIES}

MONTHLY AND ANNUAL MEAN DISCHARGES 1915-82

\begin{tabular}{|c|c|c|c|c|c|c|}
\hline MONTH & $\begin{array}{l}\text { MINIMUM } \\
\text { (CFS) }\end{array}$ & $\begin{array}{c}\text { MAXIMUM } \\
\text { (CFS) }\end{array}$ & $\begin{array}{l}\text { MEAN } \\
\text { (CFS) }\end{array}$ & $\begin{array}{l}\text { STAN- } \\
\text { DARD } \\
\text { DEVIA- } \\
\text { TION } \\
\text { (CFS) }\end{array}$ & $\begin{array}{l}\text { COEFFI- } \\
\text { CIENT OF } \\
\text { VARI- } \\
\text { ATION }\end{array}$ & $\begin{array}{c}\text { PERCENT } \\
\text { OF } \\
\text { ANNUAL } \\
\text { RUNOFF }\end{array}$ \\
\hline OCTOBER & 3.4 & 39 & 8.8 & 6.0 & .68 & .8 \\
\hline NOVEMBER & 4.4 & 140 & 28 & 30 & 1.10 & 2.4 \\
\hline DECEMBER & 4.7 & 263 & 67 & 64 & .95 & 5.8 \\
\hline JANUARY & 3.5 & 338 & 74 & 80 & 1.08 & 6.4 \\
\hline FEBRUARY & 5.0 & 384 & 111 & 102 & .92 & 9.6 \\
\hline MARCH & 5.0 & 575 & 178 & 121 & .68 & 15.4 \\
\hline APRIL & 53 & 658 & 337 & 140 & .42 & 29.2 \\
\hline MAY & 19 & 925 & 246 & 162 & .66 & 21.3 \\
\hline JUNE & 4.9 & 314 & 80 & 66 & .83 & 6.9 \\
\hline JULY & 3.1 & 77 & 15 & 13 & .84 & 1.3 \\
\hline AUGUST & 2.2 & 11 & 5.0 & 1.9 & .38 & .4 \\
\hline SEPTEMBER & 2.4 & 14 & 5.3 & 2.2 & .41 & .5 \\
\hline NNUAL & 36 & 171 & 96 & 34 & .36 & 100 \\
\hline
\end{tabular}

MAGNITUDE AND PROBABILITY OF INSTANTANEOUS PEAK FLOW BASED ON PERIOD OF RECORD $1915-82$

\begin{tabular}{|c|c|c|c|c|c|c|}
\hline \multirow{2}{*}{\multicolumn{7}{|c|}{$\begin{array}{l}\text { I SCHARGE, IN CFS, FOR INDICATED RECURRENCE INTERVAL, IN } \\
\text { YEARS, AND ANNUAL EXCEEDANCE PROBABILITY, IN PERCENT }\end{array}$}} \\
\hline & & & & & & \\
\hline $\begin{array}{l}1.25 \\
80 \%\end{array}$ & $\begin{array}{c}2 \\
50 \%\end{array}$ & $\begin{array}{c}5 \\
20 \%\end{array}$ & $\begin{array}{l}10 \\
10 \%\end{array}$ & $\begin{array}{l}25 \\
4 \%\end{array}$ & $\begin{array}{l}50 \\
2 \%\end{array}$ & $\begin{array}{r}100 \\
1 \%\end{array}$ \\
\hline 695 & 1040 & 1590 & 2010 & 2580 & 3050 & 3560 \\
\hline
\end{tabular}

WEIGHTED SKEW $=.184$
MAGNITUDE AND PROBABILITY OF ANNUAL LOW FLOW BASED ON PERIOD OF RECORD 1916-82

\begin{tabular}{|c|c|c|c|c|c|c|}
\hline \multirow{3}{*}{$\begin{array}{l}\text { PERIOD } \\
\text { (CON- } \\
\text { SECU- } \\
\text { TIVE } \\
\text { DAYS) }\end{array}$} & \multicolumn{6}{|c|}{$\begin{array}{l}\text { DISCHARGE, IN CFS, FOR INDICATED RECURRENCE } \\
\text { INTERVAL, IN YEARS, AND ANNUAL NON- } \\
\text { EXCEEDANCE PROBABILITY, IN PERCENT }\end{array}$} \\
\hline & 2 & 5 & 10 & 20 & 50 & 100 \\
\hline & $50 \%$ & $20 \%$ & $10 \%$ & $5 \%$ & $2 \%$ & $1 \%$ \\
\hline 1 & 2.8 & 2.2 & 1.9 & 1.7 & 1.5 & 1.4 \\
\hline 3 & 2.9 & 2.2 & 1.9 & 1.7 & 1.5 & 1.4 \\
\hline 7 & 3.1 & 2.4 & 2.1 & 1.8 & 1.6 & 1.5 \\
\hline 14 & 3.2 & 2.6 & 2.3 & 2.1 & 1.9 & 1.7 \\
\hline 30 & 3.6 & 2.9 & 2.5 & 2.3 & 2.1 & 1.9 \\
\hline 60 & 4.3 & 3.4 & 3.0 & 2.7 & 2.4 & 2.2 \\
\hline 90 & 4.9 & 4.0 & 3.6 & 3.4 & 3.1 & 3.0 \\
\hline 120 & 5.8 & 4.7 & 4.3 & 4.1 & 3.9 & 3.8 \\
\hline 183 & 12 & 7.3 & 5.9 & 4.9 & 4.0 & 3.6 \\
\hline
\end{tabular}

MAGNITUDE AND PROBABILITY OF ANNUAL HIGH FLOW BASED ON PERIOD OF RECORD 1915-82

\begin{tabular}{|c|c|c|c|c|c|c|}
\hline $\begin{array}{c}\text { PERIOD } \\
\text { (CON- } \\
\text { SECU- }\end{array}$ & \multicolumn{6}{|c|}{$\begin{array}{c}\text { DISCHARGE, IN CFS, FOR INDICATED RECURRENCE } \\
\text { INTERVAL, IN YEARS, AND ANNUAL } \\
\text { EXCEEDANCE PROBABILITY, IN PERCENT }\end{array}$} \\
\hline TIVE & 2 & 5 & 10 & 25 & 50 & 100 \\
\hline DAYS) & $50 \%$ & $20 \%$ & $10 \%$ & $4 \%$ & $2 \%$ & $1 \%$ \\
\hline 1 & 819 & 1210 & 1500 & 1930 & 2290 & 2680 \\
\hline 3 & 706 & 1010 & 1240 & 1550 & 1800 & 2070 \\
\hline 7 & 600 & 831 & 978 & 1160 & 1280 & 1410 \\
\hline 15 & 498 & 675 & 774 & 881 & 950 & 1010 \\
\hline 30 & 398 & 553 & 646 & 755 & 829 & 899 \\
\hline 60 & 316 & 441 & 517 & 607 & 670 & 729 \\
\hline 90 & 267 & 369 & 428 & 496 & 541 & 583 \\
\hline
\end{tabular}

DURATION TABLE OF DAILY MEAN FLOW FOR PERIOD OF RECORD 1915-82

DISCHARGE, IN CFS, WHICH WAS EQUALED OR EXCEEDED FOR INDICATED PERCENT OF TIME

\begin{tabular}{|c|c|c|c|c|c|c|c|c|c|c|c|c|c|c|}
\hline $5 \%$ & $10 \%$ & $15 \%$ & $20 \%$ & $25 \%$ & $30 \%$ & $40 \%$ & $50 \%$ & $60 \%$ & $70 \%$ & $75 \%$ & $80 \%$ & $85 \%$ & $90 \%$ & $95 \%$ \\
\hline 434 & 287 & 210 & 157 & 116 & 85 & 46 & 23 & 13 & 8.1 & 6.8 & 5.8 & 4.9 & 4.1 & \\
\hline
\end{tabular}


14044000 MIDDLE FORK JOHN DAY RIVER AT RITTER, OR

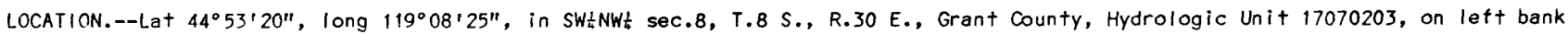

$0.2 \mathrm{mi}$ south of Ritter, $0.8 \mathrm{mi}$ downstream from Twelvemile Creek, and at mile 14.9.

DRAINAGE AREA. $--515 \mathrm{mi}^{2}$.

PERIOD OF RECORD.--October 1929 to September 1982.

GAGE.--Water-stage recorder. Datum of gage is 2,544.56 ft National Geodetic Vertical Datum of 1929 .

REMARKS.--No regulation. Diversions for irrigation above station.

AVERAGE DISCHARGE. --53 years, $247 \mathrm{ft} 3 / \mathrm{s}, 179,000$ acre- $\mathrm{ft} / \mathrm{yr}$.

EXTREMES FOR PERIOD OF RECORD.--Maximum discharge, $4,730 \mathrm{ft}^{3} / \mathrm{s}$ Jan. 30,1965 , gage height, $8.39 \mathrm{ft}$, from rating curve extended above $2,200 \mathrm{ft} 3 / \mathrm{s}$; maximum gage height, $9.13 \mathrm{ft}$ Feb. 1, 1963, ice jam; minimum discharge, 0.90 $\mathrm{ft}^{3} / \mathrm{s} \mathrm{Aug.} 19,20$, 1966.

STATISTICAL SUMMARIES

MONTHLY AND ANNUAL MEAN DISCHARGES 1930-82

\begin{tabular}{|c|c|c|c|c|c|c|}
\hline MONTH & $\begin{array}{l}\text { MINIMUM } \\
\text { (CFS) }\end{array}$ & $\begin{array}{l}\text { MAXIMUM } \\
\text { (CFS) }\end{array}$ & $\begin{array}{l}\text { MEAN } \\
\text { (CFS) }\end{array}$ & $\begin{array}{l}\text { STAN- } \\
\text { DARD } \\
\text { DEVIA- } \\
\text { TION } \\
\text { (CFS) }\end{array}$ & $\begin{array}{l}\text { COEFFI- } \\
\text { CIENT OF } \\
\text { VARI- } \\
\text { ATION }\end{array}$ & $\begin{array}{c}\text { PERCENT } \\
\text { OF } \\
\text { ANNUAL } \\
\text { RUNOFF }\end{array}$ \\
\hline OCTOBER & 17 & 92 & 42 & 15 & .35 & 1.4 \\
\hline NOVEMBER & 20 & 231 & 70 & 43 & .61 & 2.4 \\
\hline DECEMBER & 29 & 482 & 128 & 105 & .82 & 4.3 \\
\hline $\begin{array}{l}\text { JANUARY } \\
\text { FEBRUARY }\end{array}$ & 23 & $\begin{array}{l}580 \\
707\end{array}$ & $\begin{array}{l}160 \\
232\end{array}$ & $\begin{array}{l}141 \\
157\end{array}$ & .88 & $\begin{array}{l}5.4 \\
7.8\end{array}$ \\
\hline MARCH & 70 & 1214 & 416 & $\begin{array}{l}131 \\
212\end{array}$ & .081 & 14.0 \\
\hline APRIL & 175 & 1413 & 736 & 311 & .42 & 24.8 \\
\hline MAY & 79 & 1417 & 694 & 312 & .45 & 23.4 \\
\hline JUNE & 80 & 1066 & 353 & 217 & .61 & 11.9 \\
\hline JULY & 17 & 237 & 76 & 47 & .61 & 2.6 \\
\hline AUGUST & 3.8 & 74 & 29 & 17 & .58 & 1.0 \\
\hline SEPTEMBER & 10 & 71 & 29 & 12 & .42 & 1.0 \\
\hline ANNUAL & 85 & 452 & 247 & 95 & .38 & 100 \\
\hline
\end{tabular}

MAGNITUDE AND PROBABILITY OF INSTANTANEOUS PEAK FLOW BASED ON PERIOD OF RECORD 1930-82

DISCHARGE, IN CFS, FOR INDICATED RECURRENCE INTERVAL, IN YEARS, AND ANNUAL EXCEEDANCE PROBABILITY, IN PERCENT

\begin{tabular}{ccccccc}
1.25 & 2 & 5 & 10 & 25 & 50 & 100 \\
$80 \%$ & $50 \%$ & $20 \%$ & $10 \%$ & $4 \%$ & $2 \%$ & $1 \%$ \\
1080 & 1600 & 2370 & 2900 & 3580 & 4110 & 4640 \\
\hline WEIGHTED SKEW $=$ & -.062 & &
\end{tabular}

MAGNITUDE AND PROBABILITY OF ANNUAL LOW FLOW BASED ON PERIOD OF RECORD 1931-82

\begin{tabular}{|c|c|c|c|c|c|c|}
\hline $\begin{array}{l}\text { PERIOD } \\
\text { (CON- } \\
\text { SECU- }\end{array}$ & \multicolumn{6}{|c|}{$\begin{array}{l}\text { DISCHARGE, IN CFS, FOR INDICATED RECURRENCE } \\
\text { INTERVAL, IN YEARS, AND ANNUAL NON- } \\
\text { EXCEEDANCE PROBABILITY, IN PERCENT }\end{array}$} \\
\hline $\begin{array}{l}\text { TIVE } \\
\text { DAYS) }\end{array}$ & $\begin{array}{c}2 \\
50 \%\end{array}$ & $\stackrel{5}{5} \%$ & $\begin{array}{l}10 \\
10 \%\end{array}$ & $\begin{array}{l}20 \\
5 \%\end{array}$ & $\begin{array}{l}50 \\
2 \%\end{array}$ & $\begin{array}{r}100 \\
1 \%\end{array}$ \\
\hline 1 & 12 & 6.2 & 4. & 2.8 & 1.7 & 1.2 \\
\hline 3 & 14 & 7.2 & 4. & 3.2 & 1.9 & 1.3 \\
\hline 7 & 16 & 8. & 5. & 3.4 & 2.0 & 1.4 \\
\hline 14 & 18 & 9.6 & 6. & 4.5 & 2.8 & 2.0 \\
\hline 30 & 20 & 12 & 8. & 6.2 & 4.2 & 3.2 \\
\hline 60 & 24 & 16 & 12 & 9.7 & 7.4 & 6.1 \\
\hline 90 & 29 & 20 & 16 & 14 & 11 & 9.4 \\
\hline 120 & 34 & 25 & 21 & 18 & 14 & 13 \\
\hline 183 & 51 & 36 & 29 & 25 & 20 & 17 \\
\hline
\end{tabular}

MAGNITUDE AND PROBABILITY OF ANNUAL HIGH FLOW BASED ON PERIOD OF RECORD 1930-82

\begin{tabular}{|c|c|c|c|c|c|c|}
\hline $\begin{array}{l}\text { PERIOD } \\
\text { (CON- } \\
\text { SECU- }\end{array}$ & \multicolumn{6}{|c|}{$\begin{array}{c}\text { DISCHARGE, IN CFS, FOR INDICATED RECURRENCE } \\
\text { INTERVAL, IN YEARS, AND ANNUAL } \\
\text { EXCEEDANCE PROBABILITY, IN PERCENT }\end{array}$} \\
\hline $\begin{array}{l}\text { TIVE } \\
\text { DAYS ) }\end{array}$ & $\begin{array}{c}2 \\
50 \%\end{array}$ & $\begin{array}{c}5 \\
20 \%\end{array}$ & $\begin{array}{l}10 \\
10 \%\end{array}$ & $\begin{array}{l}25 \\
4 \%\end{array}$ & $\begin{array}{l}50 \\
2 \%\end{array}$ & $\begin{array}{r}100 \\
1 \%\end{array}$ \\
\hline $\begin{array}{l}1 \\
3\end{array}$ & $\begin{array}{l}1430 \\
1300\end{array}$ & $\begin{array}{l}2120 \\
1880\end{array}$ & $\begin{array}{l}2560 \\
2220\end{array}$ & $\begin{array}{l}3090 \\
2610\end{array}$ & $\begin{array}{l}3470 \\
2880\end{array}$ & $\begin{array}{l}3830 \\
3120\end{array}$ \\
\hline $\begin{array}{r}7 \\
15\end{array}$ & $\begin{array}{l}1150 \\
1010\end{array}$ & $\begin{array}{l}1610 \\
1410\end{array}$ & $\begin{array}{l}1850 \\
1620\end{array}$ & $\begin{array}{l}2100 \\
1840\end{array}$ & $\begin{array}{l}2260 \\
1970\end{array}$ & $\begin{array}{l}2390 \\
2080\end{array}$ \\
\hline $\begin{array}{l}30 \\
60 \\
90\end{array}$ & $\begin{array}{l}878 \\
743 \\
644\end{array}$ & $\begin{array}{r}1220 \\
1040 \\
885\end{array}$ & $\begin{array}{l}1400 \\
1200 \\
1020\end{array}$ & $\begin{array}{l}1580 \\
1370 \\
1160\end{array}$ & $\begin{array}{l}1700 \\
1490 \\
1250\end{array}$ & $\begin{array}{l}1790 \\
1580 \\
1320\end{array}$ \\
\hline
\end{tabular}

DURATION TABLE OF DAILY MEAN FLOW FOR PERIOD OF RECORD 1930-82

DISCHARGE, IN CFS, WHICH WAS EQUALED OR EXCEEDED FOR INDICATED PERCENT OF TIME

\begin{tabular}{|c|c|c|c|c|c|c|c|c|c|c|c|c|c|c|}
\hline $5 \%$ & $10 \%$ & $15 \%$ & $20 \%$ & $25 \%$ & $30 \%$ & 408 & $50 \%$ & $60 \%$ & $70 \%$ & $75 \%$ & $80 \%$ & $85 \%$ & $90 \%$ & $95 \%$ \\
\hline 972 & 714 & 545 & 421 & 327 & 254 & 154 & 89 & 60 & 46 & 40 & 34 & 29 & 24 & 17 \\
\hline
\end{tabular}




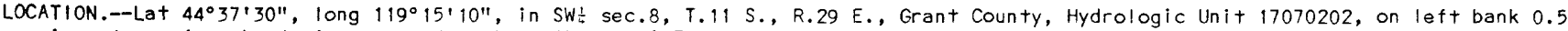
$\mathrm{mi}$ upstream from head of gorge and $6 \mathrm{mi}$ southwest of Fox.

DRAINAGE AREA.--90.2 $\mathrm{mi}^{2}$; at site used prior to June 12, 1952, $91.5 \mathrm{mi}^{2}$.

PERIOD OF RECORD.--October 1930 to September 1958.

GAGE.--Water-stage recorder. Altitude of gage is $4,240 \mathrm{ft}$ National Geodetic Vertical Datum of 1929 (from topographic map). Prior to June 12, 1952, at site $0.5 \mathrm{mi}$ downstream at different datum.

REMARKS.--Diversions for irrigation of 4,800 acres above station.

AVERAGE DISCHARGE.--28 years (water years $1931-58$ ), $25.9 \mathrm{ft}^{3} / \mathrm{s}, 18,750 \mathrm{acre}-\mathrm{ft} / \mathrm{yr}$.

EXTREMES FOR PERIOD OF RECORD.--Maximum discharge, 1,860 $\mathrm{ft}+3 / \mathrm{s} \mathrm{Mar.} \mathrm{25,} \mathrm{1952,} \mathrm{gage} \mathrm{height,} 5.85 \mathrm{ft}$, former site and datum, from rating curve extended above $200 \mathrm{ft}^{3} / \mathrm{s}$ on basis of slope-area measurement of peak tlow; no flow at times in each year.

STATISTICAL SUMMARIES

MONTHLY AND ANNUAL MEAN D!SCHARGES 1931-58

\begin{tabular}{|c|c|c|c|c|c|c|}
\hline MONTH & $\begin{array}{l}\text { MINIMUM } \\
\text { (CFS) }\end{array}$ & $\begin{array}{l}\text { MAXIMUM } \\
\text { (CFS) }\end{array}$ & $\begin{array}{l}\text { MEAN } \\
\text { (CFS) }\end{array}$ & $\begin{array}{l}\text { STAN- } \\
\text { DARD } \\
\text { DEVIA- } \\
\text { TION } \\
\text { (CFS) }\end{array}$ & $\begin{array}{l}\text { COEFFI- } \\
\text { CIENT OF } \\
\text { VARI- } \\
\text { ATION }\end{array}$ & $\begin{array}{c}\text { PERCENT } \\
\text { OF } \\
\text { ANNUAL } \\
\text { RUNOFF }\end{array}$ \\
\hline OCTOBER & 0.0 & 3.1 & .6 & .9 & 1.68 & .2 \\
\hline NOVEMBER & 0.0 & 13 & 2.2 & 3.0 & 1.35 & .7 \\
\hline DECEMBER & 0.0 & 61 & 10.0 & 15 & 1.45 & 3.2 \\
\hline JANUARY & .1 & 95 & 16 & 26 & 1.56 & 5.2 \\
\hline FEBRUARY & .1 & 130 & 35 & 34 & .98 & 19.1 \\
\hline MARCH & 3.0 & 232 & 75 & 55 & .73 & 24.0 \\
\hline APRIL & 1.8 & 180 & 87 & 44 & .51 & 27.8 \\
\hline MAY & .3 & 293 & 65 & 59 & .91 & 21.0 \\
\hline JUNE & .1 & 141 & 19 & 29 & 1.53 & 6.0 \\
\hline JULY & 0.0 & 15 & 1.9 & 3.0 & 1.56 & .6 \\
\hline AUGUST & 0.0 & 3.1 & .2 & .6 & 2.57 & .1 \\
\hline SEPTEMBER & 0.0 & 1.8 & .2 & .5 & 2.29 & .1 \\
\hline ANNUAL & 1.8 & 67 & 26 & 16 & .61 & 100 \\
\hline
\end{tabular}

MAGNITUDE AND PROBABILITY OF INSTANTANEOUS PEAK FLOW BASED ON PERIOD OF RECORD 1931-58

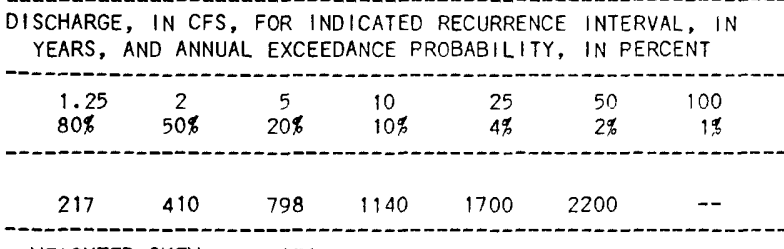

MAGNITUDE AND PROBABILITY OF ANNUAL LOW FLOW BASED ON PERIOD OF RECORD 1932-58

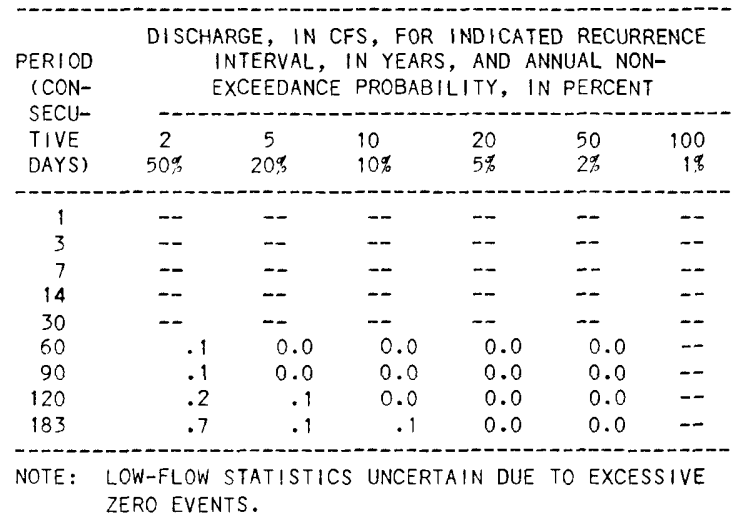

MAGN ITUDE AND PROBABILITY OF ANNUAL HIGH FLOW BASED ON PERIOD OF RECORD $1931-58$

DISCHARGE, IN CFS, FOR INDICATED RECURRENCE
INTERVAL, IN YEARS, AND ANNUAL
(CON-

DURATION TABLE OF DAILY MEAN FLOW FOR PERIOD OF RECORD 1931-58

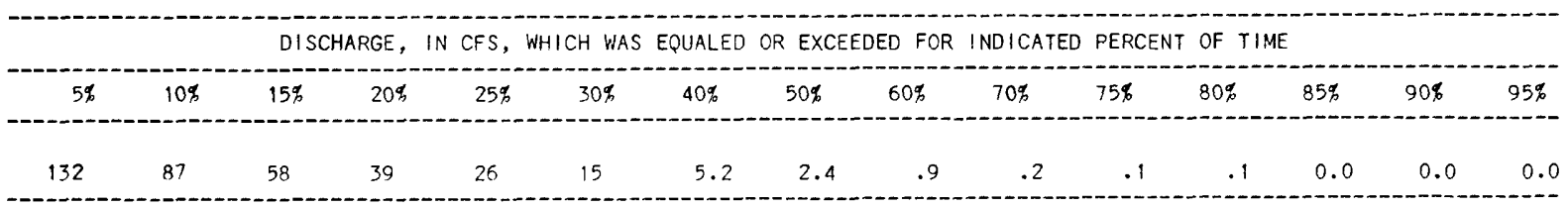


14046000 NORTH FORK JOHN DAY RIVER AT MONUMENT, OR

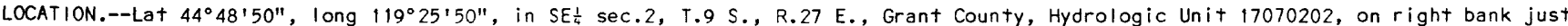
downstream trom entrance to canyon, $0.7 \mathrm{mi}$ downstream from Cottonwood Creek, $0.8 \mathrm{mi}$ west of Monument, and at mile 15.3 .

DRAINAGE AREA. $--2,520 \mathrm{mi}^{2}$, approximately.

PERIOD OF RECORD.--March 1925 to September 1982. Monthly discharge only for some periods, published in wSP 1318.

GAGE.--Water-stage recorder. Datum of gage is 1,959.64 ft National Geodetic Vertical Datum of 1929. Prior to Nov. 24, 1925, nonrecording gage and Nov. 24, 1925, to Oct. 16, 1928, water-stage recorder at datum 1.10 ft higher. 0ct. 17, 1928, to Sept. 30, 1930, water-stage recorder at datum $1.00 \mathrm{ft}$ higher.

REMARKS.--Very slight regulation by small reservoirs upstream. Many small diversions for irrigation above station.

AVERAGE DISCHARGE.--57 years, $1,248 \mathrm{ft} / \mathrm{s}, 904,200$ acre- $\mathrm{ft} / \mathrm{yr}$.

EXTREMES FOR PERIOD OF RECORD.--Maximum discharge, 33,400 $\mathrm{ft}^{3} / \mathrm{s}$ Jan. 30, 1965, gage height, $18.45 \mathrm{ft}$, from rating curve extended above 17,000 $\mathrm{ft}^{3} / \mathrm{s}$; minimum, $6 \mathrm{ft} 3 / \mathrm{s}$ sometime during period Nov. 2-13, 1936 (result of freezeup); minimum daily, $17 \mathrm{ft} 3 / \mathrm{s}$ Dec. 12 , 1932.

STATISTICAL SUMMARIES

MONTHLY AND ANNUAL MEAN DISCHARGES 1929-82

\begin{tabular}{|c|c|c|c|c|c|c|}
\hline MONTH & $\begin{array}{l}\text { MINIMUM } \\
\text { (CFS) }\end{array}$ & $\begin{array}{l}\text { MAXIMUM } \\
\text { (CFS) }\end{array}$ & $\begin{array}{l}\text { MEAN } \\
\text { (CFS) }\end{array}$ & $\begin{array}{l}\text { STAN- } \\
\text { DARD } \\
\text { DEVIA- } \\
\text { TION } \\
\text { (CFS) }\end{array}$ & $\begin{array}{l}\text { COEFFI- } \\
\text { CIENT OF } \\
\text { VARI- } \\
\text { ATION }\end{array}$ & $\begin{array}{c}\text { PERCENT } \\
\text { OF } \\
\text { ANNUAL } \\
\text { RUNOFF }\end{array}$ \\
\hline OCTOBER & 59 & 348 & 154 & 63 & .41 & 1.0 \\
\hline $\begin{array}{l}\text { NOVEMBER } \\
\text { DECEMBER }\end{array}$ & $\begin{array}{l}65 \\
97\end{array}$ & $\begin{array}{l}1621 \\
3374\end{array}$ & $\begin{array}{l}305 \\
726\end{array}$ & $\begin{array}{l}268 \\
747\end{array}$ & $\begin{array}{r}.88 \\
1.03\end{array}$ & $\begin{array}{l}2.0 \\
4.8\end{array}$ \\
\hline $\begin{array}{l}\text { JANUARY } \\
\text { FEBRUARY }\end{array}$ & $\begin{array}{r}76 \\
134\end{array}$ & $\begin{array}{l}4126 \\
4970\end{array}$ & $\begin{array}{r}931 \\
1340\end{array}$ & $\begin{array}{r}913 \\
1031\end{array}$ & $\begin{array}{l}.98 \\
.77\end{array}$ & $\begin{array}{l}6.2 \\
8.9\end{array}$ \\
\hline MARCH & 345 & 6023 & 2142 & 1096 & .51 & 14.2 \\
\hline $\begin{array}{l}\text { APRIL } \\
\text { MAY } \\
\text { JUNE }\end{array}$ & $\begin{array}{l}822 \\
378 \\
326\end{array}$ & $\begin{array}{l}6695 \\
8794 \\
5227\end{array}$ & $\begin{array}{l}3533 \\
3582 \\
1710\end{array}$ & $\begin{array}{r}1426 \\
1662 \\
993\end{array}$ & $\begin{array}{l}.40 \\
.46 \\
.58\end{array}$ & $\begin{array}{l}23.5 \\
23.8 \\
11.4\end{array}$ \\
\hline $\begin{array}{l}\text { JULY } \\
\text { AUGUST } \\
\text { SEPTEMBER }\end{array}$ & $\begin{array}{l}97 \\
37 \\
45\end{array}$ & $\begin{array}{r}1211 \\
322 \\
278\end{array}$ & $\begin{array}{l}376 \\
124 \\
114\end{array}$ & $\begin{array}{r}235 \\
65 \\
49\end{array}$ & $\begin{array}{l}.63 \\
.52 \\
.43\end{array}$ & $\begin{array}{r}2.5 \\
.8 \\
.8\end{array}$ \\
\hline ANNUAL & 441 & 2290 & 1250 & 500 & .40 & 100 \\
\hline
\end{tabular}

MAGNITUDE AND PROBABILITY OF INSTANTANEOUS PEAK FLOW BASED ON PERIOD OF RECORD 1929-82

\begin{tabular}{lcccccc} 
DISCHARGE, IN CFS, FOR IND ICATED RECURRENCE INTERVAL, IN \\
YEARS, AND ANNUAL EXCEEDANCE PROBABILITY, IN PERCENT \\
\hline 1.25 & 2 & 5 & 10 & 25 & 50 & 100 \\
$80 \%$ & $50 \%$ & $20 \%$ & $10 \%$ & $4 \%$ & $2 \%$ & $1 \%$ \\
\hline 5720 & 8930 & 14200 & 18200 & 23800 & 28400 & 33400
\end{tabular}

WEIGHTED SKEW $=.129$
MAGNITUDE AND PROBABILITY OF ANNUAL LOW FLOW BASED ON PERIOD OF RECORD 1930-82

\begin{tabular}{ccccccc} 
PERIOD & \multicolumn{2}{c}{ DISCHARGE, IN CFS, FOR INDICATED RECURRENCE } \\
(CON- & \multicolumn{5}{c}{$\begin{array}{l}\text { INTERVAL, IN YEARS, AND ANNUAL NON- } \\
\text { EXCEEDANCE PROBABILITY, IN PERCENT }\end{array}$} \\
$\begin{array}{c}\text { SECU- } \\
\text { TIVE }\end{array}$ & 2 & 5 & 10 & 20 & 50 & 100 \\
DAYS) & $50 \%$ & $20 \%$ & $10 \%$ & $5 \%$ & $2 \%$ & $1 \%$ \\
\hline 1 & 62 & 39 & 30 & 24 & 18 & 15 \\
3 & 65 & 43 & 34 & 28 & 22 & 19 \\
7 & 72 & 50 & 40 & 33 & 27 & 23 \\
14 & 79 & 56 & 45 & 38 & 30 & 26 \\
30 & 87 & 61 & 50 & 42 & 34 & 29 \\
60 & 100 & 71 & 59 & 50 & 41 & 36 \\
90 & 114 & 83 & 69 & 59 & 49 & 43 \\
120 & 132 & 96 & 80 & 69 & 58 & 51 \\
183 & 216 & 140 & 111 & 91 & 73 & 63
\end{tabular}

MAGNITUDE AND PROBABILITY OF ANNUAL HIGH FLOW BASED ON PERIOD OF RECORD 1929-82

\begin{tabular}{|c|c|c|c|c|c|c|}
\hline $\begin{array}{l}\text { PERIOD } \\
\text { ICON- } \\
\text { SECU- }\end{array}$ & \multicolumn{6}{|c|}{$\begin{array}{l}\text { DISCHARGE, IN CFS, FOR INDICATED RECURRENCE } \\
\text { INTERVAL, IN YEARS, AND ANNUAL } \\
\text { EXCEEDANCE PROBABILITY, IN PERCENT }\end{array}$} \\
\hline $\begin{array}{l}\text { TIVE } \\
\text { DAYS) }\end{array}$ & $\begin{array}{c}2 \\
50 \%\end{array}$ & $\begin{array}{c}5 \\
20 \%\end{array}$ & $\begin{array}{l}10 \\
10 \%\end{array}$ & $\begin{array}{l}25 \\
4 \%\end{array}$ & $\begin{array}{l}50 \\
2.6\end{array}$ & $\begin{array}{r}100 \\
1 \%\end{array}$ \\
\hline 1 & 7340 & 11400 & 14400 & 18600 & 21900 & 25500 \\
\hline 3 & 6630 & 9980 & 12300 & 15300 & 17600 & 19900 \\
\hline 7 & 5810 & 8440 & 10100 & 12100 & 13500 & 14800 \\
\hline 15 & 5080 & 7080 & 8190 & 9390 & 10100 & 10800 \\
\hline 30 & 4370 & 6010 & 6900 & 7830 & 8410 & 8910 \\
\hline 60 & 3710 & 5110 & 5880 & 6680 & 7190 & 7630 \\
\hline 90 & 3220 & 4420 & 5080 & 5790 & 6230 & 6620 \\
\hline
\end{tabular}

DURATION TABLE OF DAILY MEAN FLOW FOR PERIOD OF RECORD 1929-82

DISCHARGE, IN CFS, WHICH WAS EQUALED OR EXCEEDED FOR INDICATED PERCENT OF TIME

\begin{tabular}{|c|c|c|c|c|c|c|c|c|c|c|c|c|c|c|}
\hline $5 \%$ & $10 \%$ & $15 \%$ & $20 \%$ & $25 \%$ & $30 \%$. & $40 \%$ & $50 \%$ & $60 \%$ & $70 \%$ & $75 \%$ & $80 \%$ & $85 \%$ & $90 \%$ & $95 \%$ \\
\hline 4970 & 3670 & 2800 & 2190 & 1720 & 1340 & 812 & 412 & 251 & 178 & 152 & 131 & 111 & 93 & 71 \\
\hline
\end{tabular}




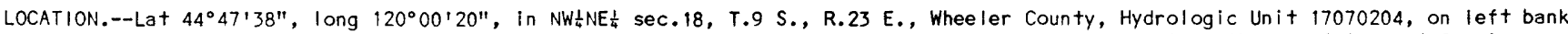
$0.2 \mathrm{mi}$ downstream from bridge on State Highway $207,0.8 \mathrm{mi}$ downstream from Service Creek, $0.5 \mathrm{mi}$ southwest of town of Service Creek, and at mile 156.7.

DRAINAGE AREA. $--5,090 \mathrm{mi}^{2}$, approximately.

PERIOD OF RECORD.--March 1925 to September 1926, October 1929 to September 1982. Monthly discharge only March 1925 to September 1926, published in WSP 1318.

GAGE.--Water-stage recorder. Datum of gage is 1,632.42 t+ National Geodetic Vertical Datum of 1929. See WSP 1738 for history of changes prior to Feb. 24, 1957.

REMARKS.--Very slight regulation by several small reservoirs above station. Many small diversions for irrigation above station. AVERAGE DISCHARGE. --53 years, $1,863 \mathrm{ft} \mathrm{t}^{3} / \mathrm{s}, 1,350,000$ acre- $\mathrm{ft} / \mathrm{yr}$.

EXTREMES FOR PERIOD OF RECORD.--Maximum discharge, 40,200 $\mathrm{ft}^{3} / \mathrm{s} \mathrm{Dec.} \mathrm{23,} \mathrm{1964,} \mathrm{gage} \mathrm{height,} 17.85 \mathrm{tt}$, from rating curve extended above $14,000 \mathrm{ft}^{3} / \mathrm{s}$ on basis of slope-area measurement of peak flow; minimum, $6.0 \mathrm{ft} / \mathrm{s}$ Aug. $23,24,1973$.

STATISTICAL SUMMARIES

MONTHLY AND ANNUAL MEAN DISCHARGES 1930-82

\begin{tabular}{|c|c|c|c|c|c|c|}
\hline MONTH & $\begin{array}{l}\text { MINIMUM } \\
\text { (CFS) }\end{array}$ & $\begin{array}{l}\text { MAXIMUM } \\
\text { (CFS) }\end{array}$ & $\begin{array}{l}\text { MEAN } \\
\text { (CFS) }\end{array}$ & $\begin{array}{l}\text { STAN- } \\
\text { DARD } \\
\text { DEVIA- } \\
\text { TION } \\
\text { (CFS) }\end{array}$ & $\begin{array}{l}\text { COEFFI- } \\
\text { CIENT OF } \\
\text { VARI- } \\
\text { ATION }\end{array}$ & $\begin{array}{c}\text { PERCENT } \\
\text { OF } \\
\text { ANNUAL } \\
\text { RUNOFF }\end{array}$ \\
\hline OCTOBER & 71 & 636 & 302 & 132 & .44 & 1.3 \\
\hline NOVEMBER & 152 & 2284 & 566 & 364 & .64 & 2.5 \\
\hline DECEMBER & 216 & 5540 & 1211 & 1125 & .93 & 5.4 \\
\hline JANUARY & 195 & 6335 & 1564 & 1420 & .91 & 6.9 \\
\hline FEBRUARY & 358 & 7930 & 2241 & 1625 & .73 & 9.9 \\
\hline MARCH & 597 & 9383 & 3331 & 1778 & .53 & 14.7 \\
\hline APRIL & 1010 & 9812 & 5136 & 2159 & .42 & 22.7 \\
\hline MAY & 491 & 12050 & 4909 & 2353 & .48 & 21.7 \\
\hline JUNE & 416 & 8327 & 2460 & 1485 & .60 & 10.9 \\
\hline JULY & 91 & 1850 & 548 & 382 & .70 & 2.4 \\
\hline AUGL & 15 & 545 & 166 & 120 & .72 & .7 \\
\hline SEPTEMBER & 31 & 492 & 165 & 99 & .60 & .7 \\
\hline NNUAL & 619 & 3485 & 1878 & 779 & .41 & 100 \\
\hline
\end{tabular}

MAGNITUDE AND PROBABILITY OF INSTANTANEOUS PEAK FLOW BASED ON PERIOD OF RECORD 1930-82 DISCHARGE, IN CFS, FOR INDICATED RECURRENCE INTERVAL, IN
YEARS, AND ANNUAL EXCEEDANCE PROBABILITY, IN PERCENT

\begin{tabular}{ccccccc}
1.25 & 2 & 5 & 10 & 25 & 50 & 100 \\
$80 \%$ & $50 \%$ & $20 \%$ & $10 \%$ & $4 \%$ & $2 \%$ & $1 \%$ \\
\hline 7410 & 11900 & 19100 & 24200 & 31200 & 36600 & 42300
\end{tabular}

WEIGHTED SKEW $=-.081$
MAGNITUDE AND PROBABILITY OF ANNUAL LOW FLOW BASED ON PERIOD OF RECORD 193 $1-82$

\begin{tabular}{|c|c|c|c|c|c|c|}
\hline \multirow{2}{*}{$\begin{array}{l}\text { PERIOD } \\
\text { (CON- } \\
\text { SECU- } \\
\text { TIVE } \\
\text { DAYS) }\end{array}$} & \multicolumn{6}{|c|}{$\begin{array}{l}\text { DISCHARGE, IN CFS, FOR INDICATED RECURRENCE } \\
\text { INTERVAL, IN YEARS, AND ANNUAL NON- } \\
\text { EXCEEDANCE PROBABILITY, IN PERCENT }\end{array}$} \\
\hline & $\begin{array}{c}2 \\
50 \%\end{array}$ & $\begin{array}{c}5 \\
20 \%\end{array}$ & $\begin{array}{l}10 \\
10 \%\end{array}$ & $\begin{array}{l}20 \\
5 \%\end{array}$ & $\begin{array}{l}50 \\
2 \%\end{array}$ & $\begin{array}{r}100 \\
1 \%\end{array}$ \\
\hline 1 & 84 & 43 & 28 & 19 & 12 & \\
\hline 3 & 85 & 44 & 29 & 20 & 12 & 8 \\
\hline 7 & 88 & 46 & 31 & 21 & 14 & 10. \\
\hline 14 & 91 & 49 & 34 & 24 & 16 & 12 \\
\hline 30 & 102 & 56 & 39 & 29 & 20 & 15 \\
\hline 60 & 128 & 73 & 53 & 40 & 29 & 23 \\
\hline 90 & 167 & 100 & 74 & 56 & 41 & 33 \\
\hline 120 & 221 & 140 & 106 & 83 & 61 & 50 \\
\hline 183 & 385 & 247 & 190 & 150 & 113 & 93 \\
\hline
\end{tabular}

MAGNITUDE AND PROBABILITY OF ANNUAL HIGH FLOW BASED ON PERIOD OF RECORD 1930-82

\begin{tabular}{ccccccc} 
DISCHARGE, IN CFS, FOR INDICATED RECURRENCE \\
INTERVAL, IN YEARS, AND ANNUAL \\
(CON- \\
$\begin{array}{c}\text { SECU- } \\
\text { TIVE }\end{array}$ & 2 & 5 & 10 & 25 & 50 & 100 \\
DAYS) & $50 \%$ & $20 \%$ & $10 \%$ & $4 \%$ & $2 \%$ & $1 \%$ \\
\hline \multicolumn{7}{c}{ EXCEEDANCE PROBABILITY, IN PERCENT } \\
1 & 10600 & 16700 & 21100 & 26900 & 31400 & 36000 \\
3 & 9650 & 15000 & 18700 & 23300 & 26600 & 30000 \\
7 & 8490 & 12700 & 15400 & 18500 & 20700 & 22700 \\
15 & 7330 & 10500 & 12200 & 14100 & 15300 & 16300 \\
30 & 6310 & 8870 & 10200 & 11600 & 12400 & 13100 \\
60 & 5330 & 7510 & 8680 & 9890 & 10600 & 11300 \\
90 & 4650 & 6520 & 7530 & 8590 & 9240 & 9800 \\
\hline
\end{tabular}

DURATION TABLE OF DAILY MEAN FLOW FOR PERIOD OF RECORD 1930-82

DISCHARGE, IN CFS, WHICH WAS EQUALED OR EXCEEDED FOR INDICATED PERCENT OF TIME

\begin{tabular}{|c|c|c|c|c|c|c|c|c|c|c|c|c|c|c|}
\hline $5 \%$ & $10 \%$ & $15 \%$ & $20 \%$ & $25 \%$ & $30 \%$ & $40 \%$ & $50 \%$ & $60 \%$ & $70 \%$ & $75 \%$ & $80 \%$ & $85 \%$ & $90 \%$ & $95 \%$ \\
\hline 7130 & 5320 & 4110 & 3260 & 2560 & 2050 & 1280 & 724 & 482 & 346 & 288 & 231 & 176 & 127 & 78 \\
\hline
\end{tabular}


14048000 JOHN DAY RIVER AT MCDONALD FERRY, OR

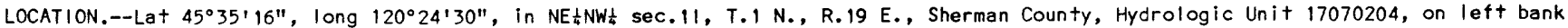
at McDonald Ferry, $0.8 \mathrm{mi}$ downstream from Rock Creek, $10 \mathrm{mi}$ east of Klondike, and at mile 20.9.

DRAINAGE AREA. $--7,580 \mathrm{mi}^{2}$, approximately.

PERIOD OF RECORD.--December 1904 to September 1982. Prior to Oct. 1, 1930, published as "at MCDonald."

GAGE.--Water-stage recorder. Datum of gage is $392.27 \mathrm{ft}$ National Geodetic Vertical Datum of 1929. Prior to Aug. 30 , 1930, nonrecording gage at same site and datum.

REMARKS.--No regulation. Many diversions for irrigation above station.

AVERAGE DISCHARGE.--77 years (water years 1906-82), 2,036 $\mathrm{ft} / \mathrm{s}, 1,475,000 \mathrm{acre}-\mathrm{ft} / \mathrm{yr}$.

EXTREMES FOR PERIOD OF RECORD.--Maximum discharge, 42,800 $\mathrm{ft}^{3} / \mathrm{s} \mathrm{Dec.} \mathrm{24,} \mathrm{1964,} \mathrm{gage} \mathrm{height,} 13.59 \mathrm{ft}$, from floodmark, from rating curve extended above $11,000 \mathrm{ft}^{3} / \mathrm{s}$ on basis of slope-area measurement of peak flow; no flow for part of Sept. 2 , 1966, Aug. 15 to Sept. 16, 1973, Aug. 13, 14, 19-25, 1977.

EXTREMES OUTSIDE PERIOD OF RECORD.--Flood of 1894 reached a stage of $12.8 \mathrm{ft}$, from floodmarks, discharge, $39,100 \mathrm{ft}{ }^{3} / \mathrm{s}$, from rating curve extended above $22,000 \mathrm{ft} / \mathrm{s}$.

STATISTICAL SUMMARIES

MONTHLY AND ANNUAL MEAN DISCHARGES 1906-82

\begin{tabular}{|c|c|c|c|c|c|c|}
\hline MONTH & $\begin{array}{l}\text { MINIMUM } \\
\text { (CFS) }\end{array}$ & $\begin{array}{l}\text { MAXIMUM } \\
\text { (CFS) }\end{array}$ & $\begin{array}{l}\text { MEAN } \\
\text { (CFS) }\end{array}$ & $\begin{array}{l}\text { STAN- } \\
\text { DARD } \\
\text { DEVIA- } \\
\text { TION } \\
\text { (CFS) }\end{array}$ & $\begin{array}{l}\text { COEFFI- } \\
\text { CIENT OF } \\
\text { VARI- } \\
\text { ATION }\end{array}$ & $\begin{array}{c}\text { PERCENT } \\
\text { OF } \\
\text { ANNUAL } \\
\text { RUNOFF }\end{array}$ \\
\hline OCTOBER & 60 & 657 & 309 & 131 & .42 & 1.3 \\
\hline $\begin{array}{l}\text { NOVEMBER } \\
\text { DECEMBER }\end{array}$ & $\begin{array}{l}157 \\
221\end{array}$ & $\begin{array}{l}2310 \\
7030\end{array}$ & $\begin{array}{r}587 \\
1199\end{array}$ & $\begin{array}{r}375 \\
1177\end{array}$ & .64 & $\begin{array}{l}2.4 \\
4.9\end{array}$ \\
\hline JANUARY & 217 & 6402 & 1664 & 1452 & .87 & 6.8 \\
\hline FEBRUARY & 374 & 8882 & 2549 & 1945 & .76 & 10.4 \\
\hline $\begin{array}{l}\text { MARCH } \\
\text { APRIL }\end{array}$ & $\begin{array}{l}557 \\
964\end{array}$ & $\begin{array}{l}10260 \\
10560\end{array}$ & $\begin{array}{l}3685 \\
5621\end{array}$ & $\begin{array}{l}2037 \\
2327\end{array}$ & $\begin{array}{l}.55 \\
.41\end{array}$ & $\begin{array}{l}15.0 \\
22.9\end{array}$ \\
\hline MAY & 533 & 13180 & 5176 & 2697 & .52 & 21.1 \\
\hline JUNE & 376 & 9531 & 2727 & 1710 & .63 & 11.1 \\
\hline $\begin{array}{l}\text { JULY } \\
\text { AUGUST }\end{array}$ & $\begin{array}{l}88 \\
5.7\end{array}$ & $\begin{array}{r}2101 \\
588\end{array}$ & $\begin{array}{l}644 \\
183\end{array}$ & $\begin{array}{l}450 \\
135\end{array}$ & $\begin{array}{l}.70 \\
.74\end{array}$ & 2.6 \\
\hline SEPTEMBER & 24 & 523 & 171 & 107 & .63 & . \\
\hline NNUAL & 603 & 3850 & 2036 & 841 & .41 & 100 \\
\hline
\end{tabular}

MAGNITUDE AND PROBABILITY OF INSTANTANEOUS PEAK FLOW BASED ON PERIOD OF RECORD 1906-82

DISCHARGE, IN CFS, FOR INDICATED RECURRENCE INTERVAL, IN YEARS, AND ANNUAL EXCEEDANCE PROBABILITY, IN PERCENT

$\begin{array}{ccccccc}1.25 & 2 & 5 & 10 & 25 & 50 & 100 \\ 80 \% & 50 \% & 20 \% & 10 \% & 4 \% & 2 \% & 1 \% \\ 7800 & 12100 & 18600 & 23100 & 28900 & 33300 & 37800\end{array}$

WEIGHTED SKEW $=-.138$
MAGNITUDE AND PROBABILITY OF ANNUAL LOW FLOW BASED ON PERIOD OF RECORD 1907-82

\begin{tabular}{|c|c|c|c|c|c|c|}
\hline \multirow{2}{*}{$\begin{array}{l}\text { PERIOD } \\
\text { (CON- } \\
\text { SECU- } \\
\text { TIVE } \\
\text { DAYS) }\end{array}$} & \multicolumn{6}{|c|}{$\begin{array}{l}\text { DISCHARGE, IN CFS, FOR INDICATED RECURRENCE } \\
\text { INTERVAL, IN YEARS, AND ANNUAL NON- } \\
\text { EXCEEDANCE PROBABILITY, IN PERCENT }\end{array}$} \\
\hline & $\stackrel{2}{50 \%}$ & ${ }_{20 \%}^{5}$ & $\begin{array}{l}10 \\
10 \%\end{array}$ & $\begin{array}{l}20 \\
5 \%\end{array}$ & $\begin{array}{l}50 \\
2 \%\end{array}$ & $\begin{array}{r}100 \\
1 \%\end{array}$ \\
\hline 1 & 88 & 40 & 24 & 15 & 8. & 5 \\
\hline 3 & 89 & 42 & 26 & 17 & 10 & 6.9 \\
\hline 7 & 93 & 45 & 28 & 19 & 11 & 7.8 \\
\hline 14 & 111 & 47 & 30 & 21 & 12 & 8.2 \\
\hline 30 & 113 & 54 & 34 & 22 & 13 & 8.5 \\
\hline 60 & 144 & 73 & 47 & 32 & 19 & 14 \\
\hline 90 & 179 & 103 & 73 & 54 & 38 & 29 \\
\hline 120 & 233 & 146 & 110 & 85 & 63 & 51 \\
\hline 183 & 398 & 262 & 207 & 169 & 133 & 112 \\
\hline
\end{tabular}

MAGNITUDE AND PROBABILITY OF ANNUAL HIGH FLOW BASED ON PERIOD OF RECORD 1906-82

\begin{tabular}{|c|c|c|c|c|c|c|}
\hline $\begin{array}{l}\text { PERIOD } \\
\text { (CON- } \\
\text { SECU- }\end{array}$ & \multicolumn{6}{|c|}{$\begin{array}{l}\text { DISCHARGE, IN CFS, FOR INDICATED RECURRENCE } \\
\text { INTERVAL, IN YEARS, AND ANNUAL } \\
\text { EXCEEDANCE PROBABILITY, IN PERCENT }\end{array}$} \\
\hline $\begin{array}{l}\text { TIVE } \\
\text { DAYS) }\end{array}$ & $\begin{array}{c}2 \\
50 \%\end{array}$ & $20 \%$ & $\begin{array}{l}10 \\
10 \%\end{array}$ & $\begin{array}{l}25 \\
4 \%\end{array}$ & $\begin{array}{l}50 \\
2 \%\end{array}$ & $\begin{array}{r}100 \\
1 \%\end{array}$ \\
\hline $\begin{array}{l}1 \\
3\end{array}$ & $\begin{array}{l}11200 \\
10300\end{array}$ & $\begin{array}{l}17000 \\
15400\end{array}$ & $\begin{array}{l}20900 \\
18600\end{array}$ & $\begin{array}{l}25900 \\
22600\end{array}$ & $\begin{array}{l}29500 \\
25300\end{array}$ & $\begin{array}{l}33200 \\
28000\end{array}$ \\
\hline $\begin{array}{r}7 \\
15\end{array}$ & $\begin{array}{l}8980 \\
7820\end{array}$ & $\begin{array}{l}13200 \\
11100\end{array}$ & $\begin{array}{l}15800 \\
12900\end{array}$ & $\begin{array}{l}18700 \\
14800\end{array}$ & $\begin{array}{l}20700 \\
15900\end{array}$ & $\begin{array}{l}22600 \\
17000\end{array}$ \\
\hline $\begin{array}{l}30 \\
60\end{array}$ & $\begin{array}{l}6710 \\
5730\end{array}$ & $\begin{array}{l}9470 \\
8120\end{array}$ & $\begin{array}{r}11000 \\
9450\end{array}$ & $\begin{array}{l}12500 \\
10900\end{array}$ & $\begin{array}{l}13500 \\
11700\end{array}$ & $\begin{array}{l}14400 \\
12500\end{array}$ \\
\hline 90 & 5040 & 7120 & 8270 & 9510 & 10300 & 11000 \\
\hline
\end{tabular}

DURATION TABLE OF DAILY MEAN FLOW FOR PERIOD OF RECORD 1906-82

\begin{tabular}{|c|c|c|c|c|c|c|c|c|c|c|c|c|c|c|}
\hline $5 \%$ & $10 \%$ & $15 \%$ & $20 \%$ & $25 \%$ & $30 \%$ & $40 \%$ & $50 \%$ & $60 \%$ & $70 \%$ & $75 \%$ & $80 \%$ & $85 \%$ & $90 \%$ & $95 \%$ \\
\hline 7890 & 5860 & 4590 & 3560 & 2850 & 2250 & 1370 & 776 & 516 & 376 & 314 & 254 & 195 & 137 & 84 \\
\hline
\end{tabular}


14050000 DESCHUTES RIVER BELOW SNOW CREEK, NEAR LA PINE, OR

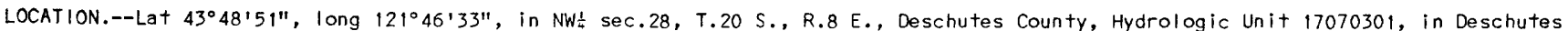
National Forest, on left bank at flow line of Crane Prairie Reservoir, $20 \mathrm{ft}$ downstream from Snow Creek, 300 ft upstream from highway bridge, and $17 \mathrm{mi}$ northwest of La Pine.

DRA INAGE AREA.--132 $\mathrm{mi}^{2}$, including Sparks, Elk, and Mud Lake basins, which have no surface outflow to Deschutes River; hydrologic drainage boundary uncertain owing to ground-water exchange.

PERIOD OF RECORD.--October 1937 to September 1982. Nonthly discharge only October 1937, published in WSP 1318. Fublished as "near Lap ine" 1937-64.

GAGE.--Water-stage recorder. Altitude of gage is 4,445 $\mathrm{ft}$, from elevation of Crane Prairie Reservoir when slack water extended to gage. Prior to Sept. 10, 1938, nonrecording gage at site $450 \mathrm{ft}$ downstream at different datum.

REMARKS.--No regulation. Crater Creek Canal diverts water to Tumalo Creek basin from tributaries of Soda Creek. Stream is spring fed and peak discharge may occur several months after the precipitation which caused it.

AVERAGE DISCHARGE. --45 years, $149 \mathrm{tt}^{3} / \mathrm{s}, 108,000$ acre- $t+\mathrm{t} / \mathrm{yr}$.

EXTREMES FOR PERIOD OF RECORD.--Maximum discharge, $480 \mathrm{ft}+\mathrm{s}$ Aug. 19, 1974, gage height, $3.17 \mathrm{ft}$; maximum gage height, $4.12 \mathrm{ft}$ Jan. 21, 1943 (ice jam); minimum discharge, $40 \mathrm{ft} 3 / \mathrm{s}$ sometime during period Dec. 22, 1959, to Mar. 2 , 1960, result of freezeup; minimum daily, $55 \mathrm{ft} / \mathrm{s}$ for many days April to June 1941 .

STATISTICAL SUMMARIES

MONTHLY AND ANNUAL MEAN DISCHARGES 1938-82

\begin{tabular}{|c|c|c|c|c|c|c|}
\hline MONTH & $\begin{array}{l}\text { MINIMUM } \\
\text { (CFS) }\end{array}$ & $\begin{array}{l}\text { MAXIMUM } \\
\text { (CFS) }\end{array}$ & $\begin{array}{l}\text { MEAN } \\
\text { (CFS) }\end{array}$ & $\begin{array}{l}\text { STAN- } \\
\text { DARD } \\
\text { DEV!A- } \\
\text { TION } \\
\text { (CFS) }\end{array}$ & $\begin{array}{l}\text { COEFFI- } \\
\text { CIENT OF } \\
\text { VARI- } \\
\text { ATION }\end{array}$ & $\begin{array}{c}\text { PERCENT } \\
\text { OF } \\
\text { ANNUAL } \\
\text { RUNOFF }\end{array}$ \\
\hline OCTOBER & 64 & 315 & 173 & 69 & .40 & 9.7 \\
\hline NOVEMBER & 62 & 236 & 146 & 51 & .35 & 8.2 \\
\hline DECEMBER & 62 & 205 & 131 & 42 & .32 & 7.3 \\
\hline JANUARY & 60 & 192 & 117 & 34 & .29 & 6.5 \\
\hline FEBRUARY & 61 & 173 & 106 & 28 & .26 & 5.9 \\
\hline MARCH & 59 & 166 & 99 & 24 & .24 & 5.5 \\
\hline APRIL & 59 & 195 & 103 & 28 & .27 & 5.8 \\
\hline MAY & 58 & 267 & 135 & 51 & .38 & 7.5 \\
\hline JUNE & 56 & 331 & 161 & 69 & .43 & 9.0 \\
\hline JULY & 58 & 419 & 183 & 87 & .47 & 10.2 \\
\hline AUGUST & 60 & 457 & 225 & 109 & .48 & 12.6 \\
\hline SEPTEMBER & 63 & 408 & 212 & 94 & .44 & 11.8 \\
\hline ANNUAL & 66 & 243 & 149 & 46 & .31 & 100 \\
\hline
\end{tabular}

MAGNITUDE AND PROBABILITY OF INSTANTANEOUS PEAK FLOW BASED ON PERIOD OF RECORD 1938-82

\begin{tabular}{|c|c|c|c|c|c|c|}
\hline \multicolumn{7}{|c|}{$\begin{array}{l}\text { DISCHARGE, IN CFS, FOR INDICATED RECURRENCE INTERVAL, IN } \\
\text { YEARS, AND ANNUAL EXCEEDANCE PROBABILITY, IN PERCENT }\end{array}$} \\
\hline $\begin{array}{l}1.25 \\
80 \%\end{array}$ & $\begin{array}{c}2 \\
50 \%\end{array}$ & $\begin{array}{c}5 \\
20 \%\end{array}$ & $\begin{array}{l}10 \\
10 \%\end{array}$ & $\begin{array}{l}25 \\
4 \%\end{array}$ & $\begin{array}{l}50 \\
2 \%\end{array}$ & $\begin{array}{r}100 \\
1 \%\end{array}$ \\
\hline 178 & 261 & 355 & 413 & 481 & 527 & 570 \\
\hline
\end{tabular}

WEIGHTED SKEW $=-.440$
MAGN I TUDE AND PROBABILITY OF ANNUAL LOW FLOW BASED ON PERIOD OF RECORD 1939-82

\begin{tabular}{|c|c|c|c|c|c|c|}
\hline \multirow{3}{*}{$\begin{array}{l}\text { PERIOD } \\
\text { (CON- } \\
\text { SECU- } \\
\text { TIVE } \\
\text { DAYS) }\end{array}$} & \multicolumn{6}{|c|}{$\begin{array}{l}\text { DISCHARGE, IN CFS, FOR INDICATED RECURRENCE } \\
\text { INTERVAL, IN YEARS, AND ANNUAL NON- } \\
\text { EXCEEDANCE PROBABILITY, IN PERCENT }\end{array}$} \\
\hline & 2 & 5 & 10 & 20 & 50 & 100 \\
\hline & $50 \%$ & $20 \%$ & $10 \%$ & $5 \%$ & $2 \%$ & $1 \%$ \\
\hline 1 & 82 & 71 & 66 & 61 & 56 & 53 \\
\hline 3 & 83 & 71 & 66 & 62 & 57 & 54 \\
\hline 7 & 84 & 72 & 67 & 63 & 58 & 54 \\
\hline 14 & 85 & 73 & 68 & 64 & 59 & 54 \\
\hline 30 & 86 & 75 & 69 & 65 & 60 & 54 \\
\hline 60 & 94 & 78 & 71 & 65 & 60 & 55 \\
\hline 90 & 100 & 81 & 72 & 66 & 60 & 55 \\
\hline 120 & 106 & 84 & 74 & 67 & 60 & 55 \\
\hline 183 & 123 & 93 & 79 & 70 & 60 & 55 \\
\hline
\end{tabular}

MAGN I TUDE AND PROBABILITY OF ANNUAL HIGH FLOW BASED ON PERIOD OF RECORD 1938-82

\begin{tabular}{|c|c|c|c|c|c|c|}
\hline $\begin{array}{l}\text { PERIOD } \\
\text { (CON- }\end{array}$ & \multicolumn{6}{|c|}{$\begin{array}{c}\text { DISCHARGE, IN CFS, FOR INDICATED RECURRENCE } \\
\text { INTERVAL, IN YEARS, AND ANNUAL } \\
\text { EXCEEDANCE PROBABILITY, IN PERCENT }\end{array}$} \\
\hline Secu- & & & & & & \\
\hline TIVE & 2 & 5 & 10 & 25 & 50 & 100 \\
\hline DAYS) & $50 \%$ & $20 \%$ & $10 \%$ & $4 \%$ & $2 \%$ & $1 \%$ \\
\hline 1 & 261 & 354 & 402 & 452 & 482 & 507 \\
\hline 3 & 259 & 352 & 401 & 451 & 482 & 508 \\
\hline 7 & 257 & 350 & 399 & 450 & 481 & 507 \\
\hline 15 & 253 & 346 & 396 & 448 & 480 & 508 \\
\hline 30 & 246 & 338 & 389 & 442 & 475 & 504 \\
\hline 60 & 231 & 320 & 369 & 424 & 459 & 490 \\
\hline 90 & 213 & 295 & 343 & 395 & 430 & 462 \\
\hline
\end{tabular}

DURATION TABLE OF DAILY MEAN FLOW FOR PERIOD OF RECORD 1938-82

\begin{tabular}{|c|c|c|c|c|c|c|c|c|c|c|c|c|c|c|}
\hline $5 \not$ & $10 \%$ & $15 \%$ & $20 \%$ & $25 \%$ & $30 \%$ & $40 \%$ & $50 \%$ & $60 \%$ & $70 \%$ & $75 \%$ & $80 \%$ & $85 \%$ & $90 \%$ & $95 \%$ \\
\hline 307 & 260 & 231 & 209 & 190 & 173 & 147 & 126 & 108 & 95 & 91 & 88 & 84 & 81 & 76 \\
\hline
\end{tabular}


14050500 CULTUS RIVER ABOVE CULTUS CREEK, NEAR LA PINE, OR

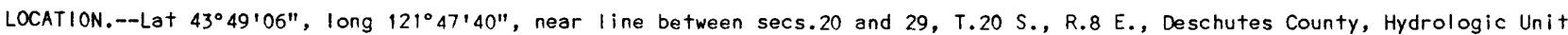
17070301, Deschutes National Forest, on left bank at highway culvert, $2 \mathrm{mi}$ upstream from Cultus Creek, and $18 \mathrm{mi}$ northwest of La Pine.

DRAINAGE AREA.-- $16.5 \mathrm{mi}^{2}$, hydrologic drainage boundry uncertain owing to ground-water exchange.

PERIOD OF RECORD.--October 1922 to September 1925, October 1937 to September 1982. Monthly discharge only October 1937, published in WSP 1318. Prior to Oct. 1, 1964, published as "near Lapine."

GAGE.--Water-stage recorder and cement bag control. Altitude of gage is 4,450 ft, by barometer. 0ct 1 , 1922, to Sept. 30, 1925, nonrecording gage at site $0.5 \mathrm{mi}$ upstream at different datum.

REMARKS.--No regulation or diversions above station.

AVERAGE DISCHARGE. -48 years, $62.3 \mathrm{ft} / \mathrm{s}, 45,140$ acre $-\mathrm{ft} / \mathrm{yr}$.

EXTREMES FOR PERIOD OF RECORD.--Maximum discharge, $178 \mathrm{ft}+3 / \mathrm{s}$ May 31 , 1956, gage height, $1.04 \mathrm{ft}$; maximum gage height, $1.32 \mathrm{ft}$ May 16 , 1972 (backwater from Crane Prairie Reservoir); minimum discharge, $26 \mathrm{ft}+\mathrm{s} / \mathrm{s}$ May 26-31, Nov. 23 to Dec. 4 , 1959.

STATISTICAL SUMMARIES

MONTHLY AND ANNUAL MEAN DISCHARGES 1923-82

\begin{tabular}{|c|c|c|c|c|c|c|}
\hline MONTH & $\begin{array}{l}\text { MIN IMUM } \\
\text { (CFS) }\end{array}$ & $\begin{array}{l}\text { MAXIMUM } \\
\text { (CFS) }\end{array}$ & $\begin{array}{l}\text { MEAN } \\
\text { (CFS) }\end{array}$ & $\begin{array}{l}\text { STAN- } \\
\text { DARD } \\
\text { DEVIA- } \\
\text { TION } \\
\text { (CFS) }\end{array}$ & $\begin{array}{l}\text { COEFFI- } \\
\text { CIENT OF } \\
\text { VARI- } \\
\text { ATION }\end{array}$ & $\begin{array}{c}\text { PERCENT } \\
\text { OF } \\
\text { ANNUAL } \\
\text { RUNOFF }\end{array}$ \\
\hline OCTOBER & 32 & 93 & 63 & 18 & .28 & 8.5 \\
\hline NOVEMBER & 31 & 91 & 60 & 16 & .27 & 8.0 \\
\hline DECEMBER & 34 & 88 & 56 & 15 & .26 & 7.5 \\
\hline JANUARY & 32 & 86 & 53 & 13 & .24 & 7.1 \\
\hline FEBRUARY & 30 & 84 & 50 & 11 & .22 & 6.7 \\
\hline MARCH & 32 & 81 & 50 & 11 & .21 & 6.7 \\
\hline APRIL & 31 & 96 & 54 & 13 & .24 & 7.2 \\
\hline MAY & 36 & 142 & 71 & 24 & .34 & 9.5 \\
\hline JUNE & 34 & 136 & 74 & 23 & .31 & 9.8 \\
\hline JULY & 37 & 126 & 75 & 21 & .28 & 10.0 \\
\hline AUGUST & 38 & 117 & 74 & 20 & .28 & 9.8 \\
\hline SEPTEMBER & 34 & 103 & 68 & 18 & .27 & 9.1 \\
\hline ANNUAL & 36 & 97 & 62 & 14 & .22 & 100 \\
\hline
\end{tabular}

MAGNITUDE AND PROBABILITY OF INSTANTANEOUS PEAK FLOW BASED ON PERIOD OF RECORD 1923-82

\begin{tabular}{lcccccc} 
DISCHARGE, IN CFS, FOR INDICATED RECURRENCE INTERVAL, IN \\
YEARS, AND ANNUAL EXCEEDANCE PROBABILITY, IN PERCENT \\
\hline 1.25 & 2 & 5 & 10 & 25 & 50 & 100 \\
$80 \%$ & $50 \%$ & $20 \%$ & $10 \%$ & $4 \%$ & $2 \%$ & $1 \%$ \\
\hline 68 & 88 & 113 & 129 & 148 & 161 & 174 \\
\hline
\end{tabular}

WEIGHTED SKEW $=-.050$
MAGNITUDE AND PROBABILITY OF ANNUAL LOW FLOW BASED ON PERIOD OF RECORD 1924-82

\begin{tabular}{|c|c|c|c|c|c|c|}
\hline $\begin{array}{l}\text { PERIOD } \\
\text { (CON- }\end{array}$ & \multicolumn{6}{|c|}{$\begin{array}{l}\text { DISCHARGE, IN CFS, FOR INDICATED RECURRENCE } \\
\text { INTERVAL, IN YEARS, AND ANNUAL NON- } \\
\text { EXCEEDANCE PROBABILITY, IN PERCENT }\end{array}$} \\
\hline $\begin{array}{l}\text { TIVE } \\
\text { DAYS) }\end{array}$ & $\stackrel{2}{50 \%}$ & $\begin{array}{c}5 \\
20 \%\end{array}$ & $\begin{array}{l}10 \\
10 \%\end{array}$ & $\begin{array}{l}20 \\
5 \%\end{array}$ & $\begin{array}{l}50 \\
2 \%\end{array}$ & $\begin{array}{r}100 \\
18\end{array}$ \\
\hline 1 & 42 & 36 & 32 & 30 & 27 & 25 \\
\hline 3 & 43 & 36 & 33 & 30 & 27 & 26 \\
\hline 7 & 43 & 36 & 33 & 30 & 28 & 26 \\
\hline 14 & 44 & 37 & 33 & 31 & 28 & 26 \\
\hline 30 & 45 & 38 & 35 & 32 & 29 & 28 \\
\hline 60 & 47 & 40 & 36 & 33 & 30 & 28 \\
\hline 90 & 49 & 40 & 36 & 34 & 31 & 29 \\
\hline 120 & 50 & 41 & 37 & 34 & 31 & 29 \\
\hline 183 & 54 & 44 & 39 & 36 & 32 & 30 \\
\hline
\end{tabular}

MAGNITUDE AND PROBABILITY OF ANNUAL HIGH FLOW BASED ON PERIOD OF RECORD 1923-82

\begin{tabular}{|c|c|c|c|c|c|c|}
\hline $\begin{array}{l}\text { PERIOD } \\
\text { (CON- } \\
\text { SECU- }\end{array}$ & \multicolumn{6}{|c|}{$\begin{array}{c}\text { DISCHARGE, IN CFS, FOR INDICATED RECURRENCE } \\
\text { INTERVAL, IN YEARS, AND ANNUAL } \\
\text { EXCEEDANCE PROBABILITY, IN PERCENT }\end{array}$} \\
\hline $\begin{array}{l}\text { TIVE } \\
\text { DAYS) }\end{array}$ & $\begin{array}{c}2 \\
50 \%\end{array}$ & $\begin{array}{c}5 \\
20 \%\end{array}$ & $\begin{array}{l}10 \\
10 \%\end{array}$ & $\begin{array}{l}25 \\
4 \%\end{array}$ & $\begin{array}{l}50 \\
28\end{array}$ & $\begin{array}{r}100 \\
1 \%\end{array}$ \\
\hline 1 & 87 & 111 & 126 & 144 & 157 & 169 \\
\hline 3 & 86 & 110 & 124 & 142 & 154 & 166 \\
\hline 7 & 85 & 108 & 122 & 138 & 150 & 161 \\
\hline 15 & 83 & 105 & 118 & 133 & 144 & 154 \\
\hline 30 & 81 & 102 & 114 & 127 & 137 & 146 \\
\hline 60 & 79 & 97 & 108 & 120 & 129 & 136 \\
\hline 90 & 77 & 95 & 106 & 118 & 126 & 134 \\
\hline
\end{tabular}

DURATION TABLE OF DAILY MEAN FLOW FOR PERIOD OF RECORD 1923-82

\begin{tabular}{|c|c|c|c|c|c|c|c|c|c|c|c|c|c|c|}
\hline $5 \%$ & $10 \%$ & $15 \%$ & $20 \%$ & $25 \%$ & $30 \%$ & $40 \%$ & $50 \%$ & $60 \%$ & $70 \%$ & $75 \%$ & $80 \%$ & $85 \%$ & $90 \%$ & $95 \%$ \\
\hline 99 & 92 & 88 & 82 & 75 & 70 & 64 & 59 & 54 & 50 & 48 & 46 & 43 & 41 & 38 \\
\hline
\end{tabular}


14051000 CULTUS CREEK ABOVE CRANE PRAIRIE RESERVOIR, NEAR LA PINE, OR

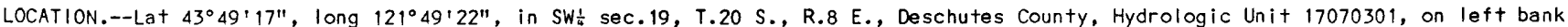
$1,000 \mathrm{ft}$ upstream from highway bridge, $1.0 \mathrm{mi}$ downstream from Cultus Lake, and $19 \mathrm{mi}$ northwest of La Pine.

DRAINAGE AREA.--33.2 $\mathrm{mi}^{2}$, hydrologic drainage boundary uncertain owing to ground-water exchange.

PERIOD OF RECORD.--March to September 1924 (published as "above Crane Prairie, near Lapine"), October 1937 to September 1982. Monthly discharge only October 1937 to September 1949, published in WSP 1318. Records for October 1923 to February 1924, published in WSP 594, have been found to be unreliable and should not be used. Published as "near Lapine" 1937-64.

GAGE.--Water-stage recorder. Altitude of gage is $4,545 \mathrm{ft}$, by barometer. Mar. 1 to Sept. 30 , 1924, nonrecording gage at site $100 \mathrm{ft}$ upstream at different datum.

REMARKS.--Some regulation by fish screens at Cultus Lake since 1962. No diversion above station.

AVERAGE DISCHARGE. -45 years (water years 1938-82), $22.6 \mathrm{ft}+3 / \mathrm{s}, 16,370$ acre- $\mathrm{ft} / \mathrm{yr}$.

EXTREMES FOR PERIOD OF RECORD.--Maximum discharge, $336 \mathrm{ft}^{3} / \mathrm{s} \mathrm{Dec.} \mathrm{25,} \mathrm{1964,} \mathrm{gage} \mathrm{height,} 4.15 \mathrm{ft}$, from floodmark, from rating curve extended above $90 \mathrm{ft}^{3} / \mathrm{s}$; no flow at times.

STATISTICAL SUMMARIES (BEFORE THE INSTALLATION OF FISH SCREENS)

MONTHLY AND ANNUAL MEAN DISCHARGES 1924-62

\begin{tabular}{|c|c|c|c|c|c|c|}
\hline MONTH & $\begin{array}{l}\text { MINIMUM } \\
\text { (CFS) }\end{array}$ & $\begin{array}{l}\text { MAXIMUM } \\
\text { (CFS) }\end{array}$ & $\begin{array}{l}\text { MEAN } \\
\text { (CFS) }\end{array}$ & $\begin{array}{l}\text { STAN- } \\
\text { DARD } \\
\text { DEVIA- } \\
\text { TION } \\
\text { (CFS) }\end{array}$ & $\begin{array}{l}\text { COEFFI- } \\
\text { CIENT OF } \\
\text { VARI- } \\
\text { ATION }\end{array}$ & $\begin{array}{c}\text { PERCENT } \\
\text { OF } \\
\text { ANNUAL } \\
\text { RUNOFF }\end{array}$ \\
\hline OCTOBER & 0.0 & 9.0 & 1.2 & 2.0 & 1.71 & .4 \\
\hline NOVEMBER & 0.0 & 49 & 6.0 & 9.8 & 1.64 & 2.2 \\
\hline DECEMBER & 0.0 & 52 & 17 & 17 & .99 & 6.4 \\
\hline JANUARY & 0.0 & 68 & 19 & 17 & .89 & 6.9 \\
\hline FEBRUARY & 2.4 & 57 & 20 & 13 & .65 & 7.3 \\
\hline MARCH & 2.7 & 37 & 18 & 8.7 & .49 & 6.4 \\
\hline APRIL & 5.6 & 52 & 22 & 11 & .48 & 8.2 \\
\hline MAY & 15 & 127 & 63 & 31 & .50 & 23.0 \\
\hline JUNE & 18 & 143 & 73 & 37 & .50 & 26.7 \\
\hline JULY & 3.9 & 66 & 27 & 17 & .63 & 9.9 \\
\hline AUGUST & .2 & 16 & 5.8 & 4.3 & .75 & 2.1 \\
\hline SEPTEMBER & 0.0 & 6.0 & 1.5 & 1.8 & 1.20 & .5 \\
\hline INNUAL & 5.1 & 44 & 23 & 9.9 & .43 & 100 \\
\hline
\end{tabular}

MAGNITUDE AND PROBABILITY OF INSTANTANEOUS PEAK FLOW BASED ON PERIOD OF RECORD 1924-62

DISCHARGE, IN CFS, FOR INDICATED RECURRENCE INTERVAL, IN YEARS, AND ANNUAL EXCEEDANCE PROBABILITY, IN PERCENT

\begin{tabular}{ccccccc}
1.25 & 2 & 5 & 10 & 25 & 50 & 100 \\
$80 \%$ & $50 \%$ & $20 \%$ & $10 \%$ & $4 \%$ & $2 \%$ & $1 \%$ \\
\hline 60 & 101 & 164 & 209 & 268 & 313 & - \\
\hline WEIGHTED SKEW & $=-211$ & &
\end{tabular}

MAGNITUDE AND PROBABILITY OF ANNUAL LOW FLOW BASED ON PERIOD OF RECORD 1939-62

\begin{tabular}{|c|c|c|c|c|c|c|}
\hline \multirow{2}{*}{$\begin{array}{l}\text { PERIOD } \\
\text { (CON- } \\
\text { SECU- } \\
\text { TIVE } \\
\text { DAYS) }\end{array}$} & \multicolumn{6}{|c|}{$\begin{array}{l}\text { DISCHARGE, IN CFS, FOR INDICATED RECURRENCE } \\
\text { INTERVAL, IN YEARS, AND ANNUAL NON- } \\
\text { EXCEEDANCE PROBABILITY, IN PERCENT }\end{array}$} \\
\hline & $\begin{array}{c}2 \\
50 \%\end{array}$ & $\begin{array}{c}5 \\
20 \%\end{array}$ & $\begin{array}{l}10 \\
10^{\circ}\end{array}$ & $\begin{array}{l}20 \\
5 \%\end{array}$ & $\begin{array}{l}50 \\
2 \%\end{array}$ & $\begin{array}{r}100 \\
1 \%\end{array}$ \\
\hline 1 & -. & -- & - & -- & - & - \\
\hline 3 & - & - & - & - & -- & - \\
\hline 7 & -- & -- & -- & -- & -- & -- \\
\hline 14 & -- & -- & -- & -- & -- & -- \\
\hline 30 & -- & -- & -- & -- & -- & -- \\
\hline 60 & .5 & .2 & .1 & .1 & 0.0 & -- \\
\hline 90 & .9 & .3 & .2 & .1 & .1 & -- \\
\hline 120 & 2.0 & .7 & .4 & .2 & .1 & -- \\
\hline 183 & 5.7 & 1.1 & .4 & .2 & .1 & -- \\
\hline
\end{tabular}

MAGNITUDE AND PROBABILITY OF ANNUAL HIGH FLOW BASED ON PERIOD OF RECORD $1924-62$

\begin{tabular}{|c|c|c|c|c|c|c|}
\hline \multirow{3}{*}{$\begin{array}{l}\text { PERIOD } \\
\text { (CON- } \\
\text { SECU- } \\
\text { TIVE } \\
\text { DAYS) }\end{array}$} & \multicolumn{6}{|c|}{$\begin{array}{c}\text { DISCHARGE, IN CFS, FOR INDICATED RECURRENCE } \\
\text { INTERVAL, IN YEARS, AND ANNUAL } \\
\text { EXCEEDANCE PROBABILITY, IN PERCENT }\end{array}$} \\
\hline & 2 & 5 & 10 & 25 & 50 & 100 \\
\hline & $50 \%$ & $20 \%$ & $10 \%$ & $4 \%$ & $2 \%$ & $1 \%$ \\
\hline 1 & 101 & 154 & 187 & 22 & 25 & -- \\
\hline 3 & 100 & 153 & 185 & 222 & 248 & -- \\
\hline 7 & 97 & 147 & 977 & 213 & 237 & -- \\
\hline 15 & 91 & 136 & 164 & 194 & 21 & - \\
\hline 30 & 82 & 122 & 145 & & 188 & -- \\
\hline 60 & 67 & 99 & 117 & 138 & 151 & -- \\
\hline 90 & 55 & 80 & 94 & 110 & 120 & -- \\
\hline
\end{tabular}

DURATION TABLE OF DAILY MEAN FLOW FOR PERIOD OF RECORD 1924-62

DISCHARGE, IN CFS, WHICH WAS EQUALED OR EXCEEDED FOR INDICATED PERCENT OF TIME

\begin{tabular}{|c|c|c|c|c|c|c|c|c|c|c|c|c|c|c|}
\hline $5 \%$ & $10 \%$ & $15 \%$ & $20 \%$ & $25 \%$ & $30 \%$ & $40 \%$ & $50 \%$ & $60 \%$ & $70 \%$ & $75 \%$ & $80 \%$ & $85 \%$ & $90 \%$ & $95 \%$ \\
\hline 90 & 63 & 47 & 37 & 30 & 25 & 18 & 13 & 8.2 & 3.8 & 2.4 & 1.3 & .5 & .2 & .1 \\
\hline
\end{tabular}


14051000 CULTUS CREEK ABOVE CRANE PRAIRIE RESERVOIR, NEAR LA PINE, OR--Continued STATISTICAL SUMMARIES (AFTER THE INSTALLATION OF FISH SCREENS)

MONTHLY AND ANNUAL MEAN DISCHARGES 1963-82

\begin{tabular}{|c|c|c|c|c|c|c|}
\hline MONTH & $\begin{array}{l}\text { MINIMUM } \\
\text { (CFS) }\end{array}$ & $\begin{array}{l}\text { MAXIMUM } \\
\text { (CFS) }\end{array}$ & $\begin{array}{l}\text { MEAN } \\
\text { (CFS) }\end{array}$ & $\begin{array}{l}\text { STAN- } \\
\text { DARD } \\
\text { DEVIA- } \\
\text { TION } \\
\text { (CFS) }\end{array}$ & $\begin{array}{l}\text { COEFFI- } \\
\text { CIENT OF } \\
\text { VARI- } \\
\text { ATION }\end{array}$ & $\begin{array}{c}\text { PERCENT } \\
\text { OF } \\
\text { ANNUAL } \\
\text { RUNOFF }\end{array}$ \\
\hline OCTOBER & 0.0 & 6.2 & .6 & 1.4 & 2.24 & .2 \\
\hline NOVEMBER & .2 & 16 & 3.8 & 4.6 & 1.22 & 1.4 \\
\hline DECEMBER & .4 & 80 & 21 & 22 & 1.04 & 8.1 \\
\hline JANUARY & .1 & 76 & 27 & 19 & .70 & 10.2 \\
\hline FEBRUARY & 0.0 & 55 & 23 & 13 & .54 & 8.9 \\
\hline MARCH & 0.0 & 68 & 21 & 15 & .73 & 7.8 \\
\hline APRIL & 1.8 & 46 & 17 & 9.6 & .57 & 6.5 \\
\hline MAY & 13 & 83 & 45 & 21 & .46 & 17.1 \\
\hline JUNE & 18 & 176 & 71 & 49 & .69 & 27.2 \\
\hline JULY & 2.5 & 71 & 27 & 22 & .83 & 10.1 \\
\hline AUGUST & 0.0 & 15 & 5.3 & 5.2 & 1.00 & 2.0 \\
\hline SEP TEMBER & 0.0 & 6.1 & 1.1 & 1.6 & 1.44 & .4 \\
\hline ANNUAL & 3.0 & 38 & 22 & 11 & .50 & 100 \\
\hline
\end{tabular}

MAGNITUDE AND PROBABILITY OF INSTANTANEOUS PEAK FLOW BASED ON PERIOD OF RECORD

DISCHARGE, IN CFS, FOR INDICATED RECURRENCE INTERVAL, IN YEARS, AND ANNUAL EXCEEDANCE PROBABILITY, IN PERCENT

$\begin{array}{ccccccc}1.25 & 2 & 5 & 10 & 25 & 50 & 100 \\ 80 \% & 50 \% & 20 \% & 10 \% & 4 \% & 2 \% & 1 \% \\ - & - & - & - & - & - & - \\ - & - & - & - & -\end{array}$

WEIGHTED SKEW $=$
MAGNITUDE AND PROBABILITY OF ANNUAL LOW FLOW BASED ON PERIOD OF RECORD $1964-82$

\begin{tabular}{|c|c|c|c|c|c|c|}
\hline \multirow{2}{*}{$\begin{array}{l}\text { PERIOD } \\
\text { (CON- } \\
\text { SECU- } \\
\text { TIVE } \\
\text { DAYS) }\end{array}$} & \multicolumn{6}{|c|}{$\begin{array}{l}\text { DISCHARGE, IN CFS, FOR INDICATED RECURRENCE } \\
\text { INTERVAL, IN YEARS, AND ANNUAL NON- } \\
\text { EXCEEDANCE PROBABILITY, IN PERCENT }\end{array}$} \\
\hline & $\stackrel{2}{50 \%}$ & $\begin{array}{c}5 \\
20 \%\end{array}$ & $\begin{array}{l}10 \\
10 \%\end{array}$ & $\begin{array}{l}20 \\
5 \%\end{array}$ & $\begin{array}{l}50 \\
2 \%\end{array}$ & $\begin{array}{r}100 \\
1 \%\end{array}$ \\
\hline 1 & -- & - & -- & -- & -- & - \\
\hline 3 & -- & -- & - & -- & -- & -- \\
\hline 7 & -- & -- & -- & -- & - & -- \\
\hline 14 & -- & -- & -- & -- & -- & -- \\
\hline 30 & -- & -- & - & -- & -- & -- \\
\hline 60 & .1 & 0.0 & 0.0 & 0.0 & -- & - \\
\hline 90 & .3 & .1 & 0.0 & 0.0 & -- & -- \\
\hline 120 & .9 & .3 & .2 & .1 & -- & -- \\
\hline 183 & 5.4 & 2.4 & 1.4 & .8 & -- & -- \\
\hline
\end{tabular}

MAGNITUDE AND PROBABILITY OF ANNUAL HIGH FLOW BASED ON PERIOD OF RECORD 1963-82

\begin{tabular}{|c|c|c|c|c|c|c|}
\hline \multirow{2}{*}{$\begin{array}{l}\text { PERIOD } \\
\text { (CON- } \\
\text { SECU- } \\
\text { TIVE } \\
\text { DAYS) }\end{array}$} & \multicolumn{6}{|c|}{$\begin{array}{l}\text { DISCHARGE, IN CFS, FOR INDICATED RECURRENCE } \\
\text { INTERVAL, IN YEARS, AND ANNUAL } \\
\text { EXCEEDANCE PROBABILITY, IN PERCENT }\end{array}$} \\
\hline & $\begin{array}{c}2 \\
50 \%\end{array}$ & $\begin{array}{c}5 \\
20 \%\end{array}$ & $\begin{array}{l}10 \\
10 \%\end{array}$ & $\begin{array}{l}25 \\
4 \%\end{array}$ & $\begin{array}{l}50 \\
2 \%\end{array}$ & $\begin{array}{r}100 \\
1 \%\end{array}$ \\
\hline $\begin{array}{l}1 \\
3\end{array}$ & $\begin{array}{l}97 \\
95\end{array}$ & $\begin{array}{l}165 \\
162\end{array}$ & $\begin{array}{l}217 \\
210\end{array}$ & $\begin{array}{l}287 \\
276\end{array}$ & -- & -- \\
\hline 7 & 91 & 153 & 198 & 257 & -- & -- \\
\hline 15 & 85 & 140 & 178 & 227 & -- & -- \\
\hline 30 & 75 & 122 & 154 & 194 & -- & -- \\
\hline 60 & 58 & 94 & 117 & 147 & -- & -- \\
\hline 90 & 46 & 73 & 90 & 110 & -- & -- \\
\hline
\end{tabular}

DURATION TABLE OF DAILY MEAN FLOW FOR PERIOD OF RECORD 1963-82

DISCHARGE, IN CFS, WHICH WAS EQUALED OR EXCEEDED FOR INDICATED PERCENT OF TIME

\begin{tabular}{|c|c|c|c|c|c|c|c|c|c|c|c|c|c|c|}
\hline $5 \%$ & $10 \%$ & $15 \%$ & $20 \%$ & $25 \%$ & $30 \%$ & $40 \%$ & $50 \%$ & $60 \%$ & $70 \%$ & $75 \%$ & $80 \%$ & $85 \%$ & $90 \%$ & $95 \%$ \\
\hline 82 & 57 & 41 & 32 & 28 & 24 & 18 & 13 & 8.3 & 3.3 & 1.8 & .8 & .3 & .1 & \\
\hline
\end{tabular}




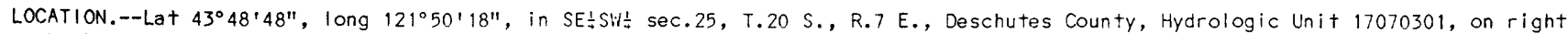
bank $150 \mathrm{ft}$ downstream trom highway bridge, $1.2 \mathrm{mi}$ downstream from Little Cultus Lake, and $19 \mathrm{mi}$ northwest of La Pine.

DRAINAGE AREA.--21.5 $\mathrm{mi}^{2}$, hydrologic drainage boundary uncertain owing to ground-water exchange.

PERIOD OF RECORD.--February to September 1924 (published as "above Crane Prairie, near Lapine"). October 1937 to September 1982. Monthly discharge only October 1937 to September 1949, published in WISP 1318. Records for October 1923 to January 1924 , published in WSP 594, have been found to be unreliable and should not be used. Published as "near Lapine" $1937-64$.

GAGE.-Water-stage recorder and sharp-crested weir control. Altitude of gage is 4,520 ft, by barometer. Feb. 1 to Sept. 30 , 1924, nonrecording gage at site $75 \mathrm{ft}$ upstream at various datums. Oct. 1, 1937, to Sept. 30, 1938, water-stage recorder at bridge 150 ft upstream at different datum. Oct. 1, 1938, to Aug. 13, 1968, water-stage recorder and wooden weir control at present site and datum $0.60 \mathrm{ft}$ higher.

REMARKS.--No regulation or diversion above station.

AVERAGE DISCHARGE. -45 years (water years 1938-82), $7.48 \mathrm{ft}+3 / \mathrm{s}, 5,420 \mathrm{acre}-\mathrm{ft} / \mathrm{yr}$.

EXTREMES FOR PERIOD OF RECORD.--Maximum discharge, $200 \mathrm{ft} / \mathrm{s}$, estimated, Dec. 25 , 1964 ; no $\mathrm{tlow}$ at times.

\section{STATISTICAL SUMMARIES}

MONTHLY AND ANNUAL MEAN OISCHARGES 1938-82

\begin{tabular}{|c|c|c|c|c|c|c|}
\hline MONTH & $\begin{array}{l}\text { MINIMUM } \\
\text { (CFS) }\end{array}$ & $\begin{array}{c}\text { MAXIMUM } \\
\text { (CFS) }\end{array}$ & $\begin{array}{l}\text { MEAN } \\
\text { (CFS) }\end{array}$ & $\begin{array}{c}\text { STAN- } \\
\text { DARD } \\
\text { DEVIA- } \\
\text { TION } \\
\text { (CFS) }\end{array}$ & $\begin{array}{l}\text { COEFFI- } \\
\text { CIENT OF } \\
\text { VARI- } \\
\text { ATION }\end{array}$ & $\begin{array}{c}\text { PERCENT } \\
\text { OF } \\
\text { ANNUAL } \\
\text { RUNOFF }\end{array}$ \\
\hline OCTOBER & 0.0 & 2.0 & .3 & .4 & 1.21 & .4 \\
\hline NOVEMBER & 0.0 & 14 & 2.1 & 2.6 & 1.24 & 2.3 \\
\hline DECEMBER & .1 & 30 & 7.1 & 7.4 & 1.04 & 7.9 \\
\hline JANUARY & .1 & 21 & 6.7 & 5.6 & .84 & 7.5 \\
\hline FEBRUARY & .1 & 24 & 6.5 & 5.1 & .78 & 7.3 \\
\hline MARCH & .6 & 32 & 6.0 & 4.8 & .81 & 6.7 \\
\hline APRIL & 1.4 & 33 & 12 & 6.1 & .52 & 12.9 \\
\hline MAY & 6.1 & 55 & 29 & 14 & .47 & 32.2 \\
\hline JUNE & .6 & 61 & 18 & 15 & .86 & 19.5 \\
\hline JULY & 0.0 & 7.4 & 2.4 & 2.1 & .89 & 2.6 \\
\hline AUGUST & 0.0 & 1.2 & .4 & .3 & .73 & .4 \\
\hline SEPTEMBER & 0.0 & .7 & .2 & .2 & .80 & .2 \\
\hline ANNUAL & 1.2 & 15 & 7.5 & 3.5 & .46 & 100 \\
\hline
\end{tabular}

MAGNITUDE AND PROBABILITY OF INSTANTANEOUS PEAK FLOW BASED ON PERIOD OF RECORD $1938-82$

\begin{tabular}{|c|c|c|c|c|c|c|}
\hline \multicolumn{7}{|c|}{$\begin{array}{l}\text { DISCHARGE, IN CFS, FOR INDICATED RECURRENCE INTERVAL, IN } \\
\text { YEARS, AND ANNUAL EXCEEDANCE PROBAB ILITY, IN PERCENT }\end{array}$} \\
\hline $\begin{array}{l}1.25 \\
80 \%\end{array}$ & $\begin{array}{c}2 \\
50 \%\end{array}$ & $\begin{array}{c}5 \\
20 \%\end{array}$ & $\begin{array}{l}10 \\
10 \%\end{array}$ & $\begin{array}{l}25 \\
4 \%\end{array}$ & $\begin{array}{l}50 \\
2,\end{array}$ & $\begin{array}{r}100 \\
1 \%\end{array}$ \\
\hline 32 & 51 & 80 & 101 & 128 & 148 & 169 \\
\hline
\end{tabular}

WEIGHTED SKEW $=-.162$
MAGNITUDE AND PROBABILITY OF ANNUAL LOW FLOW BASED ON PERIOD OF RECORD 1939-82

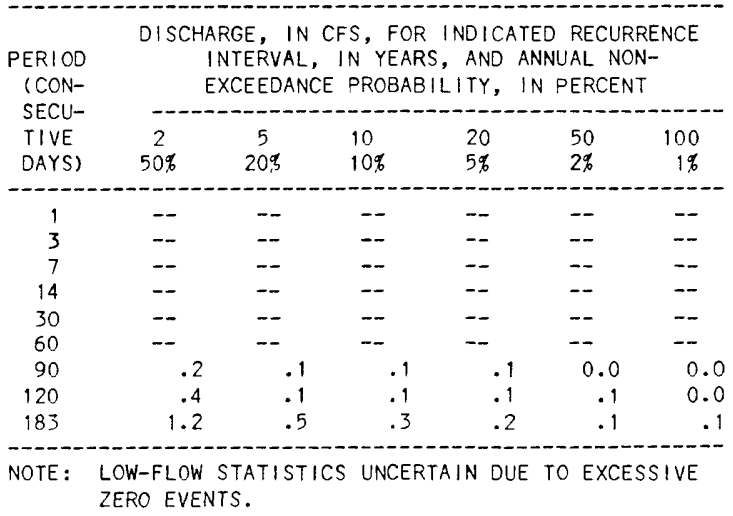

MAGN I TUDE AND PROBABILITY OF ANNUAL HIGH FLOW BASED ON PERIOD OF RECORD 1938-82

\begin{tabular}{|c|c|c|c|c|c|c|}
\hline $\begin{array}{l}\text { PERIOD } \\
\text { (CON- } \\
\text { SECU- }\end{array}$ & \multicolumn{6}{|c|}{$\begin{array}{c}\text { DISCHARGE, IN CFS, FOR INDICATED RECURRENCE } \\
\text { INTERVAL, IN YEARS, AND ANNUAL } \\
\text { EXCEEDANCE PROBABILITY, IN PERCENT }\end{array}$} \\
\hline TIVE & 2 & 5 & 10 & 25 & 50 & 100 \\
\hline DAYS) & $50 \%$ & $20 \%$ & $10 \%$ & $4 \%$ & $2 \%$ & $1 \%$ \\
\hline 1 & 51 & 74 & 86 & 97 & 104 & 109 \\
\hline 3 & 50 & 71 & 81 & 90 & 96 & 100 \\
\hline 7 & 46 & 65 & 74 & 82 & 87 & 90 \\
\hline 15 & 40 & 57 & 66 & 74 & 79 & 82 \\
\hline 30 & 34 & 49 & 58 & 66 & 71 & 75 \\
\hline 60 & 25 & 37 & 44 & 51 & 55 & 58 \\
\hline 90 & 19 & 28 & 33 & 38 & 41 & 44 \\
\hline
\end{tabular}

DURATION TABLE OF DAILY MEAN FLOW FOR PERIOD OF RECORO 1938-82

DISCHARGE, IN CFS, WHICH WAS EQUALED OR EXCEEDED FOR INDICATED PERCENT OF TIME

\begin{tabular}{|c|c|c|c|c|c|c|c|c|c|c|c|c|c|c|}
\hline $5 \%$ & $10 \%$ & $15 \%$ & $20^{\circ}$ & $25 \%$ & $30 \%$ & $40 \%$ & $50 \%$ & $60 \%$ & $70 \%$ & $75 \%$ & $80 \%$ & $85 \%$ & $90 \%$ & $95 \%$ \\
\hline 36 & 22 & 15 & 11 & 8.6 & 7.0 & 4.6 & 2.8 & 1.4 & .6 & .4 & .2 & .2 & .1 & 0.0 \\
\hline
\end{tabular}


14052500 QUINN RIVER NEAR LA PINE, OR

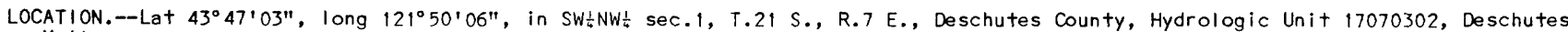
National Forest, on left bank at flow line of Crane Prairie Reservoir, 150 ft downstream from springs at head of river, and $18 \mathrm{mi}$ northwest of La Pine.

DRAINAGE AREA.-- Indeterminate, normal flow is entirely from springs 150 ft upstream.

PERIOD OF RECORD.--June 1922 to September 1925, October 1937 to September 1982. Published as "above Crane Prairie Reservoir near Lapine" 1922-25, and as "near Lapine" 1937-64. Monthly discharge only October 1937, published in wSP 1318.

GAGE.--Water-stage recorder and log control. Datum of gage is 4,442.1 ft National Geodetic Vertical Datum of i929, based on elevation of Crane Prairie Reservoir when slack water reached station. June 1, 1922, to Sept. 30, 1925, nonrecording gage at site $150 \mathrm{ft}$ downstream at different datum.

REMARKS.--No regulation or diversion above station.

AVERAGE DISCHARGE. --48 years, $23.8 \mathrm{ft}^{3} / \mathrm{s}, 17,240$ acre- $\mathrm{ft} / \mathrm{yr}$.

EXTREMES FOR PERIOD OF RECORD.--Maximum discharge, $59 \mathrm{ft}+\mathrm{s} / \mathrm{s}$ July 4 , 1949, gage height, $1.97 \mathrm{ft}$; maximum gage height, $3.92 \mathrm{ft}$ June 25 , 1943 (backwater from Crane Prairie Reservoir); practically no flow Nov. 14, 1941.

STATISTICAL SUMMARIES

MONTHLY AND ANNUAL MEAN DISCHARGES 1938-82

\begin{tabular}{|c|c|c|c|c|c|c|}
\hline MONTH & $\begin{array}{l}\text { MINIMUM } \\
\text { (CFS) }\end{array}$ & $\begin{array}{c}\text { MAXIMUM } \\
\text { (CFS) }\end{array}$ & $\begin{array}{l}\text { MEAN } \\
\text { (CFS) }\end{array}$ & $\begin{array}{l}\text { STAN- } \\
\text { DARD } \\
\text { DEVIA- } \\
\text { TION } \\
\text { (CFS) }\end{array}$ & $\begin{array}{l}\text { COEFFI- } \\
\text { CIENT OF } \\
\text { VARI- } \\
\text { ATION }\end{array}$ & $\begin{array}{c}\text { PERCENT } \\
\text { OF } \\
\text { ANNUAL } \\
\text { RUNOFF }\end{array}$ \\
\hline OCTOBER & 1.8 & 39 & 22 & 10 & .47 & 7.5 \\
\hline NOVEMBER & .8 & 36 & 19 & 9.0 & .48 & 6.6 \\
\hline DECEMBER & 2.2 & 31 & 18 & 7.7 & .42 & 6.4 \\
\hline JANUARY & 4.0 & 32 & 19 & 7.3 & .38 & 6.6 \\
\hline FEBRUARY & 4.0 & 37 & 20 & 7.5 & .38 & 6.8 \\
\hline MARCH & 5.0 & 38 & 20 & 7.6 & .37 & 7.0 \\
\hline APRIL & 5.4 & 42 & 22 & 8.2 & .37 & 7.7 \\
\hline MAY & 6.4 & 49 & 27 & 9.8 & .37 & 9.2 \\
\hline JUNE & 7.3 & 53 & 32 & 12 & .36 & 11.0 \\
\hline JULY & 7.0 & 54 & 33 & 12 & .37 & 11.4 \\
\hline AUGUST & 4.7 & 51 & $3:$ & 12 & .39 & 10.7 \\
\hline SEPTEMBER & 3.5 & 44 & 27 & 11 & .41 & 9.2 \\
\hline ANNUAL & 5.0 & 41 & 24 & 8.0 & .33 & 100 \\
\hline
\end{tabular}

MAGNITUDE AND PROBABILITY OF INSTANTANEOUS PEAK FLOW BASED ON PERIOD OF RECORD $1938-82$

DISCHARGE, IN CFS, FOR INDICATED RECURRENCE INTERVAL, IN YEARS, AND ANNUAL EXCEEDANCE PROBABILITY, IN PERCENT

\begin{tabular}{ccccccc}
1.25 & 2 & 5 & 10 & 25 & 50 & 100 \\
$80 \%$ & $50 \%$ & $20 \%$ & $10 \%$ & $4 \%$ & $2 \%$ & $1 \%$ \\
\hline 28 & 38 & 48 & 54 & 61 & 66 & 71 \\
\hline
\end{tabular}

WEIGHTED SKEW $=-.243$
MAGNITUDE AND PROBABILITY OF ANNUAL LOW FLOW BASED ON PERIOD OF RECORD 1939-82

\begin{tabular}{|c|c|c|c|c|c|c|}
\hline $\begin{array}{l}\text { PERIOD } \\
\text { (CON- } \\
\text { SECU- }\end{array}$ & \multicolumn{6}{|c|}{$\begin{array}{l}\text { DISCHARGE, IN CFS, FOR INDICATED RECURRENCE } \\
\text { INTERVAL, IN YEARS, AND ANNUAL NON- } \\
\text { EXCEEDANCE PROBABILITY, IN PERCENT }\end{array}$} \\
\hline $\begin{array}{l}\text { TIVE } \\
\text { DAYS) }\end{array}$ & $\stackrel{2}{2}$ & 5 & $\begin{array}{l}10 \\
10 \%\end{array}$ & 20 & 50 & $\begin{array}{r}100 \\
1 \%\end{array}$ \\
\hline-1 & & & & & & \\
\hline 1 & -- & -- & -- & -- & -- & -- \\
\hline 3 & -- & - & -- & - & - & -- \\
\hline 7 & -- & -- & -- & - & - & -- \\
\hline 14 & 16 & 8.9 & 5.3 & 3. & 1.5 & \\
\hline 30 & 16 & 9.2 & 5.8 & 3. & 1. & 1. \\
\hline 60 & 17 & 9.7 & 6.3 & 4.1 & 2.3 & 1. \\
\hline 90 & 18 & 10 & 6.9 & 4.7 & 2 & 1. \\
\hline 120 & 18 & 11 & 7.4 & 5.2 & 3.3 & 2. \\
\hline 183 & 19 & 12 & 8.6 & 6.3 & 4.2 & 3.1 \\
\hline
\end{tabular}

MAGNITUDE AND PROBABILITY OF ANNUAL HIGH FLOW BASED ON PERIOD OF RECORD $1938-82$

\begin{tabular}{|c|c|c|c|c|c|c|}
\hline $\begin{array}{l}\text { PERIOD } \\
\text { (CON- }\end{array}$ & \multicolumn{6}{|c|}{$\begin{array}{l}\text { DISCHARGE, IN CFS, FOR INDICATED RECURRENCE } \\
\text { INTERVAL, IN YEARS, AND ANNUAL } \\
\text { EXCEEDANCE PROBABILITY, IN PERCENT }\end{array}$} \\
\hline SECU- & & & & & & \\
\hline $\begin{array}{l}\text { TIVE } \\
\text { DAYS) }\end{array}$ & $\begin{array}{c}2 \\
50^{\alpha}\end{array}$ & $\begin{array}{c}5 \\
20 \%\end{array}$ & $\begin{array}{l}10 \\
10 \%\end{array}$ & $\begin{array}{l}25 \\
4 \%\end{array}$ & $\begin{array}{l}50 \\
2 \%\end{array}$ & $\begin{array}{r}100 \\
1 \%\end{array}$ \\
\hline
\end{tabular}

$\begin{array}{rrrrrrr}1 & 38 & 48 & 53 & 56 & 58 & 60 \\ 3 & 38 & 48 & 52 & 55 & 57 & 57 \\ 7 & 38 & 48 & 52 & 55 & 56 & 57 \\ 15 & 38 & 47 & 51 & 54 & 56 & 57 \\ 30 & 37 & 46 & 50 & 53 & 55 & 56 \\ 60 & 36 & 45 & 49 & 52 & 53 & 54 \\ 90 & 35 & 44 & 47 & 50 & 52 & 53\end{array}$

DURATION TABLE OF DAILY MEAN FLOW FOR PERIOD OF RECORD 1938-82

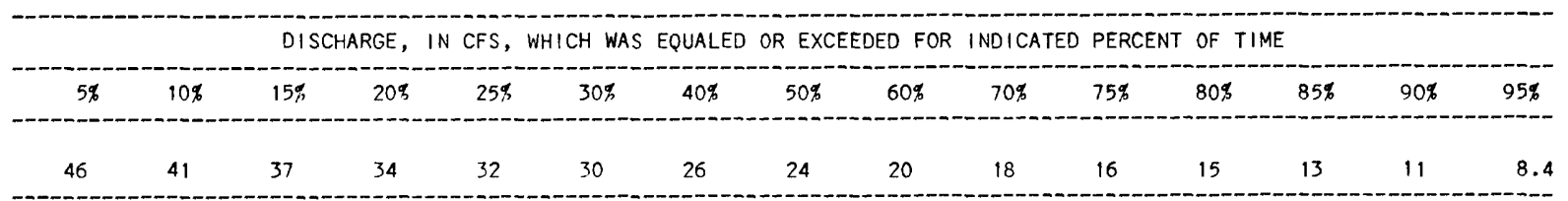




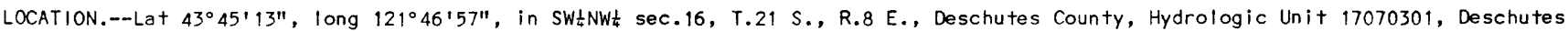
National Forest, on left bank $0.1 \mathrm{mi}$ downstream from Crane Prairie Dam, $15 \mathrm{mi}$ northwest of La Pine, and at mile 238.2 .

DRAINAGE AREA.--254 $\mathrm{mi}^{2}$, hydrologic drainage boundary uncertain owing to ground-water exchange.

PERIOD OF RECORD.--August 1907 to November 1908 and August 1912 to September 1913 (fragmentary), October 1913 to September 1917 , February 1922 to September 1982. Monthly discharge only for some periods, published in WSP 1318. Prior to October 1949, published as "at Crane Prairie, near Lapine." Published as "near Lapine" 1949-64.

GAGE.- Water-stage recorder. Datum of gage is 4,419.78 $\mathrm{ft}$ National Geodetic Vertical Datum of 1929 (Pacific Power \& Light Co. bench mark). Aug. 15, 1907, to Sept. 30, 1917, and Feb. 23 to June 8, 1922, nonrecording gage at site $0.5 \mathrm{mi}$ upstream at different datums. June 9, 1922, to May 9, 1932, nonrecording gage or water-stage recorder at present site and datum.

REMARKS.--Flow regulated since 1922 by Crane Prairie Reservoir. No diversion above station.

AVERAGE DISCHARGE. --64 years, $211 \mathrm{ft} / \mathrm{s}, 152,900$ acre $-\mathrm{ft} / \mathrm{yr}$.

EXTREMES FOR PERIOD OF RECORD.--Maximum discharge, 1,170 $\mathrm{ft}^{3} / \mathrm{s}$ July 28,1947 , gage height, $3.34 \mathrm{ft}$; no $\mathrm{flow} \mathrm{Nov}$. 15, 1978, when gates in Crane Prairie Dam were closed.

STATISTICAL SUMMARIES

MONTHLY AND ANNUAL MEAN DISCHARGES $1923-82$

\begin{tabular}{|c|c|c|c|c|c|c|}
\hline MONTH & $\begin{array}{l}\text { MINIMUM } \\
\text { (CFS) }\end{array}$ & $\begin{array}{l}\text { MAXIMUM } \\
\text { (CFS) }\end{array}$ & $\begin{array}{l}\text { MEAN } \\
\text { (CFS) }\end{array}$ & $\begin{array}{l}\text { STAN- } \\
\text { DARD } \\
\text { DEVIA- } \\
\text { TION } \\
\text { (CFS) }\end{array}$ & $\begin{array}{l}\text { COEFFI- } \\
\text { CIENT OF } \\
\text { VARI- } \\
\text { ATION }\end{array}$ & $\begin{array}{c}\text { PERCENT } \\
\text { OF } \\
\text { ANNUAL } \\
\text { RUNOFF }\end{array}$ \\
\hline OCTOBER & 13 & 675 & 206 & 117 & .57 & 8.4 \\
\hline NOVEMBER & 5.2 & 700 & 145 & 159 & 1.10 & 5.9 \\
\hline DECEMBER & 3.3 & 489 & 105 & 94 & .89 & 4.3 \\
\hline JANUARY & 3.4 & 334 & 97 & 72 & .74 & 3.9 \\
\hline FEBRUARY & 5.6 & 380 & 102 & 77 & .76 & 4.1 \\
\hline MARCH & 5.0 & 270 & 97 & 70 & .72 & 3.9 \\
\hline APRIL & 7.7 & 370 & 130 & 83 & .64 & 5.3 \\
\hline MAY & 14 & 750 & 251 & 124 & .49 & 10.2 \\
\hline JUNE & 107 & 782 & 333 & 126 & .38 & 13.5 \\
\hline JULY & 119 & 647 & 362 & 128 & .35 & 14.7 \\
\hline AUGUST & 109 & 709 & 343 & 130 & .38 & 13.9 \\
\hline SEPTEMBER & 19 & 635 & 290 & 139 & .48 & 11.8 \\
\hline NNUAL & 101 & 323 & 206 & 56 & .27 & 100 \\
\hline
\end{tabular}

MAGNITUDE AND PROBABILITY OF INSTANTANEOUS PEAK FLOW BASED ON PERIOD OF RECORD

DISCHARGE, IN CFS, FOR INDICATED RECURRENCE INTERVAL, IN YEARS, AND ANNUAL EXCEEDANCE PROBABILITY, IN PERCENT

\begin{tabular}{ccccccc}
1.25 & 2 & 5 & 10 & 25 & 50 & 100 \\
$80 \%$ & $50 \%$ & $20 \%$ & $10 \%$ & $4 \%$ & $2 \%$ & $1 \%$ \\
\hline & & & & & & \\
\hline & - & & & & & \\
\hline
\end{tabular}

MAGNITUDE AND PROBABILITY OF ANNUAL LOW FLOW BASED ON PERIOD OF RECORD $1924-82$

\begin{tabular}{|c|c|c|c|c|c|c|}
\hline $\begin{array}{r}\text { PERIOD } \\
\text { (CON- } \\
\text { SECU- }\end{array}$ & \multicolumn{6}{|c|}{$\begin{array}{l}\text { DISCHARGE, IN CFS, FOR INDICATED RECURRENCE } \\
\text { INTERVAL, IN YEARS, AND ANNUAL NON- } \\
\text { EXCEEDANCE PROBABILITY, IN PERCENT }\end{array}$} \\
\hline $\begin{array}{l}\text { TIVE } \\
\text { DAYS) }\end{array}$ & $\begin{array}{c}2 \\
50 \%\end{array}$ & $\begin{array}{c}5 \\
20 \%\end{array}$ & $\begin{array}{l}10 \\
10 \%\end{array}$ & $\begin{array}{l}20 \\
5 \%\end{array}$ & $\begin{array}{l}50 \\
2 \%\end{array}$ & $\begin{array}{r}100 \\
1 \%\end{array}$ \\
\hline 1 & 27 & 9.1 & 4.7 & 2.6 & 1. & .8 \\
\hline 3 & 28 & 9.8 & 5.2 & 2.9 & 1. & .9 \\
\hline 7 & 30 & 11 & 5.6 & 3.2 & 1. & .9 \\
\hline 14 & 33 & 13 & 7.3 & 4.4 & 2. & 1.6 \\
\hline 30 & 35 & 15 & 8.8 & 5.7 & 3. & 2.4 \\
\hline 60 & 44 & 18 & 11 & 7.0 & 4. & 2.8 \\
\hline 90 & 57 & 23 & 14 & 8.3 & 4. & 3.0 \\
\hline 120 & 70 & 29 & 16 & 9.7 & 5. & 3.2 \\
\hline 183 & 117 & 62 & 41 & 28 & 18 & 12 \\
\hline
\end{tabular}

MAGNITUDE AND PROBABILITY OF ANNUAL HIGH FLOW BASED ON PERIOD OF RECORD 1923-82

\begin{tabular}{|c|c|c|c|c|c|c|}
\hline $\begin{array}{l}\text { PERIOD } \\
\text { (CON- }\end{array}$ & \multicolumn{6}{|c|}{$\begin{array}{c}\text { DISCHARGE, IN CFS, FOR INDICATED RECURRENCE } \\
\text { INTERVAL, IN YEARS, AND ANNUAL } \\
\text { EXCEEDANCE PROBABILITY, IN PERCENT }\end{array}$} \\
\hline $\begin{array}{l}\text { TIVE } \\
\text { DAYS) }\end{array}$ & $\begin{array}{c}2 \\
50 \%\end{array}$ & $\begin{array}{c}5 \\
20 \%\end{array}$ & $\begin{array}{l}10 \\
10 \%\end{array}$ & $\begin{array}{l}25 \\
4 \%\end{array}$ & $\begin{array}{l}50 \\
2,5\end{array}$ & $\begin{array}{r}100 \\
1 \%\end{array}$ \\
\hline \multicolumn{7}{|c|}{ 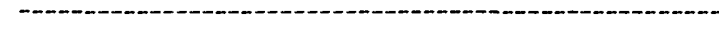 } \\
\hline $\begin{array}{l}1 \\
3\end{array}$ & $\begin{array}{l}512 \\
507\end{array}$ & $\begin{array}{l}669 \\
663\end{array}$ & $\begin{array}{l}778 \\
771\end{array}$ & $\begin{array}{l}924 \\
914\end{array}$ & $\begin{array}{l}1040 \\
1020\end{array}$ & $\begin{array}{l}1150 \\
1140\end{array}$ \\
\hline $\begin{array}{r}7 \\
15\end{array}$ & $\begin{array}{l}501 \\
481\end{array}$ & $\begin{array}{l}645 \\
618\end{array}$ & $\begin{array}{l}740 \\
709\end{array}$ & $\begin{array}{l}861 \\
826\end{array}$ & $\begin{array}{l}952 \\
915\end{array}$ & $\begin{array}{l}1040 \\
1000\end{array}$ \\
\hline $\begin{array}{l}30 \\
60\end{array}$ & $\begin{array}{l}447 \\
395\end{array}$ & $\begin{array}{l}571 \\
497\end{array}$ & $\begin{array}{l}655 \\
565\end{array}$ & $\begin{array}{l}763 \\
650\end{array}$ & $\begin{array}{l}845 \\
714\end{array}$ & $\begin{array}{l}928 \\
778\end{array}$ \\
\hline 90 & 358 & 454 & 520 & 605 & 671 & 738 \\
\hline
\end{tabular}

DURATION TABLE OF DAILY MEAN FLOW FOR PERIOD OF RECORD 1923-82

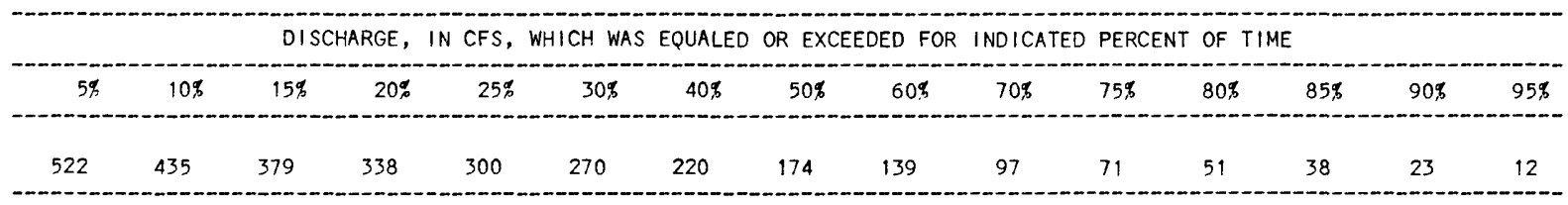


14054500 BROWN CREEK NEAR LA PINE, OR

LOCATION.--Lat $43^{\circ} 42^{\prime} 57^{\prime \prime}$, long $121^{\circ} 48^{\prime} 10^{\prime \prime}$, in NE⿺ $\frac{1}{4} W_{\frac{1}{4}}$ sec.29, T.21 S., R.8 E., Deschutes County, Hydrologic Unit 17070301, in Deschutes National Forest, on right bank at highway crossing and $15 \mathrm{mi}$ northwest of La Pine.

DRAINAGE AREA.--21 $\mathrm{mi}^{2}$, approximately, hydrologic drainage boundary uncertain owing to ground-water exchange.

PERIOD OF RECORD.--June 1922 to September 1925, July 1938 to September 1982 . Monthly discharge only July 1938 to September 1949 , published in WSP 1318. Prior to Oct. 1, 1964, published as "near Lapine."

GAGE.--Water-stage recorder. Altitude of gage is 4,370 ft, from topographic map. May 24, 1922, to Sept. 30 , 1925, nonrecording gage, and July 1, 1938, to Nov. 1, 1945, water-stage recorder at site 0.4 mi downstream at ditferent datums. Nov. 2, 1945, to Aug. 25, 1971, water-stage recorder at site $0.8 \mathrm{mi}$ upstream at datum of 4,372.94 ft National Geodetic Vertical Datum of 1929 .

REMARKS.--No regulation. No diversion above station.

AVERAGE DISCHARGE. --47 years, $38.3 \mathrm{ft}^{3} / \mathrm{s}, 27,750$ acre- $\mathrm{ft} / \mathrm{yr}$.

EXTREMES FOR PERIOD OF RECORD.--Maximum discharge, $104 \mathrm{ft} / \mathrm{s}$ Aug. 4, 1956, gage height, $1.64 \mathrm{ft}$; maximum gage height, $3.50 \mathrm{ft}$ Jan.

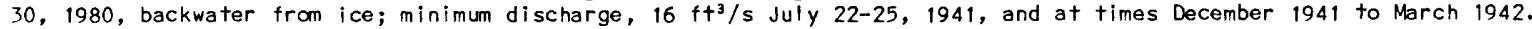

STATISTICAL SUMMARIES

MONTHLY AND ANNUAL MEAN DISCHARGES 1923-82

\begin{tabular}{|c|c|c|c|c|c|c|}
\hline MONTH & $\begin{array}{l}\text { MINIMUM } \\
\text { (CFS) }\end{array}$ & $\begin{array}{l}\text { MAXIHUM } \\
\text { (CFS) }\end{array}$ & $\begin{array}{l}\text { MEAN } \\
\text { (CFS) }\end{array}$ & $\begin{array}{l}\text { STAN- } \\
\text { DARD } \\
\text { DEVIA- } \\
\text { TION } \\
\text { (CFS) }\end{array}$ & $\begin{array}{l}\text { COEFFI- } \\
\text { CIENT OF } \\
\text { VARI- } \\
\text { ATION }\end{array}$ & $\begin{array}{c}\text { PERCENT } \\
\text { OF } \\
\text { ANNUAL } \\
\text { RUNOFF }\end{array}$ \\
\hline OCTOBER & 18 & 71 & 42 & 13 & .31 & 9.0 \\
\hline $\begin{array}{l}\text { NOVEMBER } \\
\text { DECEMBER }\end{array}$ & $\begin{array}{l}18 \\
17\end{array}$ & $\begin{array}{l}66 \\
62\end{array}$ & $\begin{array}{l}40 \\
38\end{array}$ & $\begin{array}{l}12 \\
11\end{array}$ & $\begin{array}{l}.31 \\
.30\end{array}$ & $\begin{array}{l}8.7 \\
8.2\end{array}$ \\
\hline $\begin{array}{l}\text { JANUARY } \\
\text { FEBRUARY } \\
\text { MARCH }\end{array}$ & $\begin{array}{l}16 \\
16 \\
17\end{array}$ & $\begin{array}{l}55 \\
53 \\
54\end{array}$ & $\begin{array}{l}36 \\
34 \\
34\end{array}$ & $\begin{array}{l}9.7 \\
8.9 \\
8.9\end{array}$ & $\begin{array}{l}.27 \\
.26 \\
.26\end{array}$ & $\begin{array}{l}7.7 \\
7.5 \\
7.4\end{array}$ \\
\hline APRIL & 17 & 56 & 37 & 9.8 & .27 & 7.9 \\
\hline $\begin{array}{l}\text { MAY } \\
\text { JUNE }\end{array}$ & $\begin{array}{l}18 \\
17\end{array}$ & $\begin{array}{l}69 \\
71\end{array}$ & $\begin{array}{l}37 \\
38\end{array}$ & $\begin{array}{l}12 \\
13\end{array}$ & $\begin{array}{l}.32 \\
.33\end{array}$ & $\begin{array}{l}8.1 \\
8.3\end{array}$ \\
\hline JULY & 17 & 75 & 39 & 13 & .33 & 8.5 \\
\hline AUGUST & 18 & 77 & 42 & 14 & .34 & 9.2 \\
\hline SEPTEMBER & 17 & 70 & 43 & 14 & .32 & 9.3 \\
\hline NNUAL & 18 & 59 & 38 & 10 & .26 & 100 \\
\hline
\end{tabular}

MAGNITUDE AND PROBABILITY OF INSTANTANEOUS PEAK FLOW BASED ON PERIOD OF RECORD 1923-82

DISCHARGE, IN CFS, FOR INDICATED RECURRENCE INTERVAL, IN YEARS, AND ANNUAL EXCEEDANCE PROBABILITY, IN PERCENT

\begin{tabular}{ccccccc}
1.25 & 2 & 5 & 10 & 25 & 50 & 100 \\
$80 \%$ & $50 \%$ & $20 \%$ & $10 \%$ & $4 \%$ & $2 \%$ & $1 \%$ \\
\hline 40 & 52 & 67 & 76 & 87 & 96 & 104 \\
\hline
\end{tabular}

WEIGHTED SKEW $=-.046$
MAGNITUDE AND PROBABILITY OF ANNUAL LOW FLOW BASED ON PERIOD OF RECORD 1924-82

\begin{tabular}{|c|c|c|c|c|c|c|}
\hline \multirow{3}{*}{$\begin{array}{l}\text { PERIOD } \\
\text { (CON- } \\
\text { SECU- } \\
\text { TIVE } \\
\text { DAYS) }\end{array}$} & \multicolumn{6}{|c|}{$\begin{array}{l}\text { DISCHARGE, IN CFS, FOR INDICATED RECURRENCE } \\
\text { INTERVAL, IN YEARS, AND ANNUAL NON- } \\
\text { EXCEEDANCE PROBABILITY, IN PERCENT }\end{array}$} \\
\hline & & & & & $-\infty$ & -1 \\
\hline & $\begin{array}{c}2 \\
50 \%\end{array}$ & $\begin{array}{c}5 \\
20 \%\end{array}$ & $\begin{array}{l}10 \\
10 \%\end{array}$ & $\begin{array}{l}20 \\
5 \%\end{array}$ & $\begin{array}{l}50 \\
2 \%\end{array}$ & $\begin{array}{r}100 \\
1 \%\end{array}$ \\
\hline & 20 & 23 & 20 & 18 & 16 & 15 \\
\hline 3 & 29 & 23 & 20 & $\begin{array}{l}18 \\
18\end{array}$ & 16 & 15 \\
\hline 7 & 29 & 23 & 20 & 18 & 16 & 15 \\
\hline 14 & 30 & 23 & 21 & 18 & 16 & 15 \\
\hline 30 & 30 & 24 & 21 & 19 & 17 & 15 \\
\hline 60 & 31 & 24 & 21 & 19 & 17 & 15 \\
\hline 90 & 32 & 25 & 22 & 19 & 17 & 15 \\
\hline 120 & 33 & 26 & 22 & 20 & 17 & 16 \\
\hline 183 & 35 & 27 & 23 & 21 & 18 & 16 \\
\hline
\end{tabular}

MAGNITUDE AND PROBABILITY OF ANNUAL HIGH FLOW BASED ON PERIOD OF RECORD 1923-82

\begin{tabular}{|c|c|c|c|c|c|c|}
\hline $\begin{array}{l}\text { PERIOD } \\
\text { (CON- } \\
\text { SECU- }\end{array}$ & \multicolumn{6}{|c|}{$\begin{array}{l}\text { DISCHARGE, IN CFS, FOR INDICATED RECURRENCE } \\
\text { INTERVAL, IN YEARS, AND ANNUAL } \\
\text { EXCEEDANCE PROBABILITY, IN PERCENT }\end{array}$} \\
\hline TIVE & 2 & 5 & 10 & 25 & 50 & 100 \\
\hline DAYS) & $50 \%$ & $20 \%$ & $10 \%$ & $4 \%$ & $2 \%$ & $1 \%$ \\
\hline 1 & 50 & 63 & 71 & 79 & 84 & 89 \\
\hline 3 & 49 & 61 & 69 & 76 & 81 & 86 \\
\hline 7 & 48 & 61 & 68 & 75 & 80 & 85 \\
\hline 15 & 48 & 61 & 68 & 75 & 80 & 85 \\
\hline 30 & 48 & 60 & 67 & 74 & 79 & 83 \\
\hline 60 & 47 & 59 & 66 & 73 & 78 & 82 \\
\hline 90 & 45 & 57 & 64 & 71 & 76 & 80 \\
\hline
\end{tabular}

DURATION TABLE OF DAILY MEAN FLOW FOR PERIOD OF RECORD 1923-82

DISCHARGE, IN CFS, WHICH WAS EQUALED OR EXCEEDED FOR INDICATED PERCENT OF TIME

\begin{tabular}{|c|c|c|c|c|c|c|c|c|c|c|c|c|c|c|}
\hline $5 \%$ & $10 \%$ & $15 \%$ & $20 \%$ & $25 \%$ & $30 \%$ & $40 \%$ & $50 \%$ & $60 \%$ & $70 \%$ & $75 \%$ & $80 \%$ & $85 \%$ & $90 \%$ & $95 \%$ \\
\hline 61 & 56 & 53 & 50 & 47 & 45 & 41 & 37 & 34 & 31 & 29 & 28 & 27 & 25 & 23 \\
\hline
\end{tabular}




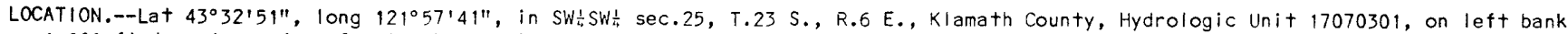
$1,000 \mathrm{ft}$ downstream from Odell Lake, $3 \mathrm{mi}$ north of town of Crescent Lake, and $14 \mathrm{mi}$ northwest of Crescent.

DRAINAGE AREA. $--39.0 \mathrm{mi}^{2}$.

PERIOD OF RECORD.--August, September 1911, August, September 1912, January, February, May to November 1913, April to August 1914, December 1923 to June 1924, May 1933 to September 1976. Gage heights and discharge measurements only August, September 1911, January 1913. Published as Jdell Lake outlet near Crescent 1911-12. Records for January to July 1912, published in WSP 332 , have been found to be unreliable and should not be used.

GAGE.--Water-stage recorder. Datum of gage is 4,799.05 ft National Geodetic Vertical Datum of 1929. Prior to June 7, 1924, nonrecording gage at several sites within $700 \mathrm{ft}$ of present site at various datums.

REMARKS.--Flow affected occasionally in winter by ice jams at outlet of Odell Lake, and slightly affected at times by seiches in Odell Lake. No diversion above station.

AVERAGE DISCHARGE.--43 years (water years 1933-76), $82.5 \mathrm{ft} / \mathrm{s}, 28.73 \mathrm{in} / \mathrm{yr}, 59.770 \mathrm{acre}-\mathrm{ft} / \mathrm{yr}$.

EXTREMES FOR PERIOD OF RECORD.--Maximum discharge, $1,100 \mathrm{ft}^{3} / \mathrm{s} \mathrm{Dec.} \mathrm{25,} \mathrm{1964,} \mathrm{gage} \mathrm{height,} 2.60 \mathrm{ft}$, from rating curve extended above $250 \mathrm{ft}^{3} / \mathrm{s}$; minimum, $9 \mathrm{ft}^{3} / \mathrm{s}$ sometime during period Sept. 7-30, 1934.

\section{STATISTICAL SUMMARIES}

MONTHLY AND ANNUAL MEAN DISCHARGES 1934-76

\begin{tabular}{|c|c|c|c|c|c|c|}
\hline MONTH & $\begin{array}{l}\text { MINIMUM } \\
\text { (CFS) }\end{array}$ & $\begin{array}{l}\text { MAXIMUM } \\
\text { (CFS) }\end{array}$ & $\begin{array}{l}\text { MEAN } \\
\text { (CFS) }\end{array}$ & $\begin{array}{l}\text { STAN- } \\
\text { DARD } \\
\text { DEVIA- } \\
T ! O N \\
\text { (CFS) }\end{array}$ & $\begin{array}{l}\text { COEFFI- } \\
\text { CIENT OF } \\
\text { VARI- } \\
\text { ATION }\end{array}$ & $\begin{array}{c}\text { PERCENT } \\
\text { OF } \\
\text { ANNUAL } \\
\text { RUNOFF }\end{array}$ \\
\hline OCTOBER & 21 & 112 & 52 & 20 & .38 & 5.3 \\
\hline NOVEMBER & 30 & 185 & 83 & 29 & .36 & 8.3 \\
\hline DECEMBER & 42 & 337 & 102 & 52 & .51 & 10.3 \\
\hline JANUARY & 47 & 218 & 103 & 38 & .37 & 10.4 \\
\hline FEBRUARY & 46 & 160 & 90 & 25 & .28 & 9.1 \\
\hline MARCH & 38 & 177 & 79 & 24 & .31 & 8.0 \\
\hline APRIL & 46 & 111 & 80 & 17 & .21 & 8.1 \\
\hline MAY & 62 & 180 & 113 & 28 & .24 & 11.4 \\
\hline JUNE & 48 & 220 & 129 & 44 & .34 & 13.0 \\
\hline JULY & 27 & 139 & 75 & 31 & .41 & 7.6 \\
\hline AUGUST & 20 & 78 & 44 & 19 & .42 & 4.5 \\
\hline SEPTEMBER & 17 & 69 & 41 & 15 & .36 & 4.1 \\
\hline ANNUAL & 47 & 120 & 83 & 20 & .25 & 100 \\
\hline
\end{tabular}

MAGNITUDE AND PROBABILITY OF INSTANTANEOUS PEAK FLOW BASED ON PERIOD OF RECORD 1933-76

DISCHARGE, IN CFS, FOR INDICATED RECURRENCE INTERVAL, IN YEARS, AND ANNUAL EXCEEDANCE PROBABILITY, IN PERCENT

$\begin{array}{lcccccc}1.25 & 2 & 5 & 10 & 25 & 50 & 100 \\ 80 \% & 50 \% & 20 \% & 10 \% & 4 \% & 2 \% & 1 \% \\ 171 & 236 & 345 & 430 & 556 & 662 & 781\end{array}$

WEIGHTED SKEW $=.574$
MAGNITUDE AND PROBABILITY OF ANNUAL LOW FLOW BASED ON PERIOD OF RECORD 1935-76

\begin{tabular}{|c|c|c|c|c|c|c|}
\hline $\begin{array}{l}\text { PERIOD } \\
\text { ( CON- } \\
\text { SECU- }\end{array}$ & \multicolumn{6}{|c|}{$\begin{array}{c}\text { DISCHARGE, IN CFS, FOR INDICATED RECURRENCE } \\
\text { INTERVAL, IN YEARS, AND ANNUAL NON- } \\
\text { EXCEEDANCE PROBABILITY, IN PERCENT }\end{array}$} \\
\hline TIVE & 2 & 5 & 10 & 20 & 50 & 100 \\
\hline DAYS) & $50 \%$ & $20 \%$ & $10 \%$ & $5 \%$ & $2 ?$ & $1 \%$ \\
\hline $1-1$ & --2 & & & & & \\
\hline 1 & 27 & 19 & 15 & 13 & 11 & \\
\hline 3 & 29 & 20 & 16 & 14 & 11 & \\
\hline 7 & 31 & 21 & 17 & 15 & 12 & 11 \\
\hline 14 & 32 & 23 & 19 & 16 & 13 & 12 \\
\hline 30 & 35 & 24 & 20 & 17 & 14 & 13 \\
\hline 60 & 37 & 27 & 22 & 19 & 16 & 14 \\
\hline 90 & 41 & 29 & 25 & 21 & 18 & 16 \\
\hline 120 & 46 & 34 & 28 & 25 & 21 & 19 \\
\hline 183 & 59 & 45 & 39 & 34 & 30 & 27 \\
\hline
\end{tabular}

MAGNITUDE AND PROBABILITY OF ANNUAL HIGH FLOW BASED ON PERIOD OF RECORD 1934-76

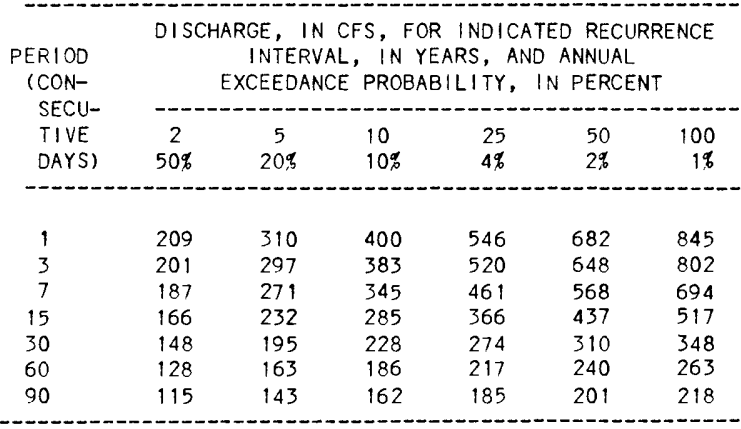

DURATION TABLE OF DAILY MEAN FLOW FOR PERIOD OF RECORD 1934-76

$\begin{array}{lllllllllllll}5 \% & 10 \% & 15 \% & 20 \% & 25 \% & 30 \% & 40 \% & 50 \% & 60 \% & 70 \% & 75 \% & 80 \% & 85 \% \\ 166 & 142 & 126 & 113 & 104 & 96 & 83 & 74 & 65 & 57 & 52 & 47 & 41\end{array}$


14055500 DESCHUTES RIVER BELOW WICKIUP RESERVOIR, NEAR LA PINE, OR

LOCATION.--Lat $43^{\circ} 41^{\prime} 10^{\prime \prime}$, long $121^{\circ} 41^{\prime} 13^{\prime \prime}$, in NW $\frac{1}{4} N E \frac{1}{4}$ sec.7, T.22 S., R.9 E., Deschutes County, Hydrologic Unit 17070301, on left bank $1,000 \mathrm{ft}$ downstream from Wickiup Dam, $9 \mathrm{mi}$ west of La Pine, and at mile 226.4 .

DRAINAGE AREA.--483 $\mathrm{mi}^{2}$, hydrologic drainage boundary uncertain owing to ground-water exchange.

PERIOD OF RECORD.--June 1938 to September 1982. Monthly discharge only June 1938, published in WSP 1318. Published as "near Lapine" 1938-64.

GAGE.--Water-stage recorder. Datum of gage is $4,257.41$ ft National Geodetic Vertical Datum of 1929 (levels by Bureau of Reclamation).

REMARKS.--Flow regulated by Crane Prairie Reservoir, and since 1942 by Wickiup Reservoir. Some leakage from Crane Prairie and Wickiup Reservoirs does not pass station. Some spill bypassed station in 1955. Crater Creek canal diverts water above station to Tuma lo Creek basin.

AVERAGE DISCHARGE.--44 years, $737 \mathrm{ft} / \mathrm{s}, 534,000$ acre- $\mathrm{ft} / \mathrm{yr}$

EXTREMES FOR PERIOD OF RECORD.--Maximum discharge, $2,280 \mathrm{ft}^{3} / \mathrm{s} \mathrm{July} 28$ to Aug. 1, 1956, July 31 , Aug. 1 , 2 , 1962; minimum, $1.9 \mathrm{ft} 3 / \mathrm{s}$ Nov. 10, 1973; minimum daily, $10 \mathrm{ft}^{3} / \mathrm{s}$ Jan. 17, 1952.

STATISTICAL SUMMARIES

MONTHLY AND ANNUAL MEAN DISCHARGES 1943-82

\begin{tabular}{|c|c|c|c|c|c|c|}
\hline MONTH & $\begin{array}{l}\text { MINIMUMM } \\
\text { (CFS) }\end{array}$ & $\begin{array}{l}\text { MAXIMUM } \\
\text { (CFS) }\end{array}$ & $\begin{array}{l}\text { MEAN } \\
\text { (CFS) }\end{array}$ & $\begin{array}{l}\text { STAN- } \\
\text { DARD } \\
\text { DEVIA- } \\
\text { TION } \\
\text { (CFS) }\end{array}$ & $\begin{array}{l}\text { COEFFI- } \\
\text { CIENT OF } \\
\text { VARI- } \\
\text { ATION }\end{array}$ & $\begin{array}{c}\text { PERCENT } \\
\text { OF } \\
\text { ANNUAL } \\
\text { RUNOFF }\end{array}$ \\
\hline OCTOBER & 112 & 1200 & 599 & 256 & .43 & 6.7 \\
\hline NOVEMBER & 14 & 1050 & 159 & 217 & 1.36 & 1.8 \\
\hline DECEMBER & 16 & 869 & 148 & 192 & 1.30 & 1.7 \\
\hline JANUARY & 18 & 851 & 187 & 239 & 1.28 & 2.1 \\
\hline FEBRUARY & 16 & 780 & 224 & 249 & 1.11 & 2.5 \\
\hline MARCH & 18 & 721 & 238 & 226 & .95 & 2.7 \\
\hline APRIL & 268 & 990 & 535 & 203 & .38 & 6.0 \\
\hline MAY & 476 & 1542 & 1029 & 227 & .22 & 11.6 \\
\hline JUNE & 638 & 1788 & 1354 & 309 & .23 & 15.2 \\
\hline JULY & 922 & 2079 & 1671 & 308 & .18 & 18.8 \\
\hline AUGU & 908 & 2123 & 1532 & 286 & .19 & 17.2 \\
\hline SEPTEMBER & 832 & 1698 & 1233 & 198 & .16 & 13.8 \\
\hline ANNUAL & 552 & 997 & 746 & 120 & .16 & 100 \\
\hline
\end{tabular}

MAGNITUDE AND PROBABILITY OF INSTANTANEOUS PEAK FLOW BASED ON PERIOD OF RECORD

DISCHARGE, IN CFS, FOR INDICATED RECURRENCE INTERVAL, IN YEARS, AND ANNUAL EXCEEDANCE PROBABILITY, IN PERCENT

\begin{tabular}{ccccccc}
1.25 & 2 & 5 & 10 & 25 & 50 & 100 \\
$80 \%$ & $50 \%$ & $20 \%$ & $10 \%$ & $4 \%$ & $2 \%$ & $1 \%$ \\
\hline- & & & & & & \\
\hline & - & - & - & - & - & \\
\hline
\end{tabular}

WEIGHTED SKEW $=$
MAGNITUDE AND PROBABILITY OF ANNUAL LOW FLOW BASED ON PERIOD OF RECORD 1944-82

\begin{tabular}{|c|c|c|c|c|c|c|}
\hline \multirow{2}{*}{$\begin{array}{l}\text { PERIOD } \\
\text { (CON- } \\
\text { SECU- } \\
\text { TIVE } \\
\text { DAYS) }\end{array}$} & \multicolumn{6}{|c|}{$\begin{array}{l}\text { DISCHARGE, IN CFS, FOR INDICATED RECURRENCE } \\
\text { INTERVAL, IN YEARS, AND ANNUAL NON- } \\
\text { EXCEEDANCE PROBABILITY, IN PERCENT }\end{array}$} \\
\hline & $\begin{array}{c}2 \\
50 \%\end{array}$ & $\begin{array}{c}5 \\
20 \%\end{array}$ & $\begin{array}{l}10 \\
10 \%\end{array}$ & $\begin{array}{l}20 \\
5 \%\end{array}$ & $\begin{array}{l}50 \\
2 \%\end{array}$ & $\begin{array}{r}100 \\
1 \%\end{array}$ \\
\hline & & & & & & \\
\hline 1 & 29 & 15 & 11 & 9.2 & 7.6 & - \\
\hline 3 & 30 & 16 & 12 & 9.9 & 8. & -- \\
\hline 7 & 36 & 18 & 13 & 11 & 8.8 & -- \\
\hline 14 & 38 & 19 & 14 & 11 & 8.8 & -- \\
\hline 30 & 43 & 20 & 15 & 11 & 9. & -- \\
\hline 60 & 47 & 22 & 15 & 12 & 9.2 & -- \\
\hline 90 & 53 & 23 & 15 & 12 & 9.6 & - \\
\hline 120 & 65 & 26 & 17 & 12 & 10 & -- \\
\hline 183 & 185 & 98 & 71 & 55 & 41 & -- \\
\hline
\end{tabular}

MAGNITUDE AND PROBABILITY OF ANNUAL HIGH FLOW BASED ON PERIOD OF RECORD 1943-82

\begin{tabular}{|c|c|c|c|c|c|c|}
\hline $\begin{array}{l}\text { PERIOD } \\
\text { (CON- } \\
\text { SECU- }\end{array}$ & \multicolumn{6}{|c|}{$\begin{array}{c}\text { DISCHARGE, IN CFS, FOR IND ICATED RECURRENCE } \\
\text { INTERVAL, IN YEARS, AND ANNUAL } \\
\text { EXCEEDANCE PROBABILITY, IN PERCENT }\end{array}$} \\
\hline TIVE & 2 & 5 & 10 & 25 & 50 & 100 \\
\hline DAYS) & $50 \%$ & $20 \%$ & $10 \%$ & $4 \%$ & $2 \%$ & $1 \%$ \\
\hline 1 & 1930 & 2120 & 2190 & 2250 & 2280 & -- \\
\hline 3 & 1920 & 2110 & 2180 & 2240 & 2270 & -- \\
\hline 7 & 1890 & 2090 & 2170 & 2240 & 2280 & -- \\
\hline 15 & 1850 & 2060 & 2150 & 2230 & 2270 & -- \\
\hline 30 & 1780 & 2000 & 2090 & 2170 & 2220 & -- \\
\hline 60 & 1690 & 1900 & 1990 & 2080 & 2120 & -- \\
\hline 90 & 1610 & 1810 & 1890 & 1970 & 2010 & -- \\
\hline
\end{tabular}

DURATION TABLE OF DAILY MEAN FLOW FOR PERIOD OF RECORD 1943-82

\begin{tabular}{|c|c|c|c|c|c|c|c|c|c|c|c|c|c|c|}
\hline $5 \%$ & $10 \%$ & $15 \%$ & $20 \%$ & $25^{\circ}$ & $30 \%$ & $40 \%$ & $50 \%$ & $60 \%$ & $70 \%$ & $75 \%$ & $80 \%$ & $85 \%$ & $90 \%$ & $95 \%$ \\
\hline 1880 & 1700 & 1540 & 1420 & 1280 & 1140 & 874 & 645 & 449 & 183 & 78 & 36 & 29 & 25 & 22 \\
\hline
\end{tabular}


14057000 DESCHUTES RIVER AT PRINGLE FALLS, NEAR LAPINE, OR

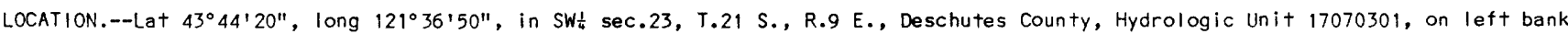
$0.5 \mathrm{mi}$ upstream from bridge at Pringle Falls, $7 \mathrm{mi}$ northwest of Lapine, and at mile 217.

DRAINAGE AREA.--507 $\mathrm{mi}^{2}$, hydrologic drainage boundary uncertain owing to ground-water exchange.

PERIOD OF RECORD,--October 1915 to September 1917, June 1922 to September 1952. Monthly discharge only for some periods, published in WSP 1318.

GAGE.--Water-stage recorder. Datum of gage is 4,243.26 ft National Geodetic Vertical Datum of 1929 (Forest Service bench mark). Prior to June 6, 1922, staft gage at practically same site at datum 3.09 ft higher. June 6, 1922, to Nov. 9, 1947, water-stage recorder at present site at datum $2.00 \mathrm{ft}$ higher.

REMARKS.--Flow regulated since 1922 by Crane Prairie Reservoir, and since 1942 by Wickiup Reservoir. Crater Creek Canal diverts water above station to Tumalo Creek basin.

AVERAGE DISCHARGE.--32 years (water years $1916-17,1923-52$ ), $727 \mathrm{ft}^{3} / \mathrm{s}, 526,300 \mathrm{acre}-\mathrm{ft} / \mathrm{yr}$.

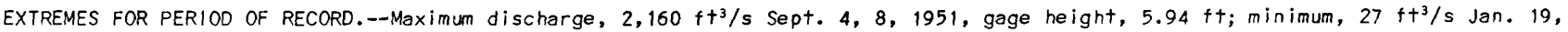
1952.

STATISTICAL SUMMARIES (BEFORE THE CONSTRUCTION OF WICKIUP RESERVOIR)

MONTHLY AND ANNUAL MEAN DISCHARGES 1923-41

\begin{tabular}{|c|c|c|c|c|c|c|}
\hline MONTH & $\begin{array}{l}\text { MINIMUM } \\
\text { (CFS) }\end{array}$ & $\begin{array}{l}\text { MAXIMUMM } \\
\text { (CFS) }\end{array}$ & $\begin{array}{l}\text { MEAN } \\
\text { (CFS) }\end{array}$ & $\begin{array}{l}\text { STAN- } \\
\text { DARD } \\
\text { DEVIA- } \\
\text { TION } \\
\text { (CFS) }\end{array}$ & $\begin{array}{l}\text { COEFFI- } \\
\text { CIENT OF } \\
\text { VARI- } \\
\text { ATION }\end{array}$ & $\begin{array}{c}\text { PERCENT } \\
\text { OF } \\
\text { ANNUAL } \\
\text { RUNOFF }\end{array}$ \\
\hline OCTOBER & 527 & 947 & 715 & 132 & .18 & 8.4 \\
\hline $\begin{array}{l}\text { NOVEMBER } \\
\text { DECEMBER }\end{array}$ & $\begin{array}{l}473 \\
469\end{array}$ & $\begin{array}{l}861 \\
883\end{array}$ & $\begin{array}{l}657 \\
629\end{array}$ & 128 & .19 & 7.7 \\
\hline JANUARY & 433 & 882 & 616 & 114 & .19 & 7.2 \\
\hline FEBRUARY & 427 & 929 & 636 & 132 & .21 & 7.5 \\
\hline MARCH & 419 & 836 & 626 & 116 & .19 & 7.3 \\
\hline APRIL & 448 & 881 & 637 & 115 & .18 & 7.5 \\
\hline MAY & 545 & 871 & 701 & 93 & .13 & 8.2 \\
\hline JUNE & 687 & 988 & 811 & 94 & .12 & 9.5 \\
\hline JULY & 660 & 1169 & 866 & 142 & .16 & 10.2 \\
\hline AUGUST & 589 & 1222 & 864 & 176 & .20 & 10.1 \\
\hline SEPTEMBER & 533 & 1085 & 773 & 154 & .20 & 9.1 \\
\hline ANNUAL & 542 & 872 & 711 & 88 & .12 & 100 \\
\hline
\end{tabular}

MAGNITUDE AND PROBABILITY OF INSTANTANEOUS PEAK FLOW BASED ON PERIOD OF RECORD 1917-41

DISCHARGE, IN CFS, FOR INDICATED RECURRENCE INTERVAL, IN YEARS, AND ANNUAL EXCEEDANCE PROBABILITY, IN PERCENT

\begin{tabular}{ccccccc}
1.25 & 2 & 5 & 10 & 25 & 50 & 100 \\
$80 \%$ & $50 \%$ & $20 \%$ & $10 \%$ & $4 \%$ & $2 \%$ & $1 \%$ \\
\hline 891 & 1000 & 1130 & 1190 & 1270 & - & -
\end{tabular}

WEIGHTED SKEW $=-.151$
MAGNITUDE AND PROBABILITY OF ANNUAL LOW FLOW BASED ON PERIOD OF RECORD 1924-41

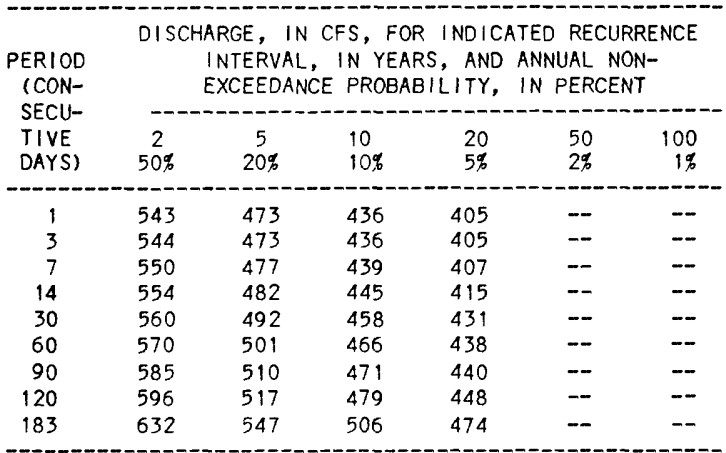

MAGNITUDE AND PROBABILITY OF ANNUAL HIGH FLOW BASED ON PERIOD OF RECORD 1923-41

PERIOD
(CON-

DURATION TABLE OF DAILY MEAN FLOW FOR PERIOD OF RECORD 1923-41

\begin{tabular}{|c|c|c|c|c|c|c|c|c|c|c|c|c|c|c|}
\hline & & DI & RGE, & CFS, & $\mathrm{ICH}$ WA & EQUALE & OR EX & DED FO & INDICA & D PERC & T OF & & & \\
\hline $5 \%$ & $10 \%$ & $15 \%$ & $20 \%$ & $25 \%$ & $30 \%$ & $40 \%$ & $50 \%$ & $60 \%$ & $70 \%$ & $75 \%$ & $80 \%$ & $85 \%$ & $90 \%$ & $95 \%$ \\
\hline 998 & 948 & 897 & 857 & 817 & 785 & 732 & 692 & 646 & 610 & 592 & 572 & 550 & 527 & 466 \\
\hline
\end{tabular}


14057000 DESCHUTES RIVER AT PRINGLE FALLS, NEAR LAPINE, OR--Continued

STATISTICAL SUMMARIES (AFTER THE COMPLETION OF WICKIUP RESERVOIR)

MONTHLY AND ANNUAL MEAN DISCHARGES 1943-52

\begin{tabular}{|c|c|c|c|c|c|c|}
\hline MONTH & $\begin{array}{l}\text { MINIMUM } \\
\text { (CFS) }\end{array}$ & $\begin{array}{l}\text { MAXIMUM } \\
\text { (CFS) }\end{array}$ & $\begin{array}{l}\text { MEAN } \\
\text { (CFS) }\end{array}$ & $\begin{array}{l}\text { STAN- } \\
\text { OARD } \\
\text { DEVIA- } \\
\text { TION } \\
\text { (CFS) }\end{array}$ & $\begin{array}{l}\text { COEFFI- } \\
\text { CIENT OF } \\
\text { VARI- } \\
\text { ATION }\end{array}$ & $\begin{array}{c}\text { PERCENT } \\
\text { OF } \\
\text { ANNUAL } \\
\text { RUNOFF }\end{array}$ \\
\hline OCTOBER & 509 & 1165 & 802 & 227 & .28 & 8.9 \\
\hline NOVEMBER & 57 & 1011 & 353 & 301 & .85 & 3.9 \\
\hline DECEMBER & 50 & 857 & 282 & 251 & .89 & 3.1 \\
\hline JANUARY & 57 & 841 & 278 & 239 & .86 & 3.1 \\
\hline FEBRUARY & 99 & 740 & 329 & 228 & .69 & 3.7 \\
\hline MARCH & 69 & 654 & 382 & 210 & .55 & 4.2 \\
\hline APRIL & 273 & 944 & 511 & 219 & .43 & 5.7 \\
\hline MAY & 475 & 1106 & 808 & 201 & .25 & 9.0 \\
\hline JUNE & 624 & 1759 & 1069 & 382 & .36 & 11.9 \\
\hline JULY & 922 & 1996 & 1423 & 418 & .29 & 15.8 \\
\hline AUGUST & 907 & 2068 & 1446 & 425 & .29 & 16.1 \\
\hline SEPTEMBER & 826 & 1715 & 1310 & 303 & .23 & 14.6 \\
\hline ANNUAL & 556 & 958 & 752 & 128 & .17 & 100 \\
\hline
\end{tabular}

MAGNITUDE AND PROBABILITY OF INSTANTANEOUS PEAK FLOW BASED ON PERIOD OF RECORD

DISCHARGE, IN CFS, FOR INDICATED RECURRENCE INTERVAL, IN YEARS, AND ANNUAL EXCEEDANCE PROBABILITY, IN PERCENT

\begin{tabular}{ccccccc}
1.25 & 2 & 5 & 10 & 25 & 50 & 100 \\
$80 \%$ & $50 \%$ & $20 \%$ & $10 \%$ & $4 \%$ & $2 \%$ & $1 \%$ \\
\hline & - & - & - & - & - & - \\
\hline & - & - & - &
\end{tabular}

WEIGHTED SKEW =

MAGNITUDE AND PROBABILITY OF ANNUAL LOW FLOW BASED ON PERIOD OF RECORD 1944-52

\begin{tabular}{|c|c|c|c|c|c|c|}
\hline \multirow{2}{*}{$\begin{array}{l}\text { PERIOD } \\
\text { (CON- } \\
\text { SECU- } \\
\text { TIVE } \\
\text { DAYS) }\end{array}$} & \multicolumn{6}{|c|}{$\begin{array}{l}\text { DISCHARGE, IN CFS, FOR INDICATED RECURRENCE } \\
\text { INTERVAL, IN YEARS, AND ANNUAL NON- } \\
\text { EXCEEDANCE PROBABILITY, IN PERCENT }\end{array}$} \\
\hline & $\begin{array}{c}2 \\
50 \%\end{array}$ & $\begin{array}{c}5 \\
20 \%\end{array}$ & $\begin{array}{l}10 \\
10 \%\end{array}$ & $\begin{array}{l}20 \\
5 \%\end{array}$ & $\begin{array}{l}50 \\
2 \%\end{array}$ & $\begin{array}{r}100 \\
1 \%\end{array}$ \\
\hline 1 & 85 & 39 & 27 & 19 & -- & $-\sim$ \\
\hline 3 & 88 & 42 & 29 & 21 & - & - \\
\hline 7 & 92 & 46 & 33 & 25 & -- & -- \\
\hline 14 & 97 & 50 & 36 & 28 & - & -- \\
\hline 30 & 115 & 59 & 43 & 34 & -- & - \\
\hline 60 & 127 & 63 & 45 & 35 & -- & -- \\
\hline 90 & 140 & 70 & 50 & 39 & -- & -- \\
\hline 120 & 188 & 101 & 75 & 59 & -- & -- \\
\hline 183 & 346 & 238 & 199 & 174 & -- & -- \\
\hline
\end{tabular}

MAGNITUDE AND PROBABILITY OF ANNUAL HIGH FLOW BASED ON PERIOD OF RECORD 1943-52

\begin{tabular}{|c|c|c|c|c|c|c|}
\hline \multirow{3}{*}{$\begin{array}{l}\text { PERIOD } \\
\text { (CON- } \\
\text { SECU- } \\
\text { TIVE } \\
\text { DAYS) }\end{array}$} & \multicolumn{6}{|c|}{$\begin{array}{l}\text { DISCHARGE, IN CFS, FOR INDICATED RECURRENCE } \\
\text { INTERVAL, IN YEARS, AND ANNUAL } \\
\text { EXCEEDANCE PROBABILITY, IN PERCENT }\end{array}$} \\
\hline & 2 & 5 & 10 & 25 & 50 & 100 \\
\hline & $50 \%$ & $20 \%$ & $10 \%$ & $4 \%$ & $2 \%$ & $1 \%$ \\
\hline 1 & 1660 & 1990 & 2180 & - & -- & - \\
\hline 3 & 1640 & 1970 & 2160 & - & - & -- \\
\hline 7 & 1590 & 1930 & 2140 & -- & - & - \\
\hline 15 & 1540 & 1910 & 2130 & -- & -- & - \\
\hline 30 & 1500 & 1870 & 2100 & -- & -- & - \\
\hline 60 & 1440 & 1810 & 2050 & -- & -- & -- \\
\hline 90 & 1390 & 1740 & 1970 & - & -- & - \\
\hline
\end{tabular}

DURATION TABLE OF DAILY MEAN FLOW FOR PERIOD OF RECORD 1943-52

OISCHARGE, IN CFS, WHICH WAS EQUALED OR EXCEEOED FOR INDICATED PERCENT OF TIME

\begin{tabular}{|c|c|c|c|c|c|c|c|c|c|c|c|c|c|c|}
\hline $5 \%$ & $10 \%$ & $15 \%$ & $20 \%$ & $25 \%$ & $30 \%$ & $40 \%$ & $50 \%$ & $60 \%$ & $70 \%$ & $75 \%$ & $80 \%$ & $85 \%$ & $90 \%$ & $95 \%$ \\
\hline 1910 & 1540 & 1350 & 1210 & 1110 & 978 & 838 & 679 & 523 & 374 & 314 & 244 & 174 & 70 & 56 \\
\hline
\end{tabular}


14057500 FALL RIVER NEAR LA PINE, OR

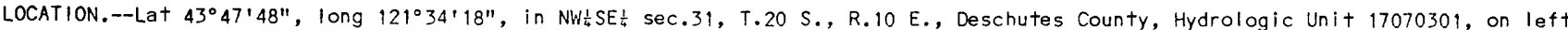
bank $50 \mathrm{ft}$ downstream from pond spillway at State fish hatchery, $9 \mathrm{mi}$ northwest of La Pine, and at $\mathrm{mile} 4.8$.

DRAINAGE AREA.- $-45.1 \mathrm{mi}^{2}$, hydrologic drainage boundary uncertain owing to ground-water exchange.

PERIOD OF RECORD.--July 1938 to September 1982. Records for May to September 1912 at site $3 \mathrm{mi}$ downstream not equivalent owing to difference in drainage area. Prior to Oct. 1, 1964, Dublished as "near Lapine."

GAGE.--Water-stage recorder. Altitude of gage is $4,220 \mathrm{ft}$, by barometer.

REMARKS.--Diversion only to ponds at tish hatchery $50 \mathrm{tt}$ above station, from which water returns to $r i v e r$ above station. Stream is spring fed and momentary extremes are caused by operation of fish hatchery.

AVERAGE DISCHARGE.--44 years, $149 \mathrm{ft}^{3} / \mathrm{s}, 108,000$ acre $-\mathrm{ft} / \mathrm{yr}$.

EXTREMES FOR PERIOD OF RECORD.--Maximum discharge, $254 \mathrm{ft}^{3} / \mathrm{s}$ June 5, 1965, gage height, $2.02 \mathrm{ft}$; minimum, $67 \mathrm{ft} \mathrm{t}^{3} / \mathrm{s}$ some $\mathrm{fime}$ during period Sept. 20-30, 1969.

STATISTICAL SUMMARIES

MONTHLY AND ANNUAL MEAN DISCHARGES 1939-82

\begin{tabular}{|c|c|c|c|c|c|c|}
\hline MONTH & $\begin{array}{l}\text { MINIMUM } \\
\text { (CFS) }\end{array}$ & $\begin{array}{c}\text { MAXIMUM } \\
\text { (CFS) }\end{array}$ & $\begin{array}{l}\text { MEAN } \\
\text { (CFS) }\end{array}$ & $\begin{array}{l}\text { STAN- } \\
\text { DARD } \\
\text { DEVIA- } \\
\text { T!ON } \\
\text { (CFS) }\end{array}$ & $\begin{array}{l}\text { COEFFI- } \\
\text { CIENT OF } \\
\text { VARI- } \\
\text { ATION }\end{array}$ & $\begin{array}{c}\text { PERCENT } \\
\text { OF } \\
\text { ANNUAL } \\
\text { RUNOFF }\end{array}$ \\
\hline OCTOBER & 83 & 207 & 147 & 31 & .21 & 8.2 \\
\hline NOVEMBER & 81 & 199 & 145 & 30 & .21 & 8.1 \\
\hline DECEMBER & 79 & 198 & 143 & 30 & .21 & 8.0 \\
\hline JANUARY & 78 & 191 & 142 & 29 & .20 & 7.9 \\
\hline FEBRUARY & 78 & 187 & 142 & 29 & .21 & 7.9 \\
\hline MARCH & 78 & 193 & 144 & 30 & .21 & 8.0 \\
\hline APRIL & 80 & 218 & 152 & 33 & .21 & 8.5 \\
\hline MAY & 80 & 233 & 159 & 37 & .23 & 8.9 \\
\hline JUNE & 82 & 233 & 157 & 36 & .23 & 8.8 \\
\hline JULY & 87 & 224 & 156 & 34 & .22 & 8.7 \\
\hline AUGUST & 86 & 218 & 153 & 32 & .21 & 8.5 \\
\hline SEPTEMBER & 86 & 208 & 150 & 32 & .22 & 8.4 \\
\hline ANNUAL & 82 & 202 & 149 & 30 & .20 & 100 \\
\hline
\end{tabular}

MAGNITUDE AND PROBABILITY OF INSTANTANEOUS PEAK FLOW BASED ON PERIOD OF RECORD

DISCHARGE, IN CFS, FOR INDICATED RECURRENCE INTERVAL, IN YEARS, AND ANNUAL EXCEEDANCE PROBABILITY, IN PERCENT

\begin{tabular}{ccccccc}
1.25 & 2 & 5 & 10 & 25 & 50 & 100 \\
$80 \%$ & $50 \%$ & $20 \%$ & $10 \%$ & $4 \%$ & $2 \%$ & $1 \%$ \\
\hline- & - & - & - & - & - & -
\end{tabular}

WEIGHTED SKEW $=$
MAGNITUDE AND PROBABILITY OF ANNUAL LOW FLOW BASED ON PERIOD OF RECORD 1940-82

\begin{tabular}{|c|c|c|c|c|c|c|}
\hline $\begin{array}{l}\text { PERIOD } \\
\text { (CON- }\end{array}$ & \multicolumn{6}{|c|}{$\begin{array}{l}\text { DISCHARGE, IN CFS, FOR INDICATED RECURRENCE } \\
\text { INTERVAL, IN YEARS, AND ANNUAL NON- } \\
\text { EXCEEDANCE PROBABILITY, IN PERCENT }\end{array}$} \\
\hline SECU- & & 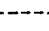 & -- & -- & - & \\
\hline TIVE & 2 & 5 & 10 & 20 & 50 & 100 \\
\hline DAYS) & $50 \%$ & $20 \%$ & 10,5 & $5 q$ & $2 \%$ & $1 \%$ \\
\hline 1 & 132 & 109 & 97 & 89 & 79 & 73 \\
\hline 3 & 32 & 109 & 98 & 89 & 79 & 73 \\
\hline 7 & 133 & 109 & 98 & 89 & 79 & 73 \\
\hline 14 & 134 & 110 & 98 & 89 & 80 & 73 \\
\hline 30 & 136 & 111 & 99 & 90 & 80 & 73 \\
\hline 60 & 138 & 113 & 101 & 91 & 81 & 74 \\
\hline 90 & 139 & 114 & 102 & 92 & 81 & 75 \\
\hline 120 & 140 & 115 & 103 & 93 & 82 & 75 \\
\hline 183 & 142 & 117 & 104 & 94 & 83 & 76 \\
\hline
\end{tabular}

MAGNITUDE AND PROBABILITY OF ANNUAL HIGH FLOW BASED ON PERIOD OF RECORD 1939-82

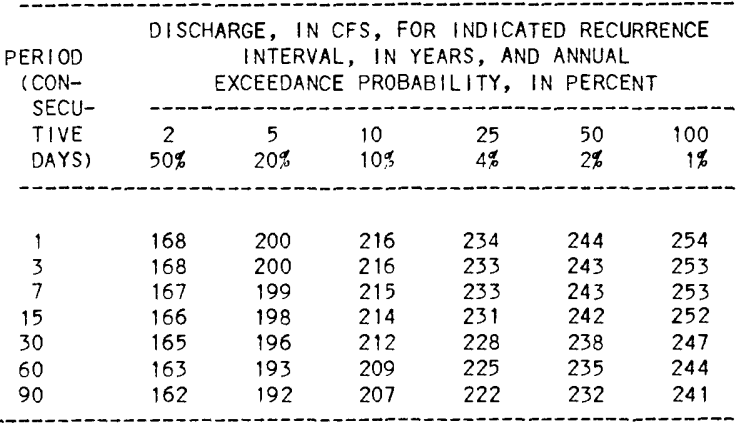

DURATION TABLE OF DAILY MEAN FLOW FOR PERIOD OF RECORD 1939-82

DISCHARGE, IN CFS, WHICH WAS EQUALED OR EXCEEDED FOR INDICATED PERCENT OF TIME

\begin{tabular}{|c|c|c|c|c|c|c|c|c|c|c|c|c|c|c|}
\hline $5 \%$ & $10 \%$ & $15 \%$ & $20 \%$ & $25 \%$ & $30 \%$ & $40 \%$ & $50 \%$ & $60 \%$ & $70 \%$ & $75 \%$ & $80 \%$ & $85 \%$ & $90 \%$ & $95 \%$ \\
\hline 204 & 191 & 184 & 178 & 174 & 169 & 159 & 149 & 141 & 131 & 125 & 119 & 114 & 108 & 99 \\
\hline
\end{tabular}


14060000 CRESCENT CREEK AT CRESCENT LAKE, NEAR CRESCENT, OR

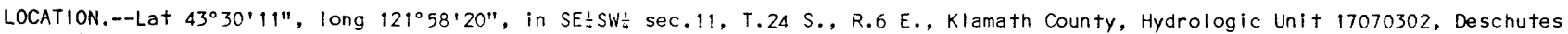
National Forest, on left bank $400 \mathrm{ft}$ downstream from Crescent Lake Dam, $0.5 \mathrm{mi}$ south of town of Crescent Lake, $14 \mathrm{mi}$ west of crescent, and at mile 29.9 .

DRAINAGE AREA.--60.7 $\mathrm{mi}^{2}$, hydrologic drainage boundary uncertain owing to ground-water exchange.

PERIOD OF RECORD.--January to September 1911 (gage heights and discharge measurements only), January 1912 to July 1915 , July to September 1927, May 1928 to September 1982. Published as Crescent Lake outlet near Crescent January 1911 to September 1912, and as Crescent Creek at outlet of Crescent Lake, near Crescent October 1913 to July 1915.

GAGE.--Water-stage recorder and Parshall flume. Datum of gage is 4,819.96 t+ National Geodetic Vertical Datum of 1929. See WSP 1935 for history of changes prior to Sept. 11, 1956.

REMARKS.--FIOW regulated since 1922 by Crescent Lake. No diversion above station.

AVERAGE DISCHARGE. -56 years (water years $1913-14,1929-82$ ), $56.9 \mathrm{ft} 3 / \mathrm{s}, 41,220$ acre- $f+f / y r$.

EXTREMES FOR PERIOD OF RECORD.-Maximum discharge, $313 \mathrm{ft} 3 / \mathrm{s} \mathrm{July} \mathrm{9,} \mathrm{1929,} \mathrm{Aug.} \mathrm{9,} \mathrm{1936;} \mathrm{no} \mathrm{flow} \mathrm{at} \mathrm{times.}$

STATISTICAL SUMMARIES

MONTHLY AND ANNUAL MEAN DISCHARGES 1929-82

\begin{tabular}{|c|c|c|c|c|c|c|}
\hline MONTH & $\begin{array}{l}\text { MINIMUM } \\
\text { (CFS) }\end{array}$ & $\begin{array}{l}\text { MAXIMUM } \\
\text { (CFS) }\end{array}$ & $\begin{array}{l}\text { MEAN } \\
\text { (CFS) }\end{array}$ & $\begin{array}{l}\text { STAN- } \\
\text { DARD } \\
\text { DEVIA- } \\
\text { TION } \\
\text { (CFS) }\end{array}$ & $\begin{array}{l}\text { COEFFI- } \\
\text { CIENT OF } \\
\text { VARI- } \\
\text { ATION }\end{array}$ & $\begin{array}{c}\text { PERCENT } \\
\text { OF } \\
\text { ANNUAL } \\
\text { RUNOFF }\end{array}$ \\
\hline OCTOBER & 0.0 & 233 & 22 & 47 & 2.12 & 3.3 \\
\hline NOVEMBER & 0.0 & 135 & 14 & 28 & 2.06 & 2.0 \\
\hline DECEMBER & 0.0 & 146 & 14 & 32 & 2.30 & 2.1 \\
\hline JANUARY & 0.0 & 148 & 16 & 33 & 2.09 & 2.3 \\
\hline FEBRUARY & 0.0 & 147 & 18 & 34 & 1.85 & 2.7 \\
\hline MARCH & 0.0 & 166 & 23 & 41 & 1.76 & 3.4 \\
\hline APRIL & 0.0 & 148 & 24 & 36 & 1.49 & 3.6 \\
\hline MAY & 0.0 & 173 & 56 & 55 & .99 & 8.3 \\
\hline JUNE & 0.0 & 261 & 104 & 76 & .73 & 15.4 \\
\hline JULY & 14 & 287 & 163 & 63 & .39 & 24.2 \\
\hline AUGUST & 11 & 276 & 151 & 66 & .44 & 22.4 \\
\hline SEPTEMBER & 0.0 & 250 & 69 & 68 & .98 & 10.3 \\
\hline ANNUAL & 8.2 & 148 & 57 & 33 & .57 & 100 \\
\hline
\end{tabular}

MAGNITUDE AND PROBABILITY OF INSTANTANEOUS PEAK FLOW BASED ON PERIOD OF RECORD

DISCHARGE, IN CFS, FOR INDICATED RECURRENCE INTERVAL, IN YEARS, AND ANNUAL EXCEEDANCE PROBABILITY, IN PERCENT

\begin{tabular}{|c|c|c|c|c|c|c|}
\hline $\begin{array}{l}1.25 \\
80 \%\end{array}$ & $\begin{array}{c}2 \\
50 \%\end{array}$ & $\begin{array}{c}5 \\
20 \%\end{array}$ & $\begin{array}{l}10 \\
10 \%\end{array}$ & $\begin{array}{l}25 \\
4 \%\end{array}$ & $\begin{array}{l}50 \\
2 \%\end{array}$ & $\begin{array}{r}100 \\
18\end{array}$ \\
\hline -- & -- & - & -- & -- & -- & -- \\
\hline
\end{tabular}

WEIGHTED SKEW =
MAGNITUDE AND PROBABILITY OF ANNUAL LOW FLOW BASED ON PERIOD OF RECORD 1930-82

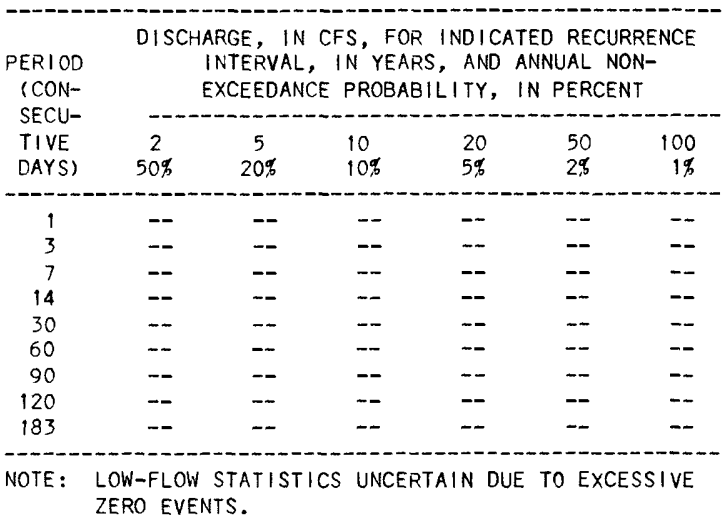

MAGNITUDE AND PROBABILITY OF ANNUAL HIGH FLOW BASED ON PERIOD OF RECORD 1929-82

\begin{tabular}{|c|c|c|c|c|c|c|}
\hline $\begin{array}{l}\text { PERIOD } \\
\text { (CON- }\end{array}$ & \multicolumn{6}{|c|}{$\begin{array}{c}\text { DISCHARGE, IN CFS, FOR INDICATED RECURRENCE } \\
\text { INTERVAL, IN YEARS, AND ANNUAL } \\
\text { EXCEEDANCE PROBABILITY, IN PERCENT }\end{array}$} \\
\hline SECU- & & & & - & -1 & \\
\hline $\begin{array}{l}\text { TIVE } \\
\text { DAYS) }\end{array}$ & $\begin{array}{c}2 \\
50 \%\end{array}$ & $\begin{array}{c}5 \\
20 \%\end{array}$ & $\begin{array}{l}10 \\
10 \%\end{array}$ & $\begin{array}{l}25 \\
4 \%\end{array}$ & $\begin{array}{l}50 \\
2,8\end{array}$ & $\begin{array}{r}100 \\
1 \%\end{array}$ \\
\hline 1 & 231 & 270 & 281 & 288 & 290 & 291 \\
\hline 3 & 229 & 267 & 278 & 285 & 287 & 288 \\
\hline 7 & 224 & 262 & 273 & 280 & 282 & 284 \\
\hline 15 & 214 & 255 & 269 & 278 & 282 & 284 \\
\hline 30 & 203 & 246 & 262 & 272 & 277 & 280 \\
\hline 60 & 179 & 227 & 245 & 259 & 265 & 269 \\
\hline 90 & 153 & 206 & 229 & 250 & 260 & 268 \\
\hline
\end{tabular}

DURATION TABLE OF DAILY MEAN FLOW FOR PERIOD OF RECORD 1929-82

\begin{tabular}{|c|c|c|c|c|c|c|c|c|c|c|c|c|c|c|}
\hline $5 \%$ & $10 \%$ & $15 \%$ & $20 \%$ & $25 \%$ & $30 \%$ & $40 \%$ & $50 \%$ & $60 \%$ & $70 \%$ & $75 \%$ & $80 \%$ & $85 \%$ & $90 \%$ & $95 \%$ \\
\hline 226 & 187 & 156 & 134 & 102 & 79 & 30 & 8.2 & 4.9 & 2.6 & 1.2 & .2 & .1 & .1 & .1 \\
\hline
\end{tabular}


14061000 BIG MARSH CREEK AT HOEY RANCH, NEAR CRESCENT, OR

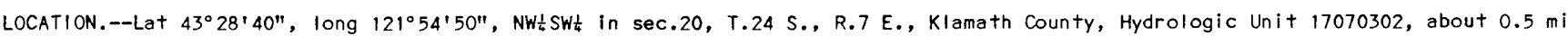

upstream from mouth, $2 \mathrm{mi}$ east of Crescent Lake, and $11 \mathrm{mi}$ west of Crescent.

DRAINAGE AREA.--51.5 $\mathrm{mi}^{2}$, hydrologic drainage boundary uncertain owing to ground-water exchange.

PERIOD OF RECORD.--April 1912 to December 1914, May 1928 to September 1930, May 1931 to September 1958.

GAGE.--Water-stage recorder. Altitude of gage is about 4,630 $\mathrm{ft}$ (from topographic map). Prior to sept. 8 , 1939, staff gages or water-stage recorder at several sites within $0.2 \mathrm{mi}$ of present site at various datums.

REMARKS.--No diversion or regulation above station.

COOPERATION.--Records for 1924, 1928-50, furnished by Oregon Water Resources Department.

AVERAGE DISCHARGE. --21 years (water years $1913,1930,1932-50$ ), $64.8 \mathrm{ft}^{3} / \mathrm{s}$.

EXTREMES FOR PERIOD OF RECORD.--Maximum discharge, $581 \mathrm{ft} / \mathrm{s} \mathrm{Apr.} \mathrm{20,} \mathrm{1943,} \mathrm{gage} \mathrm{height,} 3.79 \mathrm{ft}$; maximum gage height, $4.56 \mathrm{ft}$ Dec. 28, 30, 31, 1945 (backwater from ice); no flow Mar. 27, 1935, result of unusual regulation, but cause unknown.

STATISTICAL SUMMARIES

MONTHLY AND ANNUAL MEAN DISCHARGES 1913-58

\begin{tabular}{|c|c|c|c|c|c|c|}
\hline MONTH & $\begin{array}{l}\text { MINIMUM } \\
\text { (CFS) }\end{array}$ & $\begin{array}{c}\text { MAXIMUM } \\
(C F S)\end{array}$ & $\begin{array}{l}\text { MEAN } \\
\text { (CFS) }\end{array}$ & $\begin{array}{l}\text { STAN- } \\
\text { DARD } \\
\text { DEVIA- } \\
\text { TION } \\
\text { (CFS) }\end{array}$ & $\begin{array}{l}\text { COEFFI- } \\
\text { CIENT OF } \\
\text { VARI- } \\
\text { ATION }\end{array}$ & $\begin{array}{c}\text { PERCENT } \\
\text { OF } \\
\text { ANNUAL } \\
\text { RUNOFF }\end{array}$ \\
\hline OCTOBER & 11 & 68 & 26 & 13 & .49 & 3.0 \\
\hline $\begin{array}{l}\text { NOVEMBER } \\
\text { DECEMBER }\end{array}$ & $\begin{array}{l}10 \\
12\end{array}$ & $\begin{array}{l}133 \\
243\end{array}$ & $\begin{array}{l}43 \\
65\end{array}$ & $\begin{array}{l}28 \\
55\end{array}$ & $\begin{array}{l}.64 \\
.85\end{array}$ & $\begin{array}{l}5.1 \\
7.6\end{array}$ \\
\hline JANUARY & 4.7 & 180 & 52 & 41 & .79 & 6.1 \\
\hline FEBRUARY & 10 & 162 & 53 & 39 & .73 & 6.3 \\
\hline MARCH & 26 & 280 & 72 & 47 & .65 & 8.5 \\
\hline APRIL & 41 & 336 & 142 & 62 & .44 & 16.7 \\
\hline MAY & 56 & 329 & 180 & 81 & .45 & 21.2 \\
\hline JUNE & 31 & 249 & 119 & 63 & .53 & 14.0 \\
\hline JULY & 15 & 94 & 49 & 25 & .50 & 5.8 \\
\hline AUGUST & 10 & 47 & 27 & 12 & .43 & 3.2 \\
\hline SEPTEMBER & 8.5 & 34 & 21 & 7.9 & .37 & 2.5 \\
\hline NNUAL & 31 & 133 & 72 & 27 & .37 & 100 \\
\hline
\end{tabular}

MAGNITUDE AND PROBABILITY OF INSTANTANEOUS PEAK FLOW BASED ON PERIOD OF RECORD 1913-58

\begin{tabular}{ccccccc}
\hline $\begin{array}{c}\text { DISCHARGE, IN CFS, FOR INDICATED RECURRENCE INTERVAL, IN } \\
\text { YEARS, AND ANNUAL EXCEEDANCE PROBABILITY, IN PERCENT }\end{array}$ \\
\hline 1.25 & 2 & 5 & 10 & 25 & 50 & 100 \\
$80 \%$ & $50 \%$ & $20 \%$ & $10 \%$ & $4 \%$ & $2 \%$ & $1 \%$ \\
\hline 193 & 276 & 397 & 482 & 594 & 680 & - \\
\hline
\end{tabular}

WEIGHTEO SKEW $=.060$

MAGNITUDE AND PROBABILITY OF ANNUAL LOW FLOW BASED ON PERIOD OF RECORD 1930-58

\begin{tabular}{|c|c|c|c|c|c|c|}
\hline \multirow{2}{*}{$\begin{array}{l}\text { PERIOD } \\
\text { (CON- } \\
\text { SECU- } \\
\text { TIVE } \\
\text { DAYS) }\end{array}$} & \multicolumn{6}{|c|}{$\begin{array}{l}\text { DISCHARGE, IN CFS, FOR INDICATED RECURRENCE } \\
\text { INTERVAL, IN YEARS, AND ANNUAL NON- } \\
\text { EXCEEDANCE PROBABILITY, IN PERCENT }\end{array}$} \\
\hline & $\begin{array}{c}2 \\
50 \%\end{array}$ & $\begin{array}{c}5 \\
20 \%\end{array}$ & $\begin{array}{l}10 \\
10 \%\end{array}$ & $\begin{array}{l}20 \\
5 \%\end{array}$ & $\begin{array}{l}50 \\
2 \%\end{array}$ & $\begin{array}{r}100 \\
1 \%\end{array}$ \\
\hline 1 & 15 & 9.0 & 6.2 & 4.3 & 2.7 & - \\
\hline 3 & 15 & 9.9 & 7.5 & 5.8 & 4.3 & -- \\
\hline 7 & 16 & 11 & 8.2 & 6.4 & 4.7 & -- \\
\hline 14 & 17 & 11 & 8.5 & 6.6 & 4.8 & - \\
\hline 30 & 19 & 12 & 9.2 & 7.1 & 5.1 & -- \\
\hline 60 & 20 & 13 & 11 & 9.0 & 7.1 & -- \\
\hline 90 & 21 & 15 & 12 & 10 & 8.2 & -- \\
\hline 120 & 24 & 16 & 13 & 11 & 9.5 & -- \\
\hline 183 & 30 & 21 & 18 & 15 & 13 & - \\
\hline
\end{tabular}

MAGNITUDE AND PROBABILITY OF ANNUAL HIGH FLOW BASED ON PERIOD OF RECORD 1913-58

\begin{tabular}{|c|c|c|c|c|c|c|}
\hline $\begin{array}{l}\text { PERIOD } \\
\text { (CON- }\end{array}$ & \multicolumn{6}{|c|}{$\begin{array}{c}\text { DISCHARGE, IN CFS, FOR INDICATEO RECURRENCE } \\
\text { INTERVAL, IN YEARS, AND ANNUAL } \\
\text { EXCEEDANCE PROBABILITY, IN PERCENT }\end{array}$} \\
\hline SECU- & & & $-\cdots$ & & 步 & \\
\hline TIVE & 2 & 5 & 10 & 25 & 50 & 100 \\
\hline DAYS) & $50 \%$ & $20 \%$ & $10 \%$ & $4 \%$ & $2 \%$ & $1 \%$ \\
\hline 1 & 267 & 371 & 437 & 517 & 573 & -- \\
\hline 3 & 260 & 363 & 426 & 502 & 555 & -- \\
\hline 7 & 245 & 340 & 395 & 459 & 502 & -- \\
\hline 15 & 222 & 311 & 364 & 425 & 466 & - \\
\hline 30 & 204 & 287 & 335 & 388 & 423 & -- \\
\hline 60 & 179 & 249 & 288 & 331 & 357 & - \\
\hline 90 & 152 & 208 & 240 & 274 & 296 & -- \\
\hline
\end{tabular}

DURATION TABLE OF DAILY MEAN FLOW FOR PERIOD OF RECORD 1913-58

DISCHARGE, IN CFS, WHICH WAS EQUALED OR EXCEEDED FOR INDICATED PERCENT OF TIME

\begin{tabular}{|c|c|c|c|c|c|c|c|c|c|c|c|c|c|c|}
\hline $5 \%$ & $10 \%$ & $15 \%$ & $20 \%$ & $25 \%$ & $30 \%$ & $40 \%$ & $50 \%$ & $60 \%$ & $70 \%$ & $75 \%$ & $80 \%$ & $85 \%$ & $90 \%$ & $95 \%$ \\
\hline 240 & 180 & 140 & 115 & 94 & 77 & 56 & 43 & 34 & 28 & 25 & 21 & 19 & 16 & 13 \\
\hline
\end{tabular}


14063000 LITTLE DESCHUTES RIVER NEAR LA PINE, OR

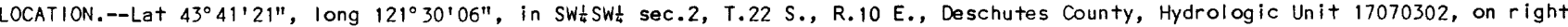
bank $10 \mathrm{ft}$ downstream from highway bridge, $1.1 \mathrm{mi}$ north of La Pine, and at mile 26.8 .

DRAINAGE AREA.--859 $\mathrm{mi}^{2}$, hydrologic drainage boundary uncertain owing to ground-water exchange.

PERIOD OF RECORD.--September 1910 to January 1911, March, April, August 1911, March to September 1912, June to October 1913, June to November 1918, August to October 1920, May 1924 to September 1982. Monthly discharge only for some periods, published in WSP 1318. Published as "Deschutes River near Lapine" 1910-12, as "East Fork Deschutes River near Lapine" 1913-20, and as "Lit+le Deschutes River near Lapine" 1924-64.

GAGE.--Water-stage recorder. Datum of gage is 4,192.81 ft National Geodetic Vertical Datum of 1929. Sept. 1, 1910, to Aug. 31, 1911, nonrecording gage at present site at different datum. Mar. 1 to Sept. 30, 1912, nonrecording gage at site 1.2 mi downstream at different datum. June 1, 1913, to Sept. 28, 1928, nonrecording gage and Sept. 29, 1928, to Sept. 30, 1931, water-stage recorder a present site at different datums.

REMARKS.--Flow regulated since 1922 by Crescent Lake. Many diversions for irrigation above station.

AVERAGE DISCHARGE. -58 years (water years $1925-82$ ), $205 \mathrm{ft} / \mathrm{s}, 148,500 \mathrm{acre}-\mathrm{ft} / \mathrm{yr}$.

EXTREMES FOR PERIOD OF RECORD.--Maximum discharge, 3,660 ft3/s Dec. 25, 1964, gage height, $8.18 \mathrm{ft}$; $\mathrm{minimum,} 8 \mathrm{ft}{ }^{3} / \mathrm{s} \mathrm{Sept.} 2$, 3, 1931.

STATISTICAL SUMMARIES

MONTHLY AND ANNUAL MEAN DISCHARGES 1925-82

\begin{tabular}{|c|c|c|c|c|c|c|}
\hline MONTH & $\begin{array}{l}\text { MINIMUM } \\
\text { (CFS) }\end{array}$ & $\begin{array}{l}\text { MAXIMUM } \\
\text { (CFS) }\end{array}$ & $\begin{array}{l}\text { MEAN } \\
\text { (CFS) }\end{array}$ & $\begin{array}{l}\text { STAN- } \\
\text { DARD } \\
\text { DEVIA- } \\
\text { TION } \\
\text { (CFS) }\end{array}$ & $\begin{array}{l}\text { COEFFI- } \\
\text { CIENT OF } \\
\text { VARI- } \\
\text { ATION }\end{array}$ & $\begin{array}{c}\text { PERCENT } \\
\text { OF } \\
\text { ANNUAL } \\
\text { RUNOFF }\end{array}$ \\
\hline OCTOBER & 17 & 350 & 83 & 61 & .73 & 3.4 \\
\hline NOVEMBER & 27 & 356 & 114 & 62 & .54 & 4.6 \\
\hline DECEMBER & 32 & 712 & 159 & 127 & .80 & 6.5 \\
\hline JANUARY & 35 & 656 & 154 & 108 & .70 & 6.3 \\
\hline FEBRUARY & 30 & 562 & 183 & 112 & .61 & 7.5 \\
\hline MARCH & 60 & 709 & 195 & 112 & .57 & 8.0 \\
\hline APRIL & 68 & 716 & 291 & 149 & .51 & 11.9 \\
\hline MAY & 83 & 990 & 389 & 201 & .52 & 15.9 \\
\hline JUNE & 63 & 788 & 329 & 172 & .52 & 13.4 \\
\hline JULY & 93 & 470 & 241 & 80 & .33 & 9.8 \\
\hline AUGUST & 30 & 342 & 197 & 72 & .36 & 8.0 \\
\hline SEPTEMBER & 12 & 320 & 118 & 75 & .64 & 4.8 \\
\hline ANNUAL & 66 & 374 & 205 & 83 & .40 & 100 \\
\hline
\end{tabular}

MAGNITUDE AND PROBABILITY OF INSTANTANEOUS PEAK FLOW BASED ON PERIOD OF RECORD 1925-82

DISCHARGE, IN CFS, FOR INDICATED RECURRENCE INTERVAL, IN YEARS, AND ANNUAL EXCEEDANCE PROBABILITY, IN PERCENT

\begin{tabular}{ccccccc}
1.25 & 2 & 5 & 10 & 25 & 50 & 100 \\
$80 \%$ & $50 \%$ & $20 \%$ & $10 \%$ & $4 \%$ & $2 \%$ & $1 \%$ \\
\hline 372 & 558 & 883 & 1150 & 1540 & 1880 & 2260
\end{tabular}

WEIGHTED SKEW $=.422$

MAGNITUDE AND PROBABILITY OF ANNUAL LOW FLOW BASED ON PERIOD OF RECORD 1926-82

\begin{tabular}{|c|c|c|c|c|c|c|}
\hline $\begin{array}{c}\text { PERIOD } \\
\text { (CON- } \\
\text { SECU- }\end{array}$ & \multicolumn{6}{|c|}{$\begin{array}{l}\text { DISCHARGE, IN CFS, FOR INDICATED RECURRENCE } \\
\text { INTERVAL, IN YEARS, AND ANNUAL NON- } \\
\text { EXCEEDANCE PROBABILITY, IN PERCENT }\end{array}$} \\
\hline $\begin{array}{l}\text { TIVE } \\
\text { DAYS) }\end{array}$ & $\begin{array}{c}2 \\
50 \%\end{array}$ & $\begin{array}{c}5 \\
20 \%\end{array}$ & $\begin{array}{l}10 \\
10 \%\end{array}$ & $\begin{array}{l}20 \\
5 \%\end{array}$ & $\begin{array}{l}50 \\
2 \%\end{array}$ & $\begin{array}{r}100 \\
1 \%\end{array}$ \\
\hline 1 & 47 & 29 & 22 & 17 & 13 & 11 \\
\hline 3 & 48 & 30 & 23 & 18 & 13 & 11 \\
\hline 7 & 50 & 31 & 23 & 18 & 14 & 11 \\
\hline 14 & 53 & 33 & 25 & 20 & 15 & 12 \\
\hline 30 & 60 & 37 & 28 & 21 & 16 & 12 \\
\hline 60 & 68 & 42 & 31 & 24 & 18 & 14 \\
\hline 90 & 79 & 50 & 39 & 31 & 24 & 20 \\
\hline 120 & 90 & 58 & 45 & 36 & 28 & 23 \\
\hline 183 & 110 & 72 & 57 & 46 & 36 & 31 \\
\hline
\end{tabular}

MAGNITUDE AND PROBABILITY OF ANNUAL HIGH FLOW BASED ON PERIOD OF RECORD 1925-82

\begin{tabular}{|c|c|c|c|c|c|c|}
\hline $\begin{array}{l}\text { PERIOD } \\
\text { (CON- } \\
\text { SECU- }\end{array}$ & \multicolumn{6}{|c|}{$\begin{array}{c}\text { DISCHARGE, IN CFS, FOR INDICATED RECURRENCE } \\
\text { INTERVAL, IN YEARS, AND ANNUAL } \\
\text { EXCEEDANCE PROBABILITY, IN PERCENT }\end{array}$} \\
\hline TIVE & 2 & 5 & 10 & 25 & 50 & 100 \\
\hline DAYSI & $50 \%$ & $20 \%$ & $10 \%$ & $4 \%$ & $2 \%$ & $1 \%$ \\
\hline 1 & 538 & 838 & 1080 & 1450 & 1760 & 2120 \\
\hline 3 & 527 & 808 & 1030 & 1350 & 1620 & 1920 \\
\hline 7 & 501 & 757 & 946 & 1210 & 1420 & 1650 \\
\hline 15 & 460 & 686 & 848 & 1070 & 1240 & 1410 \\
\hline 30 & 418 & 621 & 763 & 949 & 1090 & 1240 \\
\hline 60 & 365 & 540 & 661 & 820 & 942 & 1070 \\
\hline 90 & 329 & 478 & 580 & 710 & 809 & 908 \\
\hline
\end{tabular}

DURATION TABLE OF DAILY MEAN FLOW FOR PERIOD OF RECORD 1925-82

DISCHARGE, IN CFS, WHICH WAS EQUALED OR EXCEEDED FOR INDICATED PERCENT OF TIME

\begin{tabular}{|c|c|c|c|c|c|c|c|c|c|c|c|c|c|c|}
\hline $5 \%$ & $10 \%$ & $15 \%$ & $20 \%$ & $25 \%$ & $30 \%$ & $40 \%$ & $50 \%$ & 608 & $70 \%$ & $75 \%$ & $80 \%$ & $85 \%$ & $90 \%$ & $95 \%$ \\
\hline 546 & 406 & 337 & 298 & 258 & 241 & 199 & 166 & 132 & 103 & 91 & 79 & 68 & 56 & 41 \\
\hline
\end{tabular}




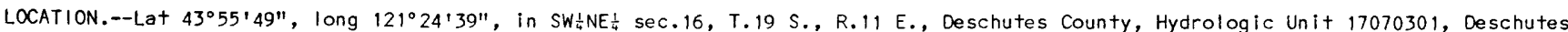
National Forest, on right bank $0.5 \mathrm{mi}$ upstream from Benham Falls, $10 \mathrm{mi}$ southwest of Bend, and at $\mathrm{mile} 181.4$.

DRAINAGE AREA. $--1,759 \mathrm{mi}^{2}$.

PERIOD OF RECORD.--April 1906 to September 1913, Adril to September 1914, August to December 1920, April to September 1921, February 1924 to September 1982. Monthly discharge only for some periods, pub! ished in WSP 1318. Published as "at West's ranch, near Lava" April 1906 to February 1909, April to Septomber 1914 . Recoras for January 1905 to llarch 1906 and October 1913 to September 1914, published under present name in WSP 370 and 394, have been found to be unreliable and should not be used.

GAGE.--Water-stage recorder. Datum of gage is 4,142.10 $\mathrm{ft}$ National Geodetic Vertical Datum of 1929 (Bureau of Reclamation bench mark). See WSP 1738 for history of changes prior to Nov. 20, 1958.

REMARKS.--Flow regulated by Crane Prairie Reservoir, Crescent Lake, and Wickiup Reservoir. Many diversions for irrigation above station.

AVERAGE DISCHARGE.--65 years (water years $1907-13,1925-82$ ) , 1,408 $\mathrm{ft}+3 / \mathrm{s}, 1,020,000 \mathrm{acre}-\mathrm{ft} / \mathrm{yr}$.

EXTREMES FOR PERIOD OF RECORD.--Maximum discharge, 5,000 $\mathrm{ft}^{3} / \mathrm{s}$, estimated, Nov. 27 , 1909 (gage height not determined); minimum, 363 $f t^{3} / \mathrm{s}$ Jan. 20,1962 .

STATISTICAL SUMMARIES (BEFORE THE CONSTRUCTION OF WICKIUP RESERVOIR)

MONTHLY AND ANNUAL MEAN DISCHARGES 1925-41

\begin{tabular}{|c|c|c|c|c|c|c|}
\hline MONTH & $\begin{array}{l}\text { MINIMUM } \\
\text { (CFS) }\end{array}$ & $\begin{array}{l}\text { MAXIMUM } \\
\text { (CFS) }\end{array}$ & $\begin{array}{l}\text { MEAN } \\
\text { (CFS) }\end{array}$ & $\begin{array}{l}\text { STAN- } \\
\text { DARD } \\
\text { DEVIA- } \\
\text { TION } \\
\text { (CFS) }\end{array}$ & $\begin{array}{l}\text { COEFFI- } \\
\text { CIENT OF } \\
\text { VARI- } \\
\text { ATION }\end{array}$ & $\begin{array}{c}\text { PERCENT } \\
\text { OF } \\
\text { ANNUAL } \\
\text { RUNOFF }\end{array}$ \\
\hline OCTOBER & 829 & 1396 & 1102 & 173 & .16 & 7.7 \\
\hline $\begin{array}{l}\text { NOVEMBER } \\
\text { DECEMBER }\end{array}$ & $\begin{array}{l}850 \\
844\end{array}$ & $\begin{array}{l}1454 \\
1519\end{array}$ & $\begin{array}{l}1075 \\
1059\end{array}$ & $\begin{array}{l}190 \\
183\end{array}$ & $\begin{array}{l}.18 \\
.17\end{array}$ & $\begin{array}{l}7.5 \\
7.4\end{array}$ \\
\hline JANUARY & 797 & 1423 & 1035 & 166 & .16 & 7.3 \\
\hline FEBRUARY & 800 & 1451 & 1067 & 189 & .18 & 7.5 \\
\hline MARCH & 864 & 1450 & 1111 & 167 & .15 & 7.8 \\
\hline APRIL & 837 & 1512 & 1197 & 183 & .15 & 8.4 \\
\hline MAY & 940 & 1763 & 1335 & 235 & .18 & 9.4 \\
\hline JUNE & 1037 & 1615 & 1373 & 178 & .13 & 9.6 \\
\hline JULY & 1029 & 1647 & 1398 & 181 & .13 & 9.8 \\
\hline AUGUST & 930 & 1671 & 1344 & 244 & .18 & 9.4 \\
\hline SEPTEMBER & 850 & 1523 & 1172 & 202 & .17 & 8.2 \\
\hline ANNUAL & 921 & 1458 & 1190 & 151 & .13 & 100 \\
\hline
\end{tabular}

MAGNITUDE AND PROBABILITY OF INSTANTANEOUS PEAK FLOW BASED ON PERIOD OF RECORD

DISCHARGE, IN CFS, FOR INDICATED RECURRENCE INTERVAL, IN YEARS, AND ANNUAL EXCEEDANCE PROBABILITY, IN PERCENT

\begin{tabular}{|c|c|c|c|c|c|c|}
\hline $\begin{array}{l}1.25 \\
80 \%\end{array}$ & $\stackrel{2}{50 \%}$ & $\begin{array}{c}5 \\
20 \%\end{array}$ & $\begin{array}{l}10 \\
10 \%\end{array}$ & $\begin{array}{l}25 \\
45\end{array}$ & $\begin{array}{l}50 \\
2 \%\end{array}$ & $\begin{array}{r}100 \\
1 \%\end{array}$ \\
\hline - & - & -- & - & -- & -- & -- \\
\hline
\end{tabular}

WEIGHTED SKEW =
MAGNITUDE AND PROBABILITY OF ANNUAL LOW FLOW BASED ON PERIOD OF RECORD 1926-41

\begin{tabular}{|c|c|c|c|c|c|c|}
\hline $\begin{array}{l}\text { PERIOD } \\
\text { (CON- }\end{array}$ & \multicolumn{6}{|c|}{$\begin{array}{l}\text { DISCHARGE, IN CFS, FOR INDICATED RECURRENCE } \\
\text { INTERVAL, IN YEARS, AND ANNUAL NON- } \\
\text { EXCEEDANCE PROBABILITY, IN PERCENT }\end{array}$} \\
\hline SECU- & & -- & --- & -- & $\cdots$ & $\ldots$ \\
\hline TIVE & 2 & 5 & 10 & 20 & 50 & 100 \\
\hline DAYS) & $50 \%$ & $20 \%$ & $10 \%$ & $5 \%$ & $2 \%$ & $1 \%$ \\
\hline 1 & 905 & 801 & 752 & 713 & -- & -- \\
\hline 3 & 922 & 815 & 768 & 732 & -- & -- \\
\hline 7 & 929 & 833 & 794 & 766 & -- & -- \\
\hline 14 & 940 & 849 & 813 & 787 & -- & -- \\
\hline 30 & 952 & 859 & 822 & 796 & - & -- \\
\hline 60 & 971 & 870 & 830 & 801 & -- & -- \\
\hline 90 & 984 & 882 & 840 & 811 & -- & -- \\
\hline 120 & 996 & 889 & 846 & 816 & $-\infty$ & -- \\
\hline 183 & 1040 & 923 & 871 & 832 & -- & -- \\
\hline
\end{tabular}

MAGNITUDE AND PROBABILITY OF ANNUAL HIGH FLOW BASED ON PERIOD OF RECORD 1925-41

\begin{tabular}{|c|c|c|c|c|c|c|}
\hline $\begin{array}{r}\text { PER!OD } \\
\text { (CON- }\end{array}$ & \multicolumn{6}{|c|}{$\begin{array}{c}\text { DISCHARGE, IN CFS, FOR INDICATED RECURRENCE } \\
\text { INTERVAL, IN YEARS, AND ANNUAL } \\
\text { EXCEEDAINCE PROBABILITY, IN PERCENT }\end{array}$} \\
\hline SECU- & & & $1-$ & -1 & -- & \\
\hline TIVE & 2 & 5 & 10 & 25 & 50 & 100 \\
\hline DAYS) & $50 \%$ & $20 \%$ & $10 \%$ & $4 \%$ & $2 \%$ & $1 \%$ \\
\hline 1 & 1560 & 1750 & 1840 & 1930 & -- & -- \\
\hline 3 & 1550 & 1730 & 1820 & 1910 & -- & - \\
\hline 7 & 1540 & 1720 & 1800 & 1880 & -- & -- \\
\hline 15 & 1530 & 1690 & 1770 & 1840 & -- & - \\
\hline 30 & 1500 & 1660 & 1740 & 1800 & -- & -- \\
\hline 60 & 1460 & 1610 & 1680 & 1740 & -- & -- \\
\hline 90 & 1420 & 1570 & 1640 & 1700 & -- & -- \\
\hline
\end{tabular}

DURATION TABLE OF DAILY MEAN FLOW FOR PERIOD OF RECORD 1925-41

\begin{tabular}{|c|c|c|c|c|c|c|c|c|c|c|c|c|c|c|}
\hline $5 \%$ & $10 \%$ & $15 \%$ & $20 \%$ & $25^{q}$ & $30 \%$ & $40 \%$ & $50 \%$ & $60 \%$ & $70 \%$ & $75 \%$ & $80 \%$ & $85 \%$ & $90 \%$ & $95 \%$ \\
\hline 1620 & 1540 & 1470 & 1420 & 1370 & 1320 & 1240 & 1170 & 1110 & 1040 & 1000 & 969 & 929 & 889 & 847 \\
\hline
\end{tabular}


14064500 DESCHUTES RIVER AT BENHAM FALLS, NEAR BEND, OR--Continued

STATISTICAL SUMMARIES (AFTER THE COMPLETION OF WICKIUP RESERVOIR)

MONTHLY AND ANNUAL MEAN DISCHARGES 1944-82

\begin{tabular}{|c|c|c|c|c|c|c|}
\hline MONTH & $\begin{array}{l}\text { MINIMUM } \\
\text { (CFS) }\end{array}$ & $\begin{array}{l}\text { MAXIMUM } \\
\text { (CFS) }\end{array}$ & $\begin{array}{l}\text { MEAN } \\
\text { (CFS) }\end{array}$ & $\begin{array}{l}\text { STAN- } \\
\text { DARD } \\
\text { DEVIA- } \\
\text { TION } \\
\text { (CFS) }\end{array}$ & $\begin{array}{l}\text { COEFFI- } \\
\text { CIENT OF } \\
\text { VARI- } \\
\text { ATION }\end{array}$ & $\begin{array}{c}\text { PERCENT } \\
\text { OF } \\
\text { ANNUAL } \\
\text { RUNOFF }\end{array}$ \\
\hline & & -- & & $=-$ & 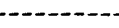 & --0 \\
\hline OCTOBER & 646 & 2089 & 1217 & 317 & .26 & 6.9 \\
\hline NOVEMBER & 487 & 1540 & 784 & 243 & .31 & 4.5 \\
\hline DECEMBER & 520 & 1360 & 824 & 241 & .29 & 4.7 \\
\hline JANUARY & 543 & 1540 & 861 & 287 & .33 & 4.9 \\
\hline FEBRUARY & 514 & 1620 & 938 & 324 & .35 & 5.3 \\
\hline MARCH & 542 & 2068 & 989 & 386 & .39 & 5.6 \\
\hline APRIL & 902 & 2103 & 1343 & 345 & .26 & 7.6 \\
\hline MAY & 1199 & 2521 & 1943 & 325 & .17 & 11.0 \\
\hline JUNE & 1264 & 3017 & 2210 & 363 & .16 & 12.6 \\
\hline JULY & 1489 & 2938 & 2392 & 353 & .15 & 13.6 \\
\hline AUGUST & 1403 & 2795 & 2221 & 326 & .15 & 12.6 \\
\hline SEPTEMBER & 1256 & 2486 & 1878 & 283 & .15 & 10.7 \\
\hline ANNUAL & 1021 & 1977 & 1470 & 239 & .16 & 100 \\
\hline
\end{tabular}

MAGNITUDE AND PROBABILITY OF INSTANTANEOUS PEAK FLOW BASED ON PERIOD OF RECORD

\begin{tabular}{|c|c|c|c|c|c|c|}
\hline \multicolumn{7}{|c|}{$\begin{array}{l}\text { DISCHARGE, IN CFS, FOR INDICATED RECURRENCE INTERVAL, IN } \\
\text { YEARS, AND ANINUAL EXCEEDANCE PROBABILITY, IN PERCENT }\end{array}$} \\
\hline $\begin{array}{l}1.25 \\
80 \%\end{array}$ & $\begin{array}{c}2 \\
50 \%\end{array}$ & $\begin{array}{c}5 \\
20 \%\end{array}$ & $\begin{array}{l}10 \\
10 \%\end{array}$ & $\begin{array}{l}25 \\
4^{\circ}\end{array}$ & $\begin{array}{l}50 \\
2 \%\end{array}$ & $\begin{array}{r}100 \\
19\end{array}$ \\
\hline -- & -- & -- & -- & -- & -- & - \\
\hline
\end{tabular}

WEIGHTED SKEW $=$
MAGNITUDE AND PROBABILITY OF ANNUAL LOW FLOW BASED ON PERIOD OF RECORD 1945-82

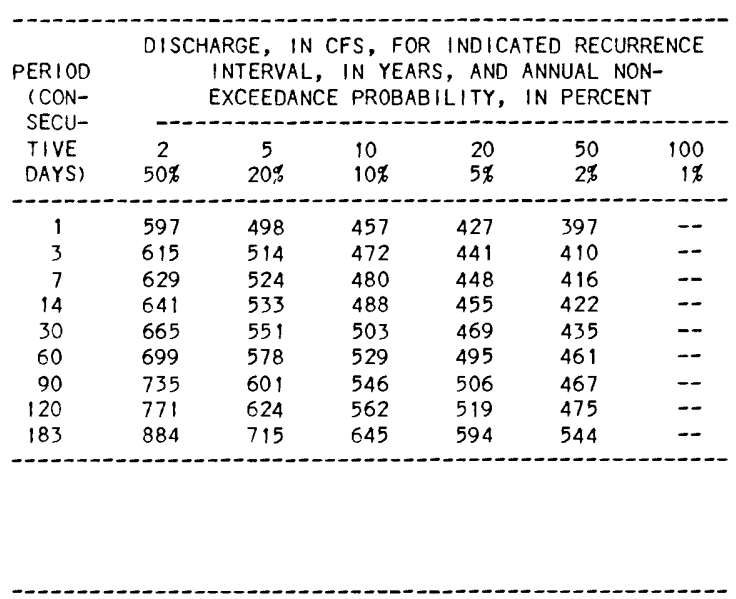

MAGNITUDE AND PROBABILITY OF ANNUAL HIGH FLOW BASED ON PERIOD OF RECORD 1944-82

\begin{tabular}{|c|c|c|c|c|c|c|}
\hline $\begin{array}{l}\text { PERIOD } \\
\text { (CON- } \\
\text { SECU- }\end{array}$ & \multicolumn{6}{|c|}{$\begin{array}{c}\text { DISCHARGE, IN CFS, FOR INDICATED RECURRENCE } \\
\text { INTERVAL, IN YEARS, AND ANNUAL } \\
\text { EXCEEDANCE PROBABILITY, IN PERCENT }\end{array}$} \\
\hline TIVE & 2 & 5 & 10 & 25 & 50 & 100 \\
\hline DAYS) & $50 \%$ & $20 \%$ & $10 \%$ & $4 \%$ & $2 \%$ & 19 \\
\hline \multicolumn{7}{|c|}{ 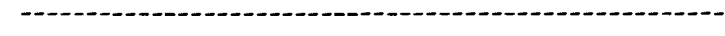 } \\
\hline 1 & 2640 & 2890 & 3000 & 3090 & 3130 & -- \\
\hline 3 & 2630 & 2870 & 2960 & 3030 & 3070 & -- \\
\hline 7 & 2600 & 2820 & 2900 & 2960 & 2990 & -- \\
\hline 15 & 2560 & 2800 & 2880 & 2950 & 2990 & - \\
\hline 30 & 2500 & 2750 & 2850 & 2930 & 2970 & -- \\
\hline 60 & 2420 & 2670 & 2780 & 2870 & 2910 & -- \\
\hline 90 & 2350 & 2600 & 2700 & 2780 & 2830 & -- \\
\hline
\end{tabular}

DURATION TABLE OF DAILY MEAN FLOW FOR PERIOD OF RECORD 1944-82

DISCHARGE, IN CFS, WHICH WAS EQUALED OR EXCEEDED FOR INDICATED PERCENT OF TIME

\begin{tabular}{|c|c|c|c|c|c|c|c|c|c|c|c|c|c|c|}
\hline $5 \%$ & $10 \%$ & $15 \%$ & $20 \%$ & $25 \%$ & $30 \%$ & $40 \%$ & $50 \%$ & $60 \%$ & $70 \%$ & $75 q$ & $80 \%$ & $85 \%$ & $90 \%$ & $95 \%$ \\
\hline 2630 & 2470 & 2330 & 2200 & 2080 & 1950 & 1650 & 1400 & 1160 & 924 & 831 & 726 & 659 & 608 & 563 \\
\hline
\end{tabular}




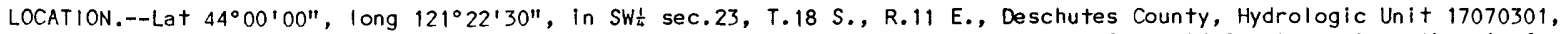
on right bank $0.8 \mathrm{mi}$ downstream from Lava $\mid \mathrm{s}$ land, $1.5 \mathrm{mi}$ downstream from intake of Arnold Canal, $5 \mathrm{mi}$ southwest of Bend, and at mile 173.0.

DRAINAGE AREA.--1,829 $\mathrm{mi}^{2}$.

PERIOD OF RECORD.--March 1925 to September 1965.

GAGE.--Water-stage recorder. Altitude of gage is 3,825 $\mathrm{ft}$ (by barometer). Prior to May 4 , 1927 , at site $0.2 \mathrm{mi}$ upstream at different datum. May 4, 1927, to Nov. 11, 1947 and Nov. 12, 1947, to Oct. 24, 1959, at present site at datums 2.00 and $1.00 \mathrm{ft}$ higher, respectively.

REMARKS.--Flow regulated by Crescent Lake and Crane Prairie Reservoir and, since 1942, by Wickiup Reservoir. Small diversions for irrigation above station. Arnold Canal diverts above station for irrigation near Bend.

AVERAGE DISCHARGE.--40 years, $1,237 \mathrm{ft}^{3} / \mathrm{s}, 895,600$ acre- $\mathrm{ft} / \mathrm{yr}$.

EXTREMES FOR PERIOD OF RECORD.--Maximum discharge, 3,360 $\mathrm{ft}^{3} / \mathrm{s} \mathrm{Dec.} 26$, gage height, $5.14 \mathrm{ft}$; minimum, $390 \mathrm{ft} / \mathrm{s}$ Jan. 20 , 1962.

STATISTICAL SUMMARIES (BEFORE THE CONSTRUCTION OF WICKIUP RESERVOIR)

MONTHLY AND ANNUAL MEAN DISCHARGES 1926-41

\begin{tabular}{|c|c|c|c|c|c|c|}
\hline MONTH & $\begin{array}{c}\text { MINIMUM } \\
\text { (CFS) }\end{array}$ & $\begin{array}{l}\text { MAXIMUM } \\
\text { (CFS) }\end{array}$ & $\begin{array}{l}\text { MEAN } \\
\text { (CFS) }\end{array}$ & $\begin{array}{l}\text { STAN- } \\
\text { DARD } \\
\text { DEVIA- } \\
\text { TION } \\
\text { (CFS) }\end{array}$ & $\begin{array}{l}\text { COEFFI- } \\
\text { CIENT OF } \\
\text { VARI- } \\
\text { ATION }\end{array}$ & $\begin{array}{c}\text { PERCENT } \\
\text { OF } \\
\text { ANNUAL } \\
\text { RUNOFF }\end{array}$ \\
\hline OCTOBER & 734 & 1243 & 959 & 147 & .15 & 7.6 \\
\hline NOVEMBER & 773 & 1256 & 956 & 157 & .16 & 7.6 \\
\hline DECEMBER & 753 & 1368 & 956 & 165 & .17 & 7.6 \\
\hline JANUARY & 709 & 1301 & 926 & 155 & .17 & 7.4 \\
\hline FEBRUARY & 727 & 1238 & 941 & 146 & .16 & 7.5 \\
\hline MARCH & 806 & 1334 & 999 & 150 & .15 & 8.0 \\
\hline APRIL & 739 & 1434 & 1056 & 167 & .16 & 8.4 \\
\hline MAY & 808 & 1594 & 1137 & 210 & .18 & 9.1 \\
\hline JUNE & 909 & 1415 & 1178 & 146 & .12 & 9.4 \\
\hline JULY & 899 & 1452 & 1229 & 154 & .13 & 9.8 \\
\hline AUGUST & 817 & 1516 & 1186 & 212 & .18 & 9.5 \\
\hline SEPTEMBER & 738 & 1379 & 1022 & 171 & .17 & 8.1 \\
\hline ANNUAL & 830 & 1305 & 1050 & 139 & .13 & 100 \\
\hline
\end{tabular}

MAGN ITUDE AND PROBABILITY OF INSTANTANEOUS PEAK FLOW BASED ON PERIOD OF RECORD 1927-41

DISCHARGE, IN CFS, FOR INDICATED RECURRENCE INTERVAL, IN YEARS, AND ANNUAL EXCEEDANCE PROBABILITY, IN PERCENT

\begin{tabular}{ccccccc}
1.25 & 2 & 5 & 10 & 25 & 50 & 100 \\
$80 \%$ & $50 \%$ & $20 \%$ & $10 \%$ & $4 \%$ & $2 \%$ & $1 \%$ \\
\hline 1240 & 1410 & 1590 & 1690 & 1800 & - & -
\end{tabular}

WEIGHTED SKEW $=-.198$
MAGNITUDE AND PROBABILITY OF ANNUAL LOW FLOW BASED ON PERIOD OF RECORD 1927-41

\begin{tabular}{|c|c|c|c|c|c|c|}
\hline $\begin{array}{r}\text { PERIOD } \\
\text { (CON- }\end{array}$ & \multicolumn{6}{|c|}{$\begin{array}{l}\text { DISCHARGE, IN CFS, FOR INDICATED RECURRENCE } \\
\text { INTERVAL, IN YEARS, AND ANNUAL NON- } \\
\text { EXCEEDANCE PROBABILITY, IN PERCENT }\end{array}$} \\
\hline SECU- & & & & & & \\
\hline TIVE & 2 & 5 & 10 & 20 & 50 & 100 \\
\hline DAYS) & $50 \%$ & $20 \%$ & $10 \%$ & $5 \%$ & $2 \%$ & $1 \%$ \\
\hline 1 & 784 & 716 & 689 & 671 & -- & -- \\
\hline 3 & 797 & 729 & 704 & 688 & -- & - \\
\hline 7 & 815 & 748 & 723 & 706 & -- & -- \\
\hline 14 & 828 & 760 & 735 & 718 & -- & -- \\
\hline 30 & 842 & 774 & 749 & 733 & -- & - \\
\hline 60 & 856 & 785 & 760 & 743 & -- & -- \\
\hline 90 & 873 & 796 & 767 & 748 & -- & -- \\
\hline 120 & 888 & 806 & 773 & 751 & - & -- \\
\hline 183 & 923 & 828 & 787 & 756 & -- & -- \\
\hline
\end{tabular}

MAGNITUDE AND PROBABILITY OF ANNUAL HIGH FLOW BASED ON PERIOD OF RECORD 1927-41

\begin{tabular}{|c|c|c|c|c|c|c|}
\hline $\begin{array}{l}\text { PERIOD } \\
\text { (CON- } \\
\text { SECU- }\end{array}$ & \multicolumn{6}{|c|}{$\begin{array}{c}\text { DISCHARGE, IN CFS, FOR INDICATED RECURRENCE } \\
\text { INTERVAL, IN YEARS, AND ANNUAL } \\
\text { EXCEEDANCE PROBABILITY, IN PERCENT }\end{array}$} \\
\hline $\begin{array}{l}\text { TIVE } \\
\text { DAYS) }\end{array}$ & $\begin{array}{c}2 \\
50 \%\end{array}$ & $\begin{array}{c}5 \\
20 \%\end{array}$ & $\begin{array}{l}10 \\
10 \%\end{array}$ & $\begin{array}{l}25 \\
4 \%\end{array}$ & $\begin{array}{l}50 \\
2 \%\end{array}$ & $\begin{array}{r}100 \\
1 \%\end{array}$ \\
\hline 1 & 1410 & 1570 & 1640 & 1710 & -- & - \\
\hline 3 & 1400 & 1550 & 1610 & 1670 & - & -- \\
\hline 7 & 1380 & 1530 & 1600 & 1650 & -- & -- \\
\hline 15 & 1370 & 1510 & 1570 & 1620 & -- & -- \\
\hline 30 & 1340 & 1490 & 1550 & 1600 & -- & -- \\
\hline 60 & 1300 & 1430 & 1490 & 1540 & - & -- \\
\hline 90 & 1260 & 1390 & 1450 & 1500 & -- & -- \\
\hline
\end{tabular}

DURATION TABLE OF DAILY MEAN FLOW FOR PERIOD OF RECORD 1927-41

DISCHARGE, IN CFS, WHICH WAS EQUALED OR EXCEEDED FOR INDICATED PERCENT OF TIME

\begin{tabular}{|c|c|c|c|c|c|c|c|c|c|c|c|c|c|c|}
\hline $5 \%$ & $10 \%$ & $15 \%$ & $20 \%$ & $25 \%$ & $30 \%$ & $40 \%$ & $50 \%$ & $60 \%$ & $70 \%$ & $75 \%$ & $80 \%$ & $85 \%$ & $90 \%$ & $95 \%$ \\
\hline 1440 & 1360 & 1300 & 1260 & 1220 & 1170 & 1100 & 1020 & 953 & 905 & 881 & 856 & 829 & 799 & 767 \\
\hline
\end{tabular}


14066000 DESCHUTES RIVER BELOW LAVA ISLAND, NEAR BEND, OR--Continued

STATISTICAL SUMMARIES (AFTER THE COMPLETION OF WICKIUP RESERVOIR)

MONTHLY AND ANNUAL MEAN DISCHARGES 1943-65

\begin{tabular}{|c|c|c|c|c|c|c|}
\hline MONTH & $\begin{array}{l}\text { MINIMUM } \\
\text { (CFS) }\end{array}$ & $\begin{array}{l}\text { MAXIMUM } \\
\text { (CFS) }\end{array}$ & $\begin{array}{l}\text { MEAN } \\
\text { (CFS) }\end{array}$ & $\begin{array}{l}\text { STAN- } \\
\text { DARD } \\
\text { DEVIA- } \\
\text { TION } \\
\text { (CFS) }\end{array}$ & $\begin{array}{l}\text { COEFF I- } \\
\text { CIENT OF } \\
\text { VARI- } \\
\text { ATION }\end{array}$ & $\begin{array}{c}\text { PERCENT } \\
\text { OF } \\
\text { ANNUAL } \\
\text { RUNOFF }\end{array}$ \\
\hline OCTOBER & 762 & 1838 & 1199 & 292 & .24 & 7.3 \\
\hline NOVEMBER & 554 & 1495 & 783 & 232 & .30 & 4.7 \\
\hline DECEMBER & 539 & 1272 & 806 & 223 & .28 & 4.9 \\
\hline JANUARY & 516 & 1293 & 811 & 236 & .29 & 4.9 \\
\hline FEBRUARY & 544 & 1575 & 916 & 297 & .32 & 5.6 \\
\hline MARCH & 543 & 1820 & 982 & 341 & .35 & 6.0 \\
\hline APRIL & 809 & 1966 & 1302 & 335 & .26 & 7.9 \\
\hline MAY & 975 & 2313 & 1749 & 377 & .22 & 10.6 \\
\hline JUNE & 1063 & 2721 & 1960 & 430 & .22 & 11.9 \\
\hline $\begin{array}{l}\text { JULY } \\
\text { AUGUST }\end{array}$ & $\begin{array}{l}1335 \\
1248\end{array}$ & $\begin{array}{l}2657 \\
2540\end{array}$ & $\begin{array}{l}2172 \\
2062\end{array}$ & $\begin{array}{l}425 \\
373\end{array}$ & .20 & $\begin{array}{l}13.2 \\
12.5\end{array}$ \\
\hline SEPTEMBER & 1114 & 2217 & 1752 & 284 & .16 & 10.6 \\
\hline ANNUAL & 904 & 1789 & 1377 & 232 & .17 & 100 \\
\hline
\end{tabular}

MAGNITUDE AND PROBABILITY OF INSTANTANEOUS PEAK FLOW BASED ON PERIOD OF RECORD

DISCHARGE, IN CFS, FOR INDICATED RECURRENCE INTERVAL, IN YEARS, AND ANNUAL EXCEEDANCE PROBABILITY, IN PERCENT

\begin{tabular}{lllllll}
1.25 & 2 & 5 & 10 & 25 & 50 & 100 \\
$80 \%$ & $50 \%$ & $20 \%$ & $10 \%$ & $4 \%$ & $2 \%$ & $1 \%$ \\
\hline- & - & - & - & - & - & -
\end{tabular}

WEIGHTED SKEW $=$
MAGNITUDE AND PROBABILITY OF ANNUAL LOW FLOW BASED ON PERIOD OF RECORD 1944-65

\begin{tabular}{|c|c|c|c|c|c|c|}
\hline \multirow{2}{*}{$\begin{array}{l}\text { PERIOD } \\
\text { (CON- } \\
\text { SECU- } \\
\text { TIVE } \\
\text { DAYS) }\end{array}$} & \multicolumn{6}{|c|}{$\begin{array}{l}\text { DISCHARGE, IN CFS, FOR INDICATED RECURRENCE } \\
\text { INTERVAL, IN YEARS, AND ANNUAL NON- } \\
\text { EXCEEDANCE PROBABILITY, IN PERCENT }\end{array}$} \\
\hline & $\begin{array}{c}2 \\
50 \%\end{array}$ & $\begin{array}{c}5 \\
20 \%\end{array}$ & $\begin{array}{l}10 \\
10 \%\end{array}$ & $\begin{array}{l}20 \\
5 \%\end{array}$ & $\begin{array}{l}50 \\
2 \%\end{array}$ & $\begin{array}{r}100 \\
1 \%\end{array}$ \\
\hline 1 & 591 & 504 & 465 & 436 & -- & -- \\
\hline 3 & 604 & 518 & 480 & 452 & -- & -- \\
\hline 7 & 621 & 530 & 490 & 460 & -- & -- \\
\hline 14 & 636 & 538 & 496 & 465 & -- & -- \\
\hline 30 & 662 & 555 & 511 & 479 & -- & - \\
\hline 60 & 695 & 579 & 531 & 496 & -- & -- \\
\hline 90 & 729 & 600 & 545 & 504 & -- & -- \\
\hline 120 & 764 & 621 & 559 & 513 & -- & -- \\
\hline 183 & 885 & 721 & 650 & 597 & -- & -- \\
\hline
\end{tabular}

MAGNITUDE AND PROBABILITY OF ANNUAL HIGH FLOW BASED ON PERIOD OF RECORD 1943-65

\begin{tabular}{|c|c|c|c|c|c|c|}
\hline \multirow{4}{*}{$\begin{array}{c}\text { PERIOD } \\
\text { (CON- } \\
\text { SECU- } \\
\text { TIVE } \\
\text { DAYS) }\end{array}$} & \multicolumn{6}{|c|}{$\begin{array}{c}\text { DISCHARGE, IN CFS, FOR INDICATED RECURRENCE } \\
\text { INTERVAL, IN YEARS, AND ANNUAL } \\
\text { EXCEEDANCE' PROBABILITY, IN PERCENT }\end{array}$} \\
\hline & & & & & & \\
\hline & 2 & 5 & 10 & 25 & 50 & 100 \\
\hline & $50 \%$ & $20 \%$ & $10 \%$ & $4 \%$ & $2 \%$ & $1 \%$ \\
\hline 1 & 2430 & 2760 & 2910 & 3040 & -- & -- \\
\hline 3 & 2420 & 2740 & 2880 & 3000 & -- & -- \\
\hline 7 & 2380 & 2660 & 2760 & 2850 & -- & -- \\
\hline 15 & 2340 & 2620 & 2740 & 2830 & -- & -- \\
\hline 30 & 2280 & 2590 & 2710 & 2820 & -- & -- \\
\hline 60 & 2210 & 2520 & 2650 & 2760 & -- & -- \\
\hline 90 & 2150 & 2450 & 2580 & 2690 & - & -- \\
\hline
\end{tabular}

DURATION TABLE OF DAILY MEAN FLOW FOR PERIOD OF RECORD 1943-65

DISCHARGE, IN CFS, WHICH WAS EQUALED OR EXCEEDED FOR INDICATED PERCENT OF TIME

\begin{tabular}{|c|c|c|c|c|c|c|c|c|c|c|c|c|c|c|}
\hline $5 q$ & $10 \%$ & $15 \%$ & $20 \%$ & $25 \%$ & $30 \%$ & $40 \%$ & $50 \%$ & $60 \%$ & $70 \%$ & $75 \%$ & $80 \%$ & $85 \%$ & $90 \%$ & $95 \%$ \\
\hline 2470 & 2320 & 2180 & 2040 & 1890 & 1760 & 1510 & 1290 & 1090 & 902 & 819 & 750 & 679 & 609 & 553 \\
\hline
\end{tabular}




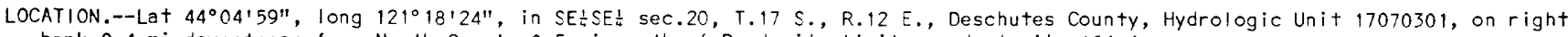
bank $0.4 \mathrm{mi}$ downstream from North Canal, $0.5 \mathrm{mi}$ north of Bend city limits, and at $\mathrm{mile} 164.4$.

DRAINAGE AREA. $--1,899 \mathrm{mi}^{2}$.

PERIOD OF RECORD.--October 1014 to September 1082.

GAGE.--Water-stage recorder. Datum of gage is z, $5,3.95$ ft ilational seodedic vertical 2atum of 1929. Prior to Cct. 1, 1931, water-stage recorder at site $200 \mathrm{ft}$ downstream at datum $1.00 \mathrm{ft}$ higher.

REMARKS.--Flow regulated by powerplant at Bend, Crescent Lake, Crane Prairie Reservoir, and Wickiup Reservoir. Six large canals and several small ditches divert water above station for irrigation.

AVERAGE DISCHARGE. --68 years, $499 \mathrm{ft}+3 / \mathrm{s}, 361,500$ acre- $\mathrm{ft} / \mathrm{yr}$.

EXTREMES FOR PERIOD OF RECORD.--Maximum discharge, 2,820 $\mathrm{ft}+3 / \mathrm{s} \mathrm{Dec.} \mathrm{27,} \mathrm{1964,} \mathrm{gage} \mathrm{height,} 4.90 \mathrm{ft}$; maximum gage height, $5.38 \mathrm{ft}$ Dec. 15, 1932 (backwater from ice); minimum discharge, $1.0 \mathrm{ft} / \mathrm{s}$ Aug. $25,1930$.

EXTREMES OUTSIDE PERIOD OF RECORD.--Maximum discharge near this site since 1905, 4,820 $\mathrm{ft}+3 / \mathrm{s}$ Nov. 27,1909 .

STATISTICAL SUMMARIES

MONTHLY AND ANNUAL MEAN DISCHARGES 1944-82

\begin{tabular}{|c|c|c|c|c|c|c|}
\hline MONTH & $\begin{array}{l}\text { MINIMUM } \\
\text { (CFS) }\end{array}$ & $\begin{array}{c}\text { MAXIMUM } \\
\text { (CFS) }\end{array}$ & $\begin{array}{l}\text { MEAN } \\
\text { (CFS) }\end{array}$ & $\begin{array}{l}\text { STAN- } \\
\text { DARD } \\
\text { DEVIA- } \\
\text { TION } \\
\text { (CFS) }\end{array}$ & $\begin{array}{l}\text { COEFFI- } \\
\text { CIENT OF } \\
\text { VARI- } \\
\text { ATION }\end{array}$ & $\begin{array}{c}\text { PERCENT } \\
\text { OF } \\
\text { ANNUAL } \\
\text { RUNOFF }\end{array}$ \\
\hline OCTOBER & 31 & 855 & 254 & 215 & .85 & 5.4 \\
\hline NOVEMBER & 289 & 1194 & 608 & 231 & .38 & 12.8 \\
\hline DECEMBER & 338 & 1186 & 676 & 218 & .32 & 14.3 \\
\hline JANUARY & 435 & 1369 & 741 & 263 & .35 & 15.7 \\
\hline FEBRUARY & 454 & 1495 & 799 & 308 & .39 & 16.9 \\
\hline MARCH & 270 & 1767 & 780 & 371 & .48 & 16.5 \\
\hline APRIL & 22 & 918 & 308 & 205 & .67 & 6.5 \\
\hline MAY & 22 & 586 & 109 & 115 & 1.06 & 2.3 \\
\hline JUNE & 24 & 799 & 131 & 168 & 1.28 & 2.8 \\
\hline JULY & 24 & 389 & 74 & 68 & .92 & 1.6 \\
\hline AUGUST & 21 & 570 & 109 & 131 & 1.20 & 2.3 \\
\hline SEPTEMBER & 19 & 523 & 143 & 152 & 1.06 & 3.0 \\
\hline ANNUAL & 205 & 718 & 392 & 154 & .39 & 100 \\
\hline
\end{tabular}

MAGNITUDE AND PROBABILITY OF INSTANTANEOUS PEAK FLOW BASED ON PERIOD OF RECORD 1944-82

DISCHARGE, IN CFS, FOR INDICATED RECURRENCE INTERVAL, IN YEARS, AND ANNUAL EXCEEDANCE PROBABILITY, IN PERCENT

\begin{tabular}{ccccccc}
1.25 & 2 & 5 & 10 & 25 & 50 & 100 \\
$80 \%$ & $50 \%$ & $20 \%$ & $10 \%$ & $4 \%$ & $2 \%$ & $1 \%$ \\
\hline 1000 & 1280 & 1670 & 1920 & 2240 & 2480 & -
\end{tabular}

MAGNITUDE AND PROBABILITY OF ANNUAL LOW FLOW BASED ON PERIOD OF RECORD 1945-82

\begin{tabular}{|c|c|c|c|c|c|c|}
\hline $\begin{array}{l}\text { PERIOD } \\
\text { (CON- } \\
\text { SECU- }\end{array}$ & \multicolumn{6}{|c|}{$\begin{array}{l}\text { DISCHARGE, IN CFS, FOR INDICATED RECURREN } \\
\text { INTERVAL, IN YEARS, AND ANNUAL NON- } \\
\text { EXCEEDANCE PROBABILITY, IN PERCENT }\end{array}$} \\
\hline $\begin{array}{l}\text { TIVE } \\
\text { DAYS) }\end{array}$ & $\stackrel{2}{20 \%}$ & $5^{5}$ & 10 & 20 & $\begin{array}{l}50 \\
2^{q}\end{array}$ & 100 \\
\hline- & & & & & & \\
\hline 1 & 21 & 14 & 11 & 9.1 & 7.4 & -- \\
\hline 3 & 25 & 17 & 14 & 12 & 9.9 & -- \\
\hline 7 & 29 & 20 & 16 & 14 & 12 & -- \\
\hline 14 & 33 & 22 & 18 & 15 & 13 & $\ldots$ \\
\hline 30 & 39 & 24 & 19 & 16 & 14 & - \\
\hline 60 & 47 & 28 & 22 & 19 & 16 & - \\
\hline 90 & 54 & 30 & 23 & 19 & 16 & - \\
\hline 120 & 59 & 32 & 24 & 20 & 16 & - \\
\hline 183 & 76 & 39 & 28 & 22 & 17 & -- \\
\hline
\end{tabular}

MAGNITUDE AND PROBABILITY OF ANNUAL. HIGH FLOW BASED ON PERIOD OF RECORD $1944-82$

\begin{tabular}{|c|c|c|c|c|c|c|}
\hline $\begin{array}{l}\text { PERIOD } \\
\text { (CON- } \\
\text { SECU- }\end{array}$ & \multicolumn{6}{|c|}{$\begin{array}{l}\text { DISCHARGE, IN CFS, FOR INDICATED RECURRE } \\
\text { INTERVAL, IN YEARS, AND ANNUAL } \\
\text { EXCEEDANCE PROBAB!LIITY, IN PERCENT }\end{array}$} \\
\hline $\begin{array}{l}\text { TIVE } \\
\text { DAYS) }\end{array}$ & $\stackrel{2}{50 \%}$ & $\begin{array}{c}5 \\
20 \%\end{array}$ & $\begin{array}{l}10 \\
10 \%\end{array}$ & $\begin{array}{l}25 \\
4 \%\end{array}$ & $\begin{array}{l}50 \\
2 \%\end{array}$ & $\begin{array}{r}100 \\
1 \%\end{array}$ \\
\hline 1 & 1180 & 1580 & 1850 & 2190 & 2440 & - \\
\hline 3 & 1140 & 1530 & 1780 & 2100 & 2340 & -- \\
\hline 7 & 1080 & 1450 & 1680 & 1980 & 2210 & - \\
\hline 15 & 988 & 1330 & 1550 & 1830 & 2050 & -- \\
\hline 30 & 876 & 1200 & 1430 & 1720 & 1950 & -- \\
\hline 60 & 796 & 1100 & 1310 & 1590 & 1810 & -- \\
\hline 90 & 750 & 1020 & 1210 & 1470 & 1670 & -- \\
\hline
\end{tabular}

DURATION TABLE OF DAILY MEAN FLOW FOR PERIOD OF RECORD $1944-82$

DISCHARGE, IN CFS, WHICH WAS EQUALED OR EXCEEDED FOR INDICATED PERCENT OF TIME

\begin{tabular}{|c|c|c|c|c|c|c|c|c|c|c|c|c|c|c|}
\hline $5 \%$ & $10 \%$ & $15 \%$ & $20 \%$ & $25 \%$ & $30 \%$ & $40 \%$ & $50 \%$ & $60 \%$ & $70 \%$ & $75 \%$ & $80 \%$ & $85 \%$ & $90 \%$ & $95 \%$ \\
\hline 1190 & 975 & 819 & 707 & 623 & 570 & 453 & 274 & 123 & 63 & 48 & 38 & 32 & 29 & 25 \\
\hline
\end{tabular}


14075000 SOUAW CREEK NEAR SISTERS, OR

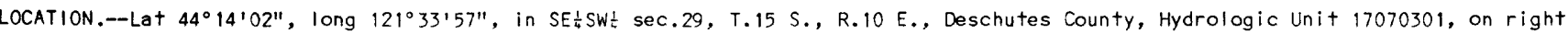
bank $800 \mathrm{ft}$ upstream from intake of McAllister ditch, $4 \mathrm{mi}$ south of Sisters, and at $\mathrm{mile} 26.8$.

DRAINAGE AREA. $--45.2 \mathrm{mi}^{2}$, revised.

PERIOD OF RECORD.--July 1906 to October 1918, June to August 1919, October 1919 to September 1920 , May 1921 to September 1924 (no winter records), April 1925 to September 1082. Monthly discharge on!y for some periods, published in WSP 1318 .

GAGE.--Water-stage recorder. Altitude of gage is 3,490 $\mathrm{ft}$, by barometer. July 1, 1906, to May 29 , 1913, nonrecording gage at site $1,000 \mathrm{ft}$ downstream at different datum, below intake of McAllister ditch (records include flow in McAllister ditch). May 30 , 1913, to Sept. 2, 1915, nonrecording gage and Mar. 24, 1916, to 0ct. 5, 1928, water-stage recorder at site 300 ft downstream at different datum. Oct. 6, 1928, to Nov. 7, 1967, water-stage recorder at site $200 \mathrm{ft}$ downstream at datum $2.64 \mathrm{ft}$ lower.

REMARKS.--No regulation. A canal near mouth of Pole Creek, a tributary above station, diverts entire flow of that creek for irrigation of lands near Sisters.

AVERAGE DISCHARGE.--70 years (water years $1907-18,1920,1926-82$ ), $105 \mathrm{ft} / \mathrm{s}, 76,070 \mathrm{acre}-\mathrm{ft} / \mathrm{yr}$.

EXTREMES FOR PERIOD OF RECORD. - Maximum discharge since $1909,2,000 \mathrm{ft} \mathrm{t}^{3} / \mathrm{s}$ Dec. 25 , 1980 , from rating curve extended above $690 \mathrm{ft}{ }^{3} / \mathrm{s}$ on basis of slope-area measurement of peak flow; a maximum gage height of 9.2 ft from water-borne ice was observed on Jan. 11 , 1979, and probably occurred on Jan. 10, 1979; previous maximum gage height, about $8.75 \mathrm{ft}$, over top of gage Nov. 22 , 1909, site and datum then in use (discharge not determined); minimum discharge, 14 $\mathrm{ft}+3 / \mathrm{s} \mathrm{Mar.} 2,1966$.

STATISTICAL SUMMARIES

MONTHLY AND ANNUAL MEAN DISCHARGES 1916-82

\begin{tabular}{|c|c|c|c|c|c|c|}
\hline MONTH & $\begin{array}{l}\text { MINIMUM } \\
\text { (CFS) }\end{array}$ & $\begin{array}{l}\text { MAXIMUM } \\
\text { (CFS) }\end{array}$ & $\begin{array}{l}\text { MEAN } \\
\text { (CFS) }\end{array}$ & $\begin{array}{l}\text { STAN- } \\
\text { DARD } \\
\text { DEVIA- } \\
\text { TION } \\
\text { (CFS) }\end{array}$ & $\begin{array}{l}\text { COEFFI- } \\
\text { CIENT OF } \\
\text { VARI- } \\
\text { ATION }\end{array}$ & $\begin{array}{c}\text { PERCENT } \\
\text { OF } \\
\text { ANNUAL } \\
\text { RUNOFF }\end{array}$ \\
\hline OCTOBER & 40 & 132 & 63 & 17 & .27 & 5.0 \\
\hline NOVEMBER & 37 & 187 & 75 & 31 & .41 & 6.0 \\
\hline DECEMBER & 35 & 220 & 85 & 44 & .52 & 6.7 \\
\hline JANUARY & 24 & 180 & 71 & 32 & .45 & 5.7 \\
\hline FEBRUARY & 24 & 200 & 68 & 31 & .46 & 5.4 \\
\hline MARCH & 34 & 158 & 60 & 21 & .35 & 4.7 \\
\hline APRIL & 37 & 133 & 75 & 22 & .29 & 6.0 \\
\hline MAY & 58 & 279 & 143 & 43 & .30 & 11.4 \\
\hline JUNE & 113 & 390 & 226 & 71 & .31 & 18.0 \\
\hline JULY & 70 & 347 & 191 & 64 & .33 & 15.2 \\
\hline AUGUST & 68 & 209 & 118 & 33 & .28 & 9.4 \\
\hline SEPTEMBER & 51 & 135 & 80 & 18 & .22 & 6.4 \\
\hline ANNUAL & 61 & 164 & 104 & 22 & .21 & 100 \\
\hline
\end{tabular}

MAGNITUDE AND PROBABILITY OF INSTANTANEOUS PEAK FLOW BASED ON PERIOD OF RECORD $1916-82$

\begin{tabular}{|c|c|c|c|c|c|c|}
\hline \multicolumn{7}{|c|}{$\begin{array}{l}\text { DISCHARGE, IN CFS, FOR INDICATED RECURRENCE INTERVAL, IN } \\
\text { YEARS, AND ANNUAL EXCEEDANCE PROBABILITY, IN PERCENT }\end{array}$} \\
\hline $\begin{array}{l}1.25 \\
80 \%\end{array}$ & $\begin{array}{c}2 \\
50 \%\end{array}$ & $\stackrel{5}{20 \%}$ & $\begin{array}{l}10 \\
10 \%\end{array}$ & 25 & $\begin{array}{l}50 \\
2 \%\end{array}$ & $\begin{array}{r}100 \\
1 \%\end{array}$ \\
\hline 366 & 557 & 887 & 1150 & 1540 & 1870 & 2250 \\
\hline
\end{tabular}

WEIGHTED SKEW $=.348$

MAGNITUDE AND PROBABILITY OF ANNUAL LOW FLOW BASED ON PERIOD OF RECORD 1917-82

\begin{tabular}{|c|c|c|c|c|c|c|}
\hline $\begin{array}{l}\text { PERIOD } \\
\text { (CON- } \\
\text { SECU- }\end{array}$ & \multicolumn{6}{|c|}{$\begin{array}{l}\text { DISCHARGE, IN CFS, FOR INDICATED RECURRENCE } \\
\text { INTERVAL, IN YEARS, AND ANNUAL NON- } \\
\text { EXCEEDANCE PROBABILITY, IN PERCENT }\end{array}$} \\
\hline $\begin{array}{l}\text { TIVE } \\
\text { DAYSI }\end{array}$ & $\stackrel{2}{50 \%}$ & $\begin{array}{c}5 \\
20 \%\end{array}$ & $\begin{array}{l}10 \\
10 \%\end{array}$ & $\begin{array}{l}20 \\
5 \%\end{array}$ & $\begin{array}{l}50 \\
2 \%\end{array}$ & $\begin{array}{r}100 \\
1 \%\end{array}$ \\
\hline 1 & 38 & 31 & 28 & 26 & 23 & 22 \\
\hline 3 & 39 & 32 & 29 & 27 & 24 & 23 \\
\hline 7 & 40 & 33 & 30 & 28 & 26 & 24 \\
\hline 14 & 42 & 35 & 32 & 29 & 26 & 24 \\
\hline 30 & 46 & 38 & 34 & 31 & 28 & 26 \\
\hline 60 & 51 & 41 & 37 & 33 & 30 & 27 \\
\hline 90 & 55 & 44 & 39 & 36 & 32 & 30 \\
\hline 120 & 60 & 47 & 42 & 38 & 34 & 32 \\
\hline 183 & 66 & 53 & 47 & 43 & 39 & 36 \\
\hline
\end{tabular}

MAGNITUDE AND PROBABILITY OF ANNUAL HIGH FLOW BASED ON PERIOD OF RECORD 1916-82

\begin{tabular}{|c|c|c|c|c|c|c|}
\hline $\begin{array}{l}\text { PERIOD } \\
\text { (CON- } \\
\text { SECU- }\end{array}$ & \multicolumn{6}{|c|}{$\begin{array}{c}\text { DISCHARGE, IN CFS, FOR INDICATED RECURRENCE } \\
\text { INTERVAL, IN YEARS, AND ANNUAL } \\
\text { EXCEEDANCE PROBABILITY, IN PERCENT }\end{array}$} \\
\hline TIVE & 2 & 5 & 10 & 25 & 50 & 100 \\
\hline DAYS) & $50 \%$ & $20 \%$ & $10 \%$ & $4 \%$ & $2 \%$ & $1 \%$ \\
\hline 1 & 411 & 598 & 742 & 946 & 1120 & 1300 \\
\hline 3 & 356 & 490 & 585 & 713 & 814 & 920 \\
\hline 7 & 308 & 404 & 463 & 533 & 583 & 630 \\
\hline 15 & 270 & 350 & 395 & 447 & 481 & 512 \\
\hline 30 & 244 & 311 & 349 & 392 & 421 & 447 \\
\hline 60 & 214 & 272 & 304 & 340 & 366 & 387 \\
\hline 90 & 190 & 238 & 264 & 294 & 313 & 331 \\
\hline
\end{tabular}

DURATION TABLE OF DAILY MEAN FLOW FOR PERIOD OF RECORD 1916-82

\begin{tabular}{|c|c|c|c|c|c|c|c|c|c|c|c|c|c|c|}
\hline & & DIS & RGE, & CFS, & $\mathrm{CH}$ WA & UALE & R EXC & ED FC & NDICA & PER & OF & & & \\
\hline $5 \%$ & $10 \%$ & $15 \%$ & $20 \%$ & $25 \%$ & $30 \%$ & $40 \%$ & $50 \%$ & $60 \%$ & $70 \%$ & $75 \%$ & $80 \%$ & $85 \%$ & $90 \%$ & $95 \%$ \\
\hline 264 & 205 & 172 & 148 & 129 & 114 & 92 & 77 & 67 & 60 & 56 & 53 & 49 & 45 & 39 \\
\hline
\end{tabular}




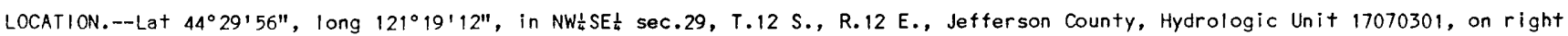
bank $2.5 \mathrm{mi}$ downstream from Squaw Creek, $6.0 \mathrm{mi}$ southwest of Culver, and at mile 120.6.

DRAINAGE AREA. $--2,705 \mathrm{mi}^{2}$.

PERIOD OF RECORD.--July 1952 to September 1982.

GAGE.--Water-stage recorder. Datum of gage is 1,980 ft National Geodetlc Vertical Datum of 1929 ( $r i v e r-p r o f i l e$ survey). July 14 , 1952, to Sept. 30, 1961, at site $4.1 \mathrm{mi}$ downstream at different datum.

REMARKS.--Flow regulated by Crescent Lake and Crane Prairle and Wickiup Reservoirs. Many diversions for irrigation above station. AVERAGE DISCHARGE.--30 years, $905 \mathrm{ft}^{3} / \mathrm{s}, 655,700$ acre- $\mathrm{ft} / \mathrm{yr}$.

EXTREMES FOR PERIOD OF RECORD.--Maximum discharge, $6,680 \mathrm{tt}^{3} / \mathrm{s}$ Dec. 24,1964 , gage height, $10.00 \mathrm{ft}$, from rating curve extended above $2,200 \mathrm{ft}^{3} / \mathrm{s}$ on basis of slope-area measurement of peak flow; minimum, $418 \mathrm{ft} / \mathrm{s} / \mathrm{July} 7,8,1964$.

STATISTICAL SUMMARIES

MONTHLY AND ANNUAL MEAN DISCHARGES 1953-82

\begin{tabular}{|c|c|c|c|c|c|c|}
\hline MONTH & $\begin{array}{l}M|N| M U M \\
\text { (CFS) }\end{array}$ & $\begin{array}{l}\text { MAXINUM } \\
\text { (CFS) }\end{array}$ & $\begin{array}{l}\text { MEAN } \\
\text { (CFS) }\end{array}$ & $\begin{array}{l}\text { STAN- } \\
\text { DARD } \\
\text { DEVIA- } \\
\text { TION } \\
\text { (CFS) }\end{array}$ & $\begin{array}{l}\text { COEFF I- } \\
\text { CIENT OF } \\
\text { VARI- } \\
\text { ATION }\end{array}$ & $\begin{array}{c}\text { PERCENT } \\
\text { OF } \\
\text { ANNUAL } \\
\text { RUNOFF }\end{array}$ \\
\hline OCTOBER & 470 & 1080 & 666 & 160 & .24 & 6.1 \\
\hline NOVEMBER & 837 & 1683 & 1161 & 207 & .18 & 10.7 \\
\hline DECEMBER & 953 & 1958 & 1288 & 281 & .22 & 11.8 \\
\hline JANUARY & 1010 & 2012 & 1360 & 305 & .22 & 12.5 \\
\hline FEBRUARY & 1010 & 2034 & 1416 & 318 & .22 & 13.0 \\
\hline MARCH & 839 & 2360 & 1325 & 401 & .30 & 12.2 \\
\hline APRIL & 510 & 1430 & 817 & 243 & .30 & 7.5 \\
\hline MAY & 457 & 1228 & 586 & 152 & .26 & 5.4 \\
\hline JUNE & 455 & 1020 & 638 & 160 & .25 & 5.9 \\
\hline JULY & 430 & 766 & 549 & 89 & .16 & 5.0 \\
\hline AUGUST & 441 & 741 & 529 & 71 & .13 & 4.9 \\
\hline SEPTEMBER & 455 & 782 & 554 & 94 & .17 & 5.1 \\
\hline NNUAL & 677 & 1195 & 905 & 163 & .18 & 100 \\
\hline
\end{tabular}

MAGNITUDE AND PROBABILITY OF INSTANTANEOUS PEAK FLOW BASED ON PERIOD OF RECORD 1953-82

\begin{tabular}{|c|c|c|c|c|c|c|}
\hline \multicolumn{7}{|c|}{$\begin{array}{l}\text { DISCHARGE, IN CFS, FOR INDICATED RECURRENCE INTERVAL, IN } \\
\text { YEARS, AND ANNUAL EXCEEDANCE PROBABILITY, IN PERCENT }\end{array}$} \\
\hline $\begin{array}{l}1.25 \\
80 \%\end{array}$ & $\begin{array}{c}2 \\
50^{\alpha}\end{array}$ & $\begin{array}{c}5 \\
20 \%\end{array}$ & $\begin{array}{l}10 \\
10 \%\end{array}$ & $\begin{array}{l}25 \\
4 \%\end{array}$ & $\begin{array}{l}50 \\
2 \%\end{array}$ & $\begin{array}{r}100 \\
1 \%\end{array}$ \\
\hline 1740 & 2170 & 2840 & 3320 & 3980 & 4510 & -- \\
\hline
\end{tabular}

MAGN ITUDE AND PROBABILITY OF ANNUAL LOW FLOW BASED ON PERIOD OF RECORD 1954-82

\begin{tabular}{|c|c|c|c|c|c|c|}
\hline \multirow{4}{*}{$\begin{array}{l}\text { PERIOD } \\
\text { (CON- } \\
\text { SECU- } \\
\text { TIVE } \\
\text { DAYS) }\end{array}$} & \multicolumn{6}{|c|}{$\begin{array}{l}\text { DISCHARGE, IN CFS, FOR INDICATED RECURRENCE } \\
\text { INTERVAL, IN YEARS, AND ANNUAL NON- } \\
\text { EXCEEDANCE PROBABILITY, IN PERCENT }\end{array}$} \\
\hline & & & & & & \\
\hline & 2 & 5 & 10 & 20 & 50 & 100 \\
\hline & $50 \%$ & $20 \%$ & $10 \%$ & $5 \%$ & $2 \%$ & $1 \%$ \\
\hline & & & & & & \\
\hline 1 & 482 & 457 & 445 & 436 & 426 & -- \\
\hline 3 & 485 & 459 & 447 & 437 & 427 & -- \\
\hline 7 & 489 & 462 & 449 & 439 & 428 & -- \\
\hline 14 & 493 & 465 & 452 & 442 & 431 & -- \\
\hline 30 & 502 & 469 & 453 & 442 & 431 & -- \\
\hline 60 & 510 & 471 & 456 & 443 & 432 & -- \\
\hline 90 & 521 & 475 & 456 & 443 & 432 & -- \\
\hline 120 & 534 & 480 & 458 & 443 & 433 & -- \\
\hline 183 & 554 & 492 & 467 & 450 & 433 & -- \\
\hline
\end{tabular}

MAGNITUDE AND PROBABILITY OF ANNUAL HIGH FLOW BASED ON PERIOD OF RECORD 1953-82

\begin{tabular}{|c|c|c|c|c|c|c|}
\hline $\begin{array}{l}\text { PERIOD } \\
\text { (CON- }\end{array}$ & \multicolumn{6}{|c|}{$\begin{array}{l}\text { DISCHARGE, IN CFS, FOR INDICATED RECURRENCE } \\
\text { INTERVAL, IN YEARS, AND ANNUAL } \\
\text { EXCEEDANCE PROBABILITY, IN PERCENT }\end{array}$} \\
\hline $\begin{array}{l}\text { SECU- } \\
\text { TIVE }\end{array}$ & 2 & 5 & 10 & 25 & 50 & 100 \\
\hline DAYS) & $50 \%$ & $20 \%$ & $10 \%$ & $4 \%$ & $2 \%$ & $1 \%$ \\
\hline 1 & 2010 & 2550 & 2930 & 3440 & 3840 & -- \\
\hline 3 & 1890 & 2400 & 2760 & 3240 & 3620 & -- \\
\hline 7 & 1790 & 2260 & 2590 & 3030 & 3380 & -- \\
\hline 15 & 1690 & 2080 & 2330 & 2640 & 2870 & -- \\
\hline 30 & 1550 & 1900 & 2120 & 2390 & 2580 & -- \\
\hline 60 & 1440 & 1750 & 1960 & 2210 & 2410 & -- \\
\hline 90 & 1380 & 1660 & 1850 & 2080 & 2260 & -- \\
\hline
\end{tabular}

DURATION TABLE OF DAILY MEAN FLOW FOR PERIOD OF RECORD 1953-82

DISCHARGE, IN CFS, WHICH WAS EQUALED OR EXCEEDED FOR INDICATED PERCENT OF TIME

\begin{tabular}{|c|c|c|c|c|c|c|c|c|c|c|c|c|c|c|}
\hline $5 \%$ & $10 \%$ & $15 \%$ & $20 \%$ & $25 \%$ & $30 \%$ & $40 \%$ & $50 \%$ & $60 \%$ & $70 \%$ & $75 \%$ & $80 \%$ & $85 \%$ & $90 \%$ & $95 \%$ \\
\hline 1830 & 1560 & 1400 & 1280 & 1190 & 1120 & 977 & 742 & 595 & 538 & 521 & 508 & 495 & 481 & 467 \\
\hline
\end{tabular}


14078000 BEAVER CREEK NEAR PAULINA, OR

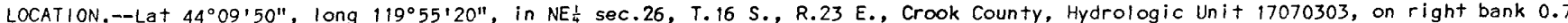
$\mathrm{mi}$ downstream from Paulina Creek, $1.7 \mathrm{mi}$ downstream from Wolt Creek, $2.7 \mathrm{mi}$ northeast of Paulina, and at mile 10.0 .

DRAINAGE AREA. $--450 \mathrm{mi}^{2}$, approximately.

PERIOD OF RECORD.--October 1942 to September 1975. Prior to October 1945 monthly discharge only, published in WSP 1318.

GAGE.--Water-stage recorder. Altitude of gage is 3,690 ft (by barometer). Oct. 1, 1942, to July 7 , 1965 at datum 1.00 higher.

REMARKS.--No regulation. Diversions for irrigation above station. Two small ditches divert above station for irrigation below.

AVERAGE DISCHARGE.--33 years, $89.1 \mathrm{ft} / \mathrm{s}, 64,550$ acre- $\mathrm{ft} / \mathrm{yr}$.

EXTREMES FOR PERIOD OF RECORD.--Maximum discharge, 12,800 ft3/s Dec. 22, 1964, gage height, $14.20 \mathrm{ft}$, from $\mathrm{floodmark}$, present datum, from rating curve extended above $1,600 \mathrm{ft} / \mathrm{s}$, on basis of slope-area measurement of peak flow; no $\mathrm{flow}$ Oct. $13-29$, 1945 .

STATISTICAL SUMMARIES

MONTHLY AND ANNUAL MEAN DISCHARGES 1943-75

\begin{tabular}{|c|c|c|c|c|c|c|}
\hline MONTH & $\begin{array}{l}\text { MINIMUM } \\
\text { (CFS) }\end{array}$ & $\begin{array}{l}\text { MAXIMUM } \\
\text { (CFS) }\end{array}$ & $\begin{array}{l}\text { MEAN } \\
\text { (CFS) }\end{array}$ & $\begin{array}{l}\text { STAN- } \\
\text { DARD } \\
\text { DEV|A- } \\
\text { TION } \\
\text { (CFS) }\end{array}$ & $\begin{array}{l}\text { COEFFI- } \\
\text { CIENT OF } \\
\text { VARI- } \\
\text { ATION }\end{array}$ & $\begin{array}{c}\text { PERCENT } \\
\text { OF } \\
\text { ANNUAL } \\
\text { RUNOFF }\end{array}$ \\
\hline OTOBER & .4 & 13 & 5.3 & 2.8 & .53 & .5 \\
\hline NOVEMBER & 5.6 & 129 & 22 & 27 & 1.22 & 2.1 \\
\hline DECEMBER & 8.0 & 432 & 83 & 109 & 1.31 & 7.7 \\
\hline JANUARY & 11 & 590 & 120 & 139 & 1.16 & 11.1 \\
\hline FEBRUARY & 16 & 858 & 198 & 165 & .83 & 18.4 \\
\hline MARCH & 35 & 889 & 279 & 194 & .70 & 25.9 \\
\hline APRIL & 1.5 & 796 & 251 & 162 & .65 & 23.3 \\
\hline MAY & .4 & 298 & 93 & 83 & .89 & 8.6 \\
\hline JUNE & .5 & 124 & 17 & 27 & 1.54 & 1.6 \\
\hline JULY & .7 & 29 & 4.9 & 5.7 & 1.17 & .5 \\
\hline AUGUST & .4 & 7.1 & 1.6 & 1.4 & .89 & .1 \\
\hline SEPTEMBER & .4 & 6.1 & 2.3 & 1.8 & .78 & .2 \\
\hline ANNUAL & 15 & 215 & 89 & 48 & .54 & 100 \\
\hline
\end{tabular}

MAGNITUDE AND PROBABILITY OF INSTANTANEOUS PEAK FLOW BASED ON PERIOD OF RECORD 1943-75

\begin{tabular}{|c|c|c|c|c|c|c|}
\hline \multicolumn{7}{|c|}{$\begin{array}{l}\text { OI SCHARGE, IN CFS, FOR INDICATEO RECURRENCE INTERVAL, IN } \\
\text { YEARS, ANO ANNUAL EXCEEOANCE PROBABILITY, IN PERCENT }\end{array}$} \\
\hline $\begin{array}{l}1.25 \\
80 \%\end{array}$ & $\begin{array}{c}2 \\
50 \%\end{array}$ & $\begin{array}{c}5 \\
20 \%\end{array}$ & $\begin{array}{l}10 \\
10 \%\end{array}$ & $\begin{array}{l}25 \\
4 \%\end{array}$ & $\begin{array}{l}50 \\
2 \%\end{array}$ & $\begin{array}{r}100 \\
1 \%\end{array}$ \\
\hline 730 & 1370 & 2750 & 4060 & 6270 & 8390 & -- \\
\hline
\end{tabular}

WEIGHTEO SKEW $=.333$
MAGNITUDE AND PROBABILITY OF ANNUAL LOW FLOW BASED ON PERIOD OF RECORD 1944-75

\begin{tabular}{|c|c|c|c|c|c|c|}
\hline \multirow{2}{*}{$\begin{array}{l}\text { PERIOD } \\
\text { (CON- } \\
\text { SECU- } \\
\text { TIVE } \\
\text { DAYS) }\end{array}$} & \multicolumn{6}{|c|}{$\begin{array}{l}\text { DISCHARGE, IN CFS, FOR IND ICATED RECURRENCE } \\
\text { INTERVAL, IN YEARS, AND ANNUAL NON- } \\
\text { EXCEEDANCE PROBABILITY, IN PERCENT }\end{array}$} \\
\hline & $\begin{array}{c}2 \\
50 \%\end{array}$ & $\begin{array}{c}5 \\
20 \%\end{array}$ & $\begin{array}{l}10 \\
10 \%\end{array}$ & $\begin{array}{l}20 \\
5 \%\end{array}$ & $\begin{array}{l}50 \\
2,6\end{array}$ & $\begin{array}{r}100 \\
1 \%\end{array}$ \\
\hline 1 & .4 & .3 & .2 & .2 & .1 & -- \\
\hline 3 & .5 & .3 & .2 & .2 & .1 & -- \\
\hline 7 & .5 & .3 & .2 & .2 & .1 & -- \\
\hline 14 & .5 & .3 & .2 & .2 & .2 & -- \\
\hline 30 & .7 & .4 & .3 & .2 & .2 & -- \\
\hline 60 & 1.0 & .6 & .4 & .4 & .3 & -- \\
\hline 90 & 1.4 & .8 & .6 & .5 & .4 & -- \\
\hline 120 & 2.0 & 1.1 & .9 & .7 & .5 & -- \\
\hline 183 & 4.8 & 2.7 & 2.0 & 1.5 & 1.1 & -- \\
\hline
\end{tabular}

MAGNITUDE AND PROBABILITY OF ANNUAL HIGH FLOW BASED ON PERIOD OF RECORD 1943-75

\begin{tabular}{|c|c|c|c|c|c|c|}
\hline \multirow{4}{*}{$\begin{array}{l}\text { PERIOD } \\
\text { (CON- } \\
\text { SECU- } \\
\text { TIVE } \\
\text { DAYS) }\end{array}$} & \multicolumn{6}{|c|}{$\begin{array}{c}\text { DISCHARGE, IN CFS, FOR INOICATED RECURRENCE } \\
\text { INTERVAL, IN YEARS, AND ANNUAL } \\
\text { EXCEEDANCE PROBABILITY, IN PERCENT }\end{array}$} \\
\hline & 2 & 5 & & 25 & & \\
\hline & 2 & 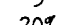 & 108 & 20 & 30 & 18 \\
\hline & & 20 & & & & \\
\hline 1 & 1140 & 2040 & 2740 & 3720 & 4510 & -- \\
\hline 3 & 901 & 1640 & 2210 & 3020 & 3680 & - \\
\hline 7 & 682 & 1210 & 1590 & 2090 & 2480 & - \\
\hline 15 & 505 & 867 & 1120 & 1440 & 1680 & - \\
\hline 30 & 389 & 645 & 812 & 1020 & 1160 & -- \\
\hline 60 & 290 & 471 & 588 & 729 & 826 & - \\
\hline 90 & 247 & 394 & 485 & 588 & 656 & - \\
\hline
\end{tabular}

DURATION TABLE OF DAILY MEAN FLOW FOR PERIOD OF RECORD 1943-75

\begin{tabular}{|c|c|c|c|c|c|c|c|c|c|c|c|c|c|c|}
\hline $5 \%$ & $10 \%$ & $15 \%$ & $20^{\circ}$ & $25 \%$ & $30 \%$ & $40 \%$ & $50 \%$ & $60 \%$ & $70 \%$ & $75 \%$ & $80 \%$ & $85 \%$ & $90 \%$ & $95 \%$ \\
\hline 421 & 264 & 181 & 125 & 87 & 60 & 25 & 13 & 7.4 & 4.4 & 2.9 & 1.6 & 1.0 & .7 & .5 \\
\hline
\end{tabular}




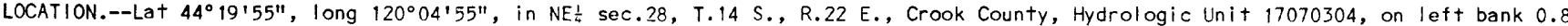

$\mathrm{mi}$ upstream from Deep Creek, $15 \mathrm{mi}$ northwest of Paulina, and $38 \mathrm{mi}$ east of Prineville.

DRAINAGE AREA.--159 $\mathrm{mi}^{2}$.

PERIOD OF RECORD.--November 1941 to December 1942, nctober 1943 to September 1954 . Prior to October 1945 monthly discharge only, published in WSP 1318.

GAGE.--Water-stage recorder. Datum of gage is $4,355.00 \mathrm{ft}$ National Geodetic Vertical Datum of 1929 (levels by Bureau of Reclamation). Prior to Oct. 1, 1946, at datum $0.33 \mathrm{tt}$ higher.

REMARKS.--No regulation. Several diversions for irrigation of about 3,600 acres above station.

AVERAGE DISCHARGE.-- 11 years (water years 1943-54), $97.0 \mathrm{ft}+\mathrm{s} / \mathrm{s}, 70,230$ acre- $\mathrm{ft} / \mathrm{yr}$.

EXTREMES FOR PERIOD OF RECORD.--Maximum discharge, 2,060 $\mathrm{ft} / \mathrm{s} \mathrm{Apr.} \mathrm{7,} \mathrm{1943,} \mathrm{gage} \mathrm{height,} 4.17 \mathrm{ft}$, from rating curve extended above $950 \mathrm{ft} / 5$; maximum gage height, $8.01 \mathrm{ft}$, last used datum, Jan. 1, 1943 (ice jam); minimum discharge, $0.5 \mathrm{ft} / \mathrm{s}$ Aug. 14,15 , 1942 , Aug. 3 to Sept. 24, 1951.

STATISTICAL SUMMARIES

MONTHLY AND ANNUAL MEAN DISCHARGES 1944-54

\begin{tabular}{|c|c|c|c|c|c|c|}
\hline MONTH & $\begin{array}{l}\text { MINIMUM } \\
\text { (CFS) }\end{array}$ & $\begin{array}{c}\text { MAXIMUM } \\
\text { (CFS) }\end{array}$ & $\begin{array}{l}\text { MEAN } \\
\text { (CFS) }\end{array}$ & $\begin{array}{c}\text { STAN- } \\
\text { DARD } \\
\text { DEVIA- } \\
\text { T!ON } \\
\text { (CFS) }\end{array}$ & $\begin{array}{l}\text { COEFFI- } \\
\text { CIENT OF } \\
\text { VARI- } \\
\text { ATION }\end{array}$ & $\begin{array}{c}\text { PERCENT } \\
\text { OF } \\
\text { ANNUAL } \\
\text { RUNOFF }\end{array}$ \\
\hline & & & & & & \\
\hline OCTOBER & 1.3 & 10 & 4.9 & 3.1 & .64 & .4 \\
\hline NOVEMBER & 4.0 & 37 & 17 & 11 & .65 & 1.4 \\
\hline DECEMBER & 4.7 & 152 & 46 & 52 & 1.13 & 3.9 \\
\hline JANUARY & 9.4 & 187 & 56 & 60 & 1.08 & 4.8 \\
\hline FEBRUARY & 19 & 210 & 95 & 71 & .74 & 8.1 \\
\hline MARCH & 58 & 488 & 189 & 122 & .65 & 16.2 \\
\hline APRIL & 160 & 911 & 465 & 239 & .51 & 39.8 \\
\hline MAY & 25 & 457 & 213 & 127 & .60 & 18.2 \\
\hline JUNE & 15 & 262 & 74 & 79 & 1.06 & 6.3 \\
\hline JULY & 1.4 & 23 & 5.8 & 6.3 & 1.09 & .5 \\
\hline AUGUST & .5 & 5.5 & 2.0 & 1.5 & .74 & .2 \\
\hline SEPTEMBER & .6 & 4.7 & 1.9 & 1.2 & .63 & .2 \\
\hline ANNUAL & 37 & 173 & 97 & 40 & .42 & 100 \\
\hline
\end{tabular}

MAGNITUDE AND PROBABILITY OF INSTANTANEOUS PEAK FLOW BASED ON PERIOD OF RECORD 1944-54

\begin{tabular}{lcccccc} 
DISCHARGE, IN CFS, FOR IND ICATED RECURRENCE & INTERVAL, IN \\
YEARS, AND ANNUAL EXCEEDANCE PROBAB IL ITY, IN PERCENT \\
\hline 1.25 & 2 & 5 & 10 & 25 & 50 & 100 \\
$80 \%$ & $50 \%$ & $20 \%$ & $10 \%$ & $4 \%$ & $2 \%$ & $1 \%$ \\
\hline 1100 & 1410 & 1820 & 2090 & - & -
\end{tabular}

WEIGHTED SKEW $=\quad .156$
MAGNITUDE AND PROBABILITY OF ANNUAL LOW FLOW BASED ON PERIOD OF RECORD 1945-54

\begin{tabular}{|c|c|c|c|c|c|c|}
\hline $\begin{array}{l}\text { PERIOD } \\
\text { (CON- }\end{array}$ & \multicolumn{6}{|c|}{$\begin{array}{l}\text { DISCHARGE, IN CFS, FOR INDICATED RECURRENCE } \\
\text { INTERVAL, IN YEARS, AND ANNUAL NON- } \\
\text { EXCEEDANCE PROBABILITY, IN PERCENT }\end{array}$} \\
\hline TIVE & 2 & 5 & 10 & 20 & 50 & 100 \\
\hline DAYS) & $50 \%$ & $20 \%$ & $10 \%$ & $5 \%$ & $2^{q}$ & $1 \%$ \\
\hline 1 & 1.0 & .7 & .6 & .5 & - & - \\
\hline 3 & 1.0 & .7 & .6 & .5 & -- & -- \\
\hline 7 & 1.1 & .7 & .6 & .5 & $\ldots$ & $\ldots$ \\
\hline 14 & 1.2 & .7 & .6 & .5 & - & - \\
\hline 30 & 1.3 & .8 & .6 & .5 & -- & -- \\
\hline 60 & 1.5 & .9 & .7 & .6 & -- & -- \\
\hline 90 & 1.7 & 1.1 & .9 & .8 & -- & -- \\
\hline 120 & 2.3 & 1.5 & 1.2 & 1.0 & - & -- \\
\hline 183 & 7.2 & 3.8 & 2.7 & 2.1 & -- & -- \\
\hline
\end{tabular}

MAGNITUDE AND PROBABILITY OF ANNUAL HIGH FLOW BASED ON PERIOD OF RECORD 1944-54

\begin{tabular}{|c|c|c|c|c|c|c|}
\hline $\begin{array}{l}\text { PERIOD } \\
\text { (CON- } \\
\text { SECU- }\end{array}$ & \multicolumn{6}{|c|}{$\begin{array}{c}\text { DISCHARGE, IN CFS, FOR INDICATED RECURRENCE } \\
\text { INTERVAL, IN YEARS, AND ANNUAL } \\
\text { EXCEEDANCE PROBABIL!TY, IN PERCENT }\end{array}$} \\
\hline $\begin{array}{l}\text { TIVE } \\
\text { DAYS) }\end{array}$ & $\begin{array}{c}2 \\
50,6\end{array}$ & $\begin{array}{c}5 \\
20 \%\end{array}$ & $\begin{array}{l}10 \\
10 \%\end{array}$ & $\begin{array}{l}25 \\
4 \%\end{array}$ & $\begin{array}{l}50 \\
2 \%\end{array}$ & $\begin{array}{r}100 \\
1 \%\end{array}$ \\
\hline 1 & 941 & 1360 & 1570 & -- & -- & -- \\
\hline 3 & 826 & 1210 & 1420 & - & -- & -- \\
\hline $\begin{array}{r}7 \\
15\end{array}$ & $\begin{array}{l}704 \\
567\end{array}$ & $\begin{array}{r}1030 \\
848\end{array}$ & $\begin{array}{l}1220 \\
1020\end{array}$ & -- & -- & -- \\
\hline 30 & 460 & 679 & 819 & - & -- & -- \\
\hline 60 & 376 & 539 & 634 & -- & -- & -- \\
\hline 90 & 300 & 422 & 490 & - & -- & $\cdots$ \\
\hline
\end{tabular}

DURATION TABLE OF DAILY MEAN FLOW FOR PERIOD OF RECORD 1944-54

\begin{tabular}{|c|c|c|c|c|c|c|c|c|c|c|c|c|c|c|}
\hline & & DI & ARGE, & CFS, & $\mathrm{CH} W$ & QUALE & $R$ EXC & ED FOF & NDICA & PERO & OF $T$ & & & \\
\hline $5 \%$ & $10 \%$ & $15 \%$ & $20 \%$ & $25 \%$ & $30 \%$ & $40 \%$ & $50 \%$ & $60 \%$ & $70 \%$ & $75 \%$ & $80 \%$ & $85 \%$ & $90 \%$ & $95 \%$ \\
\hline 504 & 317 & 212 & 148 & 101 & 65 & 32 & 15 & 7.9 & 4.5 & 3.4 & 2.2 & 1.8 & 1.4 & 1. \\
\hline
\end{tabular}


14079500 CROOKED RIVER NEAR POST, OR

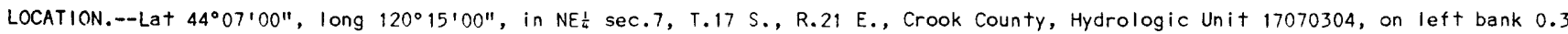
$\mathrm{mi}$ downstream from North Fork, $12 \mathrm{mi}$ southeast of Post, and at mile 113.7 .

DRAINAGE AREA.--2,160 $\mathrm{mi}^{2}$, approximately, of which $500 \mathrm{mi}^{2}$ is probably noncontributing.

PERIOD OF RECORD.--November 1908 to May 1911, December 1939 to September 1960, July 1968 to July 1973 , Records for June to August 1911, published in WSP 312, have been found unreliabie and should not be used.

GAGE.--Water-stage recorder. Datum of gage is 3,476.25 ft National Geodetic vertical Datum of 1929. Nov. 9, 1908, to Aug. 31, 1911, nonrecording gage at site $0.2 \mathrm{mi}$ downstream at different datum. Dec. 30, 1939, to Sept. 30, 1960, water-stage recorder at site $0.7 \mathrm{mi}$ downstream at different datum.

REMARKS.--No regulation. Many diversions for irrigation above station.

AVERAGE DISCHARGE.--24 years (water years 1941-60, 1969-72), $337 \mathrm{ft} / \mathrm{s}, 244,200 \mathrm{acre}-\mathrm{ft} / \mathrm{yr}$.

EXTREMES FOR PERIOD OF RECORD.--Maximum discharge, $11,500 \mathrm{ft}^{3} / \mathrm{s}$ Jan. 18, 1971, gage height, $9.61 \mathrm{ft}$, from rating curve extended above $4,800 \mathrm{ft} / \mathrm{s}$, on basis of slope-area measurement of peak flow; minimum, $2.7 \mathrm{ft} / \mathrm{s}$ Aug. 7 , 1970 .

EXTREMES OUTSIDE PERIOD OF RECORD.--Flood of Dec. 23, 1964, reached a stage of $15.00 \mathrm{ft}$ at nonequivalent site $23 \mathrm{mi}$ downstream, discharge, $19,700 \mathrm{ft}^{3} / \mathrm{s}$.

\section{STATISTICAL SUMMARIES}

MONTHLY AND ANNUAL MEAN DISCHARGES 1941-72

\begin{tabular}{|c|c|c|c|c|c|c|}
\hline MONTH & $\begin{array}{l}\text { MINIMUM } \\
\text { (CFS) }\end{array}$ & $\begin{array}{c}\text { MAXIMUM } \\
\text { (CFS) }\end{array}$ & $\begin{array}{l}\text { MEAN } \\
\text { (CFS) }\end{array}$ & $\begin{array}{l}\text { STAN- } \\
\text { DARD } \\
\text { DEVIA- } \\
\text { TION } \\
\text { (CFS) }\end{array}$ & $\begin{array}{l}\text { COEFFI- } \\
\text { CIENT OF } \\
\text { VARI- } \\
\text { ATION }\end{array}$ & $\begin{array}{c}\text { PERCENT } \\
\text { OF } \\
\text { ANNUAL } \\
\text { RUNOFF }\end{array}$ \\
\hline OCTOBER & 29 & 81 & 43 & 13 & .29 & 1.1 \\
\hline NOVEMBER & 50 & 259 & 104 & 57 & .55 & 2.6 \\
\hline DECEMBER & 56 & 753 & 208 & 185 & .89 & 5.2 \\
\hline JANUARY & 60 & 1488 & 337 & 353 & 1.05 & 8.5 \\
\hline FEBRUARY & 82 & 2000 & 522 & 379 & .73 & 13.2 \\
\hline MARCH & 188 & 2068 & 833 & 451 & .54 & 21.0 \\
\hline APRIL & 430 & 2692 & 1211 & 580 & .48 & 30.5 \\
\hline MAY & 77 & 1300 & 500 & 291 & .58 & 12.6 \\
\hline JUNE & 35 & 635 & 144 & 133 & .92 & 3.6 \\
\hline JULY & 4.7 & 108 & 31 & 22 & .71 & .8 \\
\hline AUGUST & 5.6 & 37 & 14 & 6.7 & .49 & .3 \\
\hline SEPTEMBER & 10 & 41 & 19 & 7.2 & .38 & .5 \\
\hline ANNUAL & 129 & 553 & 331 & 123 & .37 & 100 \\
\hline
\end{tabular}

MAGNITUDE AND PROBABILITY OF INSTANTANEOUS PEAK FLOW BASED ON PERIOD OF RECORD 1941-72

DISCHARGE, IN CFS, FOR INDICATED RECURRENCE INTERVAL, IN
YEARS, AND ANNUAL EXCEEDANCE PROBABILITY, IN PERCENT

\begin{tabular}{ccccccc}
1.25 & 2 & 5 & 10 & 25 & 50 & 100 \\
$80 \%$ & $50 \%$ & $20 \%$ & $10 \%$ & $4 \%$ & $2 \%$ & $1 \%$ \\
2410 & 4000 & 6560 & 8450 & 11000 & 13100 & - \\
\hline
\end{tabular}

WEIGHTED SKEW $=-.086$

MAGNITUDE AND PROBABILITY OF ANNUAL LOW FLOW BASED ON PERIOD OF RECORD 1942-72

\begin{tabular}{|c|c|c|c|c|c|c|}
\hline \multirow{2}{*}{$\begin{array}{l}\text { PERIOD } \\
\text { (CON- } \\
\text { SECU- } \\
\text { TIVE } \\
\text { DAYS) }\end{array}$} & \multicolumn{6}{|c|}{$\begin{array}{l}\text { DISCHARGE, IN CFS, FOR INDICATED RECURRENCE } \\
\text { INTERVAL, IN YEARS, AND ANNUAL NON- } \\
\text { EXCEEDANCE PROBABILITY, IN PERCENT }\end{array}$} \\
\hline & $\begin{array}{c}2 \\
50 \%\end{array}$ & $\begin{array}{c}5 \\
20 \%\end{array}$ & $\begin{array}{l}10 \\
10 \%\end{array}$ & $\begin{array}{l}20 \\
50\end{array}$ & $\begin{array}{l}50 \\
2 \%\end{array}$ & $\begin{array}{r}100 \\
1 \%\end{array}$ \\
\hline 1 & 8.7 & 5.9 & 4.7 & 3.8 & 3.0 & -- \\
\hline 3 & 9.1 & 6.2 & 5.0 & 4.1 & 3.3 & -- \\
\hline 7 & 9.8 & 6.8 & 5.5 & 4.6 & 3.7 & -- \\
\hline 14 & 10 & 7.3 & 6.0 & 5.1 & 4.2 & -- \\
\hline 30 & 11 & 8.1 & 6.8 & 5.8 & 4.9 & -- \\
\hline 60 & 14 & 10 & 8.7 & 7.8 & 7.0 & -- \\
\hline 90 & 17 & 13 & 11 & 10 & 9.2 & -- \\
\hline 120 & 23 & 17 & 15 & 13 & 11 & -- \\
\hline 183 & 44 & 33 & 29 & 26 & 24 & -- \\
\hline
\end{tabular}

MAGNITUDE AND PROBABILITY OF ANNUAL HIGH FLOW BASED ON PERIOD OF RECORD 1941-72

\begin{tabular}{|c|c|c|c|c|c|c|}
\hline \multirow{3}{*}{$\begin{array}{c}\text { PERIOD } \\
\text { (CON- } \\
\text { SECU- } \\
\text { TIVE } \\
\text { DAYS) }\end{array}$} & \multicolumn{6}{|c|}{$\begin{array}{l}\text { DISCHARGE, IN CFS, FOR INDICATED RECURRENCE } \\
\text { INTERVAL, IN YEARS, AND ANNUAL } \\
\text { EXCEEDANCE' PROBABILITY, IN PERCENT }\end{array}$} \\
\hline & $?$ & & 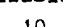 & 25 & 50 & \\
\hline & $50 \%$ & $20 \%$ & $10 \%$ & $4 \%$ & $2 \%$ & 100 \\
\hline 1 & 3170 & 5270 & 6790 & 8810 & 10400 & - \\
\hline 3 & 2730 & 4340 & 5420 & 6760 & 7750 & -- \\
\hline 7 & 2260 & 3400 & 4120 & 4950 & 5530 & -- \\
\hline 15 & 1830 & 2700 & 3230 & 3830 & 4230 & -- \\
\hline 30 & 1450 & 2090 & 2480 & 2910 & 3200 & -- \\
\hline 60 & 1130 & 1570 & 1810 & 2070 & 2220 & - \\
\hline 90 & 943 & 1290 & 1480 & 1660 & 1770 & -- \\
\hline
\end{tabular}

DURATION TABLE OF DAILY MEAN FLOW FOR PERIOD OF RECORD $1941-72$

DISCHARGE, IN CFS, WHICH WAS EQUALED OR EXCEEDED FOR INDICATED PERCENT OF TIME

\begin{tabular}{|c|c|c|c|c|c|c|c|c|c|c|c|c|c|c|}
\hline $5 \%$ & $10 \%$ & $15 \%$ & $20 \%$ & $25 \%$ & $30 \%$ & $40_{\%}^{\circ}$ & $50 \%$ & $60 \%$ & $70 \%$ & $75 \%$ & $80 \%$ & $85 \%$ & $90 \%$ & $95 \%$ \\
\hline 1480 & 960 & 671 & 498 & 367 & 270 & 150 & 89 & 63 & 43 & 32 & 24 & 19 & 15 & 11 \\
\hline
\end{tabular}




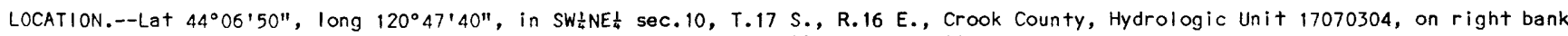
$0.4 \mathrm{mi}$ downstream from Prineville Dam, $13.6 \mathrm{mi}$ south of Prineville, and at mile 72.1 .

DRAINAGE AREA.--2,700 $\mathrm{mi}^{2}$, approximately, of which $500 \mathrm{mi}^{2}$ is probably noncontributing.

PERIOD OF RECORD.--November 1908 to September 1914, March 1941 to September 1982. Published as "near Prineville" $1908-12$, as "at Hoffman's ranch, near Prineville" 1913-14, and as "above Hoffman Dam, near Prineville" March 1941 to September 1960. The estimate of monthly mean discharge for October 1908, published in WSP 370 , has been found to be unreliable and should not be used.

GAGE.--Water-stage recorder. Datum of gage is 3,070.85 ft National Geodetic Vertical Datum of 1929 (levels by Bureau of Reclamation). Prior to September 1914, nonrecording gage at several sites from $9 \mathrm{mi}$ to $23 \mathrm{mi}$ downstream at various datums. Mar. 26, 1941, to Apr. 23, 1961, water-stage recorder at site $5.5 \mathrm{mi}$ downstream at different datum.

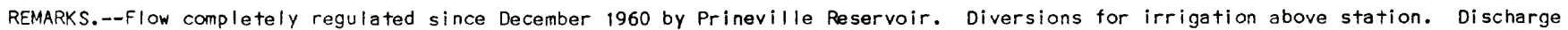
not adjusted for storage or release from Prineville Reservoir as evaporation from reservoir at times exceeds natural flow.

AVERAGE DISCHARGE.--24 years (water years 1910-14, 1942-60), $378 \mathrm{ft} / \mathrm{s}, 273,700$ acre- $\mathrm{ft} / \mathrm{yr}$; 22 years (water years $1961-82$ ), 325 $\mathrm{ft}^{3} / \mathrm{s}, 235,500$ acre- $\mathrm{ft} / \mathrm{yr}$.

EXTREMES FOR PERIOD OF RECORD.--Maximum discharge, $8,410 \mathrm{ft}+3 / \mathrm{s} \mathrm{Mar} .26$, 1952 , gage height, $8.2 \mathrm{ft}$, from $\mathrm{floodmark}$, site and datum then in use; no flow Aug. 13-21, 1959, Jan. 3-5, 1961.

STATISTICAL SUMMARIES (BEFORE THE CONSTRUCTION OF PRINEVILLE RESERVOIR)

MONTHLY AND ANNUAL MEAN DISCHARGES 1942-59

\begin{tabular}{|c|c|c|c|c|c|c|}
\hline MONTH & $\begin{array}{l}\text { MINIMUM } \\
\text { (CFS) }\end{array}$ & $\begin{array}{l}\text { MAXIMUM } \\
\text { (CFS) }\end{array}$ & $\begin{array}{l}\text { MEAN } \\
\text { (CFS) }\end{array}$ & $\begin{array}{l}\text { STAN- } \\
\text { DARD } \\
\text { DEVIA- } \\
\text { TION } \\
\text { (CFS) }\end{array}$ & $\begin{array}{l}\text { COEFFI- } \\
\text { CIENT OF } \\
\text { VARI- } \\
\text { ATION }\end{array}$ & $\begin{array}{c}\text { PERCENT } \\
\text { OF } \\
\text { ANNUAL } \\
\text { RUNOFF }\end{array}$ \\
\hline OCTOBER & 26 & 87 & 47 & 16 & .34 & 1.0 \\
\hline NOVEMBER & 63 & 207 & 110 & 44 & .40 & 2.3 \\
\hline DECEMBER & 69 & 768 & 260 & 221 & .85 & 5.6 \\
\hline JANUARY & 65 & 830 & 304 & 240 & .79 & 6.5 \\
\hline FEBRUARY & 98 & 2352 & 647 & 516 & .80 & 13.8 \\
\hline MARCH & 197 & 1880 & 901 & 497 & .55 & 19.2 \\
\hline APRIL & 455 & 3058 & 1509 & 724 & .48 & 32.2 \\
\hline MAY & 72 & 1460 & 638 & 370 & .58 & 13.6 \\
\hline JUNE & 33 & 771 & 202 & 192 & .95 & 4.3 \\
\hline JULY & 7.6 & 102 & 40 & 27 & .68 & .9 \\
\hline AUGUST & 1.4 & 38 & 11 & 9.6 & .86 & .2 \\
\hline SEPTEMBER & 2.4 & 37 & 16 & 10 & .65 & .3 \\
\hline NNUAL & 139 & 647 & 387 & 160 & .41 & 100 \\
\hline
\end{tabular}

MAGNITUDE AND PROBABILITY OF INSTANTANEOUS PEAK FLOW BASED ON PERIOD OF RECORD 1942-59

DISCHARGE, IN CFS, FOR INDICATED RECURRENCE INTERVAL, IN YEARS, AND ANNUAL EXCEEDANCE PROBABILITY, IN PERCENT

$\begin{array}{ccccccc}1.25 & 2 & 5 & 10 & 25 & 50 & 100 \\ 80 \% & 50 \% & 20 \% & 10 \% & 4 \% & 2 \% & 1 \% \\ 2260 & 3580 & 5530 & 6880 & 8620 & - & -\end{array}$

WEIGHTED SKEW $=-.195$
MAGNITUDE AND PROBABILITY OF ANNUAL LOW FLOW BASED ON PERIOD OF RECORD 1943-59

\begin{tabular}{|c|c|c|c|c|c|c|}
\hline $\begin{array}{r}\text { PERIOD } \\
\text { /CON- } \\
\text { SECU- }\end{array}$ & \multicolumn{6}{|c|}{$\begin{array}{l}\text { DISCHARGE, IN CFS, FOR INDICATED RECURRENCE } \\
\text { INTERVAL, IN YEARS, AND ANNUAL NON- } \\
\text { EXCEEDANCE PROBABILITY, IN PERCENT }\end{array}$} \\
\hline $\begin{array}{l}\text { TIVE } \\
\text { DAYS) }\end{array}$ & $\begin{array}{c}2 \\
50 \%\end{array}$ & $\begin{array}{c}5 \\
20 \%\end{array}$ & $\begin{array}{l}10 \\
10 \%\end{array}$ & $\begin{array}{l}20 \\
5 \%\end{array}$ & $\begin{array}{l}50 \\
2 \%\end{array}$ & $\begin{array}{r}100 \\
1 \%\end{array}$ \\
\hline 1 & 4.2 & 1.9 & 1. &.$\varepsilon$ & -- & -- \\
\hline 3 & 4.5 & 2.1 & 1. & 1. & -- & -- \\
\hline 7 & 4.9 & 2.5 & 1. & 1.2 & -- & -- \\
\hline 14 & 5.5 & 3.0 & 2. & 1.7 & -- & -- \\
\hline 30 & 6.9 & 3.7 & 2. & 2. & - & -- \\
\hline 60 & 10 & 5.4 & 3. & 2.8 & -- & -- \\
\hline 90 & 15 & 9.1 & 7. & 5.6 & -- & -- \\
\hline 120 & 23 & 16 & 13 & 11 & -- & -- \\
\hline 183 & 49 & 36 & 31 & 28 & -- & -- \\
\hline
\end{tabular}

MAGNITUDE AND PROBABILITY OF ANNUAL HIGH FLOW BASED ON PERIOD OF RECORD 1942-59

\begin{tabular}{|c|c|c|c|c|c|c|}
\hline $\begin{array}{l}\text { PERIOD } \\
\text { (CON- } \\
\text { SECU- }\end{array}$ & \multicolumn{6}{|c|}{$\begin{array}{c}\text { DISCHARGE, IN CFS, FOR INDICATED RECURRENCE } \\
\text { INTERVAL, IN YEARS, AND ANNUAL } \\
\text { EXCEEDANCE PROBABILITY, IN PERCENT }\end{array}$} \\
\hline TIVE & 2 & 5 & 10 & 25 & 50 & 100 \\
\hline DAYS) & $50 \%$ & $20 \%$ & $10 \%$ & $4 \%$ & $2 \%$ & $1 \%$ \\
\hline 1 & 3360 & 5140 & 6190 & 7350 & - & - \\
\hline 3 & 2910 & 4430 & 5310 & 6280 & -- & -- \\
\hline 7 & 2460 & 3680 & 4380 & 5150 & -- & -- \\
\hline 15 & 2000 & 3100 & 3790 & 4590 & -- & -- \\
\hline 30 & 1600 & 2460 & 2990 & 3610 & -- & -- \\
\hline 60 & 1310 & 1910 & 2240 & 2570 & -- & -- \\
\hline 90 & 1100 & 1590 & 1850 & 2110 & - & -- \\
\hline
\end{tabular}

DURATION TABLE OF DAILY MEAN FLOW FOR PERIOD OF RECORD 1942-59

\begin{tabular}{|c|c|c|c|c|c|c|c|c|c|c|c|c|c|c|}
\hline $5 \%$ & $10 \%$ & $15 \%$ & $20 \%$ & $25 \%$ & $30 \%$ & $40 \%$ & $50 \%$ & $60 \%$ & $70 \%$ & $75 \%$ & $80 \%$ & $85 \%$ & $90 \%$ & $95 \%$ \\
\hline 1840 & 1100 & 785 & 582 & 431 & 322 & 173 & 104 & 73 & 48 & 36 & 25 & 18 & 10 & 5.2 \\
\hline
\end{tabular}


14080500 CROOKED RIVER NEAR PRINEVILLE, OR--Continued

STATISTICAL SUMMARIES (AFTER THE COMPLETION OF PRINEVILLE RESERVOIR)

MONTHLY AND ANNUAL MEAN DISCHARGES 1961-82

\begin{tabular}{|c|c|c|c|c|c|c|}
\hline MONTH & $\begin{array}{l}\text { MINIMUM } \\
\text { (CFS) }\end{array}$ & $\begin{array}{c}\text { MAXIMUM } \\
\text { (CFS) }\end{array}$ & $\begin{array}{l}\text { MEAN } \\
\text { (CFS) }\end{array}$ & $\begin{array}{l}\text { STAN- } \\
\text { DARD } \\
\text { DEVIA- } \\
\text { TION } \\
\text { (CFS) }\end{array}$ & $\begin{array}{l}\text { COEFFI- } \\
\text { CIENT OF } \\
\text { VARI- } \\
\text { ATION }\end{array}$ & $\begin{array}{c}\text { PERCENT } \\
\text { OF } \\
\text { ANNUAL } \\
\text { RUNOFF }\end{array}$ \\
\hline OCTOBER & 26 & 270 & 144 & 78 & .54 & 3.7 \\
\hline NOVEMBER & 14 & 369 & 111 & 94 & .85 & 2.8 \\
\hline DECEMBER & 26 & 1037 & 259 & 334 & 1.29 & 6.6 \\
\hline JANUARY & .2 & 1682 & 385 & 417 & 1.08 & 9.8 \\
\hline FEBRUARY & 2.7 & 1625 & 484 & 473 & .98 & 12.4 \\
\hline MARCH & 13 & 2035 & 466 & 531 & 1.14 & 11.9 \\
\hline APRIL & 19 & 1505 & 670 & 443 & .66 & 17.1 \\
\hline MAY & 67 & 1297 & 506 & 334 & .66 & 12.9 \\
\hline JUNE & 72 & 327 & 233 & 55 & .23 & 6.0 \\
\hline JULY & 87 & 303 & 233 & 52 & .22 & 6.0 \\
\hline AUGUST & 87 & 306 & 228 & 47 & .20 & 5.8 \\
\hline SEPTEMBER & 88 & 247 & 196 & 42 & .22 & 5.0 \\
\hline NNUAL & 47 & 616 & 325 & 154 & .47 & 100 \\
\hline
\end{tabular}

MAGNITUDE AND PROBABILITY OF INSTANTANEOUS PEAK FLOW BASED ON PERIOD OF RECORD

\begin{tabular}{|c|c|c|c|c|c|c|}
\hline \multicolumn{7}{|c|}{$\begin{array}{l}\text { DISCHARGE, IN CFS, FOR INDICATED RECURRENCE INTERVAL, IN } \\
\text { YEARS, AND ANNUAL EXCEEDANCE PROBABILITY, IN PERCENT }\end{array}$} \\
\hline $\begin{array}{l}1.25 \\
80 \%\end{array}$ & $\stackrel{2}{50 \%}$ & $\begin{array}{c}5 \\
20 \%\end{array}$ & $\begin{array}{l}10 \\
10 \%\end{array}$ & 25 & $\begin{array}{l}50 \\
2 \%\end{array}$ & $\begin{array}{r}100 \\
1 \%\end{array}$ \\
\hline-- & -- & -- & -- & - & - & -- \\
\hline
\end{tabular}

MAGNITUDE AND PROBABILITY OF ANNUAL LOW FLOW BASED ON PERIOD OF RECORD 1962-82

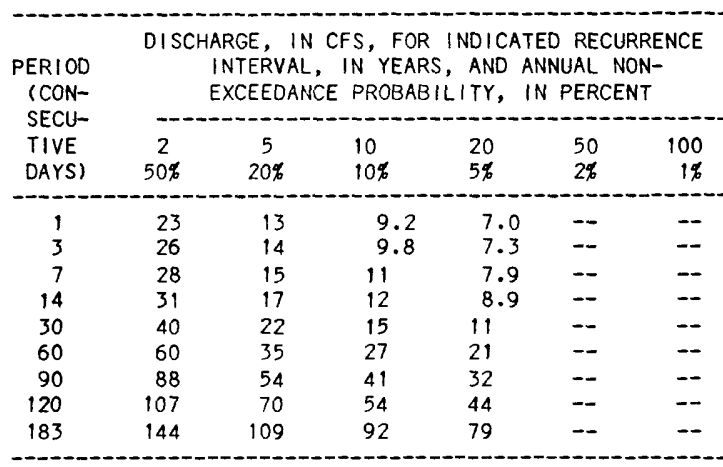

MAGNITUDE AND PROBABILITY OF ANNUAL HIGH FLOW BASED ON PERIOD OF RECORD 1961-82

\begin{tabular}{|c|c|c|c|c|c|c|}
\hline \multirow{3}{*}{$\begin{array}{l}\text { PERIOD } \\
\text { (CON- } \\
\text { SECU- } \\
\text { TIVE } \\
\text { DAYS) }\end{array}$} & \multicolumn{6}{|c|}{$\begin{array}{l}\text { DISCHARGE, IN CFS, FOR INDICATED RECURRENCE } \\
\text { INTERVAL, IN YEARS, AND ANNUAL } \\
\text { EXCEEDANCE PROBABILITY, IN PERCENT }\end{array}$} \\
\hline & $\stackrel{2}{50 \%}$ & ${ }^{5}$ & 10 & 25 & 50 & 100 \\
\hline & & & & & & \\
\hline 1 & 1760 & 2980 & 3520 & 3940 & - & -- \\
\hline 3 & 1710 & 2900 & 3440 & 3870 & - & - \\
\hline 7 & 1550 & 2690 & 3250 & 3750 & - & - \\
\hline 15 & 1300 & 2290 & 2820 & 3300 & -- & -- \\
\hline 30 & 1010 & 1780 & 2220 & 2660 & -- & - \\
\hline 60 & 741 & 1290 & 1600 & 1940 & - & -- \\
\hline 90 & 639 & 1070 & 1320 & 1590 & - & - \\
\hline
\end{tabular}

DURATION TABLE OF DAILY MEAN FLOW FOR PERIOD OF RECORD 1961-82

DISCHARGE, IN CFS, WHICH WAS EQUALED OR EXCEEDED FOR INDICATED PERCENT OF TIME

$\begin{array}{lllllllllllllll}5 \% & 10 \% & 15 \% & 20 \% & 25 \% & 30 \% & 40 \% & 50 \% & 60 \% & 70 \% & 75 \% & 80 \% & 85 \% & 90 \% & 95 \% \\ 1370 & 802 & 473 & 344 & 298 & 257 & 231 & 205 & 173 & 130 & 99 & 71 & 54 & 37 & 29\end{array}$




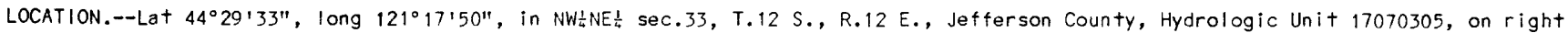
bank $0.2 \mathrm{mi}$ downstream from Opal Springs, $4.8 \mathrm{mi}$ southwest of Culver, and at mile 6.7 .

DRAINAGE AREA. - 4,300 $\mathrm{mi}^{2}$, approximately, of which $500 \mathrm{mi}^{2}$ is probably noncontributing.

PERIOD OF RECORD. - October 1961 to September 1982.

GAGE. - Water-stage recorder. Datum of gage is 1,953.60 $\mathrm{ft}$ National Geodetic Vertical Datum of 1929 (Portland General Electric Co. bench mark).

REMARKS.--Flow regulated since December 1960 by Prineville Reservoir and Ochoco Reservoir, capacity, 47,500 acre-ft. Many diversions for irrigation above station. Practically all of the summer flow comes from Opal Springs and other springs within 15 mi above station. Simultaneous records $(1961-63)$ at former gaging station 5.6 mi downstream indicated over 15 percent increase in summer flow from springs below this station.

AVERAGE DISCHARGE. - 21 years, $1,561 \mathrm{ft} / \mathrm{s}, 1,131,000$ acre $-\mathrm{ft} / \mathrm{yr}$.

EXTREMES FOR PERIOD OF RECORD.- Maximum discharge, $6,660 \mathrm{ft}^{3} / \mathrm{s} \mathrm{Dec.} 24,1964$, gage height, $9.36 \mathrm{ft}$; minimum, $836 \mathrm{ft} \mathrm{m}^{3} / \mathrm{s}$ Sept. 8 , 1981 , caused by refilling of small forebay upstream from gage.

STATISTICAL SUMMARIES

MONTHLY AND ANNUAL MEAN DISCHARGES 1962-82

\begin{tabular}{|c|c|c|c|c|c|c|}
\hline MONTH & $\begin{array}{l}\text { MINIMUM } \\
\text { (CFS) }\end{array}$ & $\begin{array}{l}\text { MAXIMUM } \\
\text { (CFS) }\end{array}$ & $\begin{array}{l}\text { MEAN } \\
\text { (CFS) }\end{array}$ & $\begin{array}{l}\text { STAN- } \\
\text { DARD } \\
\text { DEVIA- } \\
\text { TION } \\
\text { (CFS) }\end{array}$ & $\begin{array}{l}\text { COEFFI- } \\
\text { CIENT OF } \\
\text { VARI- } \\
\text { ATION }\end{array}$ & $\begin{array}{c}\text { PERCENT } \\
\text { OF } \\
\text { ANNUAL } \\
\text { RUNOFF }\end{array}$ \\
\hline OCTOBER & 1239 & 1650 & 1441 & 126 & .09 & 7.7 \\
\hline NOVEMBER & 1232 & 1724 & 1368 & 120 & .09 & 7.3 \\
\hline DECEMBER & 1179 & 2686 & 1560 & 436 & .28 & 8.3 \\
\hline JANUARY & 1182 & 3551 & 1712 & 555 & .32 & 9.1 \\
\hline FEBRUARY & 1250 & 3490 & 1868 & 620 & .33 & 10.0 \\
\hline MARCH & 1232 & 4208 & 1860 & 750 & .40 & 9.9 \\
\hline APRIL & 1192 & 3215 & 2028 & 552 & .27 & 10.8 \\
\hline MAY & 1173 & 2566 & 1663 & 422 & .25 & 8.9 \\
\hline JUNE & 1196 & 1478 & 1305 & 90 & .07 & 7.0 \\
\hline JULY & 1122 & 1389 & 1267 & 86 & .07 & 6.8 \\
\hline AUGUST & 1134 & 1593 & 1311 & 106 & .08 & 7.0 \\
\hline SEPTEMBER & 1187 & 1541 & 1379 & 102 & .07 & 7.3 \\
\hline JNUAL & 1278 & 1973 & 1561 & 213 & .14 & 100 \\
\hline
\end{tabular}

MAGNITUDE AND PROBABILITY OF INSTANTANEOUS PEAK FLOW BASED ON PERIOD OF RECORD

DISCHARGE, IN CFS, FOR INDICATED RECURRENCE INTERVAL, IN YEARS, AND ANNUAL EXCEEDANCE PROBABILITY, IN PERCENT

\begin{tabular}{lllllll}
1.25 & 2 & 5 & 10 & 25 & 50 & 100 \\
$80 \%$ & $50 \%$ & $20 \%$ & $10 \%$ & $4 \%$ & $2 \%$ & $1 \%$ \\
\hline- & & & & & & \\
\hline & - & - & - & - & - & -
\end{tabular}

WEIGHTED SKEW =

MAGNITUDE AND PROBABILITY OF ANNUAL LOW FLOW BASED ON PERIOD OF RECORD 1963-82

\begin{tabular}{|c|c|c|c|c|c|c|}
\hline $\begin{array}{l}\text { PERIOD } \\
\text { (CON- }\end{array}$ & \multicolumn{6}{|c|}{$\begin{array}{l}\text { DISCHARGE, IN CFS, FOR INDICATED RECURRENCE } \\
\text { INTERVAL, IN YEARS, AND ANNUAL NON- } \\
\text { EXCEEDANCE PROBABILITY, IN PERCENT }\end{array}$} \\
\hline $\begin{array}{l}\text { TIVE } \\
\text { DAYS) }\end{array}$ & $\begin{array}{c}2 \\
50 \%\end{array}$ & $\begin{array}{c}5 \\
20 \%\end{array}$ & $\begin{array}{l}10 \\
10 \%\end{array}$ & $\begin{array}{l}20 \\
5 \%\end{array}$ & $\begin{array}{l}50 \\
2 \%\end{array}$ & $\begin{array}{r}100 \\
1 \%\end{array}$ \\
\hline 1 & 1170 & 1130 & 1110 & 1090 & -- & -- \\
\hline 3 & 1180 & 1130 & 1110 & 1100 & -- & -- \\
\hline 7 & 1180 & 1140 & 1120 & 1110 & - & -- \\
\hline 14 & 1200 & 1150 & 1130 & 1110 & -- & -- \\
\hline 30 & 1210 & 1160 & 1140 & 1120 & -- & -- \\
\hline 60 & 1240 & 1180 & 1150 & 1130 & -- & -- \\
\hline 90 & 1260 & 1190 & 1160 & 1140 & -- & -- \\
\hline 120 & 1270 & 1210 & 1190 & 1160 & -- & -- \\
\hline 183 & 1320 & 1250 & 1220 & 1200 & -- & - \\
\hline
\end{tabular}

MAGNITUDE AND PROBABILITY OF ANNUAL HIGH FLOW BASED ON PERIOD OF RECORD 1962-82

\begin{tabular}{|c|c|c|c|c|c|c|}
\hline $\begin{array}{l}\text { PERIOD } \\
\text { (CON- } \\
\text { SECU- }\end{array}$ & \multicolumn{6}{|c|}{$\begin{array}{c}\text { DISCHARGE, IN CFS, FOR INDICATED RECURRENCE } \\
\text { INTERVAL, IN YEARS, AND ANNUAL } \\
\text { EXCEEDANCE PROBABILITY, IN PERCENT }\end{array}$} \\
\hline TIVE & 2 & 5 & 10 & 25 & 50 & 100 \\
\hline DAYS) & $50 \%$ & $20 \%$ & $10^{x}$ & $4 \%$ & $2 \%$ & 19 \\
\hline 1 & 3380 & 4550 & 5240 & 6010 & -- & -- \\
\hline 3 & 3310 & 4470 & 5150 & 5930 & - & - \\
\hline 7 & 3150 & 4260 & 4950 & 5750 & -- & - \\
\hline 15 & 2870 & 3850 & 4450 & 5180 & -- & - \\
\hline 30 & 2520 & 3310 & 3820 & 4450 & -- & -- \\
\hline 60 & 2130 & 2750 & 3170 & 3710 & - & -- \\
\hline 90 & 1990 & 2500 & 2840 & 3270 & -- & -- \\
\hline
\end{tabular}

DURATION TABLE OF DAILY MEAN FLOW FOR PERIOD OF RECORD 1962-82

DISCHARGE, IN CFS, WHICH WAS EOUALED OR EXCEEDED FOR INDICATED PERCENT OF TIME

\begin{tabular}{|c|c|c|c|c|c|c|c|c|c|c|c|c|c|c|}
\hline $5 \%$ & $10 \%$ & $15 \%$ & $20 \%$ & $25 \%$ & $30 \%$ & $40 \%$ & $50 \%$ & $60 \%$ & $70 \%$ & $75 \%$ & $80 \%$ & $85 \%$ & $90 \%$ & $95 \%$ \\
\hline 2940 & 2200 & 1820 & 1640 & 1560 & 1500 & 1430 & 1380 & 1340 & 1290 & 1270 & 1250 & 1220 & 1200 & 1150 \\
\hline
\end{tabular}


14087500 CROOKED RIVER NEAR CULVER, OR

LOCATION.--Lat $44^{\circ} 33^{\prime} 40^{\prime \prime}$, long $121^{\circ} 16^{\prime} 10^{\prime \prime}$, in sec.3 (50 ft west of $\frac{1}{4}$-corner on line between sec. 2 and 3 ), T. 12 S., R. 12 E., Jefferson County, Hydrologic Unit 17070305, on right bank $1 \mathrm{mi}$ upstream from mouth, $1.2 \mathrm{mi}$ downstream trom Cove powerplant, and $4 \mathrm{mi}$ northwest of Culver.

DRAINAGE AREA.--4,330 $\mathrm{mi}^{2}$, approximately, of which $500 \mathrm{mi}^{2}$ is probably noncontributing.

PERIOD OF RECORD.--October 1917 to September 1963.

GAGE.--Water-stage recorder. Datum of gage is 1,664.86 $\mathrm{ft}$ National Geodetic Vertical Datum of 1929. Prior to Aug. 2, 1945, staff gage at several sites within $1.2 \mathrm{mi}$ of present site at various datums.

REMARKS.--Flow slightly regulated (since 1919) by Ochoco Reservoir (capacity, 47,500 acre-ft) and since Dec. 13, 1960, by Prineville Reservoir (capacity, 152,800 acre- $f+$ ); occasional diurnal fluctuation caused by powerplant $1.2 \mathrm{mi}$ above station. Water is diverted for irrigation of land above station. The area served increased from about 30,000 acres in 1918 to 37,000 acres in 1946. Several hundred cubic teet per second of water diverted from Deschutes River for irrigation of other lands above station. Opal Springs and several other springs within about $17 \mathrm{mi}$ above station contribute about $1,000 \mathrm{ft}^{3} / \mathrm{s}$ to $\mathrm{flow}$.

AVERAGE DISCHARGE. - -46 years, $1,553+\mathrm{t}^{3} / \mathrm{s}, 1,124,000$ acre-t $t / \mathrm{yr}$.

EXTREMES FOR PERIOD OF RECORD.--Maximum discharge observed, $8,260 \mathrm{ft}^{3} / \mathrm{s} \mathrm{Mar.} \mathrm{30,} \mathrm{31,} \mathrm{1943,} \mathrm{gage} \mathrm{height,} 6.70 \mathrm{ft}$, site and datum then in use; minimum recorded, $920 \mathrm{ft}^{3} / \mathrm{s}$ Oct. 14, 1945

STATISTICAL SUMMARIES

MONTHLY AND ANNUAL MEAN DISCHARGES 1920-60

\begin{tabular}{|c|c|c|c|c|c|c|}
\hline MONTH & $\begin{array}{l}\text { MINIMUM } \\
\text { (CFS) }\end{array}$ & $\begin{array}{l}\text { MAXIMUM } \\
\text { (CFS) }\end{array}$ & $\begin{array}{l}\text { MEAN } \\
\text { (CFS) }\end{array}$ & $\begin{array}{c}\text { STAN- } \\
\text { DARD } \\
\text { DEV |A- } \\
\text { TION } \\
\text { (CFS) }\end{array}$ & $\begin{array}{l}\text { COEFFI- } \\
\text { CIENT OF } \\
\text { VARI- } \\
\text { ATION }\end{array}$ & $\begin{array}{c}\text { PERCENT } \\
\text { OF } \\
\text { ANNUAL } \\
\text { RUNOFF }\end{array}$ \\
\hline OCTOBER & 1060 & 1546 & 1285 & 131 & .10 & 6.9 \\
\hline NOVEMBER & 1116 & 1522 & 1319 & 125 & .09 & 7.0 \\
\hline DECEMBER & 1156 & 2197 & 1413 & 242 & .17 & 7.5 \\
\hline JANUARY & 1146 & 2326 & 1471 & 273 & .19 & 7.9 \\
\hline FEBRUARY & 1170 & 3977 & 1751 & 532 & .30 & 9.3 \\
\hline MARCH & 1294 & 3387 & 2097 & 590 & .28 & 11.2 \\
\hline APRIL & 1204 & 4820 & 2547 & 838 & .33 & 13.6 \\
\hline MAY & 1134 & 2966 & 1744 & 464 & .27 & 9.3 \\
\hline JUNE & 1060 & 2464 & 1376 & 269 & .20 & 7.3 \\
\hline JULY & 995 & 1491 & 1243 & 114 & .09 & 6.6 \\
\hline AUGUST & 970 & 1413 & 1229 & 112 & .09 & 6.6 \\
\hline SEPTEMBER & 1028 & 1442 & 1261 & 117 & .09 & 6.7 \\
\hline ANNUAL & 1234 & 2066 & 1559 & 237 & .15 & 100 \\
\hline
\end{tabular}

MAGNITUDE AND PROBABILITY OF INSTANTANEOUS PEAK FLOW BASED ON PERIOD OF RECORD $1920-60$

DISCHARGE, IN CFS, FOR INDICATED RECURRENCE INTERVAL, IN YEARS, AND ANNUAL EXCEEDANCE PROBABILITY, IN PERCENT

\begin{tabular}{|c|c|c|c|c|c|c|}
\hline $\begin{array}{l}1.25 \\
80 \%\end{array}$ & $\begin{array}{c}2 \\
50 \%\end{array}$ & $2^{5}$ & $\begin{array}{l}10 \\
10 \%\end{array}$ & $\begin{array}{l}25 \\
4 ?\end{array}$ & $\begin{array}{l}50 \\
2 \%\end{array}$ & $\begin{array}{r}100 \\
1 \%\end{array}$ \\
\hline 3170 & 4510 & 6280 & 7410 & 8770 & 9750 & 10700 \\
\hline
\end{tabular}

WEIGHTED SKEW $=-.228$
MAGNI TUDE AND PROBABILITY OF ANNUAL LOW FLOW BASED ON PERIOD OF RECORD 1921-60

\begin{tabular}{ccccccc} 
PERIOD & \multicolumn{1}{c}{ DISCHARGE, IN CFS, FOR INDICATED RECURRENCE } \\
ICON- & \multicolumn{5}{c}{$\begin{array}{c}\text { INTERVAL, IN YEARS, AND ANNUAL NON- } \\
\text { EXCEEDANCE PROBAB ILITY, IN PERCENT }\end{array}$} \\
$\begin{array}{l}\text { SECU- } \\
\text { TIVE }\end{array}$ & 2 & 5 & 10 & 20 & 50 & 100 \\
DAYS) & $50 \%$ & $20 \%$ & $10 \%$ & $5 \%$ & $2 \%$ & $1 \%$ \\
\hline 1 & 1200 & 1120 & 1080 & 1050 & 1010 & 987 \\
3 & 1200 & 1120 & 1080 & 1050 & 1010 & 987 \\
7 & 1200 & 1120 & 1080 & 1050 & 1010 & 987 \\
14 & 1210 & 1120 & 1080 & 1050 & 1010 & 987 \\
30 & 1210 & 1130 & 1080 & 1050 & 1010 & 987 \\
60 & 1220 & 1130 & 1090 & 1050 & 1020 & 990 \\
90 & 1230 & 1140 & 1100 & 1060 & 1030 & 1000 \\
120 & 1240 & 1150 & 1100 & 1070 & 1030 & 1010 \\
183 & 1260 & 1170 & 1130 & 1090 & 1060 & 1030 \\
\hline
\end{tabular}

MAGNITUDE AND PROBABILITY OF ANNUAL HIGH FLOW BASED ON PERIOD OF RECORD $1920-60$

\begin{tabular}{ccccccc} 
PERIOD & \multicolumn{5}{c}{ DISCHARGE, IN CFS, FOR INDICATED RECURRENCE } \\
INTERVAL, IN YEARS, AND ANNUAL \\
(CON-
\end{tabular}

DURATION TABLE OF DAILY MEAN FLOW FOR PERIOD OF RECORD 1920-60

\begin{tabular}{|c|c|c|c|c|c|c|c|c|c|c|c|c|c|c|}
\hline $5 \%$ & $10 \%$ & $15 \%$ & $20 \%$ & $25^{\circ / 5}$ & $30 \%$ & $40 \%$ & $50 \%$ & $60 \%$ & $70 \%$ & $75 \%$ & $80 \%$ & $85 \%$ & $90 \%$ & $95 \%$ \\
\hline 2840 & 2290 & 1940 & 1710 & 1580 & 1500 & 1430 & 1360 & 1300 & 1240 & 1210 & 1190 & 1160 & 1140 & 1110 \\
\hline
\end{tabular}


LOCATION.--Lat $44^{\circ} 25^{\prime} 35^{\prime \prime}$, long $121^{\circ} 43^{\prime} 30^{\prime \prime}$, in NE $\frac{1}{4}$ SW $\frac{1}{4}$ sec.24, T.13 S., R.8 E., Deschutes County, Hydrologic Unit 17070301, on left bank $300 \mathrm{ft}$ downstream from Suttle Lake and $13 \mathrm{mi}$ northwest of Sisters.

DRAINAGE AREA.--22.2 $\mathrm{mi}^{2}$.

PERIOD OF RECORD.--June to November 1911, March to September 1912, May to October 1913, April 1915 to September 1982. Monthly discharge only for some periods, published in WSP 1318.

GAGE.--Water-stage recorder. Datum of gage is 3,431.68 ft National Geodetic Vertical Datum of 1929. Prior to Apr. 1, 1916, nonrecording gage at two sites $400 \mathrm{ft}$ upstream at different datums. Apr. 1, 1916, to 0ct. 12, 1928, nonrecording gage or water-stage recorder at site $640 \mathrm{ft}$ downstream at different datum. Oct. 13, 1928, to Aug. 13, 1967, water-stage recorder at site $600 \mathrm{ft}$ downstream at datum $1.61 \mathrm{ft}$ lower.

REMARKS.--Records excellent. No regulation or diversion above station.

AVERAGE DISCHARGE.--67 years (water years 1916-82), $52.5 \mathrm{ft}^{3} / \mathrm{s}, 38,040 \mathrm{acre}-\mathrm{ft} / \mathrm{yr}$.

EXTREMES FOR PERIOD OF RECORD.--Maximum recorded discharge, $446 \mathrm{ft}^{3} / \mathrm{s}$, Dec. 15, 1977, gage height, $4.78 \mathrm{ft}$, but may have been higher during period of no gage-height record Dec. 23,$1964 ;$ minimum, $1.0 \mathrm{ft}^{3} / \mathrm{s} \mathrm{Nov.} \mathrm{4,5,1940;minimum} \mathrm{daily,} 8 \mathrm{ft}{ }^{3} / \mathrm{s} \mathrm{Nov}$. 5 , 1940, Oct. 6, 1942 .

\section{STATISTICAL SUMMARIES}

MONTHLY AND ANNUAL MEAN DISCHARGES 1918-82

\begin{tabular}{|c|c|c|c|c|c|c|}
\hline MONTH & $\begin{array}{l}\text { MINIMUM } \\
\text { (CFS) }\end{array}$ & $\begin{array}{l}\text { MAXIMUM } \\
\text { (CFS) }\end{array}$ & $\begin{array}{l}\text { MEAN } \\
\text { (CFS) }\end{array}$ & $\begin{array}{l}\text { STAN- } \\
\text { DARD } \\
\text { DEVIA- } \\
\text { TION } \\
\text { (CFS) }\end{array}$ & $\begin{array}{l}\text { COEFFI- } \\
\text { CIENT OF } \\
\text { VARI- } \\
\text { ATION }\end{array}$ & $\begin{array}{c}\text { PERCENT } \\
\text { OF } \\
\text { ANNUAL } \\
\text { RUNOFF }\end{array}$ \\
\hline OCTOBER & 17 & 67 & 33 & 7.2 & .22 & 5.2 \\
\hline NOVEMBER & 24 & 98 & 41 & 13 & .31 & 6.5 \\
\hline DECEMBER & 23 & 178 & 60 & 32 & .53 & 9.6 \\
\hline JANUARY & 23 & 147 & 65 & 30 & .46 & 10.3 \\
\hline FEBRUARY & 28 & 132 & 62 & 24 & .39 & 9.9 \\
\hline MARCH & 27 & 169 & 56 & 20 & .36 & 8.9 \\
\hline APRIL & 34 & 111 & 62 & 15 & .25 & 9.9 \\
\hline MAY & 38 & 157 & 80 & 25 & .31 & 12.7 \\
\hline JUNE & 29 & 162 & 67 & 29 & .44 & 10.6 \\
\hline JULY & 22 & 69 & 39 & 9.2 & .24 & 6.3 \\
\hline AUGUST & 22 & 43 & 32 & 5.6 & .17 & 5.1 \\
\hline SEPTEMBER & 19 & 40 & 31 & 5.1 & .17 & 4.9 \\
\hline ANNUAL & 27 & 77 & 52 & 11 & .21 & 100 \\
\hline
\end{tabular}

MAGNITUDE AND PROBABILITY OF INSTANTANEOUS PEAK FLOW BASED ON PERIOD OF RECORD 1916-82

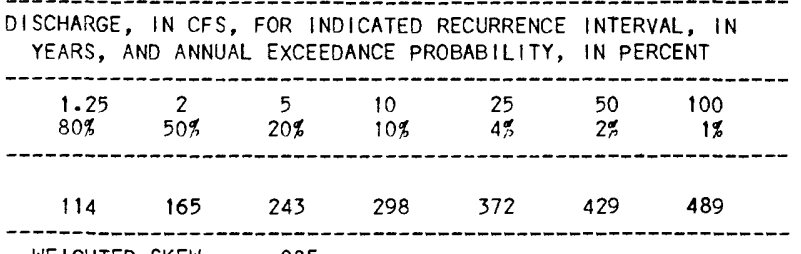

MAGNITUDE AND PROBABILITY OF ANNUAL LOW FLOW BASED ON PERIOD OF RECORD 1919-82

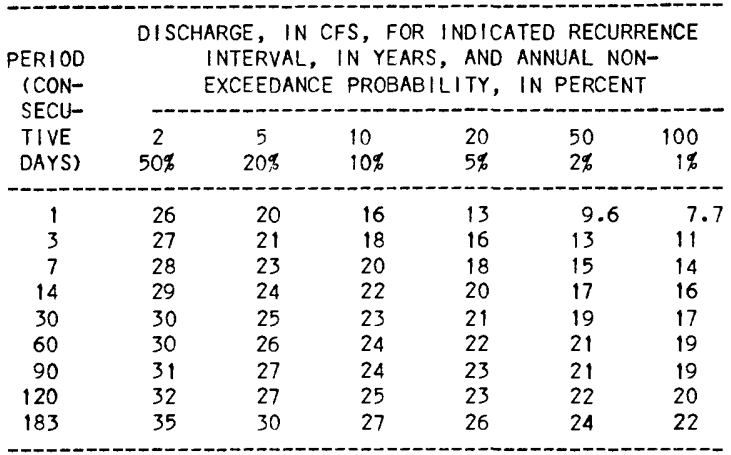

MAGNITUDE AND PROBABILITY OF ANNUAL HIGH FLOW BASED ON PERIOD OF RECORD 1918-82

\begin{tabular}{|c|c|c|c|c|c|c|}
\hline $\begin{array}{l}\text { PERIOD } \\
\text { (CON- } \\
\text { SECU- }\end{array}$ & \multicolumn{6}{|c|}{$\begin{array}{c}\text { DISCHARGE, IN CFS, FOR INDICATED RECURRENCE } \\
\text { INTERVAL, IN YEARS, AND ANNUAL } \\
\text { EXCEEDANCE PROBABILITY, IN PERCENT }\end{array}$} \\
\hline TIVE & 2 & 5 & 10 & 25 & 50 & 100 \\
\hline DAYS) & $50 \%$ & $20 \%$ & $10 \%$ & $4 \%$ & $2 \%$ & $1 \%$ \\
\hline 1 & 154 & 241 & 307 & 399 & 475 & 556 \\
\hline 3 & 149 & 229 & 287 & 367 & 431 & 498 \\
\hline 7 & 139 & 202 & 244 & 298 & 338 & 378 \\
\hline 15 & 123 & 165 & 190 & 219 & 239 & 258 \\
\hline 30 & 106 & 135 & 152 & 170 & 181 & 192 \\
\hline 60 & 90 & 110 & 120 & 131 & 137 & 142 \\
\hline 90 & 80 & 96 & 104 & 111 & 116 & 119 \\
\hline
\end{tabular}

DURATION TABLE OF DAILY MEAN FLOW FOR PERIOD OF RECORD 1918-82

DISCHARGE, IN CFS, WHICH WAS EQUALED OR EXCEEDED FOR INDICATED PERCENT OF TIME

\begin{tabular}{|c|c|c|c|c|c|c|c|c|c|c|c|c|c|c|}
\hline $5 \%$ & $10 \%$ & $15 \%$ & $20 \%$ & $25 \%$ & $30 \%$ & $40 \%$ & $50 \%$ & $60 \%$ & $70 \%$ & $75 \%$ & $80 \%$ & $85 \%$ & $90 \%$ & $95 \%$ \\
\hline 108 & 89 & 77 & 69 & 63 & 57 & 49 & 43 & 39 & 35 & 34 & 32 & 30 & 28 & 25 \\
\hline
\end{tabular}


14091500 METOLIUS RIVER NEAR GRANDVIEW, OR

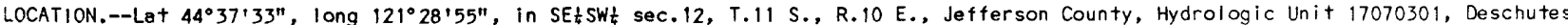
National Forest, on right bank $1.0 \mathrm{mi}$ upstream from maximum contralled pool of Lake Billy Chinook, $15.0 \mathrm{mi}$ northwest of Culver, and at mile 13.6 .

DRAINAGE AREA.--316 $\mathrm{mi}^{2}$, at cableway $1.0 \mathrm{mi}$ downstream, where all discharge measurements are made. Hydrologic drainage boundary uncertain owing to ground-water exchange.

PERIOD OF RECORD.--April 1910 to February 1912 (gage heights and discharge measurements only), March 1912 to December 1913 , October 1921 to September 1982. Published as "at Hubbard's ranch, near Sisters" 1910, and as "at Hubbard's ranch, near Grandview" 1910-13.

GAGE.--Water-stage recorder. Datum of gage is $1,974.36 \mathrm{ft}$ National Geodetic Vertical Datum of 1929 (levels by Portland General Electric Co.). Prior to Dec. 31, 1913, nonrecording gage at site $2.3 \mathrm{mi}$ upstream at different datum. Oct. 1, 1921, to May 3, 1949, nonrecording gage and May 4, 1949, to June 18, 1963, water-stage recorder at site $2.7 \mathrm{mi}$ downstream at datum 64 ft lower.

REMARKS. - No regulation. Many small diversions for irrigation above station. Stream is spring fed. Records herein are for measuring site.

AVERAGE DISCHARGE.--62 years (water years 1913, 1922-82), $1,493 \mathrm{ft}+3 / \mathrm{s}, 1,082,000$ acre- $f+$ t/yr.

EXTREMES FOR PERIOD OF RECORD.--Maximum discharge, $7,530 \mathrm{ft}^{3} / \mathrm{s}$ Dec. 24,1964 , gage height, $6.81 \mathrm{ft} ; \mathrm{minimum}, 1,080 \mathrm{ft} / \mathrm{s}$ Feb. 17 , 1932, Oct. 2-31, Nov. 6, 7, 10-14, 1942.

STATISTICAL SUMMARIES

MONTHLY AND ANNUAL MEAN DISCHARGES 1922-82

\begin{tabular}{|c|c|c|c|c|c|c|}
\hline MONTH & $\begin{array}{l}\text { MIINIAYUM } \\
\text { (CFS) }\end{array}$ & $\begin{array}{l}\text { MAXIMUM } \\
\text { (CFS) }\end{array}$ & $\begin{array}{l}\text { MEAN } \\
\text { (CFS) }\end{array}$ & $\begin{array}{l}\text { STAN- } \\
\text { DARD } \\
\text { DEV |A- } \\
\text { TION } \\
\text { (CFS) }\end{array}$ & $\begin{array}{l}\text { COEFFI- } \\
\text { CIENT OF } \\
\text { VAR!- } \\
\text { ATION }\end{array}$ & $\begin{array}{c}\text { PERCENT } \\
\text { OF } \\
\text { ANINUAL } \\
\text { RUNOFF }\end{array}$ \\
\hline OCTOBER & 1081 & 1560 & 1345 & 123 & .09 & 7.5 \\
\hline NOVEMBER & 1140 & 1816 & 1392 & 145 & .10 & 7.8 \\
\hline DECEMBER & 1110 & 2454 & 1494 & 253 & .17 & 8.3 \\
\hline JANUARY & 1154 & 2250 & 1523 & 263 & .17 & 8.5 \\
\hline FEBRUARY & 1148 & 2333 & 1556 & 274 & .18 & 8.7 \\
\hline MARCH & 1157 & 2504 & 1526 & 219 & .14 & 8.5 \\
\hline APRIL & 1162 & 1960 & 1543 & 181 & .12 & 8.6 \\
\hline MAY & 1244 & 2095 & 1606 & 192 & .12 & 9.0 \\
\hline JUNE & 1196 & 2118 & 1636 & 224 & .14 & 9.1 \\
\hline JULY & 1173 & 1910 & 1517 & 187 & .12 & 8.5 \\
\hline AUGUST & 1136 & 1746 & 1414 & 151 & .11 & 7.9 \\
\hline SEPTEMBER & 1103 & 1631 & 1364 & 131 & .10 & 7.6 \\
\hline ANNUAL & 1167 & 1845 & 1492 & 160 & .11 & 100 \\
\hline
\end{tabular}

MAGNITUDE ANO PROBABILITY OF INSTANTANEOUS PEAK FLOW BASEO DN PERIOD OF RECORD 1922-82

\begin{tabular}{|c|c|c|c|c|c|c|}
\hline \multicolumn{7}{|c|}{$\begin{array}{l}\text { DISCHARGE, IN CFS, FOR INDICATED RECURRENCE INTERVAL, IN } \\
\text { YEARS, AND ANNUAL EXCEEDANCE PROBABILITY, IN PERCENT }\end{array}$} \\
\hline $\begin{array}{l}1.25 \\
80 \%\end{array}$ & $\stackrel{2}{50 \%}$ & $20 \%$ & $\begin{array}{l}10 \\
10 \%\end{array}$ & $\begin{array}{l}25 \\
4^{g}\end{array}$ & $\begin{array}{l}50 \\
2 \%\end{array}$ & $\begin{array}{r}100 \\
10\end{array}$ \\
\hline 1980 & 2600 & 3560 & 4260 & 5220 & 6010 & 6840 \\
\hline
\end{tabular}

WEIGHTED SKEW $=.490$
MAGNITUDE AND PROBABILITY OF ANNUAL LOW FLOW BASED ON PERIOD OF RECORD 1923-82

\begin{tabular}{|c|c|c|c|c|c|c|}
\hline $\begin{array}{l}\text { PERIOD } \\
\text { (CON- }\end{array}$ & \multicolumn{6}{|c|}{$\begin{array}{l}\text { DISCHARGE, IN CFS, FOR INDICATED RECURRENCE } \\
\text { INTERVAL, IN YEARS, AND ANNUAL NON- } \\
\text { EXCEEDANCE PROBABILITY, IN PERCENT }\end{array}$} \\
\hline TIVE & & & & & & \\
\hline DAYS) & $50 \%$ & $20 \%$ & $10 \%$ & $5 \%$ & $2 \%$ & $1 \%$ \\
\hline & & & & & & \\
\hline 1 & 1280 & 1180 & 1130 & 1100 & 1050 & 1020 \\
\hline 3 & 1280 & 1190 & 1140 & 1100 & 1060 & 1030 \\
\hline 7 & 1280 & 1190 & 1140 & 1110 & 1060 & 1030 \\
\hline 14 & 1290 & 1200 & 1150 & 1110 & 1070 & 1040 \\
\hline 30 & 1300 & 1210 & 1160 & 1120 & 1080 & 1050 \\
\hline 60 & 1320 & 1220 & 1170 & 1130 & 1090 & 1060 \\
\hline 90 & 1330 & 1230 & 1180 & 1140 & 1100 & 1070 \\
\hline 120 & 1340 & 1240 & 1190 & 1150 & 1110 & 1080 \\
\hline 183 & 1370 & 1270 & 1210 & 1170 & 1120 & 1100 \\
\hline
\end{tabular}

MAGN ITUDE AND PROBABILITY OF ANNUAL HIGH FLOW BASED ON PERIOO OF RECORD 1922-82

\begin{tabular}{|c|c|c|c|c|c|c|}
\hline $\begin{array}{l}\text { PERIOD } \\
\text { (CON- } \\
\text { SECU- }\end{array}$ & \multicolumn{6}{|c|}{$\begin{array}{c}\text { DISCHARGE, IN CFS, FOR IND ICATED RECURRENCE } \\
\text { INTERVAL, IN YEARS, AND ANNUAL } \\
\text { EXCEEDANCE PROBABILITY, IN PERCENT }\end{array}$} \\
\hline TIVE & 2 & 5 & 10 & 25 & 50 & 100 \\
\hline DAYS) & $50^{d}$ & $20^{d}$ & $10^{\circ}$ & $4^{x}$ & $2:$ & $1 \%$ \\
\hline 1 & 2420 & 3230 & 3840 & 4690 & 5390 & 6150 \\
\hline 3 & 2220 & 2930 & 3460 & 4210 & 4830 & 5510 \\
\hline 7 & 2050 & 2590 & 2980 & 3520 & 3960 & 4420 \\
\hline 15 & 1910 & 2290 & 2540 & 2870 & 3110 & 3360 \\
\hline 30 & 1800 & 2070 & 2240 & 2440 & 2580 & 2720 \\
\hline 60 & 1710 & 1930 & 2050 & 2190 & 2280 & 2370 \\
\hline 90 & 1670 & 1850 & 1950 & 2060 & 2130 & 2190 \\
\hline
\end{tabular}

DURATION TABLE OF DAILY MEAN FLOW FOR PERIOD OF RECORD 1922-82

\begin{tabular}{|c|c|c|c|c|c|c|c|c|c|c|c|c|c|c|}
\hline $5 \%$ & $10 \%$ & $15 \%$ & $20 \%$ & $25 \%$ & $30 \%$ & $40 \%$ & $50 \%$ & $60^{\circ}$ & $70^{x}$ & $75 \%$ & $80 \%$ & $35:$ & $90^{\circ}$ & $95 d$ \\
\hline 1910 & 1800 & 1730 & 1680 & 1630 & 1580 & 1510 & 1460 & 1410 & 1360 & 1330 & 1300 & 1270 & 1230 & 1160 \\
\hline
\end{tabular}




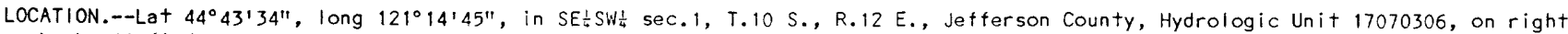
bank $400 \mathrm{ft}$ downstream from reregulating dam, $2.7 \mathrm{mi}$ downstream from Pelton Dam, $8.5 \mathrm{mi}$ northwest of Madras, and at mile 100.1 .

DRAINAGE AREA. $-7,820 \mathrm{mi}^{2}$, approximately.

PERIOD OF RECORD.--October 1923 to September 1982.

GAGE.--Water-stage recorder. Datum of gage is $1,300.25 \mathrm{ft} N a+i o n a l$ Geodetic Vertical Datun of 1929 (levels by Portland General Electric Co.). See WSP 1738 for history of changes prior to Hov. 23, 1957.

REMARKS.--Diurnal fluctuation caused by Lake Simtustus and reregulating reservoir since 1957, combined capacity for normal operation, 6,500 acre-ft. Some winter and spring runoff stored in Ochoco Reservoir, capacity, 47,500 acre-ft, in Crescent Lake, Crane Prairie and Wickiup Reservoirs, combined capacity, 354,600 acre-ft, and since 1960, in Prineville Reservoir, capacity, 152,800 acre-ft, and since 1964, in Lake Billy Chinook, capacity, 534,700 acre-ft. Large diversions in upper basin for irrigation.

AVERAGE DISCHARGE.--59 years, $4,493 \mathrm{ft} / \mathrm{s}, 3,255,000$ acre-t $t / \mathrm{yr}$.

EXTREMES FOR PERIOD OF RECORD.--Maximum discharge, 15,800 $\mathrm{ft}^{3} / \mathrm{s}$ Dec. 28 , 1964, gage height, $6.29 \mathrm{ft}$; maximum gage height, $6.89 \mathrm{ft}$ Jan. 1, 1943, site and datum then in use; minimum discharge, $916 \mathrm{tt} / \mathrm{s}$ July 4 , 1982 , caused by power company testing control gates on dam.

STATISTICAL SUMMARIES (BEFORE CONSTRUCTION OF PELTON DAM)

MONTHLY AND ANNUAL MEAN DISCHARGES 1925-56

\begin{tabular}{|c|c|c|c|c|c|c|}
\hline MONTH & $\begin{array}{l}\text { MINIMUM } \\
\text { (CFS) }\end{array}$ & $\begin{array}{l}\text { MAXIMUM } \\
\text { (CFS) }\end{array}$ & $\begin{array}{l}\text { MEAN } \\
\text { (CFS) }\end{array}$ & $\begin{array}{l}\text { STAN- } \\
\text { DARD } \\
\text { DEVIA- } \\
\text { TION } \\
\text { (CFS) }\end{array}$ & $\begin{array}{l}\text { COEFFI- } \\
\text { CIENT OF } \\
\text { VARI- } \\
\text { ATION }\end{array}$ & $\begin{array}{c}\text { PERCENT } \\
\text { OF } \\
\text { ANNUAL } \\
\text { RUNOFF }\end{array}$ \\
\hline OCTOBER & 3098 & 4773 & 3709 & 415 & .11 & 7.0 \\
\hline $\begin{array}{l}\text { NOVEMBER } \\
\text { DECEMBER }\end{array}$ & $\begin{array}{l}3570 \\
3731\end{array}$ & $\begin{array}{l}5102 \\
6520\end{array}$ & $\begin{array}{l}4227 \\
4615\end{array}$ & $\begin{array}{l}438 \\
646\end{array}$ & $\begin{array}{l}.10 \\
.14\end{array}$ & $\begin{array}{l}8.0 \\
8.7\end{array}$ \\
\hline JANUARY & 3844 & 6968 & 4735 & 750 & .16 & 3.0 \\
\hline FEBRUARY & 3900 & 8187 & 5056 & 962 & .19 & 9.6 \\
\hline MARCH & 4135 & 7155 & 5413 & 916 & .17 & 10.3 \\
\hline APRIL & 3723 & 8495 & 5496 & 1279 & .23 & 10.4 \\
\hline MAY & 3431 & 6949 & 4438 & 806 & .18 & 8.4 \\
\hline JUNE & 3239 & 5759 & 4136 & 698 & .17 & 7.8 \\
\hline JULY & 3090 & 4498 & 3715 & 406 & .11 & 7.0 \\
\hline AUGUST & 3015 & 4309 & 3582 & 384 & .11 & 6.8 \\
\hline SEPTEMBER & 3022 & 4375 & 3629 & 387 & .11 & 6.9 \\
\hline ANNUAL & 3591 & 5632 & 4391 & 542 & .12 & 100 \\
\hline
\end{tabular}

MAGNITUDE AND PROBABILITY OF INSTANTANEOUS PEAK FLOW BASED ON PERIOD OF RECORD 1925-56

DISCHARGE, IN CFS, FOR INDICATED RECURRENCE INTERVAL, IN YEARS, AND ANNUAL EXCEEDANCE PROBABILITY, IN PERCENT

\begin{tabular}{ccccccc}
1.25 & 2 & 5 & 10 & 25 & 50 & 100 \\
$80 \%$ & $50 \%$ & $20 \%$ & $10 \%$ & $4 \%$ & $2 \%$ & $1 \%$ \\
\hline 6580 & 8160 & 10200 & 11600 & 13200 & 14400 & - \\
\hline
\end{tabular}

WEIGHTED SKEW $=.174$
MAGNITUDE AND PROBABILITY OF ANNUAL LOW FLOW BASED ON PERIOD OF RECORD 1926-56

\begin{tabular}{|c|c|c|c|c|c|c|}
\hline $\begin{array}{l}\text { PERIOD } \\
\text { CCON- } \\
\text { SECU- }\end{array}$ & \multicolumn{6}{|c|}{$\begin{array}{l}\text { DISCHARGE, IN CFS, FOR INDICATED RECURRENCE } \\
\text { INTERVAL, IN YEARS, AND ANNUAL NON- } \\
\text { EXCEEDANCE PROBABILITY, IN PERCENT }\end{array}$} \\
\hline TIVE & 2 & 5 & 10 & 20 & 50 & 100 \\
\hline DAYS) & $50 \%$ & $20 \%$ & $10 \%$ & $5 \%$ & 2, & $1 \%$ \\
\hline 1 & 7,400 & 3100 & 3100 & 030 & 2960 & -- \\
\hline 3 & 3410 & 3200 & 3110 & 3040 & 2960 & -- \\
\hline 7 & 3420 & 3210 & 3120 & 3040 & 2970 & -- \\
\hline 14 & 3440 & 3220 & 3120 & 3050 & 2970 & -- \\
\hline 30 & 3460 & 3230 & 3130 & 3060 & 2980 & -- \\
\hline 60 & 3500 & 3250 & 3140 & 3060 & 2980 & -- \\
\hline 90 & 3540 & 3280 & 3160 & 3070 & 2980 & -- \\
\hline 120 & 3590 & 3320 & 3190 & 3100 & 3000 & \\
\hline 183 & 3730 & 3420 & 3280 & 3180 & 3070 & $\ldots$ \\
\hline
\end{tabular}

MAGNITUDE AND PROBABILITY OF ANNUAL HIGH FLOW BASED ON PERIOD OF RECORD $1925-56$

\begin{tabular}{|c|c|c|c|c|c|c|}
\hline $\begin{array}{l}\text { PERIOD } \\
\text { (CON- } \\
\text { SECU- }\end{array}$ & \multicolumn{6}{|c|}{$\begin{array}{c}\text { DISCHARGE, IN CFS, FOR INDICATED RECURRENCE } \\
\text { INTERVAL, IN YEARS, AND ANNUAL } \\
\text { EXCEEDANCE PROBABILITY, IN PERCENT }\end{array}$} \\
\hline TIVE & 2 & 5 & 10 & 25 & 50 & 100 \\
\hline DAYS) & $50 \%$ & $20 \%$ & $10 \%$ & $4 \%$ & $2 \%$ & $1 \%$ \\
\hline 1 & 7760 & 9780 & 11100 & 12800 & 14100 & -- \\
\hline 3 & 7420 & 9310 & 10600 & 12200 & 13400 & -- \\
\hline 7 & 6900 & 8470 & 9510 & 10800 & 11800 & - \\
\hline 15 & 6340 & 7710 & 8650 & 9870 & 10800 & -- \\
\hline 30 & 5890 & 7050 & 7840 & 8870 & 9660 & -- \\
\hline 60 & 5540 & 6480 & 7100 & 7880 & 8460 & -- \\
\hline 90 & 5310 & 6140 & 6690 & 7380 & 7890 & -- \\
\hline
\end{tabular}

DURATION TABLE OF DAILY MEAN FLOWI FOR PERIOD OF RECORD 1925-56

\begin{tabular}{|c|c|c|c|c|c|c|c|c|c|c|c|c|c|c|}
\hline $5 \%$ & $10 \%$ & $15 \%$ & $20 \%$ & $25 \%$ & $30 \%$ & $40 \%$ & $50 \%$ & $60^{\circ}$ & $70 \%$ & $75 \%$ & $80 \%$ & $85 \%$ & $90 \%$ & $95 \%$ \\
\hline 6430 & 5730 & 5310 & 4980 & 4770 & 4590 & 4370 & 4170 & 3990 & 3760 & 3650 & 3540 & 3440 & 3350 & 3230 \\
\hline
\end{tabular}


14092500 DESCHUTES RIVER NEAR MADRAS, OR--Continued

STATISTICAL SUMMARIES (AFTER COHPLETION OF ROUND BUTTE DAM)

MONTHLY AND AHMUAL MEAN! DISCHARGES 1964-82

\begin{tabular}{|c|c|c|c|c|c|c|}
\hline YONTH & $\begin{array}{l}\text { MINIIUUA } \\
\text { (CFS) }\end{array}$ & $\begin{array}{l}\text { MAXIMUM } \\
\text { (CFS) }\end{array}$ & $\begin{array}{l}\text { YEAN } \\
\text { (CFS) }\end{array}$ & $\begin{array}{l}\text { STAN- } \\
\text { DARD } \\
\text { DEVIA- } \\
\text { TION } \\
\text { (CFS) }\end{array}$ & $\begin{array}{l}\text { COEFFI- } \\
\text { CIENT OF } \\
\text { VARI- } \\
\text { ATION }\end{array}$ & $\begin{array}{c}\text { PERCENT } \\
\text { OF } \\
\text { ANNUAL } \\
\text { RUNOFF }\end{array}$ \\
\hline OCTOBER & 3085 & 5097 & 4105 & 452 & .11 & 7.4 \\
\hline NOVEMBER & 3521 & 5361 & 4620 & 420 & .09 & 8.3 \\
\hline DECEMBER & 3951 & 6655 & 5174 & 681 & .13 & 9.3 \\
\hline JANUARY & 3305 & 8097 & 5641 & 1068 & .19 & 10.2 \\
\hline FEBRUARY & 3094 & 8182 & 5243 & 1308 & .25 & 9.5 \\
\hline MARCH & 3455 & 9590 & 5259 & 1357 & .26 & 9.5 \\
\hline APRIL & 3602 & 6804 & 4895 & 861 & .18 & 8.8 \\
\hline MAY & 3554 & 5505 & 4345 & 568 & .13 & 7.8 \\
\hline JUNE & 3492 & 5134 & 4232 & 447 & .11 & 7.6 \\
\hline JULY & 3059 & 4786 & 4022 & 420 & .10 & 7.3 \\
\hline AUGUST & 3064 & 4718 & 3939 & 358 & .09 & 7.1 \\
\hline SEPTEMBER & 3021 & 4414 & 3036 & 304 & .08 & 7.1 \\
\hline NNUAL & 3558 & 5357 & 4615 & 498 & .11 & 100 \\
\hline
\end{tabular}

MAGNITUDE AND PROBABILITY OF INSTANTANEOUS PEAK FLON BASED ON PERIOD OF RECORD

DISCHARGE, IN CFS, FOR INDICATED RECURRENCE INTERVAL, IN YEARS, AND ANNUAL EXCEEDANCE PROBABILITY, IN PERCENT

\begin{tabular}{ccccccc}
1.25 & 2 & 5 & 10 & 25 & 50 & 100 \\
80, & $50 \%$ & $20 \%$ & $10 \%$ & $4^{\alpha} \frac{10}{2}$ & 2 & $1 \%$ \\
\hline- & - & - & - & - & - & -
\end{tabular}

WEIGHTED SKEW = --
MAGNITUDE AND PROBABILITY OF ANNUAL LOW FLOW BASED ON PERIOD OF RECORD 1965-82

\begin{tabular}{|c|c|c|c|c|c|c|}
\hline $\begin{array}{l}\text { PERIOD } \\
\text { (CON- }\end{array}$ & \multicolumn{6}{|c|}{$\begin{array}{l}\text { DISCHARGE, IN CFS, FOR IND ICATED RECURRENCE } \\
\text { INTERVAL, IN YEARS, AND ANNUAL NON- } \\
\text { EXCEEDANCE PROBABILITY, IN PERCENT }\end{array}$} \\
\hline SECU- & & & & & 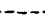 & \\
\hline TIVE & 2 & 5 & 10 & 20 & 50 & 100 \\
\hline DAYS) & $50 \%$ & $20 \%$ & $10^{2}$ & $5^{\alpha}$, & $2^{\alpha}$ & $1 \%$ \\
\hline & & & & & & \\
\hline 1 & 3240 & 3010 & 2900 & 2820 & -- & - \\
\hline 3 & 3330 & 3110 & 3000 & 2920 & -- & - \\
\hline 7 & 3460 & 3220 & 3110 & 3020 & - & - \\
\hline 14 & 3610 & 3350 & 3220 & 3110 & -- & -- \\
\hline 30 & 3820 & 3550 & 3400 & 3260 & -- & -- \\
\hline 60 & 3890 & 3610 & 3440 & 3300 & -- & -- \\
\hline 90 & 3930 & 3630 & 3460 & 3320 & - & -- \\
\hline 120 & 3970 & 3650 & 3480 & 3330 & -- & - \\
\hline 183 & 4040 & 3720 & 3550 & 3410 & -- & -- \\
\hline
\end{tabular}

MAGNITUDE AND PROBABILITY OF ANNUAL HIGH FLOW BASED ON PERIOD OF RECORD 1964-82

\begin{tabular}{|c|c|c|c|c|c|c|}
\hline \multirow{3}{*}{$\begin{array}{l}\text { PERIOD } \\
\text { (CON- } \\
\text { SECU- } \\
\text { TIVE } \\
\text { DAYS) }\end{array}$} & \multicolumn{6}{|c|}{$\begin{array}{c}\text { DISCHARGE, IN CFS, FOR INDICATED RECURRENCE } \\
\text { INTERVAL, IN YEARS, AND ANNUAL } \\
\text { EXCEEDANCE PROBABILITY, IN PERCENT }\end{array}$} \\
\hline & 2 & 5 & 10 & 25 & 50 & 100 \\
\hline & $50 \%$ & $20 \%$ & $10 \%$ & $4 \%$ & $2 \%$ & $1 \%$ \\
\hline 1 & 8210 & 10600 & 12300 & 14600 & -- & -- \\
\hline 3 & 8000 & 10200 & 11800 & 13800 & -- & -- \\
\hline 7 & 7550 & 9460 & 10800 & 12500 & -- & -- \\
\hline 15 & 6860 & 8490 & 9640 & 11200 & -- & -- \\
\hline 30 & 6270 & 7530 & 8390 & 9510 & -- & -- \\
\hline 60 & 5740 & 6700 & 7350 & 8210 & -- & -- \\
\hline 90 & 5570 & 6410 & 6930 & 7590 & -- & - \\
\hline
\end{tabular}

DURATION TABLE OF DAILY MEAN FLOW FOR PERIOD OF RECORD 1964-82

DISCHARGE, IN CFS, WHICH WAS EQUALED OR EXCEEDED FOR INDICATED PERCENT OF TIME

\begin{tabular}{|c|c|c|c|c|c|c|c|c|c|c|c|c|c|c|}
\hline $5 \%$ & $10 \%$ & $15 \%$ & $20 \%$ & $25 \%$ & $30 \%$ & $40 \%$ & $50 \%$ & $60 \%$ & $70 \%$ & $75 \%$ & $80 \%$ & $85 \%$ & $90 \%$ & $95 \%$ \\
\hline 6780 & 6080 & 5670 & 5330 & 5090 & 4870 & 4540 & 4300 & 4090 & 3930 & 3850 & 3780 & 3710 & 3610 & 3410 \\
\hline
\end{tabular}




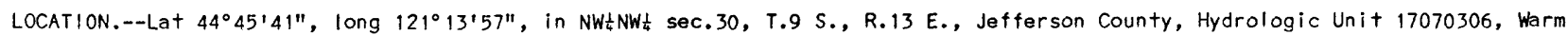

Springs Indian Reservation, on left bank $1.9 \mathrm{mi}$ east of Warm Springs and at $\mathrm{mile} 0.3$.

DRAINAGE AREA. $--105 \mathrm{mi}^{2}$.

PERIOD OF RECORD.--October 1911 to September 1916, October 1923 to September 1928, October 1972 to September 1974.

GAGE.--Nonrecording gage. Altitude of gage is $1,380 \mathrm{ft}$, from topographic map.

REMARKS.--No regulation. Some water is diverted for mill pond at polnt $0.3 \mathrm{mi}$ above station.

AVERAGE DISCHARGE.--12 years, $110 \mathrm{ft}^{3} / \mathrm{s}, 79,695$ acre- $\mathrm{ft} / \mathrm{yr}$.

EXTREMES FOR PERIOD OF RECORD.--Maximum daily discharge, 2,300 ft3/s Jan, 15, 1974; minimum daily, $20 \mathrm{ft} 3 / \mathrm{s}$ Dec. 8-15, 1972.

STATISTICAL SUMMARIES

MONTHLY AND ANNUAL MEAN DISCHARGES 1912-74

\begin{tabular}{|c|c|c|c|c|c|c|}
\hline MONTH & $\begin{array}{l}\text { MINIMUM } \\
\text { (CFS) }\end{array}$ & $\begin{array}{l}\text { MAXIMUM } \\
\text { (CFS) }\end{array}$ & $\begin{array}{l}\text { MEAN } \\
\text { (CFS) }\end{array}$ & $\begin{array}{l}\text { STAN- } \\
\text { DARD } \\
\text { DEVIA- } \\
\text { TION } \\
\text { (CFS) }\end{array}$ & $\begin{array}{l}\text { COEFFI- } \\
\text { CIENT OF } \\
\text { VARI- } \\
\text { ATION }\end{array}$ & $\begin{array}{c}\text { PERCENT } \\
\text { OF } \\
\text { ANNUAL } \\
\text { RUNOFF }\end{array}$ \\
\hline OCTOBER & 42 & 106 & 63 & 19 & .30 & 4.8 \\
\hline NOVEMBER & 50 & 167 & 96 & 32 & .34 & 7.3 \\
\hline DECEMBER & 64 & 204 & 108 & 40 & .37 & 8.2 \\
\hline JANUARY & 54 & 432 & 132 & 99 & .75 & 10.0 \\
\hline FEBRUARY & 60 & 261 & 135 & 64 & .48 & 10.2 \\
\hline MARCH & 65 & 209 & 118 & 39 & .33 & 9.0 \\
\hline APRIL & 66 & 200 & 131 & 41 & .31 & 9.9 \\
\hline MAY & 86 & 238 & 155 & 45 & .29 & 11.8 \\
\hline JUNE & 69 & 315 & 148 & 77 & .52 & 11.2 \\
\hline JULY & 47 & 213 & 105 & 52 & .50 & 8.0 \\
\hline AUGUST & 36 & 106 & 67 & 25 & .37 & 5.1 \\
\hline SEPTEMBER & 36 & 84 & 60 & 17 & .29 & 4.5 \\
\hline ANNUAL & 71 & 175 & 110 & 33 & .30 & 100 \\
\hline
\end{tabular}

MAGNITUDE AND PROBABILITY OF INSTANTANEOUS PEAK FLOW BASED ON PERIOD OF RECORD 1912-74

DISCHARGE, IN CFS, FOR INDICATED RECURRENCE INTERVAL, IN YEARS, AND ANNUAL EXCEEDANCE PROBABILITY, IN PERCENT

\begin{tabular}{ccccccc}
1.25 & 2 & 5 & 10 & 25 & 50 & 100 \\
$80 \%$ & $50 \%$ & $20 \%$ & $10 \%$ & $4 \%$ & $2 \%$ & $1 \%$ \\
\hline 306 & 524 & 934 & 1280 & - & - & \\
\hline WEIGHTED SKEW $=$ & .255 & & &
\end{tabular}

MAGNITUDE AND PROBABILITY OF ANNUAL LOW FLOW BASED ON PERIOD OF RECORD 1913-74

\begin{tabular}{|c|c|c|c|c|c|c|}
\hline $\begin{array}{l}\text { PERIOD } \\
\text { (CON- } \\
\text { SECU- }\end{array}$ & \multicolumn{6}{|c|}{$\begin{array}{l}\text { DISCHARGE, IN CFS, FOR INDICATED RECURRENCE } \\
\text { INTERVAL, IN YEARS, AND ANNUAL NON- } \\
\text { EXCEEDANCE PROBABILITY, IN PERCENT }\end{array}$} \\
\hline TIVE & 2 & 5 & 10 & 20 & 50 & 100 \\
\hline DAYS) & $50 \%$ & $20 \%$ & $10 \%$ & $5 \%$ & $2 \%$ & $1 \%$ \\
\hline \\
\hline 1 & 43 & 35 & 31 & 29 & - & - \\
\hline 3 & 45 & 36 & 32 & 29 & -- & -- \\
\hline 7 & 46 & 37 & 33 & 30 & -- & - \\
\hline 14 & 47 & 37 & 33 & 30 & -- & - \\
\hline 30 & 49 & 39 & 35 & 32 & -- & - \\
\hline 60 & 50 & 40 & 36 & 33 & -- & - \\
\hline 90 & 54 & 42 & 37 & 34 & -- & -- \\
\hline 120 & 57 & 45 & 40 & 37 & - & -- \\
\hline 183 & 67 & 56 & 51 & 48 & -- & -- \\
\hline
\end{tabular}

MAGNITUDE AND PROBABILITY OF ANNUAL HIGH FLOW BASED ON PERIOD OF RECORD 1912-74

\begin{tabular}{|c|c|c|c|c|c|c|}
\hline $\begin{array}{l}\text { PERIOD } \\
\text { (CON- } \\
\text { SECU- }\end{array}$ & \multicolumn{6}{|c|}{$\begin{array}{c}\text { DISCHARGE, IN CFS, FOR INDICATED RECURRENCE } \\
\text { INTERVAL, IN YEARS, AND ANNUAL } \\
\text { EXCEEDANCE PROBABILITY, IN PERCENT }\end{array}$} \\
\hline $\begin{array}{l}\text { TIVE } \\
\text { DAYS) }\end{array}$ & $\begin{array}{c}2 \\
50 \%\end{array}$ & $\begin{array}{c}5 \\
20 \%\end{array}$ & $\begin{array}{l}10 \\
10 \%\end{array}$ & $\begin{array}{l}25 \\
4 \%\end{array}$ & $\begin{array}{l}50 \\
2 \%\end{array}$ & $\begin{array}{r}100 \\
1 \%\end{array}$ \\
\hline $\begin{array}{l}1 \\
3 \\
7\end{array}$ & $\begin{array}{l}482 \\
388 \\
313\end{array}$ & $\begin{array}{l}867 \\
703 \\
534\end{array}$ & $\begin{array}{r}1240 \\
1000 \\
735\end{array}$ & $\begin{array}{l}-- \\
--\end{array}$ & $\begin{array}{l}-- \\
--\end{array}$ & -- \\
\hline 15 & 237 & 367 & 480 & -- & $-\infty$ & -- \\
\hline 30 & 192 & 271 & 332 & - & - & - \\
\hline 60 & 162 & 219 & 260 & - & - & -- \\
\hline 90 & 152 & 197 & 229 & -- & -- & -- \\
\hline
\end{tabular}

DURATION TABLE OF DAILY MEAN FLOW FOR PERIOD OF RECORD 1912-74

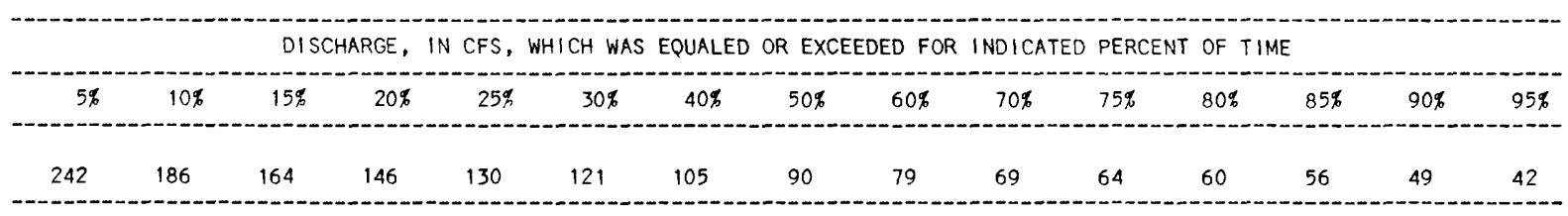


14093500 DESCHUTES RIVER AT MECCA, OR

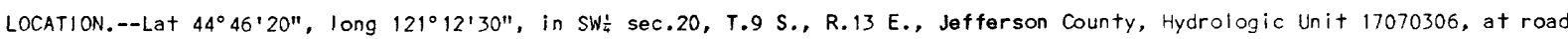
bridge, $1.5 \mathrm{mi}$ downstream from Shitike Creek.

DRAINAGE AREA. $--7,940 \mathrm{mi}^{2}$, approximately.

PERIOD OF RECORD.--June 1911 to January 1927.

GAGE.--Water-stage recorder. Altitude of gage is 1,350 ft National Geodetlc Vertical' Datum of 1929 (from river-profile map). Prior to Aug. 10, 1924, staff gage at same site and datum.

REMARKS.--Many diversions for irrigation above station (about 56,000 acres irrigated in 1911; 71,500 acres in 1927). Some regulation by cchoco Reservoir (capacity, 47,500 acre-ft) since 1919, and Crescent Lake and Crane Prairie Reservoir since 1922.

AVERAGE DISCHARGE, --15 years (water years $1912-26$ ) $, 4,891 \mathrm{ft}^{3} / \mathrm{s}$.

EXTREMES FOR PERIOD OF RECORD.--Maximum discharge, 15,200 $\mathrm{ft}^{3} / \mathrm{s}$ Jan. 6 , 1923, gage height, $6.9 \mathrm{ft}$, from rating curve extended above $11,000 \mathrm{ft}^{3} / \mathrm{s}$; minimum, 3,170 $\mathrm{ft}^{3} / \mathrm{s}$ Aug. $27-30,1920$.

STATISTICAL SUMMARIES

MONTHLY AND ANNUAL MEAN DISCHARGES 1912-27

\begin{tabular}{|c|c|c|c|c|c|c|}
\hline MONTH & $\begin{array}{l}\text { MINIMUM } \\
\text { (CFS) }\end{array}$ & $\begin{array}{l}\text { MAXIMUM } \\
\text { (CFS) }\end{array}$ & $\begin{array}{l}\text { MEAN } \\
\text { (CFS) }\end{array}$ & $\begin{array}{l}\text { STAN- } \\
\text { DARD } \\
\text { DEVIA- } \\
\text { TION } \\
\text { (CFS) }\end{array}$ & $\begin{array}{l}\text { COEFFI- } \\
\text { CIENT OF } \\
\text { VARI- } \\
\text { ATION }\end{array}$ & $\begin{array}{c}\text { PERCENT } \\
\text { OF } \\
\text { ANNUAL } \\
\text { RUNOFF }\end{array}$ \\
\hline OCTOBER & 3487 & 4595 & 4158 & 312 & .08 & 7.1 \\
\hline NOVEMBER & 4214 & 5700 & 4688 & 337 & .07 & 8.0 \\
\hline DECEMBER & 4299 & 6455 & 4914 & 560 & .11 & 8.4 \\
\hline JANUARY & 4547 & 6475 & 5177 & 637 & .12 & 8.8 \\
\hline FEBRUARY & 4469 & 7241 & 5511 & 883 & .16 & 9.4 \\
\hline MARCH & 4722 & 7710 & 5725 & 970 & .17 & 9.8 \\
\hline APRIL & 4476 & 7583 & 6380 & 1079 & .17 & 10.9 \\
\hline MAY & 3620 & 7720 & 5329 & 1130 & .21 & 9.1 \\
\hline JUNE & 3437 & 6022 & 4727 & 872 & .18 & 8.1 \\
\hline JULY & 3300 & 5606 & 4248 & 691 & .16 & 7.2 \\
\hline AUGUST & 3306 & 4550 & 3896 & 413 & .11 & 6.6 \\
\hline SEPTEMBER & 3276 & 4524 & 3963 & 391 & .10 & 6.7 \\
\hline ANNUAL & 4152 & 5507 & 4892 & 429 & .09 & 100 \\
\hline
\end{tabular}

MAGNITUDE AND PROBABILITY OF INSTANTANEOUS PEAK FLOW BASED ON PERIOD OF RECORD $1912-26$

\begin{tabular}{|c|c|c|c|c|c|c|}
\hline $\begin{array}{l}1.25 \\
80 \%\end{array}$ & $\begin{array}{c}2 \\
50 \%\end{array}$ & $\begin{array}{c}5 \\
20 \%\end{array}$ & $\begin{array}{l}10 \\
10 \%\end{array}$ & $\begin{array}{l}25 \\
4 \%\end{array}$ & $\begin{array}{l}50 \\
2 \%\end{array}$ & $\begin{array}{r}100 \\
1 \%\end{array}$ \\
\hline 8260 & 9720 & 11600 & 12700 & 14100 & -- & -- \\
\hline
\end{tabular}

WEIGHTED SKEW $=.216$
MAGNITUDE AND PROBABILITY OF ANNUAL LOW FLOW BASED ON PERIOD OF RECORD 1913-26

\begin{tabular}{|c|c|c|c|c|c|c|}
\hline $\begin{array}{l}\text { PERIOD } \\
\text { (CON- } \\
\text { SECL- }\end{array}$ & \multicolumn{6}{|c|}{$\begin{array}{l}\text { DISCHARGE, IN CFS, FOR INDICATED RECURRENCE } \\
\text { INTERVAL, IN YEARS, AND ANNUAL NON- } \\
\text { EXCEEDANCE PROBABILIITY, IN PERCENT }\end{array}$} \\
\hline $\begin{array}{l}\text { TIVE } \\
\text { DAYS) }\end{array}$ & $\begin{array}{c}2 \\
50 \%\end{array}$ & $\begin{array}{c}5 \\
20 \%\end{array}$ & $\begin{array}{l}10 \\
10 \%\end{array}$ & $\begin{array}{l}20 \\
5 \%\end{array}$ & $\begin{array}{l}50 \\
2 \%\end{array}$ & $\begin{array}{r}100 \\
1 \%\end{array}$ \\
\hline 1 & 3780 & 3490 & 3330 & 3190 & -- & -. \\
\hline 3 & 3790 & 3490 & 3330 & 3200 & -- & - \\
\hline 7 & 3800 & 3510 & 3350 & 3220 & -- & - \\
\hline 14 & 3830 & 3530 & 3380 & 3250 & -- & -- \\
\hline 30 & 3880 & 3580 & 3420 & 3290 & -- & -- \\
\hline 60 & 3930 & 3620 & 3460 & 3320 & -- & -- \\
\hline 90 & 4000 & 3690 & 3520 & 3380 & -- & - \\
\hline 120 & 4080 & 3750 & 3580 & 3430 & -- & $=-$ \\
\hline 183 & 4270 & 3920 & 3720 & 3560 & -- & -- \\
\hline
\end{tabular}

MAGN ITUDE AND PROBABILITY OF ANNUAL HIGH FLOW BASED ON PERIOD OF RECORD 1912-26

\begin{tabular}{|c|c|c|c|c|c|c|}
\hline PERIOD & DIS & $\begin{array}{l}\text { ARGE, } \\
\text { INTEP }\end{array}$ & $\begin{array}{l}\text { CFS, } \\
L, \text { iN }\end{array}$ & $\begin{array}{l}\text { INDI } \\
\text { ARS, }\end{array}$ & NNU & ENCE \\
\hline (CON- & & EXCEED & EE PROE & IL I TY, & PER & \\
\hline TIVE & 2 & 5 & 10 & 25 & 50 & 100 \\
\hline DAYS) & $50 \%$ & $20 \%$ & $10 \%$ & $4 \%$ & $2 q$ & $1 \%$ \\
\hline 1 & 9660 & 11200 & 12100 & 13200 & - & -- \\
\hline 3 & 9080 & 10400 & 11200 & 12000 & - & - \\
\hline 7 & 8290 & 9310 & 9850 & 10400 & - & -- \\
\hline 15 & 7530 & 8350 & 8770 & 9210 & - & -- \\
\hline 30 & 6990 & 7790 & 8210 & 8650 & - & -- \\
\hline 60 & 6460 & 7170 & 7560 & 7970 & -- & -- \\
\hline 90 & 6140 & 6820 & 7190 & 7600 & - & -- \\
\hline
\end{tabular}

DURATION TABLE OF DAILY MEAN FLOW FOR PERIOD OF RECORD 1912-26

DISCHARGE, IN CFS, WHICH WAS EQUALED OR EXCEEDED FOR INDICATED PERCENT OF TIME

\begin{tabular}{|c|c|c|c|c|c|c|c|c|c|c|c|c|c|c|}
\hline $5 \%$ & $10 \%$ & $15 \%$ & $20 \%$ & $25 \%$ & $30 \%$ & $40 \%$ & $50 \%$ & $60 \%$ & $70 \%$ & $75 \%$ & $80^{\circ}$ & $85 \%$ & $90 \%$ & $95 \%$ \\
\hline 7230 & 6470 & 5990 & 5600 & 5300 & 5140 & 4870 & 4690 & 4480 & 4300 & 4210 & 4050 & 3880 & 3700 & 3470 \\
\hline
\end{tabular}




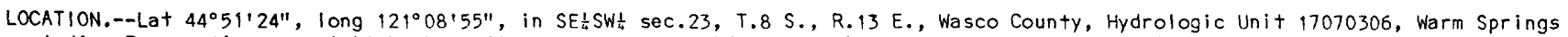

Indlan Reservation, on right bank $25 \mathrm{ft}$ upstream from bridge, $2.5 \mathrm{mi}$ east of Kahneeta Hot Springs, and at mile 4.6 .

DRAINAGE AREA. $--526 \mathrm{mi}^{2}$

PERIOD OF RECORO.--October 1972 to September 1982.

GAGE. - Water-stage recorder. Altitude of gage is $1,40 . \mathrm{ft}$, from topographic map.

REMARKS.--No regulation. Diversions above station.

AVERAGE DISCHARGE. -10 years, $442 \mathrm{ft} / \mathrm{s}, 11.41 \mathrm{in} / \mathrm{yr}, 320,200 \mathrm{acre}-\mathrm{ft} / \mathrm{yr}$.

EXTREMES FOR PERIOD OF RECORD.--Maximum discharge, $6,540 \mathrm{ft}+3 / \mathrm{s} \mathrm{Dec} .15,1977$, gage height, $8.86 \mathrm{ft} ; \mathrm{minimum} \mathrm{daily,} 160 \mathrm{ft} 3 / \mathrm{s} \mathrm{Jan}$. 1, 2,1979 .

STATISTICAL SUMMARIES

MONTHLY AND ANNUAL MEAN DISCHARGES 1973-82

\begin{tabular}{|c|c|c|c|c|c|c|}
\hline MONTH & $\begin{array}{c}\text { MIN IMUM } \\
\text { (CFS) }\end{array}$ & $\begin{array}{l}\text { MAXIMUM } \\
\text { (CFS) }\end{array}$ & $\begin{array}{l}\text { MEAN } \\
\text { (CFS) }\end{array}$ & $\begin{array}{l}\text { STAN- } \\
\text { DARD } \\
\text { DEVIA- } \\
\text { TION } \\
\text { (CFS) }\end{array}$ & $\begin{array}{l}\text { COEFFI- } \\
\text { CIENT OF } \\
\text { VARI- } \\
\text { ATION }\end{array}$ & $\begin{array}{c}\text { PERCENT } \\
\text { OF } \\
\text { ANNUAL } \\
\text { RUNOFF }\end{array}$ \\
\hline OCTOBER & 220 & 318 & 263 & 34 & .13 & 4.9 \\
\hline NOVEMBER & 244 & 396 & 308 & 48 & .15 & 5.8 \\
\hline DECEMBER & 277 & 1210 & 606 & 309 & .51 & 11.4 \\
\hline JANUARY & 201 & 1520 & 650 & 415 & .64 & 12.2 \\
\hline FEBRUARY & 264 & 1534 & 679 & 351 & .52 & 12.8 \\
\hline MARCH & 274 & 837 & 575 & 182 & .32 & 10.8 \\
\hline APRIL & 278 & 805 & 511 & 154 & .30 & 9.6 \\
\hline MAY & 278 & 819 & 518 & 198 & .38 & 9.7 \\
\hline JUNE & 246 & 803 & 410 & 176 & .43 & 7.7 \\
\hline JULY & 216 & 401 & 284 & 63 & .22 & 5.3 \\
\hline AUGUST & 212 & 323 & 260 & 42 & .16 & 4.9 \\
\hline SEPTEMBER & 215 & 301 & 255 & 34 & .13 & 4.8 \\
\hline ANNUAL & 259 & 660 & 442 & 127 & .29 & 100 \\
\hline
\end{tabular}

MAGNITUDE AND PROBABILITY OF INSTANTANEOUS PEAK FLOW BASED ON PERIOD OF RECORD 1973-82

DISCHARGE, IN CFS, FOR INDICATED RECURRENCE INTERVAL, IN YEARS, AND ANNUAL EXCEEDANCE PROBABILITY, IN PERCENT

\begin{tabular}{ccccccc}
1.25 & 2 & 5 & 10 & 25 & 50 & 100 \\
$80 \%$ & $50 \%$ & $20 \%$ & $10 \%$ & $4 \%$ & $2 \%$ & $1 \%$ \\
1880 & 3300 & 5580 & 7240 & - & - & - \\
\hline
\end{tabular}

MAGNITUDE ANO PROBABILITY OF ANNUAL LOW FLOW BASED ON PERIOD OF RECORD 1974-82

\begin{tabular}{|c|c|c|c|c|c|c|}
\hline $\begin{array}{l}\text { PERIOO } \\
\text { (CON- }\end{array}$ & \multicolumn{6}{|c|}{$\begin{array}{l}\text { DISCHARGE, IN CFS, FOR INDICATEO RECURRENCE } \\
\text { INTERVAL, IN YEARS, AND ANNUAL NON- } \\
\text { EXCEEDANCE PROBABILITY, IN PERCENT }\end{array}$} \\
\hline SECU- & & & & -- & - & - \\
\hline TIVE & 2 & 5 & 10 & 20 & 50 & 100 \\
\hline DAYS) & $50 \%$ & 20,9 & $10 \%$ & $5 \%$ & $2 \%$ & $1 \%$ \\
\hline \\
\hline 1 & 228 & 194 & 177 & 164 & -- & -- \\
\hline 3 & 230 & 197 & 181 & 167 & -- & - \\
\hline 7 & 231 & 201 & 187 & 177 & -- & - \\
\hline 14 & 231 & 205 & 193 & 184 & -- & -- \\
\hline 30 & 233 & 211 & 202 & 195 & -- & -- \\
\hline 60 & 238 & 218 & 210 & 205 & -- & -- \\
\hline 90 & 243 & 223 & 215 & 209 & -- & -- \\
\hline 120 & 247 & 226 & 217 & 211 & - & -- \\
\hline 183 & 262 & 238 & 229 & 224 & -- & - \\
\hline
\end{tabular}

MAGN I TUDE AND PROBABILITY OF ANNUAL HIGH FLOW BASED ON PERIOD OF RECORD 1973-82

\begin{tabular}{|c|c|c|c|c|c|c|}
\hline \multirow{4}{*}{$\begin{array}{l}\text { PERIOD } \\
\text { (CON- } \\
\text { SECU- } \\
\text { TIVE } \\
\text { DAYS) }\end{array}$} & \multicolumn{6}{|c|}{$\begin{array}{c}\text { DISCHARGE, IN CFS, FOR INDICATED RECURRENCE } \\
\text { INTERVAL, IN YEARS, AND ANNUAL } \\
\text { EXCEEDANCE PROBABILITY, IN PERCENT }\end{array}$} \\
\hline & \multicolumn{6}{|c|}{ 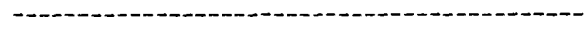 } \\
\hline & 2 & 5 & 10 & 25 & 50 & 100 \\
\hline & & $20 \%$ & $10 \%$ & & $2 \%$ & \\
\hline 1 & 2640 & 462.0 & 5690 & - & - & -- \\
\hline 3 & 2180 & 3830 & 4780 & -- & -- & -- \\
\hline 7 & 1670 & 2870 & 3600 & -- & -- & - \\
\hline 15 & 1250 & 1970 & 2380 & -- & - & -- \\
\hline 30 & 954 & 1380 & 1610 & - & - & -- \\
\hline 60 & 762 & 1050 & 1200 & $-\infty$ & -- & -- \\
\hline 90 & 696 & 934 & 1060 & -- & - & -- \\
\hline
\end{tabular}

DURATION TABLE OF DAILY MEAN FLOW FOR PERIOD OF RECORO 1973-82

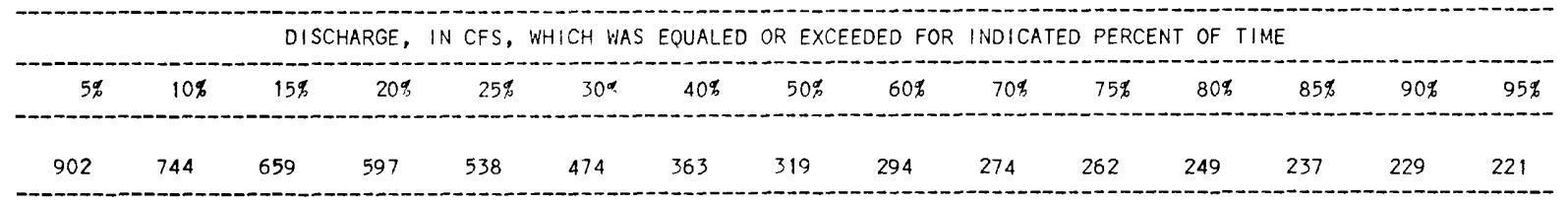


14097200 WHITE RIVER NEAR GOVERNMENT CAMP, OR

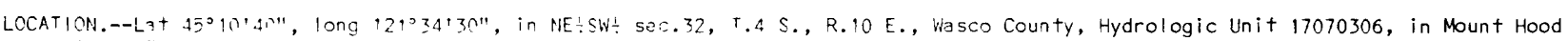
National Forest, on left bank at Faith Spring, $1.4 \mathrm{mi}$ above $\mathrm{Klip}$ Creek, and at mile 33.3 .

DRAINAGE AREA. $--40.7 \mathrm{mi}^{2}$.

PERIOD OF RECORD.--July 1969 to October 1979, July 1980 to September 1981.

GAGE. - Water-stage recorder. Altitude of gage is $2,740 \mathrm{ft}$ from topographic map.

REMARKS.--No regulation or diversion above station.

AVERAGE DISCHARGE.-- $1 \mathrm{i}$ yezrs, $165 \mathrm{ft}^{+}$'s, 55.05 in'yr, 119,500 acre-ft/yr.

EXTREMES FOR PERIOD OF RECORD.--Maximum discharge, 3,650 $\mathrm{ft}^{3} / \mathrm{s} \mathrm{Dec.} \mathrm{13,} 1977$, gage height, $6.63 \mathrm{ft}$; minimum, $20 \mathrm{ft} / \mathrm{s}$ Jan. 6 , 1977 .

STATISTICAL SUMMARIES

MONTHLY AND ANNUAL MEAN DISCHARGES 1970-81

\begin{tabular}{|c|c|c|c|c|c|c|}
\hline MONTH & $\begin{array}{l}M|N| M U M \\
\text { (CFS) }\end{array}$ & $\begin{array}{l}\text { MAXIMUM } \\
\text { (CFS) }\end{array}$ & $\begin{array}{l}\text { MEAN } \\
\text { (CFS) }\end{array}$ & $\begin{array}{l}\text { STAN- } \\
\text { DARD } \\
\text { DEVIA- } \\
\text { TION } \\
\text { (CFS) }\end{array}$ & $\begin{array}{l}\text { COEFFI- } \\
\text { CIENT OF } \\
\text { VARI- } \\
\text { ATION }\end{array}$ & $\begin{array}{c}\text { PERCENT } \\
\text { OF } \\
\text { ANNUAL } \\
\text { RUNOFF }\end{array}$ \\
\hline OCTOBER & 46 & 124 & 71 & 22 & .32 & 3.6 \\
\hline NOVEMBER & 59 & 359 & 117 & 87 & .75 & 5.9 \\
\hline DECEMBER & 49 & 593 & 229 & 155 & .68 & 11.6 \\
\hline JANUARY & 44 & 378 & 224 & 118 & .53 & 11.3 \\
\hline FEBRUARY & 48 & 300 & 185 & 82 & .44 & 9.4 \\
\hline$M A R C H$ & 60 & 595 & 183 & 123 & .67 & 9.3 \\
\hline APRIL & 127 & 252 & 182 & 41 & .22 & 9.2 \\
\hline MAY & 126 & 500 & 307 & 145 & .47 & 15.5 \\
\hline JUNE & 76 & 571 & 231 & 143 & .62 & 11.7 \\
\hline JULY & 59 & 215 & 110 & 46 & .42 & 5.6 \\
\hline AUGUST & 46 & 122 & 73 & 23 & .31 & 3.7 \\
\hline SEPTEMBER & 44 & 124 & 65 & 20 & .31 & 3.3 \\
\hline ANNUAL & 74 & 234 & 165 & 52 & .31 & 100 \\
\hline
\end{tabular}

MAGNITUDE AND PROBABILITY OF INSTANTANEOUS PEAK FLOW BASED ON PERIOD OF RECORD $1970-81$

DISCHARGE, IN CFS, FCR INDICATED RECURRENCE INTERVAL, IN YEARS, AND ANNUAL EXCEEDANCE PROBABILITY, IN PERCENT

\begin{tabular}{|c|c|c|c|c|c|c|}
\hline $\begin{array}{l}1.25 \\
80 \%\end{array}$ & $\stackrel{2}{20}$ & $\begin{array}{c}5 \\
20 \%\end{array}$ & $\begin{array}{l}10 \\
10 \%\end{array}$ & $\begin{array}{l}25 \\
4{ }_{0}^{\alpha}\end{array}$ & $\begin{array}{l}50 \\
2 \%\end{array}$ & $\begin{array}{r}100 \\
1 \%\end{array}$ \\
\hline 586 & 1280 & 2590 & 3640 & - & -- & -- \\
\hline
\end{tabular}

MAGNITUDE AND PROBABILITY OF ANNUAL LOW FLOW BASED ON PERIOD OF RECORD 1971-79

\begin{tabular}{|c|c|c|c|c|c|c|}
\hline $\begin{array}{l}\text { PERIOD } \\
\text { ICON- }\end{array}$ & \multicolumn{6}{|c|}{$\begin{array}{l}\text { DISCHARGE, IN CFS, FOR INDICATED RECURRENCE } \\
\text { INTERVAL, IN YEARS, AND ANNUAL NON- } \\
\text { EXCEEDANCE PROBABILITY, IN PERCENT }\end{array}$} \\
\hline $\begin{array}{l}\text { TIVE } \\
\text { DAYSI }\end{array}$ & $\begin{array}{c}2 \\
50 \%\end{array}$ & ${ }_{20 \%}^{5}$ & 10 & $\begin{array}{l}20 \\
5 \%\end{array}$ & $\begin{array}{l}50 \\
2 \%\end{array}$ & $\begin{array}{r}100 \\
1 \%\end{array}$ \\
\hline & & & & & & \\
\hline 1 & 46 & 41 & 38 & 35 & - & - \\
\hline 3 & 46 & 41 & 38 & 35 & - & - \\
\hline 7 & 48 & 42 & 38 & 35 & - & -- \\
\hline 14 & 50 & 44 & 40 & 38 & $\rightarrow$ & -- \\
\hline 30 & 54 & 47 & 44 & 42 & -- & -- \\
\hline 50 & 57 & 50 & 47 & 45 & -- & -- \\
\hline 90 & 61 & 52 & 49 & 48 & - & - \\
\hline 120 & 66 & 57 & 52 & 49 & - & . \\
\hline 183 & 94 & 74 & 63 & 54 & - & - \\
\hline
\end{tabular}

MAGNITUDE AND PROBABILITY OF ANNUAL HIGH FLOW BASED ON PERIOD OF RECORD 1970-81

\begin{tabular}{|c|c|c|c|c|c|c|}
\hline \multirow{2}{*}{$\begin{array}{l}\text { PERICD } \\
\text { (CON- } \\
\text { SECU- } \\
\text { TIVE } \\
\text { DAYS) }\end{array}$} & \multicolumn{6}{|c|}{$\begin{array}{l}\text { DISCHARGE, IN CFS, FOR INDICATED RECURRENCE } \\
\text { INTERVAL, IN YEARS, AND ANNUAL } \\
\text { EXCEEDANCE PROBABILITY, IN PERCENT }\end{array}$} \\
\hline & $\stackrel{2}{2}$ & $\begin{array}{c}5 \\
20 \%\end{array}$ & $\begin{array}{l}10 \\
10 \%\end{array}$ & $\begin{array}{l}25 \\
4{ }_{\rho}^{\not}\end{array}$ & $\begin{array}{l}50 \\
2 q\end{array}$ & $\begin{array}{r}100 \\
1 \%\end{array}$ \\
\hline 1 & 1200 & 1780 & 2040 & -- & -- & -- \\
\hline 3 & 915 & 1300 & 1480 & -- & -- & \\
\hline 7 & 711 & 911 & 987 & - & -- & -- \\
\hline 15 & 544 & 700 & 768 & - & - & \\
\hline 30 & 428 & 570 & 642 & -- & -- & - \\
\hline 60 & 336 & 438 & 491 & - & - & - \\
\hline 90 & 292 & 376 & 417 & -- & -- & -- \\
\hline
\end{tabular}

OURATION TABLE OF DAILY MEAN FLOW FOR PERIOD OF RECORD 1970-81

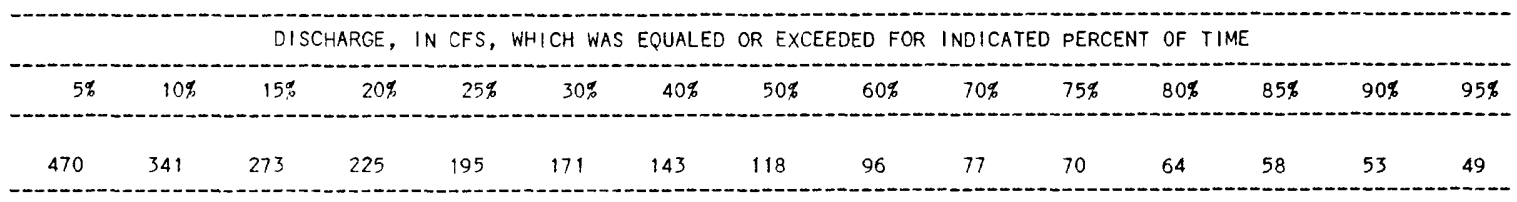




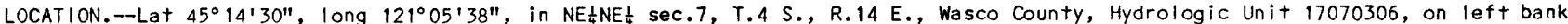
$200 \mathrm{ft}$ downstream from former Pacific Power \& Light Co. powerplant at White River Falls, $3.9 \mathrm{mi}$ east of town of Tygh Valley, and at mile 2.0.

DRAINAGE AREA. $--417 \mathrm{mi}^{2}$.

PERIOD OF RECORD.--October 1917 to September 1982.

GAGE.--Water-stage recorder. Datum of gage is $870.15 \mathrm{ft}$ National Geodetic Vertical Datum of 1929 (levels by Pacific Power \& Light Co.). Prior to July 28, 1931, at site $750 \mathrm{ft}$ downstream at different datum. July 28, 1931, to Sept. 30 , 1954, at site $700 \mathrm{ft}$ downstream at different datums.

REMARKS. --No regulation. Diversions above station for irrigation.

AVERAGE DISCHARGE.--65 years, $427 \mathrm{ft}^{3} / \mathrm{s}, 309,400$ acre $-\mathrm{ft} / \mathrm{yr}$.

EXTREMES FOR PERIOD OF RECORD.--Maximum discharge, 13,300 $\mathrm{ft}^{3} / \mathrm{s}$ Jan. 6,1923 , gage height, about $13.3 \mathrm{ft}$, site and datum then in use, from rating curve extended above $5,000 \mathrm{ft} / 3$; minimum, $7.5 \mathrm{ft}^{3} / \mathrm{s}$ Aug. 31,1961 .

\section{STATISTICAL SUMMARIES}

MONTHLY AND ANNUAL MEAN DISCHARGES 1918-82

\begin{tabular}{|c|c|c|c|c|c|c|}
\hline MONTH & $\begin{array}{l}\text { MINIMUM } \\
\text { (CFS) }\end{array}$ & $\begin{array}{c}\text { MAXIMUM } \\
\text { (CFS) }\end{array}$ & $\begin{array}{l}\text { MEAN } \\
\text { (CFS) }\end{array}$ & $\begin{array}{l}\text { STAN- } \\
\text { DARD } \\
\text { DEVIA- } \\
\text { TION } \\
\text { (CFS) }\end{array}$ & $\begin{array}{l}\text { COEFFI- } \\
\text { CIENT OF } \\
\text { VARI- } \\
\text { ATION }\end{array}$ & $\begin{array}{c}\text { PERCENT } \\
\text { OF } \\
\text { ANNUAL } \\
\text { RUNOFF }\end{array}$ \\
\hline OCTOBER & 88 & 286 & 153 & 45 & .29 & 3.0 \\
\hline NOVEMBER & 113 & 622 & 267 & 136 & .51 & 5.2 \\
\hline DECEMBER & 120 & 1683 & 517 & 387 & .75 & 10.1 \\
\hline JANUARY & 117 & 1998 & 602 & 398 & .66 & 11.7 \\
\hline FEBRUARY & 128 & 1574 & 649 & 345 & .53 & 12.6 \\
\hline MARCH & 129 & 1428 & 569 & 225 & .40 & 11.1 \\
\hline APRIL & 223 & 1445 & 695 & 259 & .37 & 13.5 \\
\hline MAY & 202 & 1818 & 761 & 342 & .45 & 14.8 \\
\hline JUNE & 132 & 1222 & 469 & 265 & .57 & 9.1 \\
\hline JULY & 96 & 392 & 199 & 69 & .34 & 3.9 \\
\hline AUGUST & 80 & 190 & 135 & 26 & .20 & 2.6 \\
\hline SEPTEMBER & 86 & 182 & 127 & 23 & .18 & 2.5 \\
\hline ANNUAL & 139 & 726 & 427 & 131 & .31 & 100 \\
\hline
\end{tabular}

MAGNITUDE AND PROBABILITY OF INSTANTANEOUS PEAK FLOW BASED ON PERIOD OF RECORD 1918-82

\begin{tabular}{lcccccc}
\hline DISCHARGE, IN CFS, FOR INDICATED RECURRENCE INTERVAL, IN \\
YEARS, AND ANNUAL EXCEEDANCE PROBABILITY, IN PERCENT \\
\hline 1.25 & 2 & 5 & 10 & 25 & 50 & 100 \\
$80 \%$ & $50 \%$ & $20 \%$ & $10 \%$ & $4 \%$ & $2 \%$ & $1 \%$ \\
\hline 1780 & 3060 & 5280 & 7010 & 9490 & 11500 & 13800
\end{tabular}

WEIGHTED SKEW $=-.005$

MAGNITUDE AND PROBABILITY OF ANNUAL LOW FLOW BASED ON PERIOD OF RECORD 1919-82

\begin{tabular}{|c|c|c|c|c|c|c|}
\hline \multirow{2}{*}{$\begin{array}{l}\text { PERIOD } \\
\text { (CON- } \\
\text { SECU- } \\
\text { TIVE } \\
\text { DAYS) }\end{array}$} & \multicolumn{6}{|c|}{$\begin{array}{l}\text { DISCHARGE, IN CFS, FOR INDICATED RECURRENCE } \\
\text { INTERVAL, IN YEARS, AND ANNUAL NON- } \\
\text { EXCEEDANCE PROBABILITY, IN PERCENT }\end{array}$} \\
\hline & $\begin{array}{c}2 \\
50 \%\end{array}$ & $\begin{array}{c}5 \\
20 \%\end{array}$ & $\begin{array}{l}10 \\
10 \%\end{array}$ & $\begin{array}{l}20 \\
5 \%\end{array}$ & $\begin{array}{l}50 \\
2 \%\end{array}$ & $\begin{array}{r}100 \\
1 \%\end{array}$ \\
\hline $\begin{array}{l}1 \\
3\end{array}$ & $\begin{array}{r}106 \\
107\end{array}$ & $\begin{array}{l}90 \\
91\end{array}$ & $\begin{array}{l}81 \\
83\end{array}$ & $\begin{array}{l}75 \\
76\end{array}$ & $\begin{array}{l}68 \\
70\end{array}$ & $\begin{array}{l}64 \\
65\end{array}$ \\
\hline 7 & 109 & 93 & 85 & 78 & 71 & 66 \\
\hline 14 & 112 & 96 & 88 & 82 & 76 & 71 \\
\hline 30 & 117 & 101 & 93 & 87 & 80 & 76 \\
\hline 60 & 124 & 106 & 98 & 91 & 84 & 79 \\
\hline 90 & 130 & 111 & 102 & 95 & 87 & 83 \\
\hline 120 & 138 & 116 & 107 & 100 & 92 & 88 \\
\hline 183 & 178 & 141 & 126 & 116 & 106 & 100 \\
\hline
\end{tabular}

MAGNITUDE AND PROBABILITY OF ANNUAL HIGH FLOW BASED ON PERIOD OF RECORD 1918-82

\begin{tabular}{|c|c|c|c|c|c|c|}
\hline \multirow{3}{*}{$\begin{array}{l}\text { PERIOD } \\
\text { (CON- } \\
\text { SECU- } \\
\text { TIVE } \\
\text { DAYS) }\end{array}$} & \multicolumn{6}{|c|}{$\begin{array}{c}\text { DISCHARGE, IN CFS, FOR INDICATED RECURRENCE } \\
\text { INTERVAL, IN YEARS, AND ANNUAL } \\
\text { EXCEEDANCE PROBABILITY, IN PERCENT }\end{array}$} \\
\hline & \multicolumn{6}{|c|}{0} \\
\hline & $\begin{array}{c}2 \\
50 \%\end{array}$ & $\begin{array}{c}5 \\
20 \%\end{array}$ & $\begin{array}{l}10 \\
10 \%\end{array}$ & $\begin{array}{l}25 \\
4 \%\end{array}$ & $\begin{array}{l}50 \\
2,6\end{array}$ & $\begin{array}{r}100 \\
1 \%\end{array}$ \\
\hline & & 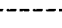 & & & & \\
\hline 1 & 2550 & 4300 & 5540 & 7170 & 8410 & 9660 \\
\hline 3 & 2150 & 3510 & 4460 & 5700 & 6640 & 7580 \\
\hline 7 & 1720 & 2620 & 3200 & 3910 & 4420 & 4910 \\
\hline 15 & 1380 & 1940 & 2260 & 2610 & 2840 & 3040 \\
\hline 30 & 1140 & 1500 & 1670 & 1830 & 1910 & 1980 \\
\hline 60 & 949 & 1220 & 1340 & 1450 & 1510 & 1550 \\
\hline 90 & 836 & 1050 & 1140 & 1220 & 1260 & 1290 \\
\hline
\end{tabular}

DURATION TABLE OF DAILY MEAN FLOW FOR PERIOD OF RECORD 1918-82

DISCHARGE, IN CFS, WHICH WIAS EQUALED OR EXCEEDED FOR INDICATED PERCENT OF TIME

\begin{tabular}{|c|c|c|c|c|c|c|c|c|c|c|c|c|c|c|}
\hline $5 \%$ & $10 \%$ & $15 \%$ & $20 \%$ & $25 \%$ & $30 \%$ & $40 \%$ & $50 \%$ & $60 \%$ & $70 \%$ & $75 \%$ & $80 \%$ & $85 \%$ & $90 \%$ & $95 \%$ \\
\hline 1180 & 928 & 767 & 658 & 574 & 499 & 373 & 272 & 206 & 164 & 150 & 139 & 129 & 118 & 105 \\
\hline
\end{tabular}


14103000 DESCHUTES RIVER AT MOODY, NEAR BIGGS, OR

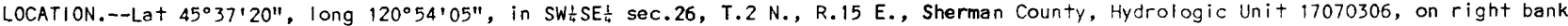
at Moody, $4.0 \mathrm{mi}$ southwest of Biggs, and at mile 1.4 .

DRAINAGE AREA.--10,500 $\mathrm{mi}^{2}$, approximately.

PERIOD OF RECORD.--October 1897 to December 1899 (published as "near Moro"), July 1906 to September 1982. Monthly discharge only for some periods, published in WSP 1318.

GAGE.--Water-stage recorder. Datum of gage is $167.54 \mathrm{ft}$ National Geodetic Vertical Datum of 1929. Oct. 19, 1897, to Dec. 31, 1899, nonrecording gage at site $10 \mathrm{mi}$ upstream at different datum. July 22, 1906, to July 18, 1930, nonrecording gage at site $300 \mathrm{ft}$ downstream at datum $0.50 \mathrm{ft}$ lower.

REMARKS.--Some fluctuation caused by regulation at Lake Simtustus since 1957. Some winter and spring runoff stored in Ochoco Reservoir, capacity, 46,420 acre-ft, in Crescent Lake, Crane Prairie and Wickiup Reservoirs, combined capacity, 323,390 acre-ft and since 1960, in Prineville Reservoir (see station 14080400), and since 1964 in Lake Billy Chinook (see station 14092100). Large diversions in upper river basin for irrigation.

AVERAGE DISCHARGE.--78 years, $5,827 \mathrm{ft} / \mathrm{s}, 4,222,000$ acre- $\mathrm{ft} / \mathrm{yr}$.

EXTREMES FOR PERIOD OF RECORD.--Maximum discharge, 75,500 $\mathrm{ft}^{3} / \mathrm{s}$ Dec. 22 , 1964, gage height, $11.80 \mathrm{tt}$, from rating curve extended above $47,000 \mathrm{ft}^{3} / \mathrm{s}$; minimum, 2,400 $\mathrm{ft}^{3} / \mathrm{s} \mathrm{Dec.} \mathrm{5,} 1957$.

STATISTICAL SUMMARIES (BEFORE CONSTRUCTION OF UPSTREAM RESERVOIRS)

MONTHLY AND ANNUAL MEAN DISCHARGES 1898-22

\begin{tabular}{|c|c|c|c|c|c|c|}
\hline MONTH & $\begin{array}{l}\text { MINIMUM } \\
\text { (CFS) }\end{array}$ & $\begin{array}{l}\text { MAXIMUM } \\
\text { (CFS) }\end{array}$ & $\begin{array}{l}\text { MEAN } \\
\text { (CFS) }\end{array}$ & $\begin{array}{l}\text { STAN- } \\
\text { DARD } \\
\text { DEVIA- } \\
\text { TION } \\
\text { (CFS) }\end{array}$ & $\begin{array}{l}\text { COEFFI- } \\
\text { CIENT OF } \\
\text { VARI- } \\
\text { ATION }\end{array}$ & $\begin{array}{c}\text { PERCENT } \\
\text { OF } \\
\text { ANNUAL } \\
\text { RUNOFF }\end{array}$ \\
\hline OCTOBER & 4029 & 6493 & 5117 & 592 & .12 & 6.5 \\
\hline NOVEMBER & 4713 & 9378 & 6059 & 1106 & .18 & 7.7 \\
\hline DECEMBER & 4804 & 10030 & 6664 & 1611 & .24 & 8.5 \\
\hline JANUARY & 5049 & 9419 & 6852 & 1136 & .17 & 8.7 \\
\hline FEBRUARY & 5305 & 13880 & 7494 & 2257 & .30 & 9.5 \\
\hline MARCH & 5672 & 14350 & 8075 & 2230 & .28 & 10.3 \\
\hline APRIL & 6424 & 11860 & 8782 & 1470 & .17 & 11.1 \\
\hline MAY & 5078 & 10570 & 7697 & 1623 & .21 & 9.8 \\
\hline JUNE & 4394 & 10660 & 6612 & 1485 & .22 & 8.4 \\
\hline JULY & 3905 & 8698 & 5588 & 1196 & .21 & 7.1 \\
\hline AUGUST & 3679 & 6746 & 4913 & 729 & .15 & 6.2 \\
\hline SEPTEMBER & 3792 & 6499 & 4913 & 659 & .13 & 6.2 \\
\hline NNUAL & 4980 & 7866 & 6517 & 832 & .13 & 100 \\
\hline
\end{tabular}

MAGNITUDE AND PROBABILITY OF INSTANTANEOUS PEAK FLOW BASED ON PERIOD OF RECORD $1898-22$

\begin{tabular}{ccccccc} 
DISCHARGE, IN CFS, FOR INDICATEO RECURRENCE INTERVAL, IN \\
YEARS, AND ANNUAL EXCEEDANCE PROBABILITY, IN PERCENT \\
\hline 1.25 & 2 & 5 & 10 & 25 & 50 & 100 \\
$80 \%$ & $50 \%$ & $20 \%$ & $10 \%$ & $4 \%$ & $2 \%$ & $1 \%$ \\
\hline 12100 & 16300 & 22200 & 26300 & 31700 & - & -
\end{tabular}

WEIGHTED SKEW $=.179$
MAGNITUDE AND PROBABILITY OF ANNUAL LOW FLOW BASED ON PERIOD OF RECORD 1899-22

\begin{tabular}{|c|c|c|c|c|c|c|}
\hline $\begin{array}{l}\text { PERIOD } \\
\text { (CON- }\end{array}$ & \multicolumn{6}{|c|}{$\begin{array}{l}\text { DISCHARGE, IN CFS, FOR INDICATED RECURRENCE } \\
\text { INTERVAL, IN YEARS, AND ANNUAL NON- } \\
\text { EXCEEDANCE PROBABILITY, IN PERCENT }\end{array}$} \\
\hline $\begin{array}{l}\text { TIVE } \\
\text { DAYS) }\end{array}$ & $\stackrel{2}{50 \%}$ & $\begin{array}{c}5 \\
20 \%\end{array}$ & $\begin{array}{l}10 \\
10 \%\end{array}$ & $\begin{array}{l}20 \\
5 \%\end{array}$ & $\begin{array}{l}50 \\
2 \%\end{array}$ & $\begin{array}{r}100 \\
1 \%\end{array}$ \\
\hline $\begin{array}{l}1 \\
3\end{array}$ & $\begin{array}{l}4530 \\
4530\end{array}$ & $\begin{array}{l}4020 \\
4020\end{array}$ & $\begin{array}{l}3770 \\
3770\end{array}$ & $\begin{array}{l}3570 \\
3570\end{array}$ & -- & - \\
\hline 7 & 4600 & 4080 & 3820 & 3620 & -- & -- \\
\hline 14 & 4680 & 4160 & 3890 & 3670 & -- & -- \\
\hline 30 & 4750 & 4230 & 3940 & 3700 & -- & - \\
\hline 60 & 4810 & 4300 & 4020 & 3790 & -- & -- \\
\hline 90 & 4870 & 4360 & 4080 & 3840 & -- & -- \\
\hline 120 & 4950 & 4440 & 4160 & 3920 & -- & -- \\
\hline 183 & 5180 & 4680 & 4400 & 4160 & -- & - \\
\hline
\end{tabular}

MAGNITUDE AND PROBABILITY OF ANNUAL HIGH FLOW BASED ON PERIOD OF RECORD 1898-22

\begin{tabular}{|c|c|c|c|c|c|c|}
\hline \multirow{2}{*}{$\begin{array}{l}\text { PERIOD } \\
\text { (CON- } \\
\text { SECU- } \\
\text { TIVE } \\
\text { DAYS) }\end{array}$} & \multicolumn{6}{|c|}{$\begin{array}{l}\text { DISCHARGE, IN CFS, FOR INDICATED RECURRENCE } \\
\text { INTERVAL, IN YEARS, AND ANNUAL } \\
\text { EXCEEDANCE PROBABILITY, IN PERCENT }\end{array}$} \\
\hline & $\underset{50 \%}{2}$ & $\begin{array}{c}5 \\
20 \%\end{array}$ & $\begin{array}{l}10 \\
10 \%\end{array}$ & $\begin{array}{l}25 \\
4 \%\end{array}$ & $\begin{array}{l}50 \\
2 \%\end{array}$ & $\begin{array}{r}100 \\
1 \%\end{array}$ \\
\hline 1 & 15900 & 21100 & 24900 & 29900 & -- & -- \\
\hline 3 & 14300 & 18700 & 22000 & 26600 & -- & -- \\
\hline 7 & 12400 & 15600 & 18100 & 21600 & -- & - \\
\hline 15 & 10900 & 13200 & 14800 & 17100 & -- & - \\
\hline 30 & 9840 & 11600 & 12800 & 14300 & - & -- \\
\hline 60 & 9140 & 10500 & 11400 & 12300 & -- & -- \\
\hline 90 & 8600 & 10000 & 10800 & 11800 & - & -- \\
\hline
\end{tabular}

DURATION TABLE OF DAILY MEAN FLOW FOR PERIOD OF RECORD $1898-22$

\begin{tabular}{|c|c|c|c|c|c|c|c|c|c|c|c|c|c|c|}
\hline & & DI & HARGE, & N CFS, & $\mathrm{HICH}$ W & EQUAL & OR EXC & EDED & INDIC & ED PERC & NT OF & & 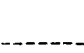 & \\
\hline $5 \%$ & $10 \%$ & $15 \%$ & $20 \%$ & $25 \%$ & $30 \%$ & $40 \%$ & $50 \%$ & $60 \%$ & $70 \%$ & $75 \%$ & $80 \%$ & $85 \%$ & $90 \%$ & $95 \%$ \\
\hline 10700 & 9410 & 8460 & 7940 & 7450 & 7010 & 6370 & 5880 & 5490 & 5220 & 5110 & 4940 & 4760 & 4480 & 4230 \\
\hline
\end{tabular}


14133000 DESCHUTES PIVER AT MCOOY, NEAR BIGGS, OR--Continued

STATISTICAL SUMMARIES (AFTER THE COMPLETION OF UPSTREAM RESERVOIRS)

MONTHLY AND ANNUAL MEAN DISCHARGES 1965-82

\begin{tabular}{|c|c|c|c|c|c|c|}
\hline MONTH & $\begin{array}{l}\text { MINIMUM } \\
\text { (CFS) }\end{array}$ & $\begin{array}{l}\text { MAXIMUM } \\
\text { (CFS) }\end{array}$ & $\begin{array}{l}\text { MEAN } \\
\text { (CFS) }\end{array}$ & $\begin{array}{l}\text { STAN- } \\
\text { DAPO } \\
\text { OEVIA- } \\
\text { TION } \\
\text { (CFS) }\end{array}$ & $\begin{array}{l}\text { CCEFEI- } \\
\text { OIE: OF } \\
\text { VARI- } \\
\text { ATION }\end{array}$ & $\begin{array}{c}\text { PERCEN! } \\
\text { OF } \\
\text { ANNUAL } \\
\text { RUNOFF }\end{array}$ \\
\hline DCTOBER & 3385 & 5594 & 4624 & 515 & .11 & 6.6 \\
\hline NOVEMBER & 3910 & 6420 & 5294 & 534 & .10 & 7.5 \\
\hline DECEMBER & 4610 & 13150 & 6939 & 2147 & .31 & 9.9 \\
\hline $\begin{array}{l}\text { JANUARY } \\
\text { FEBRUARY }\end{array}$ & $\begin{array}{l}5086 \\
4401\end{array}$ & $\begin{array}{l}11290 \\
11750\end{array}$ & $\begin{array}{l}7786 \\
7284\end{array}$ & $\begin{array}{l}1775 \\
1899\end{array}$ & $\begin{array}{l}.23 \\
.26\end{array}$ & $\begin{array}{l}11.1 \\
10.4\end{array}$ \\
\hline MARCH & 5058 & 13580 & 7029 & 1965 & .28 & 10.0 \\
\hline APRIL & 4467 & 9246 & 6439 & 1287 & .20 & 9.2 \\
\hline MAY & 4141 & 7428 & 5907 & 1025 & .17 & 8.4 \\
\hline JUNE & 4202 & 7643 & 5367 & 953 & .18 & 7.6 \\
\hline JULY & 3992 & 5917 & 4679 & 559 & .12 & 6.7 \\
\hline AUGUST & 3980 & 5359 & 4439 & 409 & .09 & 6.3 \\
\hline SEPTEMBER & 3957 & 5092 & 4459 & 321 & .07 & 6.3 \\
\hline ANNUAL & 4687 & 7171 & 5848 & 766 & .13 & 100 \\
\hline
\end{tabular}

MAGNITUDE AND PROBABILITY OF INSTANTANEOUS PEAK FLOW BASED ON PERIOD OF RECORD $1965-82$

DISCHARGE, IN CFS, FOR INDICATED RECURRENCE INTERVAL, IN YEARS, AND ANNUAL EXCEEDANCE PROBABILITY, IN PERCENT

\begin{tabular}{ccccccc}
1.25 & 2 & 5 & 10 & 25 & 50 & 100 \\
$80 \%$ & $50 \%$ & $20 \%$ & $10 \%$ & $4 \%$ & $2 \%$ & $1 \%$ \\
10600 & 15900 & 25400 & 33200 & 45100 & - & - \\
\hline WEIGHT.ED SKEW $=$ & .475
\end{tabular}

MAGNITUDE ANO PROBABILITY OF ANNUAL LOW FLOW BASED ON PERIOD OF RECORD 1966-82

\begin{tabular}{|c|c|c|c|c|c|c|}
\hline $\begin{array}{l}\text { PERICO } \\
\text { CCOH }\end{array}$ & \multirow{2}{*}{\multicolumn{6}{|c|}{$\begin{array}{l}\text { DISCHARGE, IN CFS, FOR INDICATEO RECURRENCE } \\
\text { INITERVAL, IN YEARS, ANO ANNUAL NON- } \\
\text { EXCEEDANCE PRCBABILITY, IN PERCENT }\end{array}$}} \\
\hline SECU- & & & & & & \\
\hline TIVE & 2 & 5 & 10 & 20 & 50 & 100 \\
\hline DAYS) & 50 \% & $20 \%$ & $10:$ & $5 \%$ & $2 \%$ & $1 \%$ \\
\hline & & & & & & \\
\hline 1 & 3810 & 3550 & 3430 & 3340 & -- & - \\
\hline 3 & 3880 & 3620 & 3500 & 3410 & -- & -- \\
\hline 7 & 3980 & 3730 & 3610 & 3520 & -- & -- \\
\hline 14 & 4100 & 3900 & 3810 & 3760 & -- & -- \\
\hline 30 & 4250 & 4030 & 3930 & 3860 & -- & $m$ \\
\hline 60 & 4340 & 4090 & 3980 & 3900 & -- & -- \\
\hline 90 & 4390 & 4120 & 4000 & 3910 & -- & -- \\
\hline 120 & 4470 & 4130 & 4060 & 3970 & -- & - \\
\hline 183 & 4670 & 4340 & 4190 & 4090 & -- & -- \\
\hline
\end{tabular}

MAGNITUDE AND PROBABILITY OF ANNUAL HIGH FLOW BASED ON PERIOD OF RECORD 1965-82

\begin{tabular}{|c|c|c|c|c|c|c|}
\hline $\begin{array}{l}\text { PERIOD } \\
\text { (CON- }\end{array}$ & \multicolumn{6}{|c|}{$\begin{array}{l}\text { DISCHARGE, IN CFS, FOR INOICATED RECURRE } \\
\text { INTERVAL, IN YEARS, AND ANNUAL } \\
\text { EXCEEDANCE PROBABILITY, IN PERCENT }\end{array}$} \\
\hline SECU- & & & - & $-\cdots$ & $\cdots$ & $-\infty-1$ \\
\hline TIVE & 2 & 5 & 10 & 25 & 50 & 100 \\
\hline DAYS) & $50 \%$ & $20 \%$ & $10 \%$ & $4 \%$ & $2 \%$ & $1 \%$ \\
\hline 1 & 13400 & 21100 & 28800 & 42500 & -- & $-\infty$ \\
\hline 3 & 12100 & 18600 & 24700 & 35100 & -- & -- \\
\hline 7 & 11100 & 16100 & 20600 & 27700 & -- & $\cdots$ \\
\hline 15 & 9980 & 13600 & 16400 & 20500 & $\cdots$ & - \\
\hline 30 & 8900 & 11300 & 13100 & 15400 & -- & -- \\
\hline 60 & 7950 & 9860 & 11200 & 13000 & -- & -- \\
\hline 90 & 7630 & 9210 & 10300 & 11600 & -- & -- \\
\hline
\end{tabular}

DURATION TABLE OF DAILY MEANI FLOW FOR PERIOD OF RECORD 1965-82

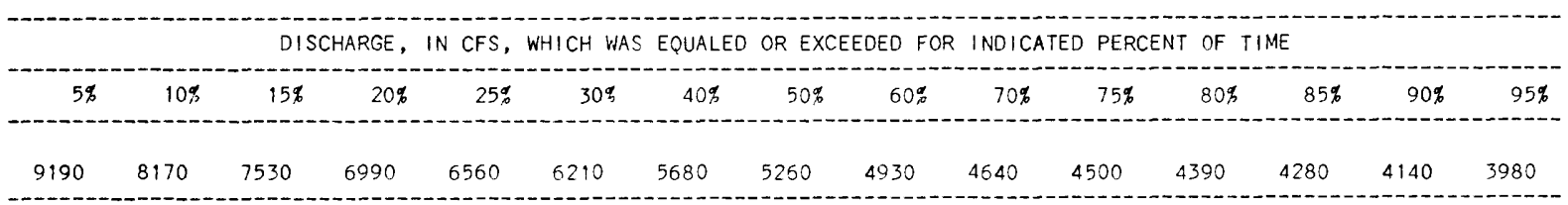


14113200 MOSIER CREEK NEAR MOSIER, OR

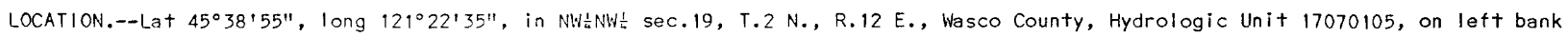
$0.1 \mathrm{mi}$ downstream from West Fork Mosier Creek, $2.5 \mathrm{mi}$ southeast of Mosier, and at mile 3.0 .

DRAINAGE AREA. $--41.5 \mathrm{mi}^{2}$.

PERIOD OF RECORD.--April 1953 to Septemider 1981.

GAGE.--Water-stage recorder. Altitude of gage is $425 \mathrm{ft}$, from topographic map. Prior to July 22, 1976, water-stage recorder at site $20 \mathrm{ft}$ upstream at datum $3.57 \mathrm{ft}$ higher. July 22, 1976, to Dec. 12, 1977, water-stage recorder at site $20 \mathrm{ft}$ upstream at datum 1.57 ft higher.

REMARKS.--No regulation. Several snall pumping diversions for irrigation above station.

AVERAGE DISCHARGE.--18 years, $23.5 \mathrm{ft}$ 's, $\mathrm{s}, 2 \mathrm{f}, 6 \mathrm{n}$ acre- $\mathrm{ft} / \mathrm{yr}$.

EXTREMES FOR PERIOD OF RECORD. - Maximum discharge, 4,790 ft3/s Dec. 23, 1964, gage height, $8.9 \mathrm{ft}$, from flood profile, from rating curve extended above $1,000 \mathrm{ft} / \mathrm{s}$ on basis of slope-area measurement of peak flow; minimum, $0.35 \mathrm{ft} / \mathrm{s} \mathrm{July} 25,26$, Aug. 6 , 7 , 1978 .

STATISTICAL SUMMARIES

MONTHIY AND ANNUAL MEAN DISCHARGES 1964-81

\begin{tabular}{|c|c|c|c|c|c|c|}
\hline MONTH & $\begin{array}{l}\text { MIN IMUM } \\
\text { (CFS) }\end{array}$ & $\begin{array}{l}\text { MAXINIUM } \\
\text { (CFS) }\end{array}$ & $\begin{array}{l}\text { MEAN } \\
\text { (CFS) }\end{array}$ & $\begin{array}{l}\text { STAN- } \\
\text { OARD } \\
\text { DEVIA- } \\
\text { TION } \\
\text { (CFS) }\end{array}$ & $\begin{array}{l}\text { COEFFI- } \\
\text { CIENT OF } \\
\text { VARI- } \\
\text { ATION }\end{array}$ & $\begin{array}{c}\text { PERCENT } \\
\text { OF } \\
\text { ANNUAL } \\
\text { RUNOFF }\end{array}$ \\
\hline OCTOBER & 2.4 & 3.7 & 3.0 & .5 & .15 & .9 \\
\hline NOVEMBER & 3.6 & 29 & 7.7 & 6.2 & .81 & 2.2 \\
\hline DECEMBER & 3.7 & 169 & 42 & 52 & 1.24 & 12.3 \\
\hline JANUARY & 3.7 & 240 & 96 & 70 & .73 & 27.9 \\
\hline FEBRUARY & 3.8 & 129 & 71 & 37 & .52 & 20.5 \\
\hline MARCH & 5.4 & $\uparrow 44$ & 65 & 44 & .67 & 18.9 \\
\hline APRIL & 4.7 & 95 & 37 & 27 & .72 & 10.9 \\
\hline MAY & 3.1 & 26 & 12 & 7.4 & .60 & 3.6 \\
\hline JUNE & 1.7 & 9.1 & 4.2 & 1.8 & .43 & 1.2 \\
\hline JULY & 1.0 & 3.7 & 2.1 & .8 & .38 & .6 \\
\hline AUGUST & 1.0 & 2.6 & 1.6 & .4 & .27 & .5 \\
\hline SEPTEMBER & 1.1 & 2.6 & 1.9 & .4 & .20 & .5 \\
\hline ANNUAL & 3.0 & 53 & 29 & 14 & .48 & 100 \\
\hline
\end{tabular}

MAGNITUDE AND PROBABILITY OF INSTANTANEOUS PEAK FLOW BASED ON PERIOD OF RECORD 1964-81

\begin{tabular}{|c|c|c|c|c|c|c|}
\hline \multicolumn{7}{|c|}{$\begin{array}{l}\text { ISCHARGE, IN CFS, FOR INDICATED RECURRENCE INTERVAL, IN } \\
\text { YEARS, AND ANNUAL EXCEEDANCE PROBABILITY, IN PERCENT }\end{array}$} \\
\hline $\begin{array}{l}1.25 \\
80 \%\end{array}$ & $\begin{array}{c}2 \\
50 \%\end{array}$ & $\begin{array}{c}5 \\
20 \%\end{array}$ & $\begin{array}{l}10 \\
10 \%\end{array}$ & $\begin{array}{l}25 \\
4 \%\end{array}$ & $\begin{array}{l}50 \\
2 \%\end{array}$ & $\begin{array}{r}100 \\
10\end{array}$ \\
\hline 290 & 670 & 1610 & 2590 & 4360 & -- & -- \\
\hline
\end{tabular}

WEIGHTED SKEW $=.168$
MAGNITUDE AND PROBABILITY OF ANNUAL LOW FLOW BASED ON PERIOD OF RECORD 1965-81

\begin{tabular}{|c|c|c|c|c|c|c|}
\hline $\begin{array}{l}\text { PERIOD } \\
\text { (CON- }\end{array}$ & \multirow{2}{*}{\multicolumn{6}{|c|}{$\begin{array}{l}\text { DISCHARGE, IN CFS, FOR INDICATED RECURRENCE } \\
\text { INTERVAL, IN YEARS, AND ANNUAL NON- } \\
\text { EXCEEDANCE PROBABILITY, IN PERCENT }\end{array}$}} \\
\hline SECU- & & & & & & \\
\hline TIVE & 2 & 5 & 10 & 20 & 50 & 100 \\
\hline DAYS) & $50 \%$ & $20 \%$ & $10 \%$ & $5 \%$ & $2 \%$ & $1 \%$ \\
\hline 1 & 1.1 & 7 & 6 & 5 & - & -- \\
\hline 3 & 1.1 & .8 & .6 & 5 & -- & -- \\
\hline 7 & 1.2 & .8 & .6 & .5 & -- & -- \\
\hline 14 & 1.2 & .9 & .7 & .6 & -- & -- \\
\hline 30 & 1.3 & .9 & .8 & .7 & -- & -- \\
\hline 50 & 1.5 & 1.2 & 1.1 & 1.0 & -- & -- \\
\hline 90 & 1.7 & 1.4 & 1.3 & 1.2 & -- & -- \\
\hline 120 & 1.9 & 1.7 & 1.5 & 1.4 & -- & -- \\
\hline 183 & 2.8 & 2.4 & 2.1 & 2.0 & - & -- \\
\hline
\end{tabular}

MAGNITUDE AND PROBABILITY OF ANNUAL HIGH FLOW BASED ON PERIOD OF RECORD 1964-81

\begin{tabular}{|c|c|c|c|c|c|c|}
\hline $\begin{array}{l}\text { PERIOD } \\
\text { (CON- } \\
\text { SECH- }\end{array}$ & \multicolumn{6}{|c|}{$\begin{array}{c}\text { DISCHARGE, IN CFS, FOR INDICATED RECURRENCE } \\
\text { INTERVAL, IN YEARS, AND ANNUAL } \\
\text { EXCEEDANCE PROBABILITY, IN PERCENT }\end{array}$} \\
\hline $\begin{array}{l}\text { TIVE } \\
\text { DAYS) }\end{array}$ & $20^{\circ}$ & $\begin{array}{c}5 \\
20 \%\end{array}$ & $\begin{array}{l}10 \\
10 \%\end{array}$ & $\begin{array}{l}25 \\
4 \%\end{array}$ & $\begin{array}{l}50 \\
20\end{array}$ & $\begin{array}{r}100 \\
1 \%\end{array}$ \\
\hline 1 & 602 & 1150 & 1380 & 1540 & -- & -- \\
\hline 3 & 462 & 802 & 923 & 999 & -- & -- \\
\hline 7 & 333 & 521 & 573 & 599 & -- & -- \\
\hline 15 & 228 & 336 & 363 & 376 & -- & - \\
\hline 30 & 156 & 206 & 215 & 218 & -- & -- \\
\hline 60 & 113 & 157 & 168 & 173 & -- & \\
\hline 90 & 93 & 131 & 140 & 145 & -- & -- \\
\hline
\end{tabular}

DURATION TABLE OF DAILY MEAN FLOW FOR PERIOD OF RECORD 1964-81

\begin{tabular}{|c|c|c|c|c|c|c|c|c|c|c|c|c|c|c|}
\hline & & & RGE, & CFS, & $\mathrm{CH}$ WF & QUALED & EXCE & ED FO & NDICA & PERCE & OF T & & & \\
\hline $5 \%$ & $10 \%$ & $15 \%$ & $20 \%$ & $25 \%$ & $30 \%$ & $40 \%$ & $50 \%$ & $60 \%$ & $70 \%$ & $75 \%$ & $80 \%$ & $85 \%$ & $90 \%$ & $95 \%$ \\
\hline 119 & 75 & 53 & 38 & 28 & 21 & 9.3 & 5.3 & 3.8 & 2.9 & 2.5 & 2.1 & 1.9 & 1.6 & 1.3 \\
\hline
\end{tabular}


14113400 DOG RIVER NEAR PARKDALE, OR

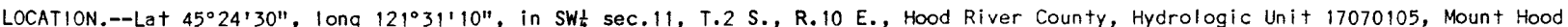
National Forest, on right bank $100 \mathrm{ft}$ upstream from city of The Dalles municipal diversion to Mill Creek basin, and $8.8 \mathrm{mi}$ south of Parkdale.

DRAINAGE AREA. $--4.50 \mathrm{mi}^{2}$.

PERIOD OF RECORD.--October 1959 to September 1971.

GAGE.--Water-stage recorder and concrete control. Datum of gage is 4,347 ft National Geodetic Vertical Datum of 1929 (levels by city of The Dalles).

REMARKS. -No regulation or diversion above station.

AVERAGE DISCHARGE. -12 years, $7.09 \mathrm{ft}^{3} / \mathrm{s}, 21.40 \mathrm{in} / \mathrm{yr}, 5,140 \mathrm{acre}-\mathrm{ft} / \mathrm{yr}$.

EXTREMES FOR PERIOD OF RECORD.- Maximum discharge, $100 \mathrm{ft} / \mathrm{s}$ May 29,1969 , gage height, $3.92 \mathrm{ft}$, from rating curve extended above 30 $\mathrm{ft}^{3} / \mathrm{s}$; minimum, $0.03 \mathrm{ft}^{3} / \mathrm{s}$ Dec. 1, 1967, result of freezeup.

STATISTICAL SUMMARIES

MONTHLY AND ANNUAL MEAN DISCHARGES 1961-71

\begin{tabular}{|c|c|c|c|c|c|c|}
\hline MONTH & $\begin{array}{l}\text { MINIMUM } \\
\text { (CFS) }\end{array}$ & $\begin{array}{l}\text { MAXIMUM } \\
\text { (CFS) }\end{array}$ & $\begin{array}{l}\text { MEAN } \\
\text { (CFS) }\end{array}$ & $\begin{array}{l}\text { STAN- } \\
\text { DARD } \\
\text { DEVIA- } \\
\text { TION } \\
\text { (CFS) }\end{array}$ & $\begin{array}{l}\text { COEFFI- } \\
\text { CIENT OF } \\
\text { VARI- } \\
\text { ATION }\end{array}$ & $\begin{array}{c}\text { PERCENT } \\
\text { OF } \\
\text { ANNUAL } \\
\text { RUNOFF }\end{array}$ \\
\hline OCTOBER & 2.1 & 3.6 & 2.5 & .4 & .17 & 2.9 \\
\hline NOVEMBER & 2.1 & 5.6 & 3.5 & 1.1 & .31 & 4.0 \\
\hline DECEMBER & 2.0 & 14 & 4.5 & 3.6 & .81 & 5.2 \\
\hline JANUARY & 2.1 & 12 & 5.5 & 2.8 & .50 & 6.3 \\
\hline FEBRUARY & 1.8 & 17 & 8.7 & 5.2 & .60 & 10.1 \\
\hline MARCH & 2.0 & 13 & 6.1 & 3.4 & .55 & 7.1 \\
\hline APRIL & 3.0 & 11 & 6.4 & 2.6 & .41 & 7.4 \\
\hline MAY & 8.4 & 40 & 16 & 8.7 & .56 & 18.1 \\
\hline JUNE & 7.5 & 36 & 18 & 8.4 & .46 & 21.1 \\
\hline JULY & 3.8 & 18 & 8.3 & 3.9 & .47 & 9.6 \\
\hline AUGUST & 2.9 & 7.2 & 4.2 & 1.2 & .29 & 4.9 \\
\hline SEPTEMBER & 2.2 & 4.6 & 2.9 & .7 & .23 & 3.4 \\
\hline ANNUAL & 4.6 & 10 & 7.2 & 1.9 & .27 & 100 \\
\hline
\end{tabular}

MAGNITUDE AND PROBABILITY OF INSTANTANEOUS PEAK FLOW BASED ON PERIOD OF RECORD $1961-71$

DISCHARGE, IN CFS, FOR INDICATED RECURRENCE INTERVAL, IN YEARS, AND ANNUAL EXCEEDANCE PROBABILITY, IN PERCENT

\begin{tabular}{ccccccc}
1.25 & 2 & 5 & 10 & 25 & 50 & 100 \\
$80 \%$ & $50 \%$ & $20 \%$ & $10 \%$ & $4 \%$ & $2 \%$ & $1 \%$ \\
\hline 24 & 36 & 55 & 70 & - & - & - \\
\hline & & & & & \\
\hline
\end{tabular}

WEIGHTED SKEW $=.300$
MAGNITUDE AND PROBABILITY OF ANNUAL LOW FLOW BASED ON PERIOD OF RECORD 1962-71

\begin{tabular}{|c|c|c|c|c|c|c|}
\hline $\begin{array}{l}\text { PERIOD } \\
\text { (CON- } \\
\text { SECI- }\end{array}$ & \multicolumn{6}{|c|}{$\begin{array}{l}\text { DISCHARGE, IN CFS, FOR INOICATED RECURRENCE } \\
\text { INTERVAL, IN YEARS, AND ANNUAL NON- } \\
\text { EXCEEDANCE PROBABILITY, IN PERCENT }\end{array}$} \\
\hline $\begin{array}{l}\text { TIVE } \\
\text { DAYS) }\end{array}$ & $\begin{array}{c}2 \\
50 \%\end{array}$ & $\begin{array}{c}5 \\
20 \%\end{array}$ & $\begin{array}{l}10 \\
10 \%\end{array}$ & $\begin{array}{l}20 \\
5 \%\end{array}$ & $\begin{array}{l}50 \\
2 \%\end{array}$ & $\begin{array}{r}100 \\
1 \%\end{array}$ \\
\hline 1 & 1.4 & .4 & .2 & .1 & -- & -- \\
\hline 3 & 1.5 & .6 & .3 & .1 & -- & -- \\
\hline 7 & 1.7 & 1.1 & .8 & .6 & -- & -- \\
\hline 14 & 1.9 & 1.5 & 1.4 & 1.2 & -- & -- \\
\hline 30 & 2.1 & 1.9 & 1.8 & 1.7 & -- & -- \\
\hline 60 & 2.2 & 2.1 & 2.0 & 2.0 & -- & - \\
\hline 90 & 2.5 & 2.2 & 2.1 & 2.0 & -- & -- \\
\hline 120 & 2.7 & 2.3 & 2.1 & 2.0 & -- & -- \\
\hline 183 & 3.2 & 2.6 & 2.4 & 2.2 & -- & - \\
\hline
\end{tabular}

MAGNITUDE AND PROBABILITY OF ANNUAL HIGH FLOW BASED ON PERIOD OF RECORD 1961-71

\begin{tabular}{|c|c|c|c|c|c|c|}
\hline $\begin{array}{l}\text { PERIOD } \\
\text { (CON- } \\
\text { SECU- }\end{array}$ & \multicolumn{6}{|c|}{$\begin{array}{c}\text { DISCHARGE, IN CFS, FOR IND ICATED RECURRENCE } \\
\text { INTERVAL, IN YEARS, AND ANNUAL } \\
\text { EXCEEDANCE PROBABILITY, IN PERCENT }\end{array}$} \\
\hline $\begin{array}{l}\text { TIVE } \\
\text { DAYS) }\end{array}$ & $\stackrel{2}{50 \%}$ & $\begin{array}{c}5 \\
20 \%\end{array}$ & $\begin{array}{l}10 \\
10 \%\end{array}$ & $\begin{array}{l}25 \\
4 \%\end{array}$ & $\begin{array}{l}50 \\
2 \%\end{array}$ & $\begin{array}{r}100 \\
1 \%\end{array}$ \\
\hline
\end{tabular}

$\begin{array}{lllllll}1 . & 31 & 46 & 58 & -- & -- & -- \\ 3 & 28 & 42 & 53 & -- & -- & -- \\ 7 & 25 & 37 & 47 & -- & -- & - \\ 15 & 22 & 32 & 41 & -- & -- & -- \\ 30 & 19 & 28 & 36 & -- & -- & -- \\ 60 & 16 & 22 & 27 & -- & -- & - \\ 90 & 13 & 18 & 22 & -- & -- & -\end{array}$

DURATION TABLE OF DAILY MEAN FLOW FOR PERIOD OF RECORD 1961-71

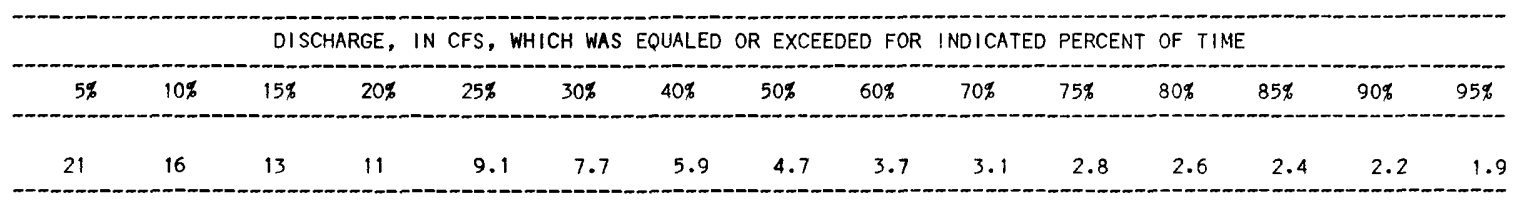


$14 \$ 18500$ WEST FORK HOOD RIVER NEAR DEE, OR

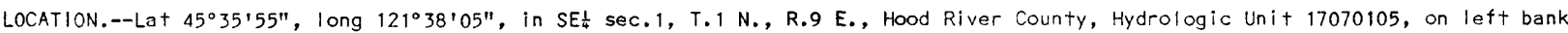
$0.3 \mathrm{mi}$ upstream from Dead Point Creek, $0.8 \mathrm{mi}$ northwest of Dee, and at mile 0.4 .

DRAINAGE AREA.---95.6 $\mathrm{mi}^{2}$.

PERIOD OF RECORD.--September 1913 to February 1916 (incomplete), June 1932 to September 1982.

GAGE.--Water-stage recorder. Datum of gage is $802.1 \mathrm{ft}$ National Geodetic Vertical Datum of 1929. Sept. 1 , 1913 , to Feb. 12, 1916, nonrecording gage at site $0.5 \mathrm{mi}$ upstream at different datum.

REMARKS.--No regulation. Dee Irrigation District canal diverts from right bank about 6 mi above station for irrigation above station and in Middle Fork basin. Diversions from Green Point Creek basin above station for irrigation near Oak Grove; water from two of these diversions is carried in Hood River Irrigation District canal.

AVERAGE DISCHARGE. --51 years (water years 1914, 1933-82), $558 \mathrm{ft}+3 / \mathrm{s}, 404,300 \mathrm{acre}-\mathrm{ft} / \mathrm{yr}$.

EXTREMES FOR PERIOD OF RECORD.--Maximum discharge not determined, Dec. 22, 1964, gage height, $27.0 \mathrm{ft} f$ rom floodmarks; maximum daily, $15,000 \mathrm{ft}^{3} / \mathrm{s}$ Dec. 23, 1964; minimum, $93 \mathrm{ft}^{3} / \mathrm{s}$ Aug. 22, 1941.

STATISTICAL SUMMARIES

MONTHLY AND ANNUAL MEAN DISCHARGES 1933-82

\begin{tabular}{|c|c|c|c|c|c|c|}
\hline MONTH & $\begin{array}{l}\text { MINIMUM } \\
\text { (CFS) }\end{array}$ & $\begin{array}{l}\text { MAXIMUM } \\
\text { (CFS) }\end{array}$ & $\begin{array}{l}\text { MEAN } \\
\text { (CFS) }\end{array}$ & $\begin{array}{l}\text { STAN- } \\
\text { DARD } \\
\text { DEVIA- } \\
\text { TION } \\
\text { (CFS) }\end{array}$ & $\begin{array}{l}\text { COEFFI- } \\
\text { CIENT OF } \\
\text { VARI- } \\
\text { ATION }\end{array}$ & $\begin{array}{c}\text { PERCENT } \\
\text { OF } \\
\text { ANNUAL } \\
\text { RUNOFF }\end{array}$ \\
\hline OCTOBER & 125 & 817 & 283 & 173 & .61 & 4.2 \\
\hline NOVEMBER & 109 & 1499 & 629 & 349 & .55 & 9.4 \\
\hline DECEMMER & 202 & 2589 & 944 & 525 & .56 & 14.1 \\
\hline JANUARY & 191 & 2362 & 858 & 483 & .56 & 12.8 \\
\hline FEBRUARY & 229 & 1918 & 785 & 370 & .47 & 11.7 \\
\hline MARCH & 386 & 1391 & 675 & 203 & .30 & 10.1 \\
\hline APRIL & 288 & 1335 & 754 & 230 & .31 & 11.2 \\
\hline MAY & 312 & 1305 & 696 & 238 & .34 & 10.4 \\
\hline JUNE & 195 & 1132 & 477 & 219 & .46 & 7.1 \\
\hline JULY & 140 & 496 & 260 & 86 & .33 & 3.9 \\
\hline AUGUST & 109 & 267 & 178 & 38 & .22 & 2.7 \\
\hline SEPTEMBER & 109 & 378 & 172 & 46 & .26 & 2.6 \\
\hline ANNUAL & 267 & 837 & 558 & 123 & .22 & 100 \\
\hline
\end{tabular}

MAGNITUDE AND PROBABILITY OF INSTANTANEOUS PEAK FLOW BASED ON PERIOD OF RECORD 1933-82

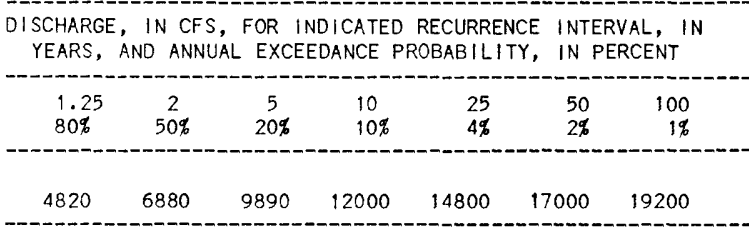

WEIGHTED SKEW $=.091$

MAGNITUDE AND PROBABILITY OF ANNUAL LOW FLOW BASED ON PERIOD OF RECORD 1934-82

\begin{tabular}{|c|c|c|c|c|c|c|}
\hline \multirow{2}{*}{$\begin{array}{l}\text { PERIOD } \\
\text { (CON- } \\
\text { SECU- } \\
\text { TIVE } \\
\text { DAYS) }\end{array}$} & \multicolumn{6}{|c|}{$\begin{array}{l}\text { DISCHARGE, IN CFS, FOR IND ICATED RECURRENCE } \\
\text { INTERVAL, IN YEARS, AND ANNUAL NON- } \\
\text { EXCEEDANCE PROBABILITY, IN PERCENT }\end{array}$} \\
\hline & $\stackrel{2}{50 \%}$ & $\begin{array}{c}5 \\
20 \%\end{array}$ & $\begin{array}{l}10 \\
10 \%\end{array}$ & $\begin{array}{l}20 \\
5 \%\end{array}$ & $\begin{array}{l}50 \\
2 \%\end{array}$ & $\begin{array}{r}100 \\
1 \%\end{array}$ \\
\hline 1 & 130 & 115 & 107 & 102 & 96 & 92 \\
\hline 3 & 132 & 116 & 109 & 103 & 97 & 93 \\
\hline 7 & 134 & 118 & 110 & 104 & 98 & 94 \\
\hline 14 & 139 & 121 & 113 & 107 & 100 & 96 \\
\hline 30 & 145 & 126 & 117 & 109 & 102 & 97 \\
\hline 60 & 157 & 134 & 123 & 115 & 107 & 102 \\
\hline 90 & 169 & 142 & 130 & 122 & 113 & 108 \\
\hline 120 & 190 & 155 & 141 & 131 & 121 & 116 \\
\hline 183 & 268 & 211 & 187 & 171 & 155 & 145 \\
\hline
\end{tabular}

MAGNITUDE AND PROBABILITY OF ANNUAL HIGH FLOW BASED ON PERIOD OF RECORD 1933-82

\begin{tabular}{ccccccc} 
PERIOD & \multicolumn{5}{c}{ DISCHARGE, IN CFS, FOR INDICATED RECURRENCE } \\
INTERVAL, IN YEARS, AND ANNUAL \\
ICON-
\end{tabular}

DURATION TABLE OF DAILY MEAN FLOW FOR PERIOD OF RECORD 1933-82

DISCHARGE, IN CFS, WHICH WAS EQUALED OR EXCEEDED FOR INDICATED PERCENT OF TIME

\begin{tabular}{|c|c|c|c|c|c|c|c|c|c|c|c|c|c|c|}
\hline $5 \%$ & $10 \%$ & $15 \%$ & $20 \%$ & $25 \%$ & $30 \%$ & $40 \%$ & $50 \%$ & $60 \%$ & $70 \%$ & $75 \%$ & $80 \%$ & $85 \%$ & $90 \%$ & $95 \%$ \\
\hline 1490 & 1090 & 894 & 766 & 683 & 612 & 494 & 403 & 316 & 238 & 210 & 188 & 168 & 150 & 133 \\
\hline
\end{tabular}


14120000 HOOD RIVER AT TUCKER BRIDGE, NEAR HOOD RIVER, OR

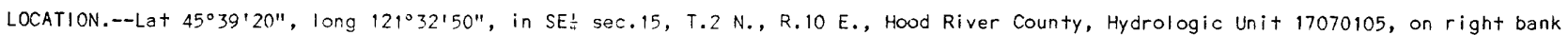
$25 \mathrm{ft}$ downstream from Tucker Bridge, $0.5 \mathrm{mi}$ upstream from Odell Creek, $4.0 \mathrm{mi}$ southwest of town of Hood River, and at mile 6.1 .

DRAINAGE AREA.--279 $\mathrm{mi}^{2}$.

PERIOD OF RECORD.--October 1897 to December 1899, September 1913 to September 1914, August 1915 to September 1917 , January 1965 to September 1982. Monthly discharge only for some periods, published in WSP 1318.

GAGE.--Water-stage recorder and crest-stage gage. Datum of gage is 383.2 tt National Geodetic Vertical Datum of 1929 (Oregon State Highway Department bench mark). Prior to July 23, 1915, nonrecording gage at bridge at various datums. July 23 to Dec. 21 , 1915, water-stage recorder at site $0.8 \mathrm{mi}$ upstream 3 t different datum. January 1916 to September 1917 , nonrecording gage at bridge at different datum. Jan. 15 to July 23,1965 , vonrecording gage at bridge.

REMARKS.--Some daily fluctuation caused by diversion dam above station and sawmill at Dee. Diversions for irrigation above station. AVERAGE DISCHARGE.--22 years (water years 1898-99, 1914, 1916-17, 1966-82), 1,089 $\mathrm{ft} / \mathrm{s}, 789,000 \mathrm{acre}-\mathrm{ft}+\mathrm{yr}$.

EXTREMES FOR PERIOD OF RECORD.--Maximum discharge, 22,300 $\mathrm{ft}^{3} / \mathrm{s} \mathrm{Dec} .13,1977$, gage height, $15.59 \mathrm{ft}$; minimum recorded, $136 \mathrm{ft} / \mathrm{s}$ Sept. 16, 1915, caused by temporary storage behind dam at Dee.

EXTREMES OUTSIOE PERIOD OF RECORO. - Flood of Dec. 22, 1964, reached a stage of $20.6 \mathrm{ft}$, present datum, discharge, $33,200 \mathrm{ft} 3 / \mathrm{s}$, from rating curve extended above $1,500 \mathrm{ft}^{3} / \mathrm{s}$ on basis of slope-area measurement of peak flow.

\section{STATISTICAL SUMMARIES}

MONTHLY AND ANNUAL MEAN DISCHARGES 1898-82

\begin{tabular}{|c|c|c|c|c|c|c|}
\hline MONTH & $\begin{array}{l}\text { MINIMUM } \\
\text { (CFS) }\end{array}$ & $\begin{array}{l}\text { MAXIMUM } \\
\text { (CFS) }\end{array}$ & $\begin{array}{l}\text { MEAN } \\
\text { (CFS) }\end{array}$ & $\begin{array}{l}\text { STAN- } \\
\text { DARD } \\
\text { DEVIA- } \\
\text { TION } \\
\text { (CFS) }\end{array}$ & $\begin{array}{l}\text { COEFFI- } \\
\text { CIENT OF } \\
\text { VARI- } \\
\text { ATION }\end{array}$ & $\begin{array}{c}\text { PERCENT } \\
\text { OF } \\
\text { ANNUAL } \\
\text { RUNOFF }\end{array}$ \\
\hline OCTOBER & 327 & 929 & 499 & 169 & .34 & 3.8 \\
\hline NOVEMBER & 431 & 1989 & 1043 & 488 & .47 & 7.9 \\
\hline DECEMBER & 438 & 4109 & 1663 & 931 & .56 & 12.6 \\
\hline JANUARY & 363 & 3313 & 1722 & 819 & .48 & 13.1 \\
\hline FEBRUARY & 430 & 3367 & 1657 & 774 & .47 & 12.6 \\
\hline MARCH & 681 & 2915 & 1352 & 532 & .39 & 10.3 \\
\hline APRIL & 704 & 2358 & 1317 & 431 & .33 & 10.0 \\
\hline $\begin{array}{l}\text { MAY } \\
\text { JUNE }\end{array}$ & $\begin{array}{l}559 \\
464\end{array}$ & $\begin{array}{l}2418 \\
2439\end{array}$ & $\begin{array}{l}1298 \\
1059\end{array}$ & $\begin{array}{l}496 \\
564\end{array}$ & $\begin{array}{l}.38 \\
.53\end{array}$ & $\begin{array}{l}9.9 \\
8.0\end{array}$ \\
\hline JULY & 256 & 1687 & 674 & 385 & .57 & 5.1 \\
\hline AUGUST & 266 & 1088 & 448 & 193 & .43 & 3.4 \\
\hline SEPTEMBER & 271 & 804 & 424 & 125 & .29 & 3.2 \\
\hline ANNUAL & 465 & 1664 & 1090 & 309 & .28 & 100 \\
\hline
\end{tabular}

MAGNITUDE ANO PROBABILITY OF INSTANTANEOUS PEAK FLOW BASED ON PERIOD OF RECORO 1898-82

\begin{tabular}{lcccccc}
\hline DISCHARGE, IN CFS, FOR INDICATEO RECURRENCE INTERVAL, IN \\
YEARS, AND ANNUAL EXCEEDANCE PROBABILITY, IN PERCENT \\
\hline 1.25 & 2 & 5 & 10 & 25 & 50 & 100 \\
$80 \%$ & $50 \%$ & $20 \%$ & $10 \%$ & $4 \%$ & $2 \%$ & $1 \%$ \\
\hline 7290 & 11800 & 18500 & 23100 & 29200 & 33800 & - \\
\hline
\end{tabular}

WEIGHTEO SKEW $=-.209$
MAGNITUDE AND PROBABILITY OF ANNUAL LOW FLOW BASED ON PERIOD OF RECORO 1899-82

\begin{tabular}{|c|c|c|c|c|c|c|}
\hline $\begin{array}{l}\text { PERIOD } \\
\text { ICON- } \\
\text { SECU- }\end{array}$ & \multicolumn{6}{|c|}{$\begin{array}{l}\text { DISCHARGE, IN CFS, FOR INDICATED RECURRENCE } \\
\text { INTERVAL, IN YEARS, AND ANNUAL NON- } \\
\text { EXCEEDANCE PROBABILITY, IN PERCENT }\end{array}$} \\
\hline $\begin{array}{l}\text { TIVE } \\
\text { DAYS) }\end{array}$ & $\stackrel{2}{50 \%}$ & $\begin{array}{c}5 \\
20 \%\end{array}$ & $\begin{array}{l}10 \\
10 \%\end{array}$ & $\begin{array}{l}20 \\
5 \%\end{array}$ & $\begin{array}{l}50 \\
2 \%\end{array}$ & $\begin{array}{r}100 \\
1 \%\end{array}$ \\
\hline 1 & 269 & 223 & 206 & 194 & 183 & - \\
\hline 3 & 273 & 228 & 211 & 200 & 190 & - \\
\hline 7 & 282 & 239 & 223 & 212 & 202 & - \\
\hline 14 & 297 & 252 & 235 & 224 & 213 & - \\
\hline 30 & 312 & 266 & 248 & 237 & 226 & - \\
\hline 60 & 338 & 287 & 267 & 255 & 243 & -- \\
\hline 90 & 363 & 310 & 292 & 279 & 268 & -- \\
\hline 120 & 400 & 340 & 318 & 304 & 291 & -- \\
\hline 183 & 520 & 430 & 394 & 369 & 345 & -- \\
\hline
\end{tabular}

MAGNITUDE AND PROBABILITY OF ANNUAL HIGH FLOW BASED ON PERIOD OF RECORD 1898-82

\begin{tabular}{|c|c|c|c|c|c|c|}
\hline \multirow{3}{*}{$\begin{array}{l}\text { PERIOD } \\
\text { (CON- } \\
\text { SECU- } \\
\text { TIVE } \\
\text { DAYS) }\end{array}$} & \multicolumn{6}{|c|}{$\begin{array}{c}\text { DISCHARGE, IN CFS, FOR INDICATED RECURRENCE } \\
\text { INTERVAL, IN YEARS, AND ANNUAL } \\
\text { EXCEEDANCE PROBAB!LITY, IN PERCENT }\end{array}$} \\
\hline & 2 & 5 & 10 & 25 & 50 & 100 \\
\hline & $50 \%$ & $20 \%$ & $10 \%$ & $4 \%$ & $2 \%$ & $1 \%$ \\
\hline 1 & 8690 & 13100 & 15300 & 17300 & 18400 & - \\
\hline 3 & 6420 & 9400 & 10900 & 12300 & 13000 & -- \\
\hline 7 & 4780 & 6760 & 7710 & 8580 & 9050 & -- \\
\hline 15 & 3490 & 4750 & 5360 & 5920 & 6240 & - \\
\hline 30 & 2780 & 3590 & 3940 & 4240 & 4390 & -- \\
\hline 60 & 2270 & 2850 & 3090 & 3300 & 3400 & \\
\hline 90 & 2010 & 2460 & 2640 & 2780 & 2850 & -- \\
\hline
\end{tabular}

DURATION TABLE OF DAILY MEAN FLOW FOR PERIOD OF RECORD $1898-82$

DISCHARGE, IN CFS, WHICH WAS EQUALEO OR EXCEEDED FOR INDICATED PERCENT OF TIME

\begin{tabular}{|c|c|c|c|c|c|c|c|c|c|c|c|c|c|c|}
\hline $5 \%$ & $10 \%$ & $15 \%$ & $20 \%$ & $25 \%$ & $30 \%$ & $40 \%$ & $50 \%$ & $60 \%$ & $70 \%$ & $75 \%$ & $80 \%$ & $85 \%$ & $90 \%$ & $95 \%$ \\
\hline 2660 & 2060 & 1740 & 1510 & 1350 & 1220 & 987 & 829 & 685 & 540 & 475 & 426 & 383 & 341 & 298 \\
\hline
\end{tabular}


STATION NO.

13330500

14078000

15061000

14025000

10397000

14054500

13226500

13227000

13275000

13274200

13273000

14032000

10370000

14042000

14042500

13320000

10384000

11340500

14060000

14087400

14087500

14080500

14079500

14051000

14050500

10371500

14052000

14064500

14093500

14103000

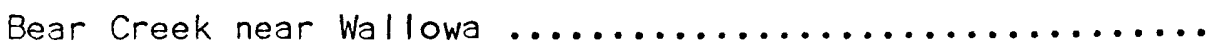

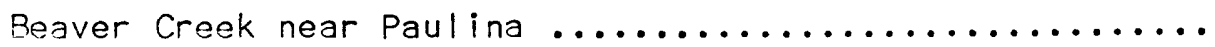

Big Marsh Creek at Hoey Ranch, near Crescent ............

Birch Creek at Rieth

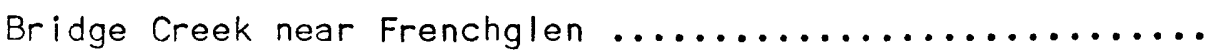

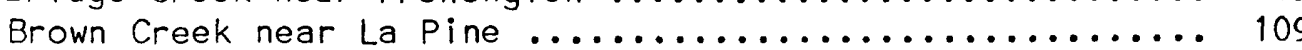

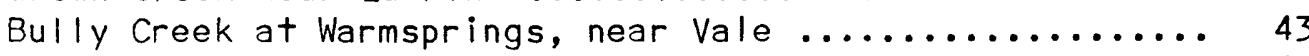

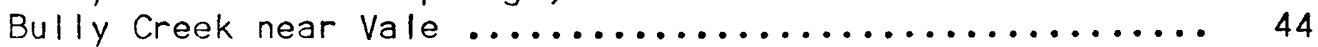

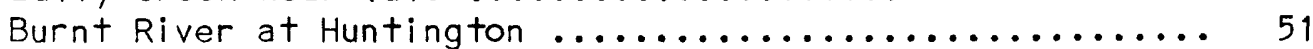

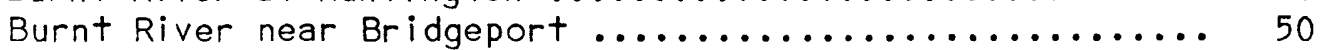

Burnt River near Hereford:

before the construction of Unity Dam ............. 48

after the completion of Unity Dam .................. 49

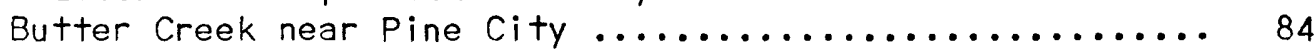

Camas Creek near Lakeview ......................... 12

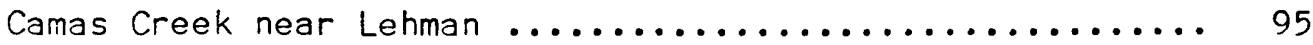

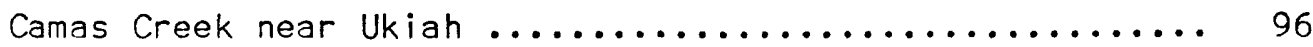

Catherine Creek near Union:

before the construction of Big Creek Diversion ........ 62

after the completion of Big Creek Diversion ........... 63

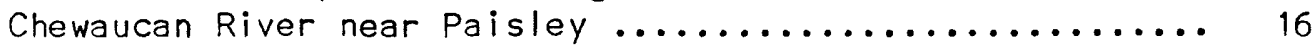

Cottonwood Creek near Lakeview:

before the construction of Cottonwood Dam ............ 23

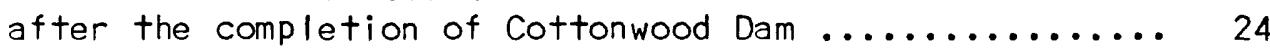

Crescent Creek at Crescent Lake, near Crescent .......... 115

Crooked River below Opal Springs, near Culver .......... 130

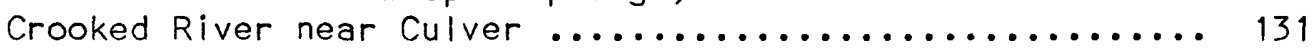

Crooked River near Prineville:

before the construction of Prineville Dam ........... 128

after the completion of Prineville Dam ................. 129

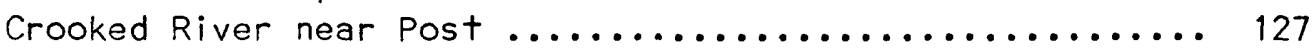

Cultus Creek above Crane Prairie Reservoir, near La Pine:

before the installation of fish screens ............... 104

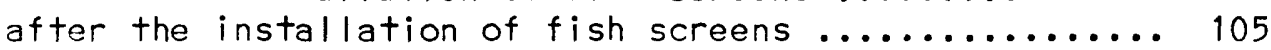

Cultus River above Cultus Creek, near La Pine .......... 103

Deep Creek above Adel ............................ 14

Deer Creek above Crane Prairie Reservoir, near La Pine ... 106

Deschutes River at Benham Falls, near Bend:

before the construction of Wickiup Dam .............. 118

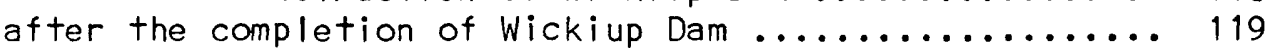

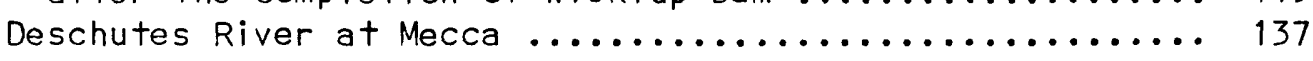

Deschutes River at Moody, near Biggs:

before the construction of upstream reservoirs ........ 141

after the completion of upstream reservoirs ........... 142

\section{9}

25

16

81

19

9

43

4

0

8

9

84

12

5

96

62

3

6

23

24

30

28

27

4

103

4

8


STATION NO.

14057000 Deschutes River at Pringle Falls, near La Pine:

before the construction of Wickiup Dam ............ 112

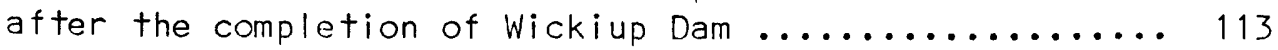

14070500

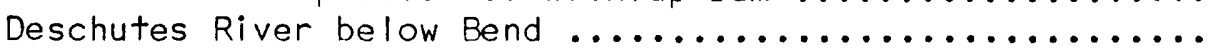

122

14054000

Deschutes River below Crane Prairie Reservoir, nr La Pine.

108

14056500

Deschutes River below Wickiup Reservoir, near La Pine ....

111

14066000

14050000

Deschutes River below Lava Island, near Bend:

before the construction of Wickiup Dam ............. 120

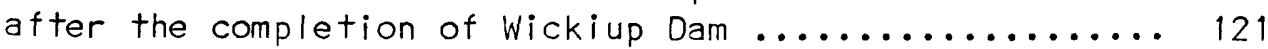

Deschutes River below Snow Creek, near La Pine ......... 102

14076500

14092500

Deschutes River near Culver

Deschutes River near Madras:

before the construction of Round Butte Dam ........... 134

after the completion of Round Butte Dam ............. 135

14113400

10396000

10371000

11339500

Dog River near Parkdale.

144

Donner und Blitzen River near Frenchglen ................ 18

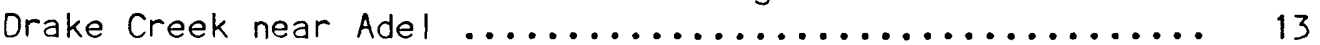

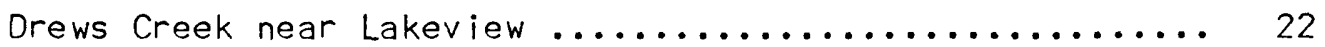

13288200 Eagle Creek above Skull Creek, near New Bridge ........ 56

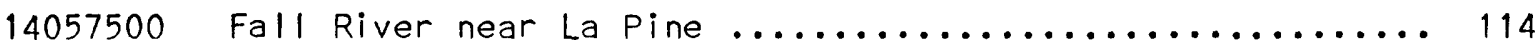

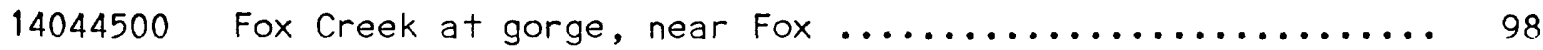

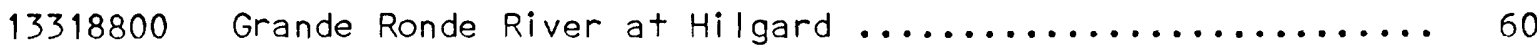

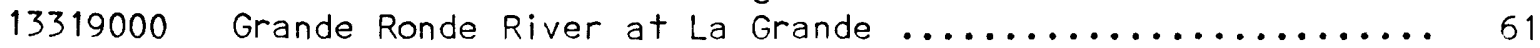

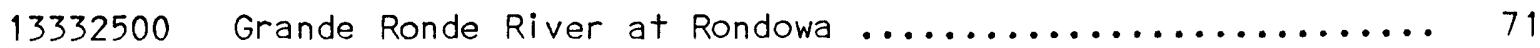

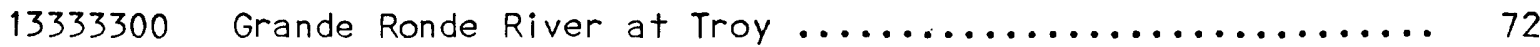

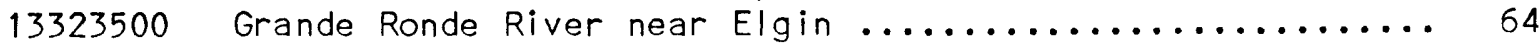

13318500 Grande Ronde River near Hilgard .................. 59

10378500 Honey Creek near Plush ........................... 15

14120000 Hood River at Tucker Bridge, near Hood River ........... 146

13329500 Hurricane Creek near Joseph ...................... 67

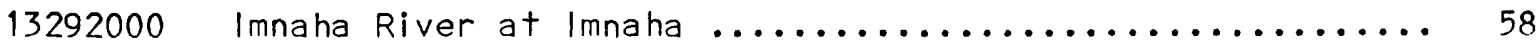

13323600 Indian Creek near Imbler ........................... 65

14048000 John Day River at McDonald Ferry ................... 101

14040500 John Day River at Picture Gorge, near Dayville ......... 93

John Day River at Prairie City:
during power canal diversion .................... 90

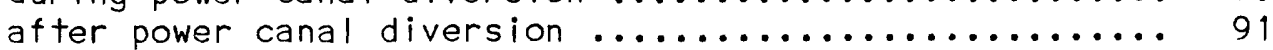

14046500 John Day River at Service Creek .................... 100

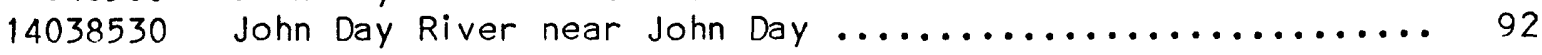

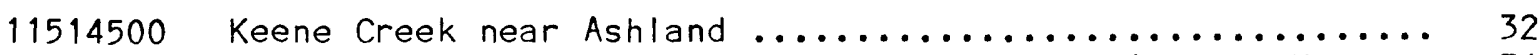

$11510700 \quad$ Klamath River below John C. Boyle Powerplant, near Keno... 31

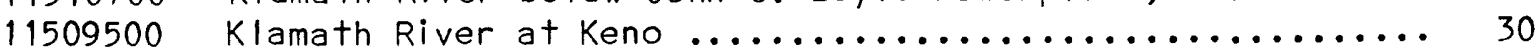


STATION NO.

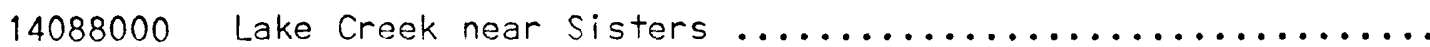

132

14063000

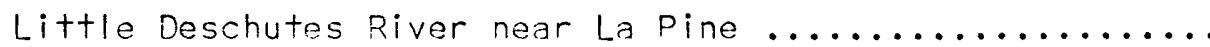

117

13330000

Lostine River near Lostine...

68

13220000

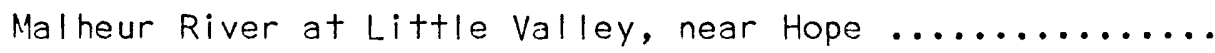

13228000

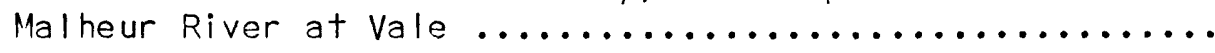

13215000

Mal heur River below Warmsprings Reservoir, near Riverside.

13214000

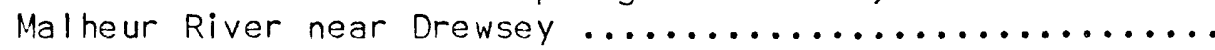

13220500

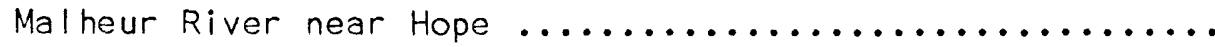

14022500

14091500

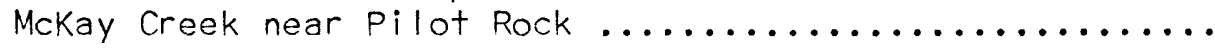

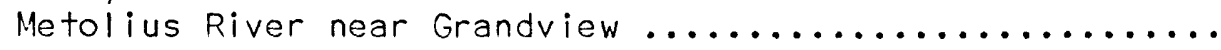

14044000

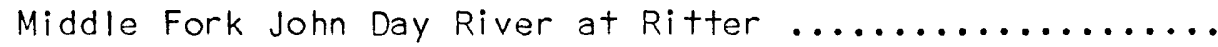

13331500

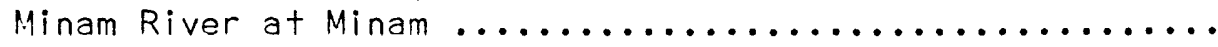

14113200

Mosier Creek near Mosier

41

45

38

37

42

80

133

97

70

143

13269300

North Fork Burnt River near Whitney ................ 46

14078500

North Fork Crooked River above Deep Creek ...............

126

14046000

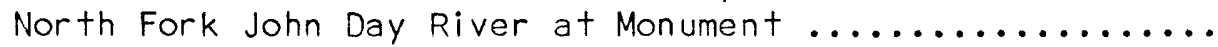

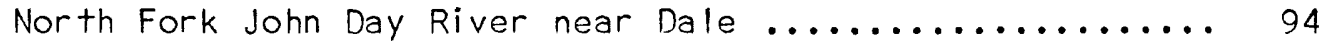

14041500

North Fork Mal heur River above Beulah Res. near Beulah ...

13217500

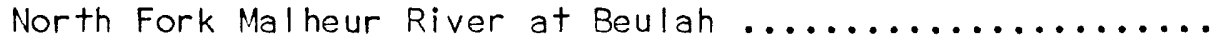

14010800

North Fork Walla Walla River near Milton Freewater .......

14011000

North Fork Walla Walla River near Milton

39

40

75

76

14055500

Odell Creek near Crescent

110

13182000

13184000

13183000

13181000

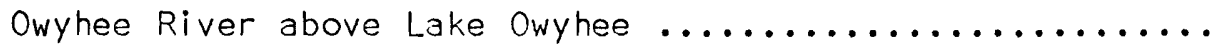

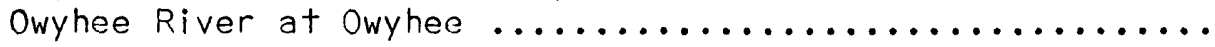

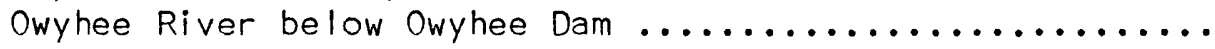

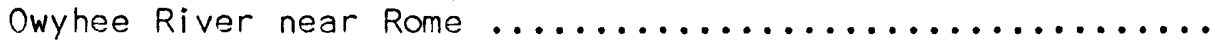

13275500

13286700

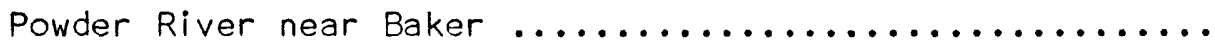

Powder River near Richland:

before the construction of Phillips Dam ............ 54

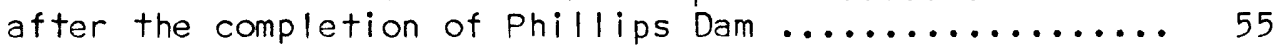

13289500

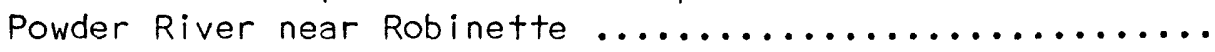

13275300

Powder River near Sumpter

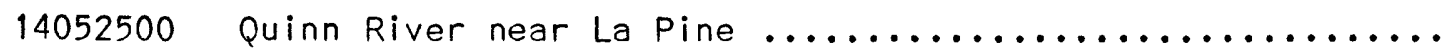

14034800

Rhea Creek near Heppner

14093000

Shitike Creek at Warmsprings

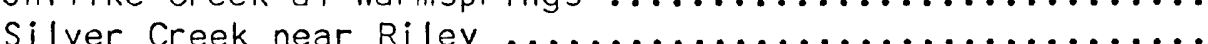

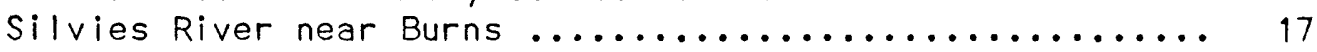

13270800

South Fork Burnt River above Barney Creek, near Unity .... 
STATION NO.

PAGE

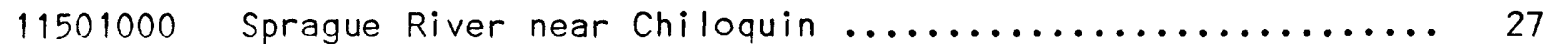

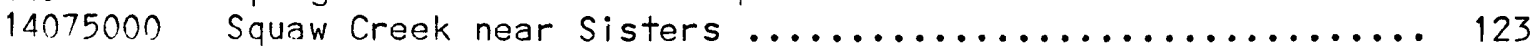

14037500 Strawberry Creek above Slide Creek, near Prairie City .... 89

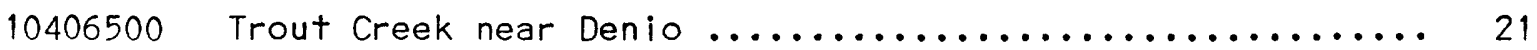

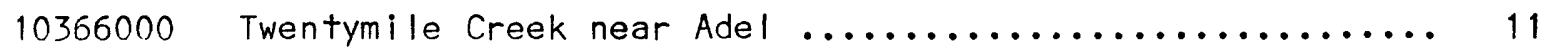

$14022000 \quad$ Umatilla River above McKay Creek, near Pendleton ....... 79

14020000 Umatilla River above Meacham Creek, near Gibbon ........ 77

$14021000 \quad$ Umatilla River at Pendleton .................... 78

14026000 Umatilla River at Yoakum:

before the construction of McKay Dam ............. 82

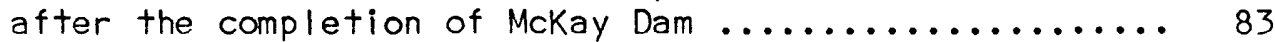

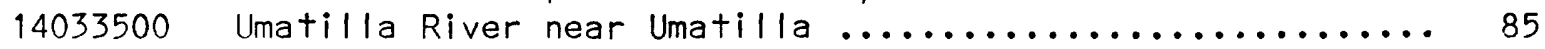

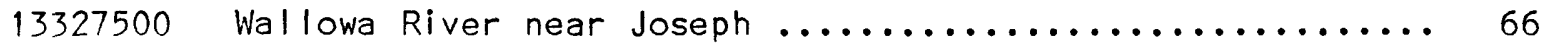

14097100 Warm Springs River near Kahneeta Hot Springs .......... 138

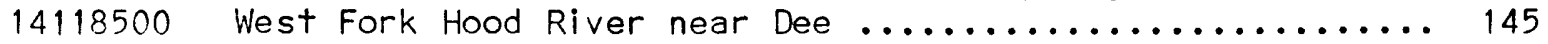

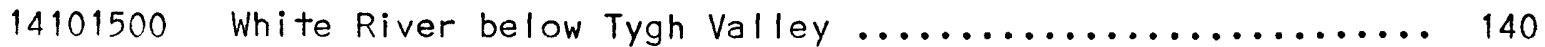

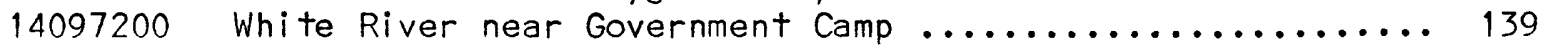

11502500 Williamson River below Sprague River, near Chiloquin ..... 28

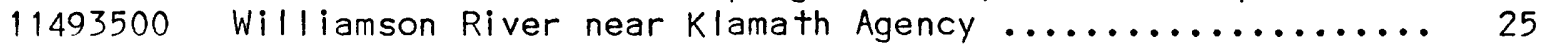

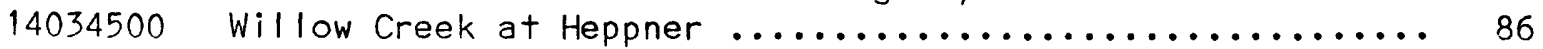

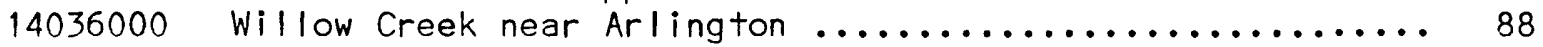

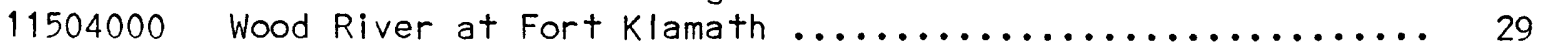

